Bernhard Manzke

\title{
Kinderlastenausgleich versus verstärkte Einwanderung
}

Alternative Ansätze zur langfristigen Sicherung der Gesetzlichen Rentenversicherung 


\section{Kinderlastenausgleich versus verstärkte Einwanderung}

Die Arbeit befaßt sich mit den Finanzierungsproblemen, die durch die prognostizierte Bevölkerungsentwicklung für die Gesetzliche Rentenversicherung entstehen. Zunächst wird herausgearbeitet, daß nur Instrumente, die an den demographischen Ursachen der Finanzierungsprobleme ansetzen, eine wirkliche Reduzierung der Lasten bewirken können. Hier bieten sich eine Erhöhung des Kinderlastenausgleichs und eine verstärkte Einwanderung an. Als Ergebnis einer Wirkungsanalyse des Kinderlastenausgleichs auf der Basis der neoklassischen Fertilitätstheorie wird eine Aufstockung der Mittel um gut 70.000 DM pro Kind für erforderlich gehalten, um den Beitragssatz im Jahr 2030 gegenüber einem laissez-faire Szenario um 1,5 Prozentpunkte zu senken. Durch eine verstärkte Einwanderung ließe sich die gleiche Beitragssatzreduzierung kostengünstiger erreichen.

Bernhard Manzke wurde 1966 in Lübeck geboren. Von 1987 bis 1992 studierte er Volkswirtschaftslehre an der Universität Göttingen und an der University of California, Los Angeles. Seit 1992 ist er als wissenschaftlicher Mitarbeiter am Lehrstuhl für Volkswirtschaftslehre/ Finanzwissenschaft der Universität Jena angestellt. Das im Rahmen dieser Tätigkeit begonnene Promotionsvor haben schloß er im Sommer $1996 \mathrm{ab.}$ 
Kinderlastenausgleich versus verstärkte Einwanderung 


\section{FINANZWISSENSCHAFTLICHE SCHRIFTEN}

Herausgegeben von den Professoren

Albers, Krause-Junk, Littmann, Oberhauser, Pohmer, Schmidt

Band 79

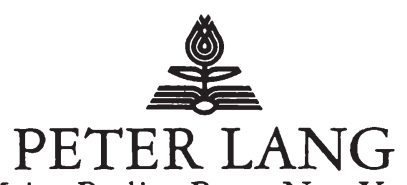

Frankfurt am Main · Berlin · Bern · New York · Paris - Wien

Bernhard Manzke - 978-3-631-75262-3

Downloaded from PubFactory at 01/11/2019 06:34:40AM

via free access 


\section{Bernhard Manzke}

\section{Kinderlastenausgleich versus verstärkte Einwanderung}

Alternative Ansätze zur langfristigen Sicherung der Gesetzlichen Rentenversicherung

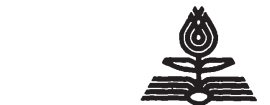

\section{PETER LANG}

Europäischer Verlag der Wissenschaften 
Die Deutsche Bibliothek - CIP-Einheitsaufnahme

Manzke, Bernhard:

Kinderlastenausgleich versus verstärkte Einwanderung :

alternative Ansätze zur langfristigen Sicherung der Gesetzlichen Rentenversicherung / Bernhard Manzke. - Frankfurt am Main ; Berlin ; Bern ; New York ; Paris ; Wien : Lang, 1997

(Finanzwissenschaftliche Schriften ; Bd. 79)

ISBN 3-631-31195-8

NE: GT

Open Access: The online version of this publication is published on www.peterlang.com and www.econstor.eu under the international Creative Commons License CC-BY 4.0. Learn more on how you can use and share this work: http://creativecommons.org/ licenses/by/4.0.

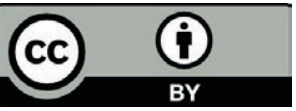

This book is available Open Access thanks to the kind support of ZBW - Leibniz-Informationszentrum Wirtschaft.

\author{
ISSN 0170-8252 \\ ISBN 3-631-31195-8 \\ ISBN 978-3-631-75262-3 (eBook) \\ (C) Peter Lang GmbH \\ Europäischer Verlag der Wissenschaften \\ Frankfurt am Main 1997 \\ Alle Rechte vorbehalten.
}

Das Werk einschließlich aller seiner Teile ist urheberrechtlich geschützt. Jede Verwertung außerhalb der engen Grenzen des

Urheberrechtsgesetzes ist ohne Zustimmung des Verlages unzulässig und strafbar. Das gilt insbesondere für

Vervielfältigungen, Übersetzungen, Mikroverfilmungen und die Einspeicherung und Verarbeitung in elektronischen Systemen.

Printed in Germany 123457 


\section{Vorwort}

Das vorliegende Buch ist eine leicht überarbeitete Fassung einer Arbeit, die im Juni 1996 von der wirtschaftswissenschaftlichen Fakultät der Friedrich-Schiller-Universität als Dissertation angenommen wurde. Es entstand während meiner Tätigkeit als wissenschaftlicher Mitarbeiter am Lehrstuhl für Volkswirtschaftslehre/Finanzwissenschaft der Friedrich-Schiller-Universität Jena.

Meinem Doktorvater Herrn Prof. Dr. Rupert Windisch danke ich für die Freiheit, die er mir bei der Themenwahl ließ, und für die intensive Betreuung und Unterstützung besonders während der Endphase der Entstehungszeit. Profitiert hat das Werk aber auch von anregenden Diskussionen mit Kollegen und Freunden. Danken möchte ich insbesondere Frau Dipl.-Vw. Antje Stobbe, Herm Dr. Michael Schubert, Herrn Dr. Lambert Koch und Herrn Dipl.-Kfm. Oliver Rokohl, die sich der Mühe unterzogen haben, Teile des Manuskripts zu lesen und einer konstruktiven Kritik zu unterwerfen, sowie vor allem meiner Frau, Dipl.-Vw. Elisabeth Andreae, auf deren fachlichen Rat und persönliche Unterstützung ich während des langwierigen Entstehungsprozesses dieser Arbeit stets zählen durfte. In schwierigen computer-technischen Fragen ist mir v.a. Herr Andreas Schumann eine zuverlässige Hilfe gewesen. Alle noch verbliebenen Fehler und Mängel gehen selbstverständlich zu meinen Lasten. 
Bernhard Manzke - 978-3-631-75262-3

Downloaded from PubFactory at 01/11/2019 06:34:40AM

via free access 


\section{Inhaltsübersicht}

Teil A: Einleitung

Teil B: Die demographische Krise der Gesetzlichen Rentenversicherung

1. Der Generationenvertrag in der Gesetzlichen Rentenversicherung

2. Empirische Entwicklung

3. Lösungsansätze für die demographische Krise des Systems der Gesetzlichen Rentenversicherung

4. Zusammenfassung

Teil C: Der Kinderlastenausgleich als Instrument zur Stabilisierung der Gesetzlichen Rentenversicherung

1. Einleitung

2. Erläuterung und Kritik verschiedener Ansätze zur Erklärung des generativen Verhaltens

3. Die neoklassische Theorie des generativen Verhaltens.

4. Versuch einer Abschätzung der Kosteneffizienz des Kinderlastenausgleichs

5. Zusammenfassung

Teil D: Verstärkte Einwanderung als Instrument zur Stabilisierung der Gesetzlichen Rentenversicherung

1. Einleitung und Begriffsklärung.

2. Theoretische Analyse der Zuwanderung

3. Kosten-Nutzen-Analyse der Einwanderung

4. Die Kosteneffizienz einer verstärkten Einwanderung

5. Zusammenfassung und Schlußfolgerung....

Teil E: Abschließender Vergleich von Kinderlastenausgleich und verstärkter Einwanderung 
Bernhard Manzke - 978-3-631-75262-3

Downloaded from PubFactory at 01/11/2019 06:34:40AM

via free access 


\section{Inhaltsverzeichnis}

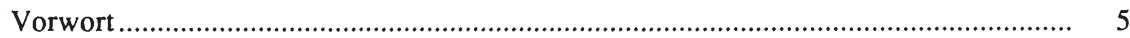

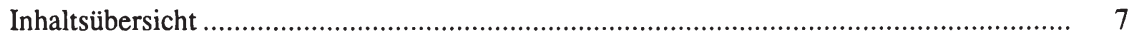

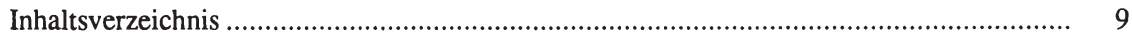

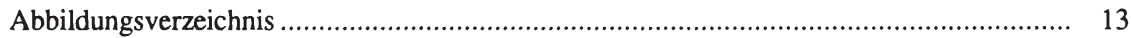

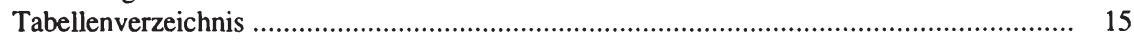

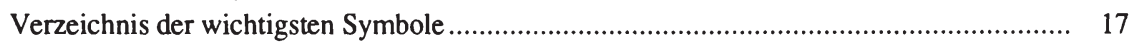

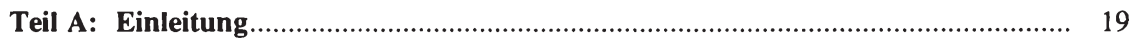

Teil B: Die demographische Krise der Gesetzlichen Rentenversicherung................. 21

1. Der Generationenvertrag in der Gesetzlichen Rentenversicherung ........................... 21

1.1 Grundtypen von Alterssicherungssystemen ......................................... 21

1.2 Die Entwicklung des Generationenvertrags in der Gesetzlichen Rentenversicherung .............................................................................. 22

1.3 Fundamentalarithmetik umlagefinanzierter Alterssicherungssysteme............... 28

2. Empirische Entwicklung ...................................................................................... 32

2.1 Der Einfluß demographischer Variablen auf die Finanzlage der Gesetzlichen Rentenversicherung ..................................................................... 33

2.1.1 Die Mortalitätsentwicklung ….............................................. 33

2.1.2 Die Fertilitätsentwicklung...................................................... 36

2.1.3 Die Entwicklung der Wanderungsbewegungen .......................... 41

2.1.4 Bevölkerungsprognosen ............................................................ 43

2.2 Der Einfluß anderer Variablen auf die Finanzlage der Gesetzlichen Rentenversicherung................................................................................. 48

2.3 Simulationsrechnungen zur langfristigen Finanzierbarkeit der Gesetzlichen

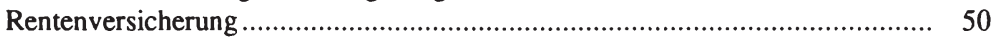

3. Lösungsansätze für die demographische Krise des Systems der Gesetzlichen

Rentenversicherung

3.1 Lösungsansätze innerhalb des Systems der Gesetzlichen Rentenversicherung.

3.1.1 Anrechnung von Kindererziehungszeiten in der Gesetzlichen Rentenversicherung ............................................................. 55

3.1.2 Verlängerung der durchschnittlichen Lebensarbeitszeit ................. 57

3.2 Lösungsansätze durch Alternativen zum System der Gesetzlichen Rentenversicherung ..................................................................... 58

3.2.1 Grundrentenvorschläge ..................................................... 58

3.2.2 Übergang zum Kapitaldeckungsverfahren.................................. 60

3.2.3 Elternrente .......................................................................... 64

3.3 Lösungsansätze durch bevölkerungspolitische Maßnahmen.......................... 67

3.3.1 Die zukünftige Entwicklung bei erhöhter Fertilität....................... 67

3.3.2 Die zukünftige Entwicklung bei verstärkter Einwanderung ............. 69

4. Zusammenfassung...... 
Teil C: Der Kinderlastenausgleich als Instrument zur Stabilisierung der Gesetzlichen Rentenversicherung ......................................................... 77

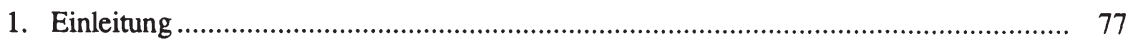

2. Erläuterung und Kritik verschiedener Ansätze zur Erklärung des generativen Verhaltens

2.1 Zur Rechtfertigung einer ökonomischen Analyse des generativen

Verhaltens..... 80

2.2 Die historische Entwicklung vor Malthus................................................ 82

2.3 Die Position von Malthus ........................................................................... 83

2.4 Die weitere Entwicklung bis 1957 ........................................................ 84

2.5 Soziologische und Demographische Ansätze ........................................... 85

2.6 Sozio-ökonomische Ansätze ................................................................. 88

3. Die neoklassische Theorie des generativen Verhaltens........................................... 92

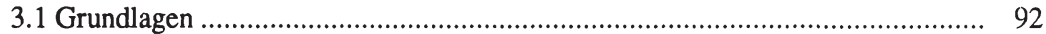

3.1.1 Anwendung der traditionellen mikroökonomischen Theorie auf die Fertilitätsentscheidung .................................................... 92

3.1.2 Erweiterung um die New Home Economics.................................... 97

Mathematischer Anhang .......................................................... 102

3.2 Fertilitätsentscheidung im Rahmen des Modells ........................................... 105

3.2.1 Die Opportunitätskosten des Kinderaufziehens ............................. 106

3.2.1.1 Theoretische Analyse der Wirkung eines steigenden Frauenlohnsatzes......................................................... 107

3.2.1.2 Empirische Größenordnung der Opportunitätskosten ........ 110

3.2.1.3 Bedeutung der Opportunitätskosten von Kindern für die Wirkungsweise des Kinderlastenausgleichs ................ 127

3.2.2 Der Zusammenhang zwischen Quantität und Qualität von Kindern ............................................................................. 128

3.2.2.1 Theoretische Analyse des Zusammenhangs zwischen Quantität und Qualität von Kindern.....

3.2.2.2 Empirische Bedeutung der Substitution von Kinderquantität durch Kinderqualität.

3.2.2.3 Bedeutung des Zusammenhangs zwischen Kinder quantität und -qualität für die Wirkungsweise des Kinderlastenausgleichs

3.2.3 Die direkten Kosten von Kindern.

Kinderkosten .

3.2.3.2 Empirische Größenordnung der direkten Kinderkosten...... 138

3.2.3.3 Bedeutung der direkten Kinderkosten für den Kinderlastenausgleich ........................................................... 153

3.2.4 Zusammenfassung ............................................................ 155

4. Versuch einer Abschätzung der Kosteneffizienz des Kinderlastenausgleichs ................ 156

4.1 Theoretische Überlegungen zu den Wirkungen unterschiedlicher Maßnahmen des Kinderlastenausgleichs. 
4.2 Empirische Abschätzung der demographischen Wirkung pronatalistischer

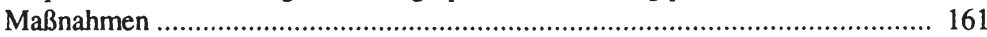

4.2.1 Zeitlicher Vergleich der Kosten von Kindern .............................. 162

4.2.2 Fallbeispiele ....................................................................... 167

4.2.3 Regressionsanalyse ..................................................... 179

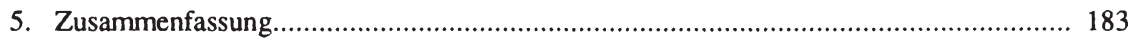

Teil D: Verstärkte Einwanderung als Instrument zur Stabilisierung der Gesetzlichen Rentenversicherung ......................................................... 185

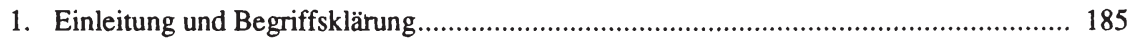

2. Theoretische Analyse der Zuwanderung................................................................ 186

2.1 Analyse des Wanderungspotentials ........................................................ 188

2.1.1 Makroökonomische Erklärungsansätze.................................... 188

2.1.2 Mikroökonomische Erklärungsansätze .................................... 191

2.1.2.1 Nachfrageorientierte Erklärungsansätze des

Wanderungspotentials .............................................. 191

2.1.2.2 Angebotsorientierte Erklärungsansätze des

Wanderungspotentials ................................................. 192

2.2 Analyse der Nachfrage nach Zuwanderern durch das Einwanderungsland ......... 197

2.2.1 Ökonomische Bestimmungsgründe der Nachfrage ......................... 197

2.2.2 Politisch-ökonomische Bestimmungsgründe der Nachfrage............. 198

3. Kosten-Nutzen-Analyse der Einwanderung ...................................................... 203

3.1 Der Einfluß der Einwanderer auf Arbeitseinkommen und Arbeitslosigkeit von Einheimischen............................................................................ 204

3.1.1 Theoretische Analyse der Arbeitsmarktwirkungen von Einwanderern ........................................................................ 204

3.1.2 Die empirische Größenordnung der Arbeitsmarktwirkungen von Einwanderern .................................................................. 209

3.1.3 Die Bedeutung der Arbeitsmarktwirkungen von Einwanderern für ein Einwanderungsgesetz ............................................... 217

3.2 Der Einfluß der Einwanderer auf die Kapitaleinkommen der Einheimischen ...... 218

3.2.1 Theoretische Analyse der Wirkungen auf die Kapitaleinkommen...... 218

3.2.2 Empirische Größenordnung der Wirkungen auf die Kapitaleinkommen ............................................................................. 220

3.2.3 Bedeutung der Wirkungen auf die Kapitaleinkommen für ein Einwanderungsgesetz........................................................... 221

3.3 Der Einfluß von Einwanderern auf die öffentlichen Haushalte ......................... 222

3.3.1 Die Assimilation der Einwanderer in den Arbeitsmarkt des

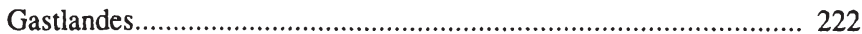

3.3.1.1 Theoretische Analyse der Assimilation .......................... 222

3.3.1.2 Empirische Analyse der Assimilation .............................. 224

3.3.1.3 Bedeutung der Assimilation für ein Einwanderungsgesetz ............................................................. 231 
3.3.2 Der Einfluß der Einwanderung auf die Haushalte der Gebietskörperschaften

3.3.2.1 Die Einnahmenseite................................................ 232

3.3.2.2 Die Ausgabenseite.................................................... 234

3.3.2.3 Bedeutung für ein Einwanderungsgesetz ....................... 237

3.3.3 Der Einfluß der Einwanderung auf die Finanzlage der

Sozialversicherungen................................................................. 238

3.3.3.1 Der Einfluß der Gastarbeiter auf die Sozial-

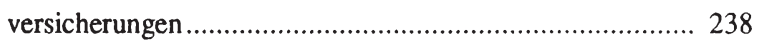

3.3.3.2 Der Einfluß der Aussiedler auf die Sozialversicherungen ......................................................... 247

3.3.3.3 Bedeutung für ein Einwanderungsgesetz ........................ 248

3.4 Der Einfluß der Einwanderer auf die Produktivitätsentwicklung...................... 250

3.4.1 Theoretische Analyse der Produktivitätseffekte ............................ 251

3.4.2 Empirische Größenordnung der Produktivitätseffekte................... 252

3.4.3 Bedeutung der Produktivitätseffekte für ein Einwanderungs-

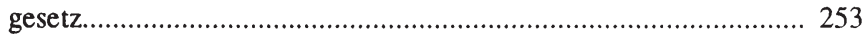

3.5 Makroökonomische Auswirkungen der Zuwanderung ................................ 254

3.5.1 Theoretische Analyse der makroökonomischen Auswirkungen ........ 254

3.5.2 Empirische Größenordnung der makroökonomischen Auswirkungen .......................................................................... 255

3.5.3 Die Bedeutung der makroökonomischen Auswirkungen für ein Einwanderungsgesetz .......................................................... 259

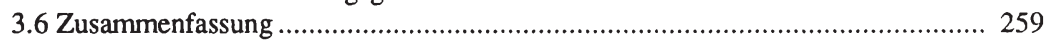

4. Die Kosteneffizienz einer verstärkten Einwanderung ............................................ 262

4.1 Kriterien für eine verstärkte Einwanderung ............................................. 262

4.2 Instrumente einer verstärkten Einwanderung ................................................ 267

4.3 Die Kosten der Zuwanderung bei Realisierung eines
Einwanderungsgesetzes .................................................................... 273

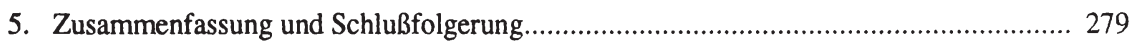

Teil E: Abschließender Vergleich von Kinderlastenausgleich und verstärkter Einwanderung 281

Anhang 1: Die biographische Theorie der Fertilität 285

Anhang 2: Überblick über wichtige Änderungen im System des Kinderlastenausgleichs der Bundesrepublik Deutschland 287

Anhang 3: Bevölkerung und Gesundheitskosten nach Altersklassen ................................ 289

Anhang 4: Klassifizierung der Zuwanderer. 291

Literaturverzeichnis 293 


\section{Abbildungsverzeichnis}

Abb. B.1: Intergenerationale Belastungswirkungen von Zwei-Generationenund Drei-Generationen-Vertrag im Vergleich

Abb. B.2: Bevölkerungsaufbau in Gesamtdeutschland 1990 und 2030 34

Abb. B.3: Anstieg der weiteren Lebenserwartung von Frauen im Alter von 20, 40 und 60 Jahren seit 1961

Abb. B.4: Altersspezifischen Mortalitätsraten für Männer im Alter von 60 bzw. 70 Jahren im früheren Bundesgebiet

Abb. B.5: Lebendgeborene in Westdeutschland zwischen 1950 und 1994 39

Abb. B.6: Zusammengefaßte Geburtenrate im früheren Bundesgebiet zwischen 1950 und 1994

Abb. B.7: Endgültige Kinderzahl der Frauenjahrgänge 1930 bis 1954 im früheren Bundesgebiet.

Abb. B.8: Zuzüge, Fortzüge und Wanderungssaldo für die frühere Bundesrepublik Deutschland, ab 1990 für Gesamtdeutschland.

Abb. B.9: Vergleich der Altersstruktur der inländischen Bevölkerung und des Zuwanderungsüberschusses 1992

Abb. B.10: Prognostizierte Entwicklung der Gesamtbevölkerung Deutschlands zwischen 1993 und 2030 46

Abb. B.11: Die Entwicklung des Altenquotienten zwischen 1993 und 2030. 46

Abb. B.12: Prognostizierter Beitragssatz zur Gesetzlichen Rentenversicherung 52

Abb. B.13: Die Entwicklung der Beitragssätze der Sozialversicherungen 54

Abb. B.14: Die Entwicklung des Altenquotienten bei unterschiedlichen Annahmen über die Altersstruktur der Zuwanderer

Abb. C.1: Phasen des demographischen Übergangs

Abb. C.2: Der Zusammenhang zwischen Kinderzahl (N) und Einkommen (Y) in den Ansätzen von Freedman und Leibenstein

Abb. C.3: Wirkung einer Erhöhung des Frauenlohnsatzes auf die Kinderzahl 109

Abb. C.4: Einkommensverluste infolge einer Unterbrechung der Erwerbsarbeit

Abb. C.5: Einkommensverluste im Lebensverlauf 122

Abb. C.6: Der Zusammenhang zwischen Quantität und Qualität von Kindern 131 
Abb. C.7: Wirkung einer Einkommenserhöhung auf die Kinderzahl unter Berücksichtigung des Zusammenhangs zwischen Kinderquantität und -qualität.

Abb. C.8: Bestimmung der direkten Kinderkosten nach Rothbarth

Abb. C.9: Das System des Kinderlastenausgleichs

Abb. C.10: Zeitliche Entwicklung der zusammengefaßten Geburtenraten verschiedener europäischer Länder .

Abb. C.11: Zeitliche Entwicklung der zusammengefaßten Geburtenrate in verschiedenen Ländern Osteuropas.

Abb. C.12: Zeitliche Entwicklung der zusammengefaßten Geburtenrate in der DDR und der Bundesrepublik Deutschland.....

Abb. D.1: Wirkung einer Verschärfung der Einwanderungspolitik auf die Zahl der Zuwanderer. 200

Abb. D.2: Arbeitsmarkteffekte von Arbeitskräftewanderungen in einem Zwei-Länder-

Modell mit homogener Arbeit. 205

Abb. D.3: Die Arbeitsmarktwirkung von Einwanderern für Einheimische in einem Modell mit Einheimischen und Einwanderern als unterschiedlichen Produktionsfaktoren. 208

Abb. D.4: Die Arbeitsmarktwirkung von Einwanderern für Einheimische in einem Modell mit qualifizierten und unqualifizierten Arbeitskräften als unterschiedlichen Produktionsfaktoren

Abb. D.5: Einfluß der Einwanderer auf das Kapitaleinkommen im Fall ohne Änderung des Kapital-Arbeit-Verhältnisses

Abb. D.6: Die Einkommensentwicklung von Einheimischen und Einwanderern gleicher Ausbildung und Berufserfahrung im Zeitablauf .... 225

Abb. D.7: Der Kohorteneffekt bei sinkender Qualität der Einwanderer..... 226

Abb. D.8: Sozialversicherungsbeiträge von Vollzeit-Erwerbstätigen 1984 240

Abb. A3.1: Bevölkerung und Gesundheitskosten nach Altersklassen in der Bundesrepublik Deutschland für die Jahre 1990, 2010 und 2050 


\section{Tabellenverzeichnis}

Tab. B.1: Die Entwicklung der Altenquote bis zum Jahr 2030 bei unterschiedlicher Nettozuwanderung und Fertilität.....

Tab. C.1: Einkommensschätzung für unselbständig beschäftigte Frauen

Tab. C.2: Lebenseinkommensverluste nach Unterbrechungsdauer, Unterbrechungszeitpunkt, Schulbildung und Art der Unterbrechung ....

Tab. C.3: Altersspezifische Erwerbsquoten von Frauen im Alter von 15 Jahren und mehr im Mai 1992 nach Altersgruppen und Altersgruppen der Kinder in der Familie

Tab. C.4: Prozentuale Anteile verschiedener Wochenarbeitszeitklassen für Frauen mit Kindern und Kinderlose zwischen 25 und unter 45 Jahren

Tab. C.5: Die Entwicklung des Preisindexes für die einfache Lebenshaltung eines Kindes im Vergleich zu der Entwicklung des Preisindexes für die Lebenshaltung eines Arbeitnehmerhaushalts mit mittlerem Einkommen.

Tab. C.6: Monetäre Kinderkosten nach Kinderzahl bzw. Haushaltsnettoeinkommen 1992

Tab. C.7: Kinderkosten in Abhängigkeit von Kinderzahl und Einkommensgruppe der Eltern

Tab. C.8: Durchschnittlicher Bruttomonatsverdienst weiblicher Arbeiter und Angestellter in Industrie bzw. Industrie, Handel, Kreditinstituten und Versicherungsgewerbe (altes Bundesgebiet)

Tab. C.9: Zusammengefaßte Geburtenraten der Frauenjahrgänge 1930-1955 für die alten Bundesländer - je 1000 Frauen bis zum Alter von 49 Jahren

Tab. C.10: Zusammengefaßte Geburtenraten 1951-1985 für die alten Bundesländer

- je 1000 Frauen bis zum Alter vom 49 Jahren

Tab. C.11 Plätze in Kindergärten, Kinderkrippen und Kinderhorten in den alten Bundesländern 1963-1986

Tab. D.1: Arbeitsplatztypen deutscher und ausländischer Arbeitnehmer.

Tab. D.2: Transfers und Beiträge zu den Sozialversicherungen pro Haushalt 1984 239

Tab. D.3: Transfers und Beiträge zu den Sozialversicherungen pro Person 1984

Tab. D.4: Gesamtwirtschaftliche Wirkungen der Zuwanderung (Abweichungen gegenüber Szenario ohne Zuwanderung)

Tab. D.5: Durchschnittliches Alter, Ausbildung und Sprachfertigkeit amerikanischer und kanadischer Einwanderer nach Herkunftsregion und Einwanderungsperiode 
Bernhard Manzke - 978-3-631-75262-3

Downloaded from PubFactory at 01/11/2019 06:34:40AM

via free access 


\section{Verzeichnis der wichtigsten Symbole}

\section{in Teil B:}

$N_{t} \quad$ Zahl der Beitragszahler; der tiefgestellte Index $t$ bezeichnet die jeweilige Periode

$L_{t} \quad$ durchschnittlicher versicherungspflichtiger Bruttolohn

$b_{t} \quad$ Beitragssatz zur Gesetzlichen Rentenversicherung vom versicherungspflichtigen Bruttolohn

$Z_{t} \quad$ Bundeszuschuß zu den Einnahmen der Rentenversicherung

$A_{t} \quad$ Zahl der Rentenempfänger

$A_{t} / N_{t}$ Rentnerquotient

$R_{t} \quad$ durchschnittliche Pro-Kopf-Rente

$q \quad$ Nettorentenniveau

$z \quad$ Prozentsatz des Bundeszuschusses zu den Ausgaben der Rentenversicherung

$n_{t} \quad$ Wachstumsrate der Beitragszahler

$g_{t} \quad$ Wachstumsrate des durchschnittlichen beitragspflichtigen Bruttoverdienstes

$r_{t} \quad$ interne Rendite des Umlageverfahrens aus Sicht des Versicherten

$i \quad$ interne Rendite eines Kapitaldeckungsverfahrens (Marktzinssatz)

$R_{E} \quad$ Elternrente

$N_{E} \quad$ Anzahl der elternrentenbegründenden Kinder

$N_{p} \quad$ Anzahl der potentiellen Beitragszahler

\section{in Teil $\mathbf{C}$ :}

$Z_{i} \quad$ Basisgut $i, i=1 \ldots m$

$x_{i} \quad$ Marktgüterbündel, das zur Herstellung einer Einheit des Basisguts $Z_{i}$ benötigt wird

$p_{i} \quad$ Preis des Marktgüterbündels $x_{i}$

w Lohnsatz

$t \quad$ Zeitbudget

$t_{w} \quad$ Arbeitszeit

$t_{h_{1}} \quad$ Summe aller zur Herstellung des Basisguts $i$ im Haushalt verbrachten Zeiten

$\pi_{i} \quad$ Schattenpreis des Basisguts $Z_{i}$,

I Geldeinkommen

$S \quad$ implizites Einkommen

$U \quad$ Nutzen 


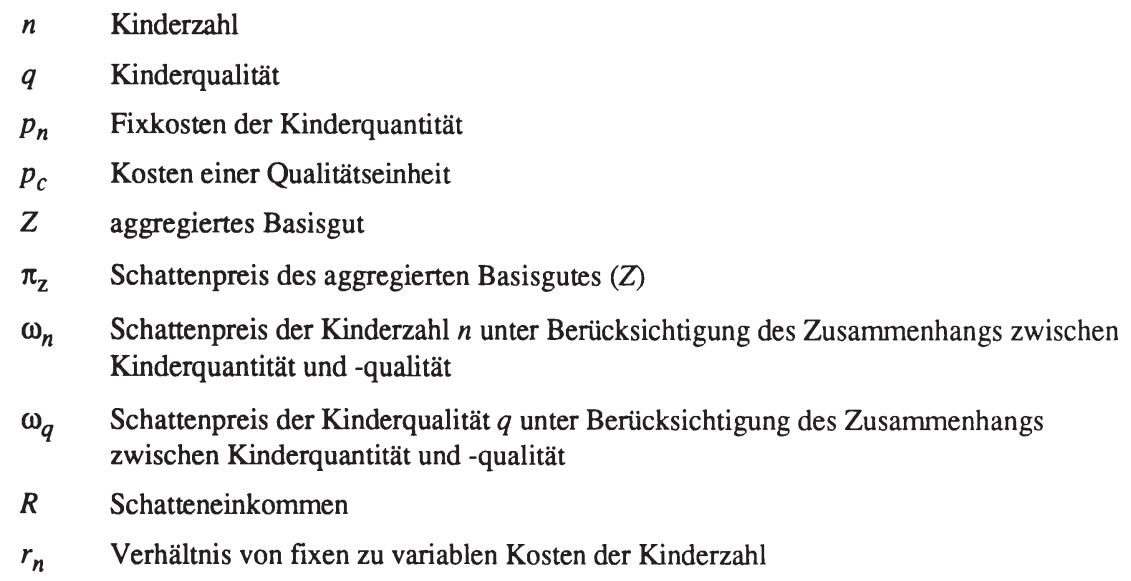

\section{in Teil D:}

$N \quad$ Herkunftsland

$H \quad$ Zielland

$K_{N H} \quad$ Wanderungskosten für eine Wanderung von Land $N$ nach Land $H$

$U \quad$ Nutzen

C Konsum

$X \quad$ Variable, die mit individuell unterschiedlichen Gewichten in die Nutzenfunktion eingehen (z.B. Freizeitwert, Sicherheit, Zufriedenheit mit der sozialen Stellung etc.)

$T \quad$ zeitlicher Planungshorizont

$r \quad$ individuelle Zeitpräferenzrate

$w_{N} \quad$ Lohnsatz im Herkunftsland

$w_{H} \quad$ Lohnsatz im Zielland

$p_{N} \quad$ Wahrscheinlichkeit, daß im Herkunftsland keine Arbeitslosigkeit eintritt

$p_{H} \quad$ Wahrscheinlichkeit, daß im Zielland keine Arbeitslosigkeit eintritt 
Teil A:

\section{EINLEITUNG}

Große Teile der deutschen Bevölkerung sind besorgt über die zukünftige Entwicklung der Gesetzlichen Rentenversicherung. Sie fürchten, entweder im Alter mit wesentlich geringeren Renten auskommen zu müssen als sie nach gegenwärtiger Rechtslage erwarten dürfen oder aber - falls sie dann noch erwerbstätig sind - mit stark steigenden Beitragssätzen belastet zu werden. Ursache dieser Befürchtungen ist die prognostizierte Bevölkerungsentwicklung, die in den nächsten Jahrzehnten zu einem zunehmenden Anteil der Personen im Rentenalter und einem abnehmenden Anteil der Personen im Erwerbsalter führen wird. Damit müssen künftig mehr Rentner von einem Beitragszahler unterstützt werden als bisher. An dieser Situation ist durch die Rentenreform im Jahr 1992 nichts Grundsätzliches geändert worden. Die aus der erwarteten demographischen Entwicklung erwachsenden Belastungen, die bis dahin allein von den Beitragszahlern zu tragen gewesen wären, wurden lediglich teilweise auf Rentner und Steuerzahler umverteilt. Es bleibt daher eine lohnende Aufgabe, nach Instrumenten zu suchen, die eine wirkliche Reduzierung der zukünftigen Belastungen bewirken. Ziel dieser Arbeit ist es zunächst, solche belastungsreduzierenden Instrumente $\mathrm{zu}$ identifizieren und sie anschließend hinsichtlich ihrer Kosteneffizienz, d.h. hinsichtlich der zur Erreichung einer jeweils gleichen Reduzierung der zukünftigen Belastungen der Gesetzlichen Rentenversicherung aufzuwendenden Kosten, miteinander zu vergleichen. Dazu wird die Arbeit in drei inhaltliche Teile untergliedert.

In Teil B wird einführend erläutert, welche Zusammenhänge zwischen den Bestimmungsfaktoren der Finanzlage der umlagefinanzierten Gesetzlichen Rentenversicherung, d.h. zwischen Beitragssatz, Rentenniveau und demographischen Variablen, bestehen. Anhand der prognostizierten Entwicklung dieser Einflußfaktoren werden dann die für die Mitglieder der Gesetzlichen Rentenversicherung zu erwartenden Belastungen herausgearbeitet. Anschließend werden verschiedene Ansätze diskutiert, die zur Lösung dieser "demographischen Krise" der Gesetzlichen Rentenversicherung vorgeschlagen wurden. Der einzige Weg, der eine tatsächliche Reduzierung der Belastungen erwarten läßt und nicht nur eine Umverteilung bewirkt, ist ein Ansatz an der Ursache des Problems, d.h. an der demographischen Entwicklung. Eine Beeinflussung der Bevölkerungsstruktur in für die Finanzlage der Gesetzlichen Rentenversicherung vorteilhafter Weise ist auf zwei Wegen möglich: erstens über eine Steigerung der Geburtenrate durch einen Ausbau des Kinderlastenausgleichs und zweitens über eine Erhöhung der (Netto-)Einwanderung. Welche Kosten diese beiden Instrumente jeweils verursachen wird in den Teilen C und D dieser Arbeit untersucht. So kann im Schlußteil eine vergleichende Gegenüberstellung der Kosteneffizienz einer Ausweitung des Kinderlastenausgleichs und einer Erhöhung der Einwanderung erfolgen, aus der dann eine Politikempfehlung ableitbar ist.

Zunächst wird in Teil $\mathrm{C}$ dieser Arbeit auf das Instrument des Kinderlastenausgleichs eingegangen. Anhand der neoklassischen Theorie der Fertilität wird analysiert, ob eine Beeinflussung der Geburtenrate über eine Verstärkung des Kinderlastenausgleichs überhaupt erreicht werden kann und welche finanziellen Mittel gegebenenfalls für die Erreichung eines bestimmten Geburtenanstiegs aufzuwenden wären. Dazu werden die Bestimmungsfaktoren der Fertilitätsentscheidung herausgearbeitet, ihre empirischen Größenordnungen bestimmt und 
Implikationen für die Gestaltung des Kinderlastenausgleichs abgeleitet. Darauf aufbauend wird anschließend mit Hilfe verschiedener Methoden eine Wirkungsanalyse des Kinderlastenausgleichs durchgeführt und so schließlich die Kosteneffizienz dieses Instruments bestimmt.

In Teil D der Arbeit wird eine Verstärkung der Einwanderung als Instrument zur Reduzierung der künftigen Probleme der Gesetzlichen Rentenversicherung untersucht. Für die absehbare Zukunft kann davon ausgegangen werden, daß die Zahl der Einwanderungswilligen größer sein wird als die Aufnahmebereitschaft des Einwanderungslandes. Deshalb stehen hier nicht wie bei der Analyse des Kinderlastenausgleichs die Kosten im Vordergrund, die durch die Schaffung von Anreizen zu höherer Fertilität hervorgerufen werden, sondern die Wirkungen, die durch die im Vergleich zu den Einheimischen andersartigen Charakteristika der Einwanderer entstehen. Dazu zählen als Primärwirkungen neben dem Einfluß der Einwanderer auf die Arbeitsmarktsituation und die Kapitaleinkommen der Einheimischen die Wirkungen, die sich durch die umverteilende Staatstätigkeit ergeben, sowie in langfristiger Perspektive die Auswirkungen auf die Produktivitätsentwicklung. Auch auf Sekundärwirkungen der gegenüber den Einheimischen andersartigen Merkmale der Einwanderer wird kurz eingegangen. Aus der Kosten-Nutzen-Analyse der Einwanderung wird abgeleitet, welche Merkmale die Zuwanderer aufweisen müssen, um für die Einheimischen möglichst günstige Wirkungen zu entfalten und welches Instrument der Einwanderungspolitik daher besonders vorteilhaft ist. Schließlich wird die Kosteneffizienz einer verstärkten Einwanderung untersucht.

Bei dem abschließenden Vergleich des Kinderlastenausgleichs und einer verstärkten Einwanderung werden die Kosteneffizienzen der beiden Instrumente einander gegenübergestellt. Das Ergebnis des Vergleichs bildet die Grundlage für eine Politikempfehlung. 
Teil B:

\section{DIE DEMOGRAPHISCHE KRISE DER GESETZLICHEN RENTENVERSICHERUNG}

\section{Der Generationenvertrag in der Gesetzlichen Rentenversicherung}

Das Grundproblem, das eine Alterssicherung erforderlich macht, besteht darin, daß für jedes einzelne Individuum über die Lebensspanne gesehen produktive und konsumtive Phasen zeitlich auseinanderfallen können. Vor allem während der Kindheit und im Alter (aber auch bei Krankheit und Berufs- bzw. Erwerbsunfähigkeit) sind die Fähigkeit und der Wille, zur Produktion beizutragen, im Vergleich zum angestrebten Konsumniveau gering oder überhaupt nicht vorhanden. Kinder und Jugendliche sind noch nicht, ältere Menschen oft nicht mehr in der Lage, ihren Lebensunterhalt durch eine produktive Tätigkeit in der gleichen Periode zu decken. Dennoch mußte und muß in jeder Gesellschaft die Versorgung dieser Altersgruppen auf die eine oder andere Weise gesichert werden.

\subsection{Grundtypen von Alterssicherungssystemen}

Beschränkt man sich auf das Problem der Versorgung der nicht mehr produktiv Tätigen, so lassen sich nach den folgenden Kriterien verschiedene Grundtypen von Alterssicherungssystemen unterscheiden: ${ }^{1}$

Nach dem Träger der Alterssicherung können staatliche Rentensysteme und private Alterssicherungsformen unterschieden werden. In einem staatlichen Rentensystem erfolgt die Verwaltung und die Festsetzung der Höhe von Beiträgen und Leistungen durch eine staatliche oder parafiskalische Institution, wobei diese auch ein eingeschränktes Selbstverwaltungsrecht haben kann.

Eine private Alterssicherung beruht dagegen auf eigener Ersparnis (Selbstversicherung) oder Verträgen mit Dritten. Bei diesen Verträgen kann es sich zum einen um Lebensversicherungsverträge handeln, die mit Finanzunternehmen abgeschlossen werden, zum anderen aber auch um Verträge mit Privatpersonen (z.B. mit den eigenen Kindern), wobei die Verträge im letztgenannten Fall nicht immer explizit formuliert sein müssen.

Nach dem Finanzierungsverfahren unterscheidet man zwischen dem Kapitaldeckungsverfahren und dem Umlageverfahren. Beim Umlageverfahren werden die Beiträge der Aktiven sofort wieder für Ausschüttungen in Form von Rentenzahlungen an die nicht mehr Aktiven verwendet. Einzahlungen und Auszahlungen einer Periode entsprechen einander, so daß der Träger der Alterssicherung keinen Kapitalbestand ansammelt.

1 Zu ähnlichen Einteilungen vgl. Breyer (1990) S. 4ff. und Homburg (1988) S. 5ff. Zu den Kombinationsmöglichkeiten der einzelnen Merkmale vgl. Homburg (1988) S. $8 \mathrm{ff}$. 
Beim Kapitaldeckungsverfahren (auch Anwartschaftsdeckungsverfahren genannt) dagegen werden die Beiträge der aktiven Generation verzinslich angelegt, so daß sich für jede Altersgruppe ein Kapitalstock ansammelt, dessen Auflösung die Zahlung von Renten an diese Generation im Alter ermöglicht.

Als weiteres Unterscheidungskriterium von Alterssicherungssystemen läßt sich der Grad des Beitrittszwangs heranziehen. Bei einem obligatorischen System sind gewisse Mindestbeträge für Beiträge und Renten gesetzlich vorgeschrieben, während in einem auf Freiwilligkeit beruhenden System jeder Einzelne selbst entscheiden kann, wie und in welcher Höhe er eine Alterssicherung betreibt.

Schließlich können noch Systeme mit und ohne Risikoausgleich unterschieden werden, wobei sich der Risikobegriff hier ausschließlich auf individuell unterschiedliche Lebensdauern beziehen soll. ${ }^{2}$ Ohne Risikoausgleich in diesem Sinne wird dem Versicherten bei Erreichen eines bestimmten Lebensalters eine genau festgelegte Gesamtsumme ausgezahlt, so daß er bei einem unerwartet frühen Tod eine ungeplant hohe Erbschaft hinterläßt, bei einem unerwartet langen Leben dagegen im hohen Alter möglicherweise unzureichend versorgt ist. In Alterssicherungssystemen mit Risikoausgleich erhält der Versicherte im Ruhestandsalter einen bestimmten Betrag pro Periode bis zu seinem Lebensende. Es muß sich dann notwendigerweise um ein kollektives System handeln, da nur bei mehreren Versicherten der gleichen Altersklasse eine derartige Umverteilung zu Lasten von Versicherten mit einer geringen Lebensdauer und zu Gunsten von Versicherten mit einer hohen Lebensdauer erfolgen kann.

\subsection{Die Entwicklung des Generationenvertrags in der Gesetzlichen Ren- tenversicherung}

Im Laufe der Geschichte haben sich verschiedene Alterssicherungssysteme aus unterschiedlichen Kombinationen von Ausprägungen der oben angeführten Kriterien herausgebildet. Bis in die noch weitgehend bäuerlich geprägte frühe Neuzeit hinein hat die Großfamilie, d.h. die Hausgemeinschaft aus Verwandten und Gesinde, die Aufgabe der Versorgung im Alter übernommen. Die jeweils erwerbstätigen ${ }^{3}$ Personen einer Großfamilie mußten aus ihrem Einkommen die nicht mehr aktive ältere Generation und die noch nicht produktiv tätige junge Generation mitversorgen. Da das Einkommen nicht zur Ansparung eines Kapitalstocks verwendet wurde, sondern sofort der Versorgung der Nicht-Erwerbstätigen diente, handelte es sich um ein Alterssicherungssystem mit innerfamiliärem Umlageverfahren, allerdings im Gegensatz zu

In der Gesetzlichen Rentenversicherung Deutschlands wird neben dem Risiko eines überdurchschnittlich langen Lebens (Altersrente) auch das Risiko einer geminderten Fähigkeit zur Einkommenserzielung (Berufs- bzw. Erwerbsunfähigkeitsrente) und in Form der Hinterbliebenenrente das Risiko des Todes von Angehörigen versichert (vgl. Rosen/Windisch (1992) S. 427). Entsprechend des in dieser Arbeit zu behandelden Problems der zunehmenden Alterung erfolgt im weiteren allerdings eine Eingrenzung auf die Versicherung des erstgenannten Risikos.

3 Zur Vereinfachung soll im folgenden davon abgesehen werden, daß nicht alle erwerbsfähigen Personen auch tatsächlich erwerbstätig sind und deshalb auch innerhalb der potentiell erwerbsfähigen Generation eine Umverteilung stattfinden muß. 
dem heutigen System ohne formellen Beitrittszwang, ohne Risikoausgleich ${ }^{4}$ und auf privater Basis. $^{5}$

Diese Form der Versorgung der Nicht-Erwerbstätigen läßt sich als Drei-GenerationenVertrag bezeichnen, wobei dieser "Vertrag" allerdings nicht im juristischen Sinne zu verstehen ist, sondern als eine von der großen Mehrheit der Bevölkerung akzeptierte Institution. Die gerade erwerbstätige Generation versorgt sowohl die ältere Generation als auch die nachfolgende Generation aus ihrem laufenden Einkommen. Sie tilgt damit einerseits die Schuld gegenüber der älteren Generation, die durch die Versorgung während der Kindheit der jetzt Erwerbstätigen entstanden ist. Andererseits erwirbt sie durch die Leistungen an die jüngere Generation einen eigenen Anspruch auf Unterstützung durch die Kinder im Alter.

Im Zuge der Industrialisierung und der mit ihr verbundenen Verstädterung, der Zunahme der Lebenserwartung sowie der Zunahme der Mobilitätserfordernisse zerbrach die Hausgemeinschaft der Großfamilie und damit auch der Generationenvertrag. Die nicht (mehr) Erwerbsfähigen waren zunehmend auf die kommunale Armenfürsorge angewiesen, die sich aber rasch als überfordert erwies. Die "soziale Frage" führte schließlich zu der Ankündigung einer neuen, im wesentlichen auf den Reichskanzler Bismarck zurückgehenden Sozialpolitik in der "Kaiserlichen Botschaft" vom 17.11.1881 (Bismarck'sche Sozialgesetzgebung). Nach dem Gesetz zur Krankenversicherung (1883) und zur Unfallversicherung (1884) wurde als dritte Säule der Sozialversicherung 1889 auch das Gesetz über die Invaliditäts- und Altersversicherung (Rentenversicherung) verabschiedet und ab 1891 in Kraft gesetzt.

Wesentliche Merkmale haben sich bis heute erhalten: die Selbstverwaltung durch öffentlichrechtliche Versicherungsanstalten, die Finanzierung durch gleichhohe Beiträge von Arbeitgebern und Arbeitnehmern sowie durch einen Reichszuschuß, die Versicherungspflicht für weite Teile der Erwerbstätigen und die lohnabhängige Beitragshöhe. Zunächst lag das Schwergewicht der Versicherung allerdings nicht bei der Altersrente, sondern bei der Invaliditätsrente. Auch das relative Bruttorentenniveau ${ }^{6}$ lag mit durchschnittlich $10 \%$ (1910) nur bei einem Bruchteil des heutigen Wertes (41,9\% 1990). Das Finanzierungsverfahren wurde zunächst als Kapitaldeckungsverfahren konzipiert.

Es handelte sich also von der Anlage her um ein staatliches Alterssicherungssystem nach dem Kapitaldeckungsverfahren mit Beitrittszwang und Risikoausgleich ${ }^{7}$. Der innerfamiliäre

4 Die alte Generation kann zwar auf eine Versorgung hoffen, deren auf eine Periode bezogene Höhe unabhängig von der individuellen Lebensdauer ist. Das Risiko haben aber die Erwerbstätigen der Großfamilie zu tragen, es wird nicht durch eine Verteilung auf eine größere Versicherungsgemeinschaft diversifiziert.

$5 \quad$ Einen plastischen Eindruck von der Altersversorgung und den potentiellen Konflikten innerhalb der Großfamilie vermittelt die Schilderung des Rückzugs einer Witwe auf das Altenteil im Jahre 1858 durch Borchert (1993) S. 39ff.

6 Als relatives Bruttorentenniveau wird das Verhältnis von durchschnittlicher Rente zu durchschnittlichem gegenwärtigen Bruttoerwerbseinkommen bezeichnet. Vgl. Rosen/Windisch (1992) S. 391f. Dabei werden für die durchschnittliche Rente in der Regel 45 Versicherungsjahre bei durchschnittlichem Arbeitsentgelt zugrundegelegt.

7 Unter der hier als erfüllt anzusehenden Voraussetzung von Risikoaversion ist in dem Risikoausgleich ein großer Vorteil gegenüber dem bis dahin geltenden System zu sehen. Denn auch bei einem überdurchschnittlich langen Leben bzw. Ausfall der Transfers von den eigenen Kindern (z.B wegen Krankheit oder deren frühzeitigem Tod) war so eine Minimalversorgung im Alter sichergestellt. Aufgrund des Versicherungseffektes stellen sich nach wohlfahrtstheoretischen Überlegungen Wohlfahrtssteigerungen ein. Vgl. beispielsweise Rothschild/Stiglitz (1976) oder Schneider (1991) S. $376 \mathrm{ff}$. 
Drei-Generationenvertrag sollte ergänzt werden - die Altersrente ging über die Sicherung eines Mindestbedarfs nicht hinaus und war daher nicht als vollständiger Ersatz für innerfamiliäre Transfers gedacht - durch eine staatlich organisierte Vorsorge der erwerbstätigen Generation für sich selber. Im Umfang der Renten der älteren Generation konnten die Transfers der erwerbstätigen Generation an die Ruheständler reduziert werden. Dafür mußten nun allerdings von der erwerbstätigen Generation Beiträge an die Gesetzliche Rentenversicherung abgeführt werden, um so eigene Rentenansprüche zu erwerben. Die durch die Unterstützung in der Kindheit gegenüber der älteren Generation entstandenen Schulden mußten nicht mehr voll bezahlt werden und die Abhängigkeit von Unterstützung durch die (eigenen) Kinder im Alter wurde verringert.

Ein reines Kapitaldeckungsverfahren wurde allerdings nie realisiert. Durch Fehlberechnungen und die Entwertung des angesparten Kapitalstocks in der Hyperinflation von 1923 vollzog sich in der Folgezeit faktisch ein allmählicher Übergang zum Umlageverfahren.

Durch die Rentenreform 1957 wurde das Umlageverfahren auch rechtlich verankert. Dem Ziel der Sicherstellung einer Lohnersatzfunktion der Rente diente die Einführung der sogenannten "dynamischen Rente". Sie sollte das relative Bruttorentenniveau (im Rentenreformgesetz 1992 erfolgte eine Umstellung auf die Nettoanpassung) auf einem stark angehobenen Niveau stabilisieren und der Rentner-Generation somit eine Teilhabe an den in Lohnsteigerungen zum Ausdruck kommenden Produktivitätsfortschritten sichern. Statt einer Minimalsicherung wird seither also eine Lebensstandardabsicherung im Alter durch die Gesetzliche Rentenversicherung angestrebt (Lohnersatzfunktion der Rente).

Damit wurde der innerfamiliäre Drei-Generationen-Vertrag praktisch aufgehoben und durch einen Zwei-Generationen-Vertrag ersetzt: ${ }^{8}$ Die Generation der jeweils Erwerbstätigen verpflichtet sich, mit ihren Beiträgen für die materielle Versorgung der Rentner aufzukommen und erwirbt dadurch das Anrecht, im Ruhestand durch die Beiträge der nachfolgenden, dann erwerbstätigen Generation versorgt zu werden. ${ }^{9}$ Während im Drei-Generationen-Vertrag die Leistungen an die ältere Generation eine Rückzahlung von Schulden darstellte und erst mit dem Aufziehen von Kindern Ansprüche auf eine eigene Alterssicherung erworben wurden, werden im Zwei-Generationen-Vertrag die Leistungen an die ältere Generation doppelt gezählt: sowohl als Tilgung der Schuld gegenüber der Eltern-Generation als auch als Erwerb von Ansprüchen gegenüber der Kinder-Generation. Die Kindererziehungsleistung wird dagegen im Rahmen des Alterssicherungssystems gar nicht mehr honoriert. ${ }^{10}$

Diese Ausgestaltung der Gesetzlichen Rentenversicherung durch die Rentenreform 1957 hat zur Folge, daß die im Alter zu erwartenden Rentenzahlungen von dem Beitragsvolumen der gesamten nachfolgenden Generation und der relativen Höhe der geleisteten Beiträge abhängen und nicht mehr von den Leistungen der von den Eltern selbst aufgezogenen Nachkommen innerhalb der eigenen Familie, wie noch im Drei-Generationen-Vertrag. Während die Kosten für das Aufziehen von Kindern nach wie vor privat von den Eltern getragen werden müssen, wird die Verpflichtung der Kinder, im Alter für die Lebenshaltung ihrer Eltern aufzukommen, auf die ganze Eltern-Generation ausgeweitet. Damit kommen die Versorgungsleistungen der Kinder-Generation auch Kinderlosen in der Generation der Eltern zugute, obwohl diese

8 Es handelt sich bei dem seitdem bestehenden System um eine staatliche Zwangsversicherung nach dem Umlageverfahren mit Risikoausgleich.

Vgl. Külp (1981) S. 131.

10 Dieser Unterschied zwischen dem Drei- und dem Zwei-Generationen-Vertrag wird allerdings erst dann deutlich, wenn die nachfolgende Generation nicht mehr ausreichend groß ist. 
während ihrer Erwerbsphase keine Kinderkosten zu tragen hatten. Die Kinderkosten bleiben privatisiert, der Alterssicherungsanspruch gegenüber den Kindern wird dagegen sozialisiert. ${ }^{11}$

Die Verteilung des von der nachfolgenden Generation aufgebrachten Beitragsvolumens innerhalb der Rentner-Generation erfolgt dabei - von Umverteilungskomponenten abgesehen nach dem Prinzip der Teilhabeäquivalenz ${ }^{12}$ lohn- bzw. beitragsabhängig. Das bedeutet, daß die individuelle Rangstelle in der Lohnskala, die ein Rentner während der Erwerbsphase gegenüber seinen Altersgenossen eingenommen hat, auch im Alter erhalten bleiben soll. Damit erhalten aber diejenigen, die ihre Erwerbstätigkeit aufgrund der Kindererziehung unterbrochen haben, unter sonst gleichen Bedingungen eine geringere Rente als Kinderlose mit ununterbrochener Erwerbskarriere. ${ }^{13}$ Die Wirkungen des Drei- bzw. Zwei-Generationenvertrages auf die Altersversorgung von Eltern und Kinderlosen wird in Abb. B.1 dargestellt. Unterschiedliche Pfeildicken kennzeichnen dabei unterschiedlich hohe Zahlungsströme.

Die Ausgestaltung des Zwei-Generationen-Vertrages in der Rentenreform von 1957 setzt daher Anreize zum Trittbrettfahren. Auch Kinderlose können jetzt durch Beitragszahlungen einen Anspruch auf Versorgung durch die nachfolgende Generation erwerben, ohne sich an den Kosten für das Aufziehen dieser Generation zu beteiligen. ${ }^{14}$ "Der naive 'Versicherte' stellt sich vor, seine Beiträge würden zwar zunächst einmal an die heutigen Rentenbezieher ausgeschüttet, nichtsdestoweniger flössen sie in seinem Alter in Gestalt der alsdann von ihm selbst bezogenen Rente an ihn wieder zurück. $\mathrm{Da}$ man die gleichen Gelder nicht zweimal (konsumtiv) ausschütten kann, soweit denkt er nicht." 15

11 Vgl. Nell-Breuning (1978) S. 79. In konsequenter Verfolgung des Gedankens des Drei-GenerationenVertrags kritisiert Nell-Breuning die Bezeichnung "Versicherung", da sie "unvermeidbar den Irrtum zu nähren [scheint], die Versicherungs-'Beiträge' finanzierten die Altersversicherung des Beitragszahlers, und diese irrige Vorstellung zeugt den weiteren Irrtum, der ganze Prozeß spiele sich zwischen nur zwei Generationen ab...". Nell-Breuning (1978) S. 78.

Homburg (1988) S. 7 definiert Teilhabeäquivalenz als eine Situation, in der die "... Leistungen an die Mitglieder einer Alterskohorte strikt proportional zu deren vergangenen Beiträgen sind. wobei unter 'Beitrag' nur eine tatsächlich geleistete Geldzahlung verstanden wird." Eine Umverteilung innerhalb einer Altersgruppe wird damit durch Teilhabeäquivalenz ausgeschlossen, nicht aber eine intergenerationale Umverteilung, denn der Barwert der Leistungen, die eine Kohorte erhält, muß nicht notwendig dem Barwert ihrer früher gezahlten Beiträge entsprechen.

13 Nell-Breuning (1980) S. 375 kritisiert die durch die lohnbezogene Rente gesetzten Anreize: "Indem unsere gesetzliche Regelung darauf verzichtet, den Aufwand für 'Infrastruktur', den jede denkbare Art von Alterssicherung unvermeidlich 'kostet', von den Versicherten einzufordern, vielmehr es den einzelnen völlig überläßt, ob oder in welchem $A u s m a ß$ sie sich daran beteiligen oder ihn auf andere abschieben, ohne da $\beta$ sich dies in der Höhe der zu erwartenden Rente irgendwie auswirkt, übt sie einen ungemein starken Anreiz aus, sich der Beteiligung an diesem gewichtigen Teil der Kosten zu entziehen und durch Entrichten des bloßen Versicherungsbeitrags sich die Rente auf völlig legale Weise zu erschleichen, nach dem bekannten Wort von Amd Jessen seine Sicherung 'auf die Kinder anderer aufzubauen'." (Hervorhebungen im Original).

14 Sofern nicht anders angegeben, wird in dieser Arbeit davon abstrahiert, daß nicht alle Mitglieder einer Generation auch in der Gesetzlichen Rentenversicherung versichert sind. Die grundlegenden Aussagen bleiben von dieser Abstraktion unberührt.

15 Nell-Breuning (1978) S. 78. Der hier dargestellten Sichtweise des Drei-Generationen-Vertrages und der Bedeutung des "Naturalbeitrages" der Kinderversorgung für die Gesetzliche Rentenversicherung hat sich auch das Bundesverfassungsgericht im Grundsatz angeschlossen (vgl. BVG v. 07.07.1992). Eine Gegenposition vertreten beispielsweise Ebert (1993) S. 103ff. oder Hase (1992). 
Abb. B.1: Intergenerationale Belastungswirkungen von Zwei-Generationen- und Drei-Generationen-Vertrag im Vergleich

\section{Drei-Generationen-Vertrag}

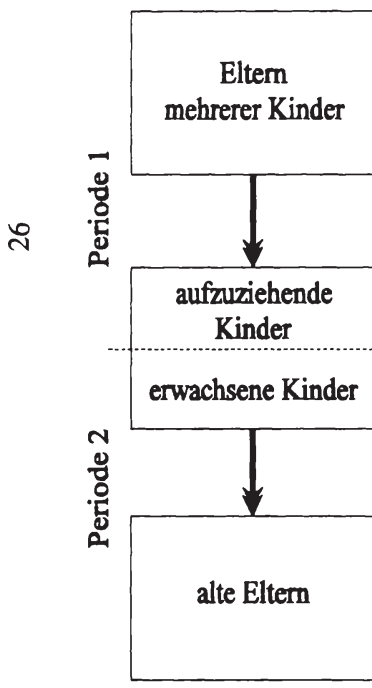

Eltern und Kinderlose haben annahernd gleiches Lebensniveau

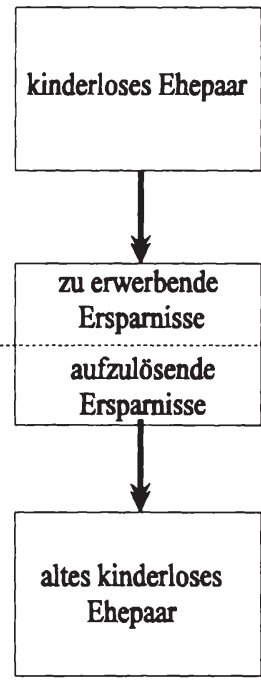

\section{Zwei-Generationen-Vertrag}

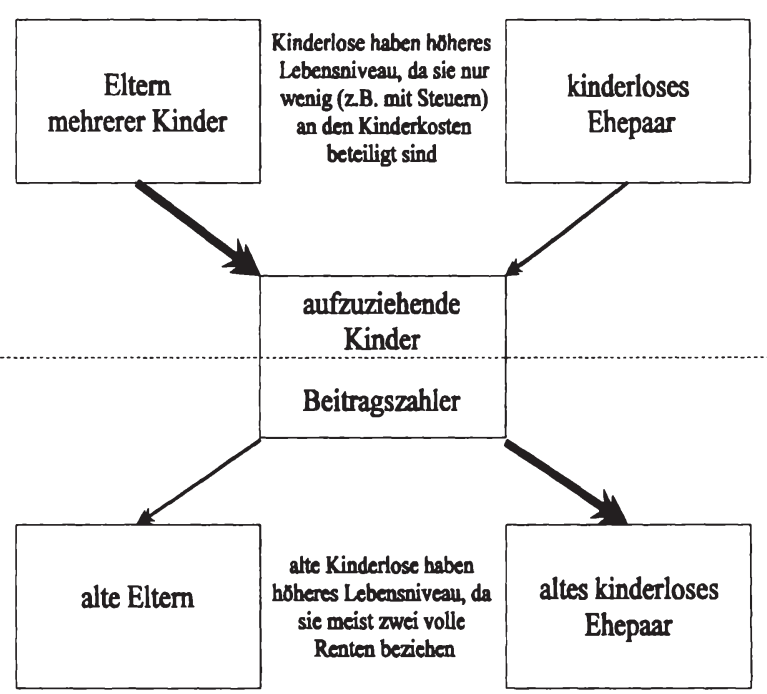


Die Folgen dieser von Borchert als "Kardinalfehler" bezeichneten Ausgestaltung des Generationenvertrags ${ }^{16}$ traten erst Jahrzehnte nach der Reform 1957 zutage. Zunächst erlaubte die Umstellung auf das Umlageverfahren eine Abweichung von dem Grundsatz der versicherungsmathematischen Äquivalenz ${ }^{17}$ und damit eine sofortige starke Anhebung des Rentenniveaus. So wurde der Kriegsgeneration, die nur in geringem Maße eigene Altersvorsorge treffen konnte, eine verbesserte finanzielle Position im Ruhestand geschaffen.

Gleichzeitig bestanden bei wachsender Bevölkerung und schnell steigenden Lohnsätzen polit-ökonomische Anreize zu einer ständigen Leistungsausweitung: Jede Erweiterung des Kreises der Leistungsempfänger und jede Erhöhung des Leistungsniveaus muß von der Erwerbstätigen-Generation nur noch während der bis zum Renteneintritt verbleibenden Jahre über höhere Beiträge finanziert werden. Danach werden für die gesamte Ruhestandszeit die höheren Rentenleistungen in Anspruch genommen. Besonders für die älteren Erwerbstätigen und die Rentner ist eine Leistungsausweitung also annähernd oder vollständig gegenleistungsfrei. Die zur Finanzierung herangezogene junge Generation ist aber größtenteils noch nicht stimmberechtigt, so daß nach dem Medianwählertheorem eine ständige Ausweitung des Systems zu erwarten ist. ${ }^{18}$

Eine derartige Erweiterung des Kreises der Leistungsberechtigten und Erhöhung des Leistungsniveaus ist bis Mitte der siebziger Jahre - v.a. im Rentenreformgesetz 1972 - zu beobachten. Seither wurde der Systemumfang durch verschiedene Konsolidierungsanläufe wieder etwas eingeschränkt. Der vorerst letzte dieser Konsolidierungsversuche war das Rentenreformgesetz 1992, in dessen Vorfeld mit der Anrechnung von Kindererziehungszeiten auch wieder ein erstes Element eines Drei-Generationen-Vertrages eingeführt wurde. Die drei Hauptelemente des Rentenreformgesetzes 1992 bestehen in einer Umstellung von der Bruttoauf die Nettoanpassung der Renten - die Rentenerhöhungen orientieren sich jetzt an der Lohnentwicklung nach Abzug von Steuern und Sozialversicherungsbeiträgen -, in einer schrittweisen Anhebung des Renteneintrittsalters und in einem nicht mehr allein an der Lohnentwick-

16 Vgl. Borchert (1993) S. 82f.

17 Unter versicherungsmathematischer Äquivalenz wird die Entsprechung des Barwertes der vom Versicherten gezahlten Beiträge und des Barwertes der erwarteten Leistungen an den Versicherten verstanden.

Das Medianwählertheorem besagt, daß unter gewissen Voraussetzungen die Menge einer eindimensional darstellbaren öffentlichen Leistung, die bei Mehrheitswahlrecht gewählt wird, den Präferenzen des Medianwählers entspricht. Für den Medianwähler gilt dabei, daß die Anzahl derjenigen Wähler, die mehr von dieser Leistung wünschen, genauso hoch ist, wie die Anzahl derjenigen Wähler, die eine geringere Bereitstellungsmenge präferieren würden. Vgl. Rosen/Windisch (1992) S. 169ff.

Eine grundlegende Studie zur Anwendung des Medianwählertheorems auf ein umlagefinanziertes Rentenversicherungssystem wurde von Browning (1975) verfaßt. In späteren Modellen wurde die Annahme einer direkten Demokratie aufgegeben (vgl. z.B. Weizsäcker (1990)), zwischen einzelnen Individuen und Familien als Entscheidungseinheiten unterschieden (vgl. z.B. Breyer/Schulenburg (1987)) und eine begrenzte Geltungsdauer der Entscheidung - und damit die Möglichkeit, daß die nachfolgende Generation bei einer zu hohen Belastung möglicherweise die Verpflichtung aus dem Generationenvertrag nicht erfüllt - berücksichtigt (vgl. z.B. Verbon 1987). Unter diesen Voraussetzungen ist eine übermäßige Ausweitung des Systems nicht mehr zwingend. Bei nachlassendem Wirtschaftswachstum oder einem Bevölkerungsrückgang ergibt sich in den meisten Modellen eine Rückführung des Systems durch sinkende Beitragssätze und noch stärker sinkende Rentenniveaus. Einen knappen Überblick über die verschiedenen polit-ökonomischen Modelle liefert Breyer (1992), eine ausführlichere aber zeitlich weiter zurückliegende Darstellung findet sich bei Homburg (1988) Kap. 8. 
lung orientierten, sondern zusätzlich proportional zum Beitragssatz steigenden Bundeszuschuß. Während die beiden erstgenannten Maßnahmen tatsächlich den Systemumfang beschränken, hat die Koppelung des Bundeszuschusses an die Beitragssatzentwicklung bei steigenden Beiträgen nur eine teilweise Verlagerung der Lasten von den Beitragszahlern zu den Steuerzahlern zur Folge. ${ }^{19}$

Die Gründe, die zu dieser Gegenentwicklung geführt haben, lassen sich am besten anhand der Budgetgleichung einer umlagefinanzierten Rentenversicherung darstellen.

\subsection{Fundamentalarithmetik umlagefinanzierter Alterssicherungssysteme}

Anhand einfacher mathematischer Umformungen der Budgetgleichung - Rosen/Windisch sprechen in diesem Zusammenhang von der Fundamentalarithmetik umlagefinanzierter Alterssicherungssysteme ${ }^{20}$ - lassen sich wesentliche Zusammenhänge zwischen den verschiedenen Variablen darstellen, die die Finanzlage der Gesetzlichen Rentenversicherung bestimmen. Da bei Anwendung des Umlageverfahrens definitionsgemäß keine Deckungsreserven vorhanden sind - von einer Schwankungsreserve soll hier abgesehen werden -, müssen die Einnahmen eines Rentenversicherers bei Anwendung des Umlageverfahrens in einer bestimmten Periode $t$ genau den Ausgaben dieser Periode entsprechen. Dabei sollen hier unter "Einnahmen" nur Beitragseinnahmen und Bundeszuschüsse, unter "Ausgaben" die in der jeweiligen Periode bestehenden Verpflichtungen zu Rentenauszahlungen verstanden werden.

\section{B.1) Einnahmen in $t=$ Ausgaben in $t$}

Die Einnahmen setzen sich zusammen aus der Zahl der Beitragszahler $\left(N_{t}\right)$ multipliziert mit dem durchschnittlichen versicherungspflichtigen Bruttolohn $\left(L_{t}\right)$ und dem Beitragssatz $\left(b_{t}\right)$ zuzüglich des Bundeszuschusses $\left(Z_{t}\right)$, wobei der tiefgestellte Index die jeweilige Periode bezeichnet. Die Ausgaben ergeben sich aus dem Produkt der Zahl der Rentenempfänger in $t$ $\left(A_{t}\right)$ und der durchschnittlichen Pro-Kopf-Rente $\left(R_{t}\right)$. Als Budgetgleichung für die Periode $t$ erhält man damit

$$
b_{t} N_{t} L_{t}+Z_{t}=A_{t} R_{t}
$$

Nach dem Rentenreformgesetz 1992 gilt der Grundsatz der Nettoanpassung, d.h. das Nettorentenniveau (q) soll entsprechend dem Konzept der dynamischen Rente stabil gehalten werden. In dem hier zugrundegelegten Modell ohne Steuern und Beiträge an andere Sozial-

19 Nicht nur zwischen Beitragszahlern und Steuerzahlern wurden Lastverschiebungen vorgenommen, sondern auch zwischen den einzelnen Sozialversicherungszweigen. So bemessen sich beispielsweise die Beitragszahlungen der Bundesanstalt für Arbeit zur Gesetzlichen Rentenversicherung für Arbeitslose seit 1995 nach 80 Prozent des letzten Bruttoentgelts und nicht wie bisher nach den Leistungen der Bundesanstalt. Vgl. Bundesregierung (1993) S. 59.

Vgl. Rosen/Windisch (1992) S. 391. Eine Darstellung der mathematischen Zusammenhänge für Umlage- und Kapitaldeckungsverfahren bei verschiedenen Politiken (konstanter Beitragssatz, konstantes Rentenniveau, Wahrung des Äquivalenzprinzips) findet sich auch bei Homburg (1988) S. $15 \mathrm{ff}$. 
versicherungszweige ergibt sich der Nettolohnsatz aus der Minderung des Bruttolohnsatzes um die Beiträge zur Rentenversicherung. Das Nettorentenniveau ${ }^{21}$ erhält man dann durch

$$
\text { B.3) } q_{t}=\frac{R_{t}}{\left(1-b_{t}\right) L_{t}} \text {. }
$$

Die Höhe des Bundeszuschusses zu den Ausgaben der Rentenversicherung ist gesetzlich festgeschrieben und ändert sich "im jeweils folgenden Kalenderjahr in dem Verhältnis, in dem die Bruttolohn- und -gehaltssumme je durchschnittlich beschäftigtem Arbeitnehmer im vergangenen Kalenderjahr zur entsprechenden Bruttolohn- und -gehaltssumme im vorvergangenen Kalenderjahr steht. Bei Veränderungen des Beitragssatzes ändert sich der Bundeszuschuß zusätzlich in dem Verhältnis, in dem der Beitragssatz des Jahres, für das er bestimmt wird, zum Beitragssatz des Vorjahres steht."22 Vereinfachend soll hier unterstellt werden, daß der Bundeszuschuß einen gleichbleibenden Prozentsatz ( $z$ ) der Ausgaben der Rentenversicherung deckt. Die Budgetgleichung B.2) läßt sich dann umformen zu

$$
\text { B.4) } \quad b_{t} N_{t} L_{t}=(1-z) A_{t} R_{t} \text {. }
$$

Nach Umstellung ergibt sich das Nettorentenniveau aus

$$
\text { B.5a) } \quad q_{t}=\frac{b_{t}}{1-b_{t}} \frac{1}{1-z} \frac{N_{t}}{A_{t}}
$$

Es wird offenbar von drei Variablen beeinflußt: dem Beitragssatz, dem Anteil des Bundeszuschusses und dem Verhältnis von Beitragszahlern zu Rentenempfängern. Sieht man von Änderung des Prozentsatzes des Bundeszuschusses und des Nettorentenniveaus ab, so müssen demographische Änderungen, die sich in einer Änderung des reziproken Rentnerquotienten ${ }^{23}$ niederschlagen, durch eine Veränderung des Beitragssatzes ausgeglichen werden. Bei konstantem Nettorentenniveau führt eine Verschlechterung des Verhältnisses von Beitragszahlern zu Rentenempfängern zwangsläufig zu einer Erhöhung des Beitragssatzes, wie auch bei Auflösung von Gleichung B.5a) nach $b$ deutlich wird (vgl. Gleichung $B .5 b)$ ).

21 Während $R_{t}$ in Gleichung B.2) die tatsächliche durchschnittliche Pro-Kopf-Rente bezeichnet, gibt $R_{t}$ in Gleichung $B .3$ ) gemäß der oben angeführten Definition für das Nettorentenniveau die Rente eines Eckrentners an, d.h. die Rentenhöhe eines Versicherten mit 45 Beitragsjahren und durchschnittlichem Arbeitsentgelt. Da unterstellt werden kann, daß sich beide Größen parallel entwickeln und ein gleichbleibender relativer Abstand zwischen ihnen für die hier vorgenommene Betrachtung unerheblich ist, stellt ihre Gleichstellung keine Einschränkung der Gültigkeit der abgeleiteten Ergebnisse dar.

$\S 213$ II SGB VI. Als Fortschreibungsformel für den absoluten Betrag des Bundeszuschusses ergibt sich aus dieser Bestimmung

$Z_{t}=Z_{t-1} \frac{\mathrm{L}_{t-1}}{\mathrm{~L}_{\mathrm{t}-2}} \frac{b_{t}}{b_{t-1}}$.

Vgl. Rosen/Windisch (1992) S. 388 FN 33.

23 Als Rentnerquotient $\left(A_{t} / N_{t}\right)$ wird das Verhältnis von Rentenempfängern zu Beitragszahlem bezeichnet. Er gibt an, wieviele Beitragszahler einen Rentner finanzieren. Vgl. Breyer (1990) S. 10. 
B.5b)

$$
b_{t}=\frac{(1-z) q_{t} A_{t} / N_{t}}{1+(1-z) q_{t} A_{t} / N_{t}}
$$

Um diesen Zusammenhang auch in Wachstumsraten ausdrücken und Aussagen über Einflußfaktoren auf die Rendite für die Versicherten machen zu können, muß die Analyse die einperiodige Sichtweise verlassen. Einen geeigneten Ansatz zur Untersuchung intergenerationaler Fragestellungen bietet das overlapping generations model. ${ }^{24}$ In jeder Periode $t$ leben zwei Generationen, die Erwerbstätigen $\left(N_{t}\right)$ und die Rentenempfänger $\left(A_{t}\right)$. Kinder werden hier nicht betrachtet. In der folgenden Periode $(t+l)$ existiert die Generation der Ruheständler aus der Vorperiode nicht mehr, aber die Erwerbstätigen der Vorperiode sind zu Rentenempfängern geworden $\left(N_{t}=A_{t+1}\right)^{25}$ und eine neue Generation befindet sich im Erwerbsleben. ${ }^{26}$ Das Nettorentenniveau erhält man jetzt in der umlagefinanzierten Rentenversicherung durch

$$
\text { B.6) } \quad q_{t}=\frac{b_{t}}{1-b_{t}} \frac{1}{1-z} \frac{N_{t}}{N_{t-1}} \text {. }
$$

Halbiert sich das Verhältnis von Beitragszahlern zu Rentnern - und das ist für die Entwicklung in den nächsten 40 Jahren keine unrealistische Annahme, wie noch zu zeigen ist ${ }^{27}$-, dann muß sich der Term $\frac{b_{t}}{1-b_{t}}$ verdoppeln, wenn das Nettorentenniveau und der prozentuale Bundeszuschuß konstant gehalten werden sollen. Der Beitragssatz muß um den Faktor $\frac{2}{b_{t}+1}$ steigen, was für einen Ausgangssatz von 20 Prozent des Bruttolohnes eine Steigerung um 67 Prozent auf 33,3 Prozent erfordern würde.

Bezeichnet man die Wachstumsrate der Beitragszahler $\left(N_{t} / N_{t-1}-l\right)$ mit $\left(n_{t}\right)$ so sieht man, daß bei konstantem Nettorentenniveau und Bundeszuschuß die Höhe des Beitragssatzes allein durch diese Wachstumsrate festgelegt wird:

B.7) $\quad q_{t}=\frac{b_{t}}{1-b_{t}} \frac{1}{1-2}\left(1+n_{t}\right)$.

Eine Analyse mit Hilfe von steady-state-Modellen, die sich an die neoklassische Wachstumstheorie anlehnen, ist hier wenig sinnvoll, weil die Probleme der Gesetzlichen Rentenversicherung gerade daraus resultieren, da $B$ die Wirtschafts- und Bevölkerungsentwicklung nicht mit konstanten Wachstumsraten wie in einem steady state verläuft. Vgl. Homburg (1988) S. 1 und S. 15 sowie Rosen/Windisch (1992) S. $397 \mathrm{f}$.

Es herrsche eine Lebensdauer von genau zwei Perioden, so daß eine Änderung der Generationengröße aufgrund vorzeitiger Todesfälle nicht berücksichtigt zu werden braucht.

Jede Generation durchlebt also zwei gleich lange Perioden. In der Realität ist natürlich die durchschnittliche Phase des Rentenbezugs wesentlich kürzer als die der Erwerbstätigkeit, so daß das Einsetzen konkreter Werte für die jeweiligen Generationengrößen in die Modellgleichungen keine sinnvollen Ergebnisse liefert (vgl. Homburg (1988) S. 16). Die unten vorgenommene Untersuchung der Auswirkungen einer Halbierung des Verhältnisses von Beitragszahlern zu Rentenempfängern bleibt davon unberührt, weil nur die relative Veränderung der Generationengrößen, nicht aber ihre absolute Größe in diese Betrachtung eingeht. 
Die Wachstumsrate des durchschnittlichen beitragspflichtigen Bruttoverdienstes - sie soll hier mit $g_{t}$ bezeichnet werden - hat in dem Modell keinen Einfluß auf das Nettorentenniveau, da dieses relativ zum Lohnniveau gemessen wird und Lohnsteigerungen sich sowohl in einem höheren Durchschnittsverdienst als auch in einer höheren Pro-Kopf-Rente niederschlagen. ${ }^{28}$

Die Wachstumsrate des durchschnittlichen beitragspflichtigen Bruttoverdienstes beeinflußt allerdings die interne Rendite $\left(r_{t}\right)$ des Umlageverfahrens aus Sicht des Versicherten. Diese ist hier interessant, weil die Höhe der Verzinsung, die auf Beiträge eines Versicherten erreicht wird, ein Maßstab für die Vorteilhaftigkeit des Umlageverfahrens gegenüber dem Kapitaldeckungsverfahren ist. Außerdem beeinflußt die Rendite die Akzeptanz des Umlageverfahrens und damit die Sicherheit des Fortbestands des Generationenvertrags nicht unwesentlich. Errechnet wird die interne Rendite, indem man die Rückflüsse an die Versicherten in Periode $t+1$ durch die in Periode $t$ geleisteten Einzahlungen dividiert, ${ }^{29}$ wobei hier ein konstanter Beitragssatz unterstellt wird: ${ }^{30}$

$$
\begin{aligned}
r_{t} & =\frac{b L_{t}\left(1+g_{t}\right) N_{t}\left(1+n_{t}\right)}{b L_{t} N_{t}}-1 \\
& =\left(1+g_{t}\right)\left(1+n_{t}\right)-1 \cong g_{t}+n_{t} .
\end{aligned}
$$

Die interne Rendite des Umlageverfahrens ergibt sich also näherungsweise aus der Summe der Wachstumsrate der durchschnittlichen beitragspflichtigen Bruttolöhne und der Wachstumsrate der Beitragszahler. Die interne Rendite eines Kapitaldeckungsverfahrens entspricht dagegen dem Marktzinssatz ( $i$ ), so daß ein Umlageverfahren für die Versicherten solange vorteilhaft ist, wie

$$
\text { B.9) } 1+i<\left(1+g_{t}\right)\left(1+n_{t}\right)
$$

gilt. Die Tatsache, daß ein Umlageverfahren bei konstanten Wachstumsraten und bei einer stabilen Bevölkerung eine höhere Verzinsung erlaubt als das Kapitaldeckungsverfahren, wenn die obige Ungleichung erfüllt ist, wird als Sozialversicherungsparadoxon von Aaron bezeichnet. ${ }^{31}$

28 Abweichungen zwischen der Steigerungsrate der Pro-Kopf-Rente auf der Ausgabenseite und der beitragspflichtigen Bruttoverdienste auf der Einnahmenseite können sich dann ergeben, wenn sich Netto- und Bruttoverdienste unterschiedlich entwickeln.

Der Bundeszuschuß soll hier vernachlässigt werden, weil er den Vergleich der Renditen zwischen Umlage- und Kapitaldeckungsverfahren unnötig verkomplizieren würde. Außerdem müßte dann berücksichtigt werden, daß die jeweils erwerbstätige Generation den Bundeszuschuß in Form von Steuern finanzieren muß.

Läßt man im Zeitablauf unterschiedliche Beitragssätze zu. dann erkennt man aus B.8) sofort, daß eine Erhöhung der Beitragssätze die interne Rendite der Versicherten im Umlageverfahren erhöht. Hierin liegt die Ursache für die oben erwähnten polit-ökonomischen Anreize zur Ausweitung des Systems. Beim Kapitaldeckungsverfahren ist dagegen die interne Rendite unabhängig von der Höhe des Beitragssatzes und es bestehen daher keine Anreize zur Systemausweitung.

31 Vgl. Rosen/Windisch (1992) S. 396. Als "Paradoxon" wird die Tatsache angesehen. daß das Umlageverfahren eine "Verzinsung" erlaubt, obwohl kein Kapitalstock vorhanden ist. und diese "Verzinsung" zudem noch höher ist als die eines Kapitaldeckungsverfahrens. 
Als Ergebnis dieses Abschnittes ist festzuhalten, daß der mit der Rentenreform 1957 weitgehend abgeschlossene Übergang von einem Drei-Generationen-Vertrag zu einem Zwei-Generationen-Vertrag in Form des Umlageverfahrens in der Gesetzlichen Rentenversicherung zu einer starken Anfälligkeit des Systems gegenüber demographischen Verschiebungen geführt hat. Die Gleichungen B.5) bis B.7) verdeutlichen diesen Zusammenhang. Gleichzeitig zeigen sie auf, welche Ansatzpunkte für politische Maßnahmen zur Stabilisierung des Beitragssatzes ${ }^{32}$ der Gesetzlichen Rentenversicherung bei ungünstiger demographischer Entwicklung bestehen, wenn man von dem Prinzip der dynamischen Rente und damit von einem konstanten Nettorentenniveau nicht abgehen will. Prinzipiell kommen folgende Ansatzpunkte in Betracht:

- $\quad$ Erhöhung des Bundeszuschusses

- $\quad$ Erhöhung der Zahl der Beitragszahler (z.B. über eine erhöhte Erwerbsbeteiligung, eine pronatalistische Bevölkerungspolitik oder über verstärkte Einwanderung von Erwerbstätigen)

- $\quad$ Minderung der Zahl der Rentner (z.B. über eine Heraufsetzung der Ruhestandsaltersgrenze)

Dabei ist darauf zu achten, welche Rückwirkungen die einzelnen Maßnahmen gegebenenfalls auf andere Einflußfaktoren haben, denn diese werden durch den hier vorgestellten partialanalytischen Modellrahmen nicht erfaßt. So kann beispielsweise eine Erhöhung des Rentenalters zu höherer Arbeitslosigkeit und dadurch zu einer geringeren Erwerbsbeteiligung führen als zunächst vermutet. Eine Erhöhung der Zahl der Beitragszahler führt möglicherweise über eine Erhöhung des Arbeitsangebots zu einer Minderung des Lohnsatzes und damit der Lohnsumme, so daß sich ein negativer Rückkoppelungseffekt auf die interne Verzinsung im Umlageverfahren ergibt. ${ }^{33}$ Auch die Wirkung auf wichtige makroökonomische Zielgrößen wie z.B. die Wachstumsrate des Sozialprodukts sollte berücksichtigt werden.

Eine genauere Darstellung von verschiedenen denkbaren Maßnahmen wird in Kapitel 3 erfolgen. Vorher wird in Kapitel 2 zunächst die gegenwärtige und die für die nächsten Jahrzehnte prognostizierte demographische Entwicklung sowie die daraus resultierende Finanzkrise der Gesetzlichen Rentenversicherung erläutert.

\section{Empirische Entwicklung}

Für die Betrachtung der empirischen Entwicklung ist es sinnvoll, die oben herausgearbeiteten Einflußfaktoren auf den Beitragssatz zur Gesetzlichen Rentenversicherung noch etwas weiter aufzugliedern. Aus den Gleichungen $B .5 a$ ) und $B .5 b$ ) wurde deutlich, daß der Beitragssatz von dem Prozentsatz des Bundeszuschusses, dem Nettorentenniveau und dem Rentnerquotienten bestimmt wird. Nimmt man an, daß der Prozentsatz des Bundeszuschusses konstant bleibt und das auch das Nettorentenniveau wegen des Prinzips der Nettoanpassung nicht geändert wird, dann ist die Höhe des Beitragssatzes nur noch von dem Rentnerquotienten abhängig, also von dem Verhältnis von Rentenempfängern zu Beitragszahlern. In einem ersten

32 Wie unten ausgeführt wird, ist eine alleinige Konzentration auf die Beitragssatzentwicklung unter Umständen nicht ausreichend, um die finanziellen Lasten aufzuzeigen, die aus der demographischen Entwicklung für die Gesetzliche Rentenversicherung erwachsen.

Vgl. hierzu Kapitel 1 in Teil C dieser Arbeit und Homburg/Gräff (1988) S. 24ff. 
Schritt wird in Unterkapitel 2.1 angenommen, daß sich die Zahl der Rentenempfänger proportional zu der Zahl der Personen im Alter über 60 Jahren entwickelt und die Zahl der Beitragszahler sich genauso verändert wie die Zahl der Personen im Alter zwischen 20 und 60 Jahren. Es wird also angenommen, daß das Verhältnis von Rentenempfängern zu Beitragszahlern (Rentnerquotient) nur von dem Verhältnis von über 60jährigen zu den 20 - bis 60 jährigen - dem sogenannten Altenquotienten ${ }^{34}$ - abhängt. In Unterkapitel 2.1 werden also zunächst die Auswirkungen demographischer Größen auf den Beitragssatz analysiert.

In Unterkapitel 2.2 wird dann untersucht, inwieweit Abweichungen zwischen Rentnerquotient und Altenquotient aufgrund einer Änderung der Erwerbsbeteiligung, des Renteneintrittsalters etc. zu erwarten sind, die die Einflüsse demographischer Größen auf den Beitragssatz verstärken oder abschwächen. Im darauf folgenden Abschnitt (2.3) wird der kombinierte Effekt der demographischen und anderen Variablen auf die Finanzlage der Gesetzlichen Rentenversicherung unter Berücksichtigung der vielfältigen Interdependenzen untersucht.

\subsection{Der Einfluß demographischer Variablen auf die Finanzlage der Ge- setzlichen Rentenversicherung}

In diesem Abschnitt wird untersucht, wovon die Entwicklung des Altenquotienten abhängt. Entscheidend für den Altenquotienten ist der Aufbau der Bevölkerungsstruktur. Diese kann anschaulich durch eine Alterspyramide dargestellt werden, wie dies beispielhaft für den Bevölkerungsaufbau in Deutschland für das Jahr 1990 geschehen ist (vgl. Abb. B.2). Deutlich sind die durch die beiden Weltkriege verursachten Einschnürungen im Bereich der 73- bis 76jährigen bzw. der 43- bis 49jährigen und die geburtenstarken Jahrgänge der sechziger Jahre zu erkennen.

Die Altersstruktur einer Bevölkerung ist abhängig von den altersspezifischen Sterbefällen, von der Zahl der Geburten und von den Wanderungen. Diese Einflußfaktoren sollen im folgenden separat untersucht werden.

\subsubsection{Die Mortalitätsentwicklung}

Der Einfluß der Mortalitätsentwicklung auf den Altenquotienten hängt davon ab, in welcher Altersgruppe die Mortalitätsänderung auftritt. Sterben relativ viele Personen kurz nach der Geburt wirkt dies wie eine Reduzierung der Geburten. Ist die Mortalität der Personen ab 60 Jahren besonders hoch, dann ist die Zählergröße des Altenquotienten klein und der Quotient selbst folglich eher niedrig.

34 Nach der Definition des Statistischen Bundesamtes, der hier gefolgt werden soll, umfaßt die Gruppe 20bis 60 jährigen genaugenommen die Altersjahrgänge 20 bis 59. die Gruppe der der über 60jährigen alle Altersjahrgänge ab 60. Gelegentlich finden sich auch andere Altersbegrenzungen. Für Deutschland erscheinen die gewählten Abgrenzungen sinnvoll, da die typische Erwerbsphase in etwa im Alter von 20 Jahren beginnt und mit ungefähr 60 Jahren endet. 
Abb. B.2: Bevölkerungsaufbau in Gesamtdeutschland 1990 und 2030
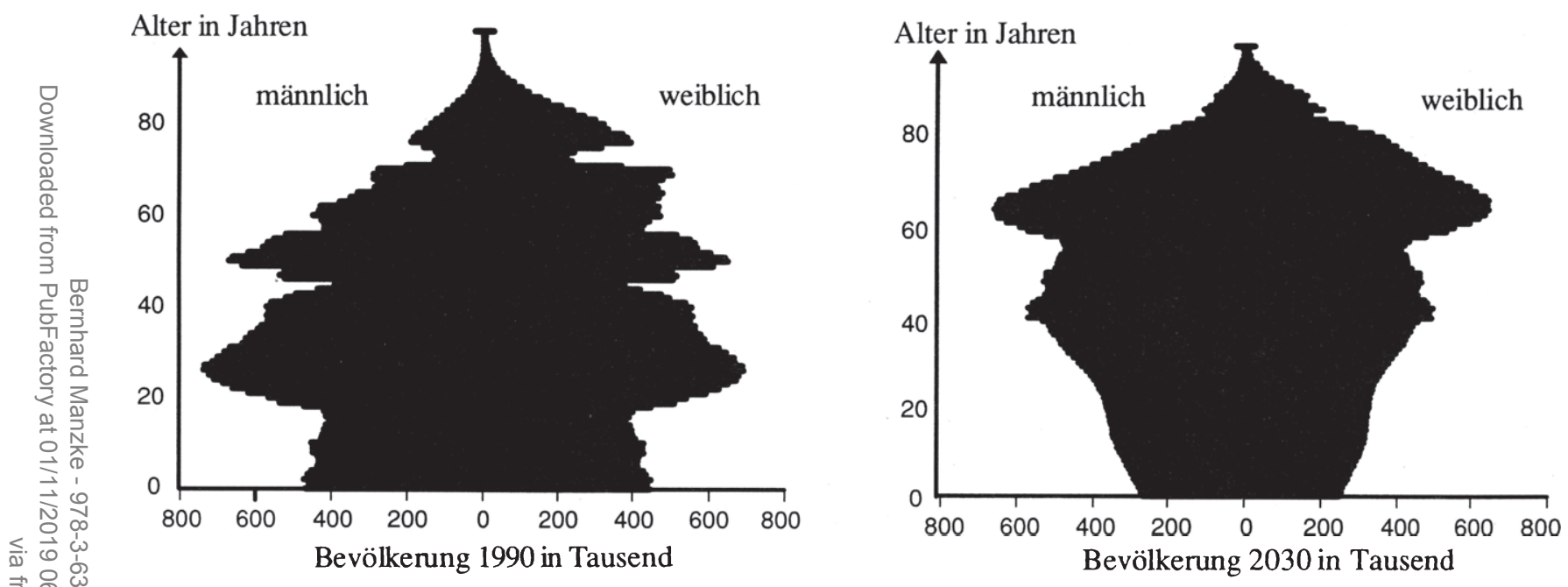

Quelle: Angaben des Statistischen Bundesamtes 
Abb. B.3: Anstieg der weiteren Lebenserwartung von Frauen im Alter von 20, 40 und 60 Jahren seit 1961

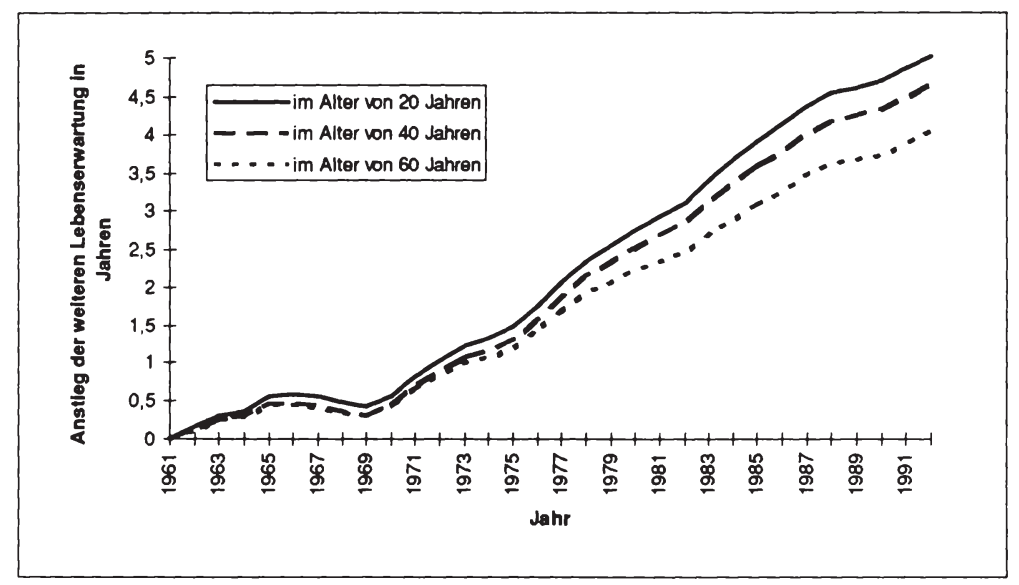

Quelle: Angaben des Statistischen Bundesamtes

Abb. B.4: Altersspezifischen Mortalitätsraten für Männer im Alter von 60 bzw. 70 Jahren im früheren Bundesgebiet

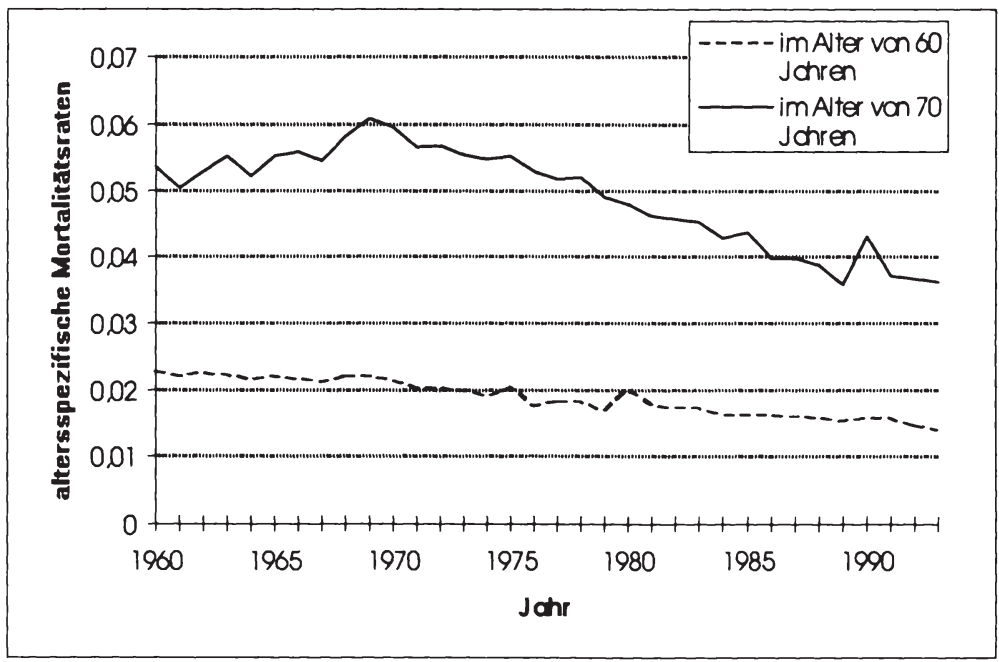

Quelle: Angaben des Statistischen Bundesamtes 
Es ist daher unmittelbar einsichtig, daß altersunspezifische Meßgrößen wie die absolute Zahl der Todesfälle eines Jahres für die Entwicklung des Altenquotienten keine Aussagekraft haben. In dem hier betrachteten Zusammenhang interessieren vielmehr altersspezifische Meßgrößen. Als Beispiel mögen die durchschnittliche restliche Lebenserwartung eines Sechzigjährigen oder altersspezifische Mortalitätsraten ${ }^{35}$ dienen. Letztere geben das Verhältnis der Todesfälle in einer Altersgruppe zu der Gesamtzahl der Personen in dieser Altersgruppe an.

Sieht man von Ausnahmeerscheinungen wie beispielsweise Kriegssituationen, Seuchen oder Hungersnöten ab, verläuft die tatsächliche Mortalitätsentwicklung ohne große Schwankungen und ist daher recht genau prognostizierbar. In den Abb. B. 3 und B. 4 kommt der generelle Trend zu einer geringeren Mortalität und daher einer steigenden Lebenserwartung zum Ausdruck. Erwähnenswert ist die wesentlich höhere Lebenserwartung der Frauen. Während Männer im Jahr 1990 bei der Geburt eine Lebenserwartung von 72,5 Jahren hatten, konnten Frauen eine Lebensdauer von 79,0 Jahren erwarten. ${ }^{36}$ Der Hauptgrund für die gestiegene Lebenserwartung in den letzten Jahrzehnten ist nicht mehr wie noch zu Beginn dieses Jahrhunderts in einer Reduktion der Säuglingssterblichkeit zu sehen, sondern in einer verminderten Sterblichkeit gerade der älteren Personen. ${ }^{37}$ Wie aus Abb. B.3 zu erkennen ist, hat sich die weitere Lebenserwartung der 60jährigen Frauen zwischen 1960 und 1992 mit einem Zuwachs von 4,03 Jahren nur geringfügig weniger erhöht als die weitere Lebenserwartung 20(Zunahme von 5,02 Jahren) bzw. 40jähriger Frauen (Zunahme von 4,65 Jahren).

$\mathrm{Zu}$ erkennen ist dieser Trend auch an den sinkenden Mortalitätsraten für 60- und 70jährige Männer in den letzten drei Jahrzehnten. Diese Entwicklung der Mortalität dürfte sich im wesentlichen in der Zukunft fortsetzen und zu einer weiteren Erhöhung des Altenquotienten beitragen.

\subsubsection{Die Fertilitätsentwicklung}

Neben der Mortalitätsentwicklung hat auch die Geburtenentwicklung einen großen Einfluß auf den Altenquotienten. Ein durch eine hohe Geburtenrate hervorgerufenes hohes natürliches Bevölkerungswachstum hat eine relativ starke Besetzung der unteren Altersgruppen zur Folge. Die jeweils nachfolgenden Jahrgänge sind stärker als die vorausgehenden, der Bevölkerungsaufbau entspricht der klassischen Pyramidenform. Daraus folgt, daß der Altenquotient relativ niedrig ist. Die Zählergröße (die Gruppe der über 60jährigen) ist klein, die Nennergröße (Bevölkerung im erwerbsfähigen Alter) dagegen groß. Nimmt die Geburtenrate aber ab, wird der Altenquotient tendenziell zunehmen.

35 Die altersspezifischen Mortalitätsraten beziehen die Zahl der Sterbenden eines Jahrgangs auf die jahresdurchschnittliche Zahl der Lebenden des Jahrgangs, wobei sich letztere aus der Halbierung der Summe von Jahresanfangs- und Jahresendbestand ergibt. Die Sterbewahrscheinlichkeit als weiteres Maß setzt dagegen den Anfangsbestand eines Jahrgangs zum Endbestand nach Ablauf eines Jahres in Beziehung. Vgl. Dinkel (1989) S. 69f.

36 Vgl. Enquete-Kommission "Demographischer Wandel" (1994) S. 26. Die Zahlen beziehen sich auf das frühere Bundesgebiet. In den neuen Bundesländern liegt die Lebenserwartung gegenwärtig noch um über zwei Jahre niedriger, aber im Zuge der Angleichung der Lebensverhältnisse ist dort mit einer Erhöhung der Lebenserwartung zu rechnen.

Vgl. Enquete-Kommission "Demographischer Wandel" (1994) S. 29. 
Natürlich treten diese Wirkungen auf den Altenquotienten nicht sofort nach einer Änderung der Geburtenrate auf. Zieht man die geburtenstarken Jahrgänge der sechziger Jahre als Beispiel heran, dann wird deutlich, daß sie den Altenquotienten bis Anfang der achtziger Jahre nicht beeinflussen. Danach führen sie ceteris paribus zu einem stärkeren Anstieg der Gruppe der 20bis 60jährigen und damit zu einer Abnahme des Altenquotienten. Nach dem Jahr 2020 schließlich werden diese Jahrgänge das sechzigste Lebensjahr überschreiten und damit gleichzeitig zu einem Rückgang der Zahl der 20- bis 60jährigen und einem Anstieg der Zahl der Älteren führen, so daß dann der Altenquotient drastisch steigen wird.

Weiterhin ist zu beachten, daß die Frauen der geburtenstarken Jahrgänge ihrerseits zwischen Mitte der achtziger und Ende der neunziger Jahre die meisten ihrer Kinder bekommen werden. Auch wenn die Kinderzahl pro Frau nicht überdurchschnittlich hoch ist, wird allein aufgrund der großen Zahl der Frauen im gebärfähigen Alter in diesem Zeitraum die absolute Geburtenzahl ansteigen. Ab dem Jahr 2005 überschreiten diese Kinder die Altersgrenze von 20 Jahren und haben dann eine entlastende Wirkung auf den Altenquotienten.

Die Geburtenentwicklung hat also einen verzögerten Einfluß auf den Altenquotienten, der sich zudem auch noch im Zeitablauf der Richtung nach ändern kann.

Wie sah die Geburtenentwicklung ${ }^{38}$ in der Vergangenheit aus? Zur Beantwortung dieser Frage muß man zunächst festlegen, welche Meßgrößen für die Fertilität herangezogen werden sollen. Die verschiedenen Meßkonzepte werden hier recht ausführlich dargestellt, damit in Teil $\mathrm{C}$ der Arbeit, in dem es um die Beeinflußbarkeit des generativen Verhaltens geht, auf sie zurückgegriffen werden kann.

Zuerst sollen verschiedene Querschnittsmaße erläutert werden. Für die Entwicklung des Altenquotienten ist sicherlich die absolute Zahl der Geburten in einem Kalenderjahr entscheidend. Will man allerdings langfristige Vorhersagen der Geburtenzahl treffen, dann ist es sinnvoll, die verschiedenen Faktoren, die die Geburtenzahl beeinflussen (z.B. Änderungen des Altersaufbaus der Bevölkerung und des generativen Verhaltens), zu isolieren.

Dazu dient in einem ersten Schritt die rohe Geburtenrate. Sie bezieht die Geburtenzahl auf 1000 Personen und schaltet so Schwankungen in der absoluten Bevölkerungsgröße aus. Allerdings wird die Struktur der Bevölkerung nicht berücksichtigt. Wenn unter 1000 Personen in einem bestimmen Zeitraum besonders viele Frauen im fruchtbaren, d.h. gebärfähigen Alter ${ }^{39}$ sind, wird die rohe Geburtenrate steigen, auch wenn die Zahl der Kinder pro Frau unverändert geblieben ist.

Um diesen Effekt auszuschalten, bezieht die allgemeine Fertilitätsrate die Zahl der Geburten auf 1000 Frauen im gebärfähigen Alter. Von speziellen Fertilitätsraten spricht man, wenn nur eine bestimmte Gruppe von Frauen in die Nennergröße eingeht (z.B. nur Ausländerinnen oder nur Verheiratete).

Längsschnittmaße erfassen im Gegensatz zu den oben beschriebenen Querschnittsmaßen nicht die Situation während eines bestimmten Zeitraumes für Frauen unterschiedlicher Geburts-

Korrekterweise müßte jeweils von der "Geborenenentwicklung" gesprochen werden, da die Zahl der Geburten und die Zahl der Geborenen aufgrund von Mehrlingsgeburten auseinanderfallen kann (vgl. Zameck-Glyscinski (1985) S. 15 FN2). Da diese Unterscheidung in der Literatur nicht eingeführt ist, wird dennoch im folgenden von "Geburten" gesprochen. 
jahrgänge, sondern messen die Fertilität im Laufe des Lebens einer (hypothetischen) Kohorte ${ }^{40}$. Sie sind eher als Querschnittsmaße dazu geeignet, Änderungen des generativen Verhaltens zu verdeutlichen.

Die altersspezifischen Fertilitätsraten geben das Verhältnis der Lebendgeborenen, die in einem Kalenderjahr von Frauen eines bestimmten Alters zur Welt gebracht wurden, zur jahresdurchschnittlichen Anzahl der Frauen dieses Alters an. Summiert man die altersspezifischen Fertilitätsraten eines Kalenderjahres für die verschiedenen Altersjahrgänge der Frauen, dann erhält man die zusammengefaßte Geburtenrate ${ }^{41}$ (auch totale Fertilitätsrate genannt). Sie gibt an, wieviele Kinder eine Frau durchschnittlich zur Welt bringen würde, wenn sie während ihres gesamten Lebens den altersspezifischen Geburtenraten des betreffenden Kalenderjahres unterläge. Es handelt sich somit um die Kinderzahl einer hypothetischen Kohorte.

Die Nettoreproduktionsrate als weiteres gebräuchliches Fertilitätsmaß liefert die durchschnittliche Zahl der lebendgeborenen Mädchen während der Lebenszeit einer Frau, welche den altersspezifischen Mortalitäts- und Fertilitätsraten unterliegt, die in einem bestimmten Kalenderjahr ermittelt wurden. Sie gibt damit an, inwieweit eine Frauengeneration unter den Mortalitäts- und Fertilitätsverhältnissen eines bestimmten Kalenderjahres durch die von ihr geborene Töchtergeneration ersetzt wird. Im Unterschied zu der zusammengefaßten Geburtenrate ist in der Nettoreproduktionsrate berücksichtigt, daß erstens ein lebendgeborenes Mädchen mit einer gewissen Wahrscheinlichkeit das Ende des gebärfähigen Alters gar nicht erreicht und zweitens, daß die Zahl der Knabengeburten regelmäßig über der Zahl der Mädchengeburten liegt. Deshalb wird bei den gegenwärtigen Sterblichkeitsverhältnissen eine zusammengefaßte Geburtenrate von etwa 2,1 benötigt, um das Bestandserhaltungsniveau und damit eine Nettoreproduktionsrate von 1,0 zu erreichen.

Die durchschnittliche Kinderzahl einer Frauenkohorte nach Abschluß der Fruchtbarkeitsphase bezeichnet man als endgültige Kinderzahl. Da es sich hierbei um ein Kohortenmaß handelt, ist die endgültige Kinderzahl immer nur für einen bestimmten Geburtsjahrgang, nicht aber für ein Kalenderjahr angebbar. Die endgültige Kinderzahl ist zur Darstellung von Änderungen des generativen Verhaltens am besten geeignet, weil sie nicht wie die Periodenmaße "zusammengefaßte Geburtenrate" und "Nettoreproduktionsrate" durch Vor-bzw. Nachholeffekte verzerrt wird. Letztere können entstehen, weil die Periodenmaße auf den Fertilitäts(und Mortalitäts-) -verhältnissen eines bestimmten Kalenderjahres aufbauen und diese dann auf eine hypothetische Frauenkohorte übertragen. So kann es zu einem überhöhten Ausweis der Fertilität kommen, wenn in dem betrachteten Kalenderjahr ältere Frauen Geburten nachholen, die sie z.B. aufgrund eines Krieges in ihren jüngeren Lebensjahren verschoben haben. Wenn dann gleichzeitig die jüngeren Frauen Geburten vorverlagern (z.B. infolge besonderer Maßnahmen des Kinderlastenausgleichs), wird eine hohe Periodenfertilität ausgewiesen, obwohl die endgültige Kinderzahl aller beteiligten Kohorten unverändert geblieben ist. ${ }^{42} \mathrm{Nach}$ Dinkel erklärt alleine ein derartiger kombinierter Vor- und Rückverlagerungseffekt einen Anstieg der Geburten um 20 bis 25 Prozent in den Babyboom-Jahren. ${ }^{43}$ Die Geburten-

40 Mit dem Begriff "Kohorte" wird ein bestimmter Jahrgang, hier ein bestimmter Geburtsjahrgang, bezeichnet.

41 In der Literatur ist auch der Ausdruck "Geburtenziffer" eingeführt. Da das Wort "Ziffer" einstellige Zahlen bezeichnet, ist die Bezeichnung "zusammengefaßte Geburtenrate" vorzuziehen.

42 Synonym zu Vor- und Nachholeffekten wird auch von timing und spacing Effekten gesprochen, wobei "timing" das Alter der Mutter bei der Geburt (des ersten Kindes) und "spacing" den Abstand zwischen den Geburten einer Frau bezeichnet. 
verlagerung zeigt sich auch darin, daß das durchschnittliche Alter der Mutter bei der Geburt von 27,9 Jahren für die Kohorte 1930 auf 25,6 Jahre für die Kohorte 1946 zurückgegangen ist und danach wieder anstieg. ${ }^{44}$

Die endgültige Kinderzahl wird zwar nicht durch derartige Vor- und Nachholeffekte verzerrt, hat aber leider den Nachteil, daß sie erst nach Abschluß der Kohortenfertilität zur Verfügung steht - für den Geburtsjahrgang 1970 beispielsweise erst im Jahr 2015. Deshalb muß zur Analyse der aktuellen Entwicklung des generativen Verhaltens auf die theoretisch unbefriedigenderen Periodenmaße der zusammengefaßten Geburtenrate und der Nettoreproduktionsrate ausgewichen werden.

Die tatsächliche Fertilitätsentwicklung nach dem Zweiten Weltkrieg ist für die Bundesrepublik Deutschland in den Abbildungen B.5 bis B.7 anhand des Verlaufs der absoluten Geburtenzahlen, der zusammengefaßten Geburtenrate und der endgültigen Kinderzahl der Kohorten 1930 bis 1950 dargestellt. ${ }^{45}$ Deutlich zu erkennen ist in Abb. B.5 der "Geburtenberg" Mitte der sechziger Jahre, der nachfolgende dramatische Rückgang und das Verharren auf niedrigem Niveau seit Anfang der siebziger Jahre mit einem leichten Anstieg ab Mitte der achtziger Jahre bei der Zahl der Geborenen.

Die zusammengefaßte Geburtenrate zeigt einen ähnlichen Verlauf, allerdings sind die Schwankungen weniger ausgeprägt und der Geburtenanstieg Ende der achtziger Jahre ist kaum noch zu erkennen. Das spricht dafür, daß er ausschließlich auf einen "Echoeffekt" der gebur-

Abb. B.5: Lebendgeborene in Westdeutschland zwischen 1950 und 1994

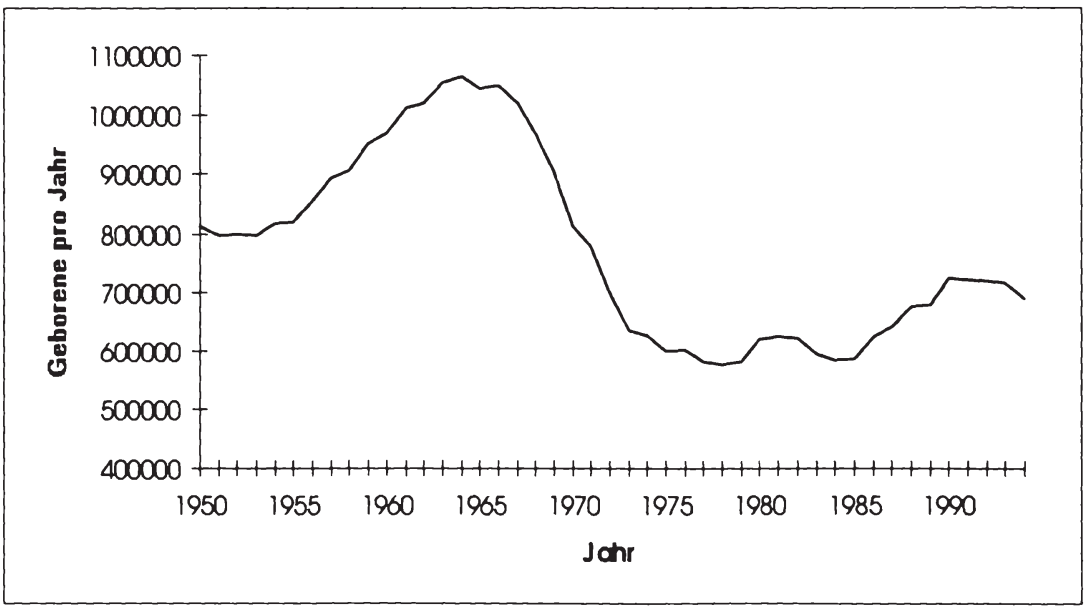

Quelle: Angaben des Statistischen Bundesamtes

44 Vgl. Eurostat (1992) S. 35.

45 Alle Daten in diesem Unterkapitel beziehen sich auf die Bundesrepublik Deutschland nach dem Gebietsstand vor dem 3.10.1990. 
Abb. B.6: Zusammengefaßte Geburtenrate im früheren Bundesgebiet zwischen 1950 und 1994

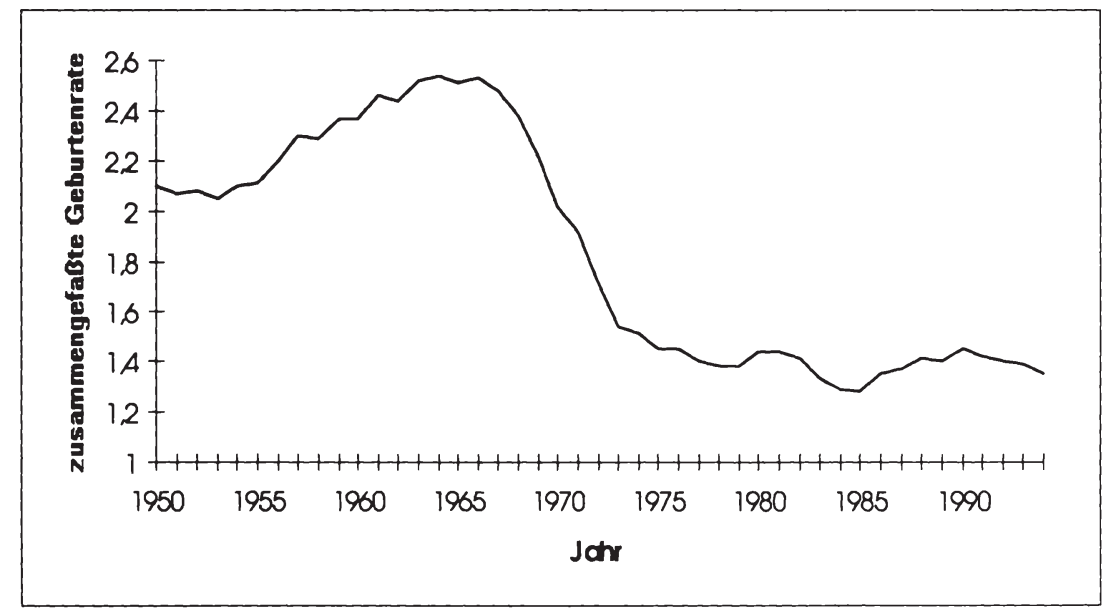

Quelle: Angaben des Statistischen Bundesamtes

Abb. B.7: Endgültige Kinderzahl der Frauenjahrgänge 1930 bis 1954 im früheren Bundesgebiet

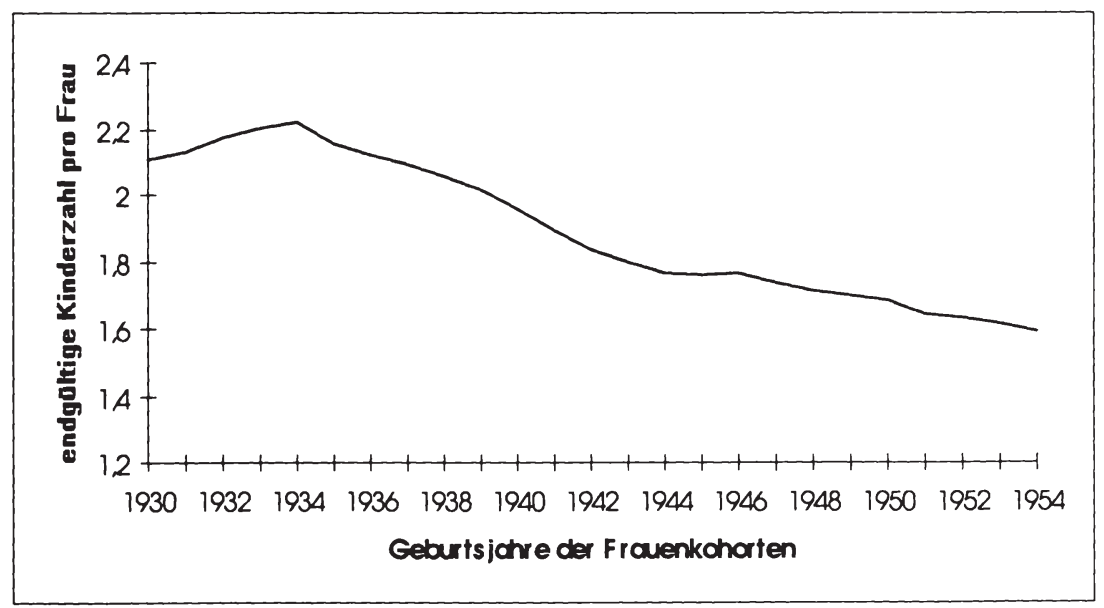

Quelle: Angaben des Statistischen Bundesamtes 
tenstarken Jahrgänge der Sechziger zurückzuführen ist, die in diesem Zeitraum in das fruchtbare Alter eintreten, und nicht etwa auf eine Änderung des generativen Verhaltens.

Kaum noch zu erkennen ist die oben beschriebene Entwicklung, wenn man die Kohortendaten in Abb. B.7 betrachtet. Nach einem leichten Anstieg bis zur Kohorte 1934 folgt ein recht gleichmäßiger Rückgang der endgültigen Kinderzahl pro Frau. Dies spricht dafür, daß die bei der absoluten Geborenenzahl und in schwächerem Maße auch bei der zusammengefaßten Geburtenrate zu beobachtenden Schwankungen hauptsächlich auf Vor- und Nachholeffekten beruhen. Der durch das Kohortenmaß erfaßte langfristige Trend des Fertilitätsverhaltens zeigt dagegen ohne große Ausschläge nach unten, so daß für die Zukunft ein von kurzfristigen Schwankungen überlagertes weiteres Absinken auch der Periodenmaße zu erwarten ist.

\subsubsection{Die Entwicklung der Wanderungsbewegungen}

Der dritte Einflußfaktor auf die Bevölkerungsentwicklung sind die Wanderungsbewegungen. Die Messung der Wanderungsbewegungen ist relativ einfach. ${ }^{46}$ Allerdings ist die absolute Zahl der Zuwanderer und der Auswanderer für die Analyse der Wirkung auf den Altenquotienten kaum geeignet, sondern es spielen, wie schon bei der Beurteilung der Mortalitätsentwicklung, die jeweiligen Altersgruppen eine entscheidende Rolle. Wandern v.a. Personen im höheren Alter $a b$, um im sonnigen Süden einen angenehmen Lebensabend zu verbringen, während die Zuwanderer überwiegend Personen jüngeren Alters sind, dann kann der Altenquotient auch bei einem Außenwanderungssaldo $0^{47}$ von Null gravierend zurückgehen. ${ }^{48}$ Dabei ist $\mathrm{zu}$ beachten, daß auch junge Zuwanderer irgendwann das sechzigste Lebensjahr überschreiten und dann den Altenquotienten erhöhen.

Die tatsächliche Entwicklung der Wanderungsbewegungen zeigt, daß die Bundesrepublik Deutschland faktisch seit ihrer Gründung ein Zuwanderungsland ist. Nur während der kurzen Phasen wirtschaftlicher Rezession hat es in der Vergangenheit einen leicht negativen Außenwanderungssaldo gegeben, da in Jahren mit geringem Wirtschaftswachstum die sonst recht konstante Zahl der Fortzüge leicht anstieg und die weit stärker schwankende Zahl der Zuzüge stark zurückging (vgl. Abb. B.8).

Im einzelnen lassen sich die folgenden Phasen unterscheiden: ${ }^{49}$ Als Folge des Zweiten Weltkrieges wurden bis 1950 etwa 12 Mio Deutsche aus Osteuropa vertrieben, von denen etwa 8 Mio nach Westdeutschland kamen. ${ }^{50}$ In den folgenden Jahren bis zum Mauerbau ging

46 Probleme bereiten v.a. illegale und daher nicht registrierte Grenzübertritte, deren Zahl nach dem Fall des Eisernen Vorhangs stark gestiegen sein dürfte.

47 Der Außenwanderungssaldo gibt die Differenz zwischen Zuwanderern und Abwanderem während einer Periode, meistens einem Jahr, an. Er wird oft auch als Nettozuwanderung bezeichnet.

48 Eine Änderung des Rentnerquotienten aus der Abwanderung älterer Personen ergibt sich nur, insofern diese durch die Wanderung Rentenansprüche verlieren (vgl. dazu §§ 110ff. SGB VI sowie Abschnitt 3.3.3 in Teil D dieser Arbeit).

Vgl. Zimmermann (1991) S. 4f. und Barabas (1992) S. 136.

Die folgenden Zahlenangaben sind Daten des Statistischen Bundesamtes oder stammen aus Barabas u.a. (1992) und beziehen sich bis 1990 auf das alte Bundesgebiet. 
Abb. B.8: Zuzüge, Fortzüge und Wanderungssaldo für die frühere Bundesrepublik Deutschland, ab 1990 für Gesamtdeutschland

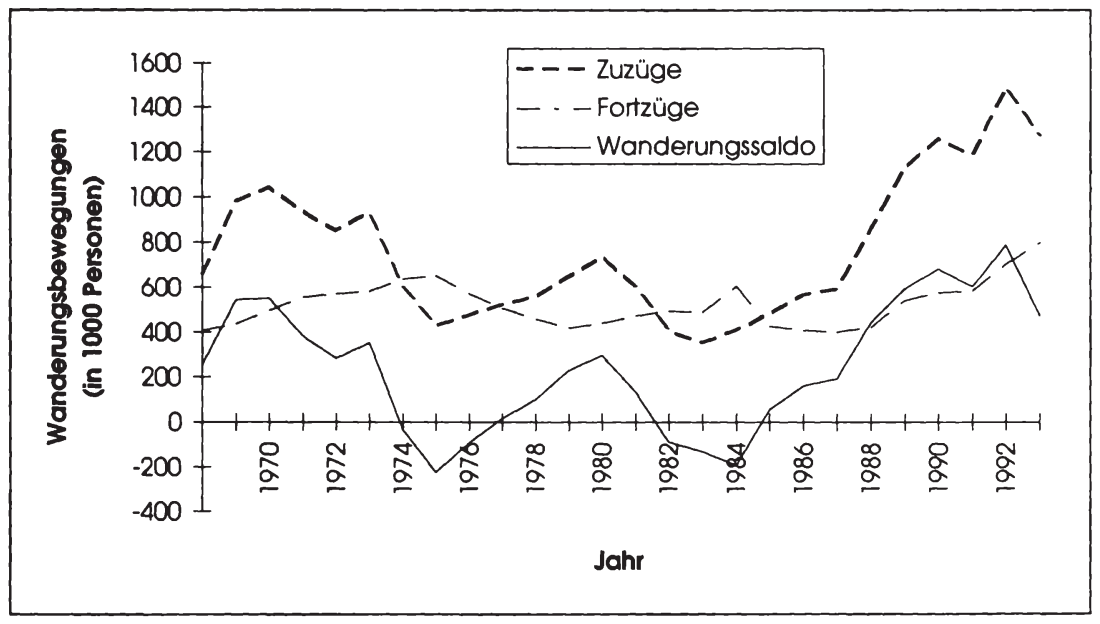

Quelle: Eigene Berechnungen aus Angaben des Statistischen Bundesamtes

Abb. B.9: Vergleich der Altersstruktur der inländischen Bevölkerung und des Zuwanderungsüberschusses (1992)

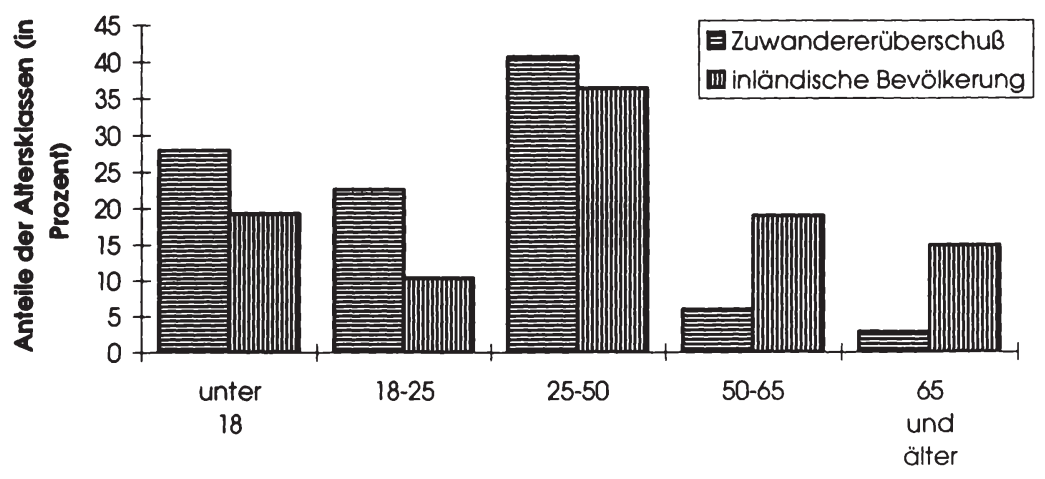

Altersklassen (in Jahren)

Quelle: Eigene Berechnungen aus Angaben des Statistischen Bundesamtes 
die Höhe des Wanderungsüberschusses etwas zurück. Dennoch ergab sich zwischen 1950 und 1961 ein kumulierter positiver Wanderungssaldo von fast 3,5 Mio, der v.a. auf die große Zahl der Übersiedler aus der ehemaligen DDR zurückzuführen ist.

Der wirtschaftliche Aufschwung führte schon Ende der fünfziger Jahre und verstärkt nach dem Mauerbau zu einem Arbeitskräftemangel in der Bundesrepublik, weshalb in den sechziger Jahren systematisch ausländische Arbeitskräfte angeworben wurden. Bis zum Anwerbestopp im Jahr 1973 stieg im Rahmen dieser Gastarbeiterwelle die ausländische Bevölkerung auf nahezu 4 Mio an.

Die restriktivere Zuwanderungspolitik ab 1973 reduzierte den Zustrom ausländischer Arbeitskräfte. Dennoch stieg die ausländische Bevölkerung durch die verstärkte Neigung zum Daueraufenthalt in der Bundesrepublik und die Familienzusammenführung bis Mitte der achtziger Jahre auf etwa 4,4 Mio an.

Ausgelöst durch die politischen Umwälzungen in Osteuropa setzte dann ab 1988 eine neue Zuwanderungswelle ein, die v.a. aus Übersiedlern aus der ehemaligen DDR, Aussiedlem aus Osteuropa und einer steigenden Zahl von Asylbewerbern bestand, und zwischen 1988 und 1991 über 3,6 Mio Zuwanderer in die Bundesrepublik trug. Durch die faktische Begrenzung des Zuzugs von Aussiedlern, die Verschärfung des Asylrechts und den Wegfall der Übersiedler aus der Statistik ist die Zahl der Zuwanderer für Gesamt-Deutschland in den letzten Jahren zwar zurückgegangen, dennoch ist auch für die nächsten Jahre mit hohen positiven Wanderungssalden zu rechnen.

Die Wirkung des positiven Wanderungssaldos auf den Altersaufbau der Bevölkerung wird erkennbar, wenn man die Altersstruktur des Wanderungsüberschusses der Altersstruktur der Gesamtbevölkerung gegenüberstellt. Aus Abb. B.9 wird deutlich, daß durch die Außenwanderungsbewegungen eine Verjüngung der Gesamtbevölkerung erfolgt. Der Überschuß der Zuzüge über die Fortzüge ist gerade in den jungen Jahrgängen recht hoch, sein Anteil in den Altersklassen ab 50 Jahren dagegen wesentlich geringer als bei der heimischen Bevölkerung.

Verstärkt wird diese verjüngende Wirkung durch zwei Sekundäreffekte. Zum einen wandern besonders viele Personen im reproduktiven Alter zu, so daß sich durch die Veränderung der Altersstruktur die Geburtenzahl erhöht. Zum anderen weisen die Zuwanderer zumindest in der ersten Generation ein anderes Fertilitätsverhalten auf, d.h. ihre Nettoreproduktionsrate ist höher, wodurch die Geburtenzahl zusätzlich erhöht wird. ${ }^{51}$

\subsubsection{Bevölkerungsprognosen}

Für die zukünftige Höhe des Altenquotienten und damit ceteris paribus des Beitragssatzes der Versicherten zur Gesetzlichen Rentenversicherung ist das Zusammenspiel von Mortalität, Fertilität und Wanderungen entscheidend. Die am weitesten verbreitete Verfahrensweise für Bevölkerungsprognosen ist daher die sogenannte Komponentenmethode ${ }^{52}$, nach der die Berechnung der Gesamtentwicklung der Bevölkerung in die oben genannten Komponenten zerlegt wird. Ausgangspunkt dieser Vorausschätzungsmethode ist der tatsächliche Bevölkerungsaufbau nach Alter und Geschlecht zu einem bestimmten Zeitpunkt, der

51 Vgl. Enquete-Kommission "Demographischer Wandel" (1994) S. 32.

52 Die Komponentenmethode wird bei nahezu allen neueren demographischen Prognosen verwandt. 
normalerweise den Ergebnissen der letzten Volkszählung oder einer Bevölkerungsfortschreibung entnommen wird. Dieser Bevölkerungsaufbau wird dann von Jahr zu Jahr fortgeschrieben.

Die Besetzung der einzelnen Altersjahrgänge von Frauen und Männern im jeweils folgenden Kalenderjahr kann bestimmt werden, indem die Besetzung des Vorjahres um die Todesfälle in

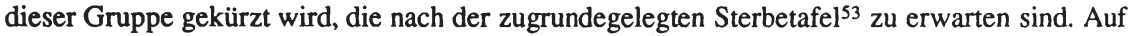
diese Weise lassen sich von wanderungsbedingten Änderungen abgesehen alle Jahrgangsstärken bis auf den der unter Einjährigen ermitteln. Letzterer wird in drei Schritten bestimmt. Zuerst multipliziert man die Besetzung der gebärfähigen Altersjahrgänge der weiblichen Bevölkerung mit den entsprechenden altersspezifischen Fertilitätsraten und erhält so die Geburtenzahl des entsprechenden Kalenderjahres. Die so ermittelte Gesamtzahl der Geburten wird dann entsprechend der Sexualproportion bei der Geburt in Mädchen und Jungen aufgeteilt. Um zur Besetzung des jüngsten Jahrganges zu gelangen, muß dann in einem dritten Schritt die Mortalitätsrate bei Säuglingen berücksichtigt werden.

Um den Aufbau der Gesamtbevölkerung um ein Jahr fortzuschreiben, muß dann nur noch die dritte Komponente, nämlich die alters- und geschlechtsspezifischen Wanderungssalden, eingerechnet werden.

Die z.T. beträchtlichen Unterschiede zwischen den Ergebnissen verschiedener Bevölkerungsprognosen sind weniger auf die Verwendung unterschiedlicher Prognosemethoden und unterschiedlicher Datensätze zurückzuführen als auf unterschiedliche Annahmen bezüglich der Mortalität, der Fertilität und der Migration, ohne die die Komponentenmethode nicht auskommt. ${ }^{54}$ Am geringsten sind die Abweichungen aufgrund unterschiedlicher Mortalitätsannahmen, denn in "normalen" Zeiten ohne Kriege, Epidernien und Hungersnöte ändern sich die Sterbetafeln nur wenig. Die Änderungen entsprechen zudem langfristigen, recht stabilen Trends. In der unten dargestellten achten koordinierten Bevölkerungsvorausberechnung des Statistischen Bundesamtes wird beispielsweise die Periodensterbetafel ${ }^{55}$ von 1992 verwendet und angenommen, daß die Sterblichkeit bis zum Jahr 2000 entsprechend dem Trend weiter sinkt und anschließend konstant bleibt. ${ }^{56}$ Demgegenüber verwendet Bomsdorf Generationen-

53 Sterbetafeln zeichnen die Erlebenswahrscheinlichkeiten von je 100.000 Personen, die in einem Jahr geboren wurden, über die Lebensaltersstufen von 0 bis 100 Jahren nach. Vgl. Dinkel (1989) S. 71.

Die Annahmen zu den einzelnen Einflußfaktoren können nicht unabhängig voneinander festgelegt werden, da gegenseitige Abhängigkeiten bestehen. So hat z.B. ein höherer Wanderungssaldo einen Einfluß auf die Fertilitätsentwicklung, wenn das Fertilitätsverhalten der Zuwanderer zumindest für eine gewisse Zeit systematisch von dem Fertilitätsverhalten der übrigen Bevölkerung abweicht. Umgekehrt ist nicht auszuschließen, daß eine dauerhaft geringe Fertilität als pull-Faktor bezüglich der Zuwanderung wirkt, wie der in Teil D dieser Arbeit analysierte Vorschlag der Erhöhung der Zuwanderung zur Stabilisierung der umlagefinanzierten Sozialversicherungssysteme verdeutlicht.

Periodensterbetafeln spiegeln die Mortalitätsverhältnisse eines bestimmten Zeitraums, beispielsweise des Jahres 1992 wider. Bei der Verwendung von Periodensterbetafeln für Prognosezwecke wird damit implizit unterstellt, daß die Sterblichkeit nur vom Alter abhängt und nicht z.B. auch von der unterschiedlichen Biographie verschiedener Geburtsjahrgänge. Nur dann sind nämlich die Sterblichkeitsverhältnisse einer Generation auf eine andere übertragbar. Will man dagegen den Einfluß unterschiedlicher Lebensläufe verschiedener Geburtsjahrgänge (z.B. durchlittene Gesundheitsbeeinträchtigungen während des letzten Krieges) berücksichtigen, dann ist die Verwendung von Kohortensterbetafeln (Bomsdorf spricht von Generationensterbetafeln) vorzuziehen, die die Absterbeordnung eines bestimmten Geburtsjahrganges wiedergeben. Vgl. Bomsdorf (1994) S. 91 und Dinkel (1984a) S. 54. allmählichen Angleichung an die Verhältnisse in Westdeutschland bis zum Jahr 2030 ausgegangen. 
sterbetafeln ${ }^{57}$ und geht von einem kontinuierlichen Rückgang der Mortalität aus. ${ }^{58}$ Aufgrund dieser unterschiedlichen Annahmen hinsichtlich der Mortalitätsentwicklung erhält er für die Gruppe der über 60jährigen einen um 15\% höheren Bevölkerungsumfang im Jahr 2050.59

Die altersspezifischen Fertilitätsraten ändern sich im Vergleich zu den altersspezifischen Mortalitätsraten recht kurzfristig und zudem auch relativ stark. Sie stellen daher eine große potentielle Fehlerquelle dar, v.a. wenn die Prognose über einen Zeitraum von über 20 Jahren hinausgeht und damit der Eintritt von Geburtsjahrgängen, deren Besetzung bereits auf Prognosewerten beruht, in das gebärfähige Alter zu einer Verstärkung der Abweichungen führt. In der achten koordinierten Bevölkerungsvorausberechnung wurde unterstellt, da $B$ die altersspezifischen Geburtenraten von 1992 auch in Zukunft Gültigkeit haben und die zusammengefaßte Geburtenrate daher konstant bei 1,4 Kindern je Frau liegt. ${ }^{60}$

Noch volatiler als die Fertilitätsraten sind die altersspezifischen Wanderungssalden, wie oben gezeigt wurde. Oft werden daher in Bevölkerungsprognosen verschiedene Szenarien bezüglich der Geburten- und Wanderungsentwicklung berechnet. So ist es möglich im Sinne einer Sensitivitätsanalyse den Einfluß bestimmter Annahmen zu verdeutlichen. Die im folgenden ausführlicher dargestellten Ergebnisse der achten koordinierten Bevölkerungsvorausberechnung beinhalten drei verschiedene Szenarien hinsichtlich des Wanderungsüberschusses von Ausländern. In Variante 1 wird ein Rückgang des Überschusses auf 100.000 , in Variante 2 auf 200.000 und in Variante auf 300.000 Personen pro Jahr ab der Jahrtausendwende unterstellt. Bei der Zuwanderung von Aussiedlern werden im Gegensatz zur Entwicklung der Wanderungen von Ausländern keine Szenarien unterschieden, sondern die unterstellte $\mathrm{Zu}$ wanderung beträgt einheitlich etwa 220.000 Personen in den ersten Jahren und sinkt dann allmählich ab, so daß für die deutsche Bevölkerung ab 2011 ein ausgeglichener Wanderungssaldo besteht. ${ }^{61}$

Nachdem die Verfahrensweise und die Bedeutung der Annahmen bei Bevölkerungsvorausberechnungen geklärt worden sind, wird nun auf die Ergebnisse der letzten, d.h. der achten koordinierten Bevölkerungsvorausberechnung des Statistischen Bundesamtes eingegangen. ${ }^{62}$ Die absolute Größe der Bevölkerung wird aufgrund des hohen positiven Außenwanderungssaldos bis in das erste Jahrzehnt des kommenden Jahrtausends hinein zunehmen. Danach wird der Wanderungssaldo durch den immer höher werdenden Überschuß der Sterbefälle über die Geburten überkompensiert, so daß die Gesamtbevölkerung immer schneller sinkt und in der Variante 1 im Jahr 2040 mit 67,6 Mio (Variante 2: 72,4 Mio; Variante 3: 77,1 Mio) um

\section{$57 \quad$ Vgl. Fußnote 55.}

$58 \quad$ Vgl. Bomsdorf (1994) S. $90 f$.

59 Vgl. Bomsdorf (1994) S. 93ff. Bomsdorf bezieht sich bei dem Vergleich auf die Ergebnisse der siebten koordinierten Bevölkerungsvorausberechnung, die in Sommer (1992) wiedergegeben sind.

60 Für die neuen Bundesländer wurde angenommen, daß nach dem Geburteneinbruch Anfang der neunziger Jahre bis 2005 (in zwei Ländern erst bis 2010) eine Angleichung an das westdeutsche Niveau stattfindet. Vgl. Sommer (1994) S. 497.

61 Vgl. Sommer (1994) S. 498.

62 Eine von der Prognos AG durchgeführte Bevölkerungsprognose kommt genauso wie eine Prognose des DIW bei leicht unterschiedlichen Annahmen bezüglich der Geburtenentwicklung und der Mortalität zu ähnlichen Resultaten für zwei hinsichtlich der Wanderungssalden unterschiedliche Szenarien (vgl. Prognos AG (1995) bzw. o.V. (1993a)). Auch die Modellrechnung von Birg/Flöthmann (1993) weicht nicht wesentlich von den Ergebnissen des Statistischen Bundesamtes ab. 
Abb. B.10: Prognostizierte Entwicklung der Gesamtbevölkerung Deutschlands zwischen 1993 und 2030

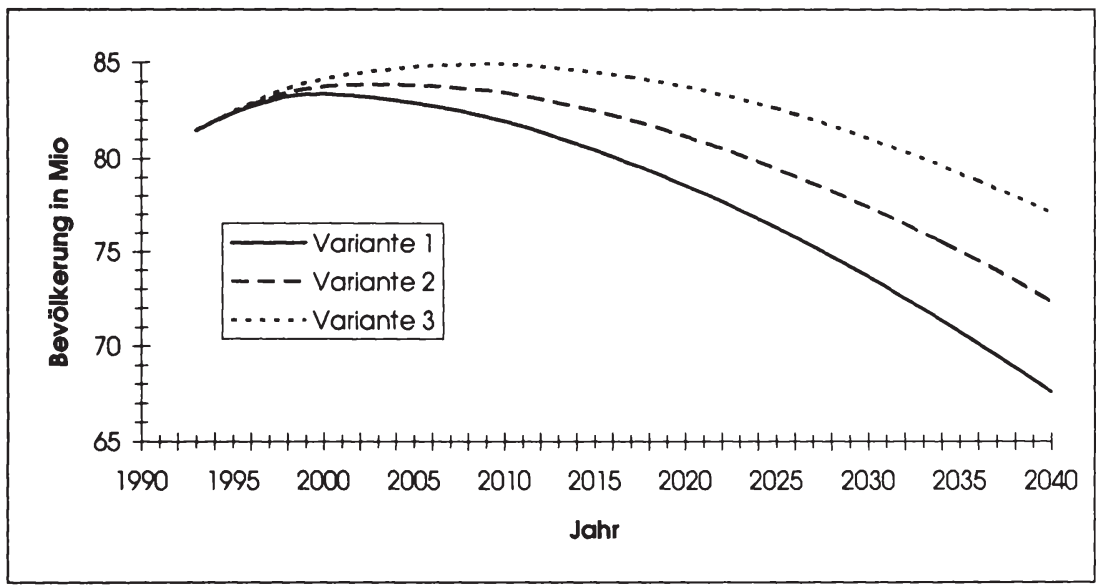

Quelle: Angaben des Statistischen Bundesamtes (Daten der achten koordinierten Bevölkerungsvorausberechnung)

Abb. B.11: Die Entwicklung des Altenquotienten zwischen 1993 und 2040

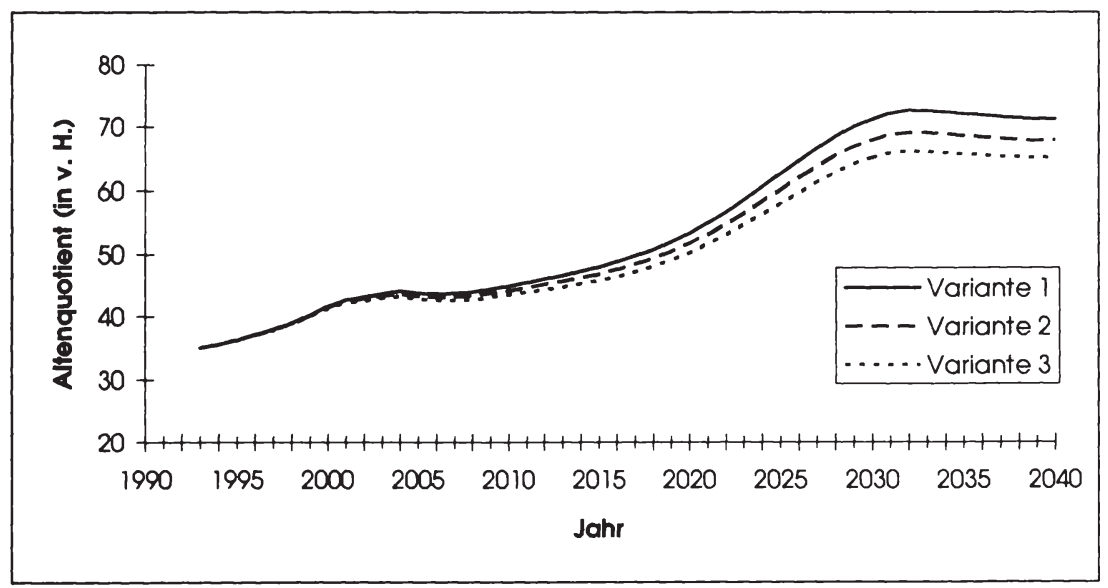

Quelle: Angaben des Statistischen Bundesamtes (Daten der achten koordinierten Bevölkerungsvorausberechnung) 
15,7 Mio (11,4 Mio; 7,8 Mio) unter dem Höchststand von 83,3 Mio (83,8 Mio; 84,9 Mio) im Jahr 2000 (2003; 2010) liegt (vgl. Abb. B.10).

Wichtiger als die Entwicklung der absoluten Bevölkerungsgröße sind in bezug auf die Alterssicherung die Verschiebungen im Altersaufbau. Bei dem Vergleich der Alters"pyramiden" von 1990 und 2030 zeigt sich die drastische Veränderung der Altersstruktur (vgl. Abb. B.2). 1990 liegt das Schwergewicht bei den Altersgruppen der 25- bis 35jährigen, den geburtenstarken Jahrgängen der sechziger Jahre. Deutlich erkennbar ist auch der nachfolgende Geburtenrückgang, der zu einer geringen Besetzung der Altersjahrgänge unter 20 führt. Im Jahr 2030 ist diese Struktur - um 40 Altersjahre verschoben - noch wiederzuerkennen. Die geburtenstarken Jahrgänge verursachen den Schirm des Pilzes im Bereich der 60- bis 70jährigen, der Geburtenrückgang ab etwa 1970 führt - unterbrochen durch den leichten Geburtenanstieg in den achtziger Jahren - zu einer zunehmenden Verjüngung des Pilzstils nach unten. Das zunehmende Gewicht der Älteren zeigt sich auch in der Entwicklung des Altenquotienten. Während die Zahl der Jüngeren (unter 20jährigen) im Jahr 2030 um 29,4\% (25,2\%; 21,4\%) niedriger ist als 1992 und die Zahl der Personen mittleren Alters um 23,8\% (18,5\%; $13,2 \%)$ fällt, nimmt die Zahl der 60jährigen und älteren um 54,7\% (57,7\%; 60,8\%) zu. Daraus ergibt sich eine Verdoppelung des Altenquotienten von 35,0 (1992) auf $71,1(67,8 ; 65,0)$ im Jahr 2030, wobei der Hauptanstieg nach 2010 zu erwarten ist und ab 2030 eine Stabilisierung auf dem sehr hohen Niveau einsetzt (vgl. Abb. B.11).

Berücksichtigt man die oben angeführte Kritik von Bomsdorf an den Annahmen des Statistischen Bundesamtes, dann ergibt sich eine noch stärkere Zunahme des Anteils der Älteren an der Bevölkerung. Es muß deshalb an dieser Stelle eindringlich davor gewarnt werden, die Zahlen als hochgradig verläßlich anzusehen. Gerade für die weit in der Zukunft liegenden Jahre können die Abweichungen zwischen den prognostizierten und den tatsächlichen Werten sehr groß sein. ${ }^{63}$ Wegen der grundlegenden Offenheit der Zukunft ist überspitzt formuliert "das einzig sichere an demographischen Prognosen .. die Tatsache, daß sie sich ex post immer als falsch erweisen." 64

Als Fazit dieses Unterkapitels kann festgehalten werden, daß der Altenquotient bei mäßig hohem Wanderungssaldo und bei unverändertem Fertilitätsverhalten auf etwa das Doppelte seines gegenwärtigen Wertes steigen wird. Entsprechend der Budgetgleichung der Gesetzlichen Rentenversicherung ist infolge dieser Entwicklung ceteris paribus mit einem Anstieg des Beitragssatzes zur Gesetzlichen Rentenversicherung um mehr als zwei Drittel auf etwa 33 Prozent zu rechnen. ${ }^{65}$ Das gilt natürlich nur unter der oben getroffenen Annahme, daß die anderen Einflußgrößen auf den Beitragssatz sich nicht ändern.

63 Nach einer Untersuchung von Keilman (1990) S. 105 nimmt der mittlere prozentuale Fehler von Fertilitätsprognosen für die Niederlande alle fünf Jahre um etwa 7 bis 8 Prozentpunkte zu und beträgt nach 15 Jahren bereits rund zwanzig Prozent.

64 Feichtinger (1990) S. 72. Feichtinger weist in den folgenden Sätzen allerdings darauf hin, daß er diese Kritik nicht zu seinem Motto machen wolle, da die Aussagekraft einer Wissenschaft nicht (nur) von der Güte ihrer Prognosen abhänge.

Tatsächlich wird der Anstieg des Beitragssatzes etwas geringer ausfallen, weil in der hier vorgenommenen Vereinfachung einige institutionelle Regelungen nicht berücksichtigt sind, die die Beitragszahler entlasten. So wird z.B. der Bundeszuschuß bei steigendem Beitragssatz überproportional steigen, so da $B$ die Steuerzahler einen höheren Anteil an den Gesamtausgaben der Gesetzlichen Rentenversicherung 
Welche Entwicklung bei den anderen Einflußfaktoren für die nächsten vierzig Jahre zu erwarten ist und inwieweit dadurch der zu erwartende Anstieg des Beitragssatzes gebremst wird oder sich noch verstärkt, wird im folgenden Unterkapitel untersucht.

\subsection{Der Einfluß anderer Variablen auf die Finanzlage der Gesetzlichen Rentenversicherung}

Nachdem im vorangegangenen Unterkapitel über die Entwicklung des Altenquotienten der Einfluß der demographischen Entwicklung auf den Beitragssatz zur Gesetzlichen Rentenversicherung untersucht wurde, interessiert jetzt die Wirkung auf die Beitragssatzentwicklung, die von Variablen ausgeht, die das Verhältnis zwischen Alten- und Rentnerquotient verändern. Dazu ist zunächst zu klären, welche nicht altersstrukturbedingten, d.h. nicht auf eine Änderung des Verhältnisses zwischen 20 - und 60jährigen und den über 60jährigen zurückzuführenden Faktoren einen Einfluß auf die Relation zwischen Rentenempfänger und Beitragszahlern ausüben.

Das Verhältnis zwischen Beitragszahlern und 20- bis 60jährigen wird beeinflußt durch die Erwerbsbeteiligung und die Änderung der Erwerbstätigenstruktur. Die Erwerbsbeteiligung ist gegensätzlichen Einflüssen ausgesetzt. Einerseits führten in der Vergangenheit längere Ausbildungszeiten und Frühverrentung zu einer Verkürzung der Erwerbsphase und damit zu einer geringeren Erwerbsbeteiligung der unteren und oberen Altersgruppen. Andererseits stieg die Erwerbsbeteiligung der Frauen stetig an.

In Zukunft ist bei der Erwerbsbeteiligung der oberen Altersgruppen allerdings eher mit einer Zunahme zu rechnen statt mit einer weiteren Abnahme, weil ein Rückgang der Frühverrentung aus arbeitsmarktpolitischen Motiven $\mathrm{zu}$ erwarten ist ${ }^{66}$ und weil die gesetzliche Altersgrenze durch das Rentenreformgesetz 1992 schrittweise heraufgesetzt wird. Die Wirksamkeit der Heraufsetzung der Altersgrenze wird allerdings dadurch beeinträchtigt, daß etwa 38 Prozent aller Rentenneuzugänge allein aufgrund von Berufs- oder Erwerbsunfähigkeit und Arbeitslosigkeit schon vor dem Erreichen des gesetzlichen Renteneintrittsalters erfolgen. ${ }^{67}$ Dagegen ist auch in Zukunft von einer weiteren Verlängerung der Ausbildungszeiten auszugehen, weil die Anforderungen der Wirtschaft an die Qualifikation der Erwerbstätigen weiter zunehmen werden. Bei der Erwerbsbeteiligung von Frauen zeichnet sich für die Zukunft eine Fortsetzung des ansteigenden Trends der Vergangenheit ab, u.a. weil die Frauenerwerbsquote in Deutschland im internationalen Vergleich noch immer recht niedrig ist. ${ }^{68}$

tragen müssen. Weiterhin senkt ein Anstieg der Steuer- und Beitragssätze die Nettoeinkommen und damit aufgrund der Nettoanpassung die Rentenausgaben, nicht aber die von den Bruttoeinkommen erhobenen Beitragszahlungen. Diese und ähnliche Regelungen werden in den in Kapitel 2.3 dargestellten Simulationsstudien erfaßt. Sie verteilen allerdings die Belastung, die durch die demographische Entwicklung hervorgerufenen wird, nur um und reduzieren sie nicht. S. 283 . 
Insgesamt geht die Prognos AG in einem Gutachten für den Verband Deutscher Rentenversicherungsträger davon aus, daß die Beschäftigtenquote ${ }^{69}$ infolge der gegenläufigen Entwicklungstendenzen von 64 Prozent 1992 auf 72,3 Prozent im Jahr 2040 (69,1 Prozent in der unteren Variante) steigen wird. ${ }^{70}$

Von der Änderung der Erwerbstätigenstruktur gehen Wirkungen auf das Verhältnis von Beitragszahlern zu den 20- bis 60jährigen aus, wenn der Anteil der Nicht-Versicherten an den Erwerbstätigen zu- oder abnimmt. ${ }^{71}$ Eine Minderung des Prozentsatzes der Beamten wirkt vermindernd auf den Anteil der Nicht-Versicherten, eine Zunahme des Anteils der nicht-versicherten Selbständigen und der Schwarzarbeiter erhöhend. Von derartigen Änderungen der Erwerbstätigenstruktur sind für die Zukunft allerdings keine gravierenden Auswirkungen zu erwarten.

Die Relation von Rentenempfängern zu den über 60jährigen wird v.a. von Änderungen des tatsächlichen Renteneintrittsalters beeinflußt. Gelingt es, nicht nur das gesetzliche, sondern auch das tatsächliche mittlere Renteneintrittsalter zu erhöhen, ergibt sich damit ein doppelter Entlastungseffekt für den Beitragssatz zur Gesetzlichen Rentenversicherung. Einerseits erhöht sich die Zahl der Beitragszahler relativ zu den 20- bis 60jährigen, andererseits vermindert sich das Verhältnis von Rentnern zu den über 60jährigen. Hier sind durch die Erhöhung der gesetzlichen Altersgrenze im Rahmen des Rentenreformgesetzes 1992 Entlastungen zu erwarten.

Weitere Entlastungen ergeben sich aus einer geringeren Zahl bzw. Höhe der Hinterbliebenenrenten. Die zukünftig höhere Erwerbsbeteiligung der Frauen hat höhere eigene Rentenansprüche zur Folge, die bei der Ermittlung der Hinterbliebenenrente ab einem bestimmten Nettoeinkommen zu $40 \%$ angerechnet werden und die Hinterbliebenenrente daher mindern oder gar gänzlich aufzehren.

Ein weiterer Einflußfaktor, der durch die Gleichungen in Unterkapitel 1.3 aufgrund der Abstraktion von Steuern sowie Beiträgen zu anderen Sozialversicherungen neben der Gesetzlichen Rentenversicherung nicht erfaßt wurde, ist die Veränderung der Nettoquote ${ }^{72}$. Sinkt die Nettoquote, erhöht sich also die Spanne zwischen Brutto- und Nettolöhnen z.B. aufgrund von überproportionalen Steuererhöhungen oder einer Anhebung des Beitragssatzes zur Arbeitslosenversicherung, dann wird der Beitragssatz zur Gesetzlichen Rentenversicherung dadurch tendenziell gesenkt. Während nämlich die Einnahmen der Gesetzlichen Rentenversicherung durch die Beiträge der Versicherten vom Bruttolohn abhängen, sind die Ausgaben in Form der Rentenzahlungen seit der Einführung der Nettoanpassung im Rahmen des Rentenreformgesetzes 1992 durch den durchschnittlichen Nettolohn bestimmt. Durch eine Erhöhung des Abstandes zwischen Brutto- und Nettolohn ergibt sich damit eine finanzielle Entlastung der Gesetzlichen Rentenversicherung.

Bundesländer abzeichnet. Die Auswirkungen eines Anstiegs der Frauenerwerbsquote auf die Entwicklung der Zahl der Erwerbspersonen untersuchen Chruscz/Höpcke (1993) S. 28ff.

69

Die Beschäftigtenquote gibt die Relation der abhängig Beschäftigten (ohne Beamte) zur Zahl der 20- bis 60 jährigen an.

Vgl. Prognos AG (1995) S. 11.

Vgl. Schmähl (1994a) S. 393.

Als Nettoquote bezeichnet man den Quotienten aus Nettolohnsumme und Bruttolohnsumme. Sie gibt an, welcher Prozentsatz der Bruttolöhne den Arbeitnehmern nach Abzug von Steuem und Sozialversicherungsbeiträgen tatsächlich zufließt. 
Zieht man als Beispiel für die Senkung der Nettoquote nicht eine Erhöhung der Steuerquote, sondern eine Anhebung des Beitragssatzes zur Gesetzlichen Kranken- oder Pflegeversicherung heran, dann muß zusätzlich berücksichtigt werden, daß dadurch die Beiträge der Gesetzlichen Rentenversicherung zur Krankenversicherung der Rentner bzw. zur Pflegeversicherung der Rentner steigen und der Verbesserung der Finanzlage der Gesetzlichen Rentenversicherung entgegenwirken.

Bei der Darstellung der Fundamentalarithmetik umlagefinanzierter Alterssicherungssysteme wurde weiterhin nicht berücksichtigt, daß der Bundeszuschuß zur Gesetzlichen Rentenversicherung seit dem Rentenreformgesetz 1992 nicht mehr ausschließlich an der Lohnentwicklung orientiert ist, sondern zusätzlich proportional zu Beitragssatzänderungen verändert wird. Damit erfährt der demographisch bedingte Anstieg des Beitragssatzes eine Dämpfung durch die Rückkoppelung mit dem Bundeszuschuß, die allerdings von den Steuerzahlern finanziert werden muß.

Insgesamt ist davon auszugehen, daß die nicht-demographischen Einflußfaktoren einen dämpfenden Einfluß auf die Entwicklung des Beitragssatzes zur Gesetzlichen Rentenversicherung ausüben werden. Die bedeutensten positiven Wirkungen gehen dabei von dem erwarteten Anstieg der Beschäftigtenquote und den Hauptelementen des Rentenreformgesetzes 1992 (Erhöhung der Altersgrenze, Nettoanpassung, beitragssatzabhängige Anpassung des Bundeszuschusses) aus.

\subsection{Simulationsrechnungen zur langfristigen Finanzierbarkeit der Ge- setzlichen Rentenversicherung}

In diesem Unterkapitel wird auf der Basis der Ergebnisse aus den beiden vorangegangenen Abschnitten untersucht, welche Wirkung die Entwicklung der demographischen und ökonomischen Einflußfaktoren zusammengenommen auf die zukünftige Finanzlage der Gesetzlichen Rentenversicherung haben, wenn Fertilität und Wanderungen im Bereich der erwarteten Entwicklung liegen.

Der Verband Deutscher Rentenversicherungsträger (VDR) hat bei der Prognos AG ein Gutachten zu den Perspektiven der Gesetzlichen Rentenversicherung in Auftrag gegeben, um die Auswirkungen der Wiedervereinigung Deutschlands und des Rentenreformgesetzes 1992 auf die zukünftige Entwicklung der Finanzlage der Gesetzlichen Rentenversicherung besser abschätzen zu können. ${ }^{73}$ Dieses Gutachten wurde im Frühjahr 1995 vorgelegt und bildet hier die Basis für die Untersuchung der Entwicklung, die ohne größere Änderungen des Kinderlastenausgleichs und der Einwanderungsgesetzgebung zu erwarten ist. Von den zwei Varianten der Studie wird im folgenden nur die "untere" wiedergegeben, weil die "obere" Variante auf der Annahme einer gesteuerten Zuwanderung (und auch eines höheren Wirtschaftswachstums) beruht und ihre Darstellung damit dem in 3.3.2 zu analysierenden Einfluß der Einwanderungs-

73 Vgl. Prognos AG (1995). Die Ergebnisse hinsichtlich der Entwicklung der Beitragssätze zur Gesetzlichen Renten- und Krankenversicherung entsprechen etwa den Resultaten einer Simulationsrechnung von Felderer (1994a), die in den beiden folgenden Unterabschnitten ausführlicher erläutert wird. Köpp (1995) kommt dagegen zu geringeren Beitragssatzsteigerungen. 
politik vorgreifen würde. Zudem entsprechen die demographischen Annahmen der unteren Variante recht genau den Annahmen der oben erläuterten dritten Variante der achten koordinierten Bevölkerungsvorausberechnung des Statistischen Bundesamtes, so daß eine weitgehende Vergleichbarkeit gewährleistet ist. ${ }^{74}$

Der vielfältigen Interdependenz ökonomischer und demographischer Einflußfaktoren auf die Finanzsituation der Gesetzlichen Rentenversicherung wurde in der folgenden Weise Rechnung getragen: Die demographischen Änderungen wurden mittels der oben beschriebenen Komponentenmethode prognostiziert und dienten gleichzeitig als Grundlage für die Schätzung des Erwerbspersonenpotentials. Das Erwerbspersonenpotential ging als eine wichtige exogene Größe in ein makroökonomisches Gesamtmodell ein, mit dessen Hilfe gesamtwirtschaftliche Variablen geschätzt wurden. Diese wurden in das Rentenmodell des VDR übernommen, das als Ergebnis das undynamisierte Rentenvolumen ${ }^{75}$ liefert. Die Dynamisierung erfolgt durch das Rentenfinanzmodell des VDR, in dem die Rentenanpassungssätze errechnet werden. Mit der Hilfe dieser Anpassungsätze wurden dann die Einnahmen-, Ausgaben-, und Vermögenspositionen in der Gesetzlichen Rentenversicherung bestimmt. Zur Abstimmung mit den Daten des makroökonomischen Modells, das auch die anderen Sozialversicherungssysteme detailliert abbildet, wurden die Ergebnisse des Rentenfinanzmodells wieder in das Makromodell eingespeist und auf diese Art und Weise so viele Iterationen durchgeführt, bis kaum noch Veränderungen der Ergebnisse feststellbar waren. ${ }^{76}$

Die Annahmen der Studie sollen hier nicht im einzelnen aufgeführt werden. Sie betreffen v.a. den zukünftigen Verlauf der internationalen Arbeitsteilung und der technischen Entwicklung, die Wirtschaftspolitik und das Erwerbsverhalten. ${ }^{77}$ Die demographischen Annahmen bezüglich Fertilität, Mortalität und Wanderungen unterscheiden sich, wie oben erwähnt, nicht wesentlich von den Annahmen der dritten Variante der achten koordinierten Bevölkerungsvorausberechnung des Statistischen Bundesamtes.

Die Ergebnisse der Modellrechnung bezüglich der Entwicklung des Beitragssatzes zur Gesetzlichen Rentenversicherung sind in Abb. B.12 dargestellt. Bis zum Jahr 2000 ist der Anstieg ausgehend von einem Wert von 17,7 Prozent im Jahr 1992 relativ gering, aber danach steigt der Beitragssatz um nahezu 3 Prozentpunkte pro Jahrzehnt auf 28,5 Prozent im Jahr 2030 und stabilisiert sich anschließend auf diesem Niveau.

Der Anstieg des Beitragssatzes gibt für sich genommen allerdings noch keine genaue Auskunft über die Finanzlage der Gesetzlichen Rentenversicherung. Die zukünftigen Belastun-

74 Für die zusammengefaßte Geburtenrate der Deutschen wird von der Prognos AG ein Anstieg von 1,362 im Jahr 1991 auf 1,488 im Jahr 2010 unterstellt, während das Statistische Bundesamt für den gesamten Prognosezeitraum einen Wert von 1,4 zugrundelegt. In der unteren Variante des Prognos-Gutachtens wird mit rund 74.000 Personen ab dem Jahr 2010 ein etwas geringerer Wanderungssaldo angenommen als in der dritten Variante der achten koordinierten Bevölkerungsvorausberechnung, wo ab dem Jahr 2010 von 100.000 Nettozuwanderern ausgegangen wird. Vgl. Prognos AG (1995) S. 31ff. und Sommer (1994) S. 497f.

Das Rentenvolumen umfaßt den Zahlbetrag aller Renten, die in dem jeweiligen Jahr zu leisten sind. Wird dieses Rentenvolumen nicht mit dem aktuellen Rentenwert dieses Jahres, sondern mit dem aktuellen Rentenwert des Ausgangsjahres berechnet, erhält man das undynamisierte Rentenvolumen. 


\section{Abb. B.12: Prognostizierter Beitragssatz zur Gesetzlichen Rentenversicherung (unteres Szenario)}

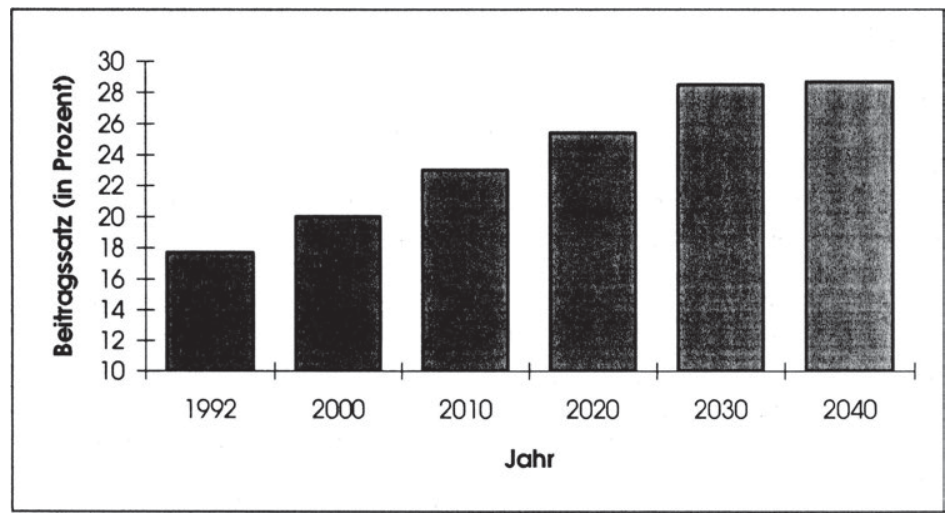

Quelle: Prognos AG (1995) S. 11 und S. U-83 (Tabellenband)

gen, die durch die veränderte Altersstruktur entstehen, sind nämlich durch das Rentenreformgesetz 1992 so umverteilt worden, daß sie sich nur zum Teil bei den Beitragszahlern niederschlagen. V.a. die drei bereits in Unterkapitel 1.2 erwähnten Maßnahmen (Nettoanpassung, Anhebung der Altersgrenze, Koppelung des Bundeszuschusses an die Beitragssatzentwicklung) haben zu einer erheblichen Modifizierung der Lastverteilung beigetragen. Die Nettoanpassung, d.h. die jährliche Erhöhung der Renten entsprechend des Zuwachses der Nettoverdienste, hat zur Folge, daß die Rentenhöhe bei einem Anstieg der Abgabenhöhe ceteris paribus zurückgeht und so die Rentner einen Teil der demographisch verursachten Last übernehmen. Die Anhebung der Altersgrenze belastet die jetzigen Beitragszahler und zukünftigen Rentner, weil sie länger im Erwerbsleben bleiben müssen. Diese Leistungskürzung der Gesetzlichen Rentenversicherung stellt die jetzigen beitragszahlenden Mitglieder schlechter, ohne daß diese Belastung in einem höheren Beitragssatz sichtbar würde. Die Koppelung des Bundeszuschusses nicht nur an die Entwicklung der Bruttoverdienste, sondern auch an Änderungen des Beitragssatzes zur Gesetzlichen Rentenversicherung schließlich entlastet zwar die Beitragszahler zur Gesetzlichen Rentenversicherung, belastet aber gleichzeitig und in gleichem Umfang die Steuerzahler.

Welche Bedeutung die Lastverschiebungen durch das Rentenreformgesetz 1992 auf die Beitragssatzentwicklung haben, wird deutlich, wenn man den prognostizierten Verlauf des Eckrentnerquotienten, d.h. die Relation der Zahl der Eckrentner ${ }^{78}$ zu der Zahl der potentiellen Beitragszahler, mit und ohne die Maßnahmen des Rentenreformgesetzes von 1992 vergleicht. Der Eckrentnerquotient kann ähnlich wie der Altenquotient als Indikator für die demo-

Die Zahl der Eckrentner erhält man durch Division des Rentenvolumens eines Jahres durch die BruttoStandardrente dieses Jahres. Vgl. Barth/Hain/Müller (1994) S. 241. Die Brutto-Standardrente, d.h. die Rente eines Eckrentners, errechnet sich nach der Rentenformel unter Zugrundelegung von 4.5 Entgeltpunkten. Die Brutto-Standardrente entspricht daher der Rentenhöhe bei einer Biographie mit 45 Beitragsjahren und durchschnittlichem Arbeitsentgelt. 
graphische Entwicklung angesehen werden. Ohne Berücksichtigung der Gesetzesänderungen würde er nach einer Modellrechnung von Barth, Hain und Müller von 1993 bis 2030 um 129 Prozent ansteigen (der Altenquotient um 118 Prozent). Modifiziert man den Eckrentnerquotienten so, daß die Entlastungswirkungen der Reform sichtbar werden, beträgt der Anstieg in dem betrachteten Zeitraum nur noch 76 Prozent. ${ }^{79}$ Ein Großteil der Differenz zwischen der Beitragssatz-Prognose der Prognos AG von 198780, die einen Anstieg auf 37 bis 42 Prozent im Jahr 2030 vorhersagte, und der Prognose von 1995, die den Beitragssatz 2030 zwischen 26,3 und 28,5 Prozent sieht, ist also auf Lastverschiebungen und nur ein geringer Teil auf geänderte Annahmen über die demographische und wirtschaftliche Entwicklung zurückzuführen. Der im Vergleich zu früheren Prognosen relativ geringe erwartete Anstieg des Beitragssatzes nach der Wiedervereinigung, dem unerwarteten Zuwandererstrom und dem Rentenreformgesetz 1992 darf daher nur zu einem kleinen Teil als echte Entlastung interpretiert werden.

Unter Einengung der Betrachtungsweise auf die Beitragssatzentwicklung wird oft argumentiert, daß der höheren Belastung der Erwerbstätigen durch höhere Beiträge zur Gesetzlichen Rentenversicherung Entlastungen in anderen Bereichen, v.a. durch einen geringeren Anteil von Jugendlichen und durch geringere Beiträge zur Arbeitslosenversicherung gegenüberstehen. Letztlich ist für mögliche Ausweichhandlungen der erwerbstätigen Generation aber die Gesamtbelastung durch Steuern und Sozialabgaben entscheidend, so daß eine Konzentration allein auf die Gesetzliche Rentenversicherung nicht problemadäquat ist. Die Ergebnisse der Prognos-Studie zeigen allerdings deutlich, daß die günstigere Entwicklung der Finanzlage der Arbeitslosenversicherung bei weitem nicht ausreicht, um für eine Kompensation der negativen Entwicklung in der Gesetzlichen Rentenversicherung zu sorgen. Bezieht man alle Sozialversicherungssysteme in die Betrachtung ein, zeigt sich im Gegenteil, daß die Veränderungen der Altersstruktur auch die Beitragssätze der Gesetzlichen Krankenkassen und der Gesetzlichen Pflegeversicherung ansteigen lassen (vgl. Abb. B.13). Der Gesamtbeitragssatz zu den Sozialversicherungen erhöht sich von 36,7 Prozent 1992 auf 52,7 Prozent im Jahr 2040. Auch die Steuerquote wird nach dieser Prognose im Jahr 2040 etwas über dem Wert von 1992 liegen, nachdem sie zwischenzeitlich leicht sinkt. ${ }^{81}$

Obwohl ein Teil der demographisch bedingten Lasten durch das Rentenreformgesetz 1992 auf die Rentnergeneration verschoben wurde, ist damit der Anstieg der Gesamtbeiträge zu den Sozialversicherungen für die Beitragszahler noch so groß, daß mit effizienzmindernden Ausweichreaktionen gerechnet werden muß. Auf kollektiver Ebene ist eine Aufkündigung des Generationenvertrages durch die erwerbstätige Generation nicht auszuschließen. Auf individueller Ebene muß davon ausgegangen werden, daß schattenwirtschaftliche Aktivitäten zunehmen werden und möglicherweise auch eine Substitution von Arbeit durch Freizeit vorgenommen wird, um den hohen Abgabesätzen zu entgehen. ${ }^{82}$

79 Vgl. Barth/Hain/Müller (1994) S. 256.

80 Vgl. Prognos AG (1987). Eine Gegenüberstellung von Prognosen mit und ohne die Einführung von Maßnahmen, die den Beitragssatzanstieg dämpfen, findet sich bei Schmähl (1988) S. $31 \mathrm{ff}$.

81 Vgl. Prognos AG (1995) S. 10.

82 Vgl. Frey (1981) S. 221. Eine Substitution von Arbeit durch Freizeit ist nur dann zu erwarten, wenn für die Mehrzahl der Individuen der Substitutionseffekt einer relativen Verbilligung der Freizeit den entgegengerichteten Einkommenseffekt überwiegt. 
Abb. B.13: Die Entwicklung der Beitragssätze der Sozialversicherungen (in Prozent)

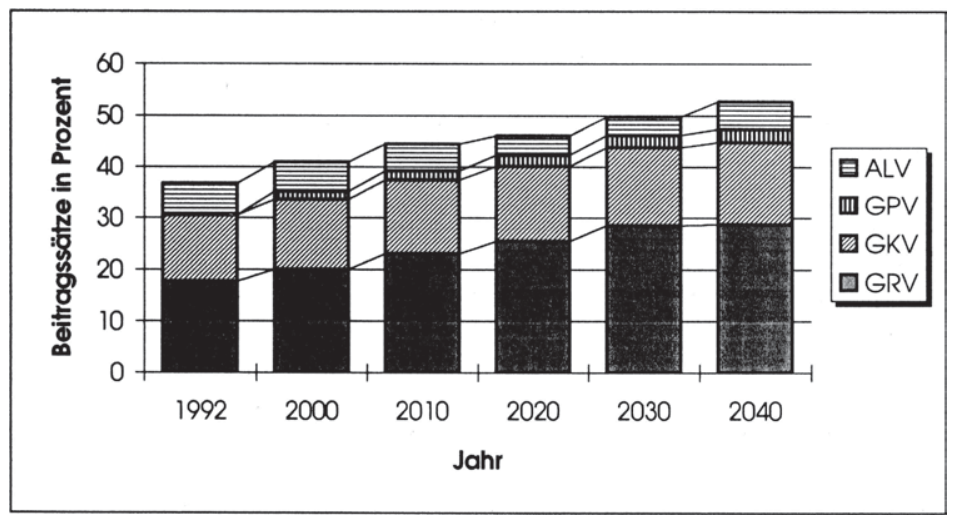

Quelle: Prognos AG (1995) S. U-83 (Tabellenband)

Welche Vorschläge zur Vermeidung derartiger Ausweichreaktionen der Beitragszahler unterbreitet wurden und wie ihr Beitrag zur Reduzierung der demographisch bedingten Krise der Gesetzlichen Rentenversicherung zu bewerten ist, wird im folgenden Kapitel untersucht.

\section{Lösungsansätze für die demographische Krise des Systems der Ge- setzlichen Rentenversicherung}

Aufbauend auf die Erläuterung der zu erwartenden demographisch bedingten Schwierigkeiten des Systems der Gesetzlichen Rentenversicherung werden in diesem Kapitel verschiedene Vorschläge untersucht, die zur Entschärfung der demographischen Krise unterbreitet wurden.

Das Kapitel gliedert sich in drei Teile. Im ersten Unterkapitel (3.1) wird auf Lösungsvorschläge eingegangen, die das grundlegende System der Gesetzlichen Rentenversicherung unverändert lassen, aber durch Änderungen im Rahmen dieses Systems eine Milderung der demographischen Krise zu erreichen suchen. Entsprechend der in 1.1 entworfenen Einteilung werden zu den grundlegenden Eigenschaften des Systems der Gesetzlichen Rentenversicherung die staatliche Trägerschaft, das überwiegend beitragsfinanzierte Umlageverfahren, der Beitrittszwang für den weitaus größten Teil der Bevölkerung und der Risikoausgleich gezählt. Im zweiten Teil (3.2) werden radikalere Umgestaltungsvorschläge analysiert, die wesentliche Merkmale des bestehenden Alterssicherungssystems verändern. Im dritten Unterkapitel (3.3) werden schließlich Lösungsansätze betrachtet, die weder Reformen im Rahmen des Systems der Gesetzlichen Rentenversicherung noch einen Ersatz dieses Systems durch alternative Alterssicherungformen beinhalten, sondern eine Korrektur der demographischen Entwicklung 
durch bevölkerungspolitische Maßnahmen anstreben. Die gegenwärtig im Bereich der Altersvorsorge gültigen institutionellen Regelungen werden dabei beibehalten. Behandelt werden hier nur die wichtigsten der derzeit diskutierten Konzepte, wobei die Darstellung entsprechend der Themenstellung dieser Arbeit auf Grundaussagen beschränkt wird.

Das Ziel dieses Kapitels ist es zu verdeutlichen, inwieweit die untersuchten Vorschläge zur Reduktion der zukünftigen demographischen Belastungen geeignet sind oder sich weitgehend auf die Umverteilung der entstehenden Lasten zwischen Rentnern, Beitragszahlern und Steuerzahlern beschränken. Dadurch wird es möglich, die in den Teilen C und D genauer behandelten Vorschläge einer Erhöhung des Kinderlastenausgleichs bzw. einer verstärkten Einwanderung mit anderen Lösungsvorschlägen zu vergleichen und zu beurteilen, welches Gewicht ihnen gegebenenfalls in einer aus einem Instrumenten-Mix bestehenden Lösung zufallen sollte.

Bevor auf die einzelnen Lösungskonzepte eingegangen werden kann, muß zunächst Klarheit darüber geschaffen werden, welche Kriterien zur Beurteilung der Vorschläge herangezogen werden sollen. Herkömmlicherweise wird in ökonomischen Arbeiten v.a. auf das Effizienzkriterium abgestellt, daneben oft auch auf das Kriterium "Gerechtigkeit". Beide Kriterien müssen in dem hier behandelten Zusammenhang präzisiert werden. Das Effizienzkriterium ergibt sich aus dem ökonomischen Prinzip, nach dem ein möglichst hoher Zielerreichungsgrad (hier z.B. Maximierung der Wohlfahrt) mit den zur Verfügung stehenden Mitteln angestrebt werden soll. Im intergenerationalen Zusammenhang spricht man von einer Pareto-Verbesserung, wenn jeder einzelnen Generation durch eine bestimmte Maßnahme ein mindestens gleich hohes und wenigstens einer Generation ein höheres Konsumniveau gegenüber der Ausgangssituation gesichert werden kann. ${ }^{83}$ Unter Gerechtigkeit wird meistens Verteilungsgerechtigkeit im Sinne einer ergebnisbezogenen Betrachtung (im Gegensatz zu "Verfahrensgerechtigkeit" bei einer regelbezogenen Sichtweise) verstanden. Hier ist neben den Auswirkungen einzelner Maßnahmen auf die Einkommens- und Vermögensverteilung innerhalb einer Generation auch die intergenerationale Verteilungsgerechtigkeit zu beachten. Eng mit dem Kriterium Gerechtigkeit ist die politische Durchsetzbarkeit verknüpft. Sie soll hier als weiteres Kriterium herangezogen werden, um die Realisierungschancen einzelner Vorschläge im Prozeß der politischen Willensbildung besser beurteilen zu können. Dazu erscheint auch die Analyse etwaiger Übergangsprobleme sinnvoll.

\subsection{Lösungsansätze innerhalb des Systems der Gesetzlichen Rentenver- sicherung}

\subsubsection{Anrechnung von Kindererziehungszeiten in der Gesetzlichen Rentenversicherung}

Seit 1986 werden im Rahmen der Gesetzlichen Rentenversicherung Kindererziehungszeiten nach § 55 SGB VI als Beitragszeiten angerechnet, wobei die Dauer der Anrechnung von zunächst einem Jahr pro Kind bis auf drei Jahre für nach dem 31.12.1991 geborene Kinder

83 Vgl. Breyer (1990) S. 19f. 
ausgeweitet wurde. Während der Kindererziehungszeit gelten Beiträge von einem Lohn in Höhe von $75 \%$ des Durchschnittsentgelts aller Versicherten als bezahlt und diese fingierten Beiträge wirken sowohl rentenerhöhend als auch rentenbegründend. Allerdings werden Beiträge aus Erwerbseinkommen während der Kindererziehungszeiten auf diese angerechnet, so $\mathrm{da} B$ sich für erziehende Erwerbstätige mit einem entsprechend hohen Einkommen keine Vorteile aus dieser Regelung ergeben. ${ }^{84}$

Die Einführung von Kindererziehungszeiten trägt dem Drei-Generationen-Gedanken Rechnung, indem auf der Leistungsseite eine Differenzierung nach der Kinderzahl vorgenommen wird. So wird (wieder) eine Anreizstruktur geschaffen, die das Aufziehen von Kindern belohnt und daher in Richtung einer Erhöhung der Kinderzahl wirkt. Übergangsprobleme traten bei der Einführung - abgesehen von der Ungleichbehandlung aufgrund der Stichtagsregelung - nicht auf, da im Gegensatz zu vielen anderen Lösungsvorschlägen kein Eingriff in Besitzstände erforderlich war. Darin liegt gleichzeitig aber auch ein Nachteil, denn es handelt sich bei der Anrechung von Erziehungsjahren um zusätzliche Leistungen, so daß der Versichertengemeinschaft vermehrte Finanzlasten auferlegt werden. Diese zusätzlichen Lasten sind zudem im zweiten Quartal des nächsten Jahrhunderts besonders hoch, weil dann die Mitglieder der geburtenstarken Jahrgänge, die Kinder erzogen haben, in das Rentenalter eintreten. In diesen Jahren ist die Gesetzliche Rentenversicherung aber ohnehin aufgrund der demographischen Einflüsse starken Belastungen ausgesetzt. Die bereits durch hohe Beitragssätze zur Gesetzlichen Rentenversicherung stark beanspruchte Kindergeneration muß zusätzlich noch die Rentenerhöhung aufgrund der Kindererziehung bezahlen, die kinderlosen Mitglieder der Elterngeneration dagegen werden nicht zur Finanzierung herangezogen. ${ }^{85}$

Als Beitrag zur Lösung der demographischen Krise der Gesetzlichen Rentenversicherung können die sogenannten "Babyjahre" daher nicht gelten. Dazu müßten von dieser Maßnahme substantielle geburtenfördernde Wirkungen ausgehen. Dies ist aber - wie in Teil C erläutert wird - kaum zu erwarten, zumal die Leistungen nicht etwa zu einer Entlastung der Eltern in der finanziell besonders angespannten Zeit der ersten Jahre nach der Geburt führen, sondern sich erst Jahrzehnte später im Rentenalter bemerkbar machen. Als weiterer Nachteil wird die einseitige Förderung der Hausfrauenehe bzw. die Benachteiligung erwerbstätiger Erziehender durch die Anrechung auf Beiträge aus Erwerbseinkommen genannt. ${ }^{86}$ Ohne eine solche Anrechnung würden jedoch die Finanzierungsprobleme noch gravierender und es könnte für durchgehend Erwerbstätige zu einer verteilungspolitisch problematischen Überversorgung im Alter kommen, d.h. für diese Gruppe könnte das Renteneinkommen höher liegen als das vorausgegangene Erwerbseinkommen. ${ }^{87}$ Als isolierte Maßnahme ist die Anrechnung von Erziehungszeiten zur Bewältigung der zukünftigen Finanzierungsprobleme der Gesetzlichen Rentenversicherung daher ungeeignet.

84 Stellvertretend für viele ähnliche Ausgestaltungen sei der Vorschlag von Richter/Weimann (1987) genannt, das Renteneintrittsalter kinderzahlabhängig zu gestalten. Die Kosten verschiedener Verbesserungen bei der Anerkennung oder Bewertung von Kindererziehungszeiten (erweiterte Anrechnung, Erhöhung der Zahl der gutgeschriebenen Erziehungsjahre, Erhöhung der Bewertung der gutgeschriebenen Erziehungsjahre) wurden von Müller (1992) untersucht.

85 Borchert (1993) S. 265 spricht in diesem Zusammenhang von "In-sich-Transfers", da die zusätzlichen Leistungen nur zwischen unterschiedlichen Generationen innerhalb der Familien umverteilt werden, während den Kinderlosen kein Beitrag abverlangt wird.

86 Vgl. Borchert (1993) S. 166.

87 Vgl. Burkhardt (1985) S. 102. 


\subsubsection{Verlängerung der durchschnittlichen Lebensarbeitszeit}

Eine Verlängerung der durchschnittlichen Lebensarbeitszeit und damit der beitragspflichtigen Versicherungsjahre kann auf unterschiedliche Weise erreicht werden, z.B. über eine Verkürzung der Ausbildungszeiten, über eine höhere Erwerbsbeteiligung insbesondere von Frauen und über eine Erhöhung des Renteneintrittsalters. Exemplarisch für diese Gruppe von Vorschlägen soll hier die letztgenannte Variante erörtert werden.

Als Rechtfertigung für eine Heraufsetzung des Renteneintrittsalters dient neben den zukünftigen Finanzierungsproblemen v.a. die gestiegene Lebenserwartung und damit verbunden die höhere Leistungsfähigkeit älterer Personen, die eine Erwerbsarbeit auch im fortgeschrittenen Alter zumutbar erscheinen läßt. Tendenziell bewirkt eine Heraufsetzung des Rentenzugangsalters eine Veränderung des Rentnerquotienten sowohl aufgrund einer Zunahme der Zahl der Beitragszahler als auch aufgrund einer Abnahme der Zahl der Rentenempfänger. Altersjahrgänge, die nach gegenwärtiger Rechtslage bereits im Ruhestand wären, gehören nach einer Erhöhung des Rentenzugangsalters noch zur potentiell erwerbstätigen und damit beitragspflichtigen Bevölkerung. Umstritten ist dabei allerdings, inwieweit sich eine derartige Erhöhung des Erwerbspersonenpotentials tatsächlich in einer Erhöhung der Zahl der Beitragszahler niederschlägt. Gerade in Zeiten wirtschaftlicher Rezession und hoher Arbeitslosigkeit muß damit gerechnet werden, daß zumindest ein Teil der gegenüber der jetzigen Regelung zusätzlich dem Arbeitsmarkt zur Verfügung stehenden Erwerbspersonen lediglich eine Erhöhung der Zahl der Arbeitslosen bewirkt - zumal wenn man bedenkt, daß es sich bei dem Zuwachs an Erwerbstätigen um Arbeitnehmer höheren Alters handelt, also um eine Gruppe, die auf dem Arbeitsmarkt oft einen besonders schweren Stand hat.

Auch die Minderung der Zahl der Rentenempfänger wird sich nach einer Heraufsetzung der gesetzlichen Altersgrenze nicht im vollen Umfang in einem Anstieg des tatsächlichen durchschnittlichen Rentenzugangsalters niederschlagen. V.a. die hohe Zahl der Erwerbs- und Berufsunfähigkeitsrenten und die vom Verhalten der Individuen abhängige Inanspruchnahme der flexiblen Altersgrenze ${ }^{88}$ haben dazu geführt, daß das tatsächliche durchschnittliche Rentenzugangsalter mit 59,67 Jahren (1990) deutlich unter der gesetzlichen Altersgrenze liegt. Eine Anhebung der gesetzlichen Altersgrenze wirkt daher nur insoweit entlastend auf den Rentnerquotienten, als dadurch eine Anhebung des tatsächlichen durchschnittlichen Rentenzugangsalters erreicht wird. ${ }^{89}$

Warlick/Burkhauser (1984) S. 367 führen aus, daß eine Heraufsetzung des gesetzlichen Renteneintrittsalters wie eine konstante Reduzierung der Rentenhöhe wirkt, wenn das tatsăchliche durchschnittliche Rentenzugangsalter sich aufgrund verstärkter Ausschöpfung der Regelungen zum vorzeitigen Ruhestand nicht ändert.

Eine verstärkte Tendenz zu vorzeitigem Eintritt in den Ruhestand nach einer Anhebung der gesetzlichen Altersgrenze würde den Einfluß auf den Rentnerquotienten zwar verkleinern, gleichzeitig aber wegen der Rentenabschläge bei Vorverlegung des Ruhestands zu einem niedrigeren Rentenniveau - hier im Gegensatz zu dem "Eckrentenniveau" verstanden als Relation von tatsächlichen Durchschnittsrenten zu durchschnittlichem Arbeitsentgelt - führen und somit zu einer finanziellen Entlastung der Gesetzlichen Rentenversicherung beitragen.

Buslei/Schmidt (1994) führen aus, da $B$ durch einen Übergang zu versicherungsmathematischen Abschlägen bei vorzeitigem Renteneintritt eine Erhöhung des durchschnittlichen Rentenzugangsalters um $0.8(1,1)$ Jahre bei einer unterstellten Zeitpräferenzrate der Versicherten von 5\% (7\%) erreicht werden könnte. Der Einfluß auf den zukünftigen Beitragssatz ist mit einer Reduzierung um etwa einen Prozentpunkt im Jahr 2030 allerdings nicht sehr groß. 
Die quantitativen Auswirkungen einer Erhöhung des durchschnittlichen Rentenzugangsalters in Form eines dann geringeren Beitragssatzes hängen von der ursprünglichen Höhe des Rentnerquotienten und der Höhe des Rentenniveaus ab. Je höher der Rentnerquotient ist, desto größer wird im Normalfall auch die Zahl der Personen sein, die von einer einjährigen Anhebung des durchschnittlichen Renteneintrittsalters betroffen sind, d.h. umso stärker sinkt ceteris paribus der Beitragssatz. Je höher das Rentenniveau, umso mehr Ausgaben können durch eine Anhebung des durchschnittlichen Rentenzugangsalters um ein Jahr eingespart werden und umso deutlicher wird ceteris paribus der Beitragssatz zurückgehen..$^{90} \mathrm{Schmähl} \mathrm{errechnet} \mathrm{für}$ das Jahr 2030 unter plausiblen Annahmen eine Beitragssatzminderung um etwa drei Prozentpunkte für jedes Jahr, um welches das durchschnittliche Rentenzugangsalter erhöht wird. ${ }^{91}$ Allerdings geht er dabei von der Beibehaltung des Leistungsumfangs Ende der achtziger Jahre aus. Inzwischen hat das Rentenreformgesetz 1992 aber zu einer nicht unbeträchtlichen Einschränkung zukünftiger Leistungen geführt und sieht zudem bereits eine schrittweise Erhöhung der gesetzlichen Altersgrenze vor, so daß der Effekt einer weiteren Anhebung mit einer Beitragssatzsenkung von etwa 1,5 Prozentpunkten pro Jahr nur noch halb so groß ist wie von Schmähl errechnet. ${ }^{92}$

Die Anhebung des Rentenzugangsalters kann einen Beitrag zur Minderung der demographischen Krise leisten. Allerdings sollten die Möglichkeiten nicht überschätzt werden, zumal eine starke Anhebung polit-ökonomisch schwer durchsetzbar sein wird - schließlich wird ähnlich wie bei einer Reduzierung der zukünftigen Leistungen die jetzige Generation der Beitragszahler und damit der Medianwähler durch eine Anhebung der Altersgrenze drastisch schlechter gestellt. Das Problem einer in Zukunft geringen, eventuell sogar negativen Verzinsung der Rentenversicherungsbeiträge für die Erwerbstätigen und damit der geringen Akzeptanz des Systems wird durch diesen Vorschlag nicht gemindert.

\subsection{Lösungsansätze durch Alternativen zum System der Gesetzlichen Rentenversicherung}

\subsubsection{Grundrentenvorschläge}

Ein ganzes Bündel von Vorschlägen zielt auf die Ablösung des Prinzips des konstanten Rentenniveaus durch eine bloße Mindestsicherung ab. Statt der Lebensstandardabsicherung im Alter durch die dynamische Rente soll nur noch eine einheitliche Sockelversorgung durch den Staat gesichert werden, deren Aufstockung durch zusätzliche Alterssicherungsformen (Betriebsrenten, Vorsorge der privaten Haushalte) dem privaten Sektor überlassen bleibt. Systematisieren läßt sich die Vielzahl der Vorschläge nach der Finanzierungsart (Steuern oder Beiträge), danach, ob das neue System auf die Alterssicherung beschränkt werden soll

\footnotetext{
90 Vgl. Schmähl (1988) S. 199f.

91 Vgl. Schmähl (1988) S. 200.

92 Vgl. Bundesregierung (1995) S. 227. Schmähl (1994b) S. 513 schätzt die Beitragssatzminderung durch ein Hinausschieben des durchschnittlichen Renteneintrittsalters um ein Jahr auf 1,5 bis 2,5 Prozentpunkte.
} 
(Grundrentenvorschläge) oder auch andere Felder der sozialen Sicherung umfaßt (Grundeinkommensvorschläge) und nach der Bedarfsorientierung, d.h. danach, ob der Leistungsanspruch unabhängig vom Bedarf besteht oder durch eine Bedürftigkeitsprüfung festgestellt wird. ${ }^{93}$

Da Grundeinkommensvorschläge nicht primär als Reaktion auf die demographische Krise in der Gesetzlichen Rentenversicherung konzipiert worden sind, sondern einen allgemeinen Umbau des gesamten Sozialsystems zum Ziel haben, wird auf diese unter den Schlagworten "negative Einkommensteuer" und "Bürgergeld" bekanntgewordenen Vorschläge im folgenden nicht näher eingegangen. ${ }^{94}$ Stattdessen wird der seit längerem von verschiedenen Seiten intensiv diskutierte Vorschlag einer steuerfinanzierten, bedarfsunabhängig gewährten Grundrente analysiert.

Miegel und Wahl' ${ }^{95}$ begründen ihren Vorschlag einer solchen Grundrente damit, daß eine Fortführung des jetzigen Systems weder nötig noch möglich ist. Unmöglich sei die Fortführung, weil auch nach der Rentenreform 1992 die Abgabenlast durch Steuern und Sozialversicherungsbeiträge demographisch bedingt unerträglich hoch steigen werde und damit zu einer Aufkündigung des Generationenvertrags durch die künftig Aktiven führen würde.96 Gleichzeitig sei eine Fortführung aber auch unnötig, da im Gegensatz zu den Verhältnissen zu Zeiten der Einführung der Gesetzlichen Rentenversicherung oder auch zur Zeit der grundlegenden Reform im Jahr 1957 inzwischen ein großer Teil der Bevölkerung - Miegel spricht von zwei Dritteln - aufgrund der stark gestiegenen Privatvermögen im Alter nicht mehr ausschließlich auf die staatliche Alterssicherung angewiesen sei. ${ }^{97}$ Als Konsequenz aus einer ordnungspolitischen Sichtweise fordern Miegel und Wahl daher eine Rückführung des Staatseinflusses durch eine steuerfinanzierte Grundrente in Höhe von $40 \%$ des durchschnittlichen Nettoarbeitseinkommens der abhängig Beschäftigten für alle Staatsbürger, die mindestens 25 Jahre in Deutschland steuerpflichtig gewesen sind. Um diskretionären Eingriffen, die zu einer Unterminierung des Vertrauens in dieses System führen würden, vorzubeugen, schlagen Miegel und Wahl eine verfassungsrechtliche Verankerung der entscheidenden Merkmale des Grundrentensystems vor. ${ }^{98}$

Kritisiert wird an den Systemen der steuerfinanzierten Grundrente ohne Bedürftigkeitsprüfung, daß der Versicherungsgedanke und mit ihm die Beitragsäquivalenz aufgehoben und damit zumindest für den Sockelbetrag die Umverteilung und der Staatseinfluß ausgeweitet

93 Vgl. zu dieser Einteilung Schmähl (1990) S. 188.

94 Vgl. zu Grundeinkommensvorschlägen beispielsweise Rosen/Windisch (1992) S. 344ff. und S. $419 f$. sowie Pelzer (1994) und Institut der deutschen Wirtschaft (1994) S. 4f.

95 Vgl. Miegel/Wahl (1985).

96 Vgl. Miegel (1989) S. 76.

97 Vgl. ebenda. Miegel weist an dieser Stelle darüber hinaus darauf hin, daß das gegenwärtige Rentensystem trotz der Rente nach Mindesteinkommen Altersarmut nicht immer verhindern kann.

98 Der Grundrentenvorschlag der Partei der GRÜNEN sieht gegenüber dem Vorschlag von Miegel/Wahl ein höheres Niveau der Grundrente und eine Ergänzung durch eine am Einkommen orientierte Zusatzrente vor. Vgl. DIE GRÜNEN (1985) und zu einer knappen Darstellung Rosen/Windisch (1992) S. 419. Auch der Bundeskongre $B$ der Arbeitsgemeinschaft Sozialdemokratischer Frauen hat ein Grundrentenmodell (mit obligatorischer Zusatzversicherung) beschlossen. 
wird. ${ }^{99}$ Dem Gedanken eines Drei-Generationen-Vertrages und damit dem grundlegenden demographischen Problem wird keine Rechnung getragen. Weiterhin sind schwierige Übergangsprobleme zu lösen. Während des Aufbaus des Systems müssen die bereits erworbenen Ansprüche der alten Generation noch befriedigt werden, was eine Doppelbelastung der Erwerbstätigen durch hohe Beitragszahlungen und gleichzeitigen Aufbau einer eigenen, privaten Zusatzversorgung zur Folge hätte. Alternativ könnte natürlich auch die Rentnergeneration belastet werden, indem das Rentenniveau drastisch gekürzt wird. Dem sind aber insofern Grenzen gesetzt, als die erworbenen Rentenansprüche nach Art. 14 GG grundsätzlich dem Eigentumsschutz unterliegen. ${ }^{100}$

Verteilt man die Lasten auf beide Generationen, die Erwerbstätigen und die Rentner, dann erscheint eine Einführung des Grundrentensystems unter dem Blickwinkel der politischen Ökonomie als illusorisch. Die Vorteile des Systems greifen erst nach einer längeren Übergangsphase, wirken sich also erst für die jetzt noch nicht wahlberechtigte Generation aus. Die gegenwärtigen Wähler, die ja über die Einführung des Systems zu entscheiden haben, sind dagegen durch die Kumulierung der Lasten in der Übergangsperiode größtenteils Verlierer bei einem Systemwechsel, so daß sich dafür kaum eine Mehrheit finden wird. ${ }^{101}$ Insgesamt können dem Vorschlag einer Ablösung des gegenwärtigen Alterssicherungssystems durch ein Grundrentensystem daher kaum Realisierungschancen eingeräumt werden.

\subsection{2 Übergang zum Kapitaldeckungsverfahren}

Besonders vor der Verabschiedung des Rentenreformgesetzes 1992 wurden verschiedene Varianten von Kapitaldeckungsverfahren als Ersatz oder Ergänzung für das gegenwärtige Umlageverfahren zur Entschärfung der demographischen Krise der Gesetzlichen Rentenversicherung vorgeschlagen. Ausgangspunkt ist dabei die Überlegung, daß die Rendite und das Rentenniveau im Kapitaldeckungsverfahren - zumindest auf den ersten Blick - unabhängig von demographischen Einflüssen sind und bei der zu erwartenden Bevölkerungs- und Wirtschaftsentwicklung dieses Verfahren zudem eine höhere Verzinsung der Beiträge verspricht als das Umlageverfahren. ${ }^{102}$ Die Varianten unterscheiden sich v.a. darin, daß im einen Fall ein vollständiger Ersatz des gegenwärtigen Systems vorgesehen wird, während es sich im anderen Fall um einen Teilersatz handelt, bei dem die Gesetzliche Rentenversicherung im Umlageverfahren eine Grundversorgung sicherstellt und nur eine Zusatzversorgung im Kapitaldeckungsverfahren erfolgt (Teilkapitaldeckungsverfahren). ${ }^{103}$ In beiden Varianten soll die Bewältigung der zukünftigen Finanzierungsprobleme durch die Ansparung eines Kapitalstocks in den nächsten

99 Dieser Kritikpunkt gilt besonders für den Grundrenten-Vorschlag der GRÜNEN. Hier wird in einem noch stärkeren Maße Anspruchshaltungen gegenüber dem Staat Vorschub geleistet.

100 Vgl. Rosen/Windisch (1992) S. 381ff.

101 Zu dieser Einschätzung vgl. auch Ebert (1993) S. 99.

102 Das Kapitaldeckungsverfahren ist dann vorteilhaft, wenn der Realzins größer ist als das Wachstum der Lohnsumme. Vgl. Unterkapitel 1.3.

103 Wird dieses Teilkapitaldeckungsverfahren privatwirtschaftlich organisiert, ergeben sich sowohl in der grundsätzlichen ordnungspolitischen Ausrichtung als auch in den Wirkungen starke Übereinstimmungen mit Grundrentenvorschlägen. Der Hauptunterschied zu dem oben diskutierten Vorschlag von Miegel/Wahl liegt dann darin, daß dort eine Steuerfinanzierung vorgesehen ist. während die Grundversorgung im Teilkapitaldeckungsverfahren nach wie vor über Beiträge sicher gestellt wird. 
zwei bis drei Jahrzehnten und seine sukzessive Auflösung in den Jahren mit dem höchsten Rentnerquotienten erfolgen.

Zunächst wird untersucht, inwiefern ein derartiger Transfer in die Zukunft gelingen kann, denn dieser Ansatz steht im Widerspruch zu der als Mackenroth-These bekanntgewordenen Behauptung, "... daß aller Sozialaufwand immer aus dem Volkseinkommen der laufenden Periode gedeckt werden muß. Es gibt gar keine andere Quelle und hat nie eine andere Quelle gegeben, aus der Sozialaufwand fließen könnte, es gibt keine Ansammlung von Fonds, keine Übertragung von Einkommensteilen von Periode zu Periode, kein 'Sparen' im privatwirtschaftlichen Sinne". ${ }^{104}$ Mackenroth geht von einer güterwirtschaftlichen Sichtweise aus. Danach können Ersparnisse nur durch Realkapitalbildung gebildet werden. Dieses Realkapital in Form von Maschinen, Gebäuden etc. kann dann aber nicht in einer späteren Periode in Konsumgüter umgewandelt werden. Unabhängig vom Finanzierungsverfahren der Rentenversicherung - also auch beim Kapitaldeckungsverfahren - muß daher die jeweils erwerbstätige Generation die Rentenausgaben derselben Periode erwirtschaften.

Beim Kapitaldeckungsverfahren geschieht dies dadurch, daß die Rentnergeneration ihre Ersparnisse auflöst, d.h. ihre Anteile am Realkapital der Volkswirtschaft an die nachfolgende Generation verkauft. Diese Generation erwirbt die Ansprüche von den Rentnern und verzichtet im Gegenzug auf einen Teil der in dieser Periode erwirtschafteten Konsumgüter. Existiert keine nachfolgende Generation oder will diese keine Ersparnisse bilden, dann ist der angesammelte Kapitalstock für die Rentner wertlos. ${ }^{105}$ Das Entsparen in Form einer Umwandlung von Kapital- in Konsumgüter kann nur mit Hilfe der erwerbstätigen Generation gelingen, indem diese erstens Konsumgüter produziert und zweitens bereit ist, diese gegen den Erwerb von Ansprüchen auf Kapitalgüter aufzugeben. "In langdauernd nutzbare Gebrauchsgüter investiertes Kapital läßt sich nur desinvestieren, indem wir es im Gebrauch abnutzen, die Wohnungen 'abwohnen' und die nicht mehr bewohnbaren abreißen; in Produktionsmittel investiertes Kapital wird desinvestiert, indem wir es im Betrieb verschleißen und die nicht mehr betriebsfähigen (oder technisch überholten) Produktionsmittel verschrotten. Im ersten Fall ergibt die Desinvestition einen Haufen Schutt, im letzteren Fall einen Haufen Schrott - aber niemals Güter des laufenden Verbrauchs, die für den Lebensunterhalt verwendbar wären." 106

Auf welche Arten läßt sich dieser güterwirtschaftliche Zusammenhang umgehen? Drei Wege kommen in Betracht: Lagerhaltung, Unterlassung von Ersatzinvestitionen und die Anlage von Vermögen im Ausland. $\mathrm{Zu}$ unterscheiden ist dabei jeweils zwischen der technischen und der wirtschaftlichen Realisierbarkeit.

Technisch ist eine Lagerung von Gütern auch über mehrere Jahrzehnte aufgrund der fortgeschrittenen Konservierungstechnik durchaus denkbar, wenn man von Dienstleistungen und einigen anderen Güterarten absieht. Allerdings ist ein zeitlicher Einkommenstransfer durch Lagerung wirtschaftlich kaum sinnvoll. Dagegen sprechen zum einen Nachfragefaktoren: Im Laufe der Zeit ändern sich - von Präferenzänderungen und der Markteinführung neuer Produkte abgesehen - die Einkommensverhältnisse und die relativen Preise der Güter, so daß sich die Nachfragestruktur grundlegend ändert. Das Angebot kann sich aber dieser geänderten

104 Mackenroth (1952) S. 41 (Hervorhebung im Original).

105 In monetärer Sicht käme es auf den Kapitalmärkten zu massiven Verkaufsaufträgen, denen keine Kaufaufträge gegenüberständen. Der dadurch bewirkte Kursverfall führt zu einer Entwertung der Ersparnisse der Rentnergeneration.

106

Nell-Breuning (1986) S. 738. 
Nachfragestruktur nur dann durch höhere Produktqualitäten und die Entwicklung neuerer Produkte anpassen, wenn die Produktion der Güter und ihr Absatz zeitlich nahe beieinander liegen.

Zum anderen stehen einer langfristigen Lagerung von Gütern auch Effizienzüberlegungen im Wege, denn erstens verursacht die Lagerhaltung Kosten, z.B. durch die Konservierung und damit meistens einhergehende Qualitätsverluste, die Einlagerung, Raummieten und die Überwachung ${ }^{107}$, und zweitens liegen die (realen) Produktionskosten wegen des technischen und organisatorischen Fortschritts in der Zukunft voraussichtlich unter denjenigen der Gegenwart. Die Lagerung bietet daher allenfalls für sehr wenige Güterarten eine wirtschaftlich sinnvolle Möglichkeit des Transfers von Einkommen in die Zukunft.

Die Unterlassung von Ersatzinvestitionen ist zu einem Einkommentransfer in die Zukunft schon eher geeignet. Voraussetzung dafür ist allerdings, daß die gegenwärtigen Ersparnisse auch tatsächlich in Kapitalgüter investiert werden und ein Entsparen in der Zukunft möglich ist. Ein Entsparen in der Zukunft, also die Umwandlung der Kapitalgüter in Konsumgüter, ist im Gegensatz zu der oben zitierten Aussage von Nell-Breuning in begrenztem Umfang möglich, indem auf Reinvestitionen verzichtet wird. Dadurch kann der bisher für Bruttoinvestitionen verwendete Teil des laufenden (Brutto-)Sozialprodukts ${ }^{108}$ von etwa 20 Prozent zur Herstellung von Konsumgütern umgeschichtet werden. Eine völlige Unterlassung von Netto- und Ersatzinvestitionen ist allerdings kaum zu erwarten und wäre aufgrund negativer Rückwirkungen auf den Wirtschaftsprozeß infolge einer durch Überalterung der Produktionsanlagen sinkenden Produktivität und internationalen Wettbewerbsfähigkeit auch kaum wünschenswert. In geringem Umfang ist gleichwohl ein Entsparen möglich.

Die dazu in der Gegenwart erforderliche Anlage der Ersparnisse in Kapitalgütern könnte sich als recht schwierig erweisen, wenn die Unternehmen auf die durch das gestiegene Kapitalangebot sinkenden Zinsen nicht mit einer entsprechenden Investitionsausweitung reagieren. Da die Vorteilhaftigkeit einer Investition außer vom Zinsniveau entscheidend von den zukünftigen Absatz- und Gewinnerwartungen abhängt, diese aber zumindest im Inland durch die schrumpfende Bevölkerung gedämpft werden, müßten die (Real-)zinsen sehr stark fallen, um die Unternehmen dennoch zu verstärkter Investitionstätigkeit anzuregen. Ein derartiger Zinsverfall würde aber die Vorteilhaftigkeit des Kapitaldeckungsverfahrens gegenüber dem Umlageverfahren und damit einen Grund des Systemwechsels in Frage stellen.

Berücksichtigt man die internationale Wirtschaftsverflechtung, dann ist ein starker Zinsverfall allerdings nicht zu erwarten. Bei einer genügend großen Differenz in den erwarteten Renditen zugunsten des Auslands werden die Ersparnisse aus dem Inland abfliessen und im Ausland zu einer Vermehrung des Realkapitals führen.

Diese Überlegung leitet unmittelbar über zu der Anlage im Ausland als dem dritten Weg eines Transfers von Einkommen in die Zukunft. Prinzipiell ist es möglich, einen Kapitalstock im Ausland anzulegen und später mittels der Zins- und Tilgungszahlungen Importe zu finanzieren, die dann die Versorgungslücke im Inland decken. Dieser Weg ist allerdings mit drei Problemen verbunden: Bei einer Anlage im Ausland besteht ein hohes Wechselkursrisiko, das durch den langen Anlagezeitraum noch verstärkt wird. Darüber hinaus besteht ein

107 Vgl. Külp (1993) S. 82.

108 Homburg (1988) S. 67f. weist daraufhin, daß deshalb das Bruttosozialprodukt und nicht wie von Mackenroth behauptet das Nettosozialprodukt (Volkseinkommen) als Obergrenze für den Konsum einschließlich des Sozialaufwands anzusehen ist. 
politisches Risiko, das sich in Anlagebeschränkungen für Ausländer während der Zeit des Aufbaus des Kapitalstocks, in Enteignungen während seines Bestandes und in Kapitalexportbeschränkungen während der Zeit der Auflösung zeigen kann. Das politische Risiko steigt dabei mit der Höhe des Anlagebetrages, da hohe Investitionen von Ausländern von den Inländern oft als "nationaler Ausverkauf" angesehen werden und hohe Kapitalabflüsse die Vermögenspositionen der Inländer berühren. Das dritte Problem besteht darin, daß die demographische Entwicklung in den meisten Industriestaaten - wenngleich oft in abgeschwächter Form - ähnlich wie in Deutschland verläuft. Sie scheiden daher als potentielle Anlageländer mit einiger Sicherheit aus. Bei einer Anlage des Kapitalstocks in weniger entwickelten Ländern steigen aber sowohl das Wechselkursrisiko als auch das politische Risiko.

Als Ergebnis der Diskussion der Mackenroth-These ist festzuhalten, daß grundsätzlich ein Einkommenstransfer in die Zukunft möglich ist, diese Möglichkeit mit steigendem Transfervolumen aber mit wachsenden Schwierigkeiten verbunden ist. Inwieweit ein Systemwechsel zum Kapitaldeckungsverfahren einen Beitrag zur Lösung des zu erwartenden Finanzierungsproblems der Gesetzlichen Rentenversicherung leisten kann, hängt damit maßgeblich von dem notwendigen Transfervolumen $\mathrm{ab}$.

Nach einer empirischen Schätzung von Grohmann für die Bundesrepublik Deutschland nach dem Gebietsstand vor dem 3.10 .1990 beliefen sich die Ansprüche von Rentnern und Aktiven gegenüber der Gesetzlichen Rentenversicherung Ende 1984 auf 5,0 bis 5,4 Billionen DM, also etwa auf die Höhe des gesamten reproduzierbaren Sachvermögens beziehungsweise des Vierfachen des Volkseinkommens in diesem Jahr. ${ }^{109}$ Der Aufbau eines derartigen Kapitalstocks ist unrealistisch, weshalb die meisten Reformvorschläge nur ein Teilkapitaldeckungsverfahren propagieren. ${ }^{110}$ Buttler/Jäger ${ }^{111}$ haben die Höhe des Kapitalstocks errechnet, der bei Aufbau eines Teilkapitaldeckungsverfahrens nötig wäre, um den Beitragssatz in den kritischen Jahren um drei Prozentpunkte zu senken. Zum Zweck der Kapitalakkumulation müßten bis zum Jahr 2010 jährlich um drei Prozentpunkte höhere Beitragssätze entrichtet werden. Danach wird dieser Zusatz-Beitragssatz jährlich um 0,2 Prozentpunkte gesenkt. Der Anteil des Alterskapitalstocks am gesamten Kapitalstock würde bei dieser Regelung sieben Prozent nicht übersteigen und die Größenordnung des zeitlichen Einkommenstransfers bewegt sich damit in einem realisierbaren Bereich. Allerdings läßt sich dadurch der Anstieg des Beitragssatzes auch nur mäßig bremsen.

Aber schon die Verwaltung eines relativ "geringen" Kapitalstocks birgt die Gefahr politischer Manipulation. Politische Einflußnahme könnte aus wahltaktischen Gründen zu einer konsumtiven Verwendung der Beträge führen, indem beispielsweise eine Anlage in Staatsschuldtiteln vorgeschrieben wird und der Staat die daraus verfügbaren Mittel für Transferzahlungen verwendet. ${ }^{112}$ Ein Einkommenstransfer in die Zukunft ist aber nur bei investiver Verwendung der Mittel möglich. Anderenfalls handelt es sich lediglich um die Umwandlung

109 Vgl. Grohmann (1987) S. 74ff. Müller/Roppel (1990) erhalten mit etwa 7 Billionen DM (in Preisen von 1988) vergleichbare Werte, Dinkel (1986) nennt dagegen sogar weit höhere Werte bis zum 18 fachen des Volkseinkommens.

110 Ein derartiges Teilkapitaldeckungsverfahren wird beispielsweise seit einer grundlegenden Reform im Jahr 1983 in den Vereinigten Staaten von Amerika angewandt. Vgl. Harrison/Raffelhüschen (1989).

111 Vgl. Buttler/Jäger (1988).

112 Zweifel an einer investiven Verwendung des zu bildenden Kapitalstocks in einem staatlichen Alterssicherungssystem äußem Rosen/Windisch (1992) S. 423. 
von impliziter Staatsverschuldung in Form von Rentenansprüchen in explizite Staatsverschuldung, ohne daß dadurch intergenerative Wirkungen erzielt werden. ${ }^{113}$ Außerdem kann die höhere Belastung aufgrund des Aufbaus eines Teilkapitaldeckungsverfahrens in der Gesetzlichen Rentenversicherung zu einer Reduzierung der privaten Vorsorge führen.

Vorteile hätte ein Teilkapitaldeckungsverfahren gegenüber dem Umlageverfahren unter dem Gesichtspunkt der intergenerativen Gerechtigkeit. Während beim Umlageverfahren mit den in 40 Jahren Aktiven eine Generation zur Finanzierung der Rentenlast herangezogen wird, die keinerlei Verantwortung für die Finanzprobleme trifft, wird beim Kapitaldeckungsverfahren die spätere Rentnergeneration an der Aufbringung der Mittel zur Deckung ihrer Renten beteiligt und so eine nicht verursachergerechte Belastung der nachfolgenden Generation von Beitragszahlern vermieden. ${ }^{114}$

Aus polit-ökonomischer Sicht ist die Einführung eines Teilkapitaldeckungsverfahrens kaum zu erwarten, die Einführung eines Systems mit vollständiger Kapitaldeckung nahezu ausgeschlossen. Die Generation der Erwerbstätigen hätte während einer Jahrzehnte umfassenden Übergangsperiode neben der Finanzierung der im Umlageverfahren erworbenen Altansprüche zusätzlich Mittel für eine eigene Alterssicherung bereitzustellen. Die dadurch entlastete nachfolgende Generation ist aber noch nicht geboren oder zumindest noch nicht stimmberechtigt, so daß ein Systemwechsel auch dann unwahrscheinlich ist, wenn er - wie neuere Untersuchungen $^{115}$ nahelegen - intergenerational pareto-superior wäre. Je näher die Zeit der demographischen Belastungen rückt, desto höher wird außerdem der jährliche Zusatzbeitrag, der für eine Entlastung der zukünftigen Beitragszahler um beispielsweise fünf Prozentpunkte erforderlich ist. Das (Teil-)Kapitaldeckungsverfahren kann daher als Lösungsmöglichkeit für die anstehende demographische Krise der Gesetzlichen Rentenversicherung bereits jetzt nur noch eine begrenzte Wirkung entfalten.

\subsubsection{Elternrente}

Ein gegenwärtig besonders häufig diskutierter systemverändernder Vorschlag einer Differenzierung der Rentenleistung nach der Kinderzahl wurde von Borchert unter der Bezeichnung "duales Modell" entworfen. ${ }^{116}$ Ziel dieses Vorschlags ist eine Vollversorgung im Ruhestand für Elternpaare mit mindestens zwei Kindern und einem vollerwerbstätigen Partner, eine eigenständige Altersversorgung für Erziehende und eine Grundversorgung für Kinderlose.

Erreicht werden sollen diese Ziele durch eine Halbierung der Rentenleistungen bei sonst unveränderter Rentenformel, d.h. ohne daß die Beiträge gekürzt würden. Die dadurch verfügbare zweite Hälfte der Beitragseinnahmen soll zur Finanzierung von "Elternrenten" verwendet

113 Die Gefahr einer rein konsumtiven Verwendung der in Staatsschuldtiteln anzulegenden Überschüsse des amerikanischen Alterssicherungssystems hat zu verschiedenen Reformvorschlägen Anlaß gegeben. Vgl. Harrison/Raffelhüschen (1989) S. 424ff.

114 Vgl. Suntum (1989) S. 209.

115 Vgl. Homburg (1990) und Breyer/Straub (1993). Die Fachdiskussion über die Effizienzwirkungen verschiedener Alterssicherungssysteme bezieht sich meistens auf stabile Bevölkerungen und ist daher für den hier betrachteten Fall von Verschiebungen in der Altersstruktur weniger relevant.

116 Vgl. Borchert (1981) S. 225ff., Borchert (1989) S. 120ff. und Borchert (1993) S. 269ff. sowie ähnlich Renesse (1992). Befürwortet wurde dieser Vorschlag außerdem von Oeter (1992). 
werden. Die Höhe der Elternrente $\left(R_{E}\right)$ soll etwa die Hälfte des Produkts aus Durchschnittsbeitrag und reziprokem Rentnerquotient betragen:

$$
\text { B.10) } R_{E}=\frac{1}{2} b L \frac{N}{A}
$$

Borchert errechnet für 1993 bei einem durchschnittlichen Beitrag von 724 DM pro Monat und einem reziproken Rentnerquotient von 2 eine Elternrente von 724 DM pro Kind und Monat. ${ }^{117}$ Die Verteilung der Elternrente auf die Eltern erfolgt umgekehrt proportional zu den von jedem Elternteil monetär erworbenen Rentenansprüchen. Auf diese Art und Weise hat jeder Elternteil eigenständige Rentenansprüche, weshalb die Hinterbliebenenrente entfallen kann und die dadurch eingesparten Mittel zur Finanzierung des Systemübergangs zur Verfügung stehen. Sie dürften aber kaum ausreichen, um einen Wechsel ohne Eingriffe in Besitzstände zu ermöglichen.

Das duale Modell von Borchert stellt einen Ansatz zur Verwirklichung des Drei-Generationen-Gedankens dar, kann aber zumindest für die monetären Beiträge auch nicht auf die rechtlich abgesicherte - Illusion verzichten, durch Beitragszahlungen würden ökonomische Anwartschaften erworben. Kritisiert wird weiterhin, daß das System in seiner gegenwärtig diskutierten Form einige innere Inkonsistenzen aufweist. Zunächst ist die Bezeichnung "Rentnerquotient" in der Formel zur Berechnung der Höhe der Elternrente irreführend. Aufgabe dieses Faktors in der Formel ist es, die Höhe der Ansprüche auf Elternrente und die zu ihrer Finanzierung erbrachten Beiträge zum Ausgleich zu bringen. Dazu wird das zur Verfügung stehende Beitragsvolumen - die Hälfte des Durchschnittsbeitrags multipliziert mit der Menge der Beitragszahler $\left(\frac{1}{2} b L N\right)$ - durch die Zahl der Anspruchsberechtigten $A$ (der "Rentner" im Rentnerquotienten) dividiert. So gelangt man zum Rentenanspruch pro Kind $\left(R_{E}\right)$. Da aber ein Rentner durchaus Ansprüche aus der Erziehung mehrerer Kinder haben kann, muß hier unter den "Rentnern" $(A)$ des reziproken "Rentnerquotienten" der "einzeln auf jedes Kind bezogene Anspruch auf Elternrente" 118 verstanden werden. Anderenfalls wäre eine Deckung der Rentenansprüche durch die Beitragszahlungen rein zufällig und würde nur auftreten, wenn sich die Zahl der Rentner und die Zahl der rentenbegründenden Kinder dieser Rentner gerade entsprechen.

Die in dieser Weise korrigierte Formel zur Berechnung der Höhe der Elternrente müßte lauten: Hälfte des Durchschnittsbeitrags mal Zahl der Beitragszahler dividiert durch die Zahl der eine Elternrente begründenden Kinder $N_{E}$

$$
\text { B.11) } R_{E}=\frac{1}{2} b L \frac{N}{N_{E}}
$$

Zur Vereinfachung sei angenommen, daß die Nennergröße, d.h. die Anzahl der elternrentenbegründenden Kinder $\left(N_{E}\right)$, der Anzahl der potentiellen Beitragszahler $\left(N_{p}\right)$ entspricht. In diesem Fall wird die Hälfte des Durchschnittsbeitrags multipliziert mit einem Bruch, in

117 Analog zur Beitragsbemessungsgrenze für monetäre Beiträge sieht Borchert (1993) S. 271 auch eine Obergrenze für Elternrenten - z.B. bei vier Kindern - vor.

118 Gallon (1993) S. 11. Hervorhebung im Original. 
dessen Zähler die tatsächlich beitragszahlenden Kinder stehen und dessen Nenner die Anzahl der potentiellen Beitragszahler bildet:

$$
\text { B.12) } R_{E}=\frac{1}{2} b L \frac{N}{N_{p}} \text {. }
$$

Damit wird die Hälfte des Durchschnittsbeitrags multipliziert mit einem Indikator für die Erwerbsbeteiligung der Kindergeneration. Bei einer hohen Erwerbsbeteiligung liegt die Elternrente pro Kind gleichfalls recht hoch, bei einer niedrigen Erwerbsbeteiligung ist sie dagegen geringer. Das Elternrentensystem setzt aber gerade Anreize, die Erwerbsbeteiligung zu reduzieren, so daß hier mit negativen Rückwirkungen auf die Höhe der Elternrente zu rechnen ist. ${ }^{119}$ Ebert bemängelt in diesem Zusammenhang die Subventionierung der traditionellen Arbeitsteilung in der Hausfrauenehe. Statt sich dem Trend einer zunehmenden Erwerbsbeteiligung von Frauen entgegenzustellen, solle der Staat durch geeignete institutionelle Maßnahmen die Erwerbstätigkeit beider Elternteile ermöglichen. ${ }^{120}$

$\mathrm{Zu}$ Kritik Anlaß gibt auch die willkürliche Festlegung des Anteils des Elternrentenvolumens am Gesamtrentenvolumen auf exakt die Hälfte. Die qualitative Gleichgewichtigkeit von monetärem Beitrag und Naturalbeitrag wird hier unbegründet in eine quantitative Gleichwertigkeit überführt. Als Konsequenz ergibt sich unter den gegenwärtigen Verhältnissen eine Überbewertung der Kindererziehungsleistung, da die Höhe der Elternrente für vier Kinder durch monetäre Beiträge nicht zu erreichen ist, selbst wenn 50 Jahre lang Beiträge von einem über der Beitragsbemessungsgrenze liegenden Einkommen gezahlt würden. ${ }^{121}$

Neben den Formeln für die Höhe der Elternrente und für die Aufteilung des Gesamtrentenvolumens auf Elternrentenvolumen und lohnbezogene Renten ist auch die Verteilung der Elternrentenansprüche zwischen den Eltern nicht konsequent durchdacht. Bei der Aufteilung im reziproken Verhältnis zu den monetären Anwartschaften wird einseitig auf die Betreuung der Kinder und damit auf den zeitlichen Aufwand abgestellt. Die aus dem Erwerbseinkommen bestrittene Finanzierung der Aufwendungen für die Kinder wird dagegen nicht honoriert. Als Folge können sich deutlich geringere Rentenansprüche für den überwiegend erwerbstätigen Elternteil ergeben, obwohl er durch die Finanzierung der Kinder wesentlich an dem Naturalbeitrag beteiligt gewesen ist. ${ }^{122}$

119 Vgl. zu dieser Kritik Ebert (1993) S. 104 und Gallon (1993) S. 11f. Als weiteren Kritikpunkt führt Gallon an, daß durch das Finanzierungsverfahren keine intragenerationelle Umverteilung von kinderarmen zu kinderreichen Mitgliedern der aktiven Generation vorgenommen wird. Dagegen läßt sich allerdings einwenden, daß durch die Halbierung der Rentenanwartschaft für Kinderlose ein erhöhter Zwang zu privater Vorsorge besteht und dadurch die verteilungspolitisch problematische ungleiche Verteilung der Konsummöglichkeiten von Kinderreichen und Kinderarmen während ihrer aktiven Lebensphase zumindest reduziert wird.

Vgl. Ebert (1993) S. 102.

Vgl. Gallon (1993) S. 14. Hier läßt sich einwenden, daß Kinder hohe monetäre Kosten für die Eltern verursachen und ein Teil der Elternrenten daher als Erstattung dieser monetären Kosten anzusehen ist. Allerdings erscheint es wenig sinnvoll, die monetären Aufwendungen für Kinder erst Jahrzehnte nach ihrer Entstehung zu erstatten. Zweckmäßiger wäre hier sicherlich ein ausgebauter Kinderlastenausgleich, der den Eltern finanzielle Mittel zu dem Zeitpunkt verschafft, zu dem auch die Kosten anfallen.

Vgl. Gallon (1993) S. 15. 
Insgesamt muß der Elternrentenvorschlag als noch nicht vollständig ausgereift angesehen werden. Einen Beitrag zur Entschärfung der demographischen Krise in der Alterssicherung kann er in jedem Fall nur dann leisten, wenn die kinderzahlabhängige Rentenhöhe zu einem Anstieg der Geburtenrate und damit der Zahl der zukünftigen Beitragszahler führt. Wie in Teil $\mathrm{C}$ dieser Arbeit ausgeführt wird, entfalten aber kinderzahlabhängige Leistungen im Rentenalter eine vergleichsweise geringe pronatalistische Wirkung. Der hier analysierte Vorschlag hat dann aber nur eine Umverteilung der aufgrund der demographischen Entwicklung zu erwartenden Lasten zur Folge: Rentner mit Kindern werden besser gestellt, der Rentenanspruch kinderloser Rentner dagegen halbiert. Hält man an der (Netto-)Lohnbindung fest, ergibt sich für die Beitragszahler keine Entlastung.

\subsection{Lösungsansätze durch bevölkerungspolitische Maßnahmen}

Die bisher in den Unterkapiteln 3.1 und 3.2 analysierten Vorschläge zur Lösung der demographischen Krise der Gesetzlichen Rentenversicherung haben sich als letztlich wenig geeignet erwiesen. Der Grund dafür liegt darin, daß diese Vorschläge nicht an der Korrektur der demographischen Ursachen dieser Belastung ansetzen, sondern sich weitgehend auf die Umverteilung der entstehenden Lasten zwischen Rentnern, Beitragszahlern und Steuerzahlern beschränken. Ob eine Erhöhung der Fertilität oder eine verstärkte Zuwanderung eher einen Beitrag zur Reduzierung der Belastung von Rentner sowie Beitrags- und Steuerzahlern leisten können, wird in diesem Unterkapitel untersucht. Dabei geht es hier zunächst nur darum, anhand von Simulationsrechnungen zu analysieren, welche Wirkungen von einer Erhöhung der Fertilitätsrate bzw. einer verstärkten Einwanderung auf den Altersaufbau der Bevölkerung und damit indirekt auch auf die Finanzlage der Gesetzlichen Rentenversicherung ausgehen. Inwieweit eine Erhöhung der Fertilitätsrate oder eine verstärkte Zuwanderung realisierbar sind und ob diese Realisierung ökonomisch sinnvoll ist, wird in den Teilen C und D dieser Arbeit untersucht.

\subsubsection{Die zukünftige Entwicklung bei erhöhter Fertilität}

$\mathrm{Zu}$ den Auswirkungen unterschiedlicher Fertilitätsraten auf die zukünftige Entwicklung der Finanzlage der Gesetzlichen Rentenversicherung liegen meines Wissens nur wenige Simulationsergebnisse vor. Deshalb muß hier auch auf Untersuchungen zurückgegriffen werden, die den Einfluß unterschiedlicher Annahmen über das Fertilitätsverhalten auf vorgelagerte Einflußgrößen der Finanzlage der Gesetzlichen Rentenversicherung wie den Altenquotienten oder auch den Eckrentnerquotienten analysieren.

Barth, Hain und Müller benutzen das Rentenmodell des Verbands Deutscher Rentenversicherungsträger, um u.a. mittels zweier Varianten die Sensitivität der Entwicklung des Eckrentnerquotienten bezüglich der Fertilitätsannahmen einschätzen zu können. ${ }^{123}$ In dem Grundszenario gehen die Autoren für die alten Bundesländer von dem durchschnittlichen

12.3 Vgl. Barth/Hain/Müller (1994). 
Fruchtbarkeitsniveau der Jahre 1988 bis 1990 aus, d.h. von einer Nettoreproduktionsrate von 0,682. Für die neuen Bundesländer wird ausgehend von einem niedrigeren Anfangswert eine Angleichung an das Fertilitätsverhalten in den westlichen Ländern unterstellt. In einem zweiten Szenario wird angenommen, daß ab dem Jahr 1996 die Nettoreproduktionsrate im Verlauf einer Dekade auf 0,8 steigt.

Welche Wirkungen ergeben sich durch einen derartigen Anstieg der Nettoreproduktionsrate um 17 Prozent auf den Eckrentnerquotienten? Bis zum Jahr 2010 sind keine Änderungen gegenüber dem Grundszenario erkennbar, weil die zusätzlich Geborenen bis dahin noch nicht zu einem Anstieg der potentiellen Beitragszahler geführt haben. Im Jahr 2040 wird der Eckrentnerquotient gegenüber dem Ausgangsszenario um etwa ein Prozent gesenkt. Ein allmählich einsetzender und zudem nicht allzu starker Anstieg der Geburten ist daher kaum geeignet, die sich ab 2010 zuspitzende Finanzsituation der Gesetzlichen Rentenversicherung zu entschärfen.

Eine weitere Simulationsrechnung mit unterschiedlichem Fertilitätsverhalten wurde von Steinmann vorgenommen. In dem Grundszenario ${ }^{124}$ unterstellt er eine konstante Mortalität nach der Allgemeinen Sterbetafel 1986-88 und geht von einer zusammengefaßten Geburtenrate von 1,34 für einheimische und von 1,9 für eingewanderte Frauen aus, was den Werten des Jahres 1987 für das alte Bundesgebiet entspricht. In dem Szenario mit erhöhter Fertilität wird ein Anstieg der zusammengefaßten Geburtenrate der einheimischen Frauen um 27 Prozent auf 1,7 Kinder pro Frau bis zum Jahr 2000 unterstellt, das Fertilitätsverhalten der eingewanderten Frauen bleibt dagegen annahmegemäß unverändert. Während im Grundszenario die Altenquote, die den Anteil der über 65jährigen an der Wohnbevölkerung angibt, von 13,99 Älteren pro 100 Einwohnern im Jahr 1991 auf 24,10 (2030) steigt, wird diese Zunahme in dem Szenario mit erhöhter Fertilität auf 21,98 beschränkt. Der Anstieg der Altenquote fällt also durch die höhere Fertilität um 21 Prozent niedriger aus. Die im Vergleich zu dem Ergebnis von Barth, Hain und Müller wesentlich höhere Entlastungswirkung ist v.a. darauf zurückzuführen, daß durch Steinmann ein stärkerer und v.a. auch früher einsetzender Geburtenanstieg simuliert wird. Darüber hinaus werden durch die Meßgröße der Altenquote auch die zusätzlichen Geborenen erfaßt, die im Jahr 2030 noch nicht potentielle Beitragszahler geworden sind und die daher nicht in den Eckrentnerquotienten eingehen.

Felderer unterstellt einen moderaten Anstieg der Lebenserwartung über die nächsten zehn Jahre sowie einen Rückgang des Einwanderungsüberschusses auf 76.000 Personen pro Jahr. Hinsichtlich der Altersstruktur der Wanderer wird von den Werten der achtziger Jahre ausgegangen. ${ }^{125}$ Im Grundszenario mit einer Nettoreproduktionsrate von 0,68 ergibt sich ein Beitragssatz zur Gesetzlichen Rentenversicherung, der mit etwa 27 Prozent im Jahr 2030 zwischen der oberen und der unteren Variante des Prognos-Gutachtens liegt. ${ }^{126}$ Für Nettoreproduktionsraten von 0,5 bzw. 0,8 errechnet Felderer für das Jahr 2020 einen Beitragssatzunterschied von weniger als 2 Prozentpunkten, für die Jahre nach 2030 dagegen von mehr als 5 Prozentpunkten. ${ }^{127}$ Auch hier macht sich also wieder die verzögerte Wirkung von

\footnotetext{
124 Ausgegangen wird hier jeweils von der Variante ohne Zuwanderung (Variante A).

125 Vgl. Felderer (1994a) S. 202f.

126 Vgl. Felderer (1994a) S. 203. Legt man den drastischen Anstieg des Bundeszuschusses von sieben Prozent der Steuereinnahmen auf über zwanzig Prozent auf die Beitragszahler um, ergibt sich ein Beitragssatz von 33 Prozent.
}

Vgl. Felderer (1994a) S. 205. 
Fertilitätsänderungen bemerkbar, die dadurch entsteht, daß Neugeborene erst nach etwa zwei Jahrzehnten zu Beitragszahlern werden.

Als Ergebnis der Simulationsstudien über die Auswirkungen unterschiedlicher Fertilitätsraten kann festgehalten werden, daß ein Geburtenanstieg allein nicht in der Lage ist, die Verschlechterung der finanziellen Situation der Gesetzlichen Rentenversicherung zu verhindern. Soll durch ein geändertes Fertilitätsverhalten in dem kritischen Zeitraum ab 2020 eine merkliche Entlastungswirkung erzielt werden, muß ein deutlicher und kurzfristig einsetzender Anstieg der Geburtenrate erreicht werden.

\subsubsection{Die zukünftige Entwicklung bei verstärkter Einwanderung}

Bevor auf die Ergebnisse verschiedener Simulationen zu den Auswirkungen verstärkter Einwanderung eingegangen wird, soll zunächst geklärt werden, welche grundsätzlichen Unterschiede in den demographischen Wirkungen zwischen dauerhaft erhöhter Fertilität und dauerhafter, positiver Nettowanderung bestehen. Dabei kann die Betrachtung auf den weiblichen Bevölkerungsteil eingeengt werden, weil nur Frauen Kinder gebären und weil Frauen auf absehbare Zeit in den reproduktiven Altersstufen das knappe Geschlecht darstellen. ${ }^{128}$

Mit Blick auf die absolute Bevölkerungszahl haben eine pro Periode zusätzlich geborene bzw. eingewanderte Person kurzfristig, d.h. ohne Berücksichtigung der dadurch hervorgerufenen weiteren Geburten und Sterbefälle, den gleichen Effekt. Aber die Altersstruktur wird auch dann unterschiedlich beeinflußt, wenn man die zweite und spätere Generationen zunächst außer acht läßt. Während die Erhöhung der Fertilität erst nach 20 Jahren Auswirkungen auf den Altenquotienten hat, schlägt sich eine positive Nettowanderung sofort in einer Änderung dieser demographischen Kennzahl nieder, wenn nicht nur Personen unter 20 Jahren wandern. Je nach dem, ob die Altersstruktur der Zuwanderer jünger oder älter als die der Einheimischen ist, sinkt oder steigt der Altenquotient im Vergleich zur Situation ohne Zuwanderung. Sogar bei einem Wanderungssaldo von Null wird sich im Normalfall der Altenquotient durch Wanderungsbewegungen verändern. Wandern beispielsweise v.a. junge Personen zu, während hauptsächlich Ältere abwandern, sinkt der Altenquotient auch dann, wenn keine Nettozuwanderung erfolgt. Die Altersstruktur der Wanderungsbewegungen hat daher schon in kurzfristiger Perspektive einen gewichtigen Einfluß auf deren demographische Wirkungen.

Noch deutlicher wird der entscheidende Einfluß der Altersstruktur der Zugewanderten in langfristiger Sicht, also unter Einbeziehung der durch die Wanderungsbewegungen verursachten Änderungen der Geburten und Todesfälle. Drei Extremfälle mögen zur Veranschaulichung dienen, wobei zur Vereinfachung zunächst unterstellt wird, daß die altersspezifischen Fertilitäts- und Mortalitätsraten von Zuwanderern und Einheimischen identisch sind und keine Abwanderung erfolgt. Wandern nur Neugeborene zu, dann hat das die gleichen Auswirkungen wie eine Erhöhung der Fertilität der Einheimischen, wie sofort einsichtig ist. Wandern dagegen nur 15jährige $\mathrm{zu}$, dann hat das ähnliche Folgen wie eine entsprechende

Vgl. Dinkel/Lebok (1993) S. 390. 
Abb. B.14: Die Entwicklung des Altenquotienten bei unterschiedlichen Annahmen über die Altersstruktur der Zuwanderer ${ }^{129}$

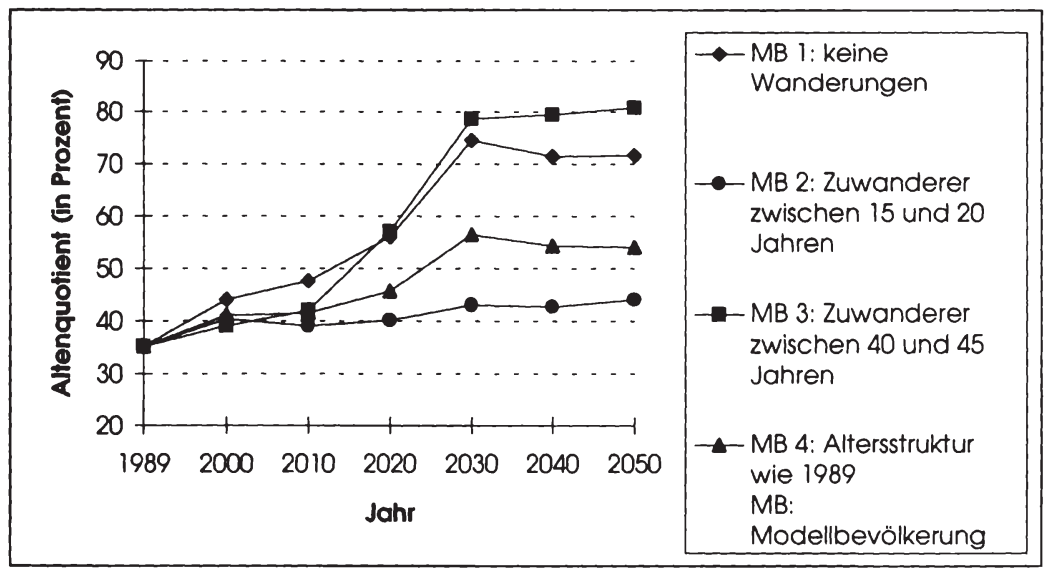

Quelle: Dinkel/Lebok (1993) S. 398

Erhöhung der Fertilität zu einem 15 Jahre zurückliegenden Zeitpunkt. ${ }^{130}$ Der Altersquotient wird früher gesenkt und das Bevölkerungswachstum, das aus der Geburt der zweiten Generation resultiert, setzt früher ein.

Wandern dagegen nur 45jährige ein, also Frauen, die ihre reproduktive Lebensphase abgeschlossen haben, dann ändert sich dadurch langfristig das Bevölkerungswachstum nicht, weil keine zusätzlichen Geburten induziert werden (vgl. Abb. B.14). Es kommt nur zu einem einmaligen Wachstumsschub, der dann beendet ist, wenn die ersten Generationen der Zuwanderinnen so gealtert sind, $\mathrm{da} \beta$ sich Sterbefälle und neue Zuwanderung in dieser Bevölkerungsgruppe die Waage halten. ${ }^{131}$ Der Altenquotient sinkt zunächst aufgrund der Zunahme der 20- bis 60jährigen, steigt aber kurz nachdem die ersten Einwanderinnen das sechzigste Lebensjahr überschritten haben an und liegt schließlich dauerhaft über dem Altenquotienten, der sich ohne Zuwanderung eingestellt hätte.

Ein weiterer Unterschied zwischen erhöhter Fertilität und Zuwanderung ergibt sich, wenn die zusammengefaßte Fertilitätsrate der Zuwanderer von derjenigen der Einheimischen abweicht. Je höher die Kinderzahl der zuwandernden Frauen im Vergleich zu derjenigen der Einheimischen ist, desto schneller wird ceteris paribus die Gesamtbevölkerung wachsen und

129 Für alle Modellbevölkerungen (MB) wurden konstante Fertilitäts- und Mortalitätsraten mit den Werten von 1989 und ein positiver Wanderungssaldo von 500.000 Personen jährlich unterstellt. Vgl. Dinkel/Lebok (1993) S. 389ff.

130 Dinkel (1990) S. 50 weist darauf hin, daß Neugeborene stets weniger Kinder haben werden als 15jährige, weil die Neugeborenen das fruchtbare Alter entsprechend der altersspezifischen Mortalitätsraten nur mit einer unter Eins liegenden Wahrscheinlichkeit erreichen. Der "reproductive value". der den Gegenwartswert der zukünftigen Geburten einer Frau in einem bestimmten Alter mit dem einer Neugeborenen vergleicht, ist daher für 15 jährige Zuwanderinnen größer als für Neugeborene. 
desto jünger wird die Altersstruktur sein. In dem Fall abweichender Fertilitätsraten ist die Angabe der Höhe der Nettozuwanderung nicht mehr ausreichend, um die demographischen Konsequenzen abschätzen zu können, sondern es sind auch die Bruttoströme und der zeitliche Verlauf der Anpassung an die Fertilitätsverhältnisse der Einheimischen von Bedeutung. Wandern beispielsweise 600.000 Frauen höherer Fertilität zu und 300.000 mit niedrigerer Fertilität $a b$, dann ergibt sich bei gleicher Höhe der Nettowanderungen ceteris paribus ein höheres Bevölkerungswachstum und eine jüngere Altersstruktur als bei Zuwanderung von 300.000 Frauen ohne Abwanderung. ${ }^{132}$

Als Quintessenz dieses knappen Vergleichs der demographischen Effekte einer erhöhten Fruchtbarkeit und einer Nettozuwanderung ist festzuhalten, daß ein positiver Wanderungssaldo schneller Einfluß auf die Altersstruktur nimmt, dieser Einfluß nach Richtung und Größenordnung aber neben der absolaten Höhe der Wanderungen entscheidend von der Altersstruktur der Migranten geprägt wird.

Im Unterschied $\mathrm{zu}$ Fertilitätsänderungen existieren bezüglich einer verstärkten Einwanderung mehr Simulationsstudien, die nicht nur die Auswirkungen der Wanderungen auf vorgelagerte Kennzahlen wie z.B. den Eckrentnerquotienten deutlich machen, sondern auch die daraus resultierende Änderung des Beitragssatzes zur Gesetzlichen Rentenversicherung abzuschätzen versuchen.

Börsch-Supan legt seiner Simulationsstudie das demographische Modell des Statistischen Bundesamtes zugrunde und benutzt die Annahmen und Ergebnisse der siebten koordinierten Bevölkerungsvorausberechnung als Ausgangsbasis für die Untersuchung des Einflusses der Wanderungsbewegungen. ${ }^{133}$ Die zusammengefaßte Fertilitätsrate wird mit 1,39 angenommen, die Mortalität sinkt leicht bis zum Jahr 2010 und bleibt nachfolgend konstant. Der Wanderungssaldo geht gleichfalls bis zum Jahr 2010 auf eine Anzahl von 45.000 Personen jährlich zurück und verharrt dann auf diesem Niveau. Im Unterschied zur Prognos-Studie berücksichtigt Börsch-Supan weder die Heraufsetzung der gesetzlichen Altersgrenze durch das Rentenreformgesetz 1992 noch eine in Zukunft steigende Erwerbsbeteiligung und kommt daher mit einem Maximum von über 34 Prozent zu wesentlich höheren Werten für den Beitragssatz. Ein konstanter Einwanderungsüberschuß von 300.000 Personen pro Jahr genügt, um den Beitragssatz in den besonders problematischen Jahren nach 2030 um etwa 7 Prozentpunkte zu senken und seinen Anstieg damit in etwa zu halbieren. ${ }^{134}$ Börsch-Supan schließt aus diesen Ergebnissen, daß bereits Einwanderung in Größenordungen, die die Bundesrepublik Deutschland schon über längere Zeiträume erfahren hat, einen wichtigen Beitrag zur Stabilisierung des Systems der Gesetzlichen Rentenversicherung und auch der anderen Sozialversicherungszweige leisten kann. ${ }^{135}$

Die oben bereits erwähnten Simulationsrechnungen von Steinmann beziehen sich zwar nicht auf den Beitragssatz zur Gesetzlichen Rentenversicherung, sondern auf die Altenquote als vorgelagerte demographische Einflußgröße, aber sie haben den Vorteil, daß sie explizit die

\footnotetext{
132 Vgl. Dinkel/Lebok (1994) S. 33.

133 Vgl. Börsch-Supan (1994) S. 121ff.

134 Vgl. Börsch-Supan (1994) S. 128.

135 Vgl. Börsch-Supan (1994) S. 129.
} 
Ergebnisse bei erhöhter Fertilität und verstärkter Einwanderung unter gleichem Annahmenkranz gegenüberstellen. Unterschieden werden vier Einwanderungsvarianten mit Aufnahmekontingenten von 0 Personen (Variante A), 150.000 Personen (Variante B), 300.000 Personen (Variante C) und 500.000 Personen (Variante D). Dabei wird hinsichtlich der Altersstruktur der Zuwanderer durchgehend von den Verhältnissen des Jahres 1987 ausgegangen und eine unveränderte Mortalität unterstellt. Durch die Zuwanderung von 150.000 Personen läßt sich die Altenquote im Jahr 2030 um über zwei Prozentpunkte von 24,10 auf 22,03 senken. Mit zunehmender Einwanderung fält die Senkung der Altenquote immer geringer aus. So wird bei Erhöhung der Zuwanderung um weitere 150.000 auf 300.000 Personen die Altenquote nur noch um 1,66 Prozentpunkte reduziert (vgl. Tab. B.1).

Aus der Gegenüberstellung der Ergebnisse für die einzelnen Wanderungsvarianten bei unveränderter und erhöhter Fertilität ist zu ersehen, daß ein Anstieg der zusammengefaßten Geburtenrate um 0,36 oder 27 Prozent für die einheimischen Frauen (bei Variante A) eine nur leicht höhere Wirkung auf die Altenquote im Jahr 2030 besitzt als ein jährlicher Einwanderungsstrom von 150.000 Migranten (Variante B bei einer zusammengefaßten Geburtenrate von 1,34$).{ }^{136}$

Tab. B.1: Die Entwicklung der Altenquote bis zum Jahr 2030 bei unterschiedlicher Nettozuwanderung und Fertilität

\begin{tabular}{|c|c|c|c|c|c|c|c|c|}
\hline \multicolumn{4}{|c|}{$\begin{array}{l}\text { zusammengefaßte Geburtenrate } \\
\text { von 1,34 }\end{array}$} & \multicolumn{3}{l|}{$\begin{array}{l}\text { zusammengefaßte Geburtenrate } \\
\text { ansteigend auf } 1,7\end{array}$} \\
\hline Jahr & Variante & $\begin{array}{c}\text { Variante } \\
\text { B }\end{array}$ & $\begin{array}{c}\text { Variante } \\
\text { C }\end{array}$ & $\begin{array}{c}\text { Variante } \\
\text { D }\end{array}$ & $\begin{array}{c}\text { Variante } \\
\text { A }\end{array}$ & $\begin{array}{c}\text { Variante } \\
\text { B }\end{array}$ & $\begin{array}{c}\text { Variante } \\
\text { C }\end{array}$ & $\begin{array}{c}\text { Variante } \\
\text { D }\end{array}$ \\
\hline 1991 & 13,99 & 13,99 & 13,99 & 13,99 & 13,99 & 13,99 & 13,99 & 13,99 \\
1995 & 14,43 & 14,33 & 14,23 & 14,10 & 14,39 & 14,29 & 14,19 & 14,06 \\
2000 & 15,03 & 14,78 & 14,54 & 14,11 & 14,86 & 14,61 & 14,37 & 14,07 \\
2010 & 18,37 & 17,67 & 17,02 & 16,24 & 17,82 & 17,15 & 16,55 & 15,81 \\
2020 & 19,51 & 18,32 & 17,28 & 16,10 & 18,48 & 17,41 & 16,48 & 15,41 \\
2030 & 24,10 & 22,03 & 20,37 & 18,60 & 21,98 & 20,27 & 18,88 & 17,38 \\
\hline
\end{tabular}

Quelle: Steinmann (1993) S. 19

Felderer kommt unter den oben erwähnten Annahmen zu dem Ergebnis, daß zu einer Beitragssatzsenkung um drei Prozentpunkte in den Jahren der höchsten Belastung eine Nettoeinwanderung von etwa 400.000 Personen nötig ist. Zu einer Stabilisierung des Beitragssatzes sind unter den Annahmen des Grundszenarios allerdings jährlich schwankende Nettozuwanderungen von bis über vier Millionen Personen pro Jahr erforderlich. ${ }^{137}$

\footnotetext{
136 Vgl. Steinmann (1993) S. 13.
}

137 Vgl. Felderer (1994a) S. 206. In einer Grafik auf der vorhergehenden Seite gibt Felderer im Widerspruch zu den Aussagen im Text sogar maximale Wanderungssalden von knapp 6 Mio Personen pro Jahr an. 
Gelingt es, die Einwanderung auf Personen zu beschränken, die nicht älter als dreißig Jahre sind, genügt ein Wanderungssaldo von etwa einer Million, um den Beitragssatz auf dem Niveau von 1992 zu stabilisieren. ${ }^{138}$

Erhebt man eine Einwanderungsabgabe, muß deren Höhe bei einer jährlichen Nettozuwanderung von einer Million Personen ab etwa 2025 ungefähr das Zwanzigfache des durchschnittlichen Monatseinkommens betragen, um den Beitragssatz zur Gesetzlichen Rentenversicherung mit den Einnahmen konstant halten zu können. Liegt der Wanderungssaldo nur bei 200.000 Personen, ist um das Jahr 2030 rund das 140 fache durchschnittliche Monatseinkommen zu verlangen. ${ }^{139}$

Welche Schlußfolgerungen lassen sich aus den Ergebnissen der unterschiedlichen Simulationsstudien ziehen? Offenbar sind sowohl eine Erhöhung der Kinderzahl als auch eine verstärkte Zuwanderung in der Lage, einen Beitrag zur Minderung der demographischen Lasten zu leisten, die der Gesetzlichen Rentenversicherung und den anderen Sozialversicherungszweigen in den nächsten Jahrzehnten bevorstehen. Dabei wirkt sich eine bis zur Jahrtausendwende abgeschlossene Erhöhung der zusammengefaßten Geburtenrate um 0,3 Kinder je Frau in den Jahren mit besonders hohen Belastungen um 2030 in etwa gleich aus wie ein Anstieg der Nettozuwanderungen um 200.000 Personen jährlich. ${ }^{140}$ Legt man die Studie von Felderer zugrunde, ist in beiden Fällen mit einer Reduktion des Beitragssatzes zur Gesetzlichen Rentenversicherung um etwa 1,5 Prozentpunkte im Jahr 2030 zu rechnen. ${ }^{141}$

Der Entlastungseffekt einer höheren Fertilitätsrate hängt entscheidend von dem Zeitpunkt $a b, z u$ dem die Geburtenerhöhung einsetzt. Der um etwa 20 Jahre verzögert einsetzende Anstieg der Beitragszahler hat zur Folge, daß nennenswerte Entlastungswirkungen in den Jahren mit besonders hohen Beitragssätzen nur zu erreichen sind, wenn die zusammengefaßte Geburtenrate bis zur Jahrtausendwende auf ein deutlich höheres Niveau angehoben werden kann.

Die Entlastungswirkung einer verstärkten Nettozuwanderung hängt neben der absoluten Zahl der Zuwanderer entscheidend von ihrer Altersstruktur ab. Eine gezielte Selektion der Zuwanderer nach ihrem Alter ist daher in der Lage die Entlastungswirkung deutlich zu steigern. Daneben spielt auch die zeitliche Verteilung der Zuwanderung eine Rolle. Gelingt es, die Wanderungen so zu steuern, daß in den Jahren der größten Belastungen für die Gesetzliche

138 Vgl. Felderer (1994a) S. 207f. Die demographischen Auswirkungen der Altersstruktur der Zuwanderer werden mit Bezug auf die USA auch durch Arthur/Espenshade (1988) analysiert.

139 Vgl. Felderer (1994a) S. 206f.

$140 \mathrm{Zu}$ beachten ist allerdings, daß die Entlastungseffekte einer steigenden Fertilität nach 2030 weiter zunehmen, während die Wirkung einer verstärkten Einwanderung nicht mehr nennenswert steigt.

141 Für eine Zunahme des Wanderungssaldos um 400.000 Personen erhielt Felderer eine Beitragssatzsenkung von drei Prozentpunkten, für 200.000 Personen also 1.5 Prozentpunkte. Für einen Anstieg der Nettoreproduktionsrate um 0,3 Mädchengeburten je Frau, der einer Zunahme der zusammengefaßten Geburtenrate um 0,63 entspricht, erhält er für das Jahr 2020 eine Beitragssatzreduktion von weniger als 2 Prozentpunkten, für die Jahre nach 2030 aber von über 5 Prozentpunkten. Berücksichtigt man, daß Felderer eine sofortige Änderung der Nettoreproduktionsrate im Jahre 1992 zugrundelegt, durch eine Rückverlagerung der Werte um fünf Jahre, erhält man für das Jahr 2030 einen Wert von etwa 3.5 Prozentpunkten. Für einen Anstieg der zusammengefaßten Geburtenrate um 0,3 ergibt sich damit eine Beitragssatzsenkung um 1,67 Prozentpunkte. Dieser Wert ist allerdings leicht überhöht. weil Felderer mit einer Nettoreproduktionsrate von 0,5 von einem sehr niedrigen Basiswert ausgeht. Der im Vergleich zu der Studie von Felderer geringere Effekt der Fertilitätserhöhung bei Steinmann kann dadurch erklärt werden, daß letzterer die zusammengefaßte Geburtenrate von Einwanderinnen konstant hält. während Felderer auch für diese Bevölkerungsgruppe einen Anstieg unterstellt. 
Rentenversicherung möglichst viele im Ausland Geborene im erwerbsfähigen Alter und möglichst wenige im Rentenalter sind, sind zusätzliche Entlastungseffekte gegenüber einem Szenario mit jährlich gleichhoher Nettowanderung zu erwarten. ${ }^{142}$

\section{Zusammenfassung}

Ziel dieses Teils der Arbeit war es, den theoretischen und empirischen Hintergrund der prognostizierten Krise der Gesetzlichen Rentenversicherung aufzuzeigen und eine Begründung für die Konzentration auf die in den Teilen $C$ und $D$ eingehender untersuchten zwei Lösungsansätze zu liefern.

Dazu wurden einleitend verschiedene Grundtypen von Alterssicherungssystemen und die historische Entwicklung zu dem gegenwärtigen System der Gesetzlichen Rentenversicherung in Deutschland erläutert und dabei die Konzeption dieses Systems auf der Basis eines ZweiGenerationen-Vertrags kritisiert. In Unterkapitel 1.3 wurden anhand der grundlegenden Budgetgleichung umlagefinanzierter Alterssicherungssysteme die Zusammenhänge zwischen Beitragssatz und anderen, insbesondere demographischen Faktoren für den Fall eines konstanten Rentenniveaus herausgearbeitet, der dem im Rentenreformgesetz 1992 festgelegten Prinzip der Nettoanpassung der Renten an die Lohnentwicklung entspricht.

Die Darstellung der empirischen Entwicklung dieser Einflußfaktoren auf den Beitragssatz erfolgte in Kapitel 2. Es wurde deutlich, daß trotz der mit dem Rentenreformgesetz 1992 ergriffenen Maßnahmen aufgrund der prognostizierten Entwicklung der Bevölkerungsgröße und v.a. der Altersstruktur, die sich aus dem Zusammenspiel von Fertilität, Mortalität und Wanderungen ergibt, mit einer drastischen Erhöhung der Beiträge zur Gesetzlichen Rentenversicherung zu rechnen ist. Die zu erwartende Entwicklung anderer Einflußgrößen kann diese Entwicklung nicht entscheidend bremsen. Da neben der Gesetzlichen Rentenversicherung auch andere Systeme der sozialen Sicherung wie die Gesetzliche Krankenversicherung, die Beamtenpensionen und die Pflegeversicherung bei zunehmender Vergreisung der Bevölkerung immer höhere Finanzmittel benötigen, werden die zukünftigen Beitragszahler eine Abgabenlast zu tragen haben, deren Höhe Ausweichreaktionen wie Schwarzarbeit und Leistungsreduzierung wahrscheinlich macht.

Einige Lösungsansätze zur Entschärfung der demographischen Krise der Gesetzlichen Rentenversicherung wurden in Kapitel 3 diskutiert. Dabei wurden nur wenige Vorschläge, die besondere Aufmerksamkeit auf sich gezogen haben, knapp untersucht, da eine eingehende Diskussion der vielfältigen Ansätze den Rahmen dieser Arbeit gesprengt hätte. Dennoch wurde deutlich, daß sowohl die dargestellten Lösungsansätze innerhalb des Systems der Gesetzlichen Rentenversicherung als auch die behandelten Lösungsvorschläge, die eine Änderung systemkonstituierender Merkmale vorsehen, letztlich der Generation der zukünftig Erwerbstätigen und/oder den zukünftigen Rentnern hohe Lasten auferlegen. Der Grund hierfür ist darin zusehen, daß die Vorschläge ihr Hauptaugenmerk nicht auf die Behebung der demo-

142 Welche Nettowanderungen zur Stabilisierung der Zahl der aktiven Bevölkerung henötigt werden, untersucht Steinmann (1991) S. 352ff. 
graphischen Ursachen der Krise richten. Stattdessen verlagern sie lediglich die infolge dieser Krise entstehenden Lasten durch Umverteilungsmaßnahmen innerhalb und zwischen den Generationen auf andere Schultern. ${ }^{143}$

Inwieweit durch bevölkerungspolitische Maßnahmen eine Entlastung der Gesetzlichen Rentenversicherung erreicht werden kann, war Thema des Unterkapitels 3.3 Dabei wurde nur die "technische" Seite betrachtet, d.h. es wurde mit Hilfe von Simulationsrechnungen untersucht, welche Wirkungen von einer bestimmten Erhöhung der Fertilitätsrate bzw. des Wanderungsaldos auf die Entwicklung des Beitragssatzes zur Gesetzlichen Rentenversicherung ausgehen, ohne da $B$ thematisiert wurde, ob und wie derartige Änderungen der Bevölkerungsentwicklung bewirkt werden können. Als Ergebnis konnte festgehalten werden, daß eine Erhöhung des Einwanderungssaldos um 200.000 Personen bei der Ende der achtziger Jahre festzustellenden Altersstruktur der Migranten mit einer Senkung des Beitragssatzes um etwa 1,5 Prozentpunkte im Jahr 2030 einen ähnlichen Effekt hat, wie eine Erhöhung der zusammengefaßten Geburtenrate um 0,3 Kinder pro Frau. Grundsätzlich kann damit sowohl über eine Erhöhung der Fertilität als auch über verstärkte Einwanderung eine ins Gewicht fallende Reduzierung der demographisch bedingten Belastungen erreicht und damit ein Beitrag zur Stabilisierung der Gesetzlichen Rentenversicherung geleistet werden.

Die Frage, inwieweit eine Milderung der demographischen Ursachen der Krise der Gesetzlichen Rentenversicherung durch eine Beeinflussung des Fertilitätsverhaltens bzw. der internationalen Wanderungen möglich ist und welche Kosten damit verbunden wären, ist Gegenstand der verbleibenden Teile dieser Arbeit. In Teil $\mathrm{C}$ werden die Möglichkeiten einer Einflußnahme auf die Geburtenzahl über einen erhöhten Kinderlastenausgleich untersucht, in Teil D die Erhöhung des Wanderungsüberschusses durch eine gezielte Zuwanderungspolitik. Abschließend erfolgt ein Vergleich dieser beiden Instrumente hinsichtlich ihrer Wirksamkeit und Kosteneffizienz. 
Bernhard Manzke - 978-3-631-75262-3

Downloaded from PubFactory at 01/11/2019 06:34:40AM

via free access 
Teil C:

\section{DER KINDERLASTENAUSGLEICH ALS INSTRUMENT ZUR STABILISIERUNG DER GESETZLICHEN RENTEN- VERSICHERUNG}

\section{Einleitung}

In Teil B dieser Arbeit wurde eine Begründung für die Konzentration auf Lösungsansätze der demographischen Krise der Gesetzlichen Rentenversicherung geliefert, die auf eine Beeinflussung der demographischen Entwicklung abzielen. In den Teilen $\mathrm{C}$ und $\mathrm{D}$ werden nun die Kosten untersucht, die bei einer Erhöhung der Geburtenrate durch eine Ausweitung des Kinderlastenausgleichs bzw. bei einer verstärkten Einwanderung für die einheimische Bevölkerung entstehen. Dabei liegt das Schwergewicht der Analyse bei der Behandlung des Kinderlastenausgleichs auf den Kosten, die durch die Schaffung von Anreizen zu einer Erhöhung der Kinderzahl hervorgerufen werden. Bezüglich der Zuwanderung steht dagegen die Änderung der Wohlfahrtsposition der Einheimischen durch die Einwanderer im Vordergrund, weil davon ausgegangen werden kann, daß die Schaffung zusätzlicher Einwanderungsanreize durch kostenträchtige Maßnahmen aufgrund der hohen Zahl der Einwanderungswilligen auf absehbare Zeit nicht notwendig sein wird.

Beiden Teilen gemeinsam ist hingegen die Vernachlässigung von Wirkungen, die sich aus einer Verlangsamung des absoluten Bevölkerungsrückgangs ergeben. Die Beziehung zwischen Wirtschafts- und Bevölkerungsentwicklung war lange Zeit kaum Gegenstand wissenschaftlicher Abhandlungen. Erst in den letzten Jahrzehnten befaßt sich - wie schon zu Zeiten der Klassiker - eine umfangreichere Literatur mit der Beziehung zwischen Bevölkerungsentwicklung und Wachstum des Pro-Kopf-Einkommens. Dabei lassen sich vier Effekte einer Bevölkerungszunahme unterscheiden: ${ }^{1}$

1) der Kapitalverdünnungseffekt (capital widening effect)

2) der Einfluß der Bevölkerungsentwicklung auf den technischen Fortschritt

3) der Einfluß auf die Finanzierung öffentlicher Güter

4) der Einfluß auf die Umweltbedingungen

ad 1): Ein Bevölkerungszuwachs hat eine Erhöhung des Faktors Arbeit zur Folge, der (zunächst) keine Änderung der anderen Produktionsfaktoren gegenübersteht. Bei einer neoklassischen Produktionsfunktion mit konstanten Skalenerträgen und abnehmenden Grenzerträgen bei partieller Faktorvariation ergibt sich daraus, daß der Lohnsatz sinkt und der Zinssatz steigt. Dieser auch als Kapitalverdünnung bezeichnete Effekt hat seine Ursache darin, daß durch eine Bevölkerungserhöhung weniger Kapital pro Arbeitskraft zur Verfügung steht und deshalb die Grenzproduktivität der Arbeit zurückgeht, während die Grenzproduktivität des Kapitals steigt. Für die Gesetzliche Rentenversicherung hat das zur Folge, daß die Summe der

1 Einen Überblick über die verschiedenen Effekte einer Bevölkerungszunahme geben Felderer/Sauga (1988) Teil III. 
Beiträge zwar aufgrund der höheren Zahl der beitragspflichtigen Mitglieder steigt, gleichzeitig aber wegen des gesunkenen Lohnsatzes zurückgeht. Welcher Effekt überwiegt hängt dann von der Preiselastizität der Arbeitsnachfrage ab. Ein positiver Einfluß eines Bevölkerungszuwachses auf die Rendite der Gesetzlichen Rentenversicherung ist demnach nicht zwangsläufig. ${ }^{2}$

Gegen diese Argumentation werden allerdings mehrere Einwände angeführt. So ist es keineswegs zwingend, daß das Verhältnis von Kapital- zu Arbeitseinsatz sich infolge eines Bevölkerungsanstiegs verschlechtert. Denkbar ist nämlich auch, daß die Eltern ihre Ersparnis erhöhen werden, um ihre Kinder mit einem gewissen Kapitalstock (zu dem auch Humankapital zählt) ausstatten zu können. Da diese Kapitalbildung freiwillig aus privaten Mitteln erfolgt, kann der daraus resultierende Rückgang des Pro-Kopf-Konsums der Eltern auch nicht als Wohlfahrtseinbuße betrachtet werden. Außerdem steigt bei einem Bevölkerungsanstieg der Anteil der jüngeren Erwerbstätigen an der Gesamtbevölkerung. Diese Bevölkerungsgruppe hat aber nach der Lebenszyklus-Hypothese eine besonders hohe Sparneigung, so daß sich die durchschnittliche Kapitalbildung in der Volkswirtschaft erhöht. Weiterhin wird die höhere Grenzproduktivität des Kapitals bei internationaler Kapitalmobilität zu Kapitalzuflüssen aus dem Ausland führen, die einem negativen Effekt des Bevölkerungswachstums auf die Lohneinkommen entgegenwirken. Schließlich geht es im Rahmen dieser Arbeit nicht um die Induzierung eines absoluten Bevölkerungszuwachses, sondern um die Verlangsamung des Bevölkerungsrückgangs. Bei einem schnellen Schrumpfen der absoluten Bevölkerungsgröße wäre aber ein Teil des vorhandenen Kapitals gar nicht mehr produktiv nutzbar und könnte damit auch nicht die Produktivität der Arbeitskräfte erhöhen. Im Gegensatz zur Situation bei einer absoluten Bevölkerungszunahme müssen nicht zusätzliche Investitionen in langfristiges Sachkapital vorgenommen werden, sondern dieses Sachkapital ist z.T. ohnehin vorhanden. Es würde bei einem Bevölkerungsrückgang nicht vollständig ausgelastet und damit teilweise entwertet. ${ }^{3}$

ad 2) Hinsichtlich der Wirkung der Bevölkerungsentwicklung auf die technische Entwicklung werden v.a. zwei Thesen diskutiert. Die erste These besagt, daß durch eine große Bevölkerung schneller Effizienzsteigerungen aufgrund von Lernkurveneffekten erzielt werden können. Die größere Zahl der Abnehmer erlaubt eine schnellere Zunahme des kumulierten Absatzes eines Gutes. Die Höhe des kumulierten Absatzes und die damit gewonnenen Erfahrungen bei der Produktion eines Gutes sind nach dieser These aber entscheidend für die Realisierung von effizienzsteigernden Kosteneinsparpotentialen. Dem kann allerdings entgegengehalten werden, daß für viele Güter der Absatz nicht auf den nationalen Markt beschränkt ist und daher die Größe der nationalen Bevölkerung nur einen eingeschränkten Einfluß auf die Lernkurveneffekte ausübt.

Die zweite These lautet, daß immer ein bestimmter Prozentsatz der Bevölkerung (die "Genies") in der Lage ist, die technische Entwicklung voranzutreiben. Je mehr Menschen vorhanden sind, desto höher wäre demnach die Rate des technischen Fortschritts. Die absolute Bevölkerungszahl ist allerdings kaum allein bestimmend für die Rate des technischen Fortschritts, sondern auch die Ausbildung und damit der Kapitalbestand spielen eine bedeutende Rolle.

2 Der Einfluß auf die Finanzlage der Gesetzlichen Rentenversicherung ist in jedem Fall positiv, weil aufgrund der Nettoanpassung mit den Lohnsätzen auch die Rentenhöhe zurückgeht und daher der Einnahmeeffekt aufgrund der höheren Zahl der Beitragszahler voll durchschlägt.

3 Vgl. die Diskussion zur Mackenroth-These in Abschnitt 3.2.2 des Teils B dieser Arbeit. 
ad 3) Einige öffentliche Ausgaben wie z.B. die Verteidigungsausgaben und die Bedienung der öffentlichen Schulden fallen unabhängig von der Größe der Bevölkerung an. Eine wachsende Bevölkerung ermöglicht die Verteilung dieser Ausgaben auf mehr Schultern, so daß entweder die Belastungen pro Kopf reduziert oder aber die Leistungen ausgeweitet werden können.

ad 4) Mit zunehmender Bevölkerungsgröße und damit auch -dichte nimmt bei gleicher ProKopf-Verschmutzung die Umweltschädigung pro Flächeneinheit zu. Ist die Grenze der Selbstregenerierungsfähigkeit der natürlichen Ressourcen überschritten, dann müssen mit zunehmender Bevölkerungszahl immer kostspieligere Umweltschutzmaßnahmen getroffen oder Einbußen bei der Umweltqualität hingenommen werden. Gleichzeitig werden nicht erneuerbare Ressourcen schneller erschöpft.

Dieser knappe Überblick über die Auswirkungen einer Änderung der Bevölkerungsgröße macht deutlich, daß es sich hier um ein kontrovers diskutiertes Themenfeld handelt. Es wirken offenbar eine Reihe gegenläufiger Effekte. Einer Quantifizierung der Gesamtwirkung stellen sich schwerwiegende Bewertungs-, Daten- und Methodenprobleme entgegen. Die meisten empirischen Studien kommen aber zu dem Ergebnis, da $\beta$ der Zusammenhang zwischen Bevölkerungswachstum und Pro-Kopf-Einkommen statistisch nicht signifikant ist. ${ }^{4}$ Deshalb konzentriert sich diese Arbeit auf die Primärwirkungen der hier diskutierten bevölkerungspolitischen Instrumente. In diesem Teil der Arbeit wird daher untersucht, wie hoch die Ausgaben für den Kinderlastenausgleich ausfallen müßten, um die Fertilitätsrate in einem bestimmten Ausmaß zu beeinflussen. Sekundärwirkungen, die sich aufgrund einer Änderung der absoluten Bevölkerungszahl gegenüber der Situation ohne Ausweitung des Kinderlastenausgleichs ergeben, bleiben dagegen unberücksichtigt.

Die Analyse des Kinderlastenausgleichs wird dadurch erschwert, daß dieser Teilbereich der Familienpolitik in der politischen Diskussion kaum von anderen Bereichen des Familienlastenausgleichs getrennt wird. Unter Kinderlastenausgleich sollen alle Maßnahmen verstanden werden, die im Sinne des Begriffs der Differentialinzidenz eine Änderung der Verteilung des verfügbaren Einkommens in Abhängigkeit von der Kinderzahl bewirken. ${ }^{5}$ Der Familienlastenausgleich umfaßt als Oberbegriff darüber hinaus auch Maßnahmen, die - wie beispielsweise das bei der Berechnung der Einkommensteuerschuld angewandte Ehegattensplitting - am Tatbestand Ehe ansetzen. Ehebezogene Maßnahmen entfalten aber keine pronatalistischen, d.h. fertilitätsfördernden Wirkungen. Bevölkerungspolitische Wirkungen ${ }^{6}$ hat nur der Kinderlastenausgleich als kindbezogener Teilbereich des Familienlastenausgleichs. ${ }^{7}$

$4 \quad$ Vgl. Felderer/Sauga (1988) S. 120.

5 Vgl. zu dieser Definition Schmidt/Frank/Müller-Rohr (1985) S. 30.

6 In der politischen Diskussion in Deutschland wird ein bevölkerungspolitisches Ziel kaum offen angeführt, weil bevölkerungspolitische Maßnahmen durch die nationalsozialistische Politik vorbelastet sind. Dagegen wird es in anderen demokratischen Staaten offen propagient und hat eine lange geschichtliche Tradition. Schon Aristoteles (1978) S. 82f. befürwortete eine staatliche Begrenzung der Kinderzahl, weil er soziale Konflikte durch Verarmung infolge von Überbevölkerung befürchtete.

Es wäre sicherlich falsch, die Bevölkerungspolitik - aufgefaßt als "zielgerichtetes, begründetes Handeln zum Zweck der planmäßigen Beeinflussung demographischer Tatbestände, wie Größe, Altersaufbau, regionale Verteilung und Wachstumsintensität der Bevölkerung" (Schubnell (1990) S. 34) - generell als Teilbereich der Familienpolitik zu verstehen. Dagegen spricht schon, daß Familienpolitik ein recht junger Zweig der Sozialpolitik ist, während Bevölkerungspolitik schon vor Christi Geburt betrieben wurde 
In diesem Teil der Arbeit ist also zu untersuchen, wie ein an dem Ziel der Erhöhung der Fertilitätsrate ausgerichteter Kinderlastenausgleich gestaltet sein müßte. ${ }^{8}$ Die Kosten einer solchen Politik sind zu ermitteln, um sie dann den in Teil D der Arbeit zu ermittelnden Kosten, die durch das alternative Instrument einer verstärkten Einwanderung induziert werden, gegenüberstellen zu können.

Im nächsten Abschnitt (Kapitel 2) werden zunächst verschiedene Theorien des generativen Verhaltens hinsichtlich ihrer Eignung als Grundlage für die Analyse der geburtenerhöhenden Wirkungen des Kinderlastenausgleichs diskutiert. Anhand der neoklassischen Theorie der Fertilität werden dann die Bestimmungsgründe der Fertilitätsentscheidung herausgearbeitet und Anhaltspunkte über die Mittel gewonnen, die zu ihrer Beeinflussung aufgewendet werden müßten (Kapitel 3). In Kapitel 4 wird auf dieser Grundlage die Kosteneffizienz des Kinderlastenausgleichs abgeschätzt. Die Ergebnisse dieses Teils der Arbeit werden in Kapitel 5 zusammengefaßt.

\section{Erläuterung und Kritik verschiedener Ansätze zur Erklärung des ge- nerativen Verhaltens}

\subsection{Zur Rechtfertigung einer ökonomischen Analyse des generativen Verhaltens}

Zunächst mag es verwunderlich erscheinen, daß Ökonomen sich mit dem generativen Verhalten beschäftigen. Faßt man als Erkenntnisobjekt der Wirtschaftswissenschaften die "Wirtschaft" auf, so wären in der Tat eher Soziologen und Psychologen aufgerufen, eine Fertilitätstheorie zu entwickeln. Ökonomen hätten dann auf deren Ergebnisse zurückzugreifen und die Fertilitätsentwicklung als exogen zu betrachten. Diese Abgrenzung ist jedoch aus zwei Gründen problematisch. Zum einen ist die Festlegung dessen, was alles unter "wirtschaftlichen" Faktoren verstanden werden soll, nicht einheitlich. Zum anderen sind die realen Probleme ihrer Natur nach komplex. ${ }^{9}$ Ist das Betriebsklima Gegenstand der Wirtschaftswissenschaften oder der Psychologie und Soziologie? Die Abgrenzung ist nicht immer eindeutig und schließt zudem

und bei einer auf Begrenzung des Bevolkerungszuwachses zielenden antinatalistischen Ausrichtung, die in vielen Entwicklungsländern anzutreffen ist, einer Familienpolitik entgegengerichtet sein kann. Darüber hinaus umfaßt die Bevolkerungspolitik neben der Einflußnahme auf die Geburtenentwicklung auch die Mortalitătsraten und die Wanderungen als Ansatzpunkte. In bezug auf die Beeinflussung der Fertilität unterscheiden sich die beiden Politikbereiche hauptsächlich durch ihre Zielsetzungen - Beeinflussung der Bevölkerungsentwicklung bzw. Unterstützung der Familien zur besseren Erfüllung ihrer Aufgaben und Funktionen. Schränkt man die Familienpolitik auf den Kinderlastenausgleich ein, sind die Maßnahmen dagegen kaum voneinander zu trennen. Maßnahmen des Kinderlastenausgleichs haben bevölkerungspolitische Nebenwirkungen und umgekehrt. $\mathrm{Da}$ in der Bundesrepublik Deutschland offiziell keine Bevölkerungspolitik betrieben wird, ist es sinnvoll, hier vom Kinderlastenausgleich auszugehen. politischen Intentionen im besonderen in einer Demokratie zulässig ist, vgl. Dinkel (1985). 
wichtige Beiträge benachbarter Wissenschaften aus. Sinnvoller als eine Abgrenzung nach dem Analysebereich ist daher die Abgrenzung nach dem spezifischen Ansatz. Demnach beschäftigen sich Wirtschaftswissenschaftler mit Formen, in denen sich menschliches Handeln bei der Verwendung knapper Mittel zur Erreichung eines Zielbündels vollzieht. ${ }^{10}$ Der ökonomische Aspekt menschlichen Verhaltens wird nach Robbins ${ }^{11}$ immer dann sichtbar, wenn

- es eine Vielzahl angestrebter Ziele gibt,

- eine Hierarchie der nach ihrer Wichtigkeit geordneten Ziele besteht,

- die Mittel wahlweise für verschiedene Ziele eingesetzt werden können,

- die Mittel zur Erreichung der Ziele knapp sind.

Diese Bedingungen treffen auf die Fertilitätsentscheidung zu. Das Oberziel Nutzenmaximierung läßt sich mit Hilfe der Unterziele Nutzen aus Marktgütern und Nutzen aus Kindern befriedigen. Dabei muß der jeweilige Nutzen aus der Befriedigung der Unterziele, also die relative Bedeutung der Unterziele, festgelegt werden. Die Mittel Geld und Zeit stehen nur in begrenztem Umfang zur Verfügung und können alternativ zur Erzielung von Nutzen aus Kindern oder aus Marktgütern verwendet werden. Eine ökonomische Analyse der Fertilität ist somit begründbar.

Die Konzentration auf die Fertilitätsentwicklung bei der Analyse der Änderungen von Bevölkerungsgröße und -struktur rechtfertigt sich ebenfalls aus dem ökonomischen Ansatz. Zwar werden diese Größen auch von der Mortalitätsentwicklung beeinflußt - man denke beispielsweise an die große Zahl von Todesfällen v.a. von Männern während der beiden Weltkriege oder die drastische Verringerung der Säuglingssterblichkeit -, aber sie ist weniger auf individuelle menschliche Entscheidungen zurückzuführen und daher durch bevölkerungspolitische Maßnahmen auch schwerer zu beeinflussen. ${ }^{12}$ Die Wanderungsbewegungen als dritter Bestimmungsfaktor von Bevölkerungsgröße und -struktur werden in Teil D untersucht.

Im folgenden sollen die wichtigsten Gedanken zur Bevölkerungsentwicklung von der Antike bis zur Gegenwart dargestellt und die verschiedenen Theorien zur Erklärung der demographischen Entwicklung kritisch beleuchtet werden. Erst auf Grundlage dieser Diskussion ist es möglich, die Wahl eines spezifischen Ansatzes zu begründen, aber auch seine Schwächen zu erkennen. Der ausgewählte Ansatz bildet dann den Rahmen für die Analyse der Wirkung des Kinderlastenausgleichs auf die Bevölkerungsentwicklung und -struktur.

$10 \quad$ Vgl. Becker (1993) S. 2.

11 Vgl. Robbins (1972) S. 15ff.

12 Natürlich ist auch das Sterbealter nicht unabhängig von individuellen menschlichen Entscheidungen. So nimmt ein Kettenraucher, Stuntman oder Motorradfahrer ohne Helm bewußt eine Reduzierung seiner durchschnittlichen Lebenserwartung gegenüber jemandem in Kauf, der auf ausgewogene Ernährung. körperliche Fitness und eine gesundes Wohn- und Arbeitsumfeld achtet. Eine staatliche Beeinflussung der Mortalitătsentwicklung zur Verbesserung der Situation der Rentenversicherung könnte zum einen auf eine Vorverlegung des Sterbealters von Rentnem gerichtet sein, eine Maßnahme, die aus ethischen Gründen ausscheidet. Zum anderen könnte versucht werden, die Zahl der Berufs- und Erwerbsunfähigen zu senken. Derartige Maßnahmen können aber die grundlegenden demographischen Probleme nur kurzfristig kompensieren. Spätestens wenn es keine Berufs- und Erwerbsunfähigen mehr gibt, ist eine Kompensation der demographischen Probleme mit diesem Instrument nicht mehr möglich. Aus diesem Grund und weil diese Arbeit sich hauptsächlich auf die Alters- und weniger die Erwerbs- und Berufsunfähigkeitsrenten konzentriert, soll eine genauere Untersuchung dieses Instruments unterbleiben. 


\subsection{Die historische Entwicklung vor Malthus}

Bei der Darstellung der geschichtlichen Entwicklung bietet es sich an, in chronologischer Reihenfolge vorzugehen, da sich neue Gesichtspunkte sowohl aus empirischen Impulsen als auch aus der Fortentwicklung oder Kritik der vorangegangenen Hypothesen und Theorien entwickeln.

Der Anfang der Behandlung von Fragen der Bevölkerungsentwicklung wird dogmenhistorisch oft in der Veröffentlichung "An Essay on the Principle of Population..." (1798) von Thomas R. Malthus oder gar erst in der Ende der fünfziger und Anfang der sechziger Jahre unseres Jahrhunderts mit Leibenstein (1957) und Becker (1960) beginnenden Integration von Bevölkerungsfragen in die neoklassische Theorie gesehen. Aber schon in der Antike finden sich Gedanken über den Zusammenhang zwischen Einkommensentwicklung und Kinderzahl. Meistens wurde ein positiver Zusammenhang angenommen. Aus makroökonomischer Sicht wird dabei das Einkommen mit den Subsistenzmitteln, also v.a. den pro Kopf zur Verfügung stehenden Nahrungsmitteln, umschrieben. Bei begrenzter Nahrungsmittelversorgung müssen steigende Einkommen und damit steigende Kinderzahlen unweigerlich wieder zu einem Rückgang der pro Kopf zur Verfügung stehenden Subsistenzmittel führen. Um zu verhindern, daß das Bevölkerungswachstum erst durch Nahrungsmittelmangel und bittere Armut gestoppt wird, befürworten daher sowohl Aristoteles als auch Plato eine antinatalistische Politik. ${ }^{13}$ Mit dieser Argumentation nehmen sie schon viel der Malthusianischen Sichtweise vorweg, auch wenn sie die Mikroebene kaum beachten. Andererseits finden sich in der Antike - v.a. kurz nach Christi Geburt - aber auch Autoren, die auf eine negative Beziehung zwischen Einkommen und Kinderzahl auf der Mikroebene hinweisen. ${ }^{14}$

Im Mittelalter wurde wie schon im römischen Reich überwiegend eine pronatalistische Position vertreten. Kinderreichtum wurde als Gnade Gottes betrachtet und die von den griechischen Philosophen vorausgesehenen und von Malthus später als positive checks bezeichneten Begrenzungen des Bevölkerungswachstums durch Hungersnöte, Seuchen und Kriege als Strafe Gottes interpretiert. Ein Zusammenhang mit einer Überbevölkerungstendenz wurde nicht gesehen. ${ }^{15}$

Auch in der frühen Neuzeit bis zur industriellen Revolution setzte sich diese Tradition zumindest in Frankreich und Deutschland fort. Die theologische Begründung entsprechend des

13 Konkret werden Abtreibung, Kindesaussetzung und -tötung sowie Homosexualität erwähnt (vgl. Hirsch (1992) S. 57). Zu dieser Zeit herrschte in vielen griechischen Stadtstaaten ein hoher Bevölkerungsdruck, der auch zu Wanderungsbewegungen in Form der Gründungen neuer griechischer Siedlungen beigetragen haben mag (vgl. Hutchinson (1967) S. 8ff.).

14 Ovid (43 v. Chr. - 17 n. Chr.), Seneca (4 v. Chr. - 65 n. Chr.) und Juvenal (ca. 60 n. Chr. - 127 n. Chr.) berichten von der Kinderarmut der reichen römischen Bürgerfamilien (nach Brentano (1909) S. 602). Polybios (ca. 200 - 120 v. Chr.) berichtet auch aus dem Bereich der griechischen Stadtstaaten von Kinderarmut. Als Begründung gibt er an: "... sie wollten nicht mehr heiraten, oder, wenn sie es taten, doch nicht alle ihre Kinder aufziehen, sondern höchstens eins oder zwei, um diese reich zu hinterlassen und üppig groß zu ziehen" (zitiert nach Brentano (1909) S. 623). Er gibt damit bereits erste Hinweise auf eine substitutionale Beziehung zwischen Kinderzahl und Kinderqualität, eine Thematik, die erst im 20. Jahrhundert von Brentano (1909) und Becker (1960) vertieft wird.

Vgl. zu dieser Einschätzung Hirsch (1992) S. 58f. und Hutchinson (1967) S. 15. 
Bibelwortes "Seid fruchtbar und mehret Euch ..." (1. Mose 1,28) wurde allerdings abgelöst von der Erwartung der Merkantilisten bzw. Kameralisten, daß eine größere Bevölkerung dem absolutistischen Herrscher auf dem Wege einer höheren Wirtschaftsleistung und größerer militärischer Macht mehr Einkommen und Einfluß sichern würde. In England und Italien finden sich dagegen eher Autoren, die einem Bevölkerungswachstum kritischer gegenüberstehen. Kurz vor dem Erscheinen der ersten Auflage des "Essay on the Principle of Population..." vertreten schließlich schon Cantillon, Townsend, Smith, und Steuart Gedanken, die der von Malthus vorgetragenen Theorie ähneln. ${ }^{16}$

\subsection{Die Position von Malthus}

Obwohl, wie erwähnt, schon andere Autoren vor Malthus ähnliche Gedanken äußerten, wird sein Werk als Meilenstein in der Demographie angesehen, da es eine der ersten geschlossenen Theorien der Bevölkerungsentwicklung enthält. Auch heute noch werden einige seiner Thesen (z.T. in modifizierter Form) vertreten. Eine grundlegende Annahme von Malthus ist, $\mathrm{da} ß$ sich die Bevölkerung solange vergrößern wird, wie es die Nahrungsmittelversorgung zuläßt. ${ }^{17}$ Er postuliert also auf der Mikroebene einen positiven Zusammenhang zwischen Einkommen bzw. Pro-Kopf-Versorgung mit Nahrungsmitteln und Kinderzahl. Je mehr Nahrungsmittel einer Familie zur Verfügung stehen, umso höher wird ihre Kinderzahl sein. Die Wachstumsrate der Bevölkerung insgesamt bestimmt sich daher aus der Differenz zwischen tatsächlichem Pro-Kopf-Einkommen und dem Existenzminimum. Da Malthus die "passion between the sexes" als konstant annimmt, wächst die Bevölkerung in geometrischer Reihe, solange keine Beschränkungen wirksam werden. Sie verdoppelt sich also in einem bestimmten Zeitraum jeweils. Demgegenüber wächst aber das Nahrungsmittelangebot seiner Meinung nach nur in arithmetischer Reihe. ${ }^{18}$ Langfristig nimmt also bei ungehindertem Wachstum die Bevölkerung immer schneller zu als die Nahrungsmittelproduktion, ${ }^{19}$ womit zwangsläufig die Nahrungsmittelversorgung pro Kopf auf das Subsistenzniveau zurückgeht (malthusianische Bevölkerungsfalle). Auch der Lohnsatz als neben der Nahrungsmittelversorgung pro Kopf alternativer Indikator für das Einkommen geht entsprechend der klassischen Argumentation zurück, weil sich die Zahl der Arbeiter in der folgenden Generation vergrößert hat.

Weiter geht Malthus davon aus, daß diese Schere zwischen Bevölkerungswachstum und Nahrungsmittelversorgung nur durch "misery" (Elend) oder "vice" (Laster) geschlossen werden kann. ${ }^{20}$ Ersteres bedeutet eine Verminderung eines bereits eingetretenen Bevölkerungswachstums durch höhere Sterblichkeit infolge von Unterernährung (positive checks), während letzteres Maßnahmen (preventive checks) sind, die aus Angst vor dem Elend

16 Vgl. Hutchinson (1967) S. 110ff.

17 Vgl. Malthus (1798) S. 36ff.

18 Sowell (1979) S. 87 kritisiert Malthus in diesem Punkt, da bei dem Vergleich der Wachstumsraten von Bevölkerung und Nahrungsmitteln nicht deutlich werde, ob theoretisch erreichbare oder tatsächliche Werte gemeint seien.

19 Malthus unterstellt implizit abnehmende Grenzerträge des Faktors Arbeit.

20 Vgl. Malthus (1798) S. 36ff. Insbesondere in späteren Auflagen hält Malthus auch eine Beschränkung des Bevölkerungswachstums durch moral restraint als weitere Form der preventive checks neben den Lastern für möglich (vgl. Malthus (1826), Book I, S. 15f.) 
getroffen werden und von Malthus sämtlich als Laster (vice) betrachtet werden. ${ }^{21}$ Dazu sind alle Maßnahmen zu rechnen, die ein höheres Bevölkerungswachstum von vornherein verhindern, also auf eine verminderte Fertilität gerichtet sind. Als Geistlicher zählt Malthus zu diesen Lastern neben Kindesaussetzung und -tötung auch die Verhütung, Abtreibung und Homosexualität. ${ }^{22}$

Nach Malthus sind die positive checks v.a. für die unteren Gesellschaftsschichten relevant. Die oberen Schichten wollen dagegen einen höheren Lebensstandard halten, ihr "Subsistenzniveau" liegt also höher. Deshalb werden sie aus Furcht, diesen Lebensstandard aufgeben zu müssen, schon zu den vorbeugenden Maßnahmen (preventive checks) greifen, bevor ihre Zahl so groß geworden ist, daß die positive checks wie Hungersnöte etc. wirksam werden. ${ }^{23}$ Diese Idee eines schichtabhängigen Anspruchsniveaus findet sich später wieder bei Freedman (1963) und Leibenstein $(1974,1975)$, auf deren Werke weiter unten noch einzugehen ist. Malthus überträgt diesen Gedanken auf die ganze Gesellschaft, indem er ausführt, daß die Kinderzahl bei gegebener Nahrungsmittelversorgung mit dem Entwicklungsgrad einer Gesellschaft sinkt. Der angestrebte Lebensstandard ist in einer weiter entwickelten Gesellschaft vergleichsweise höher und damit greifen vermehrt die preventive checks, bevor es zu einem Absinken auf das absolute Subsistenzniveau kommt. ${ }^{24}$ Bei gegebenem Anspruchsniveau erhöht aber eine Zunahme des Pro-Kopf-Einkommens auch hier die Kinderzahl durch geringere Sterblichkeit (v.a. in den unteren Schichten) und höhere Fertilität aufgrund eines geringeren Gewichts der preventive checks in den oberen Schichten.

\subsection{Die weitere Entwicklung bis 1957}

Auch andere Klassiker vertreten im wesentlichen die zentralen Punkte der Theorie von Malthus. Allerdings finden sich ebenfalls Hinweise darauf, daß ein Ausbrechen aus der malthusianischen Bevölkerungsfalle möglich ist, wenn die unteren Schichten sich an einen höheren Lebensstandard gewöhnen und dann auch vermehrt zu preventive checks greifen, um diesen halten zu können. ${ }^{25}$

21 Vgl. Hirsch (1992) S. 48ff.

22 Die Theorie von Malthus wurde später von Darwin aufgegriffen und fand Eingang in seine Evolutionstheorie. Danach ist der Anteil der Kinder fruchtbarer Eltern in ihrer Generation größer als der Anteil ihrer Eltern in deren Generation gewesen ist. Nimmt man an, daß sich die Fruchtbarkeit von Generation zu Generation vererbt, so wird die Generation der Enkel wiederum einen gestiegenen Anteil einnehmen. Als Folge dieses Selektionsprozesses ergibt sich ein sehr hoher Anteil fruchtbarer Mitglieder einer Bevölkerung und damit ein hohes Bevölkerungswachstum.

Außer acht gelassen wird dabei, daß Eltern aufgrund einer bewußten Wahlhandlung nicht immer die maximal mögliche Kinderzahl auch tatsächlich realisieren werden. Änderungen exogener Parameter, die in die Wahlentscheidung eingehen, können daher die tatsächliche Fertilitätsrate beeinflussen, wie weiter unten ausgeführt wird.

Vgl. Malthus (1798) S. 64ff.

24 Vgl. Malthus (1798) S. 27ff. 
Eine theoretische Neuorientierung erfolgt erst Anfang des zwanzigsten Jahrhunderts durch deutsche Wohlfahrtstheoretiker wie Mombert und Brentano. Sie unterstellen, daß das Fortpflanzungsverhalten nicht wie bei Malthus biologisch determiniert ist, sondern das Ergebnis einer bewußten Entscheidung darstellt. Außerdem kritisieren sie die Annahme abnehmender Grenzerträge in der Landwirtschaft, indem sie den technischen Fortschritt berücksichtigen. Auch der positive Zusammenhang zwischen Einkommen und Kinderzahl wird in Frage gestellt. Nach Brentano bewirkt zunehmender Wohlstand eine zunehmende "Konkurrenz der Genüsse und eine Verfeinerung im Gefühl der Kinderliebe"26, die zu einem Rückgang des Zeugungswillens führen. ${ }^{27}$ Es ist also nicht mehr die Armut wie bei Malthus, die zum Rückgang der Kinderzahl führt, sondern im Gegenteil der zunehmende Wohlstand. Während Zimmermann, Dinkel und Zameck-Glyscinski ${ }^{28}$ in dem Werk Brentanos eine Vorwegnahme wesentlicher Teile der späteren neoklassischen Theorie der Fertilität sehen, ist Hirsch ${ }^{29}$ zurückhaltender in der Einschätzung des Beitrags der Wohlfahrtstheoretiker.

In dem von den Neoklassikern dominierten angelsächsischen Bereich wurde die Bevölkerung lange Zeit als exogen gegeben betrachtet. Dies mag an dem Widerspruch zwischen der pessimistischen Prognose der Malthusianischen Theorie, nach der ja ein dauerhaftes Wachstum des Pro-Kopf-Einkommens unmöglich ist, und der empirischen Entwicklung im 19. Jahrhundert gelegen haben, aber auch an den Schwierigkeiten bei der Integration des Fertilitätsverhaltens in die neoklassische Theorie der Konsumwahl. Erst mit einem bedeutenden Werk von Leibenstein im Jahre 1957 und der grundlegenden Arbeit von Becker (1960) erlangten demographische Variablen auch im Rahmen der Neoklassik wieder mehr Aufmerksamkeit. ${ }^{30}$

Bevor genauer auf die neoklassische Fertilitätstheorie einzugehen ist, werden im folgenden zunächst die wichtigsten soziologischen, demographischen und sozio-ökonomischen Ansätze der letzten Jahrzehnte kurz skizziert.

\subsection{Soziologische und Demographische Ansätze}

Soziologische Arbeiten ${ }^{31}$ setzen bei der Erklärung der Fertilität überwiegend bei den Präferenzen an. ${ }^{32}$ Es werden verschiedene Faktoren (Ausbildung, Erziehung, Religions-

26 Brentano (1909) S. 602.

27 Mit dem von Brentano (1909) S. 606 als Begründung herangezogenen Gossenschen Gesetz des abnehmenden Grenznutzens kann allerdings nur ein langsamerer Anstieg, nicht aber ein Rückgang der Geburtenrate bei steigendem Einkommen erklärt werden (vgl. Felderer/Sauga (1988), S. 66). Ein Rückgang läßt sich erst durch die Substitution von Quantität durch Qualität bei steigenden Einkommen begründen.

28 Vgl. Zimmermann (1989), Dinkel (1984a) S. 297 sowie Zameck-Glyscinski (1985) S. 87.

29 Vgl. Hirsch (1992) S. 72.

$30 \quad$ Vgl. Leibenstein (1957) und Becker (1960).

31 Es seien hier Davis/Blake (1956), Blake (1966), Arnold/Fawcett (1974), Jürgens/Pohl (1975) und Schumacher (1982) genannt. 
zugehörigkeit, Status, etc.) angeführt, die die Ansichten der Menschen hinsichtlich der optimalen Kinderzahl verändern. ${ }^{33}$ Dabei ist hier unter "optimal" die Kinderzahl zu verstehen, die bei Abwesenheit aller Restriktionen gewünscht wird.

Solche Korrelationen zwischen Fertilitätsraten und den angeführten sozialen oder sozioökonomischen Merkmalen sind allerdings erst dann wertvoll, wenn diese Beziehungen erstens langfristig stabil sind und zweitens auch eine ursächliche Erklärung finden. Beides ist aber nicht der Fall. Abweichungen im Fertilitätsverhalten verschiedener Gruppen gegenüber dem Durchschnitt sind nur kurzfristig auf eben diese Gruppenzugehörigkeit zurückzuführen. Langfristig erfolgt eine Angleichung an den Durchschnitt, wenn die Abweichungen nicht durch das ökonomische Rationalprinzip zu begründen sind. ${ }^{34}$ Auch wird keine theoretische Untermauerung der je nach Gruppenzugehörigkeit unterschiedlichen Geburtenraten geliefert. Stattdessen bleiben die soziologischen Ansätze bei der Feststellung statistisch signifikanter Beziehungen stehen und setzen sich daher dem Vorwurf eines "measurement without theory" aus. ${ }^{35}$

Hirsch kritisiert darüber hinaus die oft fehlerhafte Durchführung ${ }^{36}$ der Untersuchungen. Da zudem die Interpretationen der so gewonnen Daten widersprüchlich sind, spricht er diesen Analysen nur geringe Aussagekraft zu und lehnt sämtliche Erklärungen durch sich verändernde oder unterschiedliche Präferenzen ab. ${ }^{37}$ Auch wenn diese Kritik als zu hart erscheint, sind die Ergebnisse soziologischer Untersuchungen doch theoretisch ungenügend fundiert und werden deshalb nicht als Grundlage für die Untersuchung der Kosteneffizienz des Kinderlastenausgleichs verwendet.

Die Theorie des demographischen Übergangs wurde von Bevölkerungswissenschaftlern ${ }^{38}$ aufgrund der Beobachtung entwickelt, daß die Entwicklung von Geburten- und Sterberaten und damit des Bevölkerungswachstums in verschiedenen Regionen Europas sehr ähnlich verlief. Unterschiedlich war nur der Ausgangszeitpunkt des demographischen Transformationsprozesses.

Dinkel (1984b) S. 181 sieht den Kern soziologischer Erklärungsmodelle in der Annahme, daß das generative Verhalten Ergebnis der sozialen Schichtung ist.

33 Beispielsweise wurde Katholiken eine höhere Präferenz für Kinder zugeschrieben als Protestanten. Vgl. Blake (1967) S. 200f.

Als Beispiel verweist Dinkel (1984a) S. 313 auf die schnelle Angleichung der Fruchtbarkeitsdaten von Gastarbeitern an das niedrigere deutsche Niveau. Chamie (1981) wies für den Libanon nach, daß die Religionszugehörigkeit nur während des Übergangs von der traditionellen zur modernen Gesellschaft einen spürbaren Einfluß auf die Fertilität hatte.

Vgl. Dinkel (1984a) S. 316.

Insbesondere bemängelt Hirsch (1992) S. 26ff., daß meistens nicht eindeutig zwischen Präferenzen und Restriktionen unterschieden wird. 
Abb. C.1: Phasen des demographischen Übergangs

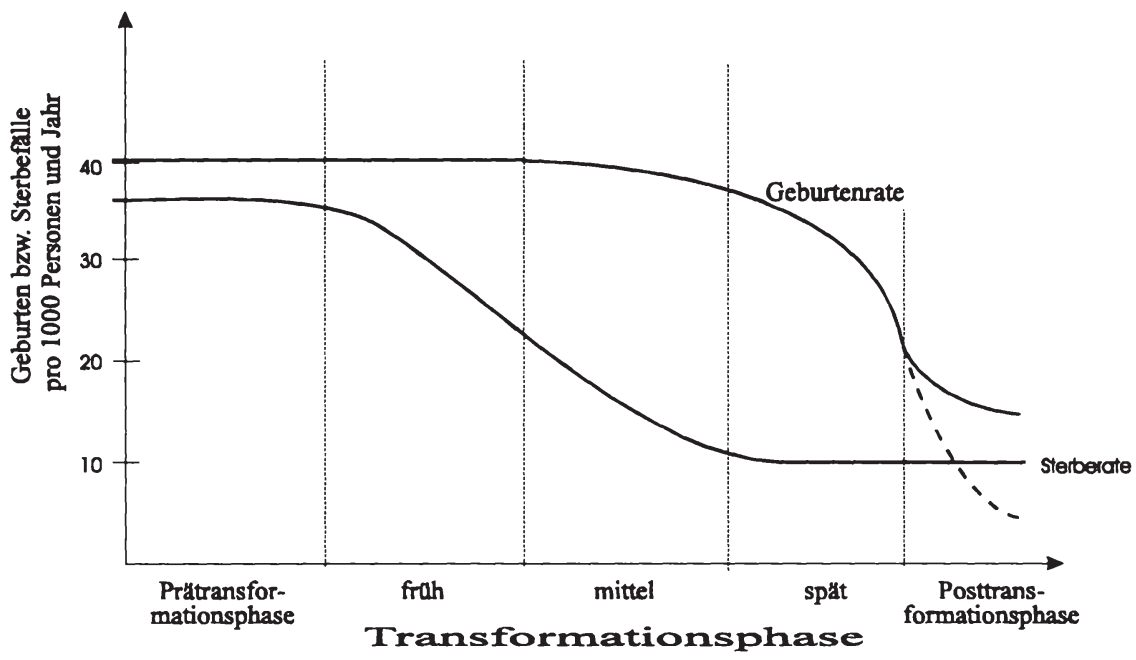

Drei verschiedene Phasen können unterschieden werden (vgl. Abb. C.1): ${ }^{39}$

- prätransformative Phase: vor der industriellen Revolution liegen Geburten- und Sterberaten auf fast gleich hohem Niveau. Das Bevölkerungswachstum und die Lebenserwartung ist gering, der "Bevölkerungsumschlag" dagegen groß.

- transformative Phase: hier kann wieder in drei Stufen untergliedert werden. In der frühtransformativen Phase fallen die Sterberaten während die Geburtenrate unverändert hoch bleibt. In der mitteltransformativen Phase sinkt die Sterberate weiter, aber auch die Geburtenrate beginnt zu fallen. In der spättransformativen Phase sinkt die Sterberate schließlich kaum noch, die Geburtenrate fällt dagegen weiter.

- posttransformative Phase: Beide Raten liegen wieder in etwa gleich hoch, allerdings auf wesentlich niedrigerem Niveau (etwa 10 Geburten bzw. Sterbefällen pro 1000 Personen und Jahr statt 35 pro 1000). Das in der transformativen Phase sehr hohe Bevölkerungswachstum tendiert wieder gegen Null, der "Bevölkerungsumschlag" ist jetzt aber gering und die Lebenserwartung hoch.

Kritisiert wird an der Theorie des demographischen Übergangs, daß es in der Realität oft Abweichungen von dem beschriebenen Schema gibt und daß es die letzte Phase falsch beschreibt. Statt eines geringen Bevölkerungswachstums scheint sich in der posttransformativen Phase aufgrund einer weiter sinkenden Geburtenrate eher ein Bevölkerungsrückgang einzustellen (gestrichelte Fortsetzung der Kurve der Geburtenrate in Abb. C.1). Hauptsächlich ist aber der geringe Erklärungswert zu bemängeln, der die Bezeichnung "Theorie" kaum rechtfertigt. Es handelt sich lediglich um eine Beschreibung der empirischen

39 Stellvertretend für viele ähnliche Darstellungen seien hier Hemmer (1988) S. 276ff. und Felderer/Sauga (1988) S. 38ff. genannt. 
Entwicklung, nicht aber um eine Analyse der dafür maßgeblichen Gründe. Außerdem ist die Annahme, daß das beschriebene Entwicklungsmuster in Zukunft für alle Länder Gültigkeit haben wird, prinzipiell nicht beweisbar. Als Grundlage für die Abschätzung der Kosteneffizienz des Kinderlastenausgleichs ist die "Theorie" des demographischen Übergangs daher ungeeignet.

\subsection{Sozio-ökonomische Ansätze}

Unter "sozio-ökonomischen" Ansätzen sollen hier Erklärungsversuche für die beobachtete negative Korrelation von Einkommen und Kinderzahl verstanden werden, die von ökonomischen Variablen abhängige Präferenzen unterstellen. ${ }^{40}$ Der Unterschied zu den im vorangehenden Unterkapitel behandelten soziologischen Ansätzen besteht also darin, daß hier nicht unterschiedliche soziale Merkmale, sondern ökonomische Variablen als Erklärungsgrößen für unterschiedliche Präferenzen herangezogen werden.

Ebenso wie in den Modellen der Chicago-School (vgl. Kapitel 3) wird in den sozioökonomischen Theorien von Freedman ${ }^{41}$, Leibenstein ${ }^{42}$ und Easterlin ${ }^{43}$ unterstellt, daß eine positive Einkommenselastizität der Nachfrage nach Kindern vorliegt und weiterhin, daß die Entscheidung für oder gegen Kinder Ergebnis eines rationalen Kalküls ist.

Die Unterschiede zwischen den einzelnen Ansätzen ergeben sich aus den verschiedenen Präferenzbildungshypothesen. Easterlin postuliert, daß das Anspruchsniveau durch den Lebensstandard der Eltern festgelegt wird. Freedman und Leibenstein nehmen dagegen an, daß die Präferenzen nach sozialem Status gruppenspezifisch differenziert sind. Da der soziale Status annahmegemäß v.a. durch den Ge- und Verbrauch an Konsumgütern gemessen wird, sollen hier auch die letztgenannten Ansätze zu den sozio-ökonomischen und nicht etwa zu den soziologischen gezählt werden.

Auf die Hypothesen von Freedman und Leibenstein soll zuerst eingegangen werden. Da angenommen wird, da $\beta$ alle Mitglieder innerhalb einer sozialen Gruppe den gleichen sozialen Status erreichen wollen und dieser soziale Status v.a. an Konsumgütern gemessen wird, bleiben für die einkommensschwächeren Mitglieder einer sozialen Gruppe entsprechend weniger Ressourcen für die Erhöhung der Kinderzahl übrig. Sie müssen bzw. wollen die gleiche Geldmenge für Konsumgüter ausgeben wie die einkommensstärkeren Mitglieder der gleichen Gruppe, so daß der finanzielle Spielraum für Ausgaben, die durch (zusätzliche) Kinder entstehen, bei ihnen entsprechend stärker eingeschränkt ist. Damit bestimmt das relative Einkommen, von Freedman definiert als "... the ratio between a man's actual income and the income customary in his socioeconomic reference group" 44 , die Kinderzahl: je höher das relative Einkommen, desto höher die Kinderzahl.

40 Vgl. Felderer/Sauga (1988) S. 80 und etwas anders Hirsch (1992) S. 32ff. Zur Kritik der Annahme unterschiedlicher bzw. veränderlicher Präferenzen vgl. Abschnitt 3.1.1.

41 Vgl. Freedman (1963).

42 Vgl. Leibenstein $(1974,1975)$.

43 Vgl. Easterlin $(1969,1973)$.

44 Freedman (1963) S. 415. 
Die insgesamt negative Beziehung zwischen Einkommen und Kinderzahl wird dann über einen positiven Zusammenhang zwischen sozialem Status und Einkommen und einen negativen Zusammenhang zwischen sozialem Status und Kinderzahl hergestellt.

Graphisch läßt sich der Zusammenhang zwischen Einkommen und Kinderzahl in den Theorien von Freedman und Leibenstein demnach wie folgt darstellen:

Abb. C.2: Der Zusammenhang zwischen Kinderzahl (N) und Einkommen ( $Y$ ) in den Ansätzen von Freedman und Leibenstein

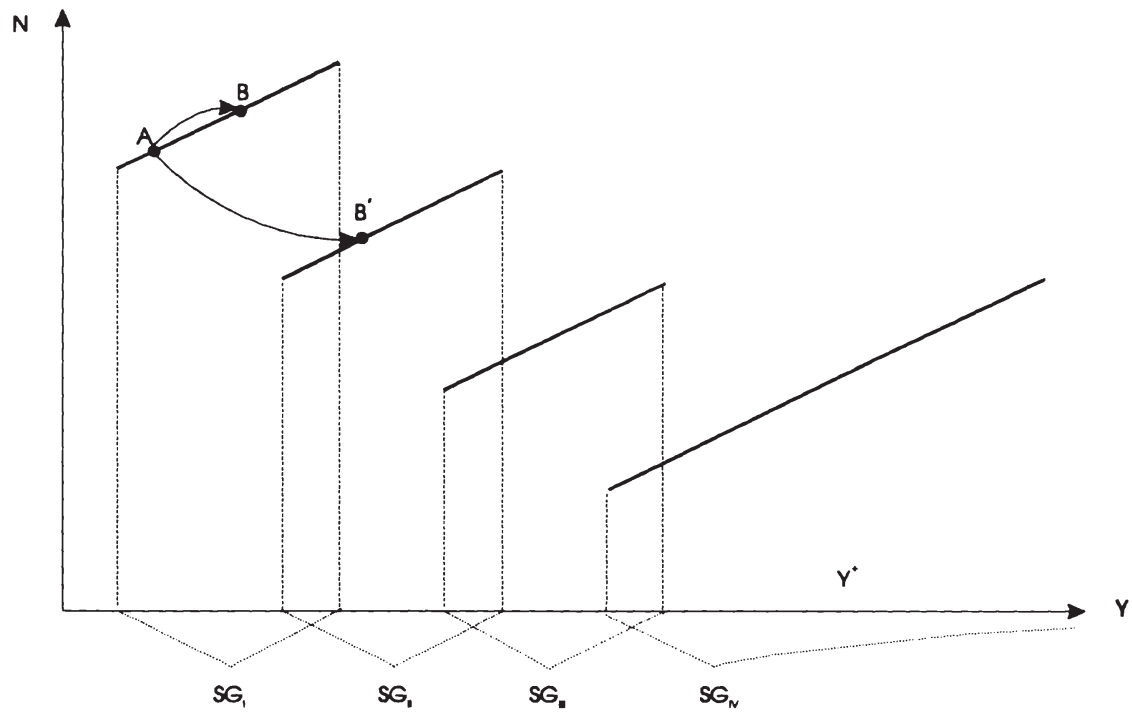

Ein Einkommensanstieg führt also solange zu einem Anstieg der Kinderzahl wie die soziale Gruppe nicht verlassen wird (Bewegung von Punkt A zu Punkt B). Führt die Einkommenserhöhung aber zu einem Aufstieg in eine höhere soziale Gruppe (hier von $S G_{I}$ in $S G_{I I}$ ), so sinkt die Kinderzahl (Bewegung von A nach B').

Dabei muß die durchschnittliche Kinderzahl höherer sozialer Gruppen niedriger sein als diejenige niedrigerer sozialer Gruppen. Nur so läßt sich die insgesamt negative Beziehung zwischen Einkommen und Fertilität erklären. Die Begründung für den negativen Zusammenhang zwischen sozialem Status und durchschnittlicher Kinderzahl sieht Freedman in mit sozialem Status steigenden Ausgaben pro Kind. Mit der Erhöhung der Konsumausgaben bei einem sozialen Aufstieg besteht ein sozialer Druck auch die Ausgaben pro Kind zu erhöhen.

Leibenstein dagegen begründet die mit höherem sozialen Status sinkende durchschnittliche Kinderzahl mit steigendem Zwang zum Konsum von Statusgütern. ${ }^{45}$ Für das Nicht-Statusgut Kind bleibt ein entsprechend geringer finanzieller Spielraum, so daß es zu einer Einschränkung

45 Der "Zwang" zum Konsum von Statusgütern äußert sich nach Leibenstein darin, daß diese Güter in einem bestimmten Bereich einen zunehmenden Grenznutzen aufweisen. Vgl. Leibenstein (1975) S. $7 \mathrm{ff}$. 
der Kinderzahl kommt. Folgt man dieser These von Leibenstein, muß es also zu einer absoluten Abnahme des nach Abzug der Ausgaben für Statusgüter noch zur Verfügung stehenden Einkommens kommen - und nicht nur zu einem sinkenden Verhältnis von schichtdurchschnittlichem Einkommen und Ausgaben für Statusgütern wie Leibenstein ${ }^{46}$ behauptet. $^{47}$ Das ist aber wohl recht realitätsfern.

Den säkularen Geburtenrückgang, d.h. die in Zeitreihendaten zurückgehenden Kinderzahlen, können diese Querschnittsbetrachtungen von Freedman und Leibenstein nicht erklären. Zwar stellt Leibenstein ${ }^{48}$ zwei Hypothesen auf, mit denen die empirischen Längsschnittdaten erklärt werden sollen, beide sind aber nicht überzeugend. Zum einen behauptet er, daß durch den Einkommensanstieg im Entwicklungsprozeß immer höhere soziale Schichten erreicht werden. Damit sinkt die durchschnittliche Fertilität, weil die höheren sozialen Schichten mit ihrer geringen Kinderzahl ein immer stärkeres Gewicht erlangen. Allerdings berücksichtigt er nicht, daß wohl auch das durchschnittliche Einkommen einer sozialen Schicht bei allgemein steigenden Einkommen entsprechend ansteigen würde. ${ }^{49}$

Zum anderen leitet er aus empirischen Daten einen "interstatus-income ratio compression effect" ab. ${ }^{50}$ Danach sinkt im Zuge der Entwicklung der relative Einkommensabstand zwischen den sozialen Gruppen. Wollen die oberen Schichten dies kompensieren und den relativen sozialen Abstand zu tieferen Schichten aufrechterhalten, müssen sie einen immer größeren Anteil ihres Einkommens für Statusgüter ausgeben. Sie können sich deswegen weniger Kinder leisten. Die Fertilität geht - v.a. in den oberen sozialen Schichten - zurück. Allerdings müßte aufgrund der gleichen Argumentation die Fertilität in den unteren Schichten steigen. Eine sich daraus ergebende Verstärkung des negativen Zusammenhangs zwischen sozialem Status und Kinderzahl steht aber im Widerspruch zur tatsächlichen Entwicklung. ${ }^{51}$

Auch Easterlin ${ }^{52}$ benutzt das Konzept des relativen Einkommens. Der Vergleichsmaßstab für das eigene Einkommen wird jetzt aber nicht mehr durch die soziale Schicht festgelegt, sondern durch den Lebensstandard der Eltern. Durch die Erfahrung des Lebensstandards im Elternhaus wird nach Easterlin das Niveau festgelegt, das jemand später selbst erreichen will. Ist das eigene Einkommen geringer als das der Eltern, wird auf Kinder verzichtet, um den Lebensstandard der Eltern über den Kauf entsprechender Konsumgüter dennoch realisieren zu können. Je höher der Lebensstandard der Eltern, desto niedriger wird die Fertilität ihrer Kinder ceteris paribus sein. Überträgt man diesen Zusammenhang auf die Makro-Ebene, dann sinkt die Fertilität also ceteris paribus mit der Höhe des Einkommens der vorangehenden ElternGeneration. ${ }^{53}$

Die Kritik an der Theorie von Easterlin richtet sich v.a. auf die folgenden drei Punkte. Erstens lassen sich mit diesem Ansatz zwar sich selbst fortpflanzende Schwankungen im

\footnotetext{
46 Vgl. Leibenstein (1975) S. 7.

47 Vgl. zu dieser Kritik Hirsch (1992) S. 35.

48 Vgl. Leibenstein (1974) S. 472ff. und (1975) S. 4ff.

49 Vgl. auch Hirsch (1992) S. 38.

so Vgl. Leibenstein (1975) S. 5 ff.

51 Zu empirischen Daten vgl. Schwarz (1982) S. 582.

52 Vgl. Easterlin (1969) und (1973).

53 Vgl. Felderer/Sauga (1988) S. 81 und Willis (1987) S. 71.
} 
Fertilitätsverhalten begründen, ${ }^{54}$ aber nicht der säkulare Trend zu weniger Kindern. ${ }^{55}$ Zweitens sprechen die Resultate verschiedener empirischer Untersuchungen gegen die von Easterlin postulierte Art der Präferenzbildung. Diese würde nämlich implizieren, da $\beta$ aufgrund der gleichen Konjunkturlage während ihrer Jugend gleiche Geburtsjahrgänge ein ähnliches Anspruchsniveau ausbilden und damit auch ein ähnliches Fertilitätsverhalten aufweisen. Tatsächlich gleichen sich aber eher die Kinderzahlen gleicher Heiratsjahrgänge. ${ }^{56}$ Drittens schließlich stellen viele empirische Untersuchungen einen nicht signifikanten oder gar negativen statt positiven Zusammenhang zwischen dem relativen Einkommen und der Kinderzahl fest. ${ }^{57}$

In einer Modifikation der Easterlin-Hypothese durch Ben-Porath ${ }^{58}$ wird unterstellt, da $\beta$ neben dem Lebensstandard der Eltern auch die Zahl der Geschwister das Anspruchsniveau der nachfolgenden Generation determiniert. Je mehr Geschwister vorhanden sind, desto weniger der Gesamtausgaben für alle Kinder entfällt auf jedes einzelne. Das Anspruchsniveau sinkt also mit der Zahl der Geschwister. ${ }^{59}$

Durch diese Ergänzung der Theorie von Easterlin werden auch säkular sinkende Fertilitätsraten erklärbar. Haben die Eltern einer Generation wenig Kinder gehabt, standen den wenigen Kindern pro Kopf relativ große Mittel zur Verfügung. Ihr Anspruchsniveau ist daher relativ hoch. Ihr relatives Einkommen (tatsächliches Einkommen im Verhältnis zum Anspruchsniveau) ist damit niedriger, als wenn sie mehr Geschwister gehabt hätten. Auch diese Generation wird aufgrund des niedrigen relativen Einkommens wenig Kinder haben, so daß sich eine intergenerative Fortschreibung des Fertilitätsverhaltens ergibt. ${ }^{60}$

Allerdings muß berücksichtigt werden, daß bei niedrigem relativen Einkommen der ElternGeneration wohl nicht nur die Zahl der Kinder reduziert wird, sondern auch die Ausgaben für andere Güter. Andernfalls müßte man für diese Güter Inferiorität unterstellen. Die in der Ausgangslage unterstellte Kombination von hohem Lebensstandard der Eltern und trotzdem geringer Geschwisterzahl ist daher unrealistisch. Ist der Lebensstandard der Eltern aber weniger hoch, sinkt auch das Anspruchsniveau in der Folgegeneration. Es wirken hier also zwei entgegengerichtete Effekte: die niedrigere Geschwisterzahl erhöht das Anspruchsniveau, der geringe Lebensstandard senkt es. Nur wenn der erste Effekt überwiegt, gibt es eine intergenerative Übertragung des Geburtenverhaltens. ${ }^{61}$

54 Dies war auch das primäre Ziel von Easterlin. Eine kleine Generation hat nach Easterlin relativ hohe Einkommen, weil die Konkurrenz am Arbeitsmarkt gering ist. Ihr relatives Einkommen ist entsprechend hoch und damit auch ihre Fertilität. Damit steigt das Anspruchsniveau der folgenden, zahlenmäßig großen Generation. Diese hat aufgrund der großen Konkurrenz am Arbeitsmarkt zudem schlechte Verdienstaussichten, so daß ihr relatives Einkommen und damit die Kinderzahl gering ist. Nach dieser Argumentation folgt also auf eine kleine Generation modellendogen eine große und umgekehrt. Die Hauptkritik an dieser These richtet sich auf die Annahme, daß die Verdienstmöglichkeiten einer Generation alleine durch die Zahl ihrer Mitglieder festgelegt wird.

s5 Vgl. Zimmermann (1986) S. 31.

56 Vgl. Smith (1981) S. 256ff.

57 Vgl. die bei Hirsch (1992) S. 44 und Zimmermann (1985) S. 139ff. bzw. Zimmermann (1986) S. 33 angegebene Literatur.

58 Vgl. Ben-Porath (1975).

59 Gleichzeitig vermutet Ben-Porath einen positiven Zusammenhang zwischen der Zahl der Geschwister und dem Wunsch nach eigenen Kindern. Vgl. Ben-Porath (1975) S. 398.

60 Vgl. Zimmermann (1986) S. 45.

61 Vgl. Hirsch (1992) S. 43. 
Die Teilung der Familienökonomie in die sozio-ökonomischen Ansätze (v.a. Easterlin) auf der einen und die Chicago-School auf der anderen Seite beruhte v.a. auf der unterschiedlichen Ansicht über die Präferenzen. Während die erstgenannte Richtung die Bildung der Präferenzen zu erklären suchte, wurden von den Vertretern der Chicago-School stabile Präferenzen unterstellt. Die Positionen haben sich aber in den achtziger Jahren so weit angenähert, daß von zwei getrennten Schulen kaum noch gesprochen werden kann. ${ }^{62}$

Als Ergebnis dieses Unterkapitels ist festzuhalten, daß die bisher dargestellten Fertilitätstheorien nicht geeignet sind, in dieser Arbeit als theoretisches Fundament für die Erklärung des generativen Verhaltens $\mathrm{zu}$ dienen. ${ }^{63} \mathrm{Daß}$ es daher gerechtfertigt ist, die neoklassische Fertilitätstheorie in dieser Arbeit der Analyse des Kinderlastenausgleichs als Instrument der Bevölkerungspolitik zugrundezulegen, wird im folgenden Unterkapitel (3.1) begründet. Dazu werden die wesentlichen Argumentationslinien der neoklassischen Theorie des generativen Verhaltens im folgenden Abschnitt (3.1.1) eingehender dargestellt.

\section{Die neoklassische Theorie des generativen Verhaltens}

\subsection{Grundlagen}

\subsubsection{Anwendung der traditionellen mikroökonomischen Theorie auf die Fertilitätsentscheidung}

Im Gegensatz zu den soziologischen und sozio-ökonomischen Theorien versucht die neoklassische Theorie der Fertilität das Geburtenverhalten bei stabilen Präferenzen zu erklären. Verhaltensänderungen sind damit allein auf Preis- und Einkommenseffekte zurückzuführen. Das Instrumentarium der neoklassischen mikroökonomischen Theorie zur Bestimmung einer Gleichgewichtsmenge für ein Konsumgut wird auf die Fertilitätsentscheidung angewandt. Zunächst wird geprüft inwieweit diese Übertragung gerechtfertigt ist, ehe dann der sich daraus ergebende Entscheidungsansatz kurz skizziert wird und die wesentlichen Annahmen des Modells auf ihre Stichhaltigkeit getestet werden.

Vgl. zu dieser Einschätzung auch Willis (1987) S. 71.

63 Zu der gleichen Einschätzung gelangen auch Dinkel (1984a) S. 317 und Hirsch (1992), S. 31. Die in der zweiten Hälfte der achtziger Jahre v.a. von Birg entwickelte biographische Theorie der Fertilität erscheint noch nicht hinreichend ausgereift und operational, um sie der Abschätzungen der Wirkungen des Kinderlastenausgleichs in dieser Arbeit zugrundezulegen. Da es sich aber um einen vielversprechenden Ansatz handelt, werden die Grundzüge der biographischen Theorie der Fertilität in Anhang 1 skizziert. 
Schon Adam Smith wies auf die Wirtschaftsgutanalogie von Kindern hin, indem er feststellte, daß "... die Nachfrage nach Arbeitskräften, wie bei jeder anderen Ware, das Wachstum der Bevölkerung ..."64 bestimme.

Dieser Ansatz geht davon aus, daß es sich wie bei dem Kauf eines normalen Guts ${ }^{65}$ bei dem Beschluß, Kinder in die Welt zu setzen, um eine freie Willensentscheidung handelt, da niemand dazu gezwungen wird. ${ }^{66}$ Auch ungeplante bzw. ungewollte Kinder können als Ergebnis einer freien Willensentscheidung unter Unsicherheit gesehen werden. ${ }^{67} \mathrm{Zu}$ jeder Zeit standen sichere "Verhütungsmittel" (im Extremfall Enthaltsamkeit) zur Verfügung, so daß die Wahl eines weniger sicheren Verhütungsmittels als Entscheidung für den daraus resultierenden Nutzen und den mit der entsprechenden Eintrittswahrscheinlichkeit gewichteten negativen Nettonutzen eines Kindes angesehen werden kann. Bei optimaler Wahl werden sich der Grenznutzen aus der Entscheidung für ein unsicheres Verhütungsmittel und die durch ein Kind hervorgerufenen Nutzeneinbußen gerade entsprechen. Insofern kann letztlich jedes Kind als geplant angesehen werden, ${ }^{68}$ solange die Möglichkeit besteht, sich über die jeweiligen Eintrittswahrscheinlichkeiten zu informieren. ${ }^{69}$

Oft wird eingewandt, daß Kindern eine besondere psychologische und ethische Bedeutung zukomme, die die Gleichsetzung von Kindern und Kühlschränken verbiete. Dabei wird nicht berücksichtigt, daß die Behandlung als Konsumgut $^{70}$ keinerlei moralische Wertung impliziert. ${ }^{71}$ Die Befriedigung, die die Eltern aus der Beziehung zu ihrem Kind erfahren, wird

64 Smith (1974) S. 69 (zitiert nach Felderer/Sauga (1988) S. 56).

65 Als "Gut" wird in den Wirtschaftswissenschaften allgemein alles, was den Menschen wertvoll ist und dessen Erlangung Ressourcen erfordert, definiert. Vgl. Dinkel (1984a) S. 64.

Vgl. Homburg/Gräff (1988) S. 14. Natürlich sind heute noch meistens zwei Personen an der Entscheidung für ein Kind beteiligt. Dies wirft aber keine Probleme auf, wenn man der neoklassischen Annahme folgt, daß Entscheidungen von Haushalten als Gesamtheiten getroffen werden.

67 Eine Ausnahme hiervon sind natürlich ausgetragene Schwangerschaften nach einer Vergewaltigung. Zahlenmäßig fallen sie aber kaum ins Gewicht.

Ähnlich auch Dinkel (1984a) S. 68.

Eine vollständige Information ist für eine rationale Entscheidung nicht erforderlich, da ein Abbruch der Informationsbeschaffung zu dem Zeitpunkt optimal ist, in dem ihre Grenzkosten den voraussichtlichen Grenznutzen übersteigen.

Im folgenden werden Kinder als Konsumgüter und nicht als Investitionsgüter bezeichnet, weil ihr Kapitalwert in Deutschland und anderen Industrieländern meistens negativ sein wird. Im übrigen ist die Unterscheidung irrelevant. Ob ein Gut ein Investitions- oder Konsumgut ist, hängt nämlich nicht von den Gütereigenschaften ab, sondern von der Nutzenfunktion. Während ein Konsumgut nur bei positivem nicht-monetären Grenznutzen gekauft wird, ist das bei einem Investitionsgut nicht der Fall. Letzteres wird auch noch bei negativem nicht-monetären Grenznutzen gekauft werden, da dem negativen nichtmonetären Grenznutzen ein positiver Kapitalwert gegenübersteht (d.h. sein monetärer (Gesamt-) Preis negativ ist). Hier wird der Käufer für den nicht-monetären Grenzschaden monetär entschädigt, bei einem Konsumgut muß er für den nicht-monetären Grenznutzen bezahlen. Ob eine marginale Guteinheit aber als nicht-monetärer Schaden oder als Nutzen betrachtet wird, hängt von der Nutzenfunktion ab. Die Unterscheidung von Investitions- und Konsumgütern ist nicht von den Gütereigenschaften abhängig, sondern von den Präferenzen. Weil Investitions- und Konsumgüter keine grundsätzlich unterschiedlichen Charakteristika haben, muB daher bei der Analyse nicht zwischen ihnen unterschieden werden. Vgl. Hirsch (1992) S. 27 und S. 107. Anders Dinkel (1984a) S. $65 f$.

Vgl. Becker (1960) S. $210 \mathrm{f}$. 
hier aber - wie auch bei anderen Gütern - nicht weiter untersucht, sondern durch die als exogen vorgegeben aufgefaßte Nutzenfunktion ausgedrückt. ${ }^{72}$

Betrachtet man Kinder also als Konsumgüter, so kann man konsequenterweise auch den Kosten-Nutzen-Ansatz auf die Fertilitätsentscheidung übertragen. Bahnbrechend in dieser Richtung waren die Gedanken von Harvey Leibenstein ${ }^{73}$, der schon 1957 folgende Nutzenund Kostenarten von Kindern unterschied:

Als Konsumnutzen bezeichnet Leibenstein die Befriedigung, die Kinder bei ihren Eltern stiften. Dazu gehört das Gefühl, gebraucht und gemocht zu werden, ebenso wie die eigene Persönlichkeitsbildung. Wie sich der Konsumnutzen im Zeitablauf verändert hat, ist schwer feststellbar. Vereinfachend geht Leibenstein deshalb von einem zeitlich konstanten Verlauf aus, einer Annahme, der auch in dieser Arbeit gefolgt wird.

Als Investitionsnutzen werden dagegen die monetären bzw. materiellen Erträge der Kinder benannt, die sie an die Eltern abführen. Hierunter fallen neben Arbeitsleistungen im Haushalt und familieneigenem Betrieb auch Beiträge zum Haushaltseinkommen aus eigenem Verdienst und Unterstützungsleistungen der Kinder bei Krankheit, Alter etc. der Eltern. Der Investitionsnutzen ist durch die längere Ausbildung der Kinder und den zunehmenden Ausbau des Sozialversicherungssystems stetig gesunken und in Industrieländern heute eher unbedeutend. Deshalb wird der Investitionsnutzen von Kindern hier vernachlässigt und das Kind als Konsumgut betrachtet, wenngleich Kindern in Entwicklungsländern durchaus noch Investitionscharakter zukommen kann.

Diesen Nutzenarten stehen die direkten und indirekten Kosten gegenüber. Als direkte Kosten werden alle Kosten bezeichnet, die tatsächlich durch die Kinder verursacht werden, indem Einkommen nicht mehr für andere Zwecke zur Verfügung steht. Es handelt sich also um monetäre Kosten von Kindern im Sinne von tatsächlich anfallenden Ausgaben. Zu nennen sind hier Unterkunft, Kleidung, Ernährung, Ausbildung, Spielzeug, etc. Die direkten Kosten haben sich im Zeitablauf v.a. wegen gestiegener Kosten für Ausbildung und im Zuge der Verstädterung auch für Wohnraum erhöht.

Gleichfalls stark gestiegen sind die indirekten Kosten, die im folgenden auch als Opportunitätskosten bezeichnet werden. Sie umfassen alle Kosten, die aus alternativen Verwendungsmöglichkeiten der Zeit resultieren. Weitaus am bedeutendsten ist hier der Verzicht auf Einkommen, wenn eine berufliche Tätigkeit aufgrund der Kindererziehung abgebrochen bzw. gar nicht erst angetreten wird. Da noch immer ein Großteil der Erziehungsarbeit von Frauen geleistet wird, wirkt sich hier der überdurchschnittlich gestiegene Lohnsatz für Frauen besonders stark aus.

Bei allen Nutzen- und Kostenarten sind für die Entscheidung für oder gegen ein Kind nicht die tatsächlich realisierten Größen maßgeblich, sondern die für die Zukunft erwarteten Größen. ${ }^{74} \mathrm{Da}$ allerdings erwartete Größen empirisch kaum beobachtbar sind und zudem bei

72 Diese Annahme wird weiter unten in diesem Abschnitt problematisiert.

73 Vgl. Leibenstein (1957) S. 159ff. Leibenstein unterscheidet neben dem Konsumnutzen zwischen "the utility to be derived from the child as a productive agent" und "the utility derived from the prospective child as a potential source of security, either in old age or otherwise" (Leibenstein (1957) S. 161), die hier zum Investitionsnutzen zusammengefaßt werden.

74 Welche Bedeutung diese Unterscheidung hat, wurde nach der Wiedervereinigung der beiden deutschen Staaten klar: Obwohl die tatsächliche ökonomische Situation (gemessen an der durchschnittlichen Kaufkraft) sich gebessert hat, ist die Geburtenrate in den fünf neuen Ländern auf etwa ein Drittel des Niveaus von 1989 zurückgegangen. Für diese dramatische Entwicklung dürfte die hohe Unsicherheit 
Gültigkeit der grundlegenden Rationalitätsannahme eine systematische Täuschung der Entscheidungsträger und damit eine längerfristige und systematische Abweichung der erwarteten von den tatsächlichen Größen unwahrscheinlich ist, wird im folgenden davon ausgegangen, daß die tatsächlichen Werte eine hinreichend genaue Approximation der eigentlich in das Entscheidungskalkül eingehenden erwarteten Werte darstellen.

Um die für den "repräsentativen" Elternhaushalt optimale Kinderzahl zu bestimmen, muß nach diesem Kosten-Nutzen-Ansatz entsprechend des Marginalprinzips der Neoklassik die Kinderzahl bestimmt werden, bei der die Summe der Grenzkosten gleich der Summe der Grenznutzen ist. Wenn die Summe der durch ein weiteres Kind verursachten direkten und indirekten Kosten gerade dem durch dieses Kind verursachten Konsumnutzen entspricht - der Investitionsnutzen wird ja vernachlässigt - ist die optimale Kinderzahl für ein Elternpaar erreicht.

Beachtet werden muß hier natürlich, daß die Kinderzahl nicht stetig variiert werden kann, sondern eine diskrete Variable ist. Die Summe der Grenzkosten wird aber in den seltensten Fällen gerade bei einer ganzzahligen Kinderzahl dem Grenznutzen entsprechen, sondern beispielsweise bei 1,6 Kindern. Daher kann mit dem Marginalprinzip strenggenommen nicht die optimale Kinderzahl bestimmt werden, sondern nur eine Eingrenzung auf die nächtshöhere und nächstniedrigere Kinderzahl erfolgen (hier ein bzw. zwei Kinder). Welche dieser beiden Kinderzahlen schließlich realisiert wird, hängt dann von der absoluten Kosten- und Nutzenänderung beim Übergang von der niedrigeren auf die höhere Kinderzahl ab. Sind die absoluten (Zusatz-)Kosten höher als der absolute (Zusatz-)Nutzen, so wird die niedrigere der beiden zur Wahl stehenden Kinderzahlen realisiert und umgekehrt. Für die Kinderzahl eines einzelnen Elternpaares mag diese Überlegung sicherlich eine große Rolle spielen, schließlich bedeutet im Beispiel die höhere Kinderzahl schon eine Verdoppelung von einem Kind auf zwei Kinder. Für eine größere Bevölkerung sind die aus der Nicht-Stetigkeit der Entscheidungsvariablen Kinderzahl resultierenden Konsequenzen dagegen dann unbedeutend, wenn man davon ausgeht, daß für etwa ebensoviele Eltern eine ganzzahlige Kinderzahl oberhalb der nach dem Marginalprinzip ermittelten stetigen Kinderzahl vorteilhaft ist wie eine unterhalb dieser stetigen Optimalgröße gelegene. Für ein einzelnes Elternpaar ist es zwar unmöglich 1,6 Kinder zu bekommen, für eine größere Gruppe kann sich dieser Wert dennoch als Durchschnitt ergeben. Trotzdem es sich bei der Kinderzahl um eine diskrete Variable handelt, ist daher eine auf dem Marginalprinzip beruhende Analyse gerechtfertigt.

Die grundlegenden Annahmen der meisten Analysen auf der Basis dieses neoklassischen Ansatzes sollen auch hier unterstellt werden. Meistens wird ein statisches mikroökonomisches Lebenszeitmodell zugrundegelegt. Mit Hilfe der komparativen Statik wird der Einfluß exogener Variablen auf die über die Lebensspanne anfallenden erwarteten Nutzen und Kosten untersucht, die ihrerseits die Fertilitätsentscheidung determinieren. Makroökonomische Rückwirkungen werden dabei vernachlässigt. Aufgrund des statischen Ansatzes können Vor- und Nachholeffekte 75 nicht untersucht werden. Kurzfristig können diese Effekte zwar bedeutend sein - Dinkel (1984) sieht in einer Vorverlagerung und anschließenden Rückverlagerung des Durchschnittsalters der Mütter einen wesentlichen Grund für den Geburtenrückgang Ende der sechziger Jahre - aber langfristig spielen sie für die Bevölkerungsentwicklung eine weniger

über die zukünftige individuelle ökonomische Situation ein wesentlicher Faktor gewesen sein. Vgl. o.V. (1993b) S. 662f.

Eine Erläuterung dieser Begriffe findet sich in Abschnitt 2.1.2 des Teils B dieser Arbeit. 
große Rolle. Vor- und Nachholeffekte müssen daher zwar bei der Interpretation der empirischen Daten beachtet werden, ein statischer Modellansatz ist aber dennoch gerechtfertigt.

Weiter wird unterstellt, daß die Nutzenfunktion neoklassische Eigenschaften besitzt, ihre einzelnen Argumente also einen positiven, aber abnehmenden Grenznutzen haben. ${ }^{76}$ Damit wird Superiorität aller Güter unterstellt und eine Erklärung des negativen Zusammenhangs zwischen Einkommen und Kinderzahl über Inferiorität von Kindern ausgeschlossen.

Am heftigsten wird an dem Ansatz der Chicago-School die Annahme stabiler Präferenzen kritisiert, die diese Schule ja gerade von den soziologischen und sozio-ökonomischen Theorien unterscheidet. In dem programmatischen Artikel "De gustibus non est disputandum"77 machen Stigler und Becker als Vertreter des neoklassischen Ansatzes klar, daß Präferenzunterschiede zwischen Personen und Präferenzänderungen zwar durchaus existieren. Aber bevor ein Rückgriff auf sie erfolgt, sollten in einer ökonomischen Analyse alle Möglichkeiten einer Erklärung bei stabilen Präferenzen untersucht worden sein. Anderenfalls würde zu schnell auf Begründungen anderer Disziplinen zurückgegriffen und nicht genug Anstrengungen unternommen, ökonomische Erklärungsmöglichkeiten auszuschöpfen. ${ }^{78}$

So erklären sie beispielsweise den zunehmenden Konsum von "guter" Musik sobald eine gewisser Mindestkonsum überschritten wurde ${ }^{79}$ nicht etwa mit sich ändernden Präferenzen, sondern mit Hilfe des Humankapital-Ansatzes und der Haushaltskonsumtechnologie ${ }^{80}$. Die Haushaltskonsumtechnologie beschreibt die Kombinationen von Einsatzfaktoren (z.B. Zeit und Humankapital), die benötigt werden, um Nutzen aus der Musik ziehen zu können. Durch das Hören guter Musik wird immer mehr musikspezifisches Humankapital gebildet. Der Genuß der Musik wird immer leichter. Der Schattenpreis der guten Musik fällt und der Konsum steigt, ohne daß eine Änderung der Präferenzen eingetreten sein muß. Die Grenzkosten des Musikkonsums haben sich durch die Akkumulation von Humankapital gesenkt, obwohl die monetären Kosten (z.B. Tonträger und Abspielgerät) und die zeitlichen Kosten (Lohnsatz) sich nicht verändert haben.

Leider sind weder Konsumtechnologie bzw. Humankapital noch Präferenzen eindeutig beobachtbar, so daß jede empirische Beobachtung durch die nicht nachprüfbare Behauptung einer Präferenz- bzw. Konsumtechnologieänderung erklärt werden kann. Da eine Integration beider Ansätze durch den Einbau einer Theorie der Präferenzbildung in die Mikroökonomik noch eine Wunschvorstellung ist, ${ }^{81}$ wird entsprechend dem ökonomischen Ansatz dieser Arbeit von stabilen Präferenzen ausgegangen.

76 Mathematisch bedeutet das, daß die erste Ableitung nach jedem Argument positiv, die zweite dagegen negativ ist.

77 Vgl. Stigler/Becker (1977).

78 Vgl. Stigler/Becker (1977) S. 76 und S. 89f.

79 Je mehr gute Musik man gehört hat, desto höher wird regelmäßig die individuelle Nachfrage nach dieser Musik. Vgl. zu diesem Beispiel Stigler/Becker (1977) S. 78ff.

80 Beide Konzepte - der Humankapital-Ansatz und die Haushaltskonsumtechnologie - werden in Abschnitt 3.1.2 ausführlich erläutert.

81 Die bisherigen Versuche einer Integration von Präferenzbildungstheorien in die Mikroökonomik beruhen meist auf nicht näher begründeten adhoc-Präferenzbildungshypothesen. Hierzu kann auf die Kritik an den sozio-ökonomischen Ansätzen in Kapitel 2.6 verwiesen werden, wonach die Präferenzen von der Gewöhnung an einen Zustand oder eine Aktivität, dem Verhalten einer Referenzgruppe oder direkt von den Preisen und Einkommen beeinflußt werden. Vgl. auch Zimmermann (1986) S. 44. 


\subsubsection{Erweiterung um die New Home Economics}

Obwohl Kinder - wie oben ausgeführt - als Konsumgüter angesehen werden können, haben sie doch Eigenschaften, die sie von vielen (nicht allen) anderen Gütern unterscheiden. So können Kinder nicht am Markt gekauft werden und es gibt für sie auch keinen Gebrauchtmarkt. ${ }^{82}$ Es fehlt also ein Markt für Kinder und damit kann auch der "Preis"83 für Kinder nicht direkt beobachtet werden. ${ }^{84}$ Kinder werden nicht gekauft, sondern unter Einsatz der Ressourcen Einkommen und Zeit im Haushalt selbst "hergestellt". Deshalb ist für die Analyse der Fertilitätsentscheidung die Berücksichtigung der Zeitrestriktion besonders wichtig. Es muß aber betont werden, daß es sich nicht um ein Merkmal handelt, das nur auf Kinder zutrifft. Auch andere Güter, wie zum Beispiel selbstgemachte Marmelade oder Mahlzeiten, werden im Haushalt hergestellt. Allerdings sind diese Güter oder zumindest enge Substitute auch am Markt verfügbar. Bei Kindern ist das kaum der Fall, so daß hier die Haushaltsproduktion eine besonders große Rolle spielt. Es ist daher angebracht in diesem Abschnitt zunächst die Theorie der Zeitallokation und der Haushaltsproduktion bzw. -konsumtion (new home economics) etwas ausführlicher zu erläutern, ehe im folgenden Unterkapitel auf dieser Grundlage die Fertilitätsentscheidung untersucht wird.

Die grundlegende Neuerung der new home economics gegenüber der traditionellen Mikroökonomik besteht darin, daß nicht mehr am Markt käufliche Güter als Argumente der Nutzenfunktion angesehen werden, sondern Basisgüter (engl. commodities), die erst mit Hilfe von Zeit und Marktgütern durch den Haushalt erstellt werden müssen. ${ }^{85}$ Selbst wenn eine Mahlzeit fertig angerichtet auf dem Teller liegt, ist eine gewisse Zeit erforderlich, um Nutzen daraus zu ziehen. Nicht das Marktgut Cordon Bleu, sondern das Basisgut Essen stiftet den Nutzen. Analog zur Produktion in einer Firma muß der Haushalt mehrere Faktoren kombinieren, um Konsumnutzen zu erzeugen. Neben dem Marktgut Fernsehgerät - um ein anderes Beispiel zu nehmen - benötigt er Zeit, um aus der Übertragung eines Fußballspieles Nutzen zu ziehen. Wie eng oder weit die Abgrenzung dieser Basisgüter zu fassen ist, wird in der Literatur unterschiedlich beurteilt. Becker führt als Beispiele für commodities die relativ weitgefaßten Kategorien "... children, prestige and esteem, health, altruism, envy, and pleasures of the senses

82 Vgl. zu weiteren Besonderheiten des Gutes "Kind" Tobin (1974) S. 293f.

83 Die Begriffe "Preis" und "Kosten" können hier synonym verwendet werden, weil Konsum und Produktion bei dem selben Wirtschaftssubjekt liegen und daher ein Gewinn - also der Unterschied zwischen Preis und Kosten - nicht auftritt.

Als Indiz dafür, daß auch ohne ein Verbot der Markt für Kinder nicht allzu umfangreich wäre, sieht Becker (1991) S. 45 die geringe Zahl von Adoptionen an. Als Gründe gibt er Verbundvorteile (Frauen können während der Schwangerschaft leichter andere Kinder aufziehen als einer Marktarbeit nachgehen), die genetische Ähnlichkeit eigener Nachkommen und die leichtere Verfügbarkeit von Informationen über eigene im Vergleich zu adoptierten Kindern z.B. über die zurückliegende Krankheitsgeschichte an.

85 Becker beschreibt diesen Ansatz wie folgt: "I have been assuming that time and goods directly provide utility, yet a more intuitive and useful assumption is that time and goods are inputs into the production of 'commodities', which directly provide utility. These commodities cannot be purchased in the marketplace but are produced as well as consumed by households using market purchases, own time, and various environmental inputs." Becker (1991) S. 23f. Dabei versteht er unter dem dritten Faktor (environmental inputs) "... household ability, human capital, social and physical climate, and other environmental variables." Becker (1991) S. 24. 
..."86 an. Bei der Analyse des Fertilitätsverhaltens im nächsten Abschnitt werden alle Basisgüter außer Kindern zu einem aggregierten Basisgut zusammengefaßt, um die Vorgehensweise anschaulich zu halten, so daß die Abgrenzung einzelner Basisgüter (außer Kindern) hier nicht interessiert.

Von der eben beschriebenen Haushaltskonsumtion kann die Haushaltsproduktion unterschieden werden. Der Vorgang des Produzierens im Haushalt erfordert gleichfalls Zeit. Das Marktgut Cordon Bleu, das dann mit Hilfe von Zeit entsprechend der Haushaltskonsumfunktion in das Basisgut Essen transformiert wird, kann nämlich auch im Haushalt hergestellt werden anstatt es am Markt (z.B. in einem Restaurant) zu kaufen. Jetzt wird das Marktgut Cordon Bleu durch den Einsatz verschiedener anderer Marktgüter (Fleisch, Fett, Herd, Salz, Strom, etc.) und Haushaltszeit unter einer bestimmten Produktionstechnik (Haushaltsproduktionstechnologie) im Haushalt hergestellt.

Allerdings ist die Abgrenzung zwischen Haushaltsproduktion und -konsum nicht immer eindeutig. Für einen Hobbygärtner mag die Tätigkeit im Garten nutzenstiftend sein. Dann wird er auch noch Gemüse in seinem Schrebergarten anbauen, wenn er vom Biobauern nebenan identisches Gemüse zu einem Preis kaufen könnte, der unter den monetären Kosten der für den Hobbygärtner notwendigen (Markt-)Inputgüter liegt. Hier ist die Gartenarbeit also offensichtlich als nutzenstiftendes Basisgut anzusehen, denn der Kapitalwert der Investition in den Gemüseanbau ist negativ. Der Anbau von Gemüse ist damit bei rationalem Verhalten nicht mehr Mittel zum Zweck (Investitionsgut), sondern das Ziel selber ${ }^{87}$ Eine andere Person kann dagegen die Gartenarbeit als notwendiges Übel zur Herstellung billigen und guten Gemüses ansehen. Hier handelt es sich offenbar um einen Haushaltsproduktionsprozeß. Der Unterschied besteht darin, daß einmal im Haushalt Marktgüter bzw. Inputgüter für die Haushaltskonsumtion (Nahrungsmittel) hergestellt werden (Haushaltsproduktion), das andere Mal im Haushalt ein nutzenstiftendes Basisgut ("Gärtnerfreude") produziert wird (Haushaltskonsumtion). ${ }^{88}$ Die Grenze hängt offensichtlich von der Nutzenfunktion ab. Stiftet schon die Gartenarbeit Nutzen, handelt es sich um einen Haushaltskonsumtionsprozeß. Ist dagegen erst der Verbrauch der produzierten Güter nutzenstiftend, handelt es sich um einen Haushaltsproduktionsprozeß. Gerade bei der Kindererziehung ist schwer feststellbar, ob bzw. wieviel der Zeit als Investition anzusehen ist und wieviel der mit den Kindern verbrachten Zeit nur deshalb aufgewendet wird, um den oben beschriebenen Konsumnutzen der Kinder zu realisieren. ${ }^{89}$

Letztlich ist die Unterscheidung zwischen der Zeit als Inputfaktor zur Haushaltsproduktion und der zum Konsum benötigten Zeit hier auch nicht entscheidend, weil nicht wie bei vielen anderen Gütern die Möglichkeit besteht, das fertige Gut am Markt zu kaufen. Beim Basisgut Essen kann man die Produktionszeit gegen Null reduzieren, indem man in ein Restaurant geht oder einen Bringdienst in Anspruch nimmt. Die Produktionszeit in Form von Einkaufszeit, Zeit fürs Kochen und Abwaschen fällt dann nicht an. Bei Kindern gibt es diese Wahlmöglichkeit

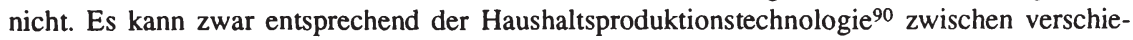

\footnotetext{
86 Becker (1991) S. 24.

87 Vgl. Fußnote 70 zur Abgrenzung von Investitions- und Konsumgut.

88 Leider werden beide Aspekte in der Literatur nicht immer eindeutig getrennt. Oft wird die Bezeichnung "Haushaltsproduktion" für beide Vorgänge verwendet und leistet so Mißverständnissen Vorschub. Vgl. Hirsch (1992) S. 95f. und Dinkel (1984a) S. 77f.
}

89 Dinkel (1984a) S. 77f. benutzt als Beispiel neben dem Aufziehen von Kindern ein Fondue, bei dem Produktionszeit und Konsumzeit fast vollständig identisch sind.

90 Die Haushaltsproduktionstechnologie legt den durch die Haushaltsproduktionsfunktion beschriebenen Zusammenhang zwischen Input- und Outputgrößen fest. 
den kapital- und zeitintensiven Produktionsmöglichkeiten für Kinder gewählt werden (z.B. Kindergartenplatz, Tagesmutter, etc. statt eigener Betreuung), aber es ist nicht möglich, Kinder in einer Form am Markt zu kaufen, die die Produktionszeit gänzlich überflüssig macht. Im Gegensatz zu vielen anderen Gütern ist daher für Kinder eine Unterscheidung zwischen Haushaltsproduktions- und -konsumtionstechnologie weder nötig noch möglich. ${ }^{91}$ Deshalb wird in der folgenden Diskussion analog zu der Vorgehensweise von Dinkel ${ }^{92}$ die Konsumzeit mit unter der Produktionszeit erfaßt. Der Begriff Haushaltsproduktion umfaßt daher im folgenden sowohl die Produktion von Marktgütern im Haushalt als auch die Herstellung von Basisgütern.

Aus der Feststellung, daß nicht Marktgüter nutzenstiftend sind, sondern sie erst durch Zeiteinsatz im Haushalt zu Basisgütern weiterverarbeitet werden müssen, ergibt sich, daß offensichtlich auch nicht der Preis der Marktgüter allein dem Nutzenmaximierungskalkül eines rationalen Haushalts zugrundeliegen wird. Stattdessen werden die Haushalte auch die Knappheit des zweiten Produktionsfaktors, nämlich der Zeit berücksichtigen. Der Preis für ein Basisgut ist daher im Gegensatz zu dem Preis eines Marktgutes nicht am Markt beobachtbar. Das Basisgut wird ja nicht zwischen verschiedenen Wirtschaftseinheiten gehandelt, sondern innerhalb eines Haushalts hergestellt. Sein Preis ist daher nur über die marginalen Herstellungskosten, den sogenannten Schattenpreis, bestimmbar. Dieser setzt sich zusammen aus den marginalen Kosten für den Inputfaktor Zeit und den marginalen Kosten für den Inputfaktor Marktgüter. Letztere entsprechen unter den Bedingungen der vollständigen Konkurrenz dem Preis $\left(p_{i}\right)$ des Bündels $\left(x_{i}\right)$ von Marktgütern, die zur Herstellung einer Einheit des Basisguts $Z_{i}$ benötigt werden.

Nimmt man als Preis einer Einheit der zur Erstellung des i-ten Basisgutes benötigten (Haushalts-)Zeittypen ${ }^{93}\left(t_{h_{1}}\right)$ die Opportunitätskosten in Form des entgangenen Arbeitslohnes $(w)$ an, ${ }^{94}$ so setzt sich der Schattenpreis $\left(\pi_{i}\right)$ eines beliebigen Basisgutes $\left(Z_{i}\right)$ zusammen aus den direkten und den indirekten Kosten:95

$$
\pi_{i}=\frac{p_{i} x_{i}}{Z_{i}}+\frac{w t_{h_{i}}}{Z_{i}}
$$

Das Gewicht der indirekten Kosten wird dabei bestimmt durch die Zeitintensität $\left(t_{h_{1}}\right)$ einerseits und die Kosten der Zeiteinheit $(w)$ andererseits. Entgegen den Aussagen der traditionellen Mikroökonomik sind es die Schattenpreise $\left(\pi_{i}\right)$, die zusammen mit der Haushaltsproduktionsfunktion $Z_{i}=Z_{i}\left(t_{h_{i}}, x_{i}\right)$ über die optimale Wahlhandlung entscheiden und nicht etwa ausschließlich die Preise der Marktgüter.

91 Vgl. Hirsch (1992) S. 100.

92 Vgl. Dinkel (1984a) S. 77f. Allerdings soll hier diese Zeit nicht wie bei Dinkel als eigenständiges Argument mit in die Nutzenfunktion aufgenommen werden, weil die Zeit dann gleichzeitig Input- und Outputgröße wäre.

93 Ein Haushaltszeittyp kann beispielsweise Gartenarbeit, ein anderer Geschirrspülen sein.

94 Später wird mit Hilfe des Humankapitalansatzes erläutern, warum der Ansatz des Lohnsatzes zu einer Unterschätzung der Zeitkosten führen kann (vgl. Unterkapitel 3.2.1).

95 In dieser Formel werden statt der marginalen die durchschnittlichen Produktionskosten ausgewiesen. Diese entsprechen aber den marginalen, wenn die Produktionsfunktionen homogen vom ersten Grad sind und keine Verbundproduktion bei den Basisgütern vorliegt. 
Bei der Analyse der Fertilitätsentscheidung ist zu berücksichtigen, daß die Schattenpreise normalerweise weder bei Änderungen des Lohnsatzes noch des Nicht-Arbeitseinkommens oder der zur Verfügung stehenden Gesamtzeit konstant bleiben werden. Eine Lohnsatzerhöhung erhöht die Zeitkosten, so daß eine Substitution hin zu weniger zeitintensiven Basisgütern erfolgen wird und gleichzeitig auf eine weniger zeitintensive Produktionsweise dieses Basisgutes übergegangen wird. Eine Erhöhung des Nicht-Arbeitseinkommens mindert die Einkommensrestriktion. Der Faktor Zeit wird dadurch relativ knapper, d.h. die Zeitrestriktion tendenziell vor der Einkommensrestriktion bindend. Die Folgen sind die gleichen wie oben. Der Schattenpreis kapitalintensiver Basisgüter wird sinken, der Schattenpreis zeitintensiver Basisgüter dagegen steigen. Gebremst werden die dadurch ausgelösten Substitutionseffekte wieder durch den Übergang zu anderen Produktionsverfahren.

Grundlegend für die Argumentationsweise in den folgenden Abschnitten sind zwei Prämissen: Zum ersten wird angenommen, daß Kinder ein relativ zeitintensives Basisgut darstellen. Das heißt, daß bei der Erstellung des Basisgutes Kind im Vergleich zur Erstellung anderer Basisgüter relativ viel Zeit und relativ wenig Marktgüter benötigt werden. Eine Erhöhung des Schattenpreises der Zeit wird somit zu einer Reduktion der Kinderzahl führen, weil dadurch ein Substitutionsprozeß hin zu weniger zeitintensiven Basisgütern ausgelöst wird.

Zweitens wird angenommen - und das soll ausführlicher begründet werden -, daß ein Ehepartner besonders stark mit der Kindererziehung beschäftigt ist, während der andere relativ mehr Zeit auf die Erzielung eines Markteinkommens verwendet ${ }^{96}$ Eine derartige Arbeitsteilung ist unter Effizienzgesichtspunkten einer gleichmäßigen Verteilung der Aufgaben überlegen, wenn man von der - sicherlich realistischen - Annahme ausgeht, daß beide Tätigkeiten spezifisches Humankapital erfordern. Dann hat der Partner einen komparativen Vorteil bei der Erzielung eines Markteinkommens, für den das Verhältnis von Grenzprodukt im Marktsektor zu Grenzprodukt im Haushaltssektor höher ist als für den anderen. Die Höhe dieser Grenzprodukte hängt positiv von dem verfügbaren Bestand an sektorspezifisch relevantem Humankapital ab. Hat beispielsweise der eine Partner nach einem Hauptschulabschluß eine Hauswirtschaftsschule besucht und damit relativ viel haushaltsspezifisches, aber wenig marktspezifisches Humankapital erworben, so verfügt er über einen komparativen Vorteil bei der Haushaltsproduktion gegenüber einem Partner, der durch Studium, Praktika und bisherige Berufserfahrung relativ viel marktspezifisches Humankapital gebildet hat. Da die Rendite des spezifischen Humankapitals davon abhängt, wieviel Stunden sein Träger in dem jeweiligen Sektor arbeitet, kann das Nutzenniveau des Haushalts durch eine dem komparativen Vorteil entsprechende Arbeitsteilung gesteigert werden.

Verstärkt wird die Arbeitsteilung dadurch, daß die überwiegende Tätigkeit in einem Sektor Anreize schafft, in das für diesen Sektor spezifische Humankapital zu investieren, da es ja überdurchschnittlich genutzt wird. Dadurch werden anfängliche komparative Vorteile in diesem Sektor noch vergrößert.

Die nach wie vor häufig zu beobachtende traditionelle Rollenverteilung, in der die Frau das Aufziehen der Kinder und die Hausarbeit, der Mann die Erzielung des Erwerbseinkommens übernimmt, kann nach Becker mit den folgenden drei Argumenten begründet werden: erstens mit biologischen Unterschieden zwischen den Geschlechtern, zweitens mit einer Diskriminierung gegen Frauen, die sich in einem ceteris paribus geringeren Lohnsatz niederschlägt, und drittens mit einer unterschiedlichen Investition in Humankapital. 
Die biologischen Unterschiede sind v.a. während der Zeit der Schwangerschaft der Frau von Bedeutung. In dieser Zeit ist die Frau in ihrer Fähigkeit, einer Marktarbeit nachzugehen, eingeschränkt und zwar vergleichsweise stärker als bei der Betreuung älterer Kinder. Diese "complementarity between bearing and rearing children"97 führt dazu, daß die Frau - auch wenn sie über das gleiche Humankapital verfügt wie der Mann - einen komparativen Vorteil im Haushaltssektor hat. Allerdings wird dieser Vorteil mit sinkender Kinderzahl immer geringer, weil dann die oben beschriebenen Verbundvorteile von Kinderaufziehen und -austragen weniger ins Gewicht fallen, so daß ihm heute wegen geringerer Geburtenraten weit weniger Bedeutung zukommt als etwa noch Ende des letzten Jahrhunderts.

Weiterhin wird die traditionelle Rollenverteilung durch eine Diskriminierung gegen Frauen auf dem Arbeitsmarkt gefördert. ${ }^{98}$ Wenn deshalb die Frau bei gleicher beruflicher Qualifikation am Markt weniger verdienen kann als der Mann, ist es rational - d.h. für den Haushalt nutzenmaximierend - wenn in erster Linie der Mann für das Erwerbseinkommen sorgt.

Die unterschiedlichen sektorspezifischen Investitionen aufgrund des komparativen Vorteils infolge von Diskriminierung und biologischen Unterschieden verstärken diese Rollenverteilung noch, weil sie von der in der jeweiligen Tätigkeit verbrachten Zeit abhängen. ${ }^{99}$ Auf diese Weise können anfänglich geringe komparative Nachteile der Frau, die auf biologische Unterschiede und Diskriminierung zurückzuführen sind, zu großen tatsächlich beobachteten Unterschieden führen. ${ }^{100}$ Etwas gemildert wird diese Spezialisierung dagegen durch eine komplementäre statt einer substitutiven Beziehung zwischen Frauen- und Männerzeit bei der Erstellung einiger Basisgüter. ${ }^{101}$ So stellt sich beispielsweise der volle Nutzen eines Wochenendausfluges möglicherweise erst dann ein, wenn beide Partner an ihm teilnehmen.

97

\section{Becker (1991) S. 38.}

Vgl. Becker (1991) S. 62ff. In diesem Anhang zu Kapitel 2 seines Buches führt Becker allerdings anhand eines Modells aus, daß die auch nach Anstieg der Erwerbsbeteiligung und bei gleicher Qualifikation von Frauen nach wie vor zu beobachtenden Lohnunterschiede nicht unbedingt auf eine Diskriminierung zurückgehen müssen. Die zentrale Annahme ist dabei, daß die Frau auch dann weiterhin den Großteil der Hausarbeit erledigt, wenn beide Partner berufstätig sind. Unter dieser Prämisse werden die Frauen einen Teil ihrer Anstrengungen (effort) auf den Haushaltssektor richten und entsprechend weniger intensiv im Erwerbsleben arbeiten können. Sie ermüden infolge der Doppelrolle schneller, müssen eventuell der Arbeit fernbleiben, um kranke Kinder zu betreuen, können schlechter Schichtarbeitsplätze oder Tätigkeiten mit häufigen Reisen übernehmen. Da die Unternehmen aber die Gesamtleistung der Arbeitnehmer bestehend aus Arbeitszeit multipliziert mit dem jeweiligen "Anstrengungsniveau" (effort intensity) entlohnen, wird eine Frau aufgrund der geringeren effort intensity einen geringeren Lohnsatz erhalten. Durch diesen Ansatz läßt sich auch begründen, warum Frauen eher Arbeiten übermehmen, die eine geringere effort-intensity erfordern bzw. besser mit der Hausarbeit kombinierbar sind. Eine Arbeitsteilung entgegen der traditionellen Rollenverteilung würde dementsprechend zu vergleichbaren Effekten bei dem Mann führen.

Da spezifisches Humankapital schon ab der frühen Kindheit gebildet wird, ist es für Eltern rational, Mädchen mehr mit haushaltsspezifischem als mit marktspezifischem Humankapital auszustatten, solange die Wahrscheinlichkeit, daß sie dieses später brauchen werden größer ist, als die Wahrscheinlichkeit für eine Markttätigkeit. Durch dieses spezifische Humankapital haben Frauen dann später einen komparativen Vorteil in der Haushaltsproduktion. Aufgrund dieser rationalen Entscheidung unter Unsicherheit setzt sich die traditionelle Rollenverteilung fort, obwohl die ursprünglichen Gründe dafür heutzutage nicht mehr so relevant sind wie früher. Vgl. Becker (1991) S. $40 \mathrm{f}$.

Vgl. Becker (1991) S. 63.

Ein weiterer Hinderungsgrund für die Spezialisierung ist die mit zunehmender Arbeitsteilung abnehmende Kontrollierbarkeit der Handlungen der anderen Haushaltsmitglieder. Durch die Spezialisierung kann ein Haushaltsmitglied nicht mehr beurteilen, mit welcher Intensität sich ein anderes Haushaltsmitglied seinen Aufgaben widmet. Vgl. Becker (1991) S. 48ff. 
Da die komparativen Nachteile der Frau infolge von Diskriminierung und biologischer Unterschiede aufgrund der niedrigeren Kinderzahl zurückgehen, die Investition in spezifisches Humankapital aber geschlechtsunabhängig ist, wird zwar weiter eine Arbeitsteilung bestehen bleiben, aber die konkrete Aufteilung wird unabhängiger vom Geschlecht werden. Der Trend zu häufigerer Hausarbeit von Männern wird sich also noch verstärken. ${ }^{102}$

\section{Mathematischer Anhang}

Der im Vorangehenden beschriebene Nutzenmaximierungskalkül läßt sich formal folgendermaßen darstellen:

Die Nutzenfunktion eines repräsentativen Elternhaushalts enthält als Argumente die $m$ Basisgüter $Z_{i}$, von denen eines auch Kinder sein können:

$$
\text { C.1) } \quad U=U\left(Z_{1}, \ldots, Z_{m}\right)
$$

Die Geldeinkommensrestriktion besagt, daß das ganze Einkommen $(I)$ notwendigerweise mit den aus den Preisen $\left(p_{i}\right)$ und den Mengen $\left(x_{i}\right)$ errechneten Ausgaben für verschiedene Güter bzw. Verwendungszwecke (wozu hier u.a. auch das Sparen gerechnet wird) übereinstimmt:

$$
\text { C.2) } \quad I=\sum_{i=1}^{m} p_{i} x_{i} \text {. }
$$

Neben dieser Einkommensrestriktion ist auch eine Zeitrestriktion zu erfüllen: die 24 Stunden eines Tages $(t)$ werden in die Summe aller zur Herstellung verschiedener Basisgüter im Haushalt verbrachten Zeiten $\left(\sum_{i=1}^{m} t_{h_{i}}\right)$ und in die Arbeitszeit $\left(t_{w}\right)$ aufgeteilt:

$$
\text { C.3) } t=\sum_{i=1}^{m} t_{h_{t}}+t_{w} \text {. }
$$

Da das Einkommen $(I)$ festgelegt wird durch die Summe aus Nicht-Arbeitseinkommen und dem Produkt aus Arbeitszeit $\left(t_{w}\right)$ und Lohnsatz $(w)$, sind Einkommensrestriktion und Zeitrestriktion nicht unabhängig voneinander. Sie können daher zusammengefaßt werden zur Budgetrestriktion des impliziten Einkommens $(S)$ (Becker spricht von "full income"),

$$
\text { C.4) } \sum_{i=1}^{m} p_{i} x_{i}+w \sum_{i=1}^{m} t_{h_{i}}=S \text {, }
$$

die unter Beachtung der oben im Text erläuterten Definition der Schattenpreise $\left(\begin{array}{ll}\pi & i\end{array}\right)$

102 Vgl. Becker (1991) S. 78. 
C.5) $\pi_{i}=\frac{p_{i} x_{i}}{Z_{i}}+\frac{w t_{h_{i}}}{Z_{i}}$

auch als Summe der mit ihren Schattenpreisen $\left(\pi_{i}\right)$ bewerteten Basisgüter $\left(Z_{i}\right)$ aufgefaßt werden kann:

$$
\text { C.6) } S=\sum_{i=1}^{m} \pi_{i} Z_{i}
$$

Optimiert man die Zielfunktion $C .1$ ) unter der Nebenbedingung $C .6$ ) mit Hilfe eines LagrangeAnsatzes

$$
L\left(Z_{1}, \ldots, Z_{m}, \lambda\right)=U\left(Z_{1}, \ldots, Z_{m}\right)-\lambda\left(\sum_{i=1}^{m} \pi_{i} Z_{i}-S\right)
$$

so erhält man für das Basisgut $\mathrm{j}^{103}$

$$
\begin{gathered}
\frac{\partial L}{\partial Z_{j}}=\frac{\partial U}{\partial Z_{j}}-\lambda \pi_{j}=0 \\
\frac{\partial U}{\partial Z_{j}}=\lambda \pi_{j} \\
\frac{\partial L}{\partial \lambda}=-\left(\sum_{i=1}^{m} \pi_{i} Z_{i}-S\right)=0 .
\end{gathered}
$$

Führt man die gleiche Optimierung für ein zweites Basisgut $Z_{k}$ durch, so erhält man durch Division aus $C .8$ ), daß im Konsumoptimum das Verhältnis der Grenznutzen zweier Basisgüter dem Verhältmis ihrer Schattenpreise entsprechen muß:

$$
\text { C.9) } \frac{\frac{\partial U}{\partial Z_{j}}}{\frac{\partial U}{\partial Z_{k}}}=\frac{\pi_{j}}{\pi_{k}} \text {. }
$$

Steigt ceteris paribus der Schattenpreis für das Basisgut $Z_{j}$, so geht die Nachfrage nach diesem Basisgut und damit auch die Nachfrage nach seinen Einsatzfaktoren $x_{j}$ und $t_{h}$ zurück, denn mit einer Abnahme von $Z_{j}$ steigt der Grenznutzen dieses Basisgutes (es gilt $\frac{\partial^{2} U}{\partial Z_{j}^{2}}<0$ ). Der Rückgang der Nachfrage nach $Z_{j}$ setzt sich fort, bis infolge des durch diesen Nachfragerückgang

103 Wie bereits erwähnt, wird angenommen, daß alle Haushaltsproduktionsfunktionen homogen vom ersten Grad sind. Daraus folgt, daß der Schattenpreis $\pi_{i}$ nicht von der konsumierten Menge eines Basisgutes abhängt, d.h. $\frac{\partial \pi_{i}}{\partial Z_{i}}=0$. 
ausgelösten Anstiegs des Grenznutzens dieses Basisgutes die Optimalitätsbedingung C.9) auch nach der Schattenpreiserhöhung wieder erfüllt ist.

Um das optimale Einsatzverhältnis der beiden zur Herstellung der Basisgüter $Z_{i}$ eingesetzten Inputfaktoren $t_{h_{i}}$ und $x_{i}$ bestimmen zu können, müssen die Haushaltsproduktionsfunktionen

$$
\text { C.10) } Z_{i}=f_{i}\left(x_{i}, t_{h_{i}}\right) \quad \text { mit } 1 \leq i \leq m
$$

in der Nutzenfunktion berücksichtigt werden. Sie legen fest, welche Kombinationen von Inputs gewählt werden können, um eine gegebenen Menge des jeweiligen Basisgutes zu erzeugen.

Das optimale Faktoreinsatzverhältnis ergibt sich dann aus der Maximierung der Zielfunktion

$$
\text { C.II) } U=U\left(Z_{i}\left(x_{i}, t_{h_{i}}\right)\right) \quad \text { mit } 1 \leq i \leq m
$$

unter der Nebenbedingung C.4).

Der Lagrange-Ansatz für ein beliebiges Basisgut $Z_{j}$ lautet folglich:

$$
L\left(x_{j}, t_{h_{j}}, \lambda\right)=U\left(Z_{1}\left(x_{1}, t_{h_{h}}\right), \ldots, Z_{m}\left(x_{m}, t_{h_{m}}\right)\right)+\lambda\left(S-\sum_{i=1}^{m} p_{i} x_{i}-w \sum_{i=1}^{m} t_{h_{i}}\right) .
$$

Nullsetzen der ersten Ableitungen liefert

$$
\begin{aligned}
& \frac{\partial L}{\partial x_{j}}=\frac{\partial U}{\partial Z_{j}} \frac{\partial Z_{j}}{\partial x_{j}}-\lambda p_{j}=0 \\
& \frac{\partial L}{\partial t_{h_{j}}}=\frac{\partial U}{\partial Z_{j}} \frac{\partial Z_{j}}{\partial t_{h_{j}}}-\lambda w=0 \\
& \frac{\partial L}{\partial \lambda}=S-\sum_{i=1}^{m} p_{i} x_{i}-w \sum_{i=1}^{m} t_{h i}=0
\end{aligned}
$$

und hieraus folgt die Optimalitätsbedingung

$$
\text { C.12) } \frac{\frac{\partial Z_{j}}{\partial x_{j}}}{\frac{\partial Z_{j}}{\partial t_{h_{j}}}}=\frac{p_{j}}{w} \text {. }
$$


Unterstellt man für beide Einsatzfaktoren positive aber abnehmende Grenzprodukte, so macht C.11) sofort einsichtig, daß ein Anstieg des Lohnsatzes $w$ bei konstantem Preis $p_{j}$ zu einem höheren Einsatz von Marktgütern - dann sinkt $\partial Z_{j} / \partial x_{i}$ - und einem niedrigeren Einsatz von Zeit - dann steigt $\partial Z_{j} / \partial t_{h_{j}}$ - bei der Erstellung des j-ten Basisgutes führt. Dieses Ergebnis gilt für alle Basisgüter.

Eine Lohnsatzerhöhung führt also erstens immer zu einer Verminderung des Einsatzverhältnisses von Haushaltszeit zu Marktgütern. Zweitens ist aus C.9) ersichtlich, daß der relative Schattenpreis von zeitintensiven Basisgütern sich aufgrund einer Lohnsatzerhöhung tendenziell erhöhen würde und es somit zu einem Substitutionsprozeß weg von zeitintensiven und hin zu güterintensiven Basisgütern kommt.

\subsection{Fertilitätsentscheidung im Rahmen des Modells}

In diesem Abschnitt sollen die Bestimmungsgründe für die Fertilitätsentscheidung im Rahmen des neoklassischen Modells untersucht werden. Die Fertilitätsentscheidung läßt sich dabei auf die Veränderung der oben unter 3.1.1 aufgeführten marginalen Kosten und Nutzen von Kindern zurückführen.

Wie schon festgestellt, hat der Investitionsnutzen von Kindern in entwickelten Industrieländern mit einem ausgebauten sozialen Sicherungssystem kaum noch Bedeutung. Der Konsumnutzen wurde als stabil angenommen, weil es auf theoretischer Ebene keine Gründe für eine Änderung gibt und Meßprobleme die Feststellung des tatsächlichen zeitlichen Verlaufs verhindern. Zur Erklärung der empirischen Fertilitätsentwicklung muß daher v.a. auf die Änderung der direkten und indirekten Kosten von Kindern eingegangen werden.

Auch als Ansatzpunkte staatlicher Einflußnahme auf die Fertilitätsentscheidung kommen in einer liberal eingestellten Gesellschaft nur die beiden Kostenkategorien in Frage. Eine Beeinflussung des Konsumnutzens durch den Staat - z.B. durch öffentliche Appelle - würde auf eine paternalistische Beeinflussung der Präferenzen hinauslaufen und damit auf eine Bevormundung, die mit den auf der Autonomie des Individuums aufbauenden Verfassungen der "westlichen" Demokratien nicht vereinbar ist. Eine gezielte Erhöhung des Investitionsnutzen von Kindern scheidet als Instrument staatlicher Politik ebenfalls aus, weil dazu die Sozialversicherungssysteme (gesetzlich und privat) derart beschnitten werden müßten, daß der Einzelne in Notlagen (Alter, Krankheit, Arbeitslosigkeit, Invalidität) in erheblichem Umfang auf die Unterstützung seiner Kinder angewiesen ist. Diese Rückkehr zur sozialen Absicherung durch eigene Kinder ist aber politisch nicht durchsetzbar und aufgrund der gegenüber früheren Jahrhunderten wesentlich anderen sozialen Verhältnisse (höhere Lebenserwartung, außerhäusliche Erwerbsarbeit, hohe Scheidungsrate, etc.) auch kaum durchführbar. ${ }^{104}$

Im Mittelpunkt der folgenden Analyse steht daher die Entwicklung der beiden genannten Kostenkategorien. Die indirekten Kosten können als Kosten des Inputfaktors Zeit bei der Produktion des Basisgutes Kind verstanden werden, die direkten Kosten als Kosten des Input-

104 Es waren ja gerade diese sozialen Umbrüche, die Ende des 19. Jahrhunderts die Einführung des sozialen Sicherungssystems dringlich erscheinen ließen (vgl. Mitterauer/Sieder (1991) S. 188f.). Außerdem führt die durch soziale Sicherungssysteme vergrößerte Risikostreuung zu Effizienzgewinnen. Diese können allerdings durch eine größere Mißbrauchsneigung in unüberschaubaren gesellschaftlichen Sicherungssystemen wieder zunichte gemacht werden, wie u.a. Biedenkopf (1993) ausführt. 
faktors Marktgüter. Die im Rahmen der neoklassischen Theorie angestellten Überlegungen konzentrieren sich dabei darauf, Erklärungsmöglichkeiten für eine bei steigendem Einkommen rückläufige Fertilität unter der Prämisse zu finden, daß das Basisgut Kind ein Normalgut darstellt. Drei grundsätzliche Erklärungsmuster lassen sich abgrenzen:105 erstens eine Erklärung über mit dem Lohnsatz steigende Opportunitätskosten, zweitens der Zusammenhang zwischen Quantität und Qualität bei der Fertilitätsentscheidung ${ }^{106}$ und drittens eine Zunahme der direkten qualitätsunabhängigen Kosten des Kinderaufziehens. Auf diese Gründe wird in den folgenden Abschnitten einzeln eingegangen, um - auf diese Erklärungen des Geburtenrückgangs aufbauend - Ansatzpunkte für pronatalistische Maßnahmen zu finden und im nächsten Kapitel eine Wirkungsanalyse des Kinderlastenausgleichs vornehmen zu können.

\subsubsection{Die Opportunitätskosten des Kinderaufziehens}

Als eine Ursache des starken Geburtenrückgangs in den Industrieländern werden von Vertretern der New Home Economics die steigenden indirekten Kosten des Kinderaufziehens genannt. Die Höhe dieser Kosten bestimmt sich nach dem Wert, den die auf die Kindererziehung verwandte Zeit in der nächstbesten alternativen Verwendung hat. Die indirekten Kosten werden deshalb auch als Opportunitätskosten bezeichnet. Hier soll zunächst der theoretische Zusammenhang zwischen Geburtenentwicklung und indirekten Kosten erläutert werden (Unterabschnitt 3.2.1.1), ehe ein Versuch unternommen wird, die Größenordnung dieser Kosten wenigstens annähernd empirisch abzuschätzen (Unterabschnitt 3.2.1.2). Aus diesen Überlegungen werden dann in Unterabschnitt 3.2.1.3 erste Schlußfolgerungen für Ansatzpunkte staatlicher Instrumente zur Geburtenförderung abgeleitet.

Die indirekten Kosten von Kindern bestehen neben der Einschränkung der Mobilität der Eltern v.a. in den durch Einschränkung oder Aufgabe der Marktarbeit entstehenden Einkommensverlusten und der damit oft verbundenen höheren Abhängigkeit vom Partner sowie möglicherweise einem subjektiv empfundenen Verlust an Selbstwertgefühl und sozialer Integration. Die Kosten der Mobilitätsbeschränkung entziehen sich ebenso einer Quantifizierung wie die immateriellen Kosten einer Einschränkung der Erwerbsarbeit. Zum einen dürfte beispielsweise der Grad der Einschränkung von Mobilität höchst unterschiedlich sein, je nachdem ob die Eltern die Betreuung der Kinder vorübergehend nahe wohnenden Verwandten oder Bekannten übertragen können und wollen. Zum anderen wird eine gleiche Mobilitätsbeschränkung sicherlich von verschiedenen Personen unterschiedlich bewertet. Die immateriellen Opportunitätskosten werden daher im folgenden nicht näher untersucht. Gleichwohl sollte im Auge behalten werden, daß sie die indirekten Kosten noch erhöhen können.

Von einigen Autoren ${ }^{107}$ wird neben den Einkommensverlusten wegen Aufgabe einer Erwerbsarbeit zusätzlich die Kinderbetreuungszeit zu den Opportunitätskosten gerechnet. Das hat eine Doppelzählung zur Folge, denn die bisher für Erwerbsarbeit aufgebrachte Zeit kann

$105 \mathrm{Vgl}$. Hirsch (1992) S. 223ff. Oft wird allerdings auch auf die Untersuchung der direkten Kinderkosten als Begründung verzichtet.

106 Der substitutive Zusammenhang zwischen Qualität und Quantität macht eine Aufteilung der direkten Kosten in qualitätsunabhängige direkte Kosten und Kosten, die auf einer nutzenstiftenden Qualitätssteigerung beruhen, notwendig (vgl. dazu Abschnitt 3.2.2).

Erwähnt seien hier beispielsweise Vottlerer (1987) S. 18f. und Lampert (1986) S. 19. 
jetzt ja für die Kinderbetreuung verwendet werden. Die Kosten der mit der Kinderbetreuung verbrachten Zeit - die unmittelbar einleuchtende Tatsache, daß mit dem Vorhandensein von Kindern ein höherer Umfang an Eigenarbeit im Haushalt einhergeht, wurde von Glatzer ${ }^{108}$ im Rahmen einer multivariaten Analyse bestätigt - sind also schon durch die Einkommensverluste erfaßt. Im folgenden werden daher allein die durch die Kindererziehung verursachten Einkommensverluste als Maß für die einer Frau entstehenden Opportunitätskosten verwendet. ${ }^{109}$

Diese Einkommensverluste können natürlich sowohl beim Mann als auch bei der Frau auftreten. Wenn hier im folgenden auf die einer Frau entstehenden Opportunitätskosten abgestellt wird, so allein deshalb, weil die empirischen Studien aufgrund mangelnden Datenmaterials ${ }^{110}$ durchweg die Opportunitätskosten für Frauen untersuchen. Dieses Vorgehen ist insofern gerechtfertigt, als die Kinderbetreuung immer noch hauptsächlich von Frauen geleistet wird. " gesprochen werden. Dabei ist wiederum zu beachten, daß die Opportunitätskosten auch bei beiden Partnern auftreten können, wenn z.B. beide wegen der Geburt eines Kindes von einer Vollzeit- zu einer Teilzeitbeschäftigung wechseln oder Überstunden einschränken. ${ }^{112}$

\subsubsection{Theoretische Analyse der Wirkung eines steigenden Frauenlohnsatzes}

Nach der Theorie der New Home Economics ist ein wesentlicher Grund für den Geburtenrückgang in steigenden Löhnen von Frauen zu sehen. Die Auswirkungen steigender Frauenlohnsätze auf die Kinderzahl lassen sich in den Einkommenserhöhungseffekt und den Schattenpreisänderungseffekt zerlegen.

108 Vgl. Glatzer (1983) S. 13 (zitiert nach Vottlerer (1987) S. 16).

109 Von manchen Autoren wie z.B. Schubnell (1973) wird auch in der Zunahme der Erwerbsbeteiligung der Frauen eine Opportunitätskostenerhöhung gesehen, die einen Rückgang der Geburtenzahl verursacht. Hier muß allerdings beachtet werden, da $B$ die Erwerbsbeteiligung der Frauen bei der Maximierung des Nutzenniveaus eines Haushalts keine exogene Größe ist und die Kausalitätsrichtung deshalb genauso von der Kinderzahl zur Erwerbsbeteiligung laufen kann, weil eine geringere Kinderzahl ein stärkeres Marktengagement ermöglicht. Zimmermann (1985) S. 64 stellt fest, daß sich die Determinanten der Fertilität und des Arbeitsangebots weitgehend überschneiden und findet empirisch keine Bestätigung für die eine oder andere Kausalrichtung (S. 347ff.). Gegen eine Erhöhung der Opportunitätskosten durch eine steigende Erwerbsbeteiligung spricht außerdem, daß bei einer höheren Erwerbsbeteiligung der Nutzen der im Haushalt verbrachten Zeit entfältt, der mit dem Lohnsatz approximiert wird. Die Verlagerung der Zeitverwendung führt daher nicht zu einer Erhöhung der Opportunitätskosten.

110 Daten über die Erwerbsbeteiligung von Frauen bzw. Männern in Abhängigkeit von der Kinderzahl sind erhältlich, nicht aber die eigentlich interessierenden Datenreihen, die die Einschränkung der Erwerbsarbeit des oder der kinderbetreuenden Partner in Abhängigkeit von der Kinderzahl ausweisen.

111 Einen vergleichbaren Schluß zieht Hirsch (1992) S. 179f. Von den fast 800.000 Erziehungsgeldempfängern waren 1991 nur etwa ein Prozent Mănner (vgl. Bundesministerium für Arbeit und Sozialordnung (1993) S. 11). Zu einer mikroökonomischen Begründung dieser Rollenverteilung vgl. den vorangegangenen Abschnitt. Dort wird auch ausgeführt, warum eine (weitere) Lockerung dieser traditionellen Arbeitsteilung für die Zukunft zu erwarten ist.

112 Wie oben ausgeführt, ist dieser Fall aber in sofern relativ selten, als dadurch Effizienzverluste infolge des Verzichts auf Spezialisierungsvorteile (v.a. durch spezifisches Humankapital) entstehen. 
Der Einkommenserhöhungseffekt wird durch den Einkommensanstieg infolge der höheren Markteinkommen von Frauen nach der Lohnsatzsteigerung hervorgerufen. Das höhere Einkommen resultiert dabei nicht nur aus dem gestiegenen Lohnsatz, sondern auch aus einer Erhöhung der Arbeitszeit. Denn bei einer Arbeitsangebotsfunktion mit positiver Steigung - d.h. die Wirkung des Substitutionseffekts auf die angebotene Arbeitszeit ist größer als die des entgegengerichteten Einkommenseffektes - wird die Erwerbsarbeitszeit zu Lasten der im Haushalt verwandten Zeit erhöht. Da Kinder annahmegemäß als superiores Gut anzusehen sind, führt dieser Einkommensanstieg zu einer steigenden Nachfrage nach Kindern, die Geburtenzahl steigt.

Der Schattenpreisänderungseffekt resultiert aus einer Erhöhung der Schattenpreise der Basisgüter. Die steigenden Lohnsätze für Frauen führen zu einem Anstieg der Opportunitätskosten der Zeit der Frau. ${ }^{113} \mathrm{Da}$ die Zeit neben Marktgütern ein Inputfaktor bei der Produktion von Basisgütern ist, erhöhen sich die Schattenpreise aller Basisgüter aufgrund der gestiegenen Opportunitätskosten der Frau. Diese Preiserhöhung hat einen Einkommenseffekt zur Folge, der infolge der Superioritätsannahme eine Reduzierung der Kinderzahl bewirkt.

Zusätzlich entsteht ein nachfrageseitiger Substitutionseffekt bei der Wahl der Basisgüter, denn die relative Erhöhung der Schattenpreise ist für die einzelnen Basisgüter unterschiedlich. Das relative Preisverhältnis der zur Produktion der Basisgüter nötigen Inputs (Marktgüter und Zeit) verändert sich zu ungunsten der Zeit. In der Haushaltsproduktion verbrachte Zeit wird relativ teurer, so daß auch Basisgüter, die relativ zeitintensiv sind, relativ teurer werden als marktgut-intensive Basisgüter. Da Kinder aber ein sehr zeitintensives Basisgut sind, steigt ihr relativer (Schatten-)Preis und die Nachfrage nach Kindern sinkt dementsprechend.

Gleichzeitig kommt es bei Substitutionalität der Produktionsfaktoren Zeit und Marktguiter zu einem zweiten, angebotsseitigen Substitutionseffekt, nämlich bei der Produktion der Basisgüter im Haushalt. Alle Basisgüter werden aufgrund der gestiegenen relativen Kosten des Produktionsfaktors Zeit jetzt mit Hilfe einer weniger zeitintensiven Produktionstechnik erstellt. Dieser Effekt bremst den relativen Preisanstieg von Kindern umso stärker, je größer ceteris paribus die Substitutionalität von Zeit und Marktgütern bei ihrer Erstellung ist. Im Extremfall einer unendlichen Substitutionselastizität erhöht sich der Preis von Kindern überhaupt nicht, da die Erhöhung der Kosten der Zeit dazu führt, daß der Inputfaktor Zeit gar nicht mehr benutzt wird. Dann wäre sogar denkbar, daß das Basisgut Kind vermehrt nachgefragt wird, wenn nämlich bei der Herstellung anderer Basisgüter eine geringere Substitutionselastizität besteht und ihr relativer Schattenpreis damit ansteigt. In diesem Fall wäre der zusammengefaßte Substitutionseffekt positiv. Im folgenden wird unterstellt, daß die Substitutionselastizität bei der Erstellung aller Basisguiter in etwa gleich ist, so daß ein insgesamt positiver Substitutionseffekt ausgeschlossen ist. Der aus den beiden gegenläufigen Einzeleffekten zusammengesetzte gesamte Substitutionseffekt bewirkt daher einen Rückgang der Geburtenrate aufgrund steigender Opportunitätskosten. Da der Einkommenseffekt in die gleiche Richtung wirkt, verursacht der Schattenpreisänderungseffekt insgesamt eine Reduzierung der Kinderzahl.

Einkommenserhöhungs- und Schattenpreisänderungseffekt haben demnach gegenläufige Wirkungen auf die Geburtenentwicklung. Ohne empirische Untersuchungen ist daher nicht feststellbar, ob die positive Wirkung des Einkommenserhöhungs- oder die negative Wirkung

113 Die Opportunitätskosten der Zeit der Frau enthalten neben dem Lohnsatz auch noch Humankapitalverluste (vgl. dazu unten). Da sich Humankapitalverluste und Lohnsatz aber annähernd proportional entwickeln, ist für die hier vorgenommene Betrachtung von Veränderungsrichtungen die Beachtung des Lohnsatzes ausreichend. 
des Schattenpreisänderungseffektes überwiegt. In den zahlreichen empirischen Untersuchungen zu dem Zusammenhang zwischen Frauenlohnsatz und Geburtenentwicklung wird meistens ein hoch signifikanter negativer Zusammenhang gefunden. ${ }^{114}$ Die zugrundeliegende Theorie wird zusätzlich dadurch gestützt, daß der Einfluß des Einkommens des Mannes eher positiv als negativ zu sein scheint. Nimmt man nämlich an, daß die Zeit des Mannes nicht oder nur geringfügig als Inputfaktor des Basisgutes Kind dient, dann hat ein höheres Einkommen des Mannes einen Einkommenserhöhungs-, aber kaum einen Schattenpreisänderungseffekt zur Folge. ${ }^{115} \mathrm{Da}$ der Einkommenserhöhungseffekt die Kinderzahl positiv beeinflußt, ist aufgrund theoretischer Überlegungen ein positiver Gesamteinfluß zwingend.

Abb. C.3: Wirkung einer Erhöhung des Frauenlohnsatzes auf die Kinderzahl

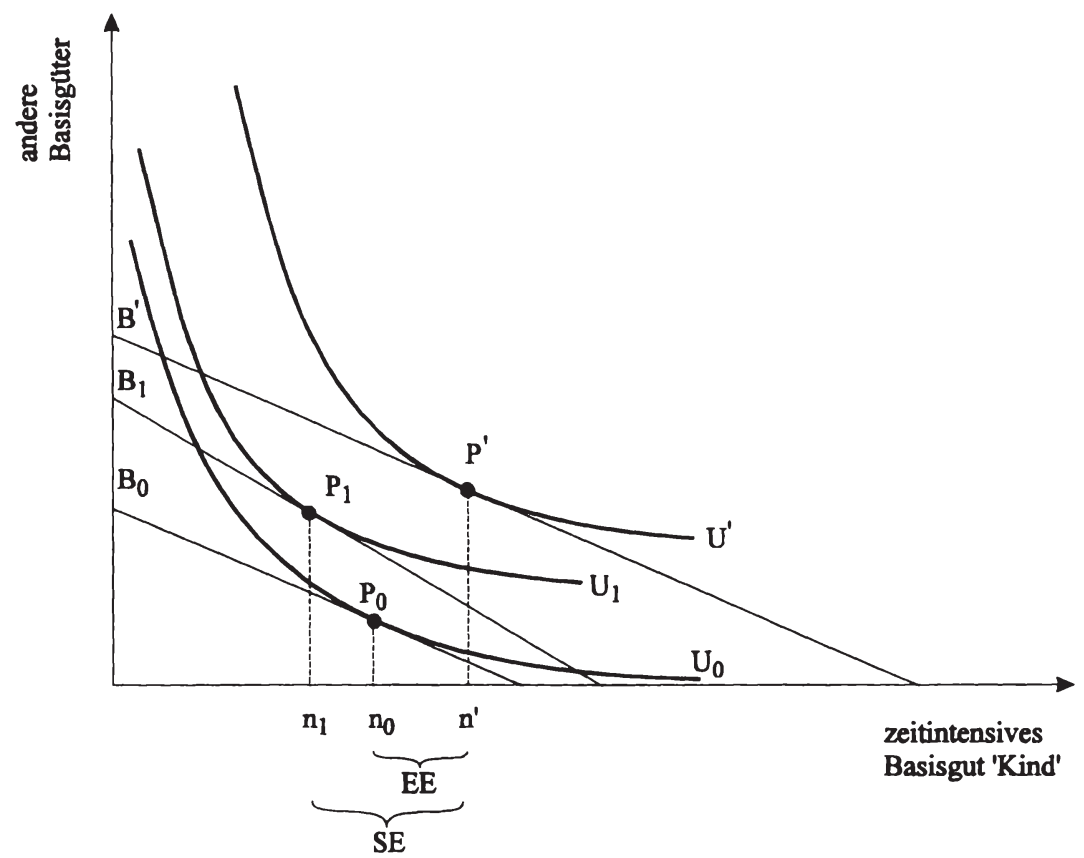

In Abb. C. 3 sind diese Zusammenhänge graphisch dargestellt. Die Ausgangslage wird durch $P_{0}$ beschrieben. Durch den exogenen Anstieg des Lohnsatzes für Frauen wird die Budgetbeschränkung des impliziten Einkommens $\left(B_{0}\right)$ parallel nach außen verschoben $(B)$, wenn man zunächst von der Änderung der Schattenpreise der beiden Basisgütergruppen absieht. Der

114 Vgl. Felderer/Sauga (1988) S. 78.

115 Hier soll außer acht bleiben, daß auch ein Einkommensanstieg eine Änderung der relativen Schattenpreise auslösen kann, weil zwar die Einkommensrestriktion nicht aber die Zeitrestriktion gelockert wird und damit der Inputfaktor Zeit relativ zum Inputfaktor Marktgüter knapper wird. 
Einkommenserhöhungseffekt (EE) bewirkt also eine Erhöhung der auf der Abszisse abgetragenen Kinderzahl von $n_{0}$ auf $n^{\prime}$.

Man hat nun jedoch zusätzlich die Wirkung des gestiegenen Lohnsatzes auf die Schattenpreise der Basisgütergruppen zu berücksichtigen: Der Schattenpreis des auf der Ordinate abgetragenen aggregierten Basisgutes hat sich nur leicht erhöht, da zu seiner Herstellung neben Marktgütern nur relativ wenig des im Preis gestiegenen Inputfaktors Zeit benötigt wird. Der Schattenpreis des zeitintensiven Basisgutes Kind hat sich dagegen stark erhöht. Das relative Schattenpreisverhältnis hat sich also insgesamt zu ungunsten von Kindern verändert, so daß es zu einer Drehung der Budgetgeraden kommt (Substitutionseffekt der Schattenpreisänderung).

Neben dieser Drehung bewirkt der gestiegene Lohnsatz auch eine Verschiebung der Budgetgeraden von $\mathrm{B}^{\prime}$ ausgehend nach innen, denn der Inputfaktor Zeit hat sich für beide Basisgüterarten verteuert (Einkommenseffekt der Schattenpreisänderung). Das hat zur Folge, da $\beta$ der betrachtete Haushalt sich bei gleichem impliziten Einkommen weniger von beiden Gütern leisten kann. Der Schattenpreisänderungseffekt (SE) als Kombination dieser beiden Teileffekte bewirkt in Abb. C.3 somit eine Verlagerung der Budgetgeraden von $B^{\prime}$ nach $B_{I}$ und damit einen Rückgang der Kinderzahl von $n^{\prime}$ auf $n_{l}$. Da der Schattenpreisänderungseffekt hier größer ist als der Einkommenserhöhungseffekt, geht die Kinderzahl infolge der Erhöhung des Lohnsatzes für Frauen von $n_{0}$ auf $n_{l}$ zurück. Der Gesamteffekt ist damit negativ, obwohl der eigentliche Einkommenseffekt - Kinder stellen, wie gesagt, ein superiores Gut dar - positiv ist.

\subsubsection{Empirische Größenordnung der Opportunitätskosten}

Um die empirische Größenordnung der Opportunitätskosten korrekt zu erfassen, müssen diese zunächst operationalisiert werden. Zwei Operationalisierungsvarianten sind denkbar. Zum einen kann man die Betreuungszeit messen und dann mit den Zeitkosten (meistens wird der Lohnsatz genommen) bewerten. Tendenziell werden Kinder mit zunehmendem Alter weniger Betreuungszeit beanspruchen ${ }^{116}$ und der zusätzliche Arbeitsaufwand, der mit dem ersten Kind einhergeht, wird größer sein als die Anforderungen an das Zeitbudget der Eltern durch ein zweites Kind. ${ }^{117}$ Außerdem können Kinder mit zunehmendem Alter auch selbst mehr im Haushalt mithelfen. Es muß daher bei dieser Operationalisierungsvariante beachtet werden, daß die Opportunitätskosten nicht etwa den gesamten zusätzlichen Zeitaufwand umfassen, der durch ein zusätzliches Kind anfällt, sondern nur den Mehraufwand für die Eltern, also nicht etwa den Beitrag der Kinder selbst. Humankapitalverluste, die nach einer kindbedingten Unterbrechung der Erwerbstätigkeit zu Einkommenseinbußen führen, werden bei dieser Operationalisierungsvariante nicht berücksichtigt.

Dieses Problem kann durch die hier gewählte zweite Operationalisierungsvariante vermieden werden. Danach werden die einer Frau entstehenden Opportunitätskosten durch den Einkommensausfall gemessen, den die Eltern (hier annahmegemäß die Frauen) durch die Aufgabe einer Erwerbsarbeit wegen des Vorhandenseins eines Kindes erleiden. Die Lebenseinkommen von Frauen mit unterschiedlicher Kinderzahl werden mit den Lebenseinkommen

\footnotetext{
116 VgI. Blosser-Reisen/Seifert (1984) S. 135f.

117 Vgl. Kössler (1984) S. 33f.
} 
verglichen, die diese Frauen ohne Kinder hätten erzielen können. ${ }^{118}$ In einer Längsschnittstudie fanden Buhr/Strack/Strohmeier, daß 69\% der zwischen 1983 und 1987 befragten Frauen vor der Geburt ihres ersten Kindes erwerbstätig waren, nachher aber nur 36\%. Von diesen wiederum wechselte etwa die Hälfte auf eine Teilzeittätigkeit. ${ }^{119}$ Die Differenz der Lebenseinkommen von Frauen mit und ohne Kinder gibt dann die Opportunitätskosten an, die einer Frau aufgrund der kindbedingten Erwerbsunterbrechung entstehen. Anschließend müssen diese einer Frau entstehenden Opportunitätskosten durch die Zahl der Kinder dividiert werden, die diese Frau versorgt, um zu den Opportunitätskosten eines Kindes zu gelangen. Da aber für eine einzelne Frau mit kindbedingt unterbrochener Erwerbskarriere der Alternativfall einer ununterbrochenen Erwerbsbiographie nicht beobachtbar ist, muß zunächst ein Modell für die Lebenseinkommensentwicklung von Frauen entwickelt werden. Die bei diesem Vorgehen implizit vorgenommene Unterstellung, daß Frauen ihre Erwerbstätigkeit ausschließlich aufgrund der Kindererziehung unterbrechen, wird durch neuere empirische Studien gerechtfertigt. So gelangt Krombholz beispielsweise zu dem Resultat, daß für Frauen familiäre Gründe ausschlaggebend sind für die Unterbrechung bzw. Reduktion ihrer Berufstätigkeit. ${ }^{120}$

Bevor auf die drei Operationalisierungsschritte - Aufstellung des Humankapitalmodells, Berechnung der einer Frau entstehenden Opportunitätskosten und schließlich Berechnung der Opportunitätskosten eines Kindes - genauer eingegangen wird, sollen zunächst noch zwei Annahmen diskutiert werden.

Bei der Abschätzung der einer Frau entstehenden Opportunitätskosten ist zu beachten, daß bei Vorliegen bestimmter Rationierungen die Opportunitätskosten von der Differenz der Einkommen abweichen. Ist z.B. nur die Wahl zwischen Vollzeitarbeit und vollständiger Unterbrechung möglich, so wird auch eine Frau die Erwerbsarbeit ganz einstellen (müssen), die gerne noch einige Stunden pro Woche arbeiten würde. In diesem Fall ist der Lohnausfall größer als die Opportunitätskosten und der Grenznutzen der Zeit liegt unter dem Lohnsatz. Die freie Zeit kann verwendet werden, um mehr Basisgüter herzustellen und/oder ihre Herstellung zeitintensiver zu gestalten. Damit kann direkt oder über die Ersparnis von Ausgaben für Marktgüter ein Nutzenzuwachs erzielt werden, der die durch den Lebenseinkommensrückgang gemessenen Opportunitätskosten reduziert. Wenn hier dennoch eine Gleichsetzung von Opportunitätskosten und Einkommensausfällen vorgenommen wird, liegt dem die Annahme zugrunde, daß sich Überschätzungen aufgrund von geringerer realisierter als gewünschter Arbeitszeit und Unterschätzungen aufgrund einer größeren tatsächlichen als gewünschten Arbeitszeit in etwa ausgleichen. ${ }^{121}$

Becker weist außerdem darauf hin, daß die Opportunitätskosten der Zeit auch von der Anstrengungsintensität (effort intensity) der jeweiligen Tätigkeit abhängen. ${ }^{122}$ Erfordert eine bestimmte Aktivität ein geringeres Anstrengungsniveau als die Erwerbsarbeit, so liegt auch der Schattenpreis der auf diese Aktivität verwandten Zeit unter dem Lohnsatz, weil die Einsparung

\footnotetext{
118 Makroökonomische Rückwirkungen über ein steigendes Arbeitsangebot und einen infolgedessen sinkenden Lohnsatz bei einer höheren Frauenerwerbsquote können hier außer Acht gelassen werden, weil sie für die individuelle Entscheidung für oder gegen Kinder keine Rolle spielen.

119 Vgl. Buhr/Strack/Strohmeier (1987) S. 22.

120 Vgl. Krombholz (1991) S. 206.

121 Vgl. Dinkel (1984a) S. 90.

122 Vgl. Becker (1991) S. 68.
} 
an Anstrengung gegenüber der Arbeitszeit Nutzen stiftet. Allerdings geht Becker davon aus, daß sowohl Erwerbsarbeit als auch die Betreuung von Kindern in ihren ersten Lebensjahren ein hohes Anstrengungsniveau erfordern, so daß eine Berücksichtigung unterschiedlicher Anstrengungsniveaus hier unnötig erscheint. So können auch die kaum überwindbaren Probleme bei einer Operationalisierung dieses Konzepts vermieden werden.

In Abbildung C. 4 werden die sich aus dem Humankapitalansatz ergebenden Opportunitätskosten, die für eine Frau aus der Kindererziehung entstehen, graphisch dargestellt. Die obere durchgezogene Linie gibt die Periodeneinkommen $(E)$ bei durchgehender Vollerwerbstätigkeit an. Typischerweise wird aber die Erwerbstätigkeit der Frau bei der Geburt eines Kindes stark eingeschränkt oder sogar ganz eingestellt (Zeitpunkt $t_{l}$ in Abb. C.4). Diese Entwicklung ist durch die untere durchgezogene Linie (vollständige Unterbrechung) bzw. die gestrichelte Linie (bei Übergang zu Teilzeitarbeit) in Abb. C.4 gekennzeichnet. Sobald die Kinder älter werden und damit weniger Zeit beanspruchen bzw. auch selbst Aufgaben im Haushalt übernehmen, wird die Erwerbstätigkeit oft wieder aufgenommen (Zeitpunkt $t_{2}$ ). Allerdings besteht auch dann noch ein Einkommensnachteil im Vergleich mit der Situation ohne Kinder. Wegen der Unterbrechung der Erwerbskarriere wird nämlich beim Wiedereinstieg in den Beruf jetzt nicht der gleiche Lohnsatz erzielt, der bei ununterbrochener Laufbahn erlangt worden wäre. Neben den kurzfristigen Einkommenseinbußen in Höhe von $I_{I}$ entstehen also zusätzliche Einkommensverluste in Höhe der Fläche $I_{2}$. Der Grund hierfür kann

\section{Abb. C.4: Einkommensverluste infolge einer Unterbrechung der Erwerbsarbeit}

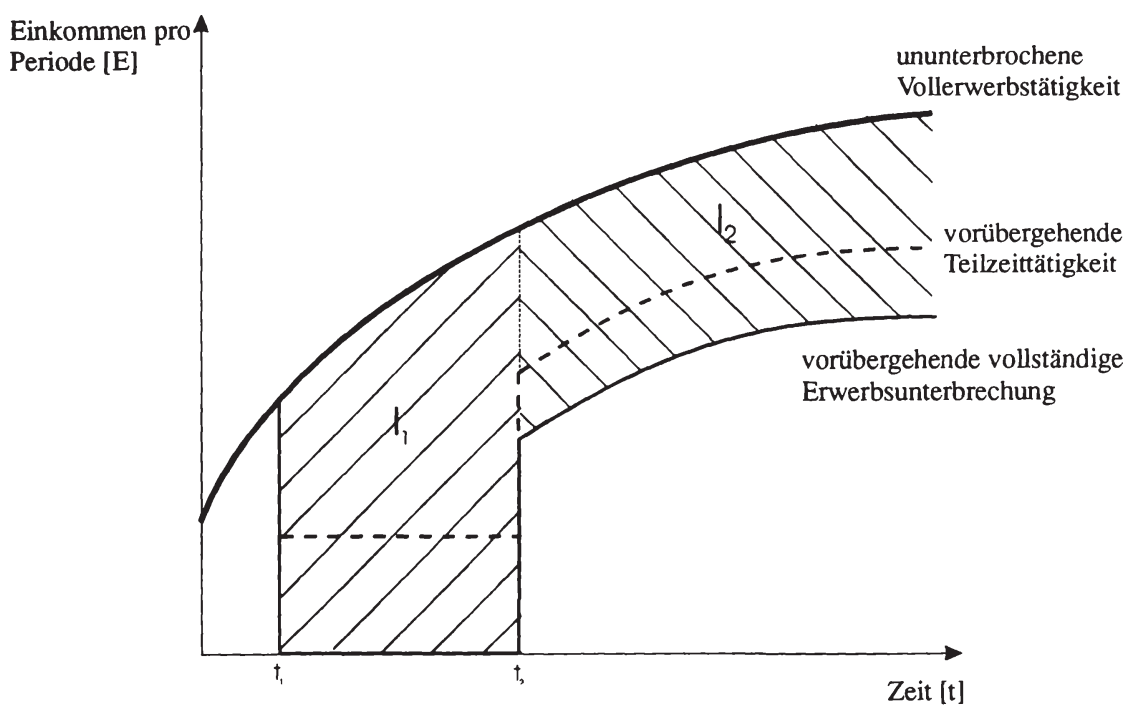

Quelle: Modifiziert nach Dinkel (1984) S. 89 bzw. Galler (1988) S. 103 
zum einen in einer ungünstigeren Entwicklung des berufsspezifischen Humankapitals gesehen werden, die wiederum auf das Vergessen und Verlernen bereits besessenen Humankapitals (Abschreibung) und auf nicht erfolgte Erhöhung des Humankapitals durch learning by doing aufgeteilt werden kann. ${ }^{123}$ Zum anderen kann das zu Beginn der Unterbrechung vorhandene Humankapital aber auch an den zu diesem Zeitpunkt besetzten Arbeitsplatz gebunden gewesen sein. Es handelt sich dann um arbeitsplatzspezifisches Humankapital, das bei jedem Arbeitsplatzwechsel unabhängig von einer Unterbrechung der Erwerbstätigkeit verloren geht. Solch arbeitsplatzspezifisches Humankapital kann z.B. in der Kenntnis der Abläufe in einem bestimmten Betrieb mit seinen spezifischen formellen und informellen Organisationsstrukturen und Außenbeziehungen bestehen.

Die Unterscheidung zwischen dem Verlust des arbeitsplatzspezifischen Humankapitals und der Entwertung des berufsspezifischen Humankapitals ist hier von Bedeutung, weil ersterer unabhängig von der Länge der Erwerbsunterbrechung auftritt, während letztere mit der Dauer der Marktarbeitsunterbrechung zunimmt. Infolgedessen wird der Lohnsatz für eine Erwerbstätigkeit mit arbeitsplatzspezifischem Humankapital auch schon nach einer kurzen Unterbrechung stark sinken, wenn eine Rückkehr an den alten Arbeitsplatz nicht möglich ist, während der Lohnsatz für einen Arbeitsplatz mit berufsspezifischem Humankapital erst mit zunehmender Dauer der Unterbrechung sinkt. Die Höhe der einer Frau entstehenden Opportunitätskosten hängt damit von der Art des Humankapitals und der Länge der Unterbrechung der Erwerbstätigkeit ab.

Um den Einkommensausfall infolge eines kindbedingten Ausscheidens einer Frau aus dem Erwerbsleben empirisch abzuschätzen, wird hier auf Berechnungen von Galler ${ }^{124}$ zurückgegriffen, die auf Daten der ersten Erhebungswelle des sozio-ökonomischen Panels von 1984 für die damalige Bundesrepublik beruhen. Die Schätzungen fußen auf einem einfachen Humankapitalmodell, daß aber die Unterscheidung nicht nur von Phasen der Vollzeittätigkeit und Erwerbsunterbrechungen, sondern darüber hinaus auch von Phasen der Teilzeitbeschäftigung zuläßt. Dabei wird angenommen, daß während der Zeit der formalen Ausbildung das bisher akquirierte Humankapital gänzlich für die weitere Humankapitalproduktion und nicht zur Einkommenserzielung eingesetzt wird. Die Akkumulationsrate des Humankapitals ist in den $s$ Jahren der formalen Ausbildung also gleich eins. In den Jahren der Berufstätigkeit wird nur noch auf einen Teil des potentiell erzielbaren Einkommens zu Investitionszwecken verzichtet. Weil sich die Humankapitalinvestitionen mit fortschreitendem Alter der Erwerbstätigen über immer kürzer werdende Zeiträume amortisieren müssen, wird unterstellt, daß der Investitionsanteil (d.h. die Akkumulationsrate) linear fällt und am Ende der geplanten Erwerbstätigkeit den Wert Null annimmt. Die logarithmierte Lohnrate als abhängige Variable ergibt sich dann aus

$$
\log w(t)=r s+\sum_{i=1}^{n}\left(r a_{i} e_{i}+r b_{i} e_{i}^{2}\right)
$$
Effizienzlohnsatz geeignet, die Entwicklung individueller Einkommensprofile im Lebenszyklus zu erklären. Wie Galler (1991) in einer Folgestudie feststellt, sind sich die drei Ansätze hinsichtlich der Implikationen für die Auswirkungen einer Erwerbsunterbrechung auf den Lebenseinkommensverlauf aber recht ähnlich. Der Rückgriff auf den Humankapitalansatz stellt daher keine Beeinträchtigung der Aussagefähigkeit der Ergebnisse dar. 
wobei mit $r$ die als konstant angenommene Rendite des Humankapitals und mit $a_{i}$ bzw. $b_{i}$ die Parameter der $n$ einzelnen Erwerbsphasen $\left(e_{i}\right)$ bezeichnet werden. ${ }^{125}$

Um dieses Modell schätzen zu können, wird für alle Perioden außer der letzten angenommen, daß die Parameter $a_{i}$ den gleichen Wert haben und der nicht-lineare Term vernachlässigbar ist. Außerdem wird als abhängige Variable aufgrund des Datenmaterials nicht der Lohnsatz, sondern das Bruttomonatseinkommen unselbständig beschäftigter Frauen gewählt, und es werden verschiedene zusätzliche Variable eingeführt, um den Einfluß von Teilzeitarbeit und Erwerbsunterbrechungen erfassen zu können. Die Ausbildungszeit wurde unterteilt in die Schuljahre $(s)$ und die Zahl der Jahre der Weiterbildung nach dem ersten Schulabschluß $\left(s_{w}\right)$. Die Dummy-Variablen $H_{T}$ und $H_{G}$, die jeweils den Wert 1 bei Teilzeit- bzw. bei geringfügiger Beschäftigung annehmen und anderenfalls Null sind, sollen dem Einfluß unterschiedlicher Arbeitszeiten auf das Einkommen Rechnung tragen. Die Variablen $v_{l}$ bzw. $v_{2}$ geben für Frauen mit unterbrochener Erwerbskarriere die Zahl aller Jahre mit Vollzeitbeschäftigung vor $\left(v_{l}\right)$ bzw. während $\left(v_{2}\right)$ der letzten Erwerbsphase an. Bei Teilzeitbeschäftigung (Erwerbsunterbrechungen) werden die entsprechenden Jahre durch $t_{l}\left(u_{l}\right)$ und $t_{2}\left(u_{2}\right)$ erfaßt. Für Frauen ohne Erwerbsunterbrechung gibt bei Wechsel zwischen Voll- und Teilzeitarbeit die Variable $v_{3}$ die Zeit in Vollzeitbeschäftigung, $t_{3}$ die Jahre in Teilzeitbeschäftigung an. Bei ununterbrochener Vollzeitarbeit wird die Zahl der Jahre durch $v_{4}$ gemessen. Die DummyVariable $T Z(U N)$ erfaßt den Verlust arbeitsplatzspezifischen Humankapitals beim Übergang von Vollzeit- zu Teilzeitarbeit (Erwerbsunterbrechung).

Die Ergebnisse der Schätzung sind in Tabelle C.1 wiedergegeben. Dabei wurde der öffentliche Dienst aufgrund abweichender institutioneller Regelungen ${ }^{126}$ getrennt ausgewiesen. Die Dauer früherer $\left(t_{1}\right)$ oder der letzten $\left(t_{2}\right)$ Teilzeitbeschäftigungen oder Erwerbsunterbrechungen $\left(u_{1}\right.$ bzw. $\left.u_{2}\right)$ tragen nicht zur Erklärung der Höhe des Brutto-Monatseinkommens bei. Allerdings ist die Akkumulationsrate bei Vollerwerbstätigkeit nach einer Unterbrechung etwas geringer als nach einer Teilzeitbeschäftigung (der Koeffizient von $v_{2}$ beträgt 0,0343 , derjenige von $v_{3}$ dagegen 0,0362). Auch der Verlust arbeitsplatzspezifischen Humankapitals ist, allerdings nur insoweit er durch die Dummy-Variablen erfaßt wird, weder bei Übergang zu einer Teilzeitbeschäftigung noch bei vollständiger Unterbrechung statistisch signifikant ( $T Z$ und $U N){ }^{127}$ Die Dauer früherer Erwerbstätigkeit $\left(v_{l}\right)$ hat keinen Einfluß auf die Höhe des Einkommens. Entscheidend ist vielmehr neben der Bildung (v.a. der Schulbildung) die Dauer der letzten Vollerwerbsphase $\left(v_{2}\right)$. Damit scheint arbeitsplatzspezifisches Humankapital eine große Rolle zu spielen, denn weder die Dauer einer Unterbrechung der Vollerwerbsarbeit noch die Dauer zurückliegender Beschäftigungsphasen hat größeren Einfluß auf das BruttoMonatseinkommen. ${ }^{128}$ Das durch frühere Erwerbstätigkeit erworbene Humankapital wird also offenbar bei Aufnahme einer anderen Beschäftigung nicht honoriert, was dafür spricht, daß es

125 Vgl. Galler (1988) S. 98. Zu einer ausführlicheren Darstellung vgl. Franz (1991) S. $102 \mathrm{ff}$.

126 Der Lohnsatz ist beispielsweise im öffentlichen Dienst meistens altersabhängig und es bestehen vom privaten Sektor abweichende Regelungen bezüglich der Unterbrechungsmöglichkeiten.

127 Der im Gegensatz zu den theoretischen Ergebnissen stehende positive Wert von UN wird von Galler (1988) S. 102 mit einem Reparatur-Effekt begründet, der nach einer Unterbrechung zu einem teilweisen Aufholen des Einkommensrückstands durch besonders hohe Akkumulationsraten führt.

Diese Schlußfolgerung läßt sich auch aus den Ergebnissen einer Untersuchung von Helberger (1984) insbesondere S. 18ff. ziehen. Helberger erhielt mit einem modifizierten Ansatz Koeffizienten in der gleichen Größenordnung wie Galler. 
Tab. C.I: Einkommensschätzung für unselbständig beschäftigte Frauen ( $t$-Werte in Klammern)

\begin{tabular}{|c|c|c|c|}
\hline & insgesamt & ohne öffentl. Dienst & öffentl. Dienst \\
\hline Fälle & 1273 & 881 & 392 \\
\hline$R^{2}$ & 0,645 & 0,628 & 0,690 \\
\hline adj. $R^{2}$ & 0,640 & 0,620 & 0,675 \\
\hline \multirow[t]{2}{*}{ Konstante } & 6,6218 & 6,6515 & 6,7724 \\
\hline & $(99,5)$ & $(64,1)$ & $(79,6)$ \\
\hline \multirow[t]{2}{*}{$s$} & 0,0746 & 0,0675 & 0,0698 \\
\hline & $(15,1)$ & $(8,1)$ & $(12,4)$ \\
\hline \multirow[t]{2}{*}{$s_{w}$} & 0,0155 & 0,0363 & $-0,0163$ \\
\hline & $(0,9)$ & $(1,5)$ & $(-0,8)$ \\
\hline \multirow[t]{2}{*}{$v_{1}$} & 0,0001 & 0,0037 & $-0,0114$ \\
\hline & $(0,0)$ & $(0,8)$ & $(-2,2)$ \\
\hline \multirow[t]{2}{*}{$v$, } & 0,0343 & 0,0295 & 0,0531 \\
\hline & $(5,0)$ & $(3,5)$ & $(3,7)$ \\
\hline \multirow{2}{*}{$v,^{2}$} & $-0,0009$ & $-0,0007$ & $-0,0016$ \\
\hline & $(-3,4)$ & $(-2,3)$ & $(-2,5)$ \\
\hline \multirow[t]{2}{*}{$v^{3}$} & 0.0362 & 0,0404 & 0,0244 \\
\hline & $(4,7)$ & $(4,1)$ & $(2,1)$ \\
\hline \multirow[t]{2}{*}{$v_{3}^{2}$} & $-0,0008$ & $-0,0008$ & $-0,0007$ \\
\hline & $(-2,9)$ & $(-2,4)$ & $(-1,7)$ \\
\hline \multirow[t]{2}{*}{$v_{4}$} & 0,0391 & 0,0435 & 0,0301 \\
\hline & $(7,1)$ & $(6,0)$ & $(4,0)$ \\
\hline \multirow[t]{2}{*}{$v_{4}^{2}$} & $-0,0007$ & $-0,0009$ & $-0,0005$ \\
\hline & $(-5,0)$ & $(-4,3)$ & $(-2,6)$ \\
\hline \multirow[t]{2}{*}{$T Z$} & $-0,0408$ & $-0,0081$ & $-0,0907$ \\
\hline & $(-0,9)$ & $(-0,1)$ & $(-1,3)$ \\
\hline \multirow[t]{2}{*}{$t_{1}$} & $-0,0057$ & $-0,0075$ & $-0,0016$ \\
\hline & $(-0,9)$ & $(-0,9)$ & $(-0.2)$ \\
\hline \multirow[t]{2}{*}{$t$} & 0,0099 & 0,0062 & 0.0163 \\
\hline & $(2,7)$ & $(1,3)$ & $(3,0)$ \\
\hline \multirow[t]{2}{*}{$t_{3}$} & 0,0038 & 0,0015 & 0,0103 \\
\hline & $(1,1)$ & $(0,3)$ & $(1,8)$ \\
\hline \multirow[t]{2}{*}{$U N$} & 0,0906 & 0,1018 & 0,0496 \\
\hline & $(1,4)$ & $(1,3)$ & $(0,5)$ \\
\hline \multirow[t]{2}{*}{$u_{l}$} & 0,0015 & $-0,0015$ & 0,0147 \\
\hline & $(0,2)$ & $(-0,2)$ & $(1,3)$ \\
\hline \multirow[t]{2}{*}{$u$, } & $-0,0032$ & $-0,0050$ & 0,0018 \\
\hline & $(-1,1)$ & $(-1,4)$ & $(0,4)$ \\
\hline \multirow[t]{2}{*}{$H T$} & $-0,4640$ & $-0,4985$ & $-0,3994$ \\
\hline & $(-12,0)$ & $(-10,1)$ & $(-6,9)$ \\
\hline \multirow[t]{2}{*}{$H G$} & $-1,3153$ & $-1,3461$ & $-1,1508$ \\
\hline & $(-26,9)$ & $(-22,0)$ & $(-14,9)$ \\
\hline
\end{tabular}

Quelle: Galler (1988) S. 99 
spezifisch an den aufgegebenen Arbeitsplatz gebunden war. ${ }^{129}$ Galler ${ }^{130}$ bietet als weitere Erklärung ein Ausklinken aus innerbetrieblichen Karriereleitern an. Unabhängig davon, ob nur weniger oder gar nicht mehr gearbeitet wird, führt der Ausstieg aus der Karriere dazu, daß bei einem Wiedereinstieg ein niedrigeres Anfangseinkommen hingenommen werden muß. Insgesamt bestätigen die empirischen Ergebnisse den in Abb. C. 4 dargestellten Verlauf der Lebenseinkommenskurven, wenn man annimmt, daß die Querschnittsdaten auch im Längsschnitt Gültigkeit haben. ${ }^{131}$

Aus den Ergebnissen in Tab. C.1 lassen sich prinzipiell durch die Differenz der Integrale zwischen dem Einkommensverlauf bei ununterbrochener Erwerbskarriere und bei verschieden langen Unterbrechungen die jeweiligen Lebenseinkommensverluste einer Frau (d.h. die einer Frau entstehenden Opportunitätskosten) berechnen, wenn man davon ausgeht, daß sich die Rahmenbedingungen in Zukunft nicht wesentlich verändern werden. ${ }^{132}$ Aufgrund der dabei auftretenden mathematischen Probleme berechnet Galler stattdessen die in Tab. C.2 angegebenen Verluste an Lebenseinkommen während und nach der Unterbrechung für ausgewählte Erwerbsverläufe (Unterbrechung nach 1, 3, 6 oder 10 Jahren für verschiedene Qualifikationen unterteilt in völlige Aufgabe der Erwerbstätigkeit und Übergang zu Teilzeitarbeit). Diese Berechnungen beziehen sich allerdings nur auf Einkommensverluste, die vom Beginn der Erwerbstätigkeit bis zum fünfzigsten Lebensjahr auftreten, weil für ältere Jahrgänge die Datenbasis keine verläßliche Schätzung erlaubt. Damit werden die absoluten Verluste tendenziell noch unterschätzt und zwar umso mehr, je weniger sich nach einer Unterbrechung der Humankapitalbestand und damit das Periodeneinkommen im fünfzigsten Lebensjahr dem Zustand bei ununterbrochener Vollerwerbskarriere wieder angeglichen hat. ${ }^{133}$

Ein weiterer Grund für eine Unterschätzung der Frauen entstehenden Opportunitätskosten nach dem Ansatz von Galler ist darin zu sehen, daß für Frauen, die ihre Erwerbskarriere trotz der Geburt eines oder mehrerer Kinder nicht einschränken, überhaupt keine Opportunitätskosten entstehen, da sie keine Einkommenseinbußen erleiden. Anstelle eines Einkommensverzichts infolge einer Erwerbsunterbrechung können beide Eltern ja auch nach der Geburt eines Kindes in unverminderter Intensität weiter arbeiten und so Verluste an Lebenseinkommen

129 Bei einer Befragung durch Gaugler u.a. (1984) S. 204 gaben etwa ein Viertel der Frauen an, beim Wiedereinstieg einen beruflichen Abstieg erfahren zu haben, zwei Drittel hatten keine Veränderung erfahren (zitiert nach Galler (1988) S. 86).

Vgl. Galler (1988) S. 102.

131 Für bestimmte Kohorten besonders günstige oder ungünstige Arbeitsmarktbedingungen können $\mathrm{zu} A b-$ weichungen der Querschnitts- von Längsschnittdaten führen. Für die folgende Berechnung wurden die Querschnittsdaten nicht diskontiert, weil angenommen wird, daß sich die individuelle Zeitpräferenzrate und die Einkommenswachstumsrate gerade entsprechen und damit gegenseitig kompensieren. Der Barwert des Lebenseinkommens ist dann gerade gleich der Summe der Periodeneinkommen aus Querschnittsdaten. Vgl. Galler (1988) FN 26 S. 107.

132 Da die Fertilitätsentscheidung vor dem Hintergrund der aufgrund der im Entscheidungszeitpunkt verfügbaren Informationen erwarteten Opportunitätskosten getroffen wird, ist die Annahme eines konstanten Bedingungsrahmens wohl nicht so realitätsfern, wie sie auf den ersten Blick erscheinen mag.

133 Eine weitere Unterschätzung resultiert daraus, daß in den hier betrachteten Einkommensausfällen die Minderung der Rentenansprüche aufgrund einer Erwerbseinschränkung nur teilweise enthalten ist. Der hier als Meßgröße verwendete Brutto-Monatslohn enthält nämlich nicht den Arbeitgeberbeitrag zur Rentenversicherung. Nimmt man vereinfachend individuelle Beitragsäquivalenz an, so wird also nur die Hälfte der Rentenminderung berücksichtigt. Bei einem Beitragssatz von knapp $20 \%$ liegen die tatsächlichen Opportunitätskosten also um etwa $10 \%$ höher als hier berechnet. 
vermeiden. ${ }^{134}$ In diesem Fall manifestieren sich - so wird besonders von konservativer Seite argumentiert - die indirekten Kosten nicht in einem Einkommensverzicht, sondern in einer geringeren Qualität des Erziehungsergebnisses. Bei Teilzeitarbeit tritt ein Teil der indirekten Kosten in Form von Einkommenseinbußen, der andere Teil in Form gesunkener Zeit für die Beschäftigung mit dem Kind auf. Vorausgesetzt wird dabei, daß zusätzliche Zeit der Eltern für das Kind sich positiv auf dessen Entwicklung auswirkt. Die indirekten Kosten sind dann nur im Fall der völligen Unterbrechung der Erwerbstätigkeit durch die Einkommensverluste vollständig erfaßt. Anderenfalls müßten die Einbußen an Erziehungsergebnis berücksichtigt werden.

Führt man diesen Gedanken fort, kommt man zu der Überlegung, daß Eltern generell bemüht sein werden, die Grenzkosten eines Einkommensverzichts dem Grenzschaden durch Qualitätsverlust der Erziehung anzugleichen. Mit steigenden marginalen Opportunitätskosten der Zeit ist daher davon auszugehen, daß die Qualität der Kindererziehung ceteris paribus sinkt. Dann wird nämlich eine Substitution von Marktgütern für Zeit im Haushaltsproduktionsprozeß zu einer weniger zeitintensiven Art der Kindererziehung führen. ${ }^{135}$ Diese Substitution kann z.B. durch vermehrten Kauf von Spielzeug als Ersatz für mit dem Kind verbrachter Zeit, in der Beschäftigung eines Babysitters, dem Besuch eines Kindergartens oder Internats bestehen. Allen Formen gemeinsam ist, daß durch sie weniger Zeit und mehr Marktgüter bei der Produktion des Basisgutes Kind in dem Haushalt aufgewandt wird. Allerdings ist es wahrscheinlich, daß die Qualität der Kindeserziehung bei diesem Substitutionsprozeß nicht konstant bleibt. Erfolgt gar keine Kompensation für den aufgrund höherer Opportunitätskosten gesunkenen Zeitinput (der Eltern), so ist der Qualitätsverlust offensichtlich. Aber auch eine Kompensation mittels Spielzeug, Kindergärten etc. wird oft nicht als vollwertiger Ersatz für die Zeit der Eltern bzw. hier der Mutter angesehen. Den Vorteilen aufgrund geringerer Opportunitätskosten, die Frauen entstehen, die ihre Erwerbstätigkeit nur geringfügig einschränken, würde dann die gesunkene Qualität des Erziehungsergebnisses gegenzurechnen sein. ${ }^{136}$

Allerdings lassen sich auch Argumente für eine gegenteilige Auffassung vertreten. So kann der Sozialisationsprozeß im Kindergarten gerade für Einzelkinder als wichtiges Erziehungsergebnis angesehen und eine übermäßige Beschäftigung der Eltern mit dem Kind als eher hinderlich für eine eigenständige Entwicklung betrachtet werden. ${ }^{137}$ Außerdem ist zu fragen, ob die pro Kind aufgewendete Erziehungszeit heute wirklich deutlich niedriger ist als in Zeiten, in denen die Erwerbstätigkeit von Frauen noch eher die Ausnahme war. Schließlich war damals

134 Werden Marktgüter wie Kindergarten oder Kindermädchen für Zeit substituiert, kommt es bei gleichem Einkommen zu einer Einschränkung der Konsummöglichkeiten der Eltern.

135 Das führt natürlich dazu, daß die direkten Kosten von Kindern in Form von Ausgaben für Marktgütern steigen werden.

136 Als Ergebnis einer Sichtung empirischer Studien kommen Haveman/Wolfe (1995) S. $1870 \mathrm{zu}$ dem Schluß, daß "growing up in a family in which the mother chooses to work appears to have a modest adverse effect on educational attainment, suggesting a negative effect of the loss of child care time. However, mother's work choices do not appear to have an effect on the probability that a girl will experience an out-of-wedlock birth in her teens, or be a welfare recipient, nor on educational attainment if the mother's work occurs during a child's teen years."

137 Moore/Hofferth (1979) S. 146 stellen fest: "... it is not at all clear that the number of hours spent with a parent is the critical determinant of a child's development. Characteristics of parent-child interaction for example, the appropriateness of the parent's stimulation of the child's developmental maturity, the variety of the stimulation, and responsiveness to the infant's behavior - are more important to child development than is the sheer quantity of interaction or stimulation." 
Tab. C.2: Lebenseinkommensverluste nach Unterbrechungsdauer, Unterbrechungszeitpunkt, Schulbildung und Art der Unterbrechung (in DM bzw. Prozent)

\begin{tabular}{|c|c|c|c|c|c|c|c|c|c|}
\hline \multirow{2}{*}{\multicolumn{2}{|c|}{$\begin{array}{l}\text { Dauer d. Schulbildung } \\
\text { Art d. Unterbrechung }\end{array}$}} & \multicolumn{2}{|c|}{ Hauptschule (8 Jahre) } & \multicolumn{2}{|c|}{ Realschule (10 Jahre) } & \multicolumn{2}{|c|}{ Abitur (13 Jahre) } & \multicolumn{2}{|c|}{ Hochschule (18 Jahre) } \\
\hline & & Teilz. & N.erw. & Teilz. & N.erw. & Teilz. & N.erw. & Teilz. & N.erw. \\
\hline \multicolumn{10}{|c|}{ Ohne Unterbrechung } \\
\hline \multicolumn{2}{|c|}{ Lebenseinkommen } & 1.243 .435 & 1.243 .435 & 1.337 .138 & 1.337 .138 & 1.474 .074 & 1.474 .074 & 1.673 .374 & 1.673 .374 \\
\hline \multicolumn{10}{|c|}{ Unterbrechung nach 3 Jahren für } \\
\hline \multirow[t]{3}{*}{$1 \mathrm{Jahr}$} & Verlust insgesamt & 79.398 & 147.013 & 86.974 & 159.288 & 98.342 & 176.934 & 115.833 & 201.915 \\
\hline & Rel. Eink.verzicht & 6,4 & 11,8 & 6,5 & 11,9 & 6,7 & 12,0 & 6,9 & 12,1 \\
\hline & davon $\mathrm{n}$. Unterbr. & 85,5 & 81,6 & 84,9 & 80,5 & 83,6 & 78,5 & 80,5 & 73,6 \\
\hline \multirow[t]{3}{*}{3 Jahre } & Verlust insgesamt & 134.656 & 223.838 & 150.895 & 248.058 & 176.639 & 286.226 & 220.928 & 352.757 \\
\hline & Rel. Eink.verzicht & 10,8 & 18,0 & 11,3 & 18,6 & 12,0 & 19,4 & 13,2 & 21,1 \\
\hline & davon n. Unterbr. & 72,3 & 62,4 & 71,7 & 61,1 & 70,4 & 58,7 & 66,8 & 53,1 \\
\hline \multirow{3}{*}{6 Jahre } & Verlust insgesamt & 215.922 & 338.893 & 243.931 & 380.224 & 288.754 & 447.474 & 366.946 & 571.856 \\
\hline & Rel. Eink.verzicht & 17,4 & 27,3 & 18,2 & 28,4 & 19,6 & 30,4 & 21,9 & 34,2 \\
\hline & davon n. Unterbr. & 61,5 & 47,7 & 61,0 & 46,7 & 59,7 & 44,5 & 55,5 & 39,2 \\
\hline \multirow{3}{*}{\multicolumn{2}{|c|}{$\begin{array}{l}10 \text { Jahre Verlust insgesamt } \\
\text { Rel. Eink.verzicht } \\
\text { davon n. Unterbr. }\end{array}$}} & 318.935 & 490.068 & 360.033 & 552.410 & 425.213 & 654.833 & 536.388 & 847.357 \\
\hline & & 25,6 & 39,4 & 26,9 & 41,3 & 28,8 & 44,4 & 32,1 & 50,6 \\
\hline & & 51,1 & 36,1 & 50,4 & 35,1 & 48,6 & 33,0 & 42,9 & 27,4 \\
\hline \multicolumn{10}{|c|}{ Unterbrechung nach 6 Jahren für } \\
\hline \multirow[t]{3}{*}{$1 \mathrm{Jahr}$} & Verlust insgesamt & 75.298 & 154.125 & 82.281 & 170.593 & 92.596 & 195.214 & 107.781 & 232.381 \\
\hline & Rel. Eink.verzicht & 6,1 & 12,4 & 6,2 & 12,8 & 6,3 & 13,2 & 6,4 & 13,9 \\
\hline & davon n. Unterbr. & 83,0 & 80,5 & 82,2 & 79,9 & 80,7 & 78,4 & 76,7 & 74,6 \\
\hline
\end{tabular}




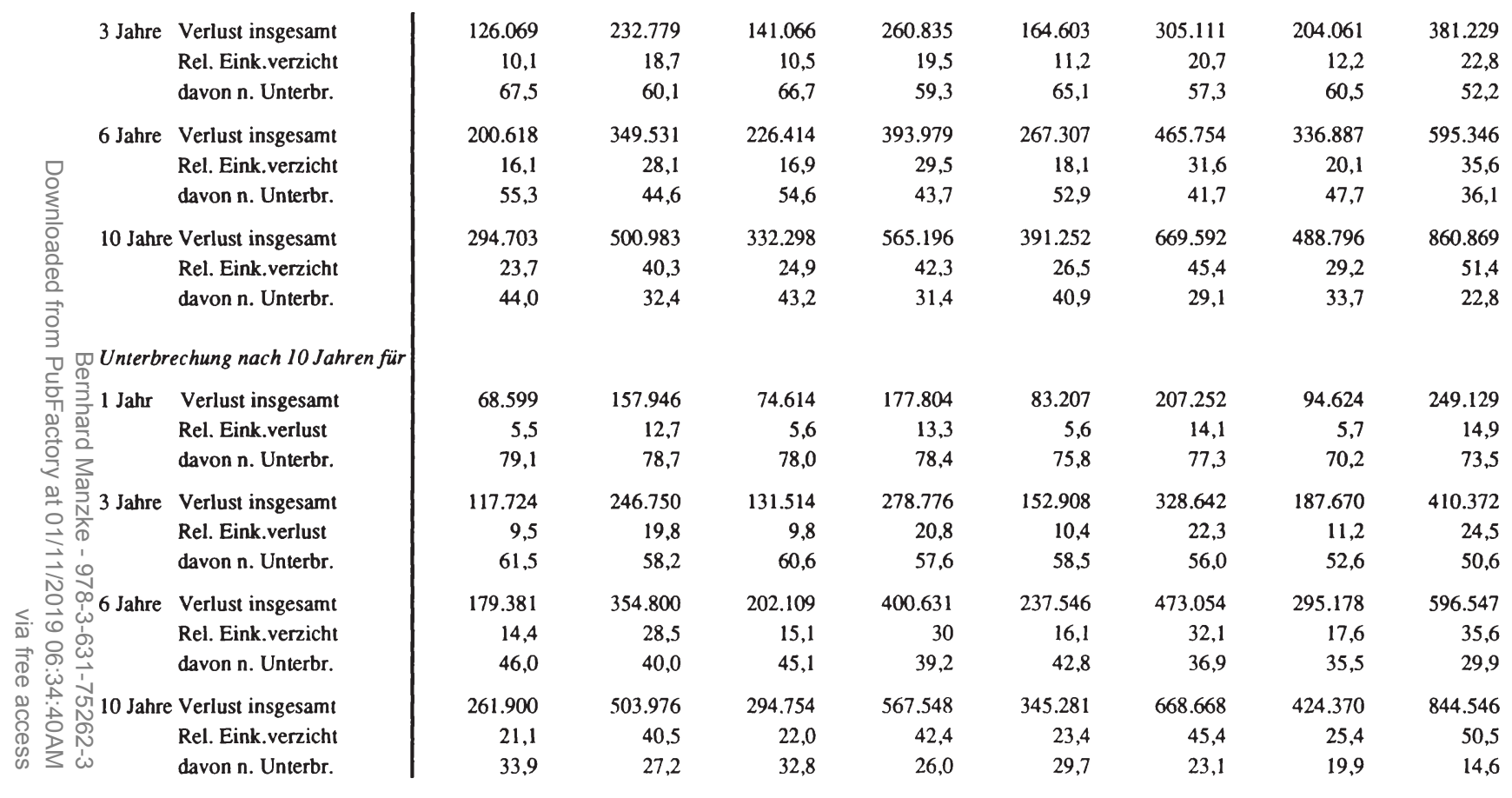

Quelle: Galler (1988) S. 104f. Die für 1984 angegebenen Werte wurden mit Hilfe der Entwicklung des durchschnittlichen Brutto monatslohns für weibliche Angestellte bis 1992 fortgeschrieben 
auch die durchschnittliche Kinderzahl einer Familie wesentlich höher und die zeitsparende Technisierung der Haushalte nicht so weit vorangeschritten.

Letztlich würde eine Bewertung des Erziehungsergebnisses dessen Quantifizierung voraussetzen. Dies scheitert schon an unterschiedlichen Auffassungen darüber, welche Ziele mit der Erziehung erreicht werden sollen (Zufriedenheit des Kindes, Zufriedenheit der Eltern, Ausbildung, soziales Verhalten, etc.), ganz abgesehen von der Gewichtung der einzelnen Ziele und Definition von adäquaten quantifizierbaren Meßgrößen. Deshalb muß es hier bei dem Hinweis bleiben, daß die Opportunitätskosten, die Frauen entstehen, die auch nach der Geburt eines Kindes weiterhin voll erwerbstätig sind, eventuell unterschätzt werden, die Quantifizierung der Fehlschätzung aber aufgrund der Unmöglichkeit der Bewertung des Erziehungsergebnisses nicht möglich ist.

Auch wenn man von der Qualität des Erziehungsergebnisses absieht, sind die Opportunitätskosten, die Frauen entstehen, die ihre Erwerbstätigkeit nicht einschränken, durch die obigen Angaben zu niedrig ausgewiesen, weil die Eltern die Zeit der Beschäftigung mit dem Kind in diesem Fall zu Lasten sonstiger Freizeitaktivitäten aufbringen müssen. ${ }^{138}$ Die folgenden Zahlen stellen daher eine recht vorsichtige Schätzung der einer Frau bei Einschränkung der Erwerbstätigkeit entstehenden Opportunitätskosten dar.

Aus der Darstellung der Lebenseinkommensverluste in Tab. C.2 ist zu erkennen, daß eine Gleichsetzung der einer Frau entstehenden Opportunitätskosten mit dem Einkommensausfall während der Zeit der Einschränkung der Erwerbsarbeit, d.h. eine Vernachlässigung der Einkommensverluste nach der Unterbrechung, zu einer gravierenden Unterschätzung der Opportunitätskosten führen würde, denn diese langfristigen Einkommensverluste machen bis über $80 \%$ der gesamten Verluste aus (bei Hauptschulabschluß und einjähriger Unterbrechung drei Jahre nach dem Beginn der Erwerbskarriere). Ein höheres Bildungsniveau führt zu einem höheren relativen und absoluten Einkommensausfall. Schon eine einjährige Unterbrechung nach drei Jahren hat bei einer Akademikerin eine Reduzierung des Lebenseinkommens um über 200.000 DM oder 12,1\% zur Folge. Mit zunehmender Länge der Unterbrechung erhöhen sich diese Werte bis auf fast $850.000 \mathrm{DM}$ bzw. über $50 \%$ bei einer zehnjährigen Unterbrechung. ${ }^{139}$

Auch bei Übergang zu einer Teilzeitbeschäftigung statt vollständiger Aufgabe der Erwerbstätigkeit bleiben die Einkommensverluste bedeutend. Für eine Abiturientin mit einer sechsjährigen Unterbrechung nach drei Jahren betragen sie beispielsweise etwa 290.000 DM oder knapp 20\% des sonst bis zum fünfzigsten Lebensjahr erreichbaren Einkommens. Der Unterbrechungszeitpunkt, d.h. die Zahl der Vollerwerbsjahre vor der Unterbrechung, hat nur auf den Anteil der langfristigen Verluste einen größeren Einfluß (sie sinken mit einer späteren Unterbrechung, weil die Zahl der Erwerbsjahre nach Unterbrechung, in denen sie sich realisieren, zurïckgeht), ändert aber die absoluten und relativen Einkommensausfälle insgesamt kaum.

138 Robinson (1987) S. 318 stellt für die USA fest, daß die Zeit für die Kindererziehung v.a. zu Lasten der Freizeit geht.

139 Mit den steigenden indirekten Kinderkosten läßt sich der empirisch gefundene negative Zusammenhang zwischen Bildungsniveau der Frau und Kinderzahl erklären. Nimmt man an, daß das Bildungsniveau entweder durch Vererbung von Intelligenz, Sozialisation oder den im folgenden Kapitel ausgeführten negativen Zusammenhang zwischen Quantität und Qualität zumindest teilweise innerhalb einer Familie übertragen wird, so ergibt sich aus dieser Entwicklung schon nach wenigen Generationen ceteris paribus ein geringerer durchschnittlicher Bildungsstand der Gesellschaft. 
Bisher wurde der Bruttolohnsatz als abhängige Variable benutzt. Sinnvoller dürfte allerdings eine Berechnung auf Basis des Nettolohnsatzes sein, denn für die meisten Haushalte wird wohl v.a. interessieren, wie hoch die Einbußen nach Berücksichtigung der steuerlichen Entlastungen aufgrund des gesunkenen Einkommens sind. Grundlage der Entscheidung eines Paares für (oder gegen) Kinder dürften die damit verbundenen Einbußen an verfügbarem Einkommen sein.

Diese Vorgehensweise hätte allerdings den gravierenden Nachteil, daß die durch Kinder entstehenden Kosten und die teilweise Erstattung dieser Kosten durch staatliche Regelungen vermengt würden. In dieser Arbeit werden die staatlichen Transfers im Rahmen des Kinderlastenausgleichs daher von den Kosten getrennt. ${ }^{140}$ Der Kinderlastenausgleich umfaßt allerdings nur die kindgebundenen Transfers und damit nicht etwa Entlastungen nach Einschränkung der Erwerbstätigkeit durch die Steuerprogression und vermehrte Vorteile aus dem Ehegattensplitting sowie geringere Sozialversicherungsbeiträge, die auch Personen ohne Kindern gewährt werden.

Für die hier vorgenommene Abschätzung der Opportunitätskosten von Kindern für ihre Eltern wäre daher ein Ansatz adäquat, der die Einkommensverluste anhand der Differenz der Nettoeinkommen vor Kinderlastenausgleich als Maßstab heranzieht. Eine derartige Schätzung wurde von Galler in einer Modifizierung und Aktualisierung der ersten Studie vorgenommen. ${ }^{141}$ Die grundlegenden Ergebnisse der Vorläuferstudie, die aus der oben in Tabelle C.1 dargestellten Schätzung für die Koeffizienten der Lohngleichung abgeleitet wurden, bleiben erhalten. Die Brutto-Einkommensverluste sind tendenziell gegenüber der Vorläuferstudie etwas angestiegen. Sie belaufen sich jetzt beispielsweise für eine Hochschulabsolventin mit einer zehnjährigen Unterbrechung auf ungefähr 1.150.000 DM (in Preisen von 1992) statt etwa 850.000 DM nach dem früher verwandten Ansatz. Diese Erhöhung dürfte v.a. darauf zurückzuführen sein, daß die Einkommensverluste jetzt bis zum sechzigsten statt nur bis zum fünfzigsten Lebensjahr berechnet wurden und jetzt außerdem Verluste während der Phase des Rentenbezugs berücksichtigt wurden.

Neben den Bruttoeinkommen wurden von Galler (1991) auch die Netto-Einkommensverluste nach Berücksichtigung von Einkommensteuer und Sozialversicherungsbeiträgen berechnet. Zugrundegelegt wurden dabei die 1990 gültigen Regelungen, bei der Berechnung der Rentenansprüche die Gesetzeslage entsprechend des Rentenreformgesetzes 1992. Um der großen Bedeutung des Ehegattensplittings Rechnung zu tragen, wurde zwischen verheirateten und unverheirateten Frauen differenziert. Außerdem wurde danach unterschieden, ob die Einkommensverluste während der Einschränkung der Erwerbsarbeit, während der nachfolgenden Vollerwerbsphase oder während der Zeit des Rentenbezugs anfallen. Bei der Berechnung des Rentenanspruchs wurden Kindererziehungszeiten für ein Kind berücksichtigt, also eine Entlastungswirkung des Kinderlastenausgleichs schon in die einer Frau entstehenden Opportu-

140 Die vom Staat im Rahmen von Realtransfers übernommenen Kinderkosten (Mitversicherung in der Gesetzlichen Krankenversicherung, Schulbildung, Jugendhilfe, etc.) werden hier bei der Darstellung der privaten Kosten nicht mit aufgeführt und dürfen daher auch nicht als kostenentlastend angesehen werden.

141 Vgl. Galler (1991). Auf die Modifikationen soll hier nicht im einzelnen eingegangen werden. Die wesentlichste Änderung gegenüber der Vorläuferstudie besteht in der Verwendung eines zweistufigen Schätzansatzes, um eventuelle Selektionseffekte hinsichtlich der Partizipationsrate der Frauen am Erwerbsleben ausschalten zu können. Selektionseffekte treten auf, wenn nicht die Erwerbsbiographie den Lohnsatz bestimmt, sondern umgekehrt der Lohnsatz die Erwerbsbiographie. So könnte beispielsweise die Erwerbsbeteiligung von Frauen mit niedrigem Einkommenspotential tendenziell unterdurchschnittlich sein. Weitere wichtige Modifikationen betreffen die Abwandlung der Lohngleichung und die Berücksichtigung von Panel-Daten zwischen 1984 und 1988. 
Abb. C.5: Einkommensverluste im Lebensverlauf (in Preisen von 1984)

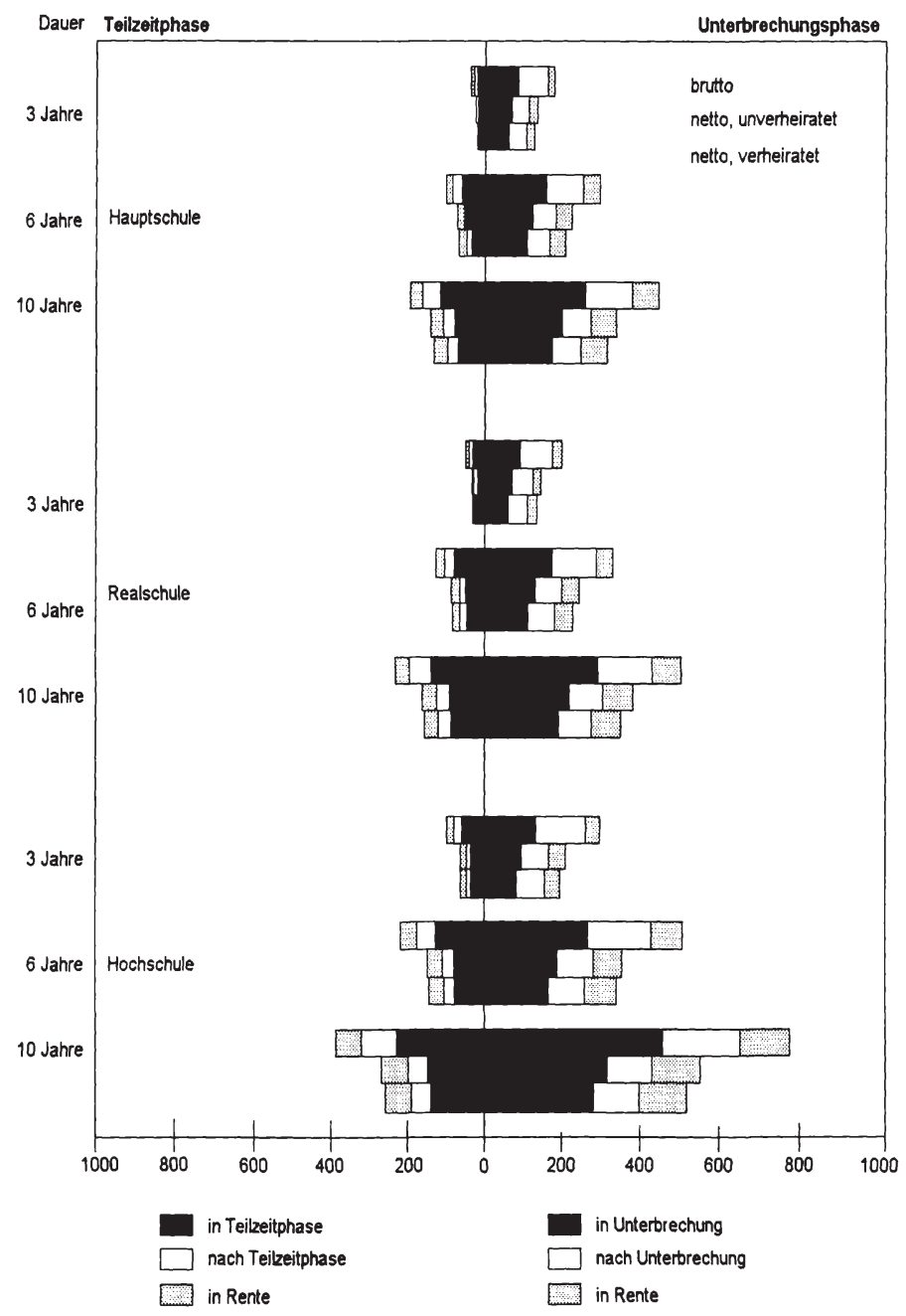

Quelle: Galler(1991) S. 142 
nitätskosten eingerechnet. Die dadurch bewirkte Unterschätzung der Opportunitätskosten ist quantitativ allerdings nicht sehr bedeutend.

Die Ergebnisse der Schätzung der Einkommensverluste sind für einige beispielhafte Lebensverläufe in Abbildung C.5 dargestellt. ${ }^{142}$ Aufgrund der Wirkung des Steuer- und Transfersystems sind die Netto-Einkommensverluste um etwa ein Drittel niedriger als die BruttoEinkommensverluste, wobei wegen des Ehegattensplittings die Netto-Einkommensverluste von verheirateten Frauen nochmals deutlich unter denen von unverheirateten Frauen liegen. Generell liegen die Netto-Einkommensverluste zwischen etwa $10 \%$ des potentiellen NettoLebenseinkommens für eine dreijährige Unterbrechung und etwa $30 \%$ für eine zehnjährige Unterbrechung und erreichen damit für den ungünstigsten Fall einer Hochschulabsolventin mit zehnjähriger Unterbrechung etwa 725.000 DM (in Preisen von 1992).

Als Schlußfolgerung aus den Studien zu den Lebenseinkommensverlusten einer Frau läßt sich daher festhalten, daß die indirekten Kosten in Form einer Reduzierung des Lebenseinkommens der Mutter infolge einer kindbedingten Einschränkung der Erwerbstätigkeit insgesamt sowohl absolut als auch in Relation zum potentiellen Lebenseinkommen sehr bedeutend sind. Die einer Frau entstehenden Opportunitätskosten werden v.a. durch ihr Bildungsniveau und die Dauer der Unterbrechung festgelegt.

$\mathrm{Zu}$ Aussagen über die durchschnittlichen Opportunitätskosten eines Kindes gelangt man aber erst, wenn man die Unterbrechungsdauer in Abhängigkeit von der Kinderzahl feststellt. Dazu müßten die unterschiedlichen Erwerbsverläufe mit ihrer relativen Häufigkeit gewichtet zu durchschnittlichen Kosten für unterschiedliche Unterbrechungsdauern zusammengefaßt werden. Dieses Vorgehen ist insofern ungenau, als die in Tabelle C. 2 berechneten Erwerbsbiographien nur einen Teil aller möglichen Einkommensverläufe aufzeigen und die Auswahl zudem nicht frei von subjektiven Einschätzungen erfolgte. Die durchschnittlichen indirekten Kosten in Abhängigkeit von der Kinderzahl erhält man, indem man die Wahrscheinlichkeiten einer bestimmten Unterbrechungsdauer bei einer bestimmten Kinderzahl mit den durchschnittlichen Kosten dieser Unterbrechungsdauer multipliziert und dann die Summe über alle Unterbrechungsdauern für die jeweilige Kinderzahl bildet. ${ }^{143}$

Dieser Weg der Bestimmung der Opportunitätskosten in Abhängigkeit von der Kinderzahl scheitert aber an einer zu geringen Besetzung der einzelnen Klassen. Es muß hier daher bei allgemeineren Aussagen über den Zusammenhang zwischen Kinderzahl und Erwerbseinschränkung bleiben.

Aus Tabelle C.3 läßt sich erkennen, daß der Anteil der Erwerbstätigen unter den Frauen mit Kindern unter 18 Jahren für die relevanten Altersgruppen ${ }^{144}$ mit einer Erwerbsquote von 55,6 Prozent für Frauen im Alter von 25 bis unter 35 und von 64,0 Prozent für Frauen von 35 bis

142 Eine detaillierte Darstellung der Einkommensverluste in Abhängigkeit von Unterbrechungsdauer, Bildungsniveau, Unterbrechungszeitpunkt wie in Tabelle C.1 ist leider für die modifizierte Studie nicht angegeben worden.

143 Die Tatsache, daß Frauen mit höherer Bildung durchschnittlich eine geringere Unterbrechungsdauer haben, ist schon durch die entsprechend niedrigere Wahrscheinlichkeit der Besetzung dieser Klasse berücksichtigt.

144 Über vier Fünftel aller Frauen mit Kindern unter 18 Jahren befinden sich im Alter von 25 bis unter 45 Jahren. 
Tab. C.3: Altersspezifische Erwerbsquoten von Frauen im Alter von 15 Jahren und mehr im Mai 1992 nach Altersgruppen und Altersgruppen der Kinder ${ }^{145}$ in der Familie

\begin{tabular}{|c|c|c|c|c|c|c|c|c|c|c|}
\hline $\begin{array}{c}\text { Alter der } \\
\text { Frauen } \\
\text { von ... bis } \\
\text { unter ... } \\
\text { Jahren }\end{array}$ & $\begin{array}{l}\text { Insge- } \\
\text { samt }\end{array}$ & $\begin{array}{l}\text { ohne } \\
\text { Kinder }\end{array}$ & $\begin{array}{c}\text { mit } \\
\text { Kindern } \\
\text { (ohne } \\
\text { Alters- } \\
\text { begren- } \\
\text { zung) }\end{array}$ & $\begin{array}{c}\text { mit } \\
\text { Kindern } \\
\text { unter } 3 \\
\text { Jahren }\end{array}$ & $\begin{array}{c}\text { mit } \\
\text { Kindern } \\
\text { unter } 6 \\
\text { Jahren }\end{array}$ & $\begin{array}{c}\text { mit } \\
\text { Kindern } \\
\text { von } 6 \\
\text { bis } \\
\text { unter } 15 \\
\text { Jahren }\end{array}$ & $\begin{array}{c}\text { mit } \\
\text { Kindern } \\
\text { unter } 18 \\
\text { Jahren }\end{array}$ & $\begin{array}{c}\text { mit } \\
\text { Kindern } \\
\text { von } 15 \\
\text { bis } \\
\text { unter } 18 \\
\text { Jahren }\end{array}$ & $\begin{array}{c}\text { mit } \\
\text { Kindern } \\
\text { von } 18 \\
\text { bis } \\
\text { unter } 28 \\
\text { Jahren }\end{array}$ & $\begin{array}{c}\text { mit } \\
\text { Kindern } \\
\text { von } 28 \\
\text { Jahren } \\
\text { und älter }\end{array}$ \\
\hline $15-25$ & 53,4 & 54,5 & 44,4 & 42,0 & 44,0 & 48,8 & 44,4 & - & - & - \\
\hline $25-35$ & 66,8 & 84,2 & 55,6 & 43,6 & 49,7 & 58,7 & 55,6 & 61,0 & 61,0 & - \\
\hline $35-45$ & 69,1 & 81,8 & 65,5 & 41,2 & 47,3 & 63,2 & 64,0 & 68,1 & 69,7 & - \\
\hline $45-55$ & 62,6 & 65,8 & 59,6 & - & 43,6 & 54,5 & 56,8 & 57,9 & 59,6 & 56,3 \\
\hline $55-65$ & 23,2 & 21,4 & 29,2 & - & - & 26,1 & 31,5 & 34,0 & 31,9 & 24,8 \\
\hline $\begin{array}{ll}65 & \text { und } \\
\text { mehr } & \end{array}$ & 1,7 & 1,5 & 4,8 & - & - & - & - & - & 5,9 & 4,6 \\
\hline $\begin{array}{l}\text { Zusam- } \\
\text { men }\end{array}$ & 44,1 & 37,5 & 55,0 & 43,0 & 48,4 & 60,3 & 58,1 & 63,7 & 56,7 & 22,9 \\
\hline
\end{tabular}

Quelle: Angaben des Statistischen Bundesamtes

Tab. C.4: Prozentuale Anteile verschiedener Wochenarbeitszeitklassen für Frauen mit Kindern und Kinderlose zwischen 25 und unter 45 Jahren (1992)

\begin{tabular}{|c|c|c|c|c|c|}
\hline & Insgesamt & Erwerbstätige & $\begin{array}{l}\text { bis einschließ- } \\
\text { lich } 20 \text { Stunden }\end{array}$ & $\begin{array}{l}\text { Wochenarbeitszeit } \\
\text { von } 21 \text { bis ein- } \\
\text { schl. } 35 \text { Stunden }\end{array}$ & $\begin{array}{c}\text { von } 36 \text { Stunden } \\
\text { und mehr }\end{array}$ \\
\hline alle Frauen & 100 & 67,9 & 13,9 & 9,4 & 44,7 \\
\hline ohne Kinder & 100 & 83,4 & 6,1 & 6,7 & 70,6 \\
\hline $\begin{array}{l}\text { mit Kindern unter } \\
18 \text { Jahren }\end{array}$ & 100 & 59,7 & 17,7 & 10,3 & 31,7 \\
\hline
\end{tabular}

Quelle: Angaben des Statistischen Bundesamtes, eigene Berechnungen

unter 45 um knapp ein Viertel $(23,3 \%)$ unter dem Anteil für kinderlose Frauen liegt $(84,2$ Prozent für 25- bis unter 35jährige bzw. 81,8 Prozent für 35- bis unter 45jährige). Dabei liegt die Erwerbsquote von Müttern mit jungen Kindern besonders weit unter derjenigen von Kinderlosen. Frauen mit Kindern unter drei Jahren im Alter von 35 bis 45 Jahren sind mit einer Erwerbsquote von 41,2 Prozent nur etwa halb so häufig erwerbstätig wie gleichaltrige Frauen ohne Kinder (81,8 Prozent). Aus Tabelle C.3 läßt sich eine durchschnittliche Unterbrechungsdauer von etwa vier bis fünf Jahren ableiten. $\mathrm{Zu}$ beachten ist dabei, daß hier nur vollständige

145 Unter Kindern werden nach der Festlegung des Statistischen Bundesamtes hier nur ledige Personen verstanden, die mit ihren Eltern oder einem Elternteil in einem Haushalt zusammenleben. 
Erwerbsunterbrechungen erfaßt sind, weil ein Übergang von Vollzeit- zu Teilzeitbeschäftigung sich nicht in einem Rückgang der Erwerbsquote widerspiegelt. Daneben ist aber auch der Anteil der Teilzeitbeschäftigten bei den Frauen mit Kindern unter 18 Jahren mit 28,0 Prozent wesentlich höher als bei Frauen ohne Kinder (12,8 Prozent, vgl. Tabelle C.4).

Um die durchschnittlichen Unterbrechungskosten, die einer Frau kindbedingt entstehen, festzustellen, werden in einem ersten Schritt die Lebenseinkommensverluste bei einer drei- und einer sechsjährigen vollständigen Unterbrechung für die verschiedenen Bildungsarten gemittelt, ${ }^{146}$ um so Werte zu erhalten, die den Werten bei einer tatsächlichen durchschnittlichen Unterbrechungsdauer von vier bis fünf Jahren möglichst nahe kommen. Multipliziert man diese Zahlen mit der relativen Häufigkeit des entsprechenden Bildungsabschlusses, so erhält man durchschnittliche Unterbrechungskosten (bei vollständiger Unterbrechung) von gut 350.000 DM pro Frau.

Hierzu müssen jetzt noch die Unterbrechungskosten aufgrund eines Übergangs zu einer Teilzeitbeschäftigung addiert werden. Führt man die Berechnung auf die gleiche Art und Weise durch wie bei einer vollständigen Unterbrechung, so ergeben sich bei durchschnittlich knapp drei Jahren Teilzeittätigkeit Lebenseinkommenseinbußen von etwa 150.000 DM aufgrund eines Übergangs zu einer Teilzeitbeschäftigung.

Die gesamten mittleren Lebenseinkommenseinbußen einer Frau aufgrund einer kindbedingten Reduzierung oder Unterbrechung der Erwerbstätigkeit setzen sich zusammen aus 350.000 DM für eine mit der entsprechenden Wahrscheinlichkeit gewichtete vollständige Erwerbsunterbrechung und etwa 150.000 DM für den Übergang zu einer Teilzeitbeschäftigung und betragen daher rund 500.000 DM.

Da Frauen mit Kindern unter 18 Jahren in der hier betrachteten Altersgruppe von 25 bis 45 Jahren durchschnittlich 1,73 minderjährige Kinder zu versorgen haben, betragen die Opportunitätskosten pro Kind rund 290.000 DM.

Dieser Wert deckt sich zumindest der Größenordung nach mit den in anderen Studien erhaltenen Werten. Lampert errechnet beispielsweise den Betreuungsaufwand, indem er anhand von Daten des Statistischen Landesamtes Baden-Württemberg die jährliche Betreuungszeit für Kinder in unterschiedlichen Altersstufen und die Zeit kinderbedingter Haushaltstätigkeiten ermittelt und diese anschließend mit dem Bruttostundenlohn einer Kindergärtnerin multipliziert. Als gesamten Betreuungsaufwand für zwei Kinder bis zu ihrem 18. Lebensjahr erhält er 638.000 DM (1992), wobei er aber den Bruttolohnsatz für die ersten Jahre ungerechtfertigterweise wesentlich niedriger ansetzt als für die folgende Zeit. Für ein Kind in einer ZweiKinder-Familie ergibt sich damit ein Betreuungsaufwand von über $320.000 \mathrm{DM}$, wobei die langfristigen Einkommenseinbußen noch nicht einmal berücksichtigt sind. ${ }^{147}$

146 Die Werte sind der Tabelle C.2 entnommen. Da die Unterschätzung gegenüber der Folgestudie (Galler $1991)$ in Höhe von ungefăhr einem Drittel der Lebenseinkommenseinbußen in etwa dadurch ausgeglichen wird, daß in Tabelle C. 2 Brutto- statt Nettowerte dargestellt werden, braucht keine Anpassung der Zahlen vorgenommen zu werden.

147 Vgl. Lampert (1992). Damit liegt der von Lampert errechnete Wert über den oben erhaltenen 290.000 DM pro Kind, obwohl durch Lampert noch nicht einmal langfristige Einkommenseinbußen erfaßt worden sind. Dies kann darauf zurückgeführt werden, daß Lampert auch die Reduzierung der Freizeit bei Erwerbstätigkeit erfaßt, die sich aus der Kinderbetreuungszeit und der Zeit für kindbedingte Haushaltstätigkeiten ergibt. 
Zwei Faktoren können die Opportunitätskosten eines Kindes über den hier erhaltenen Wert hinaus erhöhen: das Risiko von Arbeitslosigkeit nach einer kindbedingten Erwerbsunterbrechung und die relativ hohe Wahrscheinlichkeit einer Trennung vom Lebenspartner. Letztere soll zunächst untersucht werden.

Bisher ist immer davon ausgegangen worden, daß die Entscheidung über die Zahl der Kinder vom Haushalt getroffen wird. Geht man aber davon aus, daß entweder überwiegend die Frau diese Entscheidung trifft oder sich der Partner mit dem geringeren Kinderwunsch durchsetzt, ${ }^{148}$ so trägt die steigende Scheidungsrate ${ }^{149}$ zum Geburtenrückgang bei. Sie hat nämlich die einer Frau entstehenden Opportunitätskosten noch weiter erhöht, indem durch die höhere Scheidungsrate die Wahrscheinlichkeit gestiegen ist, daß eine Frau nach einer kindbedingten Unterbrechung ihrer Erwerbstätigkeit allein auf ihr dann im Vergleich zur Situation ohne Unterbrechung gesunkenes Einkommenspotential (und/oder meist geringe Unterstützungszahlungen des geschiedenen Mannes) angewiesen ist. Das erwartete zukünftige Einkommen der Frau sinkt also mit zunehmender Scheidungshäufigkeit, wenn sie kindbedingt ihre Erwerbstätigkeit unterbrochen hat, gegenüber der Situation bei ununterbrochener Erwerbskarriere. Zudem streut das tatsächliche zukünftige Einkommen stärker um den Erwartungswert, so daß bei Risikoaversion eine zusätzliche Nutzeneinbuße entsteht. Wie Galler ausführt, dürfte der Grad der Risikoaversion aufgrund der Irreversibilität der Entscheidung für ein Kind eine bedeutende Rolle spielen. ${ }^{150}$

Wenn Frauen durch die gestiegene Wahrscheinlichkeit, später wieder auf ihr eigenes Einkommen angewiesen zu sein, mehr in marktspezifisches Humankapital investieren, kann sich die Tendenz zu höheren Scheidungsraten selbst verstärken, weil dadurch das Verlassen der Ehe erleichert wird. ${ }^{151}$ Die empirische Relevanz dieser Überlegungen wird durch eine Studie von Gaugler u.a. bestätigt, derzufolge ein Drittel aller Frauen als Grund für die Wiederaufnahme einer Erwerbstätigkeit nach einer Unterbrechung auch die größere Unabhängigkeit durch eigenen Verdienst angab. ${ }^{152}$

Ganz ähnlich wie die gestiegene Scheidungsrate erhöht auch das nach einer Unterbrechung besonders hohe Risiko der Arbeitslosigkeit die Opportunitätskosten. In diesem Fall ist die Zeit des Erwerbsausfalls länger als beabsichtigt und die Einbußen an Lebenseinkommen entsprechend höher. Auch hier wirkt bei Risikoaversion die Unsicherheit über die Wiedereinstiegsmöglichkeit in das Erwerbsleben verstärkend. ${ }^{153}$

Zusammenfassend kann festgehalten werden, daß der in dieser Arbeit entwickelte Operationalisierungsansatz für die einer Frau entstehenden Opportunitätskosten über die

Fried/Udry (1979) kommen anhand verschiedener Regressionsanalysen für Weiße in den USA zu dem Ergebnis, daß die Nutzenfunktion der Frau die Fertilitätsentscheidung stark beeinflußt, während die zusätzliche Berücksichtigung der Nutzenfunktion des Mannes wenig zur Erklärung des Fertilitätsverhaltens beiträgt.

Zur Theorie der Scheidung vgl. u.a. Becker/Landes/Michael (1977).

Vgl. Galler (1988) S. 86. Allerdings ist Risikoaversion im Gegensatz zu der in Galler/Ott (1987) S. 404 vertretenen Meinung keine notwendige Voraussetzung für einen negativen Effekt zunehmender Instabilität der Ehe auf die Kinderzahl, weil schon die steigenden Opportunitătskosten durch die Minderung des erwarteten Einkommens zur Begründung eines solchen Zusammenhangs ausreichen.

Vgl. Willis (1987) S. 74. Als weiteren Grund für diesen Selbstverstärkungseffekt führt Willis die gestiegene Zahl der potentiellen Partner für eine weitere Heirat an.

Vgl. Gaugler u.a. (1984) S. 62 (zitiert nach Galler (1988) S. 86).

Vgl. Vottlerer (1987) S. 20. 
Berechnung der Lebenseinkommensverluste, die sich aus einer kindbedingten Unterbrechung bzw. Reduzierung der Erwerbstätigkeit ergeben, zu ähnlichen Ergebnissen führt, wie die von Lampert verwendete Operationalisierungsvariante, die auf eine Messung und Bewertung der für Kinder aufgewandten Zeit abstellt. Im Rahmen der hier gewählten Vorgehensweise wurde zunächst ein Humankapitalmodell vorgestellt, das die Berechnung potentieller Lebenseinkommen für Frauen verschiedenen Bildungsstandes erlaubt. Anschließend wurden die Lebenseinkommen für verschiedene Erwerbsverläufe mit einer Unterbrechung bzw. Reduzierung der Erwerbstätigkeit anhand des Humankapitalmodells bestimmt. Die Differenz dieser Lebenseinkommen zu dem bei ununterbrochener Vollerwerbstätigkeit erzielbaren Lebenseinkommen wurden als die einer Frau aus einer Erwerbsunterbrechung entstehenden Opportunitätskosten gedeutet. Um von den einer Frau entstehenden Opportunitätskosten zu den Opportunitätskosten eines Kindes zu gelangen, wurde die durchschnittliche Unterbrechungslänge anhand von Erwerbsquoten festgestellt und durch die durchschnittlich von einer Frau zu betreuende Kinderzahl dividiert. Als Ergebnis läßt sich festhalten, daß die Opportunitätskosten pro Kind mit der Dauer und Art einer Erwerbsunterbrechung durch einen Elternteil sowie dessen Bildungsstand stark schwanken. Der hier errechnete mittlere Wert von grob 300.000 DM pro Kind dürfte aus den angeführten Gründen - Nichtberücksichtigung immaterieller Opportunitätskosten, systematische Unterschätzung der Lebenseinkommensverluste durch die Vorgehensweise von Galler, Scheidungshäufigkeit, hohes Risiko von Arbeitslosigkeit nach einer Unterbrechung - die tatsächlichen indirekten Kosten eines Kindes wohl noch unterschätzen.

\subsubsection{Bedeutung der Opportunitätskosten von Kindern für die Wirkungs- weise des Kinderlastenausgleichs}

Aus den theoretischen und empirischen Ergebnissen bezüglich der indirekten Kosten lassen sich wichtige Schlußfolgerungen für Ansatzpunkte staatlicher Instrumente ziehen. Die Höhe der indirekten Kosten in der Größenordnung von bis zu mehreren hunderttausend Mark pro Kind schließt eine Kompensation über staatliche Leistungen aus. Nun ist zwar eine vollständige Kompensation auch nicht erforderlich, denn es genügt, die indirekten Kosten soweit zu reduzieren, daß die marginalen Kosten insgesamt kleiner werden als der marginale Konsumnutzen eines Kindes, aber die Zahlen machen deutlich, da $\beta$ ein derartiges Programm kaum finanzierbar wäre, auch wenn nur ein Teil dieser Kosten ersetzt werden sollte. ${ }^{154}$ Wenn der Reallohn in Zukunft weiter steigt, würden die Kosten eines solchen Programms zudem weiter ansteigen. Daher müssen die staatlichen Maßnahmen in erster Linie auf die Vermeidung von Opportunitätskosten von Kindern gerichtet sein, indem die Vereinbarkeit von Erwerbsarbeit und Kinderbetreuung verbessert wird. ${ }^{155}$ Dieser Gedanke wird in Unterkapitel 4.1 wieder aufgegriffen.

154 In Abschnitt 4.2.1 wird errechnet, daß allein die Kompensation des Anstiegs der Opportunitătskosten von etwa 1960 bis Ende der achtziger Jahre ungefähr 290 Mrd DM jährlich (in Preisen von 1992) erfordern würde. 


\subsubsection{Der Zusammenhang zwischen Quantität und Qualität von Kindern}

Neben steigenden Opportunitätskosten von Kindern wird von Vertretern der New Home Economics der Zusammenhang zwischen Quantität und Qualität bei der Fertilitätsentscheidung als Erklärung für den drastischen Geburtenrückgang herangezogen. Dieser Zusammenhang soll nach einigen allgemeinen Vorüberlegungen zunächst wieder einer theoretischen Analyse unterzogen werden, ehe auf die Empirie und die Bedeutung für den Kinderlastenausgleich eingegangen wird.

Der Erklärungsansatz über die Interdependenz von Kinderquantität und -qualität geht auf Becker (1960) zurück und gründet auf der getrennten Aufnahme von Kinderzahl und Kinderqualität in die Nutzenfunktion (C.l). Faßt man alle übrigen Basisgüter zu dem aggregierten Basisgut $(Z)$ zusammen, so erhält die Nutzenfunktion die Gestalt

$$
\text { C.14) } U=U(n, q, Z) \text {. }
$$

Hier bezeichnet $n$ die Kinderzahl und $q$ die Kinderqualität. Während in der traditionellen mikroökonomischen Nachfragetheorie implizit unterstellt wird, daß ein Gut eine homogene Qualität besitzt bzw. unterschiedliche Qualitäten als unterschiedliche Güter behandelt werden, wird hier also neben der Quantität die Qualität ausdrücklich als zweite Bestimmungsgröße in die Nutzenfunktion aufgenommen. Dieses Vorgehen ermöglicht die Berücksichtigung des Zusammenhangs zwischen diesen beiden Nutzenargumenten, zugleich entsteht aber das schwierige Problem der Operationalisierung der Qualität. Becker definiert die Qualität als "expenditure on each child"156, wobei er unter "expenditure" nicht nur monetäre Kosten, sondern auch Opportunitätskosten aufgrund alternativer Verwendungsmöglichkeiten der Zeit versteht. Qualität wird also nicht allein mit Hilfe von Marktgütern, sondern auch durch den Inputfaktor Haushaltszeit erstellt. Allerdings führt Becker abweichend von der obigen Definition auch fixe Kosten pro Kind ein, die er als "psychic and monetary expenditures on children that are largely independent of quality" 157 bezeichnet. Desweiteren spricht er von Ausgaben für Kinder, die aufgrund von Kuppelproduktion weitgehend unabhängig von der Kinderzahl sind und führt als Beispiel Kleidung an, die von jüngeren Kindern weiterverwendet wird, wenn die älteren aus ihr herauswachsen. Implizit geht er damit von der oben zitierten engen, inputbezogenen Auslegung des Qualitätsbegriffs ab und konzediert, daß die Qualität als Ergebnis der Produktion eigentlich auch outputbezogen gemessen werden müßte.

Die Vorgehensweise Beckers kann in Analogie zu dem allgemein anerkannten Verfahren zur Bewertung unentgeltlich abgegebener staatlicher Leistungen in der volkswirtschaftlichen Gesamtrechnung (VGR) gesehen werden. In beiden Fällen ist die direkte Messung des Outputs unmöglich, da die bereitgestellten Güter nicht am Markt gehandelt werden und sich daher kein Marktpreis bildet, in dem die Wertschätzung der Nachfrager für die erstellte Leistung zum Ausdruck kommt. Anstelle der nicht meßbaren Outputgröße wird deshalb auf die zur Erstellung dieses Outputs nötigen Kosten zurückgegriffen. Die eigentlich interessierende Outputgröße wird also durch eine Inputgröße approximiert. Im Falle der Qualität der Kinder müßte eigentlich das Erziehungsergebnis als Outputgröße herangezogen werden, da eine

156 Becker (1991) S. 137.

157 Becker (1991) S. 149. 
Operationalisierung des Erziehungsergebnisses aber - wie oben ausgeführt - unmöglich ist, wird stattdessen auf die Kosten seiner Erstellung zurückgegriffen. ${ }^{158}$

Im Fall der Qualität von Kindern ist v.a. von Bedeutung, daß ein empirisch beobachteter Kostenanstieg pro Kind nicht unbedingt auf eine Qualitätsverbesserung gründen und daher zu einem höheren Output bzw. zu einer Nutzensteigerung führen muß. So kann ein Kostenanstieg z.B. auch einfach auf einen Preisanstieg für Spielzeug zurückzuführen sein (der Anstieg solcher direkter Kinderkosten wird in 3.2.3 untersucht). Es muß also unterschieden werden, ob es sich um einen Kostenanstieg bei konstanter Qualität (Erhöhung der Kosten $p_{c}$ einer Qualitätseinheit) oder die Kosten einer Qualitätserhöhung (nutzenstiftende Mehrausgaben, also eine Erhöhung von $q$ ) handelt. ${ }^{159}$ Im ersten Fall handelt es sich um einen Preisanstieg, der einen entsprechenden Substitutionsprozeß von anderen Basisgütern für Kinder nach sich zieht. Der zweite Fall dagegen ist nicht Ausgangspunkt, sondern Ergebnis einer Entscheidung, nämlich der Entscheidung mehr pro Kind auszugeben, um so die Qualität zu erhöhen. Unter einer Qualitätserhöhung soll hier demnach nur der preisbereinigte Kostenanstieg verstanden werden.

Wie oben erwähnt, muß auch ein preisbereinigter Anstieg der monetären Kosten nicht unbedingt einen Anstieg des Erziehungsergebnisses zur Folge haben. Vielmehr kann er auch auf einen Substitutionsprozeß bei der Erstellung des Erziehungsergebnisses infolge gestiegener Zeitkosten zurückzuführen sein. In diesem Fall muß der verminderte Einsatz des Inputfaktors Zeit bei der Erstellung des Erziehungsergebnisses mit einem höheren Input an Marktgütern kompensiert werden, um insgesamt das Outputniveau halten zu können. Würde man den Qualitätsbegriff auf monetäre Kosten einschränken, bliebe dieser Zusammenhang unberücksichtigt.

\subsubsection{Theoretische Analyse des Zusammenhangs zwischen Quantität und Qualität von Kindern}

Um aufgrund theoretischer Überlegungen aus der getrennten Berücksichtigung von Qualität und Quantität im Rahmen des Modells einen negativen Zusammenhang zwischen Einkommenserhöhung und Kinderzahl ableiten zu können, müssen verschiedene Annahmen erfüllt sein, auf die hier kurz eingegangen werden soll.

Zunächst wird angenommen, daß die Eltern das gleiche Qualitätsniveau für alle Kinder wählen. Außerdem wird postuliert, daß die Einkommenselastizität der Kinderqualität größer ist als die Einkommenselastizität der Kinderzahl. Bei einem Einkommensanstieg wird also ceteris paribus die Kinderqualität prozentual stärker steigen als die Kinderzahl, analog einem Zusammenhang, der sich für langlebige Konsumgüter empirisch nachweisen läßt. Desweiteren

158 Obwohl diese Vorgehensweise legitim ist, um überhaupt sinnvoll mit dem Qualitătsbegriff hantieren zu können, dürfen die daraus erwachsenden Probleme nicht übersehen werden. Bei der Betrachtung von Bestandsgrößen ergeben sich Fehlerquellen, weil das Outputniveau sowohl ober- als auch unterhalb des gemessenen Inputniveaus liegen kann (je nachdem ob die Wertschöpfung positiv oder negativ ist). Aber auch bei der Betrachtung von Verănderungsraten ergeben sich Probleme und zwar immer dann, wenn sich die Outputgröße nicht proportional zur gemessenen Inputgröße verändert - im Beispiel der VGR also beispielsweise dann, wenn aufgrund zunehmender Ineffizienz im öfentlichen Sektor zwar die Staatsausgaben steigen, nicht aber die Bereitstellung öffentlicher Leistungen. 
wird unterstellt, daß die beiden Nutzenargumente $n$ und $q$ in einer substitutionalen Beziehung stehen. ${ }^{160}$

Aus diesen Annahmen ergibt sich jetzt eine nicht-lineare Budgetbeschränkung, denn die Kosten der Kinder sind das Produkt aus Kinderzahl, Qualität pro Kind und den (hier als konstant angenommenen) Kosten einer Qualitätseinheit. Mit $S$ wird das implizite Einkommen bezeichnet, daß sich aus dem monetären Einkommen und der mit dem Lohnsatz bewerteten Freizeit zusammensetzt. ${ }^{161}$ Die Budgetrestriktion lautet also

$$
\text { C.15) } \quad p_{c} n q+\pi_{z} Z=S \text {. }
$$

Die multiplikative Verknüpfung von $n$ und $q$ ist das zentrale Element der folgenden Analyse, weil sie dazu führt, daß die Schattenpreise der Qualität und Quantität voneinander abhängen. Wenn man die Nutzenfunktion $C .14$ ) unter der Budgetrestriktion $C .15$ ) maximiert, erhält man nämlich die folgenden notwendigen Optimalitätsbedingungen:

$$
\begin{aligned}
& \text { C.16a) } \frac{\partial U}{\partial n}=: M U_{n}=\lambda p_{c} q=\lambda \omega_{n} \\
& \text { C.16b) } \frac{\partial U}{\partial q}=: M U_{q}=\lambda p_{c} n=\lambda \omega_{q} \\
& \text { C.16c) } \frac{\partial U}{\partial Z}=: M U_{z}=\lambda \pi_{z}
\end{aligned}
$$$$
\omega_{n}: \text { Schattenpreis von } n
$$$$
\omega_{q}: \text { Schattenpreis von } q
$$$$
\pi_{\mathrm{z}}: \text { Schattenpreis von } Z
$$

Die Schattenpreise $\omega_{n}$ und $\omega_{q}$ bezeichnen hier nicht wie bisher die Preise eines Basisgutes unter Berücksichtigung des Inputfaktors Zeit, sondern die Preise der Basisgüter Kinderzahl und Kinderqualität unter Beachtung ihres multiplikativen Zusammenhangs. Um eine Verwechslung auszuschließen, wird deshalb das Symbol $\omega$ anstelle des früher bebrauchten Symbols $\pi$ für die Schattenpreise von Kinderquantität und -qualität verwendet.

Der Schattenpreis der Kinderzahl $\left(\omega_{n}\right)$ steigt also mit der Kinderqualität. Bei einem niedrigen Qualitätsniveau muß für das nächste Kind wenig, bei einem hohen entsprechend mehr ausgegeben werden, um das Qualitätsniveau der Geschwister zu erreichen. Der Schattenpreis der Kinderqualität $\left(\omega_{q}\right)$ steigt mit der Kinderzahl. Bei einem Kind fallen die höheren Ausgaben nur einmal an, bei vier Kindern dagegen entsprechend vierfach, so daß eine gleichgroße Qualitätserhöhung entsprechend teurer ist. Die Schattenpreise sind also nicht exogen vor-

160 Bei einer komplementären Beziehung würde eine kompensierte Preiserhöhung der Kinderquantität auch zu einer Abnahme der Qualität und damit zu einer Zunahme des Konsums sonstiger Basisgüter führen. Der Lebensstandard der Eltern müßte dann steigen, während derjenige der Kinder sinkt. Eine solche Abkoppelung des Lebensstandards der Kinder von dem der Eltern ist aber empirisch nicht zu beobachten (so auch Zameck-Glyscinski (1985) S. 173). Vottlerer (1987) S. 67 kommt beispielsweise auf Grundlage einer in Baden-Württemberg durchgeführten ausgedehnten empirischen Studie zu dem Ergebnis, daß "... von einer Zunahme des Haushaltsnettoeinkommens ... Kinder und Eltern nahezu in gleichem Maße" profitieren (Unterstreichung im Original). Die Annahme einer substitutionalen Beziehung erscheint somit empirisch begründet.

Vgl. zu einer Darstellung des folgenden Zusammenhangs beispielsweise Becker (1991) S. 145ff. 
Abb.C.6: Der Zusammenhang zwischen Quantität und Qualität von Kindern

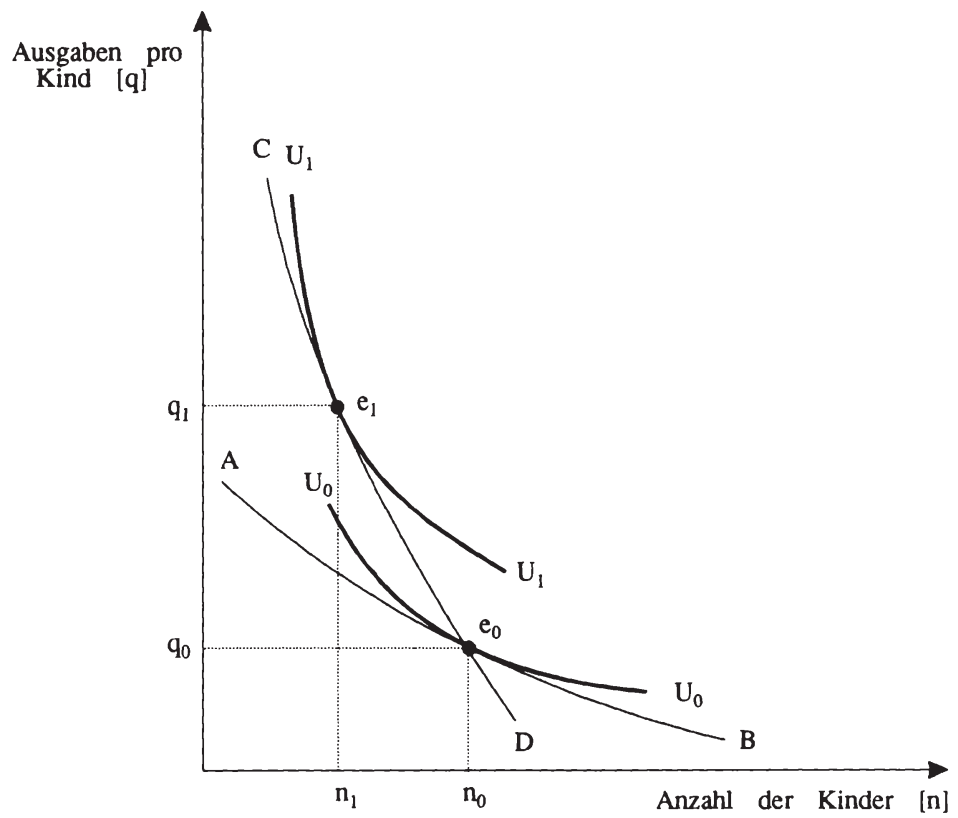

Quelle: Modifiziert nach Becker (1991) S. 147

gegeben wie Preise in der traditionellen Analyse, sondern werden durch die Entscheidungen des Haushalts beeinflußt. ${ }^{162}$

Graphisch läßt sich der Zusammenhang zwischen Kinderqualität und -quantität wie in Abb. C.6 darstellen. Aufgrund der multiplikativen Verknüpfung von $n$ und $q$ ist die Budgetbeschränkung konvex gekrümmt. $\mathrm{Da}$ auch die Indifferenzkurven wie üblich konvex verlaufen, erhält man ein inneres Optimum mit positiven Werten für $n$ und $q$ nur, wenn die Krümmung der Indifferenzkurve stärker ist als die der Budgetgeraden. Da die Krümmung der Indifferenzkurve mit abnehmender Substituierbarkeit von Kinderqualität für Kinderquantität zunimmt, ergibt sich ein inneres Optimum ausschließlich, wenn Qualiät und Quantität keine allzu engen Substitute sind. ${ }^{163}$ Findet vom Ausgangsgleichgewicht $e_{0}$ im Tangentialpunkt der Budget-

162 Das Schatteneinkommen $(R)$ erhält man aus der Summe der mit ihren Schattenpreisen multiplizierten Argumente der Nutzenfunktion:

$$
R=\omega_{q} q+\omega_{n} n+\pi_{z} Z=p_{c} n q+p_{c} q n+\pi_{z} Z=S+p_{c} q n
$$

163 Algebraisch lautet die Bedingung für den AusschluB einer Ecklösung $\sigma_{n q}<\frac{1-k_{z} \sigma_{z}}{1-k_{z}}$, wobei mit $\sigma_{n q}$ die Substitutionselastizităt zwischen $n$ und $q$ und mit $\sigma_{z}$ sowohl die Substitutionselastizităt zwischen 2 
gerade $A B$ und der Indifferenzkurve $U_{0}$ ausgehend eine kompensierte Erhöhung des relativen Schattenpreises der Kinderzahl statt, so daß man als neue Budgetgerade $C D$ erhält, dann liegt der neue Optimalpunkt bei $e_{l}$.

Aufgrund der Annahme einer geringeren Einkommenselastizität der Kinderzahl als der Kinderqualität ist jetzt ein negativer Zusammenhang zwischen Einkommen und Fertilität ableitbar: ${ }^{164}$ Steigt nämlich das Einkommen, so wird zunächst die Kinderzahl zwar steigen, aber prozentual weniger stark als die Ausgaben pro Kind. Infolge der relativ stärker gestiegenen Qualität steigt der Schattenpreis der Kinderzahl relativ zu dem Schattenpreis der Kinderqualität, wie aus den Gleichungen C.16a) und C.16b) ersichtlich ist. Damit wird der Haushalt veranlaßt, relativ billiger gewordene Qualität für relativ teurer gewordene Quantität zu substituieren. Sofern dieser in bezug auf die Quantität negative Substitutionseffekt größer ist als der positive Einkommenseffekt, erhält man das bemerkenswerte Ergebnis, daß die Fertilität sinkt, wenn das Einkommen zunimmt, obwohl der ursprüngliche Einkommenseffekt wegen der Superiorität des Gutes Kinderzahl positiv ist.

In Abb. C.7 ist dieser Zusammenhang graphisch dargestellt. Das Ausgangsgleichgewicht $E_{0}$ liegt im Berührpunkt der Budgetbeschränkung $C_{0}$ mit der Indifferenzkurve $U_{0}$. Das ursprüngliche Verhältnis der Schattenpreise $\omega_{n} / \omega_{q}$ ist durch die Steigung der Tangente $A A$ gegeben. Durch den exogenen Einkommensanstieg verlagert sich die Budgetbeschränkung nach $C_{1}$. Der neue Tangentialpunkt $\left(E_{0}\right)$ von Indifferenzkurve $\left(U_{0}^{\prime}\right)$ und unverändertem Schattenpreisverhältnis $\left(A^{\prime} A^{\prime}\right)$ liegt aufgrund der höheren Einkommenselastizität der Qualität links vom Strahl $0 a$ auf der neuen Budgetkurve $C_{l}$. Die durch diese Änderung des Verhältnisses von $q$ zu $n$ ausgelöste Änderung des Schattenpreisverhältnisses kommt in der betragsmäßig größeren Steigung der Tangente $B B$ gegenüber $A A$ bzw. $A^{\prime} A^{\prime}$ zum Ausdruck. Der neue Optimalpunkt $E_{l}$ liegt deshalb notwendigerweise links von $E_{0^{\prime}}$. Insgesamt sinkt die Kinderzahl durch die Einkommenserhöhung von $n_{0}$ auf $n_{l}$. Dieses Ergebnis ist allerdings nicht zwingend, denn ob der Substitutionseffekt - wie hier dargestellt - tatsächlich größer ist als der Einkommenseffekt ist allein auf Grundlage theoretischer Überlegungen nicht feststellbar.

Neben dieser nachfrageseitigen Begründung mittels der Annahme einer größeren Einkommenselastizität der Kinderqualität als der Kinderquantität kann die Zunahme des Verhältnisses von Qualität zu Quantität auch angebotsseitig durch den im vorangehenden Abschnitt untersuchten Anstieg der Opportunitätskosten eines Kindes erklärt werden. Ist nämlich die Erstellung der Qualität weniger zeitintensiv als die der Quantität, ${ }^{165}$ so steigt der Schattenpreis der Quantität aufgrund einer Erhöhung der Opportunitätskosten (Zeitkosten) relativ mehr als der Schattenpreis der Qualität. Auch hier wird dieser Primäreffekt wieder durch die oben beschriebenen Sekundäreffekte verstärkt. ${ }^{166}$

und $n$ als auch die zwischen $z$ und $q$ bezeichnet ist. Mit $k_{z}$ ist der Anteil der Ausgaben für die anderen Basisgüter an dem Schatteneinkommen $R$ bezeichnet. Wenn also andere Güter gute Substitute für Kinderzahl und -qualität sind, so dürfen $n$ und $q$ keine engen Substitute sein, wenn eine Ecklösung ausgeschlossen sein soll. Vgl. Becker (1991) S. 146.

Eine etwas andere Darstellung findet sich bei Zameck-Glyscinski (1985) S. 169ff.

Eine gegenüber der Quantität geringere Zeitintensität der Qualität ist wahrscheinlich, da ein zusätzliches Kind zumindest in seinen ersten Lebensjahren viel Zeit der Eltern beansprucht, eine Erhöhung der Qualităt dagegen ist eher auch durch eine Erhöhung der monetären Kinderkosten möglich, z.B. indem Nachhilfestunden bezahlt werden.

Zameck-Glyscinski (1985) S. 210ff. weist darauf hin, daß sich aus der Substitution hin zu mehr Qualität bei steigenden Opportunitätskosten eine säkular sinkende Geburtsrate ableiten läßt. Die höhere Qualität 
Abb. C.7: Wirkung einer Einkommenserhöhung auf die Kinderzahl unter Berücksichtigung des Zusammenhangs zwischen Kinderquantität und -qualität ${ }^{167}$

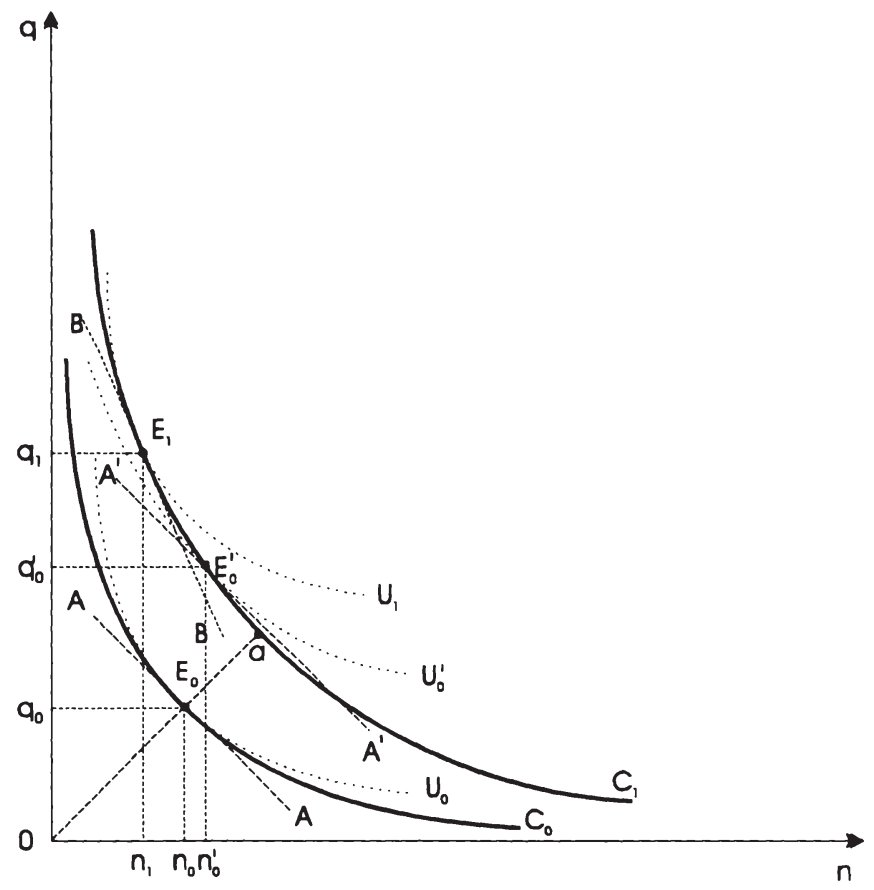

Unabhängig davon, ob die primäre Änderung der Relation von Kinderquantität zu -qualität nachfrageseitig durch eine höhere Einkommenselastizität der Qualität als der Quantität oder angebotsseitig durch weniger zeitintensive Herstellung der Qualität erzeugt wird, kommt es also durch den hier erläuterten, auf induzierte Schattenpreisänderungen zurückzuführenden Verstärkungsprozeß zu einer Erhöhung der Wirkung des ursprünglichen Effektes.

kann sich nämlich einmal direkt in besserer Ausbildung der Nachkommen niederschlagen, so daß sich deren Zeitkosten wegen besserer Verdienstmöglichkeiten im fruchtbaren Lebensalter erhöhen, sie daher wieder die Qualität zu Lasten der Kinderzahl erhöhen und es so zu von Generation zu Generation sinkender Kinderzahl und steigender Qualităt kommt. Aber auch wenn die steigende Qualität sich in besserer Ernährung und Gesundheitsvorsorge niederschlägt, kommt es letztlich zu dieser fortlaufenden Verringerung der Kinderzahl. Aufgrund der geringeren Mortalität steigt dann nämlich die erwartete Rendite von Investitionen in die Ausbildung der Kinder, so daß diese Investitionen erhöht werden und auch hier eine Generation später höhere Opportunitätskosten von Kindern resultieren.

167 In einer vergleichbaren Graphik von Zameck-Glyscinski (1985) S. 170 wird der Punkt $E_{0}$ ' fälschlicherweise oberhalb der Budgetkurve $C_{l}$ auf der Indifferenzkurve $U_{l}$ eingezeichnet. Dieser Punkt kann aber, da er außerhalb des Bereichs der möglichen Konsumpunkte liegt, nicht, wie von der Autorin behauptet, der neue Optimalpunkt bei unveränderten relativen Kosten sein. Darüber hinaus wird in ihrer Darstellung keine Reduktion der Kinderzahl als Konsequenz des Einkommensanstiegs erreicht. 
Die Bedeutung des Zusammenhangs zwischen Kinderqualität und -quantität für die Fertilitätsentwicklung wird allerdings wieder relativiert, wenn man sich den unter 3.1.1 beschriebenen grundlegenden Ansatz dieser Studie nochmals vor Augen hält. Danach wird die Entscheidung für ein weiteres Kind auf der Grundlage einer Abwägung seines erwarteten Nutzens und seiner erwarteten Kosten getroffen. Die Substitution von Quantität zu Qualität führt nun zu einem starken Anstieg der Kosten eines weiteren Kindes: Es muß viel Zeit und Geld in seine Erziehung und Ausbildung investiert werden. Aber gleichzeitig wird sich der Konsumnutzen des Kindes erhöhen, denn die gestiegene Qualität geht als Argument in die Nutzenfunktion ein. ${ }^{168}$ Durch die höhere Qualität wird der Nutzen eines weiteren Kindes für die Eltern zunehmen. Der Gesamtnutzen aller Kinder für die Eltern ändert sich zwar kaum, aber statt mit vielen Kindern geringer Qualität wird dieses Gesamtnutzenniveau jetzt mit wenig Kindern hoher Qualität erreicht. Es findet also auf der Basis einer stabilen Nutzenfunktion eine Verlagerung vom Argument $n$ zum Argument $q$ statt. In Abb. C.7 kommt diese Verlagerung in der Bewegung von $E_{0}^{\prime}$ zu $E_{l}$ zum Ausdruck: die Kinderzahl verringert sich von $n_{0}^{\prime}$ auf $n_{l}$, die Qualität steigt von $q_{0}^{\prime}$ auf $q_{1}$ und das Nutzenniveau steigt geringfügig von $U_{0}^{\prime}$ auf $U_{l}$. Der Nutzen eines (weiteren) Kindes ist dann aus zwei Gründen gestiegen: Erstens steigt nach dem ersten Gossen'schen Gesetz mit abnehmender Kinderzahl der Grenznutzen eines Kindes und zweitens, und das ist hier entscheidend, steigt der Grenznutzen aufgrund der Zunahme der Qualität.

\subsubsection{Empirische Bedeutung der Substitution von Kinderquantität durch Kinderqualität}

Wie groß ist nun die empirische Bedeutung der Substitution von Kinderquantität durch Kinderqualität für den säkularen Rückgang der Fertilitätsraten in den Industriestaaten? Leider läßt sich diese Frage noch nicht einmal annähernd beantworten, denn zu viele statistische Probleme stehen einer exakten Abschätzung im Wege. Schon die outputbezogene Operationalisierung von "Kinderqualität" ist - wie oben ausgeführt - kaum möglich und selbst der theoretisch anfechtbaren inputbezogenen Messung sind bei dem hier notwendigen Vergleich über längere Zeiträume aufgrund der schlechten Datenlage enge Grenzen gesetzt. Hinzu kommt das Problem der Isolierung von anderen Einflüssen auf Kinderzahl und -qualität.

Man ist deshalb bei der Beurteilung der Tragweite des Zusammenhangs zwischen Quantität und Qualität von Kindern auf die Betrachtung einzelner Fallbeispiele angewiesen. Dies ist insofern unbefriedigend als die Erkenntnisse nur bedingt verallgemeinerungsfähig sind und zudem den oben angeführten statistischen Problemen unterliegen.

Becker benutzt zur Illustration des negativen Zusammenhangs zwischen Kinderqualität und -quantität die starke Zunahme der Schulausbildung bei gleichzeitigem starken Rückgang der Fertilität. In den USA sind zwischen 1950 und 1960 beispielsweise die Geburtenraten um 38\% gefallen, während der Anteil der Personen zwischen 25 und 34, die die high school abgeschlossen haben, um $33 \%$ gestiegen ist. ${ }^{169}$ Als weiterer Hinweis lassen sich die hohen Humankapital-

168 Die oben getroffene Annahme eines konstanten Konsumnutzens eines Kindes gilt nur für Kinder gleicher Qualität und Parität, d.h. gleicher Zahl älterer Geschwister. Mit zunehmender Qualität wird der Konsumnutzen wie oben erläutert ebenso zunehmen, wie er nach dem Gossen'schen Gesetz mit zunehmender Kinderzahl abnehmen wird.

Vgl. Becker (1991) S. $150 f$. 
investitionen von Juden anführen, die mit im Vergleich zur restlichen Bevölkerung geringeren Geburtenraten einhergingen.

Auch die wenigen empirischen Studien zu dieser Fragestellung kommen fast durchgehend zu dem Ergebnis einer negativen Beziehung zwischen Qualität und Quantität. ${ }^{170}$

\subsubsection{Bedeutung des Zusammenhangs zwischen Kinderquantität und -quali- tät für die Wirkungsweise des Kinderlastenausgleichs}

Die Bedeutung des Zusammenhangs zwischen Kinderquantität und Kinderqualität für die Wirkungsweise des Kinderlastenausgleichs und anderer bevölkerungspolitischer Maßnahmen soll hier anhand von Instrumenten beleuchtet werden, die direkt auf die Kinderzahl $(n)$ bzw. die -qualität $(q)$ wirken. ${ }^{171}$

Als Beispiel für eine Maßnahme, die in ihrer Primärwirkung die Kinderqualität beeinflußt, mag die kostenlose staatliche Bereitstellung von Schul- und weiterführender Ausbildung oder auch die verschiedenen Leistungen im Rahmen des Bundesausbildungsförderungsgesetzes (BAFöG) dienen. Durch diese Transfers kann ein gewisses Qualitätsniveau ohne bzw. mit geringen monetären Kosten für die Eltern erreicht werden. Erst wenn die Qualität darüber hinaus gesteigert werden soll, müssen die Eltern auf private Mittel zurückgreifen. Durch die für die Eltern kostenlose Qualitätserhöhung werden die marginalen Kosten der Qualität über die durchschnittlichen angehoben. Die Kosten einer Qualitätseinheit können daher nicht mehr als konstant angesehen werden, sondern steigen mit zunehmender Qualität, weshalb die Budgetgleichung nun wie folgt zu formulieren ist:

$$
\text { C.17) } S=p_{c}(q) q n+\pi_{z} Z \text {. }
$$

Maximiert man die Nutzenfunktion $C .14$ ) unter der Nebenbedingung $C .17$ ), so erhält man jetzt folgende notwendige Optimalitätsbedingungen für $n$ und $q$ :

$$
\begin{aligned}
& M U_{n}=\lambda p_{c} q=\lambda \omega_{n} \\
& M U_{q}=\lambda\left(p_{c} n+\frac{\partial p_{c}}{\partial q} n q\right)=\lambda p_{c} n\left(1+\varepsilon_{p q}\right)=\lambda \omega_{q}
\end{aligned} \quad \text { mit } \quad \varepsilon_{p q}=\frac{\partial p_{c}}{\partial q} \frac{q}{p_{c}}
$$

Durch Division erhält man

C.18) $\frac{M U_{n}}{M U_{q}}=\frac{\omega_{n}}{\omega_{q}}=\frac{q}{n} \frac{1}{\left(1+\varepsilon_{p q}\right)}$.

\footnotetext{
170 Vgl. beispielsweise die Studien von De Tray (1978) und Rosenzweig/Wolpin (1980).

171 Der folgende Abschnitt stützt sich teilweise auf Becker (1991) S. 149ff.
} 
Das Verhältnis der Schattenpreise $\omega_{n} / \omega_{q}$ sinkt mit steigendem $\left(1+\varepsilon_{p q}\right)$, d.h. mit einem steigenden Verhältnis von marginalen zu durchschnittlichen Kosten der Qualität. ${ }^{172}$ Eine kostenlose Bereitstellung von Ausbildung hat also über die Erhöhung des Verhältnisses von marginalen zu durchschnittlichen Kosten der Qualität eine Senkung des relativen Preises der Quantität zur Folge. Der dadurch ausgelöste Substitutionseffekt von Qualität zu Quantität führt zu einem weiteren Rückgang des relativen Preises der Quantität, so daß sich im Endergebnis eine beachtliche Zunahme der Kinderzahl auch für relativ geringe Änderungen von $\varepsilon_{p q}$ ergeben kann. Dieses Ergebnis mag auf den ersten Blick paradox erscheinen, denn schließlich wird ja die Qualität subventioniert, aber es wird auch intuitiv einsichtig, wenn man bedenkt, daß hier nur die privaten Kosten und damit natürlich auch nur die privat bereitgestellte Qualität betrachtet werden. Die Qualität wird nicht insgesamt sinken, sondern aus privaten Mitteln finanzierte Qualität wird lediglich durch aus öffentlichen Mitteln finanzierte Qualität ersetzt. Obwohl das Qualitätsniveau insgesamt mindestens konstant bleibt, müssen die Eltern dadurch weniger für jedes Kind ausgeben. Der Schattenpreis der Quantität sinkt also, was die Zunahme der Kinderzahl erklärt.

Zur Illustration von Maßnahmen, die zunächst auf die Kinderzahl einwirken, sei hier ein Anstieg der Kinderzahl betrachtet, wie er beispielsweise durch die Erhöhung ungeplanter ${ }^{173}$ Geburten durch eine restriktivere Abtreibungsgesetzgebung ausgelöst werden kann. Die höhere Kinderzahl erhöht den Schattenpreis für die Kinderqualität, denn die Kinderkosten fallen jetzt für mehr Kinder an. Es kommt zu einem Substitutionseffekt mit dem Ergebnis, daß die Kinderqualität sinkt, die Zahl aber weiter steigt. Dadurch steigt der Schattenpreis der Qualität weiter, während der Schattenpreis der Kinderzahl $n$ aufgrund der niedrigeren Ausgaben pro Kind sinkt. In der nächsten Runde kommt es daher zu einer Fortsetzung des Substitutionseffektes. Wenn die Krümmung der Indifferenzkurve kleiner ist als die der Budgetgeraden, setzt sich dieser Prozeß fort, bis eine Ecklösung erreicht ist. Anderenfalls wird ein neues inneres Gleichgewicht erreicht, das umso weiter von dem alten entfernt liegt, je engere Substitute Quantität und Qualität sind. Wegen dieser Wechselwirkung kann also auch hier schon ein kleiner exogener Anstieg der Kinderzahl im Endeffekt einen drastischen Quantitätsanstieg und Qualitätsrückgang verursachen.

Als Fazit dieses Unterabschnitts läßt sich festhalten, daß die Substitution von Quantität durch Qualität aufgrund des iterativen Verstärkungseffektes über die Änderung der Schatten-

172 Der Term $\left(1+\varepsilon_{p q}\right)$ läßt sich umformen zu

$$
1+\varepsilon_{p q}=1+\frac{\partial p_{c}}{\partial q} \frac{q}{p_{c}}=1+\frac{\frac{\partial p_{c}}{\partial q}}{\frac{p_{c}}{q}}=\frac{\frac{p_{c}}{q}+\frac{\partial p_{c}}{\partial q}}{\frac{p_{c}}{q}}=\frac{p_{c}+\frac{\partial p_{c}}{\partial q} q}{p_{c}},
$$

so daß schließlich im Zähler die marginalen Kosten und im Nenner die durchschnittlichen Kosten der Kinderqualităt stehen.

173 Wie oben ausgeführt gibt es strenggenommen keine ungeplanten Kinder, da auch die Wahl unsicherer Verhütungsmethoden als Ergebnis eines rationalen Entscheidungsprozeßes betrachtet werden kann. Bessere Verhütungsmittel führen aber durch eine Senkung der materiellen und sonstigen Kosten einer Schwangerschaftsvermeidung zu geringeren Kinderzahlen, denn im Optimum entsprechen die Grenzkosten der Empfangnisverhütung genau dem mit der entsprechenden Wahrscheinlichkeit gewichteten Grenzschaden eines zusătzlichen Kindes. 
preise von Kinderzahl und -qualität einen bedeutenden Einfluß auf die Fertilitätsentwicklung gehabt haben kann. Die Verstärkungswirkung ist aber empirisch wegen statistischer Probleme nicht quantifizierbar und wird darüber hinaus durch die mit dem Anstieg der Qualität verbundene Erhöhung des marginalen Konsumnutzens eines Kindes für die Eltern wieder abgeschwächt. Dabei stellt der Zusammenhang zwischen Kinderzahl und -qualität keine eigenständige Ursache für den Geburtenrückgang dar, sondern verstärkt nur die Wirkung anderer exogener Störungen wie v.a. des säkularen Einkommensanstiegs oder auch des Anstiegs des Frauenlohnsatzes, einer kostenlosen Schulausbildung, etc. Er muß deshalb zwar bei der Wirkungsanalyse von Maßnahmen des Kinderlastenausgleichs berücksichtigt werden, die diese exogenen Größen beeinflussen, muß aber nicht eigenständig untersucht werden.

\subsubsection{Die direkten Kosten von Kindern}

Im folgenden wird die Wirkung einer Änderung der direkten Kosten von Kindern untersucht. Die direkten Kosten umfassen im Unterschied zu den indirekten Kosten, die aus alternativen Verwendungsmöglichkeiten der Zeit resultieren, die Kosten, die dadurch entstehen, daß Einkommen aufgrund für Kinder anfallender Ausgaben nicht mehr für andere Zwecke zur Verfügung steht. Die Wirkung einer Änderung der direkten Kosten soll - wie schon bei den indirekten Kosten - zunächst theoretisch behandelt werden, ehe die empirische Entwicklung und die Bedeutung für die Gestaltung des Kinderlastenausgleichs dargestellt wird.

\subsubsection{Theoretische Analyse der Wirkung steigender direkter Kinderkosten}

$\mathrm{Zu}$ den theoretischen Überlegungen kann im wesentlichen auf die Ausführungen in Abschnitt 3.2.1 verwiesen werden. Eine Erhöhung der direkten Kosten wirkt nämlich wie eine Erhöhung der indirekten Kosten. Für die Entscheidung für oder gegen Kinder sind die gesamten marginalen Kosten entscheidend, also die Summe aus marginalen direkten und marginalen indirekten Kosten, so daß nur die Größe der Gesamtänderung, nicht aber die Aufteilung auf die Kostenarten ausschlaggebend ist. Allerdings fällt im Gegensatz zu den Opportunitätskosten hier kein Einkommenserhöhungseffekt an, wie ihn die Einkommenszunahme aufgrund eines gestiegenen Lohnsatzes erzeugt. Betrachtet werden muß hier also nur der Schattenpreisänderungseffekt.

Untersucht werden hier nur die Auswirkungen der Preiserhöhung einer Qualitätseinheit $\left(p_{c}\right)$. Kommt es zu einer Erhöhung der direkten Kosten, ohne daß ein Qualitätszuwachs und damit Nutzenzuwachs entsteht - also zu einer Erhöhung der qualitätsunabhängigen, nicht-nutzenstiftenden direkten Kosten -, so steigt der Preis von Kindern relativ zu dem Preis des aggregierten anderen Basisguts Z. Das Preisverhältmis zwischen Quantität und Qualität von Kindern bleibt von der Erhöhung der direkten Kosten unberührt, wenn man eine proportionale Erhöhung der Kosten von Qualität und Quantität annimmt, d.h. wenn weder qualitätsunabhängige Kosten der Quantität noch quantitätsunabhängige Kosten der Qualität 
existieren. ${ }^{174}$ Später werden qualitätsunabhängige Fixkosten der Quantität eingeführt, um die Auswirkungen monetärer Maßnahmen des Kinderlastenausgleichs besser analysieren zu können. In diesem Fall - d.h. bei einer Erhöhung der fixen Kosten der Quantität - kommt es auch zu einer Änderung des relativen Preises von Quantität zu Qualität (hier zu einer Erhöhung). Zunächst seien aber solche Änderungen des relativen Preisverhältnisses ausgeschlossen und damit auch ein Substitutionseffekt zwischen $q$ und $n$.

Eine Preiserhöhung einer Qualitätseinheit $\left(p_{c}\right)$, beispielsweise eine relative Verteuerung von Babynahrung, hat sowohl einen Anstieg der Kosten der Qualität als auch der Quantität zur Folge, wie man aus den in $C .16 a$ ) und $C .16 b$ ) abgeleiteten Schattenpreisen ersehen kann, und zwar in einem prozentual gleichen Ausmaß. Qualitätserhöhende direkte Kosten würden sich dagegen in einer Erhöhung von $q$ niederschlagen und nur den Schattenpreis der Kinderzahl erhöhen. Hier wird deutlich, wie bedeutend die Unterscheidung zwischen einem Anstieg der qualitätserhöhenden direkten Kosten und der Erhöhung der Kosten einer Qualitätseinheit ist. ${ }^{175}$ Ersterer verändert das relative Preisverhältnis zwischen $n$ und $q$ und führt damit zu dem früher beschriebenen iterativen Substitutionsprozeß weg von der Kinderquantität und hin zu mehr Qualität, während letztere das Verhältnis der Schattenpreise von $n$ und $q$ unverändert läßt.

Eine derartige, durch steigende Kosten einer Qualitätseinheit ausgelöste proportionale Erhöhung der Schattenpreise von $n$ und $q$ erhöht das relative Preisverhältnis zwischen $n$ und $q$ einerseits und dem aggregierten Basisgut $Z$ andererseits, aber eben nicht die Qualität zu Lasten der Quantität. Der daraus resultierende Substitutionseffekt führt entsprechend den Kreuzpreiselastizitäten eindeutig zu einer Abnahme sowohl der Kinderzahl als auch der Kinderqualität und einer Zunahme sonstiger Basisgüter. Dabei muß noch einmal betont werden, daß dieses Ergebnis nicht etwa bei jeder absoluten Erhöhung des Preises einer Qualitätseinheit eintritt, sondern nur dann, wenn diese Preissteigerung über dem Preisanstieg von $Z$ liegt, es also zu einer relativen Verteuerung kommt. Aber auch dann dürfte der Rückgang von $n$ und $q$ eher gering ausfallen, weil für Kinder keine engen Substitute zur Verfügung stehen und die Preiselastizität der Nachfrage daher wohl eher niedrig ist. Selbst größere Änderungen des relativen Preises von Kinderqualität und -quantität zu anderen Basisgütern hätten also nur geringe Mengenänderungen zur Folge.

Als Ergebnis der theoretischen Analyse läßt sich also festhalten, daß eine rein preisbedingte Erhöhung der direkten Kosten keinen großen Rückgang der Kinderzahl erwarten läßt.

\subsubsection{Empirische Größenordnung der direkten Kinderkosten}

Bei der Abschätzung der empirischen Größenordnung und Entwicklung der direkten Kinderkosten sind nur die privat, d.h. von den Eltern zu tragenden, direkten Kosten zu ermitteln, denn allein diese gehen ja in das Nutzenkalkül potentieller Eltern bei der Entscheidung für oder gegen ein Kind ein. Die auch im Bereich "nachwachsende Generation"

174 Im Fall ohne Fixkosten ist aus den Gleichungen $C .16 a$ ) und $C .16 b$ ) für den Schattenpreis der Quantität folgender Zusammenhang ableitbar: $\omega_{n}=p_{c} q$. Für den Schattenpreis der Qualität gilt entsprechend $\omega_{q}=p_{c} n$. Es ist sofort erkennbar, daß eine Erhöhung des Preises einer Qualitätseinheit $\left(p_{c}\right)$ beide Schattenpreise im gleichen Verhältnis verändern würde.

Becker (1991) unterscheidet hier nicht explizit, sondern umgeht das Problem, indem er postuliert, daß der Preis einer Qualitätseinheit konstant ist (vgl. Becker (1991) S. 145). Die Wirkung einer Erhöhung qualitätsunabhängiger direkter Kosten untersucht er nur über die Änderung von Fixkosten der Quantität. 
zunehmende Übernahme von Aufgaben und damit Kosten durch den Staat braucht hier daher nicht betrachtet zu werden, sondern wird in Unterabschnitt 3.2.3.3 als Entlastung der Eltern von privaten Kinderkosten untersucht.

Im Vergleich zu der Berechnung der Opportunitätskosten eines Kindes erscheint die Ermittlung der direkten Kosten von Kindern zunächst als eine relativ einfache Aufgabe, weil es sich ja um tatsächliche monetäre Aufwendungen handelt, die damit prinzipiell beobachtbar sind. Zwei Probleme führen aber zu besonderen Schwierigkeiten bei einer Berechnung.

Zum einen müssen qualitätserhöhende, also nutzensteigernde monetäre Ausgaben ausgeschlossen werden. Von einer Preiserhöhung des Gutes Kind, die eine Substitution hin zu anderen Basisgütern auslöst, kann nur dann gesprochen werden, wenn - wie bei Preisindizes anderer Güter auch - Qualitätsänderungen herausgerechnet werden. ${ }^{176}$ Wenn aufgrund eines gestiegenen Einkommens ein höheres Qualitätsniveau gewählt wird und damit die Kosten pro Kind steigen, sagt das noch nichts über die hier interessierende Preisentwicklung einer Qualitätseinheit aus. Die Herausrechnung von Qualitätszuwächsen in einem Warenkorb muß notwendigerweise immer ungenau bleiben, weil die Änderung eines bestimmten Produktes von einer Person als große Verbesserung, von einer anderen aber als weniger bedeutend oder gar als Verschlechterung angesehen werden kann. Außerdem sind Produktneueinführungen bzw. das Auslaufen bestimmter Güter und die Änderung der Zusammensetzung des Warenkorbes Problemfelder. Diese Schwierigkeiten sind nicht spezifisch für das Gut Kind, werden jedoch bei der Verwendung eines solchen Preisindizes hier deshalb akzentuiert, weil es um säkulare Preisentwicklungen geht und die oben genannten Schwierigkeiten mit zunehmender Länge der Zeitreihe anwachsen.

Das zweite Problem resultiert aus der Berechnungsweise des "Preisindex für die einfache Lebenshaltung eines Kindes" durch das Statistische Bundesamt und vieler anderer Untersuchungen der direkten Kinderkosten. Seine Aufgabe ist es nämlich, die Kosten der Lebenshaltung des Kindes "isoliert von der Lebenshaltung derjenigen Person(en) darzustellen, mit der (bzw. denen) es in der Wirklichkeit in einem gemeinsamen Haushalt zusammenlebt". ${ }^{177}$ Es handelt sich um eine normative Bedarfsrechnung, die von einem "Nullpunkt aus alle für das Leben des Kindes notwendigen Aufwendungen"178 umfaßt. Es werden also nicht etwa die tatsächlich anfallenden Unterhaltskosten gemessen, sondern - zwangsläufig subjektiv - der Geldbetrag festgelegt, der für erforderlich gehalten wird, um den Lebensunterhalt eines Kindes zu gewährleisten (Unterhaltsbedarf). ${ }^{179}$ Hier interessiert aber nicht eine derartige Normgröße, sondern die tatsächlich durch ein "repräsentatives" Kind zusätzlich verursachten Kosten.

176 Vgl. Zameck-Glyscinski (1985) S. 128.

177 Rasch (1980) S. 236.

178 Dinkel (1984a) S. 81.

179 Vgl. Euler (1974) S. 320, Schweitzer (1979) S. 118f. und Vottlerer (1987) S. 12ff. Zu erklären ist dieser für einen Preisindex zunăchst recht merkwürdig erscheinende Rückgriff auf den Unterhaltsbedarf im Gegensatz zu den tatsächlich anfallenden Unterhaltskosten durch seine Verwendung zur Festlegung von Unterhaltszahlungen. Aus diesem Grund wird wohl auch von einer "einfachen" Lebenshaltung ausgegangen, also von etwas mehr als dem Existenzminimum. Letztlich muB eine Bedarfsfestlegung immer ermessensbehaftet bleiben (vgl. auch Schemmel (1991) S. 488) und die Entscheidung daher politisch getroffen werden. Die schematische Bedarfsmengenfestsetzung erweckt dagegen oft den Eindruck einer nach wissenschaftlichen Maßstäben ermittelten objektiven Größe, weshalb Vottlerer (1987) S. 13 von einer "Scheinobjektivität der Warenkorbfestsetzung" spricht. 
Neben diesem Bedarfscharakter macht die Aufschlüsselung auch der sogenannten familiaren Güter ${ }^{180}$ auf die Kinder die Verwendung dieses Indexes problematisch, wie im folgenden anhand der Bestimmung des Anteils der Kinder an den Haushaltsausgaben erläutert wird.

Relativ einfach ist noch die Zuordnung von Kosten, die nachweisbar für das Kind einzeln anfallen wie z.B. Säuglingsnahrung, Kinderbett, Kindergartenbeitrag etc. (in der betriebswirtschaftlichen Kostenrechnung werden solche Kosten als Einzelkosten bezeichnet).

Problematischer ist dagegen schon die Zuordnung von Kosten, die zwar dem Kind im Prinzip zurechenbar wären, bei denen diese Zuordnung aber praktisch kaum durchführbar ist (unechte Gemeinkosten). Als Beispiel mag die von allen Familienmitgliedern gemeinsam benutzte Zahnpasta oder eine gemeinsame Mahlzeit dienen. Da ein Abwiegen der Verbrauchsmengen der einzelnen Haushaltsmitglieder zu mühsam wäre, muß hier mit Schlüsselgrößen gearbeitet werden.

Besondere Schwierigkeiten macht die Zuordnung der Kosten für familiare Güter (echte Gemeinkosten). Herkömmlicherweise wird - entsprechend der Vorgehensweise der Vollkostenrechung bei dem Versuch einer möglichst verursachungsgerechten Zuordnung der

Welche Probleme die Bestimmung solcher Bedarfsgrößen bereitet, wird deutlich, wenn man die Normen für die soziokulturellen Existenzminima bezüglich des Wohnraums für unterschiedliche Haushaltstypen miteinander vergleicht, die im Wohngeldgesetz (WoGG), in den Wohnungsbindungsrichtlinien von Hessen bzw. bundesdurchschnittlich und vom Wissenschaftlichen Beirat für Familienfragen 1992 und 1975 festgelegt wurden. Wie aus der nachfolgenden Tabelle zu entnehmen ist, ergeben sich z.T. Abweichungen von über $30 \%$.

Normen für das soziokulturelle Existenzminimum bezüglich des Wohnraums (in Quadratmetern)

\begin{tabular}{l|r|r|r|r|r|r|r}
$\begin{array}{l}\text { Haushaltsgröße } \\
\text { (Pers.) }\end{array}$ & 1 & 2 & 3 & 4 & 5 & 6 & 7 \\
\hline Beirat 1975 & 35,5 & 51 & 64,5 & 80 & 97 & 112 & 125 \\
Beirat 1992 & 37 & 51 & 66 & 85 & 103 & 119,5 & 135 \\
WoGG 1990 & 48 & 62 & 74 & 86 & 98 & 110 & 122 \\
$\begin{array}{l}\text { WoBindRili 1990 } \\
\text { (Hessen) }\end{array}$ & 50 & 60 & 75 & 85 & 95 & 105 & 115 \\
$\begin{array}{l}\text { WoBindRili 1990 } \\
\text { (Bundesdurch- }\end{array}$ & 40 & 50 & 65 & 80 & 90 & 100 & 110 \\
schnitt) & & & & & & &
\end{tabular}

Quelle: Wissenschaftlicher Beirat für Familienfragen (1992) sowie die dort angegebene Literatur

Auch die Höhe des gesamten Existenzminimums von Kindern wird sehr unterschiedlich gesehen. Willeke/Onken (1991) S. 5 geben für 1990 mit 725 DM monatlich einen um 375 DM und damit über doppelt so hohen Wert an wie der niedrigste Betrag von Ross (1990) S. 612.

Allerdings ist auch die Verwendung von Ist-Größen nicht unproblematisch. So weist der wissenschaftliche Beirat für Familienfragen (1992), S. 2 richtigerweise daraufhin, daß bei einem Vergleich der IstDifferenzen der Mietkosten unterschiedlicher Haushaltsgrößen (Ist-Differenzmethode) eventuelle Einschränkungen der übrigen Haushaltsmitglieder im Wohnungsstandard nicht berücksichtigt werden und daher die kindbedingten Kosten unterschätzt werden. Es muß daher zusätzlich eine Kompensation für den gesunkenen Lebensstandard der bisherigen Haushaltsmitglieder berücksichtigt werden. 
Gemeinkosten auf die einzelnen Kostenstellen - mit Schlüsselgrößen gearbeitet, wobei für Kinder z.B. 0,7 Vollpersoneneinheiten zugrundegelegt werden. ${ }^{181}$

Die in der betriebswirtschaftlichen Literatur ${ }^{182}$ angeführten Kritikpunkte an der Vollkostenrechnung treffen auch hier zu: Die Auswahl der Schlüsselgrößen ist letztlich subjektiv und die Verteilung der Kosten für familiare Güter (Fixkosten) nicht verursachungsgerecht möglich. ${ }^{183}$ Darüber hinaus kann es zu Fehlentscheidungen bei der Auswahl des optimalen Konsumbündels (der Steuerung des Produktionsprogramms) kommen, weil nicht erkennbar ist, wie der Nutzen (Gesamtgewinn) durch eine Änderung der Konsumstruktur beeinflußt wird. Außerdem werden die Kosten der Kinder systematisch überschätzt, weil die Kosten für familiare Güter auch ohne sie anfallen würden und daher richtigerweise nicht teilweise den Kindern angelastet werden dürfen. Wurde der Fernseher beispielsweise nicht eigens des Kindes wegen angeschafft, sind seine Anschaffungskosten für die Berechnung der direkten Kinderkosten irrelevant. Es muß also eine Marginalbetrachtung entsprechend der Teilkostenrechnung vorgenommen werden, indem nur die Differenz der Lebenshaltungskosten ohne Kind mit den Lebenshaltungskosten mit Kind - also nur die Einzelkosten und unechten Gemeinkosten - betrachtet wird. ${ }^{184}$

Die Basis des Preisindexes für die einfache Lebenshaltung eines Kindes erweist sich damit für die Bestimmung der absoluten Kinderkosten ebenso als unbrauchbar wie die meisten anderen empirischen Untersuchungen.

Für die Bestimmung der Veränderung der direkten Kinderkosten soll der Preisindex für die einfache Lebenshaltung eines Kindes hier - in Ermangelung einer besseren Alternative dennoch herangezogen werden. Das erscheint insofern gerechtfertigt als die genannten Nachteile bei der Betrachtung von relativen Größen weniger stark ins Gewicht fallen als bei absoluten Größen. Nur falls die Preisentwicklung der echten Gemeinkosten von der Preis-

181 Die erste Untersuchung der direkten Kinderkosten auf der Grundlage von Äquivalenzskalen stammt von Engel (1883) (nach Turchi (1984) S. 259). Dabei werden hier - wie in den meisten Studien dieser Art nicht nur die Gemeinkosten, sondern alle Ausgaben einschließlich der Einzelkosten nach Schlüsselgrößen aufgeteilt.

182 Stellvertretend für viele Bücher zur Kostenrechnung sei Wedell (1986) genannt.

183 In Wander (1984) S. 241 wird beispielsweise bei dem Vergleich verschiedener Ländergruppen für die Nahrungsmittelausgaben folgender Verteilungsschlüssel zugrundegelegt, ohne daß eine nähere Begründung erfolgt:

$\begin{array}{llll}\text { Männer (15-64 Jahre) } & 1,00 & \text { Kinder (unter einem Jahr) } & 0,35 \\ \text { Kinder (1-4 Jahre) } & 0,50 & \text { Kinder (5-9 Jahre) } & 0,60 \\ \text { Jungen (10-14 Jahre) } & 0,90 & \text { Frauen (15 Jahre und älter) } & 0,70 \\ \text { Männer (65 Jahre und älter) } & 0,80 & & \end{array}$

Mädchen zwischen 10-14 Jahren sind in dem Schema unbegründeterweise nicht enthalten. Für die Bundesrepublik Deutschland wird in dieser Studie (Wander (1984) S. 247ff.) zwar eine wesentlich detailliertere Aufschlüsselung sowohl der Warengruppen als auch der Altersgruppen vorgenommen, an dem grundsătzlichen Problemen ändert sich dadurch allerdings nichts. Schweitzer (1979) S. 123 führt dagegen Vollpersonenskalen an, die vom Statistischen Reichsamt aufgestellt wurden und die z.T. beträchtlich von den Werten abweichen, die Wander zugrundelegt:

$\begin{array}{llll}\text { Kinder (bis 5 Jahre) } & 0,40 & \text { Kinder (5-9 Jahre) } & 0,50 \\ \text { Kinder (9-14 Jahre) } & 0,70 & \text { Kinder (14-18 Jahre) } & 0,90 \\ \text { Ehepaar } & 1,90 & & \end{array}$

$184 \mathrm{Zu}$ Problemen bei dieser Vorgehensweise vgl. Fußnote 179. 
entwicklung der übrigen Güter des Warenkorbs dieses Indizes abweicht, kommt es überhaupt zu einer verzerrten Darstellung. ${ }^{185}$ Und auch dann wird diese Verzerrung durch das relativ geringe Gewicht ${ }^{186}$ der echten Gemeinkosten in dem zugrundegelegten Warenkorb reduziert.

Vergleicht man nun die Entwicklung des Preisindexes für die einfache Lebenshaltung eines Kindes mit dem Preisindex für die Lebenshaltung eines Arbeitnehmerhaushalts mit mittlerem Einkommen (Tab. C.5), so stellt man fest, daß tatsächlich die in dem Index für die einfache Lebenshaltung eines Kindes enthaltenen Güter eine etwas größere Teuerungsrate aufweisen als der Warenkorb für einen Arbeitnehmerhaushalt mittleren Einkommens. Das relative Preisverhältnis zwischen Kindern und sonstigen Gütern hat sich also zu Lasten der Kinder verschoben. ${ }^{187}$ Allerdings ist der Unterschied mit nicht ganz fünf Prozent in den 30 Jahren zwischen 1962 und 1992 recht gering und dürfte durch die überdurchschnittlich gestiegenen Preise für familiare Güter wie Wohnungsmieten und Energie noch überzeichnet sein. Die Preiselastizität der Nachfrage nach Kindern dürfte zudem eher gering sein, da es zu ihnen keine engen Substitute gibt. Mit der geringen Verschlechterung des relativen Preisverhältnisses kann der drastische Geburtenrückgang in den letzten drei Jahrzehnten daher nicht erklärt werden.

$\mathrm{Zu}$ einer ähnlichen Einschätzung ${ }^{188}$ gelangt auch Dinkel: "Die direkten Kinderaufwendungen vor allem für Kleinkinder sind ein relativ untergeordneter Faktor und haben sich in den letzten Jahrzehnten im Vergleich zur allgemeinen Kaufkraftentwicklung der Haushalte tendenziell eher reduziert als erhöht. Die für Kinder nötigen Marktgüter (die umgangssprachlichen "Kinderkosten") allein können den säkularen Rückgang der Kohortenfertilität somit nicht erklären." 189

185 Tendenziell war die Teuerungsrate bei Warengruppen, die familiare Güter enthalten (z.B. Wohnungsmieten und Energie) etwas höher als bei den anderen Warengruppen, so daß der Preisindex die wahre Entwicklung eher überschätzen dürfte. Vgl. verschiedene Jahrgänge der Jahrbücher des Statistischen Bundesamtes.

186 Den größten Anteil nehmen mit 44,5\% bzw. 23,9\% Nahrungsmittel und alkoholfreie Getränke bzw. Bekleidung und Schuhe ein, also Warengruppen, die in ihrer Gesamtheit Einzelkosten bzw. unechte Gemeinkosten darstellen. Warengruppen, in denen ein mehr oder weniger großer Anteil an echten Gemeinkosten vermutet werden muß, wie beispielsweise Wohnungsmieten, Energie, Möbel, etc., gehen mit einer Gewichtung von weniger als $30 \%$ in den Warenkorb ein. Vgl. Statistisches Bundesamt (1993) S. 660 .

187 Da auch im Preisindex für die Lebenshaltung eines Arbeitnehmerhaushalts mit mittlerem Einkommen zwei Kinder berücksichtigt werden, ist die Verschiebung etwas größer als durch den Vergleich der beiden Indizes ausgewiesen. Besser geeignet wäre daher der Preisindex aller privaten Haushalte, in den nur 0,6 Kinder eingehen, aber dieser Index ist erst ab 1962 verfügbar.

188 Eine andere Meinung vertreten Felderer/Sauga (1988), S. 69 in bezug auf die Entwicklung seit den Anfängen der Industrialisierung. Aufgrund der überproportional steigenden Wohnungskosten und des starken Gewichts der Wohnungskosten bei der Ermittlung der Kinderkosten schließen sie auf säkular gestiegene Kinderkosten. Hier ist einzuwenden, daß das Gewicht der Wohnungskosten in den meisten Studien durch die Aufteilung mittels Schlüsselgrößen überschätzt wird. Außerdem dürften die Nahrungsmittelkosten, die einen noch höheren Anteil an den direkten Kinderkosten haben, im Laufe der letzten beiden Jahrhunderte tendenziell eher zurückgegangen sein und somit den steigenden Wohnungskosten entgegengewirkt haben.

189 Dinkel (1984a) S. 85, Hervorhebungen im Original. Da hier relative Preisveränderungen untersucht werden sollen, darf korrekterweise die Entwicklung der direkten Kosten nicht mit der allgemeinen Kaufkraftentwicklung verglichen, sondern muß der Preisentwicklung anderer Güter gegenübergestellt werden. Dinkels Einschätzung ist insofern zu relativieren. 
Tab. C.5: Die Entwicklung des Preisindexes für die einfache Lebenshaltung eines Kindes im Vergleich zu der Entwicklung des Preisindexes für die Lebenshaltung eines Arbeitnehmerhaushalts mit mittlerem Einkommen $(1962=100)$

\begin{tabular}{c|c|c|c|c|c}
\hline Jahr & $\begin{array}{c}\text { Preisindex für } \\
\text { die einfache } \\
\text { Lebenshaltung } \\
\text { eines Kindes }\end{array}$ & $\begin{array}{c}\text { Preisindex für die } \\
\text { Lebenshaltung eines } \\
\text { Arbeitnehmerhaus- } \\
\text { halts mit mittlerem } \\
\text { Einkommen }\end{array}$ & Jahr & $\begin{array}{c}\text { Preisindex für } \\
\text { die einfache } \\
\text { Lebenshaltung } \\
\text { eines Kindes }\end{array}$ & $\begin{array}{c}\text { Preisindex für die } \\
\text { Lebenshaltung eines } \\
\text { Arbeitnehmerhaus- } \\
\text { halts mit mittlerem } \\
\text { Einkommen }\end{array}$ \\
\hline 1957 & 87,7 & 90,9 & 1977 & 189,2 & 176,8 \\
1958 & 89.9 & 92,8 & 1978 & 193,6 & 181,3 \\
1959 & 91.1 & 93,5 & 1979 & 201,1 & 188,3 \\
1960 & 92,7 & 95,0 & 1980 & 211,8 & 198,1 \\
1961 & 94.8 & 97,4 & 1981 & 224,1 & 210,8 \\
1962 & 100,0 & 100,0 & 1982 & 235,1 & 221,8 \\
1963 & 103,1 & 103,1 & 1983 & 241.7 & 229,2 \\
1964 & 105,3 & 105,5 & 1984 & 247,8 & 234,4 \\
1965 & 109,7 & 109,1 & 1985 & 251,1 & 239,2 \\
1966 & 113,5 & 112,9 & 1986 & 251,8 & 238,8 \\
1967 & 114,2 & 114,6 & 1987 & 253,1 & 239,0 \\
1968 & 115,6 & 116,0 & 1988 & 255,6 & 241,6 \\
1969 & 120,4 & 118,4 & 1989 & 262,7 & 248,6 \\
1970 & 124,3 & 122,2 & 1990 & 270,4 & 255,3 \\
1971 & 130,8 & 128,5 & 1991 & 279,5 & 264,4 \\
1972 & 139,0 & 135,4 & 1992 & 288,0 & 274,9 \\
1973 & 149,9 & 144,5 & 1993 & 295,8 & 285,4 \\
1974 & 160,8 & 154,3 & 1994 & 303,1 & 293,7 \\
1975 & 173,0 & 163,6 & & & \\
1976 & 184,2 & 171,1 & & & \\
& & & & & \\
\hline
\end{tabular}

Quelle: Statistisches Bundesamt, Statistisches Jahrbuch (verschiedene Jahrgänge)

Neben der Änderung ist auch die absolute Größe der direkten Kinderkosten von Interesse, denn letztlich kommt es bei der Entscheidung für ein Kind auf die durch ein (weiteres) Kind insgesamt zusätzlich verursachten Kosten an. Auch für die Entwicklung der gesamten Kinderkosten - bestehend aus Opportunitätskosten und direkten Kinderkosten - ist die absolute Höhe der direkten Kinderkosten von Bedeutung: je größer der Anteil der kaum gestiegenen direkten Kosten an diesen gesamten Kosten ist, desto geringer fallen die stark gestiegenen Opportunitätskosten (vgl. 3.2.1) ins Gewicht.

Wie oben erläutert, können die Berechnungen des Unterhaltsbedarfs, auf die der Preisindex für die einfache Lebenshaltung eines Kindes aufbaut, wegen ihres normativen Charakters und des Einschlusses der familiaren Güter (echte Gemeinkosten) für die Abschätzung der absoluten Größenordnung der direkten Kosten nicht herangezogen werden.

Zwei unterschiedliche Wege der Berechnung der Höhe der direkten Kosten sind denkbar: erstens eine Einzelermittlung aller für das Kind anfallenden Kosten, d.h. der Einzelkosten und der unechten Gemeinkosten und zweitens ein Vergleich der Lebenshaltungskosten von 
vergleichbaren Ehepaaren ohne bzw. mit Kind. Auf den zuerst angeführten Weg wird zunächst eingegangen.

Bei einer Einzelermittlung aller für ein Kind entstehenden Kosten sind die Einzelkosten und die unechten Gemeinkosten zu berücksichtigen. Die Einzelkosten sind ohne größere Schwierigkeiten bestimmbar. Da aber eine direkte Zuordnung der unechten Gemeinkosten praktisch nicht durchführbar ist, muß für sie wieder auf Schlüsselgrößen zurückgegriffen werden. Zieht man dabei für weit abgegrenzte Warengruppen nur eine einzige Schlüsselgröße heran, um den Erhebungsaufwand zu minimieren, so geht das zu Lasten einer verursachungsgerechten Zuordnung. Nimmt man beispielsweise an, daß die Ernährungskosten eines einjährigen Kindes mit 0,3 Vollpersonenanteilen anzusetzen sind, so wird dem Kind von den Ausgaben des Haushalts für Babynahrung ein ebenso großer Prozentsatz zugerechnet wie vom Eierlikörkonsum seiner Eltern. Versucht man aber möglichst kleine Warengruppen zu bilden, steigt der Erhebungsaufwand enorm an und die Suche nach geeigneten Schlüsselgrößen wird schwieriger. Empirische Untersuchungen, die auf einer Einzelermittlung beruhen, sind mir nicht bekannt. Meistens wird wie bei den Berechnungen des Statistischen Bundesamtes nicht zwischen unechten und echten Gemeinkosten unterschieden und oft beide in ein und derselben Warengruppe erfaßt, so daß auch eine nachträgliche Trennung unmöglich ist. ${ }^{190}$

Der zweite Weg zur Bestimmung der absoluten Höhe der direkten Kinderkosten, nämlich der Vergleich der Lebenshaltungskosten von Haushalten mit unterschiedlicher Kinderzahl, läßt sich in zwei Varianten zerlegen, die sich hinsichtlich der Bestimmungsart des elterlichen Lebensstandards unterscheiden. Beiden Ansätzen gemeinsam ist der Versuch abzuschätzen, wieviel zusätzliches Einkommen ein Elternpaar als Kompensation benötigt, um trotz der Kosten für ein Kind gegenüber einem Paar ohne Kind nicht schlechter gestellt zu sein. Gesucht wird also die Höhe des Einkommensunterschieds, der den Nutzen der Eltern unverändert läßt. Diese Einkommensdifferenz gibt dann die Kinderkosten an. Im Gegensatz zur Nutzenmaximierung unter einer gegebenen Budgetrestriktion wird hier nach dem minimalen Einkommen gefragt, das die Erreichung eines vorgegebenen Nutzenniveaus für unterschiedliche Haushaltstypen erlaubt. Dabei darf der Nutzen, den das Kind den Eltern stiftet, nicht mitgerechnet werden, denn es werden hier nicht die Nettokosten - direkte Kinderkosten abzüglich des monetär bewerteten Konsumnutzens des Kindes - gesucht, sondern die tatsächlichen Ausgaben. Die Nettokosten wären zudem immer negativ, weil sich Eltern wohl nur dann für ein Kind entscheiden, wenn der Nutzen die Kosten überwiegt. ${ }^{191}$

Ein zunächst intuitiv vielleicht näherliegender Vergleich von Haushalten mit gleichem Einkommen bei unterschiedlicher Kinderzahl macht wenig Sinn, denn die zusätzlichen Ausgaben für das Kind müssen dann durch insgesamt gleich große Kürzungen bei anderen Einkommensverwendungsarten erwirtschaftet werden. ${ }^{192}$ Damit würden die Eltern aber

190 In Wander (1984) S. 240 werden beispielsweise folgende Warengruppen unterschieden: 1) food and beverages, 2) clothing and footwear, 3) housing and household, 4) health, education, social security, 5) transport and communication, 6) capital formation, 7) other expenditures. Die Ausgaben für diese Warengruppen werden dann nach einem für jede Gruppe unterschiedlichen Verteilungsschlüssel unterschiedlichen Altersklassen zugeordnet. Eine nachträgliche Aufgliederung in Einzelkosten, unechte und echte Gemeinkosten ist unmöglich.

191 Unterstellt man eine systematische Unterschätzung der Kinderkosten bzw. eine Überschätzung des Kindernutzens, so kann der für die Entscheidung ausschlaggebende erwartete Nettonutzen positiv sein, obwohl der tatsächliche Nettonutzen negativ ist.

Vgl. Deaton/Muellbauer (1986) S. 723. 
weniger Einkommen für sich selber zur Verfügung haben und damit eine Nutzeneinbuße erleiden. ${ }^{193}$ Turchi bezeichnet die zusätzlichen Ausgaben aufgrund der Anforderungen des hinzugekommenen Familienmitglieds eines gewissen Alters, Geschlechts, etc. als specific effect und die Ausgabenumschichtung aufgrund der fehlenden kompensierenden Einkommenserhöhung als redistribution effect. ${ }^{194}$ Selbst wenn es möglich wäre alle zusätzlichen Ausgaben $\mathrm{zu}$ isolieren, würde dieses Kostenmaß die auf dem redistribution effect beruhenden Wohlfahrtsverluste der Eltern nicht erfassen.

Die beiden Hauptvarianten zur Bestimmung der absoluten Höhe der direkten Kinderkosten unterscheiden sich hinsichtlich des Kriteriums für einen gleich hohen Lebensstandard. Während in der Engel'schen Variante ${ }^{195}$ dann ein gleicher Lebensstandard angenommen wird, wenn die Anteile der Nahrungsmittelausgaben an den Gesamtausgaben verschiedener Haushalte gleich hoch sind, sieht Rothbarth ${ }^{196}$ einen gleichen Lebensstandard erreicht, wenn das gleiche Ausgabenniveau für adult goods erreicht wird. Unter adult goods sollen dabei Güter verstanden werden, die ausschließlich von Erwachsenen konsumiert werden. Da die Methoden also unterschiedliche Definitionen für "gleichen Lebensstandard" implizieren, führen sie bei gleichem Datenmaterial nur zufällig zu gleichen Ergebnissen. ${ }^{197}$

In Abb. C. 8 geben $x_{0}$ und $x_{1}$ die Höhe der Gesamtausgaben ${ }^{198}$ an, die für einen Haushalt ohne Kinder bzw. einen Haushalt mit einem Kind zu dem gleichen Ausgabenniveau für adult goods $\left(a^{*}\right)$ und damit annahmegemäß zu dem gleichen Lebensstandard der Eltern führt. Die Differenz zwischen $x_{1}$ und $x_{0}$ (Strecke $c$ ) gibt die zusätzlichen Ausgaben an, die der Haushalt mit Kind benötigt, um diesen Lebensstandard für die Eltern zu sichern und ist daher das Maß für die direkten Kinderkosten. Für die Vorgehensweise nach Engel ist die Bezeichnung der Ordinate in "Anteil der Nahrungsmittel an den Gesamtausgaben" zu ändern und die beiden Kurven werden negative Steigungen aufweisen. Voraussetzung für beide Methoden ist also die Bestimmung der Engelkurven, also des Zusammenhangs zwischen Einkommen und Nahrungsmittelanteil bzw. zwischen Einkommen und Konsumniveau der adult goods für Haushalte mit unterschiedlicher Kinderzahl.

Die Engel'sche Methode beruht auf den empirischen Beobachtungen, daß einerseits für Haushalte gleicher demographischer Zusammensetzung der Anteil der Nahrungsmittel an den Gesamtausgaben sinkt, wenn die Gesamtausgaben steigen, dieser Anteil andererseits aber bei gleichem Ausgabenniveau mit zunehmender Kinderzahl steigt. Die letztere Beobachtung läßt

193 Im Gegensatz zur in der neoklassischen Mikroökonomie gewöhnlichen Betrachtungsweise wird hier also nicht der Nutzen des gesamten Haushalts betrachtet, sondern nur der Nutzen der Eltern. Erstens ist nur durch den Ausschluß des Nutzens, der Kindern entsteht, ein sinnvoller Vergleich von Haushalten unterschiedlicher Kinderzahl möglich, und zweitens sind es ja die Eltern, die die Entscheidung über die Kinderzahl fällen und bei dieser Entscheidung wird der Nutzen der noch gar nicht existierenden Kinder sicherlich keine Rolle spielen.

194 Vgl. Turchi (1984) S. 261.

195 Die erste Analyse der Kinderkosten auf dieser Grundlage wurde von Engel (1895) vorgenommen (nach Deaton/Muellbauer (1986) S. 727).

196 Vgl. Rothbarth (1943) zu den theoretischen Grundlagen.

197 Vgl. Turchi (1984) S. 260.

198 Die Gesamtausgaben sollen hier alle Verwendungsmöglichkeiten für das Einkommen umfassen, also auch das Sparen, so daß Gesamtausgaben und Gesamteinnahmen synonym verwendet werden können. 
Abb. C.8: Bestimmung der direkten Kinderkosten nach Rothbarth

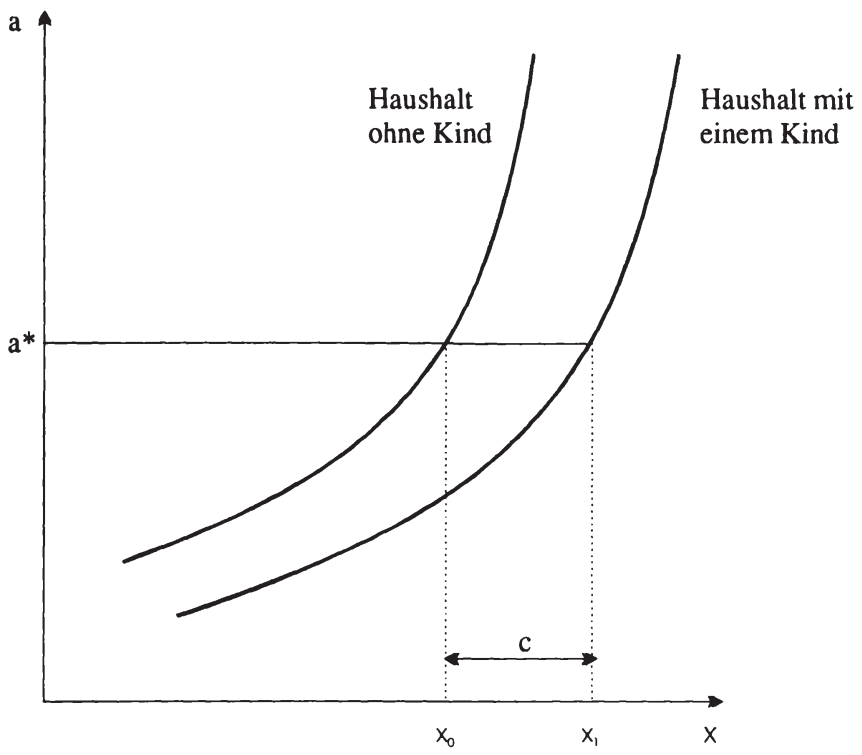

sich einmal mit dem sinkenden Ausgabenniveau pro Kopf begründen, aber auch mit einem höheren Anteil der Nahrungsmittel an allen für Kinder getätigten Ausgaben. Insoweit die letztgenannte Begründung greift, d.h. der Anteil der Nahrungsmittelausgaben für Kinder höher ist als für Erwachsene, führt die Wiederherstellung des alten Nahrungsmittelanteils für den gesamten Haushalt zu einer Überschätzung der Kinderkosten. Denn wenn der Nahrungsmittelanteil für den gesamten Haushalt auf das alte Niveau gesunken ist und der Anteil der Nahrungsmittel an den Ausgaben für das Kind überdurchschnittlich hoch ist, muß der Anteil der Nahrungsmittel an den Ausgaben für die Eltern schon unter dem alten Niveau liegen. Damit birgt die Engel'sche Methode die Gefahr der Überkompensation, d.h. der Überschätzung der Kinderkosten. ${ }^{199}$

Während die Engel'sche Methode also den Geldbetrag errechnet, der den Nahrungsmittelanteil ohne Kind wiederherstellt, beruht die Methode von Rothbarth auf der Berechnung des Geldbetrages, der das Ausgabenniveau für adult goods wiederherstellt. Problematisch bei der letztgenannten Methode ist die Annahme, daß nur der Einkommenseffekt das Ausgabenniveau für adult goods beeinflußt. Sobald nämlich Kinder bei einigen Güterarten mitkonsumieren und dadurch die Konsummöglichkeiten der Eltern einschränken, werden diese Güter für die Eltern relativ teurer. Diese Änderung der relativen Preise kann dann zu einer Substitution weg von gemeinsam konsumierbaren Gütern und hin zu ausschließlich von den Eltern konsumierten Gütern - eben den adult goods - führen. Dieser Substitutionseffekt hat zur Folge, daß das Niveau der adult goods schon früher wieder erreicht wird und daher die Kinderkosten tenden- 
ziell unterschätzt werden. Ein weiterer Nachteil dieser Methode bei der in empirischen Studien meist recht engen Abgrenzung von adult goods - oft werden hierzu nur Tabak- und Alkoholprodukte gezählt - besteht in der geringen Einkommenselastizität dieser Güter. Damit erhöht sich das Risiko gravierender Fehlschätzungen der Kinderkosten. Turchi kommt beispielsweise bei einer Schätzung mit entweder Alkohol oder Tabak oder Erwachsenenkleidung als "adult good" zu sehr unterschiedlichen Erwachsenenäquivalenten, ${ }^{200}$ obwohl sich theoretisch für jede Schätzung die gleichen Werte ergeben müßten. Als Äquivalzenzzahlen für Kinder bis zu fünf Jahren errechnet er 0,581 Erwachseneneinheiten mit Alkohol als adult good, aber nur 0,306 für Erwachsenenkleidung und gar nur 0,084 für Tabak.

Während also die Methode nach Engel eher zu einer Überschätzung der Kinderkosten führt, werden letztere durch die zuerst von Rothbarth vorgeschlagene Vorgehensweise tendenziell zu niedrig ermittelt.

Beide Methoden gehen implizit von der Annahme aus, daß das ganze Haushaltseinkommen einer Periode für Konsumzwecke ausgegeben wird. Berücksichtigt man die Möglichkeit des Sparens oder Entsparens, so kann ein bestimmtes Konsumniveau eventuell nach der Geburt eines Kindes nur zu Lasten der Ersparnisbildung aufrechterhalten werden. Diesem Effekt der Änderung des Sparverhaltens aufgrund von Kindern läßt sich zwar relativ leicht Rechnung tragen, indem die Ersparnis als weitere Einkommensverwendungsmöglichkeit in die Haushaltsausgaben aufgenommen wird. Allerdings werden die empirischen Ergebnisse auch dann noch verzerrt sein, denn die Sparquote eines kinderlosen Paares mag gerade wegen der in der Zukunft erwarteten Kinderkosten besonders hoch sein. Umgekehrt kann auch die Sparneigung einer Familie ansteigen, um künftige Ausbildungskosten abdecken zu können. ${ }^{201}$ Will man die Ersparnis nicht als reine Residualgröße ansehen, sondern als auch von den erwarteten zukünftigen Belastungen abhängig betrachten, liegt hier also eine mögliche Fehlerquelle beider dargestellten Methoden. Weiterhin steht zu vermuten, daß kinderlose Haushalte sich oft in einer anderen Lebensphase (Kinder haben den Elternhaushalt bereits verlassen) befinden werden als Familien mit Kindern. Sofern dieses unterschiedliche durchschnittliche "Alter" der Familien die Konsumstruktur beeinflußt, dürften nur Elternpaare gleicher Altersklassen miteinander verglichen werden, da sonst Struktureffekte den Einfluß von Kindern auf die Konsumstruktur verfälschen. Dieser Einwand gilt zwar auch für die Engel'sche Methode, aber es kann angenommen werden, daß der Konsum von adult goods als Gütern des gehobenen Bedarfs eher von der Lebensphase des Paares abhängt als Grundbedarfsgüter wie Nahrungsmittel.

Da alle Verfahren zur Ermittlung der absoluten Höhe der direkten Kinderkosten spezifische Mängel haben, ist es zur Erhöhung der Verläßlichkeit zweckmäßig, die Ergebnisse mehrerer empirischer Studien mit möglichst unterschiedlicher Vorgehensweise heranzuziehen und zu vergleichen. Auch auf Studien, die die Ausgaben für familiare Güter mittels Schlüsselgrößen auf die Kinder verteilen, soll deshalb zurückgegriffen werden, weil sie im Gegensatz zu den anderen Ansätzen auch für Deutschland durchgeführt wurden. ${ }^{202}$ Ihre Ergebnisse können

200 Vgl. Turchi (1984) S. 264f.

201 Vgl. Gaag (1982) S. 101f.

202 Auf die Darstellung der Ergebnisse der OECD und des Statistischen Amtes der Europäischen Gemeinschaften soll trotzdem verzichtet werden, da die Aufteilung der Haushaltsausgaben mittels Äquivalenzziffern von 1,0 für den ersten Erwachsenen, 0,7 für jeden weiteren Erwachsenen und 0,5 für jedes Kind erfolgte. Die sich aus dieser willkürlichen und über alle Verbrauchsgruppen einheitlichen Festlegung er- 
aufgrund der in ihrer Vorgehensweise angelegten Überschätzung der direkten Kinderkosten als Obergrenze gelten.

Zunächst werden die Ergebnisse von Studien resümiert, die Schlüsselgrößen verwenden. Das Statistische Landesamt Baden-Württemberg hat im Auftrag des Landesministeriums für Arbeit, Gesundheit, Familie und Sozialordnung im Rahmen einer Zusatzerhebung zur Einkommens- und Verbrauchsstichprobe 1983 eine umfangreiche Untersuchung der direkten Kinderkosten vorgenommen. ${ }^{203}$ Dabei wurde eine Kombination von Einzelermittlung und Verbrauchseinheitenrechnung gewählt, wobei die Zurechnung der Anteile an den familiaren Gemeinkosten auf die einzelnen Haushaltsmitglieder von den Familien selbst vorgenommen wurde. Rechtfertigen läßt sich dieses Vorgehen mit dem Hinweis, daß letztlich nicht die objektive Belastung der Eltern durch Kinder für die Fertilitätsentscheidung ausschlaggebend ist, sondern die subjektiv durch potentielle Eltern empfundene Belastung.

Als Ergebnis dieser Studie ist festzustellen, daß die direkten Kinderkosten (pro Kind) mit dem Einkommen der Eltern stark steigen, mit der Kinderzahl sinken - wobei insbesondere beim Übergang von einem zu zwei Kindern die Ausgaben pro Kind stark zurückgehen - und mit dem Alter der Kinder ansteigen. Die absoluten Werte lagen 1992 (fortgeschrieben mit dem Preisindex für die einfache Lebenshaltung eines Kindes) zwischen knapp 600 DM pro Monat für das dritte Kind einer Drei-Kinder-Familie der mittleren Einkommensgruppe ${ }^{204}$ und über $1.300 \mathrm{DM}$ pro Monat für das Kind einer Ein-Kind-Familie aus der oberen Einkommensgruppe (vgl. Tab. C.6 und C.7). Der gewichtete Durchschnittswert über alle Einkommensgruppen, Kindesalter und Geschwisterzahlen betrug etwa 830 DM pro Monat. Rechnet man diesen Durchschnittswert hoch, so erhält man als monetäre Kosten eines Kindes für die Eltern von der Geburt bis zum 18. Lebensjahr etwa 180.000 DM. ${ }^{205}$ Die Kosten des ersten Kindes liegen dabei mit etwa 204.000 DM wesentlich über denen des zweiten mit 129.000 DM und des dritten mit 150.000 DM. ${ }^{206} \mathrm{Daß}$ der Wert für das dritte Kind entgegen den theoretischen Erwartungen sogar über dem Wert für das zweite Kind liegt, dürfte darauf zurückzuführen sein, daß die untere Einkommensklasse bei Familien mit drei Kindern in dieser Studie sehr schwach besetzt war und somit ein höheres Durchschnittseinkommen der Drei-Kinder-Familien zu einem überhöhten Ausweis der monetären Kinderkosten führt.

Auch von Engelbert wird nach einer Durchsicht der wichtigsten Studien zu den Kinderkosten in Deutschland dieses Ergebnis als beste Schätzung angenommen. ${ }^{207}$

Gleichfalls eine Kombination aus Einzelermittlung und Verbrauchseinheitenrechnung liegt der Studie der Arbeitsgruppe "Lebenshaltungsaufwendungen Kinder" des Bundesministeriums für Familie und Senioren zugrunde. ${ }^{208}$ Als Datenbasis diente die Einkommens- und Ver-

gebenden Fehlerquellen machen die Resultate dieser Studien für die hier behandelte Fragestellung wertlos. Vgl. auch Euler (1993) S. 767f.

Vgl. Vottlerer (1987).

Die untere Einkommensgruppe ist bei Familien mit drei Kindern zu gering besetzt und daher nicht ausgewiesen.

Eine Abdiskontierung ist hier nicht erforderlich, da die Ergebnisse aus Querschnittsdaten gewonnen wurden.

Eigene Berechnungen auf Grundlage der Daten in Vottlerer (1987).

Vgl. Engelbert (1992) S. 15.

Die Ergebnisse der Arbeitsgruppe werden in Euler (1993) dargestellt. 
Tab. C.6: Monetäre Kinderkosten nach Kinderzahl bzw. Haushaltsnettoeinkommen 1992

\begin{tabular}{c|c}
\hline Kinderzahl & $\begin{array}{c}\text { Ausgaben (DM pro } \\
\text { Monat und Kind) }\end{array}$ \\
\hline 1 & 946 \\
2 & 771 \\
3 & 746
\end{tabular}

\begin{tabular}{c|c}
\hline \hline $\begin{array}{c}\text { Haushaltsnettoein- } \\
\text { kommen }\end{array}$ & $\begin{array}{c}\text { Ausgaben (DM pro } \\
\text { Monat und Kind) }\end{array}$ \\
\hline $\begin{array}{c}\text { untere } \\
\text { Einkommensgruppe } \\
\text { mittlere } \\
\text { Einkommensgruppe } \\
\text { obere }\end{array}$ & 667 \\
Einkommensgruppe & 764 \\
\end{tabular}

Quelle: eigene Berechnungen auf Grundlage von Daten aus Vottlerer (1987)

Tab. C.7: Kinderkosten in Abhängigkeit von Kinderzahl und Einkommensgruppe der Eltern ${ }^{209}$

\begin{tabular}{c|c|c|c|c}
\hline Kinderzahl & $\begin{array}{c}\text { Haushaltsnetto- } \\
\text { einkommen }\end{array}$ & $\begin{array}{c}\text { Ausgaben für das erste } \\
\text { Kind in DM }\end{array}$ & $\begin{array}{c}\text { Ausgaben für das } \\
\text { zweite Kind in DM }\end{array}$ & $\begin{array}{c}\text { Ausgaben für das } \\
\text { dritte Kind in DM }\end{array}$ \\
\hline $\begin{array}{c}\text { untere Einkom- } \\
\text { mensgruppe } \\
\text { mittlere Einkom- } \\
\text { mensgruppe } \\
\text { obere Einkom- } \\
\text { mensgruppe }\end{array}$ & $\begin{array}{c}\text { untere Einkom- } \\
\text { mensgruppe } \\
\text { zwei Kinder }\end{array}$ & 1307 & 536 \\
$\begin{array}{c}\text { mintlere Einkom- } \\
\text { mensgruppe } \\
\text { obere Einkom- } \\
\text { mensgruppe }\end{array}$ & 865 & 693 \\
\hline drei Kinder & $\begin{array}{c}\text { mittlere Einkom- } \\
\text { mensgruppe } \\
\text { obere Einkom- } \\
\text { mensgruppe }\end{array}$ & 7613 & 939 \\
\hline
\end{tabular}

Quelle: eigene Berechnung auf Grundlage von Daten aus Cornelius/Linder/Rückert (1986)

209 Die Zahlen wurden aus Comelius/Linder/Rückert (1986) S. 167 entnommen und basieren ebenso wie die Zahlen in Tab. C.6 auf Daten aus der Zusatzerhebung zur Einkommens- und Verbrauchsstichprobe des Statistischen Landesamtes Baden-Württemberg 1983. Sie wurden gleichfalls mit den Preisindex für die einfache Lebenshaltung eines Kindes fortgeschrieben auf 1992. 
brauchsstichprobe 1988. Die Schlüsselgrößen wurden weitgehend auf mikroökonomisch ermittelte Daten gestützt und nur in einigen Fällen normativ festgelegt. So wurde beispielsweise die Ausgabenkategorie Energieverbrauch auf alle Haushaltsmitglieder gleichmäßig verteilt. Die Aufwendungen für ein Kind wurden für drei Familientypen (Alleinerziehende, Ehepaare mit einem bzw. mit zwei Kindern) differenziert nach Einkommensklassen, Alter der Kinder und sozialer Stellung der Bezugsperson ermittelt: Diese Aufwendungen betragen für Alleinerziehende mit nur einem Kind und eigenem Haushalt fortgeschrieben auf 1992 durchschnittlich 613 DM, für ein Ehepaar mit einem Kind 779 DM und für ein Ehepaar mit zwei Kindern pro Kind 554 DM. Der geringe Wert für den dritten Familientyp dürfte v.a. auf die geringeren monetären Kosten des zweiten Kindes zurückzuführen sein, die die durchschnittlichen Kosten pro Kind senken. Als monetäre Kosten eines Kindes bis zum 18. Lebensjahr erhält man etwa 132.000 DM für Alleinerziehende und 168.000 DM bzw. 120.000 DM für Ehepaare mit ein bzw. zwei Kindern.

Lampert schätzt die von ihm als "monetärer Aufwand" bezeichneten direkten Kinderkosten für zwei 1983 bzw. 1985 geborene Kinder von ihrer Geburt bis zur Volljährigkeit auf zusammen knapp über 300.000 DM. ${ }^{210}$

Van der Gaag kommt nach einer Darstellung der Ergebnisse unterschiedlicher Studien über die direkten Kinderkosten in den USA zu dem Schluß, daß in der Literatur ein "surprising lack of consensus about the 'true' number" 211 existiert und außerdem diese hohe Spannweite der Ergebnisse auch bei Anwendung der gleichen Methode auftrat. Unter starken Vorbehalten meint er dennoch für die USA feststellen zu können, daß die Kosten des ersten Kindes etwa 25\% des Einkommens der Eltern betragen, die des zweiten und dritten Kindes jeweils etwa halb so hoch liegen und sich für weitere Kinder nochmals halbieren. Würde man diese Ergebnisse auf die Bundesrepublik übertragen können, so erhielte man 1992 bei einem durchschnittlichen verfügbaren Einkommen je Haushalt von $53.700 \mathrm{DM}^{212}$ (ohne nicht entnommene Gewinne der Unternehmen ohne eigene Rechtspersönlichkeit) monetäre Kinderkosten von der Geburt bis zum 18. Lebensjahr von etwa 242.000 DM für das erste, 121.000 DM für das zweite und dritte und 60.500 DM für weitere Kinder.

Für die Bundesrepublik Deutschland wurde meines Wissens eine Untersuchung der absoluten Kinderkosten mit Hilfe eines Vergleichs der Lebenshaltungskosten von Haushalten mit unterschiedlicher Kinderzahl in den letzten beiden Jahrzehnten nicht durchgeführt. Deshalb sollen hier Vorgehensweise und Ergebnisse einer sehr detaillierten und umfangreichen Studie über die direkten monetären Kinderkosten in den USA dargestellt werden, die 1984 von Espenshade durchgeführt wurde. ${ }^{213}$

Bei der Auswahl einer Verhältniszahl als Indikator für den Lebensstandard, wurde aus neun Variablen für den Zähler und drei Variablen für den Nenner nach folgenden fünf Kriterien jeweils eine Variable ausgewählt. Erstens sollte Monotonität vorliegen, d.h. für steigende Familiengröße sollte bei konstantem Einkommen die Änderung des Indikators ebenso monoton

210 Vgl. Lampert (1992) S. 135 und S. 140. Um zu dieser Summe zu gelangen, addiert Lampert allerdings für den Zeitraum von 1983 bis 1991 nominale Werte. Daraus ergibt sich eine Unterschätzung der Kosten, die noch dadurch verstärkt wird, daß Lampert das Jahr 1991 als Basisjahr benutzt.

211 Gaag (1982) S. 78.

212 Laut telefonischer Auskunft des Statistischen Bundesamtes vom 21.4.1994.

213 Vgl. Espenshade (1984). 
sein wie auch für steigendes Einkommen bei konstanter Kinderzahl. Zweitens sollte die Elastizität des Indikators bezüglich Kinderzahl und Einkommen möglichst groß sein. Drittens sollte die im Zähler stehende Ausgabengruppe einen möglichst großen Anteil an den Gesamtausgaben besitzen, um den Einfluß zufälliger Schwankungen möglichst gering zu halten. Viertens sollte der Indikator in dem Sinne konsistent sein, daß ein Anstieg der Familiengröße ihn entgegengesetzt beeinflußt wie eine Erhöhung des Einkommens. Schließlich wird auch der Lebensstandard mit steigender Kinderzahl negativ, mit steigendem Einkommen aber positiv beeinflußt. Fünftens sollten die Ergebnisse mit Resultaten früherer Studien in Einklang stehen. So lassen frühere Forschungsresultate beispielsweise einen negativen Zusammenhang zwischen Einkommen und Anteil der Nahrungsmittel und einen positiven Zusammenhang zwischen Kinderzahl und Anteil der Nahrungsmittel erwarten. Nach diesen fünf Kriterien erwies sich der Anteil der Ausgaben für Nahrungsmittel (ohne auswärtige Verpflegung) an den Gesamtausgaben als der beste Indikator für den Lebensstandard. Das Vorgehen von Espenshade entspricht damit der Engel'schen Methode.

In der oben beschriebenen Art und Weise wurden dann die direkten Kinderkosten ermittelt, indem anhand von empirisch geschätzten Engelkurven der Einkommensausgleich errechnet wurde, der einer Familie mit einem zusätzlichen Kind genau den gleichen Lebensstandard sichert, den ein Ehepaar mit einem Kind weniger hat. Die Kinderkosten wurden dabei getrennt untersucht für verschiedene Einkommensniveaus, Familiengrößen, Geburtenfolgen, unterschiedliches Alter des Kindes und verschiedene Beschäftigungssituationen der Mütter.

Im wesentlichen entsprechen die Einflußrichtungen den Ergebnissen der Untersuchung des Statistischen Landesamtes Baden-Württembergs. Den größten Einfluß auf die durchschnittlichen monetären Kinderkosten übt demnach die Kinderzahl aus. Mit steigender Kinderzahl sinken die Kosten pro Kind schnell ab. Mit steigendem Einkommen, zunehmender Arbeitszeit der Mutter oder steigendem Alter der Kinder nehmen die Kosten dagegen zu. Der Geburtenabstand (spacing) und der Geburtszeitpunkt (timing) scheinen dagegen keinen signifikanten Einfluß aufzuweisen. Für eine von Espenshade als durchschnittlich angesehene Familie mit mittlerem Status und zwei Kindern mit einer teilzeitbeschäftigten Mutter lagen die Kosten für das Aufziehen eines Kindes nach seinen Angaben 1981 bei $\$ 82.400$. Die höchsten Kosten treten mit $\$ 135.700$ für ein Einzelkind in einer Familie mit hohem sozio-ökonomischen Status auf, dessen Mutter vollzeitbeschäftigt ist. Die niedrigsten direkten Kosten wurden mit $\$ 58.300$ pro Kind für eine Familie mit niedrigem sozio-ökonomischen Status und drei Kindern ermittelt, deren Mutter keiner Erwerbsarbeit nachgeht.

Die Übertragbarkeit dieser Ergebnisse auf Deutschland ist durch unterschiedliche Preisstrukturen und Gewohnheiten stark eingeschränkt. Erschwerend kommt hinzu, daß der Wechselkurs als Umrechnungsfaktor von US-Dollar in DM starken Schwankungen unterworfen ist und kein geeignetes Kriterium für die Auswahl eines "richtigen" UmrechnungsWechselkurses existiert. Dieses Problem soll hier umgangen werden, indem der relativ stabile Zusammenhang zwischen dem Anteil der Ausgaben für Kinder an den Gesamtausgaben einer Familie und der Zahl der Kinder ausgenutzt wird. Espenshade hat nämlich festgestellt, daß dieser Anteil für alle sozio-ökonomischen Gruppen etwa 30\% für ein Kind, 40-45\% für zwei und knapp 50\% für drei Kinder beträgt. ${ }^{214}$ Diese Werte stimmen zumindest grob mit denen des oben erwähnten Survey-Artikels von van der Gaag überein, der Anteile von etwa $25 \%$ bzw. 37,5\% und 50\% erhielt, wobei er diese Anteile allerdings auf das Einkommen und nicht auf die Ausgaben bezieht. Auch für die Bundesrepublik scheint dieser Zusammenhang Gültigkeit zu haben. Cornelius/Linder/Rückert ermittelten als Anteile der Kinderkosten am gesamten

Vgl. Espenshade (1984) S. 3 und Espenshade/Calhoun (1986) S. $814 f$. 
privaten Verbrauch für eine Ein-Kind-Familie $24 \%$ bis $26 \%$, für eine Zwei-Kinder-Familie 38$40 \%$ und für eine Familie mit drei Kindern 48-50\%. ${ }^{215}$ Hier werden Anteile von $26 \%$ bzw. 40\% und 48\% zugrundegelegt und mit durchschnittlichen monatlichen Ausgaben für den privaten Verbrauch von 3.743 DM bzw. 4.069 DM und 4.358 DM ${ }^{216}$ im Jahr 1988 multipliziert, um die monatlichen Kinderkosten zu erhalten. Diese werden dann mit dem Preisindex für die einfache Lebenshaltung eines Kindes fortgeschrieben auf das Jahr 1992.217 Anschließend werden die Kinderkosten von der Geburt bis zum 18. Lebensjahr berechnet: Es ergeben sich Kinderkosten von ungefähr 237.000 DM für ein erstes, knapp 160.000 DM für ein zweites und etwa 113.000 DM für ein drittes Kind in Deutschland. Damit liegen die Werte dieser Schätzung entgegen dem Ergebnis der theoretischen Überlegungen sogar noch über den Werten der Studien, die die familiaren Aufwendungen mittels Schlüsselgrößen auch den Kindern zurechnen. Die mit der Kinderzahl schnell sinkenden monetären Kosten können als eine Erklärung dafür dienen, daß die bedingte Geburtenwahrscheinlichkeit von zweiten Kindern höher ist als die von ersten Kindern oder mit anderen Worten dafür, daß eine gewisse Polarisierung zwischen Kinderlosen einerseits und Familien mit mindestens zwei Kindern andererseits besteht. 218,219

Als Resultat der Zusammenschau verschiedener empirischer Studien läßt sich festhalten, daß zumindest die grobe Größenordnung auch bei unterschiedlichen Ansätzen übereinstimmt und die Ergebnisse daher als einigermaßen gesichert angesehen werden können. Die monetären Kosten eines ersten Kindes liegen demnach 1992 bei grob 230.000 DM, die eines zweiten bei ungefähr 150.000 DM und die eines dritten Kindes bei etwa 115.000 DM. Dabei muß allerdings beachtet werden, daß diese Zahlenangaben nur grobe Größenordnungen angeben und die tatsächlichen Kinderkosten aufgrund der Vielzahl unterschiedlicher Einflußfaktoren aber auch aufgrund der Unzulänglichkeiten der Schätzungen um mehrere 10.000 DM von diesen Werten abweichen können. Ein Teil der Abweichungen ist sicherlich auch darauf zurückzuführen, daß den Studien unterschiedliche Basisjahre zugrundeliegen. Geht man davon aus, daß die Qualität von Kindern kontinuierlich zugenommen hat, so beziehen sich die Studien, die auf älteren Daten beruhen, auf ein geringeres Qualitätsniveau. Sie weisen damit die monetären Kinder-

215 Vgl. Cornelius/Linder/Rückert (1986) S. 168f.

216 Nach Angaben des Statistischen Bundesamtes.

217 Eine Fortschreibung der Ausgaben auf das Jahr 1992 mit nachfolgender Berechnung der Kinderkosten wäre sicherlich sinnvoller als die hier gewählte Vorgehensweise, nach der die Berechnung der Kinderkosten für 1988 erfolgt und danach in einem zweiten Schritt die Fortschreibung. Dies scheitert aber daran, daß für die Haushaltsausgaben keine Zeitreihe vorliegt, mit deren Hilfe eine Fortschreibung vorgenommen werden könnte. Es muß hier damit bei dem Hinweis bleiben, daß die hier gewählte Vorgehensweise zu einer Unterschätzung führt, wenn die Zunahme der Ausgaben in den Jahren 1988 bis 1992 über der Zunahme des Preisindexes für die einfache Lebenshaltung eines Kindes gelegen hat.

218 Vgl. u.a. Birg (1992) S. 211.

219 Bisher ist bei der Ermittlung der direkten Kosten unberücksichtigt geblieben, daß die durchschnittliche Unterhaltsdauer, also die Zeitspanne, in der die Eltern finanziell für den Lebensunterhalt ihrer Kinder aufkommen, nicht konstant geblieben ist. Im Zeitraum von 1960 bis 1990 hat sich diese Zeitspanne um etwa vier Jahre erhöht. Berücksichtigt man, daß die Aufwendungen gerade für ältere Kinder besonders hoch sind, so ergibt sich allein aus dieser Entwicklung eine Zunahme der direkten Kosten in einer Größenordnung von 25\%. Allerdings handelt es sich bei dieser Zunahme nicht um eine Erhöhung des Preises einer Qualitätseinheit, denn die längere Unterhaltsdauer ist eine Folge längerer (unbezahlter) Ausbildungszeiten, die eine Erhöhung der Kinderqualität bewirken. Die direkten Kosten aufgrund einer späteren finanziellen Selbständigkeit der Kinder sind daher für die Fragestellung dieses Abschnitts irrelevant. 
kosten tendenziell niedriger aus als Studien, die neuere Datensätze verwenden. Da die Untersuchungen von van der Gaag und von Espenshade mit Hilfe von Einkommensdaten aus dem Jahr 1992 bzw. mit Angaben über den privaten Verbrauch 1988 fortgeschrieben wurden, lassen sich so die höheren Werte dieser Studien teilweise erklären.

\subsubsection{Bedeutung der direkten Kinderkosten für den Kinderlastenausgleich}

Die Bedeutung der direkten Kinderkosten für den Kinderlastenausgleich wird oft überschätzt. Die tatsächlich durch ein Kind zusätzlich anfallenden Kosten sind - obwohl absolut gesehen recht hoch - in den meisten Fällen doch geringer als die Opportunitätskosten. Trotzdem konzentriert sich ein Großteil der Maßnahmen des Kinderlastenausgleichs in der Bundesrepublik Deutschland auf die (teilweise) Kompensation dieser direkten Kosten. Deshalb soll die Wirkung solcher Maßnahmen auf die Fertilitätsentscheidung hier genauer untersucht werden. Dabei kann auf die Überlegungen im vorangegangenen Abschnitt zurückgegriffen werden. Allerdings muß das Modell aus 3.2.2 um Fixkosten $\left(p_{n}\right)$ erweitert werden, die pro Kind unabhängig von der Qualität anfallen. ${ }^{220}$ Es handelt sich also um Fixkosten der Quantität. Diese Fixkosten können einerseits aus "time, expenditure, discomfort, and risk spent in pregnancy and delivery..."221 und anderen monetären und psychischen Kosten bestehen, die keinen Einfluß auf die Qualität haben. Andererseits können darunter aber auch kindgebundene staatliche Transfers - wie beispielsweise das Kindergeld - als "negative Kosten" verstanden werden. Eine Kindergelderhöhung ist dann gleichzusetzen mit einer Minderung der qualitätsunabhängigen Fixkosten. Betrachtet werden also im folgenden nur solche Maßnahmen des Kinderlastenausgleichs, die in ihrer Primärwirkung unabhängig von der Qualität allein die Kinderzahl subventionieren. Nur solche Instrumente können im Modell als eine Minderung der qualitätsunabhängigen Fixkosten verstanden werden. Hinsichtlich der Instrumente, die die Kosten einer Qualitätseinheit reduzieren - einer Subventionierung der zur Erstellung des Basisgutes Kind erforderlichen Einsatzfaktoren also - kann auf die theoretischen Überlegungen zu Beginn dieses Abschnitts verwiesen werden. Dort wurde festgestellt, daß eine Erhöhung des Preises einer Qualitätseinheit allenfalls einen geringen Einfluß auf die Kinderzahl hat. Eine Senkung dieses Preises durch Maßnahmen des Kinderlastenausgleichs hat dann gleichfalls wenig Einfluß auf die Quantitätsentscheidung.

Die Budgetgleichung lautet unter Einschluß qualitätsunabhängiger Fixkosten

$$
p_{n} n+p_{c} q n+\pi_{z} Z=S
$$

und die Optimalitätsbedingungen, die man aus der Maximierung der Nutzenfunktion

$$
\text { C.14) } U=U(n, q, Z)
$$

unter der Budgetbeschränkung C.19) erhält

220 Vgl. zu den folgenden Ausführungen beispielsweise Becker (1991) S. 149ff. oder Zameck-Glyscinski (1985) S. 135ff.

221 Becker (1991) S. 149. 


$$
\begin{aligned}
& \text { C.20) } M U_{n}=\lambda\left(p_{n}+p_{c} q\right)=\lambda p_{c} q\left(1+r_{n}\right)=\lambda \omega_{n} \quad r_{n}=\frac{p_{n}}{p_{c} q} \\
& \text { C.21) } M U_{q}=\lambda p_{c} n=\lambda \omega_{q} \\
& \text { C.22) } M U_{z}=\lambda \pi_{z} .
\end{aligned}
$$

Mit $r_{n}$ ist dabei das Verhältnis von fixen zu variablen Kosten der Kinderzahl bezeichnet. Durch Division der Gleichungen C.20) und C.21) erhält man

C.23) $\frac{M U_{n}}{M U_{q}}=\frac{\omega_{n}}{\omega_{q}}=\frac{q\left(1+r_{n}\right)}{n}$.

Das Verhältnis der Schattenpreise von Quantität $(n)$ und Qualität $(q)$ hängt jetzt nicht mehr nur von dem Verhältnis von $q$ zu $n$ ab, sondern auch von dem Verhältnis von fixen zu variablen Kosten.

Ein preisbedingter Anstieg des fixen Teils der direkten Kosten hat einen relativen Anstieg des Schattenpreises der Kinderzahl $\left(\omega_{n}\right)$ gegenüber $\omega_{q}$ und $\pi_{z}$ zur Folge, so daß $n$ substituiert wird durch mehr $q$ und Z. Diese Erhöhung des Verhältnisses von Qualität $(q)$ zu Quantität $(n)$ zieht dann einen weiteren Anstieg des Schattenpreisverhältnisses $\omega_{n} / \omega_{q}$ nach sich und es kommt wieder zu dem schon bekannten iterativen Substitutionsprozeß zwischen Kinderqualität und -quantität. Die Nachfrage nach anderen Basisgütern $(Z)$ bleibt von diesen Sekundäreffekten aber unberührt. Unter Umständen kann also eine kleine Änderung der direkten Fixkosten ausreichen, um eine große Änderung der Quantität und eine entgegengerichtete Änderung der Qualität zu verursachen und zwar auch, wenn die Substitutionselastizität zwischen $n$ und $q$ eher klein ist. ${ }^{222}$

Allerdings ist für die Wirkung von kindgebundenen Maßnahmen des Kinderlastenausgleichs zu beachten, daß erstens die direkten Kosten eines zusätzlichen Kindes eine eher untergeordnete Rolle bei der Fertilitätsentscheidung spielen und zweitens mit kindgebundenen Transfers zwar die Kinderzahl tendenziell erhöht werden kann, gleichzeitig aber tendenziell ein Substitutionsprozeß zu Lasten der Kinderqualität ausgelöst wird. Da die Kinderqualität zu einem großen Teil durch die Ausbildung bestimmt wird, geht durch die sinkende Qualität das Einkommenspotential der zukünftigen Generation zurück. Es besteht also die Gefahr, daß zwar die Kinderzahl durch Maßnahmen des Kinderlastenausgleichs erhöht wird, gleichzeitig aber durch diese Maßnahmen der Humankapitalbestand der Volkswirtschaft und damit ihr zukünftiges Wachstum negativ beeinflußt wird. In dem höheren Humankapitalbestand, der nicht zuletzt auf die Substitution von Kinderquantität durch -qualität zurückzuführen ist, ist ein wesentlicher Faktor für die hohen Wachstumsraten der Wirtschaft seit dem Zweiten Weltkrieg zu sehen. Letztlich ist es aber nicht allein die absolute Zahl der nachfolgenden Generation, sondern auch die von ihr erwirtschaftete Brutto-Lohnsumme, die für die Stabilisierung der gesetzlichen Rentenversicherung von Bedeutung ist. Wenn ein hohes Ausbildungsniveau und daraus resultierende hohe Lohnzuwächse eine Zunahme des Nettolohns auch bei stark steigenden

222 Eine Reihe von Beispielen, die einen starken negativen Zusammenhang zwischen Kinderquantität und Kinderqualität empirisch belegen, findet sich bei Becker (1991) S. 151ff. Vgl. auch die dort auf S. 153 angeführten empirischen Studien. 
Beitragssätzen ermöglicht, ist die Akzeptanz des Systems der gesetzlichen Rentenversicherung sicher weniger gefährdet als bei Realeinkommensrückgängen.

Als Ergebnis dieses Abschnitts kann festgehalten werden, daß die quantitative Bedeutung der direkten Kosten von Kindern - bei allen Schwierigkeiten ihrer empirischen Bestimmung absolut gesehen mit etwa 180.000 DM pro Kind ${ }^{223}$ recht hoch ist. Dennoch ist ihr Anteil an den gesamten Kosten eines (weiteren) Kindes wegen der außerordentlich hohen Opportunitätskosten eher gering, was ihren Einfluß auf die Entscheidung für oder gegen ein Kind reduziert. Darüber hinaus ist die Veränderung der direkten Kosten - gemessen durch die Veränderung des Preises einer Qualitätseinheit relativ zum allgemeinen Preisniveau - gering. Für die Erklärung des drastischen säkularen Geburtenrückgangs bietet die Entwicklung der direkten Kosten daher keinen Anhaltspunkt.

Der Einfluß von Maßnahmen des Kinderlastenausgleichs, die über eine Reduktion des Preises einer Qualitätseinheit eine Minderung der Kinderkosten bewirken, dürfte aufgrund der Höhe der Kosten für ein zusätzliches Kind niedrig sein. Kindgebundene Maßnahmen haben zwar aufgrund der durch sie ausgelösten Substitution von Kinderqualität durch Kinderquantität eine größere Wirkung, gleichzeitig reduzieren sie aber über ihren negativen Einfluß auf die Humankapitalbildung die Wachstumsaussichten der Wirtschaft.

\subsubsection{Zusammenfassung}

Aufbauend auf die Darstellung der Grundlagen der neoklassischen Theorie des generativen Verhaltens wurden in diesem Unterkapitel die Wirkungen verschiedener exogener Parameteränderungen auf die Entscheidung für oder gegen ein Kind untersucht. Im Vordergrund standen dabei drei große Bereiche: die Erhöhung der indirekten Kosten eines Kindes durch steigende Frauenlohnsätze, der Zusammenhang zwischen Quantität und Qualität, dessen Wirkung v.a. anhand einer Änderung des Einkommens untersucht wurde, und schließlich die Erhöhung der direkten Kosten.

In Hinblick auf die Opportunitätskosten ergaben sich aufgrund des säkularen Wachstums des (Frauen-)Lohnsatzes und der damit zunehmenden Verknappung des Faktors Zeit Größenordnungen für zeitliche Kosten eines Kindes von im Normalfall mehreren hunderttausend DM durch die mit der Erziehung zusammenhängenden direkten und indirekten Einkommensausfälle. Diese jetzt schon enormen Kosten werden in der Zukunft mit weiter steigenden Lohnsätzen noch zunehmen und zusätzlich durch das gestiegene Scheidungsrisiko erhöht. Damit kann im Rahmen des Kinderlastenausgleichs nicht angestrebt werden, den Eltern diese Kosten zu erstatten, denn schon eine teilweise Erstattung würde außerordentlich hohe Summen erfordern. Stattdessen sollte versucht werden, durch Maßnahmen des Kinderlastenausgleichs die Entstehung dieser Kosten zu reduzieren. Welche Maßnahmen dazu geeignet erscheinen, wird im Unterkapitel 4.1 untersucht.

Dieser Wert wurde errechnet aus den Kosten für ein erstes bzw zweites und drittes Kind multipliziert mit dem jeweiligen Anteil der Gruppe an allen Kindern. Die Prozentzahlen für Erstgeborene (46,8\%), Zweitgeborene $(35,7 \%)$ und Drittgeborene $(17,5 \%)$ wurden nach Angaben des Statistischen Bundesamtes für im Jahr 1992 ehelich geborene Kinder in Gesamtdeutschland errechnet. Dabei wurden Kinder höherer Parităt mit zu den Drittgeborenen gezählt. 
Der Zusammenhang zwischen Quantität und Qualität von Kindern stellte sich als empirisch besonders schwer faßbar heraus. Deshalb blieb es bei einigen recht allgemeinen Bemerkungen über die Einflußrichtung insbesondere der säkularen Einkommenserhöhung. Grundsätzlich führt die Interdependenz von Qualität und Quantität dazu, daß die Wirkung einer exogenen Störung, die eine Änderung des Schattenpreisverhältnisses dieser beiden Argumente der Nutzenfunktion bewirkt, durch einen iterativen Substitutionsprozeß verstärkt wird. Allerdings wird die Bedeutung dieses "Verstärkers" dadurch eingeschränkt, daß der marginale Konsumnutzen eines Kindes mit der Qualität steigt, den gestiegenen marginalen Kosten also auch ein gestiegener marginaler Nutzen gegenübersteht. Auch für Maßnahmen des Kinderlastenausgleichs bietet der Zusammenhang zwischen Quantität und Qualität wenig Anhaltspunkte. Zwar werden Maßnahmen, die das Schattenpreisverhältnis zugunsten der Kinderquantität beeinflussen, verstärkt, gleichzeitig wird dadurch aber die Qualität und damit auch das Humankapital gemindert und so das für spätere Rentenversicherungsbeiträge entscheidende Einkommenserzielungspotential der nächsten Generation eingeschränkt.

Eine Erhöhung der direkten Kosten eines Kindes, der Kosten einer Qualitätseinheit also, wurde in ihrer Wirkung als wenig bedeutend eingestuft, weil es erstens zu keinem iterativen Verstärkungsprozeß kommt und zweitens diese Kosten in den letzten Dekaden kaum schneller gestiegen sind als diejenigen anderer Güter. Die Größenordnung der monetären Kosten eines Kindes erwies sich als schwer bestimmbar und abhängig v.a. vom Alter des Kindes, der Zahl der Geschwister und dem Einkommen der Eltern. Die tatsächlichen monetären Kosten eines Kindes dürften aber im Durchschnitt bei allen Vorbehalten bei etwa 180.000 DM liegen.

Diese Ergebnisse fallen ganz anders aus, wenn man, wie Becker und Barro, davon ausgeht, da $\beta$ Eltern sich gegenüber ihren Kindern altruistisch verhalten. ${ }^{224}$ In diesem Fall berücksichtigen die Eltern bei der Entscheidung über die Kinderzahl schon das Nutzenniveau, das dieses Kind später einmal erreichen wird. Da der Nutzen dieses Kindes wiederum von dem Nutzenniveau abhängt, das seine Kinder (die Enkel der Eltern) erreichen, usw., müssen die Eltern bei ihrer Entscheidung schon alle zukünftigen Nutzen berücksichtigen. Die Annahme, daß Eltern bei der Entscheidung für oder gegen ein Kind statt der Qualität des Kindes das zukünftig erreichbare Nutzenniveau des Ungeborenen berücksichtigen, erscheint recht unrealistisch. Während nämlich die Qualität des Kindes über den Ressourceneinsatz beeinflußt werden kann, hängt das erreichbare Nutzenniveau eines Kindes wiederum auch von der Zahl und dem Nutzenniveau seiner Kinder ab. Deshalb wird hier auf diesen Ansatz und seine Implikationen nicht weiter eingegangen.

\section{Versuch einer Abschätzung der Kosteneffizienz des Kinderlastenaus- gleichs}

In diesem Kapitel soll aufbauend auf die bisher gewonnenen Ergebnisse untersucht werden, welche Kosten eine Erhöhung der Fertilitätsrate durch eine Ausweitung des Kinderlastenausgleichs verursachen würde. Idealerweise sollte die Abschätzung der Kosteneffizienz des Kinderlastenausgleichs unter der Annahme erfolgen, daß die zur Geburtenförderung auf-

Vgl. Becker/Barro (1988). 
gewendeten Mittel für die wirksamsten Maßnahmen verausgabt werden. Anderenfalls wird der Vergleich der Kosteneffizienz von Kinderlastenausgleich und verstärkter Einwanderung möglicherweise dadurch verzerrt, daß die finanziellen Mittel im Rahmen des Kinderlastenausgleichs für ineffiziente Maßnahmen verwendet werden. Im Unterkapitel 4.1 wird daher erörtert, welche Maßnahmen im Rahmen des Kinderlastenausgleichs als besonders effektiv in Hinblick auf eine geburtenfördernde Wirkung einzuschätzen sind. So können mögliche Verbesserungen des bestehenden Systems erkannt werden. Leider muß es hier bei theoretischen Überlegungen bleiben, da sich einer empirischen Wirkungsanalyse einzelner $\mathrm{Maßnahmen} \mathrm{unüberwindbare} \mathrm{Schwierigkeiten} \mathrm{entgegenstellen.} \mathrm{Im} \mathrm{zweiten} \mathrm{Unterkapitel} \mathrm{(4.2)}$ wird deshalb mittels unterschiedlicher Methoden versucht, eine Wirkungsanalyse des gesamten gegenwärtigen Systems des Kinderlastenausgleichs vorzunehmen. Auf dieser Grundlage können dann wenigstens grobe Anhaltspunkte über die Kosteneffizienz des Familienlastenausgleichs gewonnen werden.

\subsection{Theoretische Überlegungen zu den Wirkungen unterschiedlicher Maßnahmen des Kinderlastenausgleichs}

Dem gegenwärtigen System des Kinderlastenausgleichs liegen unterschiedliche Zielsetzungen zugrunde. Bei der Bestimmung der Kosteneffizienz pronatalistischer Maßnahmen muß daher berücksichtigt werden, daß nicht alle Maßnahmen des Kinderlastenausgleichs dem Ziel einer Erhöhung der Geburtenzahl dienen - von offizieller Seite wird dieses Ziel schließlich nicht einmal erwähnt. Es steht daher zu vermuten, daß einige Instrumente in Hinblick auf die bevölkerungspolitische Zielsetzung effektiver, andere dagegen weniger effektiv sind. Durch eine Reallokation der Mittel auf die bevölkerungspolitisch effektivsten Instrumente könnte die Kosteneffizienz des Systems des Kinderlastenausgleichs daher gesteigert werden. In diesem Unterkapitel soll untersucht werden, welche Maßnahmen in dieser Hinsicht als besonders wirksam anzusehen sind. Da eine empirische Wirkungsanalyse einzelner Maßnahmen beim gegenwärtigen Stand der Forschung aus weiter unten zu erläuternden Gründen nicht durchführbar ist, können hier nur einige theoretische Überlegungen angeführt werden.

Zunächst ist zu untersuchen, welche Voraussetzungen eine Maßnahme erfüllen muß, damit sie überhaupt eine pronatalistische Wirkung entfaltet. Da sich die prospektiven Eltern nach dem neoklassischen Modell bei ihrer Fertilitätsentscheidung an den von ihnen erwarteten zukünftigen Nutzen und Kosten eines Kindes orientieren, muß die Maßnahme mindestens eine dieser Größen beeinflussen. Die Einwirkung auf den erwarteten Nutzen mittels politischer Maßnahmen 225 birgt - wie bereits erläutert - die Gefahr des Paternalismus und steht daher in Widerspruch zu Grundprinzipien unserer Gesellschaftsordnung, sobald sie über reine Informationsbereitstellung hinausgeht. Instrumente, die an einer Präferenzbeeinflussung ansetzen, werden hier deshalb nicht weiter betrachtet.

Wie müssen Maßnahmen beschaffen sein, die an den erwarteten Kosten ansetzen, um einen pronatalistischen Effekt zu haben? Zwei Voraussetzungen sind wesentlich: Fühlbarkeit und Verläßlichkeit. Fühlbarkeit bedeutet, daß die Maßnahme einen gewissen Mindestumfang besitzen $\mathrm{mu} ß$, um von den potentiellen Eltern als den Entscheidungsträgern überhaupt wahr- 
genommen und in das mehr oder weniger bewußt ablaufende Entscheidungskalkül einbezogen zu werden. Empirische Erhebungen ${ }^{226}$ haben gezeigt, daß viele Entscheidungsträger einen Großteil der angebotenen Maßnahmen im Rahmen des Kinderlastenausgleichs gar nicht kennen. ${ }^{227}$ Selbst Fachleuten fällt es nicht leicht, die von einer Reihe unterschiedlicher Träger gewährte Vielzahl an pronatalistisch wirkenden Maßnahmen mit den jeweiligen Sonderregelungen zu überblicken. Geht man aber davon aus, daß potentielle Eltern die Erwartungen über die Kosten eines Kindes anhand der Lebenssituation von Bekannten und Verwandten mit Kindern bilden, so ist nicht die Kenntnis einzelner Maßnahmen entscheidend, sondern die Gesamtwirkung aller Maßnahmen im Rahmen des Kinderlastenausgleichs. ${ }^{228}$ In diesem Fall ist die Voraussetzung der Fühlbarkeit als erfüllt anzusehen, denn daran, daß der Kinderlastenausgleich insgesamt einen spürbaren Einfluß auf die Kosten von Kindern hat, dürfte kaum ein Zweifel bestehen.

Als zweite Voraussetzung für die Wirksamkeit bevölkerungspolitischer Maßnahmen wurde ihre Verläßlichkeit angeführt. Nur wenn potentielle Eltern sich darauf verlassen können, daß die gegenwärtigen (und eventuell für die Zukunft in Aussicht gestellten) Instrumente auch zukünftig bestehen, werden sie voll bei der Entscheidungsfindung berücksichtigt werden. Sobald aber Unsicherheit über den Fortbestand der Leistungen besteht, werden potentielle Eltern die Vorteile aus ihnen entsprechend geringer gewichten. Diese Unsicherheit spielt gerade bei der Entscheidung für Kinder eine besonders große Rolle, weil es sich um eine sehr langfristige Festlegung ohne Revisionsmöglichkeit handelt. Betrachtet man die Geschichte des Kinderlastenausgleichs in der Bundesrepublik Deutschland (eine knapper tabellarischer Abriß findet sich in Anhang 2), dann kann von Kontinuität aber kaum die Rede sein. Einzelne Maßnahmen wurden neu eingeführt, wieder abgeschafft und dann erneut eingeführt, andere wurden einkommensabhängig gestaltet, Zuschüsse auf Darlehen umgestellt und umgekehrt, neue Leistungen wurden eingeführt, alte gekürzt oder auch stark erhöht - kurz: es fanden häufige, z.T. gegenläufige und in jedem Fall längerfristig nicht vorhersehbare Änderungen statt. Diese bewegte Vergangenheit des Kinderlastenausgleichs läßt auch für die Zukunft wenig Verläßlichkeit erwarten, obwohl einschränkend darauf hingewiesen werden muß, daß der der Umfang des Kinderlastenausgleichs insgesamt weniger großen Schwankungen ausgesetzt war als die einzelnen Maßnahmen. Dennoch dürfte die mangelnde Verläßlichkeit die demographische Wirksamkeit des Kinderlastenausgleichs in der Vergangenheit (unnötig) gesenkt haben und auch in Zukunft herabsetzen.

Die Maßnahmen des Kinderlastenausgleichs lassen sich unterteilen in reale Transfers einerseits und in monetäre Transfers andererseits. Die monetären Transfers ihrerseits bestehen aus den expliziten monetären Transfers, die Geldleistungen an die Empfänger darstellen, und den impliziten Transfers, also Vergünstigungen bei der staatlichen Abgabenerhebung (vgl. Abb. C.9). ${ }^{229}$ Unter Transfers sind dabei entsprechend der Definition der Transfer-Enquête-

226 Nach Höhn (1992) S. 151ff. konnten 42\% der Befragten in der Nordwest-Schweiz nicht die Höhe der Kinderzulage angeben. Nach Lengsfeld (1987) S. 34ff. ist auch die bundesdeutsche Bevölkerung schlecht über Leistungen im Rahmen des Kinderlastenausgleichs informiert.

227 Geht man dagegen von der neoklassischen Annahme vollständiger Information und rationaler Erwartungen aus, so kann es keine systematische Abweichung zwischen den in das Entscheidungskalkül eingehenden und den tatsächlichen Leistungen geben. Alle Maßnahmen wären dann fühlbar.

Vgl. Kaufmann (1990) S. 391.

Vgl. zu einer ähnlichen Einteilung Oberhauser (1989) S. 27. Oberhauser unterscheidet außerdem bei den expliziten monetären Transfers noch direkte und indirekte Transfers. Letztere definiert er als Trans- 
Abb. C.9: Das System des Kinderlastenausgleichs

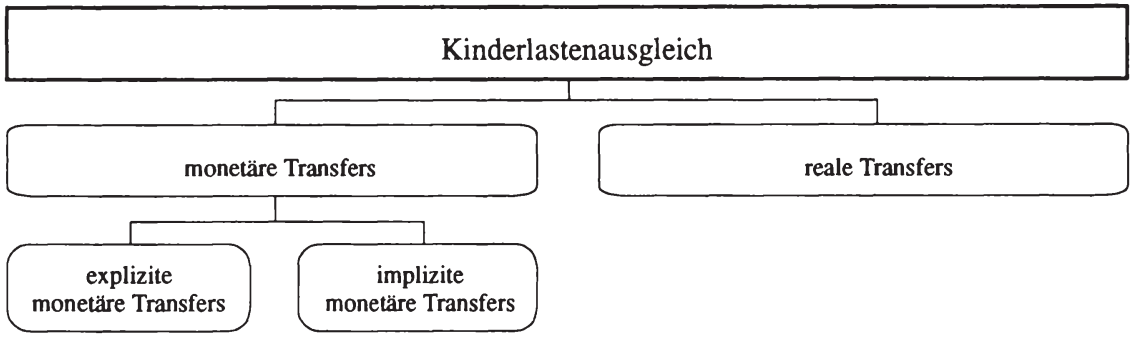

Kommission Leistungsströme zu verstehen, "denen keine spezielle Gegenleistung im Produktionsprozeß gegenübersteht und die kein Entgelt für die Nutzung von Produktionsfaktoren sind."230 Es handelt sich bei Transfers also um "Übertragungen von Ansprüchen auf Güter und Dienste außerhalb des marktmäßigenVerteilungsprozesses." 231

Welche Unterschiede bestehen in der Wirkung verschiedener Maßnahmen? Zur Beantwortung dieser Frage kann auf die in Unterkapitel 3.2 abgeleiteten Ergebnisse zurückgegriffen werden. Danach liegen die Opportunitätskosten von Kindern weit über ihren direkten Kosten. Und diese Differenz wird sich in Zukunft wahrscheinlich noch vergrößern, weil die (Frauen-) Lohnsätze wohl auch in Zukunft real steigen, die direkten Kosten sich aber - extrapoliert man

die Entwickung in der Vergangenheit - in etwa proportional zu den sonstigen Lebenshaltungskosten entwickeln werden. Außerdem sind die Kinderkosten absolut gesehen mit bis weit über eine halbe Million DM so hoch, daß eine auch nur teilweise Erstattung dieser Kosten durch den Staat schnell an Grenzen der Finanzierbarkeit stoßen würde. Unter diesem Gesichtspunkt erscheinen Maßnahmen, die von vornherein auf eine Vermeidung dieser Kosten abzielen, vorteilhafter als Maßnahmen, die eine (teilweise) nachträgliche Erstattung darstellen. Monetäre Maßnahmen haben darüber hinaus einen negativen Rückkopplungseffekt, wenn die Einkommenserhöhung über den Zusammenhang zwischen Kinderquantität und -qualität bewirkt, daß die Kinderzahl ceteris paribus zurückgeht. Weiterhin ist offenbar für potentielle Eltern in zunehmendem Maße nicht die Einkommensrestriktion bindend, sondern eher die Zeitrestriktion. Geht man davon aus, daß Zeit und Einkommen beim Aufziehen von Kindern keine vollständig substituierbaren Inputfaktoren sind, dann haben Einkommenstransfers mehr und mehr nur Mitnahmeeffekte zur Folge und tragen darüber hinaus durch die bewirkte Einkommenszunahme dazu bei, daß die Zeitrestriktion früher bindend wird als anderenfalls. Die bevölkerungspolitische Wirkung von Maßnahmen, die auf eine Verminderung der Zeitrestriktion abzielen, ist daher höher einzuschätzen.

Es spricht also vieles dafür, daß Maßnahmen zur besseren Vereinbarkeit von Familie und Beruf im Rahmen der Realtransfers wirkungsvoller sind als monetäre Maßnahmen. Alle

fers, die den Empfänger über den Umweg des Preisbildungsprozesses erreichen. Sie spielen aber im Rahmen des Kinderlastenausgleichs eine untergeordnete Rolle, weshalb die expliziten Transfers hier nicht weiter untergliedert werden.

Transfer-Enquête-Kommission (1979) S. 36.

Ebenda S. 36. 
Instrumente, die es einem Elternteil ermöglichen sollen, sich unter teilweiser oder vollständiger Aufgabe der Erwerbstätigkeit den Kindern zu widmen, gehören zu den monetären Maßnahmen. Hierzu wäre beispielsweise das Erziehungsgeld zu rechnen. Zur Gruppe der Maßnahmen zur besseren Vereinbarkeit von Familie und Beruf gehört dagegen z.B. die Bereitstellung von Kinderbetreuungseinrichtungen, Ganztagsschulen, eine Flexibilisierung der Arbeitszeiten und ihre bessere Abstimmung mit den Öffnungszeiten der Kinderbetreuungseinrichtungen, etc. Diese Maßnahmen zielen im Gegensatz zum Erziehungsgeld nicht auf eine Entscheidung zwischen entweder Kindern oder Beruf, sondern versuchen beiden Elternteilen sowohl Kinder als auch Beruf zu ermöglichen. Die Tatsache, daß in Deutschland eine im internationalen Vergleich besonders niedrige Geburtenrate mit einer niedrigen Frauenerwerbsquote einhergeht, könnte auf die hierzulande vergleichsweise schwierigere Vereinbarkeit von Kindererziehung und Beruf zurückzuführen sein.

Welche Instrumente sind innerhalb der insgesamt weniger vorteilhaften Gruppe der monetären Transfers in pronatalistischer Hinsicht als besonders günstig einzuschätzen? Wie oben schon ausgeführt, läuft der Einkommenseffekt unter gewissen Voraussetzungen dem Substitutionseffekt entgegen und schwächt somit die kinderzahlerhöhende Wirkung von monetären Transfers. Aus diesem Grund dürften paritätsabhängige ${ }^{232}$ monetäre Leistungen beispielsweise ein nach der Kinderzahl gestaffeltes Kindergeld - wirksamer sein als paritätsunabhängige Zahlungen. Bei einem paritätsabhängigen Transfer ist der Einkommenseffekt aufgrund der relativ niedrigen Leistungen für bereits geborene Kinder niedrig, die Entlastung bei einem weiteren Kind und damit der Substitutionseffekt aber groß. Desweiteren dürften einkommensabhängige monetäre Maßnahmen eine höhere Wirksamkeit besitzen, da die Kinderkosten für Geringverdienende unter denen von Höherverdienenden liegen. Die zur Entscheidung für ein weiteres Kind nötige Transfersumme wird daher in der Gruppe der Geringverdienenden geringer sein. Eine Konzentration der Leistungen auf diese Gruppe verspricht daher eine größere Steigerung der Kinderzahl als für alle Einkommensschichten gleichhohe Transferzahlungen. ${ }^{233}$

Betrachtet man den Kinderfreibetrag im Rahmen der Einkommensteuer als Beispiel für einen impliziten monetären Transfer, ist schnell festzustellen, daß er weniger wirksam ist als explizite monetäre Transfers. Die Kinderfreibetragsregelung entlastet nämlich aufgrund des progressiven Steuertarifs Höherverdienende stärker als die Bezieher niedrigerer Einkommen. Darüber hinaus sinkt die Entlastung mit der Parität, weil das zu versteuernde Einkommen und damit der Grenzsteuersatz durch jeden weiteren Kinderfreibetrag gesenkt wird. Wie oben ausgeführt, ist aber ein mit dem Einkommen zunehmender und mit der Kinderzahl abnehmender Transfer bevölkerungspolitisch besonders ineffektiv.

Als Ergebnis dieses Abschnittes bleibt festzuhalten, daß Maßnahmen zur besseren Vereinbarkeit von Familie und Beruf in Hinblick auf das Ziel einer Erhöhung der Kinderzahl als besonders wirkungsvoll einzuschätzen sind. Weniger vorteilhaft sind dagegen explizite und v.a. implizite monetäre Transfers. Durch eine Umschichtung der Mittel weg von den Steuererleichterungen und hin $\mathrm{zu}$ beispielsweise einem Ausbau der Kinderbetreuungseinrichtungen

232 Als Parität wird die Zahl der älteren Geschwister einschließlich des betrachteten Kindes bezeichnet.

233 Eine formale Herleitung der pronatalistischen Wirkung eines einkommens- und paritätsabhängigen Kindergeldes, von Kinderfreibeträgen und eines linear sinkenden Beitragssatzes in der Gesetzlichen Rentenversicherung im Vergleich zu einem paritäts- und einkommensunabhängigem Kindergeld findet sich bei Hirsch (1992) S. $279 \mathrm{ff}$. 
könnte die bevölkerungspolitische Effizienz des Kinderlastenausgleichs in der Bundesrepublik Deutschland daher wohl gesteigert werden. Betrachtet man die Entwicklung im letzten Jahrzehnt und die für die Zukunft anvisierten Änderungen, so sind die zurückgehende Bedeutung der steuerlichen Komponente im Rahmen der ab 1996 geltenden Wahlmöglichkeit zwischen Kinderfreibetrag und Kindergeld und die angestrebte Verbesserung des Versorgungsgrades mit Kindergartenplätzen positiv zu beurteilen.

\subsection{Empirische Abschätzung der demographischen Wirkung pronatalistischer Maßnahmen}

Der Versuch einer empirischen Abschätzung der demographischen Wirkungen von Maßnahmen des Kinderlastenausgleichs trifft auf eine Vielzahl schwerwiegender Probleme: Selten wird nur eine einzelne Maßnahme des Kinderlastenausgleichs isoliert eingeführt, so daß eine Zuordnung der Gesamtwirkung auf die einzelnen Teile eines Maßnahmenpaketes vorgenommen werden muß. Das würde aber die Kenntnis der Wirkungen zumindest einiger Einzelmaßnahmen voraussetzen, um dann über die Differenz zur Gesamtwirkung den Einfluß der restlichen Maßnahmen bestimmen zu können - eine Voraussetzung, die kaum gegeben ist. Verstärkt werden die Schwierigkeiten bei der Bestimmung der Wirkung einzelner Maßnahmen noch durch die unbekannten und von Maßnahme zu Maßnahme wahrscheinlich auch unterschiedlichen Wirkungsverzögerungen, die zusammen mit den Interdependenzen der einzelnen Maßnahmen zu Überlappungen, Verstärkungen oder auch Abschwächungen der isolierten Wirkung führen können. Deshalb muß hier auf eine empirische Abschätzung der Wirkung des Einsatzes von Einzelmaßnahmen verzichtet werden.

Aber auch die Wirkungsanalyse von Maßnahmenpaketen stößt auf kaum überwindbare Schwierigkeiten. So sind zunächst die Wirkungen aller anderen Einflußfaktoren auf die Fertilitätsentwicklung herauszurechnen, um die allein durch den Kinderlastenausgleich verursachte Geburtenänderung bestimmen zu können. Das scheitert schon daran, daß nicht abschließend geklärt ist, welche Faktoren denn die Fertilitätsentwicklung beeinflussen und schon gar nicht, welches Gewicht diesen einzelnen Faktoren zukommt, und ob und gegebenenfalls wie sich ihr Einfluß im Zeitablauf verändert. ${ }^{234}$ Hier liegt die schwerwiegenste Schwäche der unten dargestellten Fallstudien, da sie die Wirkungen anderer Einflußfaktoren kaum berücksichtigen. Eine Korrelation bestimmter Maßnahmen mit Änderungen der Geburtenraten stellt aber keinen ausreichenden Test der Wirksamkeit dar.

Einfacher ist hingegen die Herausrechnung bloßer Altersstruktureffekte, also von kurzfristigen Fertilitätsänderungen, die durch die Änderung der Stärken der Jahrgänge verursacht wird, die sich im reproduktiven Alter befinden. Auch bei der Betrachtung der Wirkung eines Maßnahmenbündels sind die Vorhol-, Nachhol- und Mitnahmeeffekte und der aus ihnen resultierende Unterschied zwischen kurzfristiger und langfristiger Wirkung ein Problem, das man nur durch die Betrachtung der abgeschlossenen Kinderzahl der fraglichen Kohorten umgehen kann. Da diese Daten naturgemäß erst nach einer langen Zeitspanne verfügbar sind, kann eine auf ihnen aufbauende Wirkungsanalyse aber erst Jahre oder gar Jahrzehnte nach dem Instrumenteneinsatz durchgeführt werden. Rückschlüsse auf die Wirksamkeit gegenwärtiger 
Maßnahmen können dann aber kaum noch gewonnen werden, weil sich für die Wirkung entscheidende Konstellationen von Einflußfaktoren inzwischen wahrscheinlich gewichtig verändert haben werden. Beispielsweise können Politikänderungen in anderen Bereichen (Wohnungspolitik, Arbeitsmarktpolitik, etc.) einen beträchtlichen Einfluß auf die Fertilitätsentwicklung ausüben. Die bevölkerungswirksamen Nebenwirkungen von Maßnahmen anderer Politikbereiche sind aber bisher kaum untersucht worden. ${ }^{235}$ Aus dem gleichen Grund ist neben der zeitlichen auch die räumliche Übertragbarkeit eingeschränkt: Eine Maßnahme, die in Schweden einen großen Effekt hat, kann in Frankreich nahezu unwirksam sein. ${ }^{236}$

Trotz dieser großen Vorbehalte soll im folgenden der Versuch einer Abschätzung der demographischen Wirkungen des Kinderlastenausgleichs vorgenommen werden, wobei drei unterschiedliche Vorgehensweisen herangezogen werden, die alle drei mit spezifischen Mängeln behaftet sind. Zunächst wird in Abschnitt 4.2.1 versucht, die Wirkung des Kinderlastenausgleichs durch den seit den fünfziger Jahren aufgrund der steigenden Kinderkosten zu verzeichnenden Geburtenrückgang zu ermitteln. In 4.2.2 folgt eine Untersuchung anhand von Fallstudien, ehe dann in 4.2.3 auf eine Wirkungsanalyse mit Hilfe eines Regressionsmodells eingegangen wird. Auf die Einbeziehung von Befragungen wurde verzichtet, da sie "seitens der Befragten ein Maß an Reflexionsfähigkeit und Lebensplanung voraussetzen, das im Alltag fast aller Menschen nicht vorhanden ist." 237

\subsubsection{Zeitlicher Vergleich der Kosten von Kindern}

Der erste Ansatz zur empirischen Bestimmung der Wirksamkeit des Kinderlastenausgleichs geht von der Frage aus, welche Kostensteigerung im Sinne einer Zunahme der direkten und indirekten Kinderkosten zwischen zwei Perioden festzustellen war. In einem zweiten Schritt wird dann untersucht, welchen Geburtenrückgang dieser Kostenzuwachs verursacht hat. Im Umkehrschluß ist damit auch ermittelt worden, welche finanziellen Mittel im Rahmen des monetären Kinderlastenausgleichs hätten aufgebracht werden müssen, um diesen Kostenanstieg zu kompensieren und so das Geburtenniveau auf dem Stand der Ausgangsperiode zu stabilisieren.

Zunächst müssen die beiden Vergleichsperioden festgelegt werden. Folgende Kriterien sollten bei ihrer Auswahl beachten werden: Die Perioden sollten lang genug sein, um den Einfluß zufälliger Ausschläge der Kosten und/oder Fertilitätsentwicklung zu dämpfen. Andererseits dürfen die Perioden auch nicht zu lang sein, weil sich sonst ein wesentlicher Teil der Veränderungen nicht mehr zwischen, sondern innerhalb der Perioden ergibt. Ein Zeitraum von fünf Jahren dürfte ein brauchbarer Kompromiß sein. Einerseits sollten die Perioden möglichst weit auseinander liegen, damit der Einfluß zufälliger Abweichungen der Werte dieser Perioden vom Trend möglichst klein gehalten wird. Andererseits sollte zwischen den Perioden

235 Vgl. Schubnell (1990) S. 46.

236 Eine ähnliche Darstellung der Probleme bei der Wirkungsanalyse des Kinderlastenausgleichs findet sich bei Höhn/Schubnell (1986b) S. 210f. und bei Schwarz (1988) S. 12f. Vgl. auch Kaufmann (1990) S. $387 \mathrm{ff}$.

237 Schwarz (1990) S. 495. Zu einer harschen Kritik an Versuchen, die bei Abwesenheit aller Restriktionen optimale Kinderzahl mit Hilfe von Befragungen zu ermitteln vgl. Hirsch (1992) S. $26 f$. 
aber auch kein Strukturbruch liegen, der die Vergleichbarkeit der Werte gefährdet. Es erscheint daher sinnvoll, die Anfangsperiode nicht vor Gründung der Bundesrepublik beginnen zu lassen. Weiterhin sollten beide Perioden durch eine möglichst "normale" Situation gekennzeichnet sein. D.h. weder die Kosten von Kindern noch mögliche andere Einflußfaktoren auf die Fertilität, die beispielsweise über die Erwartungen künftiger Kosten auf die Fertilitätsentwicklung Einfluß nehmen, sollten in diesem Zeitraum weit von ihrem langfristigen Trend abweichen. Da unklar ist, welche Einflußfaktoren bedeutend sind, sollten beide Perioden in wirtschaftlich und politisch ruhigen Phasen liegen. So können Verzerrungen aufgrund stark unterschiedlicher Abweichungen zwischen tatsächlichen und erwarteten Kosten minimiert werden. Sowohl die ersten Jahre nach Gründung der Bundesrepublik als auch die Zeit der Wiedervereinigung der beiden deutschen Staaten und des daraus resultierenden Wirtschaftsbooms in Westdeutschland sind daher wenig geeignet.

Sucht man anhand der genannten Kriterien eine geeignete Anfangsperiode von der Gründung der Bundesrepublik aus in die Zukunft gerichtet und die Endperiode von der Gegenwart in die Vergangenheit gerichtet, so erhält man als erste sinnvolle Anfangsperiode das Jahrfünft von 1955 bis einschließlich 1959 und als erste sinnvolle Endperiode die fünf Jahre 1985 bis 1989. Leider sind einige wichtige Zeitreihen - der Preisindex für die einfache Lebenshaltung eines Kindes und die Angaben über die Bruttoverdienste von weiblichen Angestellten erst ab 1957 verfügbar, so daß als Basisperiode die Jahre 1957 bis einschließlich 1961 verwendet werden müssen.

Nachdem die Vergleichsperioden festgelegt sind, müssen nun die Zahlenwerte der Kosten und der Fertilitätsrate für diese Zeiträume bestimmt werden. Für die Fertilitätsrate ist dies vergleichsweise einfach. Zunächst muß ein sinnvolles Fertilitätsmaß gesucht werden. ${ }^{2.38}$ Man könnte zunächst daran denken, ein Kohortenmaß zu verwenden. Das hätte den Vorteil, daß Altersstruktureffekte sowie Vor- und Nachholeffekte nicht verzerrend wirken, es also nicht aufgrund im fraglichen Zeitraum besonders stark besetzter Jahrgänge im reproduktiven Alter oder einer Verschiebung des Alters bei der Mutterschaft zwischen unterschiedlichen Frauenjahrgängen zu einem überhöhten Ausweis der Periodenfruchtbarkeit kommen kann. Kohortenmaße sind aber aufgrund von zweien ihrer Eigenschaften hier nicht anwendbar. Zum einen können sie als Längsschnittsdaten nicht für ein bestimmtes Kalenderjahr angegeben werden, sondern nur für verschiedene Geburtsjahrgänge. Zum anderen sind sie erst dann zu ermitteln, wenn die Fertilität einer Kohorte (nahezu) abgeschlossen ist, so daß sie die neuere Entwicklung nicht erfassen. Es muß also nach geeigneten Querschnittsmaßen gesucht werden.

$\mathrm{Da}$ das $\mathrm{Maß}$ keine Verzerrungen durch Altersstruktureffekte enthalten sollte, scheint sich hier zunächst die Nettoreproduktionsrate anzubieten. Sie ist aber durch die im Betrachtungszeitraum leicht rückläufige Sterbewahrscheinlichkeit verzerrt, weil dadurch in der Endperiode weniger Mädchengeburten ausreichen, um beispielsweise eine Nettoreproduktionsrate von 1,0 zu erreichen, als in der Basisperiode. Sinnvoller ist daher die Verwendung der Bruttoreproduktionsrate oder der zusammengefaßten Geburtenrate (auch als Summe der altersspezifischen Fertilitätsraten oder totale Fertilitätsrate bezeichnet). Beide haben allerdings - wie alle Periodenmaße - den Nachteil, daß sie durch eine Verschiebung des Alters bei der Geburt zwischen Frauen verschiedener Geburtsjahrgänge in der betrachteten Periode verzerrt werden. Es muß daher bei der Auswahl der Perioden darauf geachtet werden, daß dieser Einfluß in beiden Zeiträumen in etwa gleich ist oder korrigiert wird. Das läßt sich mit dem timing index 
feststellen. ${ }^{239}$ Leider ist dieser für die Endperiode noch nicht berechenbar, da dazu die Fertilität der beteiligten Kohorten bereits abgeschlossen sein müßte. Für die Jahre 1961 und 1962 lag der timing index bei 1,088 bzw. 1.087, d.h. die zusammengefaßte Geburtenrate weist als periodenbezogene Kennzahl aufgrund einer Verschiebung des Geburtszeitpunkts zwischen den Generationen einen um etwa neun Prozent überhöhten Wert aus. ${ }^{240}$ Unterstellt man, daß dieser Wert für den gesamten Zeitraum von 1957 bis 1961 Gültigkeit besitzt, so müssen die jeweiligen Werte der zusammengefaßten Geburtenraten um diesen Prozentsatz gekürzt werden. Als auf diese Art und Weise korrigierte durchschnittliche zusammengefaßte Geburtenrate ergibt sich für die Basisperiode eine Kinderzahl von 2162 je 1000 Frauen im Alter zwischen 15 und 49 Jahren.

Für die Endperiode ist - wie ausgeführt - der timing index noch nicht errechenbar. Deshalb muß hier das durchschnittliche Alter bei der Mutterschaft als grober Anhaltspunkt für die Verschiebung des Geburtszeitpunktes zwischen Frauen verschiedener Geburtsjahrgänge dienen. Im früheren Bundesgebiet stieg das durchschnittliche Alter bei der Mutterschaft leicht von 27,86 Jahren 1985 auf 28,23 Jahre 1989.241 Diese Entwicklung ist darauf zurückzuführen, daß die altersspezifischen Fertilitätsraten der älteren Jahrgänge in diesem Zeitraum leicht angestiegen sind. Dies entspricht dem längerfristigen Trend, so daß eine besondere Ballung von Geburten in diesem Zeitraum nicht zu vermuten ist. Der Durchschnitt der zusammengefaßten Geburtenraten der Endperiode von 1360 Kindern pro 1000 Frauen wird hier deshalb nicht korrigiert.

Als Ergebnis der Untersuchung der Fertilitätsentwicklung ergibt sich also ein Rückgang der (korrigierten) zusammengefaßten Geburtenrate um etwa 800 von 2160 in der Basisperiode auf 1360 Kinder je 1000 Frauen in der Endperiode.

Die Bestimmung der Zahlenwerte für die Kinderkosten erweist sich - wie in Unterkapitel 3.2 ausgeführt - als recht problematisch. Schon für die Endperiode bereitet die Berechnung einiges Kopfzerbrechen, für die Basisperiode ist sie kaum möglich. Es soll daher hier gar nicht versucht werden, die Kosten für jedes einzelne Jahr von Grund auf neu zu errechnen. Stattdessen werden die in 3.2 ermittelten Werte auf die einzelnen Jahre der relevanten Perioden zurückgerechnet.

Für die direkten Kinderkosten kann hier der Preisindex für die einfache Lebenshaltung eines Kindes herangezogen werden. ${ }^{242}$ Geht man von direkten Kinderkosten in Höhe der in Abschnitt 3.2.3 erhaltenen 180.000 DM für 1992 aus, so erhält man durch Deflationierung mit dem Preisindex für die einfache Lebenshaltung eines Kindes für die Basisperiode einen Durchschnittswert von 57.025 DM und für die Endperiode einen Wert von 159.288 DM. Um vergleichbare Werte zu erhalten, werden die jeweiligen Jahreswerte mit dem Preisindex für die Lebenshaltung eines Arbeitnehmerhaushalts mit mittlerem Einkommen ${ }^{243}$ inflationiert. ${ }^{244}$ In

239 Ist der timing index in einem bestimmten Kalenderjahr größer als eins, haben die beteiligten Kohorten in diesem Jahr einen größeren Teil ihrer Gesamtfruchtbarkeit verwirklicht als in den Jahren vorher und nachher. Eine Darstellung der Berechnungsweise des timing index befindet sich bei Dinkel (1984a) S. $32 \mathrm{f}$.

Vgl. Dinkel (1984a) S. 34. In die Berechnung einbezogen waren nur die Altersstufen 18 bis 32.

Die Daten wurden Eurostat (1992) S. 22 entnommen, weil die entsprechenden Angaben des Statistischen Bundesamtes sich nur auf eheliche Geburten beziehen.

$\mathrm{Zu}$ den damit verbundenen Problemen vgl. Abschnitt 3.2.3.2.

Der an sich geeignetere Preisindex aller privaten Haushalte wurde erst ab 1962 berechnet. 
Tab. C.8: Durchschnittlicher Bruttomonatsverdienst ${ }^{245}$ weiblicher Arbeiter und Angestellter in Industrie bzw. Industrie, Handel, Kreditinstituten und Versicherungsgewerbe (altes Bundesgebiet)

\begin{tabular}{|c|c|c|c|c|c|}
\hline Jahr & $\begin{array}{l}\text { durchschnittlicher } \\
\text { Bruttomonats- } \\
\text { verdienst weib- } \\
\text { licher Arbeiter in } \\
\text { Preisen von } 1992 \\
\text { (in DM) } \\
\end{array}$ & $\begin{array}{l}\text { durchschnittlicher } \\
\text { Bruttomonats- } \\
\text { verdienst weib- } \\
\text { licher Angestellter } \\
\text { in Preisen von } \\
1992 \text { (in DM) } \\
\end{array}$ & Jahr & $\begin{array}{l}\text { durchschnittlicher } \\
\text { Bruttomonats- } \\
\text { verdienst weib- } \\
\text { licher Arbeiter in } \\
\text { Preisen von } 1992 \\
\text { (in DM) } \\
\end{array}$ & $\begin{array}{l}\text { durchschnittlicher } \\
\text { Bruttomonats- } \\
\text { verdienst weib- } \\
\text { licher Angestellter } \\
\text { in Preisen von } \\
1992 \text { (in DM) } \\
\end{array}$ \\
\hline 1950 & 165 & & 1973 & 1.069 & 1.238 \\
\hline 1951 & 187 & & 1974 & 1.177 & 1.393 \\
\hline 1952 & 200 & & 1975 & 1.243 & 1.518 \\
\hline 1953 & 213 & & 1976 & 1.373 & 1.626 \\
\hline 1954 & 222 & & 1977 & 1.477 & 1.749 \\
\hline 1955 & 235 & & 1978 & 1.564 & 1.862 \\
\hline 1956 & 252 & & 1979 & 1.655 & 1.982 \\
\hline 1957 & 274 & 329 & 1980 & 1.755 & 2.129 \\
\hline 1958 & 287 & 346 & 1981 & 1.842 & 2.248 \\
\hline 1959 & 308 & 362 & 1982 & 1.912 & 2.366 \\
\hline 1960 & 343 & 391 & 1983 & 1.999 & 2.453 \\
\hline 1961 & 382 & 430 & 1984 & 2.073 & 2.544 \\
\hline 1962 & 426 & 473 & 1985 & 2.146 & 2.648 \\
\hline 1963 & 456 & 511 & 1986 & 2.229 & 2.764 \\
\hline 1964 & 495 & 551 & 1987 & 2.307 & 2.875 \\
\hline 1965 & 547 & 605 & 1988 & 2.394 & 2.989 \\
\hline 1966 & 587 & 658 & 1989 & 2.481 & 3.108 \\
\hline 1967 & 591 & 687 & 1990 & 2.581 & 3.265 \\
\hline 1968 & 626 & 717 & 1991 & 2.737 & 3.483 \\
\hline 1969 & 700 & 785 & 1992 & 2.859 & 3.689 \\
\hline 1970 & 782 & 887 & 1993 & 2.946 & 3.880 \\
\hline 1971 & 873 & 995 & 1994 & 3.072 & 4.012 \\
\hline 1972 & 956 & 1.099 & & & \\
\hline
\end{tabular}

Quelle: Daten des Statistischen Bundesamtes, eigene Berechnungen

Preisen von 1992 ergeben sich dann Werte von 166.900 DM für die Basisperiode und 181.360 DM für die Endperiode.

Die Rückrechnung der indirekten Kinderkosten erfolgt mit Hilfe von Zeitreihendaten für den Frauenlohnsatz. Nach den theoretischen Überlegungen in Abschnitt 3.2.1 werden die steigenden Frauenlohnsätze im betrachteten Zeitraum zu einer Substitution des Einsatzfaktors

244 Damit ergibt sich die nachfolgend angeführte Differenz zwischen den direkten Kinderkosten in der Basis- und in der Endperiode aus der unterschiedlichen Entwicklung der beiden Preisindizes.

245 Die durchschnittlichen Bruttowochenverdienste der Arbeiterinnen wurden mit dem Umrechnungsfaktor 4,345 auf Monatsbasis umgerechnet. Die nominalen Werte wurden mit dem Preisindex für die Lebenshaltung eines Arbeitnehmerhaushalts mit mittlerem Einkommen deflationiert. 
Zeit gegen den Einsatzfaktor Marktgüter bei der "Produktion" des Basisgutes Kind geführt haben. Für jedes Kind wurde in der Endperiode weniger Zeit aufgewendet als in der Basisperiode. Dieser Effekt führt zu einer leichten Überschätzung der Kostenänderung, da die niedrigen Frauenlohnsätze in den fünfziger Jahren auf den geringeren Zeiteinsatz der Erhebungsperiode bezogen werden. In Tabelle C.8 sind die durchschnittlichen Bruttomonatsverdienste weiblicher Arbeiter und Angestellter in Industrie, Handel, Kreditinstituten und Versicherungsgewerbe für das alte Bundesgebiet dargestellt.

Geht man von den in Abschnitt 3.2.1 errechneten Opportunitätskosten von 1992 nahezu 300.000 DM pro Kind aus, so ergibt sich durch Rückrechnung mit dem Lohnsatz weiblicher Angestellter ein durchschnittlicher Wert der Opportunitätskosten von 30.252 DM für die Basisperiode und von 233.966 DM für die Endperiode. Inflationiert mit dem Preisindex für die Lebenshaltung eines Arbeitnehmerhaushalts mit mittlerem Einkommen erhält man Kosten von 88.547 DM bzw. 266.390 DM in Preisen von 1992. Benutzt man statt des Lohnsatzes für weibliche Angestellte den Lohnsatz für Arbeiterinnen, ${ }^{246}$ dann ergeben sich nach Inflationierung Opportunitätskosten von 97.974 DM für die Basisperiode und 276.103 DM für die Endperiode. Da die Differenz sowohl für weibliche Angestellte wie auch für Arbeiterinnen etwa 178.000 DM beträgt, kann die weitere Berechnung auf die Werte für die Angestellten beschränkt werden. Die Summe aus direkten und indirekten Kinderkosten beläuft sich in Preisen von 1992 für die Basisperiode auf 255.400 DM und für die Endperiode auf 447.800 DM.

Dieser Kostenanstieg von $192.000 \mathrm{DM}$ in 28 Jahren hat - unter den oben genannten Einschränkungen - den Geburtenrückgang um 0,8 Kinder je Frau im gleichen Zeitraum bewirkt. ${ }^{247}$ Unterstellt man in dem relevanten Bereich einen linearen Zusammenhang zwischen Kostenanstieg und Geburtenrückgang, dann müßte der Staat pro Kind monetäre Transferleistungen von etwa $72.000 \mathrm{DM}$ finanzieren ${ }^{248}$, um die zusammengefaßte Geburtenrate um 0,3 Kinder pro Frau zu erhöhen.

Um das in der Basisperiode in etwa realisierte langfristige Bestandserhaltungsniveau wieder zu erreichen, wäre ein Anstieg um etwa 0,8 Kinder je Frau erforderlich. Es müßte somit ein Betrag von gut 190.000 DM pro Kind aufgewendet werden, was bei einer dann zu erwartenden Geburtenzahl von über 1,3 Mio eine Ausweitung des monetären Kinderlastenausgleichs um knapp 290 Mrd DM jährlich bedeuten würde. Von diesen $290 \mathrm{Mrd}$ DM sind knapp $40 \mathrm{Mrd}$ DM auf die Erhöhung der Kinderzahl bei gleichen Leistungen pro Kind (Beibehaltung der bisherigen gesetzlichen Regelungen) zurückzuführen ${ }^{249}$ und der Restbetrag von etwa 250 Mrd DM auf die Erhöhung der Leistungen pro Kind. Diese Zahlen können alternativ auch als der Betrag interpretiert werden, der nach weiteren 28 Jahren aufgewendet werden muß, um ein

246 Da das Statistische Bundesamt zu dem jeweiligen Gewicht der weiblichen Angestellten bzw. Arbeiterinnen keine Angaben machen kann, ist es leider nicht möglich, die beiden Zeitreihen zusammenzuführen.

247 Dabei ist noch nicht in Rechnung gestellt worden, daß die Ausweitung des Kinderlastenausgleichs in dem betrachteten Zeitraum den Geburtenrückgang verlangsamt hat. Ohne diese kompensierende Wirkung wäre die zusammengefaßte Geburtenrate wohl noch stärker gesunken.

248 Dieser Betrag ist als Barwert für das Jahr 1992 zu verstehen. Ob der Transfer wie hier angenommen in Form einer einmaligen Zahlungen im Geburtsjahr oder über mehrere Jahre gestreckt erfolgt, ist daher unerheblich, sofern keine Fiskalillusion vorliegt.

Tatsächlich dürfte der Betrag noch höher liegen, weil hier eine proportionale Beziehung zwischen Transfers und Kinderzahl unterstellt wurde. Da viele Transfers aber mit der Parität steigen und bei einem Anstieg der Geburtenrate wieder mehr Kinder höherer Parität geboren werden, werden die Transfers überproportional zur Kinderzahl zunehmen. 
weiteres Absacken der Geburtenrate zu verhindern und eine Stabilisierung auf dem jetzigen, schon sehr niedrigen Niveau zu erreichen. Für einen Anstieg der zusammengefaßten Geburtenrate um den als Vergleichswert mit einer verstärkten Einwanderung gewählten Wert von 0,3 Kindern je Frau wäre ein Betrag von etwa 110 Mrd DM pro Jahr aufzubringen.

Die exakten Zahlenwerte spiegeln einen Genauigkeitsgrad vor, der tatsächlich nicht annähernd gegeben ist. Die Ergebnisse sollten vielmehr nur als grobe Richtwerte aufgefaßt werden, da die Untersuchungsmethode zu umfangreicher Kritik Anlaß bietet. Eine grundlegende Annahme der letzten Interpretation der errechneten Werte ist, da $B$ die indirekten und direkten Kinderkosten sich in Zukunft genauso entwickeln werden, wie in der Vergangenheit. Das muß aber nicht unbedingt der Fall sein. Der (Frauen-)Lohnsatz als Haupteinflußfaktor der Änderung der Kinderkosten wird in Zukunft beispielsweise nur dann gleiche absolute Zuwächse der indirekten Kosten verursachen, wenn er nur in arithmetischer und nicht etwa in geometrischer Reihe steigt. Realistischer erscheint aber ein Anstieg in geometrischer Reihe (wenngleich wohl nicht mehr mit einer dem oben untersuchten Zeitraum vergleichbar hohen Wachstumsrate). Damit werden die Kosten, die zukünftig für eine Stabilisierung der Geburtenrate anfallen würden, unterschätzt.

Als weiterer Kritikpunkt ist die Nichtbeachtung anderer Einflußfaktoren anzuführen. Sollten andere Faktoren - wie etwa das höhere Wirtschaftswachstum in der Basisperiode oder die unterschiedliche Zuwanderung - neben den tatsächlichen Kosten in den Vergleichsperioden einen unterschiedlichen Einfluß auf die erwarteten Kosten genommen haben, so sind die Ergebnisse verzerrt. Die Ausweitung des Kinderlastenausgleichs könnte zu einer Unterschätzung führen. Weiterhin ist die Ermittlung der Kinderkosten mit allen in Unterkapitel 3.2 genannten Mängeln behaftet und wird zusätzlich durch die Rückrechnung mit dem nur bedingt geeigneten Preisindex für die einfache Lebenshaltung eines Kindes ungenauer. Auch die Wirkung des mit der Wiedervereinigung einhergehenden Strukturbruches ist unbekannt.

Als Ergebnis dieses Abschnitts läßt sich daher nur als grober Orientierungswert festhalten, $\mathrm{da} B$ allein die Stabilisierung des Geburtenniveaus auf dem jetzigen Niveau von etwa 1,3 Kindern pro Frau eine allmähliche Ausweitung des Kinderlastenausgleichs auf eine Höhe von 290 Mrd DM jährlich (in Preisen von 1992) in den nächsten drei Jahrzehnten erfordern würde bzw. 110 Mrd DM pro Jahr sofort aufgewendet werden müßten, um einen Anstieg der zusammengefaßten Geburtenrate um 0,3 Kinder je Frau zu erreichen.

\subsubsection{Fallbeispiele}

Zunächst sollen einige Fallbeispiele zur Wirksamkeit bevölkerungspolitischer Maßnahmen in anderen europäischen Ländern untersucht werden, später wird dann auf die Erfahrungen in Deutschland eingegangen.

Betrachtet man die Entwicklung der zusammengefaßten Geburtenraten in verschiedenen europäischen Ländern wird deutlich, daß bis nach 1945 noch relativ große Unterschiede bestehen, es im Laufe der fünfziger Jahre aber zu einer Angleichung der Reproduktionsniveaus und auch ihrer zeitlichen Entwicklung kommt: etwa Mitte der sechziger Jahre setzt in allen Ländern ein deutlicher Rückgang ein, der erst in der zweiten Hälfte der siebziger Jahre 
gestoppt wird, allerdings auf einem Niveau, das z.T. wesentlich unter dem zur Bestandserhaltung nötigen liegt.

In den Fallstudien ${ }^{250}$ soll nun untersucht werden, inwieweit erkennbare länderspezifische Besonderheiten durch Maßnahmen des Kinderlastenausgleichs bewirkt worden sein könnten. Ausgewählt wurden dabei solche Länder, die erstens in einem gewissen Umfang pronatalistische Maßnahmen eingesetzt haben (auch wenn diese nicht immer explizit als Instrumente zur Beeinflussung der Geburtenentwicklung bezeichnet wurden). Anderenfalls ist eine Wirkungsanalyse nicht sinnvoll durchführbar. Zweitens müssen die ausgewählten Länder aber auch bezüglich der Daten- und Literaturlage eine hinreichende Ausgangsbasis bieten.

Wegen des hier besonders früh einsetzenden Rückgangs der Geburtenraten hat Frankreich eine bis ins 19. Jahrhundert zürückreichende Tradition pronatalistischer Maßnahmen. ${ }^{251}$ In der Zeit nach dem Zweiten Weltkrieg kam es zunächst zu einem Babyboom, der v.a. durch das Nachholen von während des Krieges unterbliebenen Geburten, eventuell aber auch durch die geburtenfördernden Maßnahmen des 1939 erlassenen Code de la Famille und seine Ausweitung 1946 (Einbeziehung der Sozialleistungen für Familien in das Gesamtsystem der sozialen Sicherung, Familiensplitting bei der Einkommensteuer) hervorgerufen wurde. Bis Mitte der sechziger Jahre hielt das hohe Reproduktionsniveau an, was wohl auch auf die neuerlich eingeführten, v.a. monetären Maßnahmen und die hohe Bedeutung, die seitens der Politik einer wachsenden Bevölkerung zugemessen wurde, zurückzuführen ist. In dem Jahrzehnt zwischen 1965 und 1975 war die Geburtenrate stark rückläufig und sank unter das Bestandserhaltungsniveau ab. Eine Erklärung dieser Entwicklung durch das 1967 erlassene "loi Neuwirth", das das Verbot für Werbung, Herstellung, Import und Handel mit Kontrazeptiva aufhob und der 1975 erfolgten Freigabe des Schwangerschaftsabbruches greift sicherlich zu kurz, da der Geburtenrückgang schon vor diesen gesetzgeberischen Maßnahmen einsetzte und die gleiche Entwicklung auch in anderen europäischen Ländern zu beobachten war. Der starke Rückgang hat sich seit Mitte der siebziger Jahre nicht fortgesetzt, aber ein längerfristiges Ansteigen der Nettoreproduktionsrate ist gleichfalls nicht zu erkennen.

Insgesamt ist der Kinderlastenausgleich in Frankreich gekennzeichnet durch eine Vielzahl differenzierter Maßnahmen, die zusammen mit den häufigen Änderungen der Ausgestaltung dazu geführt hat, daß das System wie in Deutschland sehr unübersichtlich und kompliziert geworden ist. Hervorzuheben ist dagegen die Kontinuität des Kinderlastenausgleichs als Ganzes und sein großer Umfang. Obgleich der Anteil der staatlichen Hilfen gemessen am Familieneinkommen kontinuierlich abnahm, betrugen die Familienleistungen 1983 etwa 100 Mrd Franc. ${ }^{252}$ Die umfangreichen Leistungen führten 1982 dazu, daß das Nettoeinkommen eines verheirateten Industriearbeiters mit zwei Kindern in Frankreich um etwa $10 \%$ über

250 Die Fallstudien zu den europäischen Ländern wurden der detaillierten Analyse von Höhn/Schubnell (1986a) und (1986b) entnommen. Eine weitere sehr detaillierte Untersuchung über den Vergleich der Wirkungen der Familienpolitiken im Südwesten Baden-Württembergs, dem Nordwesten der Schweiz und dem Elsaß haben Hoffmann-Nowotny/Höhn/Fux (1992) herausgegeben. Als Ergebnis erhalten Hoffmann-Nowotny/Fux (1992) zwar eine Kovarianz von höheren Fertilitätsraten und dem Ausbaustandard der staatlichen Familienpolitik (S. 175), sind aber der Ansicht, daß der Trend sinkender Geburtenraten durch familienpolitische Maßnahmen nicht umgekehrt werden kann (S. 178).

251 Vgl. Schultheis (1988) S. 119ff. und zu einer umfassenden Darstellung der pronatalistischen Bevölkerungspolitik in Frankreich seit der Industrialisierung Schultheis (1990). 
Abb. C.10: Zeitliche Entwicklung der zusammengefaßten Geburtenraten verschiedener europäischer Länder

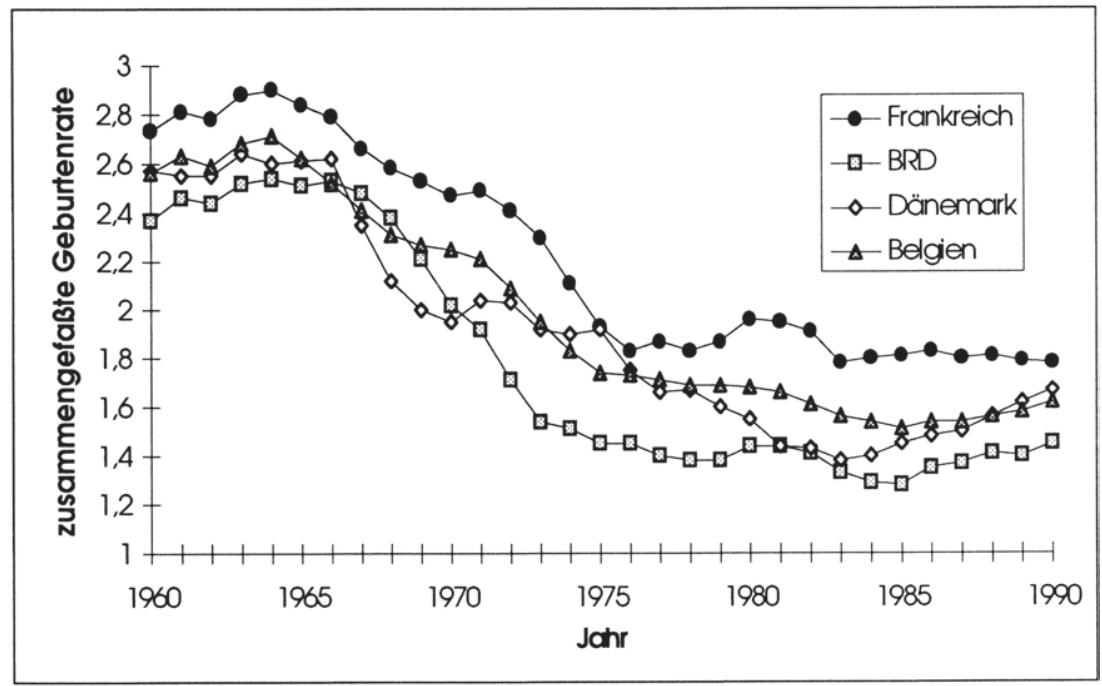

Quelle: Eurostat (1992)

seinem Bruttoeinkommen lag. ${ }^{253}$ Hervorzuheben ist auch die hohe Verfügbarkeit von z.T. kostenlosen Einrichtungen zur Betreuung von Kindern im Vorschulalter in Frankreich, die durch eine Ausrichtung auf die Bedürfnisse berufstätiger Eltern die Vereinbarkeit von Familie und Beruf in Frankreich wesentlich verbessert haben. ${ }^{254} \mathrm{Zu}$ nennen wären hier Kinderkrippen für Kinder unter drei Jahren, Vorschulkindergärten für zwei- bis sechsjährige und Kindergärten für drei- bis sechsjährige Kinder und für bereits schulpflichtige Kinder die Tatsache, daß es in Frankreich Tagesschulen gibt.

Vergleicht man die Entwicklung der zusammengefaßten Geburtenrate in Frankreich mit den Kurven für andere europäische Staaten, so läßt sich feststellen, daß in den sechziger Jahren auch dort ein deutlicher Anstieg der Geburtenraten zu verzeichnen ist, obwohl der Umfang pronatalistischer Maßnahmen in den anderen Ländern Westeuropas wesentlich geringer war.

Nimmt man an, da $\beta$ die demographischen Wirkungen des außerordentlich hohen französischen Kinderlastenausgleichs mit etwas mehr als der Differenz zwischen den Kurven zu veranschlagen ist, so erhält man eine Steigerung der Geburtenzahl um etwa zehn Prozent oder 0,2 Kinder je Frau. ${ }^{255}$

\footnotetext{
253 Vgl. Schultheis (1988) S. 114.

$254 \mathrm{Vgl}$. auch Schultheis (1988) S. 124f.

255 Vgl. Calot (1978).
} 
Abb. C.11: Zeitliche Entwicklung der zusammengefaßten Geburtenrate in verschiedenen Ländern Osteuropas

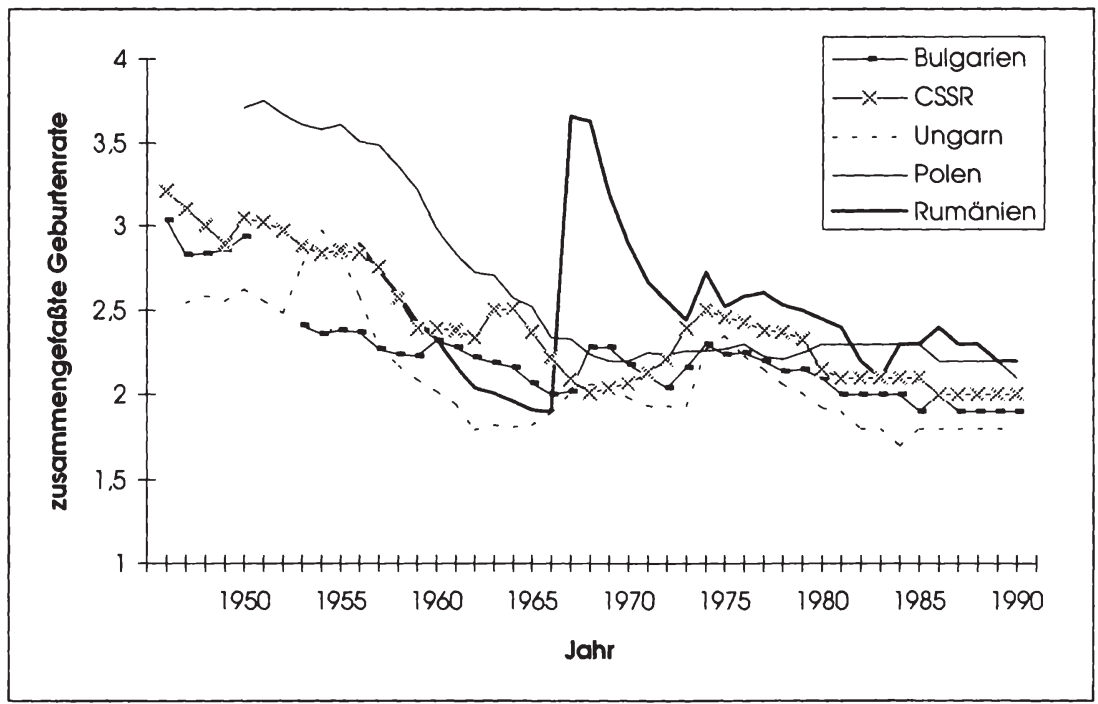

Quellen: Institut national d'études démographiques (1982), World Bank (1992)

In den anderen beiden westeuropäischen Ländern mit ausgeprägtem Familienlastenausgleich, die Höhn/Schubnell in ihre Analyse einbezogen haben - Österreich und Schweden konnte eine langfristig geburtenfördernde Wirkung weder nachgewiesen noch ausgeschlossen werden. 256

Als weitere Fallstudie soll Rumänien herangezogen werden zum einen, um die Systemabhängigkeit der Wirksamkeit von Maßnahmen des Kinderlastenausgleichs überprüfen zu können und zum anderen, weil in Rumänien besonders drastische Maßnahmen getroffen wurden. Der Unterschied zwischen der Bevölkerungspolitik in sozialistischen Staaten und westlichen Demokratien liegt v.a. darin, daß in ersteren die Festlegung der Ziele und die Wahl der Maßnahmen bedeutend weniger von der öffentlichen Meinungsbildung beeinflußt wird. Die Ausgangslage und die sich daraus ergebenden Probleme sind dagegen recht ähnlich.

Auch in Rumänien lag die zusammengefaßte Geburtenrate nach 1945 aufgrund der aufgeschobenen Geburten zunächst recht hoch, ist aber auch in dieser Zeit schon rückläufig (3,3 Kinder je Frau 1952, 2,8 1957). Der Rückgang verstärkt sich nach der Freigabe der Abtreibung 1957 und liegt Mitte der sechziger Jahre unter dem Bestandserhaltungsniveau. Als Reaktion auf diese Entwicklung wird 1966 ein Bündel drastischer bevölkerungspolitischer Maßnahmen ergriffen. Schon im folgenden Jahr hat sich die zusammengefaßte Geburtenrate mit 3,7 gegenüber 1,9 Kindern je Frau nahezu verdoppelt (vgl. Abb. C.11). Es kommt dann 
allerdings in der folgenden Zeit bis Mitte der siebziger Jahre wieder zu einem sehr steilen Rückgang, ehe sich die zusammengefaßte Geburtenrate bei etwa 2,4 annähernd stabilisiert.

Interessant an dieser Entwicklung ist v.a. der offenbar dramatische Effekt des Maßnahmenpakets von 1966, weshalb die damals eingeführten vier Veränderungen - Verbot der Abtreibung, Erschwerung der Scheidung, Besteuerung Kinderloser, sozio-ökonomische Verbesserungen - genauer beschrieben werden sollen. Die Abtreibung wurde bis auf wenige Ausnahmefälle wie z.B. schwere Erbkrankheiten und Vergewaltigung verboten. Die zusammengefaßte legale Abtreibungsrate ${ }^{257}$, die in den Jahren vorher noch bei über sieben (!) lag, ging daher auf weniger als ein Viertel zurück. ${ }^{258}$ Vor 1966 war Abtreibung in Rumänien das hauptsächliche Mittel zur Geburtenkontrolle. Der Rückgang der legalen Abtreibungen muß deshalb nicht unbedingt zu mehr Geburten geführt haben, denn an ihre Stelle können illegale Abtreibungen oder Kontrazeptiva getreten sein. Letzere waren allerdings in Rumänien nicht sehr verbreitet. ${ }^{259}$ Die Zahl illegaler Abtreibungen dürfte daher stark angestiegen sein, wofür auch die im fraglichen Zeitraum steigende Muttersterblichkeit spricht. Scheidungen wurden durch bürokratische Hindernisse und finanzielle Belastungen so sehr erschwert, daß die Scheidungsrate von knapp 2,0 je 1000 Einwohner Anfang der sechziger Jahre auf nahezu Null abstürzte, ehe sie infolge von Erleichterungen in den nachfolgenden Jahren wieder anstieg. Kinderlose über 25 Jahre müssen seit 1966 eine Steuer von ca. 1,5\% bei niedrigen und mittleren und bis zu 7\% bei hohen Einkommen zahlen. Als sozio-ökonomische Verbesserung wurde eine Geburtenbeihilfe eingeführt, eine Erhöhung des relativ geringen Kindergeldes fand dagegen nicht statt.

Welche demographischen Wirkungen wurden nun mit Hilfe dieser drakonischen Maßnahmen erzielt? Kurzfristig ist, wie oben ausgeführt und in Abb. C.11 sichtbar, ein enormer Anstieg der zusammengefaßten Geburtenrate zu verzeichnen. Versucht man die langfristige Wirkung mittels der durchschnittlichen endgültigen Kinderzahl verschiedener Kohorten abzuschätzen, so kommt man auch für den langfristigen Trend zu dem Ergebnis, daß der säkulare Rückgang der Kinderzahl je Frau gestoppt werden konnte und sich für die Geburtsjahrgänge ab 1935 ein leichter Anstieg von etwa 0,2 Kindern je Frau ergibt. ${ }^{260}$ Die langfristige Wirkung ist damit aufgrund von Vor- und Nachholeffekten wesentlich geringer als der enorme kurzfristige Anstieg.

In den anderen (ehemals) sozialistischen Staaten, die zwar auch eine pronatalistische Politik betreiben, aber mit einer geringeren Eingriffsintensität als Rumänien, sind die langfristigen Effekte noch geringer. Im Vergleich zu Staaten ohne ausgeprägte Bevölkerungspolitik (UdSSR, Polen und Jugoslawien) wird langfristig kaum eine günstigere Entwicklung erreicht, die geburtenfördernden Maßnahmen scheinen v.a. zu Vor- und Nachholeffekten zu führen, die eine ungleichmäßigere Fertilitätsentwicklung und damit auch einen ungünstigeren Altersaufbau zur Folge haben.

257 Die zusammengefaßte legale Abtreibungsrate gibt analog zur zusammengefaßten Geburtenrate die Zahl der Abtreibungen pro Frau im Verlauf ihres Lebens unter den Bedingungen des Beobachtungsjahres an.

258 Vgl. Frejka (1983) S. 499ff.

259 Vgl. Frejka (1983) S. 515f.

260 Vgl. Höhn/Schubnell (1986b) S. 198 und Trebici (1982) S. 127. 
Abb. C.12: Zeitliche Entwicklung der zusammengefaßten Geburtenrate in der DDR und der Bundesrepublik Deutschland

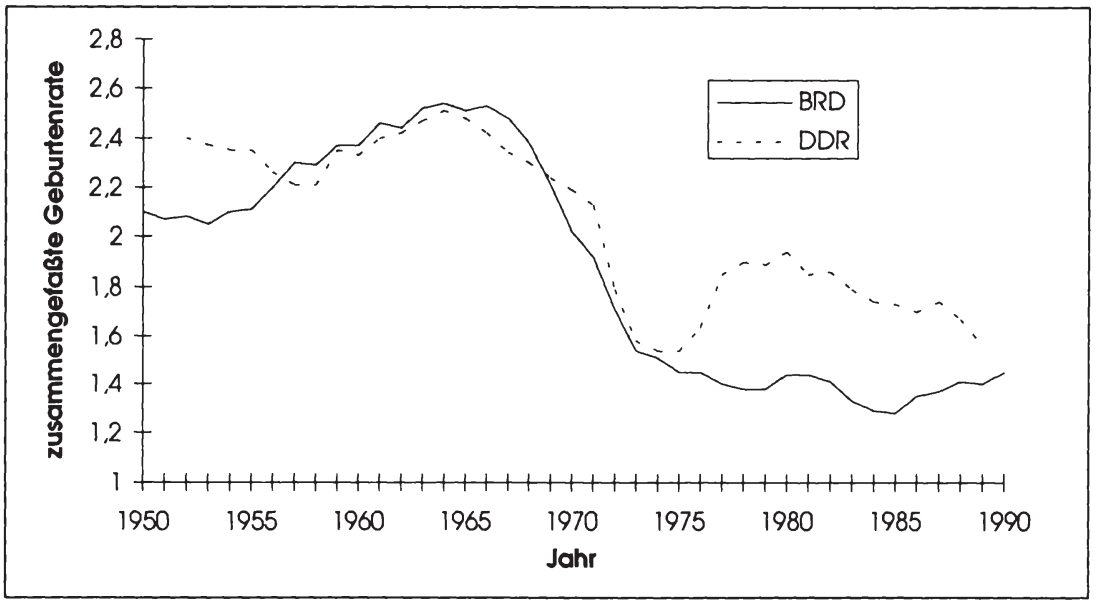

Quellen: Eurostat (1992), Statistisches Bundesamt (1993)

Neben den oben beschriebenen allgemeinen Kritikpunkten einer Wirkungsanalyse wird die Übertragbarkeit der aus Fallstudien anderer Länder gewonnenen Hypothesen über den Effekt bevölkerungspolitischer Maßnahmen dadurch beeinträchtigt, daß möglicherweise länderspezifische Traditionen, unterschiedliche Religionen und Wirtschaftssysteme dort die Geburtenentwicklung anders beeinflussen als in Deutschland. Deshalb soll nun auf Fallbeispiele aus Deutschland eingegangen werden, wobei allerdings zu beachten ist, daß aufgrund des anderen Gesellschaftssystems auch die Wirksamkeit von bevölkerungspolitischen Maßnahmen in der ehemaligen DDR nicht uneingeschränkt auf die heutige Bundesrepublik Deutschland übertragen werden können.

Zunächst sei auf die Wirkungen der pronatalistischen Maßnahmenpakete der DDR in den siebziger Jahren eingegangen.

Vergleicht man die Geburtenentwicklung in beiden deutschen Staaten seit dem letzten Weltkrieg, so ist - bis auf das erste Nachkriegsjahrzehnt - eine weitgehende Parallelität festzustellen (vgl. Abb. C.12). Von Mitte der fünfziger bis Mitte der sechziger Jahre steigt die zusammengefaßte Geburtenrate zunächst an, um dann bis 1973 drastisch abzunehmen. Auch das Niveau unterscheidet sich kaum: zunächst liegt es in der Bundesrepublik etwas höher, ab Ende der sechziger Jahre dann in der DDR. Erst ab 1973 laufen die Geburtenraten auseinander. In der Bundesrepublik setzt sich der Rückgang in abgeschwächter Form fort, während es in der DDR nach 1975 zu einem Wiederanstieg von unter 1,6 Kindern pro Frau auf etwa 1,9 kommt.

Die bevölkerungspolitischen Maßnahmen wurden in zwei Paketen 1972 und 1976 eingeführt und dann 1979 und 1984 nochmal aufgestockt und erweitert. Sie umfaßten neben 
einem Kindergeld von $20 \mathrm{M}$ für das erste und zweite und $100 \mathrm{M}$ für jedes weitere Kind und Geburtenbeihilfen (Belastung des Staatshaushalts 1975 von zusammen 1,45 Mrd M) v.a. Familiengründungsdarlehen, Unterstützung kinderreicher Familien bei Wohnungssuche und Miete, Arbeitsplätzen und Konsumgütern. Ein Schwerpunkt lag auf der Erleichterung der Berufstätigkeit von Müttern (die Erwerbsquote von Frauen lag 1983 in der DDR schon bei $88 \%$ in der Bundesrepublik dagegen erst bei $34 \%$ ). Konkrete Maßnahmen waren z.B. eine Arbeitsbefreiung bei Krankheit eines Kindes, bezahlter Urlaub vor und nach der Geburt (6 bzw. 20 Wochen; Belastung des Staatshaushalts 1975 von 296 Mio M), Zusatzurlaub, geringere Wochenarbeitszeit, Einrichtung von Krippenplätzen (Belastung 1975: 617 Mio M), etc. Auf Zwangsmaßnahmen (Abtreibungsverbot, etc.) wurde verzichtet, stattdessen wurde gerade Anfang der siebziger Jahre der Schwangerschaftsabbruch liberalisiert. Kontrazeptiva wurden kostenlos ausgegeben.

Diese kostspieligen Maßnahmen hatten kurzfristig einen großen Erfolg. Allerdings beruht die starke Zunahme der zusammengefaßten Geburtenrate Mitte der siebziger Jahre zu einem großen Teil auf Vor- und Nachholeffekten. Schon Anfang der achtziger Jahre setzt daher trotz der Aufstockungen der bevölkerungspolitisch wirksamen Leistungen 1979 und 1984 wieder ein Rückgang ein, der sich bis 1989 fortsetzt. ${ }^{261}$ Der langfristige Effekt der Maßnahmen wird von Fritsche auf etwa 0,1 Kinder pro Frau nach abgeschlossener Familiengröße geschätzt ${ }^{262}$ und reicht damit in keiner Weise aus, um das gesetzte Ziel der Bestandserhaltung zu erreichen.

Auf den ersten Blick scheint es naheliegend, die Wirkungen des Kinderlastenausgleichs in der ehemaligen DDR anhand der Geburtenänderung nach der Wiedervereinigung beider deutscher Staaten im Beitrittsgebiet zu messen, denn die Leistungen wurden recht schnell dem bundesdeutschen Niveau angepaßt. Allerdings wird die Wirkung des Kinderlastenausgleichs in dieser Umbruchsituation durch so gravierende gesellschaftliche und wirtschaftliche Änderungen überlagert, daß der Beitrag des Rückgangs des Kinderlastenausgleichs zu dem drastischen Geburtenrückgang in Ostdeutschland nicht bestimmt werden kann. Ein großer Teil der Geburtenausfälle nach 1989 dürfte auf eine zeitliche Verschiebung von Geburten zurückgehen, die zu einer Angleichung des Alters der Mutter bei der Geburt des ersten Kindes an das höhere westdeutsche Niveau führt.

In einer sehr umfangreichen Studie ${ }^{263}$ untersucht Schwarz die demographischen Wirkungen familienpolitischer Maßnahmen in der Bundesrepublik Deutschland vor der Wiedervereinigung. Er geht von einer detaillierten Darstellung der familienpolitischen Maßnahmen von Bund und Ländern aus. In einem zweiten Schritt beschreibt er dann die Entwicklung von Geburten und Eheschließungen. Aus dieser Betrachtung der bundesdurchschnittlichen demo-

261 Diese "Abnutzung" der Wirksamkeit bevölkerungspolitischer Maßnahmen tritt auch in anderen Fällen auf und wird von einigen Autoren durch einen sogenannten "Gewöhnungseffekte" erklärt (vgl. z.B. Schubnell (1990) S. 41 oder Höhn/Schubnell (1986b) S. 208). Danach werden die Leistungen nach einer gewissen Zeit als selbstverständlich angesehen und verlieren daher einen Teil ihrer Anreizwirkung. Plausibler erscheint eine Erklärung über die Unterscheidung zwischen der kurzfristigen Wirkung auf Querschnittsdaten und der langfristigen Wirkung auf die Geburtenzahl abgeschlossener Kohorten. Eine nach dem Herausrechnen kurzfristiger Effekte verbleibende Abnahme auch der langfristigen Wirksamkeit kann dann als Konsequenz der säkularen Erhöhung der indirekten Kosten verstanden werden. deren Kompensierung immer mehr bevölkerungswirksame Maßnahmen verlangt.

262 Vgl. Fritsche (1984) S. 114.

263 Vgl. Schwarz (1988). Dieser Untersuchung sind die folgenden Fallbeispiele entnommen. Einc Kurzfassung der Studie findet sich in dem von Felderer 1990 herausgegebenen Tagungsband zum Jahrestreffen des Vereins für Socialpolitik 1989 in Wien (Schwarz (1990)). 
graphischen Entwicklung leitet er vier Besonderheiten ab, die eventuell auf familienpolitische Maßnahmen des Bundes zurückzuführen sein könnten. Durch Vergleich mit den Zeitpunkten, zu denen neue Leistungen eingeführt wurden und durch Isolierung anderer Einflüsse gelangt er für die Familienpolitik des Bundes zu dem Ergebnis, daß es nicht möglich sei, "für die Nachkriegszeit Zusammenhänge zwischen der Familienpolitik des Bundes und der Entwicklung der Heiratshäufigkeit und Geburtenhäufigkeit zu entdecken."264 Dieses Ergebnis ist insofern zu relativieren, als die Betrachtung von Auffälligkeiten relativ kurzer Dauer bei Ausschaltung von Vor- und Nachholeffekten kaum ein anderes Ergebnis erwarten läßt. Inwiefern der langfristige Trend der Geburtenrate durch familienpolitische Maßnahmen des Bundes über das Niveau, das sich ohne sie eingestellt hätte, angehoben wurde, ist jedenfalls aufgrund der gewählten Vorgehensweise nicht erkennbar. ${ }^{265}$ Das Resultat der Untersuchung kann daher nur als Beleg dafür gewertet werden, daß familienpolitische Maßnahmen keine spektakulären Wirkungen hatten und die Geburtenrate nicht sprunghaft auf ein höheres Niveau anhoben, sondern allenfalls eine graduelle Trendänderung bewirkt haben.

Eine andere Vorgehensweise wählt Schwarz bei der Wirkungsanalyse von Maßnahmen auf Landesebene. Hier wird die Geburtenentwicklung in Ländern mit eigener, zusätzlich zur Familienpolitik auf Bundesebene wirkender Familienpolitik mit der Entwicklung in anderen Bundesländern und dem Bundesdurchschnitt verglichen. Dadurch sind auch langsam einsetzende Trendänderungen erkennbar. Es kann daher nicht verwundern, wenn Schwarz für die länderspezifischen Maßnahmen ein anderes Ergebnis erhält als für bundesweite Maßnahmen. In zwei Fällen läßt sich ein positiver Zusammenhang zwischen besonderen Maßnahmen und der demographischen Entwicklung feststellen. ${ }^{266}$

So geht die kontinuierliche und besonders auf eine bessere Vereinbarkeit von Familie und Beruf gerichtete Familienpolitik in West-Berlin mit einer gegenüber den anderen beiden Stadtstaaten wesentlich höheren zusammengefaßten Geburtenrate einher (vgl. Tab. C.9).

Seit 1962 werden vom Land Berlin Familiengründungsdarlehen gewährt, deren Höhe von ursprünglich 3.000 DM auf 5.000 DM aufgestockt wurde und ab 1979 bei der Geburt eines zweiten Kindes durch ein Zusatzdarlehen ergänzt wurden. Die Restschuld wird durch Geburten gemindert, ab 1979 nach der Geburt eines zweiten oder weiteren Kindes ganz erlassen. Zusätzlich erhalten Berliner seit 1983 im Anschluß an das Mutterschaftsgeld bzw. Erziehungsgeld des Bundes für weitere 12 Monate ein einkommensabhängiges Familiengeld von 400 DM. Besonders auffällig sind aber die aus Tab. C.11 ablesbare, im Vergleich zu allen

264 Schwarz (1990) S. 517.

265 Für die Existenz eines positiven Zusammenhangs zwischen familienpolitischen Maßnahmen und der Fertilitätsrate spricht die höhere Geburtenzahl von Beamten und Angestellten im öffentlichen Dienst, da die öffentliche Hand ihnen zusätzliche kinderzahlabhängige Leistungen gewährt (Kinderzulage zum Ortszuschlag, nach Kinderzahl differenzierte Beihilfesätze etc.). Eine Darstellung dieses Zusammenhangs findet sich bei Schwarz (1990) S. 498ff.

266 Schwarz (1988 und 1990) erkennt in drei Fällen eine positive Wirkung länderspezifischer Maßnahmen des Familienlastenausgleichs auf die Geburtenentwicklung. Im Fall Baden-Württembergs stützt sich dieses Ergebnis aber v.a. auf die Entwicklung der Kinderzahl pro Ehe. Bei der zusammengefaßten Geburtenrate liegt Baden-Württemberg zwar immer etwas oberhalb des Bundesdurchschnitts, ein Einfluß der erst ab Mitte der siebziger Jahre eingeführten Landesmaßnahmen ist aber nicht zu erkennen. Und auch der relative Anstieg der Kinderzahl pro Ehe setzt schon ab dem Ehejahrgang 1960 ein und kann daher kaum durch den ländereigenen Familienlastenausgleich erklärt werden. 
Tab. C.9: Zusammengefaßte Geburtenraten der Frauenjahrgänge 1930-1955 für die alten Bundesländer - je 1000 Frauen bis zum Alter von 49 Jahren

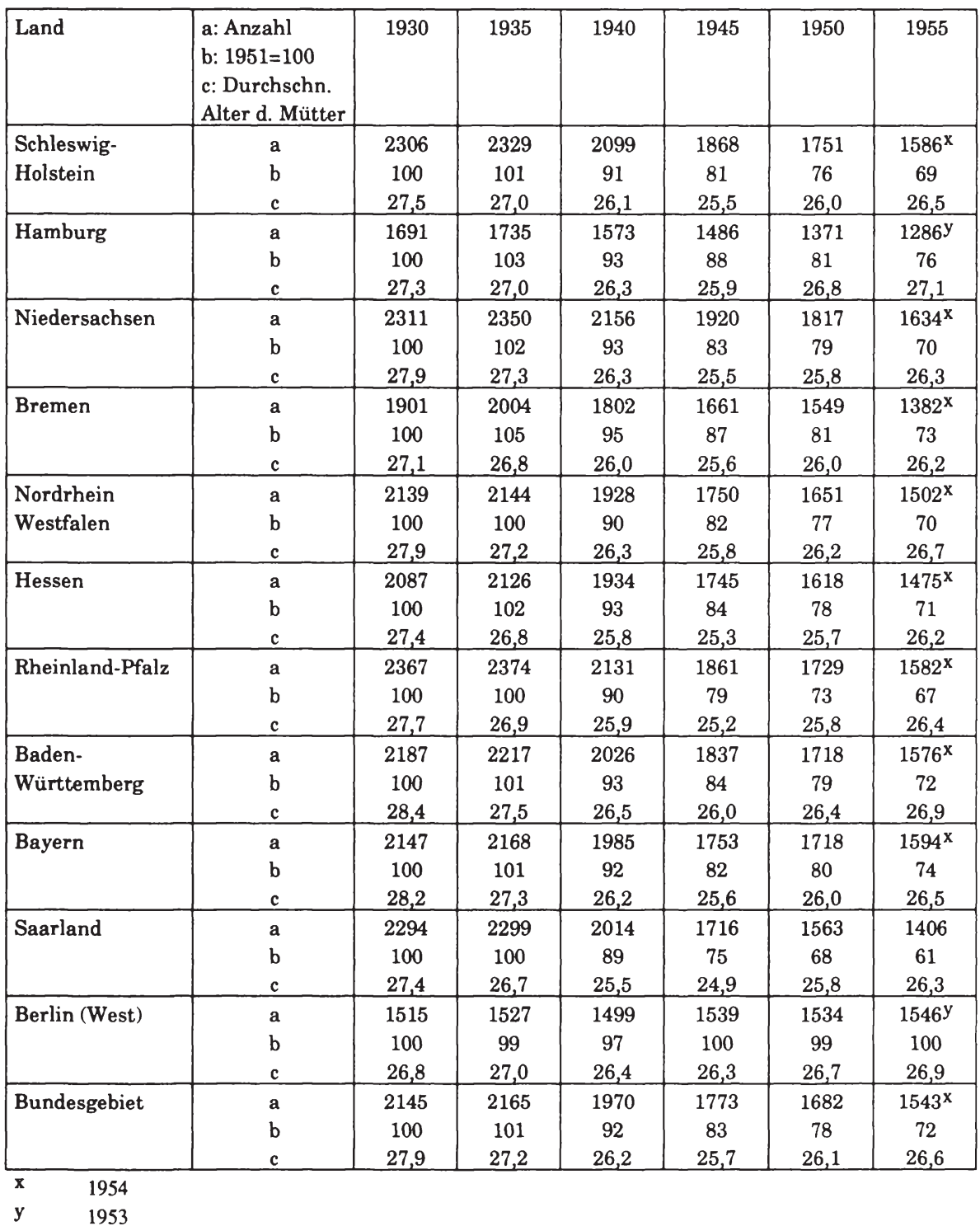

Quelle: Schwarz (1990) S. $510 f$. 
Tab. C.10: Zusammengefaßte Geburtenraten 1951-1985 für die alten Bundesländer - je 1000 Frauen bis zum Alter vom 49 Jahren

\begin{tabular}{|c|c|c|c|c|c|c|c|c|c|}
\hline Land & \begin{tabular}{|l|} 
a: Anzahl \\
b: $1951=100$ \\
c: Durchschn. \\
Alter d. Mütter \\
\end{tabular} & 1951 & 1955 & 1960 & 1965 & 1970 & 1975 & 1980 & 1985 \\
\hline $\begin{array}{l}\text { Schleswig- } \\
\text { Holstein }\end{array}$ & $\begin{array}{l}a \\
b \\
c\end{array}$ & $\begin{array}{c}2141 \\
100 \\
27,6 \\
\end{array}$ & $\begin{array}{c}2121 \\
99 \\
27,6 \\
\end{array}$ & $\begin{array}{c}2434 \\
114 \\
27,5 \\
\end{array}$ & $\begin{array}{c}2645 \\
124 \\
27,1 \\
\end{array}$ & $\begin{array}{c}2123 \\
99 \\
26,8 \\
\end{array}$ & $\begin{array}{c}1483 \\
69 \\
26,6 \\
\end{array}$ & $\begin{array}{c}1449 \\
68 \\
27,0 \\
\end{array}$ & $\begin{array}{c}1243 \\
58 \\
28,1 \\
\end{array}$ \\
\hline Hamburg & $\begin{array}{l}\mathrm{a} \\
\mathrm{b} \\
\mathrm{c}\end{array}$ & $\begin{array}{c}1554 \\
100 \\
25,8 \\
\end{array}$ & $\begin{array}{c}1508 \\
97 \\
27,4 \\
\end{array}$ & $\begin{array}{c}1807 \\
116 \\
27,5 \\
\end{array}$ & $\begin{array}{c}1948 \\
125 \\
27,1 \\
\end{array}$ & $\begin{array}{c}1483 \\
95 \\
26,9 \\
\end{array}$ & $\begin{array}{c}1164 \\
75 \\
26,7 \\
\end{array}$ & $\begin{array}{c}1226 \\
79 \\
27,4 \\
\end{array}$ & $\begin{array}{c}1101 \\
71 \\
28,2 \\
\end{array}$ \\
\hline $\begin{array}{l}\text { Nieder- } \\
\text { sachsen }\end{array}$ & $\begin{array}{l}\mathrm{a} \\
\mathrm{b} \\
\mathrm{c}\end{array}$ & $\begin{array}{c}2236 \\
100 \\
28,1 \\
\end{array}$ & $\begin{array}{c}2213 \\
99 \\
28,1 \\
\end{array}$ & $\begin{array}{c}2511 \\
112 \\
27,8 \\
\end{array}$ & $\begin{array}{c}2749 \\
123 \\
27,3 \\
\end{array}$ & $\begin{array}{c}2280 \\
102 \\
27,0 \\
\end{array}$ & $\begin{array}{c}1570 \\
70 \\
26,7 \\
\end{array}$ & $\begin{array}{c}1497 \\
67 \\
26,9 \\
\end{array}$ & $\begin{array}{c}1286 \\
58 \\
27,9 \\
\end{array}$ \\
\hline Bremen & $\begin{array}{l}\mathrm{a} \\
\mathrm{b} \\
\mathrm{c}\end{array}$ & $\begin{array}{c}1779 \\
100 \\
27,1 \\
\end{array}$ & $\begin{array}{c}1800 \\
101 \\
27,4 \\
\end{array}$ & $\begin{array}{c}2057 \\
116 \\
27,3 \\
\end{array}$ & $\begin{array}{c}2300 \\
129 \\
27,0 \\
\end{array}$ & $\begin{array}{c}1772 \\
100 \\
26,5 \\
\end{array}$ & $\begin{array}{c}1353 \\
76 \\
26,4 \\
\end{array}$ & $\begin{array}{c}1248 \\
72 \\
26,9 \\
\end{array}$ & $\begin{array}{c}1048 \\
61 \\
27,7 \\
\end{array}$ \\
\hline $\begin{array}{l}\text { Nordrhein- } \\
\text { Westfalen }\end{array}$ & $\begin{array}{l}\mathrm{a} \\
\mathrm{b} \\
\mathrm{c}\end{array}$ & $\begin{array}{c}2054^{x} \\
100 \\
28,4 \\
\end{array}$ & $\begin{array}{c}2135 \\
104 \\
28,3 \\
\end{array}$ & $\begin{array}{c}2340 \\
114 \\
28,0 \\
\end{array}$ & $\begin{array}{c}2473 \\
120 \\
27,5 \\
\end{array}$ & $\begin{array}{c}1963 \\
96 \\
27,0 \\
\end{array}$ & $\begin{array}{c}1434 \\
70 \\
26,8 \\
\end{array}$ & $\begin{array}{c}1425 \\
69 \\
27,1 \\
\end{array}$ & $\begin{array}{c}1272 \\
62 \\
27,8 \\
\end{array}$ \\
\hline Hessen & $\begin{array}{l}\mathrm{a} \\
\mathrm{b} \\
\mathrm{c}\end{array}$ & $\begin{array}{c}2012 \\
100 \\
27,7 \\
\end{array}$ & $\begin{array}{c}2012 \\
100 \\
27,5 \\
\end{array}$ & $\begin{array}{c}2295 \\
114 \\
27,2 \\
\end{array}$ & $\begin{array}{c}2413 \\
120 \\
26,8 \\
\end{array}$ & $\begin{array}{c}1898 \\
94 \\
26,4 \\
\end{array}$ & $\begin{array}{c}1388 \\
69 \\
26,4 \\
\end{array}$ & $\begin{array}{c}1362 \\
68 \\
26,8 \\
\end{array}$ & $\begin{array}{c}1188 \\
59 \\
27,7 \\
\end{array}$ \\
\hline $\begin{array}{l}\text { Rheinland- } \\
\text { Pfalz }\end{array}$ & $\begin{array}{l}a \\
b \\
c\end{array}$ & $\begin{array}{c}2397^{x} \\
100 \\
28,4 \\
\end{array}$ & $\begin{array}{c}2471 \\
103 \\
28,1 \\
\end{array}$ & $\begin{array}{c}2608 \\
109 \\
27,7 \\
\end{array}$ & $\begin{array}{c}2715 \\
113 \\
27,2 \\
\end{array}$ & $\begin{array}{c}2133 \\
89 \\
26,7 \\
\end{array}$ & $\begin{array}{c}1453 \\
61 \\
26,6 \\
\end{array}$ & $\begin{array}{c}1486 \\
62 \\
26,7 \\
\end{array}$ & $\begin{array}{c}1286 \\
54 \\
27,5 \\
\end{array}$ \\
\hline $\begin{array}{l}\text { Baden- } \\
\text { Württem- } \\
\text { berg }\end{array}$ & $\begin{array}{l}a \\
b \\
c\end{array}$ & $\begin{array}{c}2108 \\
100 \\
28,9 \\
\end{array}$ & $\begin{array}{c}2215 \\
105 \\
28,6 \\
\end{array}$ & $\begin{array}{c}2474 \\
117 \\
28,3 \\
\end{array}$ & $\begin{array}{c}2601 \\
123 \\
27,7 \\
\end{array}$ & $\begin{array}{c}2064 \\
98 \\
27,3 \\
\end{array}$ & $\begin{array}{c}1515 \\
72 \\
27,1 \\
\end{array}$ & $\begin{array}{c}1508 \\
72 \\
27,4 \\
\end{array}$ & $\begin{array}{c}1323 \\
63 \\
28,1 \\
\end{array}$ \\
\hline Bayern & $\begin{array}{l}a \\
b \\
c\end{array}$ & $\begin{array}{c}2084^{y} \\
100 \\
28,5 \\
\end{array}$ & $\begin{array}{c}2144 \\
103 \\
28,4 \\
\end{array}$ & $\begin{array}{c}2462 \\
118 \\
28,0 \\
\end{array}$ & $\begin{array}{c}2537 \\
122 \\
27,4 \\
\end{array}$ & $\begin{array}{c}2006 \\
96 \\
26,9 \\
\end{array}$ & $\begin{array}{c}1470 \\
71 \\
26,8 \\
\end{array}$ & $\begin{array}{c}1484 \\
71 \\
27 \\
\end{array}$ & $\begin{array}{c}1335 \\
64 \\
27,8 \\
\end{array}$ \\
\hline Saar & $\begin{array}{l}a \\
b \\
c\end{array}$ & $\begin{array}{c}2465^{\mathrm{x}} \\
100 \\
28,1 \\
\end{array}$ & $\begin{array}{c}2383 \\
97 \\
27,9 \\
\end{array}$ & $\begin{array}{c}2571 \\
104 \\
27,2 \\
\end{array}$ & $\begin{array}{c}2581 \\
105 \\
27,0 \\
\end{array}$ & $\begin{array}{c}1820 \\
74 \\
26,6 \\
\end{array}$ & $\begin{array}{c}1302 \\
53 \\
26,4 \\
\end{array}$ & $\begin{array}{c}1360 \\
55 \\
26,6 \\
\end{array}$ & $\begin{array}{c}1190 \\
48 \\
27,4 \\
\end{array}$ \\
\hline $\begin{array}{l}\text { Berlin } \\
\text { (West) }\end{array}$ & $\begin{array}{l}a \\
b \\
c\end{array}$ & $\begin{array}{c}1509 \\
100 \\
26,5\end{array}$ & $\begin{array}{c}1329 \\
88 \\
26,9\end{array}$ & $\begin{array}{c}1514 \\
100 \\
26,8\end{array}$ & $\begin{array}{c}1795 \\
119 \\
26,5\end{array}$ & $\begin{array}{c}1453 \\
96 \\
26,4\end{array}$ & $\begin{array}{c}1336 \\
89 \\
26,5\end{array}$ & $\begin{array}{c}1518 \\
101 \\
27,0\end{array}$ & $\begin{array}{c}1407 \\
93 \\
27,71\end{array}$ \\
\hline $\begin{array}{l}\text { Bundes- } \\
\text { gebiet }\end{array}$ & $\begin{array}{l}a \\
b \\
c\end{array}$ & $\begin{array}{c}2062 \\
100 \\
28,3\end{array}$ & $\begin{array}{c}2113 \\
102 \\
28,2\end{array}$ & $\begin{array}{c}2371 \\
115 \\
27,9 \\
\end{array}$ & $\begin{array}{c}2508 \\
122 \\
27,4 \\
\end{array}$ & $\begin{array}{c}1992 \\
97 \\
26,9 \\
\end{array}$ & $\begin{array}{c}1452 \\
70 \\
26,8 \\
\end{array}$ & $\begin{array}{c}1449 \\
70 \\
27,1 \\
\end{array}$ & $\begin{array}{c}1281 \\
62 \\
27,9 \\
\end{array}$ \\
\hline
\end{tabular}

Quelle: Schwarz (1990) S. 5I2f. 
Tab. C.11: Plätze in Kindergärten, Kinderkrippen und Kinderhorten in den alten Bundesländern 1963-1986

\begin{tabular}{|c|c|c|c|c|c|c|c|}
\hline \multirow[t]{2}{*}{ Land } & \multirow[t]{2}{*}{$\begin{array}{l}\text { Jahr } \\
19 . .\end{array}$} & \multicolumn{2}{|c|}{$\begin{array}{c}\text { Kindergärten ( } 3 \text { bis } 5 \\
\text { Jahre) }\end{array}$} & \multicolumn{2}{|c|}{$\begin{array}{c}\text { Kinderkrippen ( } 0 \text { bis } 2 \\
\text { Jahre })\end{array}$} & \multicolumn{2}{|c|}{$\begin{array}{c}\text { Kinderhorte ( } 6 \text { bis } 9 \\
\text { Jahre) }\end{array}$} \\
\hline & & $\begin{array}{c}\text { Anzahl } \\
\text { (absolut) }\end{array}$ & $\begin{array}{c}\text { pro } 1000 \\
\text { Kinder } 267\end{array}$ & $\begin{array}{c}\text { Anzahl } \\
\text { (absolut) }\end{array}$ & $\begin{array}{c}\text { pro } 1000 \\
\text { Kinder }\end{array}$ & $\begin{array}{c}\text { Anzahl } \\
\text { (absolut) }\end{array}$ & $\begin{array}{c}\text { pro } 1000 \\
\text { Kinder }\end{array}$ \\
\hline Schleswig- & 63 & 12769 & 199 & - & - & 1846 & 15 \\
\hline \multirow{3}{*}{ Holstein } & 70 & 18071 & 136 & 81 & 1 & 1449 & 9 \\
\hline & 80 & 35831 & 491 & 191 & 3 & 2700 & 23 \\
\hline & 86 & 39346 & 596 & 401 & 6 & 2624 & 28 \\
\hline \multirow[t]{4}{*}{ Hamburg } & 63 & - & - & 571 & 8 & - & - \\
\hline & 70 & 24097 & 326 & 568 & 9 & 250 & 9 \\
\hline & 80 & 20849 & 549 & 3923 & 103 & 9214 & 168 \\
\hline & 86 & 20169 & 531 & 4130 & 125 & 9336 & 187 \\
\hline \multirow[t]{4}{*}{ Niedersachsen } & 63 & 46584 & 143 & 715 & 2 & 3514 & 8 \\
\hline & 70 & 71570 & 190 & 746 & 2 & 5123 & 10 \\
\hline & 80 & 117477 & 546 & 1540 & 7 & 5990 & 18 \\
\hline & 86 & 121886 & 606 & 1841 & 10 & 6507 & 26 \\
\hline \multirow[t]{4}{*}{ Bremen } & 63 & 4982 & 163 & 58 & 2 & 1586 & 44 \\
\hline & 70 & 8288 & 237 & 55 & 2 & 1827 & 41 \\
\hline & 80 & 10897 & 621 & 79 & 5 & 2673 & 99 \\
\hline & 86 & 13238 & 735 & 142 & 7 & 588 & 28 \\
\hline \multirow{4}{*}{$\begin{array}{l}\text { Nordrhein- } \\
\text { Westfalen }\end{array}$} & 63 & 232394 & 296 & 577 & 1 & 19069 & 20 \\
\hline & 70 & 307359 & 367 & 737 & 1 & 18752 & 17 \\
\hline & 80 & 375491 & 773 & 1956 & 4 & 21646 & 29 \\
\hline & 86 & 377225 & 786 & 1816 & 4 & 20291 & 33 \\
\hline \multirow[t]{4}{*}{ Hessen } & 63 & 76082 & 341 & 600 & 2 & 5537 & 21 \\
\hline & 70 & 101228 & 395 & 814 & 4 & 5451 & 16 \\
\hline & 80 & 152807 & 961 & 1872 & 12 & 15355 & 65 \\
\hline & 86 & 144757 & 888 & 2240 & 15 & 11581 & 54 \\
\hline \multirow[t]{4}{*}{ Rheinland-Pfalz } & 63 & 73650 & 407 & 417 & 2 & 1923 & 5 \\
\hline & 70 & 87094 & 473 & 519 & 3 & 2484 & 10 \\
\hline & 80 & 102754 & 998 & 519 & 5 & 1906 & 12 \\
\hline & 86 & 110698 & 971 & 408 & 4 & 2010 & 15 \\
\hline \multirow{4}{*}{$\begin{array}{l}\text { Baden- } \\
\text { Würtemberg }\end{array}$} & 63 & 234703 & 571 & 6572 & 15 & 6450 & 13 \\
\hline & 70 & 305327 & 655 & 4113 & 10 & 8513 & 14 \\
\hline & 80 & 302446 & (1079) & 2990 & 11 & 10404 & 25 \\
\hline & 86 & 308936 & $(1047)$ & 3442 & 12 & 11947 & 33 \\
\hline \multirow[t]{4}{*}{ Bayern } & 63 & 155801 & 321 & 3581 & 7 & 19379 & 33 \\
\hline & 70 & 188911 & 360 & 3086 & 7 & 19646 & 28 \\
\hline & 80 & 218427 & 679 & 3401 & 10 & 19534 & 40 \\
\hline & 86 & 238329 & 693 & 3004 & 9 & 19431 & 47 \\
\hline
\end{tabular}

267 In Klammern Fälle, in denen die Einrichtungen offenbar auch Kinder anderer Altersgruppen als in den Fußnoten erwähnt aufnehmen. 


\begin{tabular}{|l|c|c|c|c|c|c|c|}
\hline Saarland & 63 & 24008 & 400 & - & - & 256 & 4 \\
& 70 & 29583 & 553 & 10 & 0 & 290 & 4 \\
& 80 & 29721 & 1000 & 164 & 6 & 517 & 12 \\
& 86 & 29181 & 884 & 115 & 4 & 637 & 16 \\
\hline Berlin (West) & 63 & 14415 & 244 & 4046 & 57 & 8815 & 132 \\
& 70 & 19208 & 263 & 6728 & 107 & 9152 & 96 \\
& 80 & 26988 & 540 & 9469 & 186 & 15734 & 222 \\
& 86 & 34618 & 679 & 10814 & 208 & 17920 & 326 \\
\hline Bundesgebiet & 63 & 889596 & 325 & 17137 & 6 & 68630 & 22 \\
& 65 & 925875 & 318 & 18108 & 6 & 73636 & 21 \\
& 70 & 1160736 & 385 & 17457 & 6 & 72937 & 18 \\
& 75 & 1478856 & 655 & 24251 & 13 & 82730 & 21 \\
& 80 & 1393708 & 780 & 26104 & 15 & 105673 & 39 \\
& 82 & 1354997 & 769 & 26245 & 14 & 86831 & 36 \\
& 86 & 1438383 & 798 & 28353 & 16 & 102874 & 46 \\
\hline
\end{tabular}

Quelle: Schwarz (1990) S. $506 f$.

anderen Bundesländern wesentlich höhere Verfügbarkeit von Kinderkrippen und Kinderhorten (nicht aber Kindergärten), sowie die zahlreichen mit öffentlicher Unterstützung arbeitenden Tagesmütter.

Zunächst liegen sowohl die zusammengefaßte Geburtenrate abgeschlossener Frauenjahrgänge als auch die zusammengefaßte Geburtenrate in einem bestimmten Kalenderjahr in Hamburg und Bremen deutlich über denjenigen in West-Berlin. Aber während sie in den beiden Hansestädten kontinuierlich sinken, bleiben sie in West-Berlin nahezu konstant, so daß sich Mitte der achtziger Jahre ein um etwa 0,3 Kinder pro Frau höheres Niveau ergibt. Durch den höheren Ausländeranteil in West-Berlin ist diese Differenz nur zu einem kleinen Teil zu erklären. ${ }^{268}$ Aufgrund der theoretischen Überlegungen in Unterkapitel 4.1 kann vermutet werden, daß diese doch recht beträchtliche Wirkung v.a. auf die hohe Zahl von Plätzen in Kinderbetreuungseinrichtungen zurückzuführen ist, da hierdurch die besonders hohen Opportunitätskosten des Kinderaufziehens gemindert werden können.

Besonders interessant ist auch die Fertilitätsentwicklung im Saarland. Wie aus Tab. C.9 zu entnehmen ist, sank die zusammengefaßte Geburtenrate im Saarland besonders schnell und besonders tief. Dafür mögen einerseits die wirtschaftlichen Schwierigkeiten durch den Niedergang von Kohlebergbau und Stahlindustrie verantwortlich sein. Andererseits liegt es aber auch nahe, die Verschlechterungen des Familienlastenausgleichs infolge der Wiederangliederung an die Bundesrepublik im Jahre 1957 als Erklärung heranzuziehen. Zwar war das Saarland zwischen 1948 und 1957 nicht in den vollen Genuß der Leistungen des französischen Familienlastenausgleichs gekommen, aber dennoch sank der Nettomonatslohn eines Facharbeiters im

268 Vgl. Schwarz (1988) S. 193. Welchen Einfluß die Insellage West-Berlins auf die Fertilitätsentwicklung hatte ist ungeklärt. Da die höhere Geburtenrate in West-Berlin im Vergleich zu Hamburg und Bremen aber das Ergebnis eines langfristigen Trends ist und nicht etwa in den ersten Jahren nach 1949 oder nach dem Mauerbau 1962 in Form eines sprunghaften Anstiegs ergab, ist zu vermuten, daß die Insellage keinen positiven Einfluß hatte. 
Saarland mit zwei Kindern durch die Umstellung auf den Familienlastenausgleich der Bundesrepublik ab 1960 um etwa $15 \% .^{269}$

Zieht man den Bundesdurchschnitt als Vergleichsmaßstab heran, so kann vermutet werden, $\mathrm{da} ß$ durch den Entzug dieser Leistungen das Geburtenniveau um etwa 0,2 bis 0,3 Kinder je Frau gesenkt worden ist. Diese Zahl stimmt recht genau mit den Ergebnissen überein, die oben für die Wirkung des französischen Kinderlastenausgleichs ermittelt wurde.

Als Fazit der Fallstudien läßt sich festhalten, daß trotz des oft sehr hohen Volumens des Kinderlastenausgleichs die demographischen Wirkungen recht bescheiden sind. Kurzfristig lassen sich zwar - v.a. mit repressiven Maßnahmen - auch größere Änderungen erzielen, aber meistens sind diese wohl lediglich auf Vorhol- und Nachholeffekte zurückzuführen, also auf bloße zeitliche Verlagerungen ohnehin geplanter Geburten. Langfristig dürfte selbst die Wirkung extremer Maßnahmen selten höher als 0,5 Kinder pro Frau liegen und für die Bundesrepublik eher unter 0,2 bis 0,3 Kindern je Frau betragen. Die Ergebnisse für West-Berlin und Frankreich sprechen dafür, daß entsprechend den theoretischen Überlegungen in Unterkapitel 4.1 Maßnahmen zur besseren Vereinbarkeit von Familie und Beruf tendenziell wirksamer sind als solche, die auf eine Minderung der direkten Kosten abzielen. All diese Resultate stehen allerdings unter den gewichtigen Vorbehalten, die früher gegen die Wirkungsanalyse mit Hilfe von Fallstudien vorgebracht wurden.

\subsubsection{Regressionsanalyse}

Neben Fallbeispielen sind zur Untersuchung der demographischen Wirkungen des Kinderlastenausgleichs auch Regressionsanalysen verwendet worden. Aufgrund der dargestellten Schwierigkeiten einer Wirkungsanalyse haben sich nur wenige Autoren an die Aufgabe einer ökonometrischen Umsetzung der neoklassischen Fertilitätsmodelle herangewagt und ihre Ergebnisse sind im Licht der großen Gefahr von Fehlspezifikationen zu sehen. Die Ergebnisse sollten daher nicht etwa als unumstößlich richtig interpretiert, sondern im Gegenteil als mit hoher Wahrscheinlichkeit fehlerhaft eingestuft werden. Da andererseits - wie oben ausgeführt auch die Fallstudien bestenfalls Anhaltspunkte über die Größenordnung der Wirkungen des Kinderlastenausgleichs liefern können, soll eine Regressionsanalyse als Mittel herangezogen werden, die im vorigen Abschnitt abgeleiteten Ergebnisse zu überprüfen.

Diese Vorgehensweise der Überprüfung mittels unterschiedlicher Methoden ist schon bei der Abschätzung der direkten Kosten angewandt worden. Liegen die Ergebnisse unterschiedlicher Methoden relativ nahe beieinander, so kann dies vorsichtig als eine Erhöhung der Wahrscheinlichkeit der Richtigkeit der Ergebnisse interpretiert werden. Allerdings ist auch dann noch keine Sicherheit erreicht, denn alle Studien können aus unterschiedlichen oder gleichen (etwa eine in allen getroffene, aber unrealistische Annahme) Fehlern zu einer ähnlichen Abweichung vom eigentlichen Wert führen. Die Wahrscheinlichkeit gravierend fehlerhafter Ergebnisse wird also nur gemindert, nicht aber auf Null reduziert. Liegen die Ergebnisse von Untersuchungen unterschiedlicher Vorgehensweisen weit auseinander, so bieten sich Anhaltspunkte für eine Ursachenanalyse und Fehlersuche, die im Ergebnis zu einer Verbesserung der

269 Vgl. Schwarz (1990) S. 516. Besonders einschneidend ist der Wegfall der Zahlungen der Kasse für Familienzulagen, in die $9,5 \%$ der Bruttolohnsumme abgeführt wurden. 
Schätzungen oder zum Verwerfen einer Vorgehensweise aufgrund aufgedeckter methodischer Mängel führen kann.

Liegen die Ansätze verschiedener Untersuchungen relativ dicht beieinander, d.h. weichen nur einzelne Annahmen voneinander ab (hier z.B. verschiedene Spezifikationen des Regressionsmodells), so kann dies als Sensitivitätsanalyse aufgefaßt werden. Ist dagegen die grundlegende Methodik unterschiedlich (Fallstudien und Regressionsanalyse), so handelt es sich um einen Test der Eignung dieser Methoden.

Die Regressionsanalyse von Genosko/Weber beruht auf Daten aus fünf Wellen des sozioökonomischen Panels (1984 bis 1988) und bezieht sich nur auf die ehemalige Bundesrepublik. Sie stützt sich im wesentlichen auf das Modell der neoklassischen Fertilitätstheorie, wobei allerdings einige Vereinfachungen vorgenommen wurden. ${ }^{270} \mathrm{Da}$ die Annahmen mögliche Fehlerquellen darstellen, sollen sie kurz aufgeführt werden: die Rückwirkungen des Rentenversicherungssystems auf die Fertilität wurden als für Industriestaaten unbedeutend vernachlässigt - eine sicherlich gewagte Annahme. Weiterhin wurde die Produktionsfunktion aus der Betrachtung ausgeschlossen, da durch den Kinderlastenausgleich nur die Budgetrestriktion verändert wird. Deshalb wird anstelle des aggregierten Basisgutes $Z$ ein aggregiertes Marktgut $C$ betrachtet und auch die Kinderqualität $q$ nur monetär gemessen. Problematisch ist die Annahme eines exogen gegebenen Qualitätsniveaus, denn unter 3.2.3 wurde dargestellt, daß kindgebundene Maßnahmen des Kinderlastenausgleichs über geänderte relative Schattenpreise einen iterativen Substitutionseffekt weg von der Qualität und hin zur Quantität auslösen. Dies muß bei der Interpretation der Ergebnisse beachtet werden. Die weiteren Annahmen entsprechen den oben aufgeführten Grundlagen der neoklassischen Fertilitätstheorie, so daß sich aus der Nutzenfunktion

$$
\text { C.24) } U=U(C, n, \bar{q})
$$

und der Budgetrestriktion 271

$$
\text { C.25) } E+W+\beta(n) n=C+\bar{q} n
$$

die Lagrangefunktion

$$
L=U(C, n, \bar{q})+\lambda[E+W+\beta(n) n-C-\bar{q} n]
$$

ergibt. Dabei bezeichnet $E$ das Einkommen des Mannes und das Nicht-Arbeitseinkommen, $W$ das Einkommen der Frau und $\beta(n)$ die kinderzahlabhängigen Transfers des Kinderlastenausgleichs an die Eltern.

Um die Einflußgrößen auf die Kinderzahl $(n)$ bestimmen zu können, wird die erste Ableitung nach $n$ gebildet:

C.27) $\frac{\partial L}{\partial n}=\frac{\partial U}{\partial n}+\lambda\left[\beta(n)+\frac{d \beta}{d n} n-\bar{q}\right]$

270 Die folgenden Ausführungen beruhen auf Genosko/Weber (1992).

271 Die Preise des aggregierten Konsumguts $C$ und einer Qualitätseinheit wurden jeweils auf 1 normiert. 
Genosko/Weber beziehen neben der bereits existierenden Kinderzahl und den Transferzahlungen durch den Kinderlastenausgleich als weitere Variablen auch noch die Wohnkosten, das Alter der Mutter, ${ }^{272}$ ihr Einkommen, ${ }^{273}$ das Einkommen des Mannes und die Nationalität der Eltern sowie verschiedene Variable, die das Humankapital der Mutter erfassen sollen, ${ }^{274}$ in ihr Regressionsmodell mit ein. Alle Variablen sind mit einem einjährigen lag versehen, um die Zeitverzögerung zwischen Entscheidung für ein Kind und der tatsächlichen Geburt zu erfassen.

Die Daten wurden fünf Wellen des sozioökonomischen Panels zwischen 1984 und 1988 entnommen und beziehen sich daher nur auf die alte Bundesrepublik Deutschland.

Als Ergebnis in bezug auf die Wirkung des Kinderlastenausgleichs erhalten Genosko/Weber einen hoch signifikanten positiven Einfluß des Kinderlastenausgleichs. Allerdings ist die Gefahr groß, daß mit der als Kinderlastenausgleich bezeichneten Variablen auch ganz andere Effekte erfaßt werden. Da die Berechnung der konkreten Höhe des Kinderlastenausgleichs für ein Elternpaar kaum möglich ist, wurde in der Studie nämlich für den Kinderlastenausgleich einfach eine Dummy-Variable benutzt, die für die Jahre 1984 und 1985 den Wert Null, für die folgenden drei Jahre dagegen den Wert Eins hat. Da 1986 eine Reihe von Maßnahmen des Kinderlastenausgleichs entweder ausgeweitet oder neu eingeführt wurden, 275 glauben Genosko/Weber auf diese Weise die geburtenerhöhende Wirkung dieser Maßnahmenänderung erfassen zu können. Durch diese Vorgehensweise sind in dem Koeffizienten dieser DummyVariablen allerdings die Änderungen aller Einflußfaktoren auf die Fertilität, die nicht in der Regressionsgleichung auftauchen, von 1986 bis 1988 gegenüber 1984/85 enthalten. Die Autoren dieser Studie selbst mahnen zur Vorsicht bei der Interpretation ihres Ergebnisses und weisen daraufhin, daß "the positive effect of child allowances depends decisively on the national economic stiuation (sic!) or, more accurate, on the expectations of the national economy's future development". ${ }^{276}$ Der gefundene hoch signifikante Einfluß der 1986 erfolgten

272 Als Begründung für die Einbeziehung des Alters der Mutter führen sie an: "Due to medical and other considerations it is obvious that child quality tend [sic!] to decrease with the increasing age of maternity" (Genosko/Weber (1992) S. 9). Sie spielen damit offensichtlich auf das höhere Geburtsrisiko bei älteren Frauen an und sehen darin eine Minderung der Qualität des Kindes und vermuten damit entsprechend der Nutzenfunktion auch eine geringere Fertilität mit steigendem Alter der Mutter. Das widerspricht allerdings der herkömmlichen Definition von Qualität als Aufwendungen pro Kind. die auch diese Autoren einige Seiten zuvor ihren Überlegungen zugrundelegen: " $\bar{Q}$ is an exogenous variable measured by the units of the single composite commodity which are spent for the children." (Genosko/Weber (1992) S. 4). Einen weiteren Grund für die Aufnahme des Alters der Mutter als erklärende Variable sehen Genosko/Weber unter Rückgriff auf Ermisch (1989), S. 95 darin. daß so unbeobachtete Eigenschaften der Mütter erfaßt werden können. Bei der Interpretation der Ergebnisse ist also zu beachten, daB mit dieser Variable nicht unbedingt nur das Alter der Mutter erfaßt wird.

273 Hatte die Mutter kein Einkommen, so wurde es mit Hilfe eines ähnlichen Ansatzes wie dem in 3.2.1 beschriebenen geschätzt (vgl. Genosko/Weber (1992) S. 13f.).

274 Mit Bezug auf Ermisch (1989), S. 93 und Cigno/Ermisch (1989). S. 744 weisen Genosko/Weber aber darauf hin, da $B$ mit den Humankapitalvariablen wahrscheinlich auch ein Vermögenseffekt erfaßt wird. Mit höherem Bildungsstand geht ein höheres Vermögen einher, da $B$ wiederum die Fertilität positiv heeinflußt. Es darf daher nicht überraschen, wenn statt des ansich zu erwartenden negativen Zusammenhangs zwischen Humankapital der Frau und Kinderzahl in der Studie von Genosko/Weher eine positive Korrelation ausgewiesen wird.

275 Vgl. Anhang 2.

276 Genosko/Weber (1992), S. 19. Im folgenden Satz schreiben sie allerdings "child allowances have obviously then a positive effect on the probability of births, if and only if the national economy is in a good condition" (ähnlich auch auf S. 30). Damit wird deutlich, daß sie die Wirksamkeit der Maßnahmen des Kinderlastenausgleichs als abhängig von der (erwarteten) Wirtschaftentwicklung ansehen und nicht 
Erhöhung des Kinderlastenausgleichs ist also möglicherweise zumindest teilweise Folge einer positiveren Einschätzung der künftigen wirtschaftlichen Entwicklung in den Jahren 1986 bis 1988 gegenüber den beiden vorangegangenen Jahren. Das Ergebnis wird weiter relativiert, wenn man sich vor Augen hält, daß hier die Qualität als konstant angesehen wurde. Aus den Überlegungen in Abschnitt 3.2.3 ergab sich aber ein Substitutionseffekt von Quantität zu Lasten der Qualität, so daß anstelle eines unveränderten Qualitätsniveaus mit einem Rückgang zu rechen ist. Insofern das Qualitätsniveau auch das Humankapital beinhaltet, kommt es damit zu der unerwünschten Nebenwirkung einer Minderung der zukünftigen Wachstumschancen der Volkswirtschaft. Darüber hinaus wird hier nur die kurzfristige Wirkung der Maßnahmen gemessen. Bei der Untersuchung der Fallbeispiele hat sich aber gezeigt, daß die langfristige Wirkung aufgrund der nur vorübergehend wirksamen Vorhol-, Nachhol- und Mitnahmeeffekte geringer ist als die kurzfristige.

Andererseits erfaßt die verwendete Variable nicht die demographische Wirkung des gesamten Kinderlastenausgleichs, sondern nur die Geburtenzunahme infolge seiner 1986 erfolgten Ausweitung. Demnach müßte die Wirkung des gesamten Kinderlastenausgleich noch ungleich höher sein.

Genosko/Weber führen ihre Regressionsanalyse auch getrennt für drei verschiedene Einkommensgruppen durch. Erwartungsgemäß erhalten sie eine besonders hohe Wirksamkeit, d.h. eine besonders hohe Zunahme der Wahrscheinlichkeit einer Geburt, für Frauen in der niedrigsten Einkommensklasse (Nettoeinkommen bis 2.500 DM). Die 1986 neu eingeführten oder erhöhten Transfers des Kinderlastenausgleichs sind sowohl absolut als v.a. relativ für die unteren Einkommensgruppen am bedeutensten, weshalb es nicht verwundert, wenn auch die Wirkung hier besonders groß ist. ${ }^{277}$

Zusammenfassend läßt sich feststellen, daß Regressionsanalysen zur Untersuchung des Einflußes des Kinderlastenausgleichs auf die Fertilitätsentwicklung wegen einer Vielzahl von Problemen einem hohen Risiko der Fehlspezifikation ausgesetzt sind. Das Ergebnis eines hoch signifikanten positiven Zusammenhangs in der Studie von Genosko/Weber ist entsprechend zu relativieren. ${ }^{278}$ Vor allem wegen der ungenauen Erfassung des Kinderlastenausgleichs läßt die Untersuchung keine sinnvolle Quantifizierung der Wirkung zu, sondern kann allenfalls als vorsichtige Bestätigung des theoretisch erwarteten positiven Zusammenhangs gewertet werden.

Wegen der großen Mängel aller drei betrachteten Methoden der Wirkungsanalyse ist ein Ergebnis in bezug auf die Kosteneffizienz des Kinderlastenausgleichs nur unter größten Vorbehalten ableitbar. In allen drei Vorgehensweisen wird ein pronatalistischer Effekt von Maßnahmen des Kinderlastenausgleichs bestätigt. Eine Quantifizierung der Größenordnung der

etwa einen direkten Einfluß dieser Erwartungen auf die Geburtswahrscheinlichkeit sehen, der aufgrund ihrer Modellspezifikation durch die Dummy-Variable für den Kinderlastenausgleich mit erfaßt wird.

277 Genosko/Weber (1992) S. 26 führen als Grund für die höhere Wirksamkeit in der untersten Einkommensgruppe die von Ermisch (1989) S. 84 aufgestellte Hypothese an, daß dies an den geringeren Mitteln liegt, die von Frauen dieser Einkommensklasse für fremde Kinderbetreuung aufgewendet werden können.

278 Genosko/Weber (1992) selbst ziehen auf Seite 30 den Schluß, "that the empirically stated positive impact of family allowances on demographic changes (i.e. on fertility rates) can at most be a small (perhaps only a tiny) mosaic piece for a financial release of the German Old Age Insurance". 
finanziellen Mittel, die im Rahmen des Kinderlastenausgleichs aufgewendet werden müßten, um über monetäre Anreize die Geburtenrate um 0,3 Kinder je Frau anzuheben, war nur mit Hilfe der Methode des zeitlichen Vergleichs der Kosten von Kindern möglich. Als Transferbetrag wurde ein Barwert von annähernd $72.000 \mathrm{DM}$ pro Kind bzw. etwa $110 \mathrm{Mrd}$ DM pro Jahr (jeweils in Preisen von 1992) ermittelt. Die untersuchten Fallstudien lassen eine exakte Quantifizierung ebensowenig zu, wie die im dritten Unterkapitel dargestellte Regressionsanalyse. Die vermutete Steigerung des Geburtenniveaus in Frankreich um etwa 0,2 bis 0,3 Kinder je Frau durch den umfangreichen französischen Kinderlastenausgleich und die Erfahrungen mit den pronatalistischen Maßnahmen der DDR und anderen ehemals sozialistischen Staaten lassen die aus dem Kostenvergleich abgeleiteten Ergebnisse aber ebenso plausibel erscheinen wie die Erkenntnisse, die aus den Maßnahmen einzelner Bundesländer gewonnen werden konnten. Die oben angeführten Zahlen können unter allen im Vorspann zu diesem Unterkapitel genannten Vorbehalten als ihrer Größenordnung nach bestätigt angesehen werden.

\section{Zusammenfassung}

In diesem Teil der Arbeit wurde untersucht, inwieweit mit dem Instrument des Kinderlastenausgleichs die demographische Krise der Rentenversicherung entschärft werden kann, indem über eine Ausweitung der finanziellen Leistungen im Rahmen des Kinderlastenausgleichs eine Erhöhung der Geburtenrate erreicht wird.

Dazu mußte zunächst in Kapitel 2 eine geeignete Theorie über das Fertilitätsverhalten gefunden werden. Nach der Diskussion verschiedener Ansätze wurde die neoklassische Fertilitätstheorie als Grundlage für die Untersuchung gewählt und in Kapitel 3 detailliert erläutert. Nach dieser Theorie erfolgt die Entscheidung für ein Kind ähnlich wie die Entscheidung für ein Konsumgut anhand der erwarteten Kosten und Nutzen. Der Nutzen von Kindern wurde als Ansatzpunkt für pronatalistische Maßnahmen als kaum geeignet erkannt, weshalb die weitere Analyse auf die Kosten von Kindern konzentriert wurde. Dabei wurde unterschieden zwischen den indirekten Kinderkosten (auch als Opportunitätskosten bezeichnet), die die Kosten umfassen, die aus dem Verlust alternativer Verwendungsmöglichkeiten der Zeit resultieren, und den direkten Kinderkosten, die aus dem Verzicht auf alternative Verwendungsmöglichkeiten des Einkommens entstehen.

Als Haupteinflußgröße der Opportunitätskosten eines Kindes wurde der Frauenlohnsatz identifiziert. Eine Erhöhung des Frauenlohnsatzes hat einen positiven Einkommenserhöhungsund einen diesen überkompensierenden entgegengerichteten Schattenpreisänderungseffekt auf die Kinderzahl. Unter Rückgriff auf eine Studie von Galler wurden anhand eines Humankapitalmodells die Lebenseinkommensverluste von Frauen berechnet, die aufgrund der Kinderbetreuung (vorübergehend) ihre Vollerwerbstätigkeit einschränken. Daraus wurden anschließend die durchschnittlichen Opportunitätskosten eines Kindes in einer Größenordnung von nahezu 300.000 DM errechnet.

Der Zusammenhang zwischen Quantität und Qualität von Kindern wurde als Ursache für eine Verstärkung der Wirkung mehrerer Einflußgrößen erkannt. Die säkular steigenden Einkommen führen unter bestimmten Annahmen zu einer Substitution von Quantität durch Qualität und damit zu einem Rückgang der Kinderzahl, wenn der Substitutionseffekt größer ist 
als der Einkommenseffekt. Die verstärkende Veränderung der Kinderkosten durch den Zusammenhang zwischen Quantität und Qualität wird allerdings dadurch abgemildert, daß sich gleichzeitig aufgrund der gestiegenen Qualität auch der Konsumnutzen eines Kindes gleichgerichtet verändert.

Die direkten Kosten eines Kindes betragen durchschnittlich etwa 180.000 DM pro Kind. Wie auch bei den Opportunitätskosten, ist allerdings in Abhängigkeit von verschiedenen demographischen und ökonomischen Variablen eine recht große Streuung um diesen Wert festzustellen. Die direkten Kosten haben sich preisbereinigt im Zeitablauf nur wenig verändert. Theoretische Überlegungen sprechen zudem für einen relativ geringen Einfluß dieser Kosten einer Qualitätseinheit auf die Geburtenrate, weil durch ihre Änderung kein iterativer Verstärkungsprozeß über den Zusammenhang zwischen Quantität und Qualität ausgelöst wird. Eine größere Wirkung ist dagegen von Maßnahmen des Kinderlastenausgleichs zu erwarten, die allein an der Kinderzahl ansetzten.

Aufbauend auf die Darstellung der neoklassischen Theorie des generativen Verhaltens in Kapitel 3 wurde in Kapitel 4 versucht, die Kosteneffizienz des Kinderlastenausgleichs zu bestimmen. Dazu wurde zunächst auf theoretischer Ebene die Effizienz unterschiedlicher Maßnahmen im Rahmen des Kinderlastenausgleichs untersucht. Da sich eine Abschätzung der Kosteneffizienz einzelner Maßnahmen als unmöglich erwies, wurde der nachfolgenden Wirkungsanalyse das existierende System des Kinderlastenausgleichs zugrunde gelegt. Dabei wurden drei unterschiedliche Wege beschritten. Eine genauere Quantifizierung war nur durch einen zeitlichen Vergleich der Kinderkosten möglich, der positive Einfluß des Kinderlastenausgleichs auf die Geburtenentwicklung wurde aber durch Fallbeispiele und eine Regressionsanalyse bestätigt. Die Fallbeispiele ließen auch die durch den Zeitvergleich errechnete Größenordung des Betrags plausibel erscheinen, der zu einer Anhebung der Geburtenrate um 0,3 Kinder je Frau erforderlich ist. Dieser wurde mit einem Barwert von etwa 72.000 DM pro Kind oder ungefähr 110 Mrd DM pro Jahr (in Preisen von 1992) festgestellt, wobei die Zahlenwerte allerdings aufgrund der großen Mängel der verschiedenen Methoden der Wirkungsanalyse und der Probleme bei der Erarbeitung des zugrundeliegenden Datenmaterials nur als grobe Orientierungswerte anzusehen sind. Bei dem Vergleich mit dem im folgenden Teil der Arbeit analysierten Mittel einer verstärkten Einwanderung ist außerdem zu beachten, daß die Kosteneffizienz dieser Maßnahmen sich vermutlich erheblich steigern ließe, wenn die Leistungen einkommens- und paritätsabhängig gezahlt und vermehrt Maßnahmen zur besseren Vereinbarkeit von Beruf und Familie getroffen würden. 
Teil D:

\section{VERSTÄRKTE EINWANDERUNG ALS INSTRUMENT ZUR STABILISIERUNG DER GESETZLICHEN RENTEN- VERSICHERUNG}

\section{Einleitung und Begriffsklärung}

Wie in Teil B gezeigt wurde, sind sowohl eine Erhöhung der Kinderzahl als auch eine höhere Zahl an Einwanderern grundsätzlich in der Lage, die Ausmaße der drohenden Finanzkrise der Gesetzlichen Rentenversicherung zu mindern. Beide Maßnahmen haben sich gegenüber anderen Lösungsvorschlägen als besonders vorteilhaft erwiesen, weil sie nicht nur eine Umverteilung der zukünftigen Belastungen bewirken, sondern eine Reduzierung dieser Last ermöglichen, indem sie an der Ursache der Krise, d.h. der Veränderung des Altersaufbaus der Bevölkerung, ansetzen. Ob im einzelnen eine Erhöhung der Kinderzahl oder eine verstärkte Einwanderung vorteilhafter ist, hängt von den jeweiligen Kosten ab. Nachdem oben untersucht wurde, welche Kosten mit einer Erhöhung der Fertilität durch eine Ausweitung des Kinderlastenausgleichs verbunden wären, wird in diesem Teil auf die Kosten eingegangen, die den Einheimischen durch eine verstärkte Einwanderung entstehen würden.

Die Vorgehensweise in diesem Teil der Arbeit ähnelt derjenigen in Teil C. Zunächst werden verschiedene Migrationstheorien erläutert. Leider existiert bisher kein der neoklassischen Theorie der Fertilität vergleichbarer geschlossener Theorierahmen, aus dem ableitbar wäre, welche Qualifikation die Zuwanderer aufweisen, in welchem Alter sie einwandern und wie ihr Remigrationsverhalten aussieht. Dieser Mangel wird dadurch gemildert, daß bei der Analyse der Einwanderungspolitik die Suche nach Instrumenten zur Schaffung von Einwanderungsanreizen weniger bedeutend ist. Denn für die absehbare Zukunft kann davon ausgegangen werden, daß die Zahl der Zuwanderungswilligen die Zahl derer übersteigt, die realistischerweise aufgenommen werden können. Das Fehlen eines einheitlichen Theorierahmens macht es allerdings notwendig, bei der anschließenden Analyse der verschiedenen Kosten- und Nutzenarten der Einwanderung für die Einheimischen unterschiedliche Theoriebausteine heranzuziehen. Betrachtet werden die Primärwirkungen der Einwanderung auf die Einkommensentstehung der Einheimischen, d.h. auf ihre Arbeitsmarktsituation und ihre Kapitaleinkommen, die Auswirkungen, die sich durch die umverteilende Staatstätigkeit ergeben, die Wirkung auf die Produktivitätsentwicklung und makroökonomische Folgen. Dabei wird nach der theoretischen Analyse jeweils versucht, aus empirischer Sicht die Größenordnungen der Kosten bzw. Nutzen festzustellen und Schlußfolgerungen für ein Einwanderungsgesetz zu ziehen. Abschließend wird die Kosteneffizienz einer verstärkten Einwanderung untersucht.

Bevor mit der Analyse des Migrationsverhaltens begonnen werden kann, ist zu definieren, was unter "Einwanderung" bzw. dem hier synonym gebrauchten Begriff "Zuwanderung" verstanden wird. Im Gegensatz zu einer eher formalen Definition, die an dem Erwerb der Staatsbürgerschaft anknüpft, werden hier alle Personen als Einwanderer bezeichnet, die wenigstens vorübergehend ihren Lebensmittelpunkt in das Inland verlagern. Für die ökonomischen Auswirkungen von Wanderungsbewegungen hat die Staatsbürgerschaft nur eine 
untergeordnete Bedeutung, entscheidend ist vielmehr der Wohnsitz bzw. dauernde Aufenthaltsort. ${ }^{1}$ Als Zuwanderer in diesem Sinne gelten im Jahr ihrer Einreise Asylbewerber, Asylberechtigte, De-facto-, Bona-fide- und Kontingentflüchtlinge, Gastarbeiter und deren Angehörige, EU-Ausländer, Personen ohne Aufenthaltsberechtigung, (Spät-)Aussiedler und bis zur Wiedervereinigung der beiden deutschen Staaten auch Übersiedler. ${ }^{2}$ Nicht zu den Zuwanderern gezählt werden Urlauber, Transit- und Geschäftsreisende.

Als Bestandsgröße könnte die Summe aller in der Vergangenheit zugewanderten Personen verwendet werden. Zählt man auch die Abkömmlinge der Zuwanderer zu dieser Bestandsgröße, dann besteht nahezu die gesamte Bevölkerung aus Einwanderern, weil fast jeder Einwohner mindestens einen Vorfahren besitzt, der seinen Wohnsitz aus dem Ausland in das Inland verlegt hat. Sinnvoller ist daher eine Begrenzung auf zugezogene Personen, die im Ausland geboren sind, denn ökonomisch unterscheidet sich die zweite Generation der Einwanderer oft nur noch geringfügig von den Einheimischen. Leider sind statistische Angaben über die Zahl der im Ausland Geborenen (foreign born) zwar für die USA, nicht aber für Deutschland und die meisten anderen europäischen Länder verfügbar. Deshalb wird häufig auf die Zahl der im Inland lebenden Ausländer ausgewichen. Diese Zahl kann sich aber aus mehreren Gründen stark von der Zahl der im Ausland Geborenen unterscheiden. Eine Unterschätzung ergibt sich, weil gerade für die Bundesrepublik Deutschland ein erheblicher Teil der Zuwanderer nicht Ausländer, sondern Deutsche sind (Vertriebene und Flüchtlinge, Aussiedler und Übersiedler). Außerdem wird eine wenn auch bislang relativ geringe Zahl von Ausländern der ersten Generation eingebürgert. Eine Überschätzung ergibt sich demgegenüber daraus, daß nach deutschem Staatsbürgerrecht auch die zweite und dritte Generation ausländischer Zuwanderer nicht automatisch eingebürgert werden, sondern weiterhin als Ausländer gelten. Die Zahl der in Deutschland lebenden Ausländer ist daher ein wenig befriedigendes Substitut für die Zahl der im Ausland Geborenen. ${ }^{3}$ Für internationale Vergleiche ist sie aufgrund abweichender Einbürgerungsbestimmungen in anderen Ländern nicht sinnvoll verwendbar. Die Zahl der Ausländer als Bestandsgröße wird deshalb im folgenden nur dort verwendet, wo es unumgänglich ist.

\section{Theoretische Analyse der Zuwanderung}

Wanderungen können durch verschiedene Motivationen ausgelöst werden. Neben ökonomischen sind v.a. politische, demographische und ökologische Gründe zu nennen, also z.B. die Flucht vor Verfolgung oder Krieg bzw. durch hohe Bevölkerungswachstumsraten in der

1 In der amtlichen Statistik wird unabhängig von der Staatsbürgerschaft des Migranten jede Wohnsitzverlagerung in das Gebiet der Bundesrepublik Deutschland als Zuwanderung, jeder Fortzug als Abwanderung gezählt. Die Differenz der beiden Größen gibt den Wanderungssaldo an. Vgl. Dinkel/Lebok (1994) S. 27.

2 Erläuterungen zu den verschiedenen Zuwanderergruppen finden sich in Anhang 4.

Dinkel/Lebok schreiben in bezug auf den Ausländeranteil: "Für die Bundesrepublik muß festgehalten werden, daß der sogenannte 'Ausländeranteil' an der Bevölkerung letztlich so groß ist, wie wir selbst es wünschen: Durch die Handhabung unserer Regelungen zur Gewährung der deutschen Staatsbürgerschaft bestimmen wir diese für viele Personen so 'bedrohlich' anmutende Zahl selbst." Dinkel/Lebok (1994) S. 27. 
Dritten Welt oder durch Dürreperioden induzierte Wanderungen. Demographische oder ökologische Ursachen können allerdings unter die ökonomische Motivation gefaßt werden, da sie im allgemeinen erst durch ihre ökonomischen Konsequenzen für eine Wanderungsentscheidung relevant werden. Sie werden daher hier nicht separat untersucht.

Bei einer konkreten Wanderungsentscheidung wirken die verbleibenden ökonomischen und politischen Faktoren zusammen und sind für die Behörden des Einwanderungslandes oft nur schwer voneinander unterscheidbar. Dies gilt umso mehr als Zuwanderungswillige Anreize zu strategischem Verhalten haben, wenn die Wahrscheinlichkeit der Aufnahme in das Gastland von der Angabe eines bestimmten Beweggrundes abhängt. So fält wahrscheinlich ein Großteil der Asylbewerber in Deutschland unter die Kategorie überwiegend ökonomisch motivierter Zuwanderer, die allein deshalb eine politische Begründung angeben, weil sich dadurch ihre Aufnahmechancen erhöhen. ${ }^{4}$ Trotz dieser Verflechtung von ökonomischen und politischen Wanderungsgründen ist eine Differenzierung nach Wanderungsmotiven sinnvoll, denn eine Kontingentierung der politisch induzierten Zuwanderung nach ökonomisch ausgerichteten Kriterien wie Alter und Ausbildungsstand erscheint ethisch kaum akzeptabel.

Politisch motivierte Wanderungen spielen bei der Erklärung von Wanderungsströmen eine bedeutende Rolle - man denke beispielsweise an die große Zahl der Bürgerkriegsflüchtlinge aus dem ehemaligen Jugoslawien in der ersten Hälfte der neunziger Jahre. Allerdings ist es hauptsächlich die Ausreiseentscheidung, die aufgrund politischer Motive getroffen wird. Die Wahl des Ziellandes erfolgt dagegen auch bei politischen Migranten überwiegend nach ökonomischen Kriterien.5 Darüber hinaus sind politisch motivierte Wanderungsströme starken Schwankungen unterworfen und eine langfristige Prognose über ihren Verlauf ist daher nicht möglich. ${ }^{6}$ Die langfristige Sicherung der Rentenversicherung auf die Hoffnung zu bauen, da $\beta$ in Zukunft eine große Zahl von Zuwanderern aus politischen Motiven nach Deutschland einwandert, bietet daher keine ausreichende Planungssicherheit. Außerdem können sich durch eine aktive Förderung politisch motivierter Zuwanderung unerwünschte Zielkonflikte mit einer auf Stabilität gerichteten Außenpolitik ergeben. Politisch motivierte Zuwanderung wird daher im folgenden allenfalls als Randbedingung in dem Sinne zu berücksichtigen sein, daß ein Einwanderungsgesetz flexibel genug gestaltet werden muß, um die temporär stark schwankende Zahl von aus politischen Gründen Zuwandernden in ein umfassendes Einwanderungskonzept zu integrieren.

In diesem Kapitel wird deshalb nur die ökonomisch induzierte Zuwanderung anhand verschiedener ökonomischer Wanderungsmodelle analysiert. Dabei wird zunächst in Unterkapitel 2.1 anhand makroökonomischer und mikroökonomischer Erklärungsansätze das Wanderungspotential, also die Angebotsseite, untersucht. In Unterkapitel 2.2 interessiert dann, wovon die Nachfrage nach Zuwanderern abhängt. Dabei wird unterschieden zwischen ökonomischen und politisch-ökonomischen Einflußgrößen. Die Ergebnisse dieser Modelle werden in den folgenden Kapiteln benutzt, um die Charakteristika der Einwanderer zu erklären

4 Zimmermann (1994a) S. 227 schreibt in diesem Zusammenhang: "The prevailing view is that a large share of the asylum seekers are actually economic migrants, and that they are abusing the asylum rights. This is difficult to prove, but also difficult to reject. It can be conjectured that it is the impossibility of applying for a work permit that leads many people to abuse the asylum rights. The impossibility of legal labor immigration could be a major policy failure, because as an economic migrant an individual would work and contribute to society's welfare, but as an asylum seeker waiting to be granted asylum she would normally not."

$5 \quad$ Vgl. Darvish-Lecker/Kahana (1992).

6 Eine interessante Analyse der politisch-ökonomischen Hintergründe und der Wirkungsweise der Fortzugsbeschränkungen in den früheren Ostblock-Staaten liefern Tietzel/Weber (1993). 
und Möglichkeiten zu deren Beeinflussung aufzuzeigen. Da die Merkmale der Einwanderer wiederum maßgeblich über die durch sie hervorgerufenen Kosten entscheiden, bilden die folgenden Überlegungen auch die Basis zur Erklärung der Höhe dieser Kosten und der Möglichkeiten zu ihrer Beeinflussung.

\subsection{Analyse des Wanderungspotentials}

\subsubsection{Makroökonomische Erklärungsansätze}

Die meisten makroökonomischen Ansätze zur Erklärung des Wanderungsverhaltens basieren auf dem Heckscher-Ohlin-Samuelson Modell. ${ }^{7}$ Das Modell beruht auf den folgenden sechs Grundannahmen: ${ }^{8}$

- vollständige Konkurrenz auf Güter- und Faktormärkten mit gewinnmaximierenden Unternehmen und nutzenmaximierenden Haushalten als Akteuren,

- international handelbare Güter,

- international identische, konkave Produktionsfunktionen mit konstanten Skalenerträgen und positiven, aber mit zunehmendem Einsatz eines Faktors abnehmenden Grenzerträgen,

- identische, homothetische Nachfragepräferenzen in allen Ländern,

- vollständige regionale und sektorale Mobilität der Produktionsfaktoren innerhalb eines Landes,

- international immobile Produktionsfaktoren.

In dem Zwei-Länder, Zwei-Sektoren, Zwei-Güter-Modell wird internationaler Handel durch unterschiedliche Faktorausstattungen der Länder hervorgerufen. Jedes Land wird das Gut exportieren, bei dessen Produktion der Faktor überproportional eingesetzt wird, mit dem das Land relativ reichlich ausgestattet ist. ${ }^{9}$ Der internationale Handel führt nicht nur zu international einheitlichen Güterpreisen, sondern unter den oben angeführten Prämissen wird darüber hinaus auch eine internationale Angleichung der Faktorpreise erreicht (factor-priceequalization theorem). Der internationale Handel schafft damit eine international effiziente Allokation. ${ }^{10}$ Eine Wanderung der Produktionsfaktoren Arbeit und Kapital, die annahmegemäß im Vergleich zum Warenhandel mit relativ hohen Transaktionskosten verbunden ist, kann durch freien internationalen Warenhandel vermieden werden.

Erst wenn aufgrund von rechtlichen und administrativen (Zölle, nicht-tarifäre Handelsbeschränkungen) sowie natürlichen (hohe Transportkosten, nicht handelbare Güter) Handelshemmnissen eine Faktorpreisangleichung durch internationalen Freihandel nicht möglich ist,

7 Ricardos Theorie komparativer Vorteile erwies sich aufgrund der Beschränkung auf einen Produktionsfaktor als Grundlage für Erweiterungen, die die Erklärung internationaler Wanderungen zum Ziel haben, als weniger geeignet.

$8 \quad$ Vgl. Straubhaar (1992) S. 563.

9 Eine ausführliche Darstellung des Heckscher-Ohlin-Samuelson Modells findet sich z.B. bei Rose/Sauernheimer (1992).

10 Vgl. Straubhaar (1992) S. 565. 
besteht aufgrund von Entlohnungsunterschieden ein Anreiz zu Faktorwanderungen. " Dieser Anreiz bleibt bestehen, bis der fortgesetzte Zustrom von Arbeitskräften in das relativ kapitalreiche Land sowie die Kapitalverlagerung in das Land mit relativ reichlicher Ausstattung an Arbeitskräften zu einer Angleichung der Grenzproduktivitäten und damit der Faktorpreise geführt hat. Die Faktorpreisangleichung kann also sowohl durch internationalen Handel von Gütern als auch durch internationale Faktorwanderungen oder eine Kombination von beidem erreicht werden. Handel und Faktorwanderungen sind daher in diesem Modellrahmen perfekte Substitute.

Als Beispiel für eine wanderungsinduzierende Handelsbeschränkung soll die Einführung eines Zolls auf das arbeitsintensive Importgut seitens des kapitalreichen Landes betrachtet werden. Nach dem Stolper-Samuelson-Theorem führt eine derartige Maßnahme dazu, daß der Faktorpreis desjenigen Faktors steigt, der in der Produktion des durch den Zoll verteuerten Gutes relativ intensiv genutzt wird. In dem hier betrachteten Fall kommt es also zu einer Erhöhung des Lohnsatzes in dem kapitalreichen Land. Dadurch werden internationale Wanderungen von Arbeitskräften aus dem arbeitsreichen in das kapitalreiche Land ausgelöst. ${ }^{12}$ Diese Wanderungen kommen erst zum Stillstand, wenn der Import des arbeitsintensiven Gutes aufgrund höherer inländischer Produktion in dem kapitalreichen Land völlig zum Erliegen kommt.

Wie sich dieses Ergebnis ändert, wenn die recht restriktiven Annahmen des HeckscherOhlin-Samuelson Modells gelockert werden und zunehmende Skalenerträge, unvollständiger Wettbewerb, Steuern auf Produktion und Faktoren oder unterschiedliche Produktionstechnologien in die Betrachtung einbezogen werden, hat Markusen untersucht. ${ }^{13}$ In diesen Fällen sind Faktorwanderungen und internationaler Handel komplementär: Die Produktionsfaktoren wandern in die Länder, die einen relativen Kostenvorteil bei den Gütern haben, bei deren Erstellung sie relativ intensiv genutzt werden.

Folgendes auf Arbeitskräftewanderungen bezogene Beispiel diene zur Veranschaulichung für den Fall unterschiedlicher Produktionsfunktionen. Land A habe einen technologischen Vorteil bei der Produktion des kapitalintensiven Gutes, das es daher exportiert. Bei ungehindertem Handel gelten in beiden Ländern die gleichen Güterpreise, so daß sich bei gleicher Faktorausstattung der technologische Vorteil des Landes A bei der Produktion des kapitalintensiven Gutes in einer höheren Entlohnung des Kapitals und entsprechend in einer niedrigeren Entlohnung des Faktors Arbeit im Vergleich zu Land B niederschlägt. ${ }^{14}$ Ist der Faktor Arbeit international mobil, werden daher Arbeitskräfte von A nach B wandern. Nach dem Rybcynski-Theorem wird bei Erhöhung des Mengenbestandes einer Ressource ceteris paribus die Produktion des Gutes erhöht, das diesen Faktor relativ intensiv nutzt und die Produktion des anderen Gutes gemindert. In dem hier betrachteten Fall wird daher in Land A die

11 Die Auswirkungen von Handelshemmnissen auf internationale Faktorwanderungen unter den Annahmen des Heckscher-Ohlin-Samuelson Modells wurden zuerst von Mundell (1957) untersucht.

12 Entsprechend des Untersuchungsziels werden hier Wanderungen von Arbeitskräften untersucht und angenommen, daß Kapital international nicht mobil ist. Betrachtet man Kapital als den mobilen Faktor. ergibt sich gleichfalls eine handelssubstituierende Wirkung.

13 Vgl. Markusen (1983).

14 In Land $\mathrm{A}$ wird das arbeitsintensive Gut mit weniger Kapital- und mehr Arbeitseinsatz hergestellt als in Land B, weil ein größerer Anteil des Kapitals durch die vermehrte Produktion des kapitalintensiven Gutes gebunden ist. Bei Entlohnung nach dem Wertgrenzprodukt sowie gleichen Produktionsfunktionen und Preisen für das arbeitsintensive Gut in beiden Ländern hat das höhere Arbeit/Kapital-Einsatzverhältnis in Land A einen niedrigeren Lohn und einen höheren Zinssatz als in Land B zur Folge. 
Produktion des kapitalintensiven Gutes zunehmen, in Land B die Produktion des arbeitsintensiven Gutes. In beiden Ländern wird also mehr von dem jeweiligen Exportgut hergestellt und der internationale Handel damit durch die Faktorwanderungen ausgedehnt. Internationaler Handel und Faktorwanderungen sind jetzt also nicht mehr Substitute, sondern Komplemente. ${ }^{15}$

In Modellen vom Ricardo-Viner Typ wird eine Erweiterung des Grundmodells auf viele Güter und Faktoren vorgenommen. Jedes Gut wird in einem Sektor erstellt. $\mathrm{Zu}$ seiner Erstellung wird ein sektorspezifischer Faktor benötigt, so daß es genauso viele sektorspezifische Faktoren wie Güter gibt. Daneben gibt es noch mindestens einen Faktor, der intersektoral mobil ist. In einem solchen Modellrahmen reicht internationaler Handel zur Faktorpreisangleichung nicht aus. ${ }^{16}$ Es bleiben daher auch bei Freihandel Anreize zu Faktorwanderungen erhalten.

Welche Schlußfolgerungen lassen sich aus den makroökonomischen Modellen für die internationalen Wanderungsbewegungen von Arbeitskräften ziehen? Mundells ${ }^{17}$ grundlegende Analyse führte zu dem Ergebnis, daß im Fall von Handelsbeschränkungen Faktorpreisunterschiede bestehen bleiben und daher Faktorbewegungen zu erwarten sind. Gleiches gilt bei international unterschiedlicher Arbeitsproduktivitäten sowie für den Fall, daß sektorspezifische Produktionsfaktoren vorliegen. ${ }^{18}$ Allerdings bleibt unklar, ob diese Faktorpreisunterschiede vorrangig zu internationalen Kapitalströmen oder zu Arbeitskräftewanderungen führen werden. Welcher Faktor wandert, hängt von vielen Einflüssen ab, wie z.B. den Transportkosten, der Verfügbarkeit der relevanten Informationen, Wechselkursrisiken, Steuern, politischen Unsicherheiten, etc. ${ }^{19}$ Letztlich kommt es auf die relative Attraktivität der Länder für die Produktionsfaktoren an. Eine theoretische Ableitung der tatsächlichen Kombination aus Handel, Kapitalbewegungen und Arbeitskräftewanderungen, die zu Faktorpreisangleichungen führt, erscheint derzeit nicht erreichbar. ${ }^{20}$

Dieses Ergebnis macht deutlich, daß bei dem Versuch, die restriktiven Prämissen des Heckscher-Ohlin-Samuelson Modells der Realität anzunähern und internationale Wanderungen in das Modell aufzunehmen, die eindeutigen Aussagen des Modells verlorengehen. Damit sind auf dieses Modell aufbauende makroökonomische Erklärungsansätze für internationale Migration in bezug auf das hier verfolgte Untersuchungsziel wenig hilfreich.

\footnotetext{
15 Vgl. zu diesem Beispiel Straubhaar (1988) S. 23.

16 Vgl. Dixit/Norman (1980) S. 122ff.

17 Vgl. Mundell (1957).

18 Vgl. Straubhaar (1992) S. 569.

19 Vgl. Straubhaar/Zimmermann (1992) S. 11.

20 Vgl. Straubhaar (1988) S. 27. Lucas (1990) zeigt allerdings mit Hilfe der Neuen Wachstumstheorie, daß durch auf Humankapital zurückzuführende externe Effekte auch dann ein Anreiz für Arbeitskräftewanderungen von armen Ländern mit einem geringen Humankapitalbestand in reiche Länder mit hoher Humankapitalausstattung vorliegen kann, wenn die Grenzproduktivitäten des Kapitals in den betrachteten Ländern gleich sind und daher kein Anreiz zu internationalen Kapitalbewegungen besteht.
} 


\subsubsection{Mikroökonomische Erklärungsansätze}

Wie bisher soll auch in diesem Teil der Arbeit vom methodologischen Individualismus ausgegangen werden. Angewandt auf die ökonomische Analyse von Wanderungsbewegungen in einem mikroökonomischen Modell bedeutet das, daß untersucht werden muß, unter welchen Umständen ein einzelnes Individuum einen Anreiz zur Migration hat. Ausgangspunkt ist die Annahme rationalen Verhaltens, also die Annahme, daß jedes Individuum bemüht sein wird, seine Lebensumstände zu verbessern. In bezug auf die Wanderungsentscheidung wird jedes Individuum den Gegenwartswert des Nutzens aus den zukünftig erwarteten Lebensumständen am derzeitigen Wohnort mit den an anderen Orten erwarteten Gegenwartswerten vergleichen. Es handelt sich also um einen intertemporalen Ansatz zur Maximierung des erwarteten Nutzens über die Lebenszeit unter Berücksichtigung verschiedener Restriktionen. ${ }^{21}$ Die Voraussetzung für eine Wanderung von Land $N$ nach Land $H$ ist, daß der Gegenwartswert des Nutzens in $H$ abzüglich der in Nutzeneinheiten ausgedrückten Wanderungskosten $\left(K_{N H}\right)$ größer ist als der Gegenwartswert des Nutzens in $N$.

$$
\text { D.1) } \quad \int_{t=0}^{T} U\left[C_{H}(t), X_{H}(t)\right] e^{-r t} d t-K_{N H}>\int_{t=0}^{T} U\left[C_{N}(t), X_{N}(t)\right] e^{-r t} d t
$$

In die Nutzenfunktion gehen dabei neben dem Konsum $(C(t))$ auch andere Variable $(X(t))$ mit individuell unterschiedlichen Gewichten ein (z.B. Freizeitwert, Sicherheit, Zufriedenheit mit der sozialen Stellung etc.). Zukünftig bis zum Planungshorizont $(T)$ anfallende Nutzen werden mit der individuellen Zeitpräferenzrate $(r)$ auf den Gegenwartszeitpunkt $(t=0)$ diskontiert. ${ }^{22}$ In die Wanderungskosten $\left(K_{N H}\right)$ fließen dabei nicht nur monetäre, sondern auch psychische Größen wie z.B. eine Trennung von Familienangehörigen und Freunden ein. Hervorzuheben ist, daß es jeweils um erwartete Größen geht, denn die zukünftigen Lebensumstände sind schon am Herkunftsort nicht genau prognostizierbar und für den fremden Zielort besonders schwer abschätzbar. Die daraus resultierende Unsicherheit kann durch das Einholen von Informationen gesenkt, aber nicht völlig aufgehoben werden. Daher werden sowohl die Höhe der monetären und zeitlichen Informationskosten als auch die in der Nutzenfunktion zum Ausdruck kommende individuelle Risikoneigung die Wanderungsentscheidung beeinflussen.

Die verschiedenen Ursachen von Wanderungen lassen sich in zwei Gruppen unterteilen: nachfragebezogene Bestimmungsgründe und angebotsbezogene Bestimmungsgründe. ${ }^{23}$

\subsubsection{Nachfrageorientierte Erklärungsansätze des Wanderungspotentials}

In nachfrageorientierten Ansätzen wird der potentielle Migrant weniger als Anbieter von Faktorleistungen, sondern eher als Nachfrager von verschiedensten Güterarten angesehen.

Dieser allgemeine mikroökonomische Ansatz ist damit wesentlich umfassender als die allein auf Lohnsatzdifferenzen abstellenden Makromodelle, die im vorangegangenen Abschnitt vorgestellt wurden.

23 Einen Überblick über die verschiedenen Ansätze zur Erklärung des Wanderungsverhaltens geben Delbrück/Raffelhüschen (1993). 
Diese Ansätze befassen sich daher v.a. mit den in Gleichung $D .1)$ mit $X$ bezeichneten weiteren Variablen, die neben dem Konsumniveau in die Nutzenfunktion eingehen. ${ }^{24} \mathrm{Zu}$ diesen nachfrageorientierten Bestimmungsgründen gehören neben dem Angebot an öffentlichen Gütern und Freizeitmöglichkeiten in dem jeweiligen Land auch Institutionen wie z.B. Rechtssicherheit und individuelle Freiheit sowie Umweltbedingungen (Klima, ökologische Situation). Ein Individuum wird das Land als Zielland wählen, welches ihm unter Beachtung seiner Budgetrestriktion die Erreichung des höchstmöglichen Nutzenniveaus erlaubt, wobei in die Budgetrestriktion u.a. die Preise für öffentliche Güter (Steuern bzw. Abgaben) eingehen. Letztlich handelt es sich um eine nach dem individuellen Nutzenkalkül getroffene "Abstimmung mit den Füßen" zwischen den verschiedenartigen institutionellen und natürlichen Rahmenbedingungen einzelner Staaten. ${ }^{25}$

Nachfrageseitige Bestimmungsgründe spielen mit zunehmendem Einkommen eine immer größere Rolle, da die meisten von ihnen nicht den Grundbedürfnissen zuzurechnen sind und sie daher eine Einkommenselastizität größer als Eins aufweisen. Sie sind daher v.a. für Wanderungen zwischen entwickelten Staaten mit ähnlich hohem Einkommensniveau bedeutend und weniger ausschlaggebend für individuelle Wanderungsentscheidungen zwischen Staaten mit hohem Entwicklungsgefälle.

\subsubsection{Angebotsorientierte Erklärungsansätze des Wanderungspotentials}

Die angebotsorientierten Erklärungsansätze für internationale Wanderungen von Arbeitskräften stellen die Faktorangebotsentscheidung des Individuums in das Zentrum der Analyse. Das Individuum wird also vorrangig als Anbieter von Arbeitsleistungen ${ }^{26}$ und nicht als Nachfrager von nutzenstiftenden (Basis-)Gütern angesehen. Untersucht wird daher, inwiefern das erwartete Nutzenniveau über eine Lockerung der Einkommensrestriktion beeinflußt wird, die infolge eines durch eine Wanderung steigenden Lohnsatzes eintritt. Nach den auf Sjaastad ${ }^{27}$ zurückgehenden Humankapitalmodellen wird die Migrationsentscheidung allein an der besten Verwertbarkeit des Humankapitals ausgerichtet, d.h. an die Stelle der Nutzenmaximierung tritt hier die Einkommensmaximierung. ${ }^{28}$

Unterstellt wird also, daß der Einkommensunterschied zwischen zwei Ländern auch dem Nutzenunterschied entspricht. ${ }^{29}$ In Gleichung D.1) ist entsprechend der Nutzen durch das

24 Das Konsumniveau, das v.a. durch den realen Lohnsatz determiniert wird, wird somit (implizit) als konstant angenommen.

25 Ein Beispiel für einen nachfrageseitigen Erklärungsansatz ist das aus der Finanzwissenschaft bekannte Tiebout-Modell. Vgl. Tiebout (1956).

26 Die Angebotsentscheidung der Individuen bezüglich des Faktors Kapital kann im Gegensatz zur Entscheidung über das Arbeitsangebot weitgehend unabhängig von internationalen Wanderungen getroffen werden und wird hier daher nicht weiter betrachtet.

27 Vgl. Sjaastad (1962).

28 In den von Bhagwati (1985) überblicksartig dargestellten ökonometrischen Studien wird der gegenüber anderen Einflußfaktoren herausragende Einfluß des Einkommens auf die Wanderungsbewegungen bestätigt.

29 Vgl. Siebert (1993) S. 231. Diese Annahme ist schon aus dem Grund problematisch, als die Einkommensunterschiede zwischen verschiedenen Staaten durch die in Hochlohnländern höheren Lebens- 
Einkommen zu ersetzen. Die Wanderungskosten - z.B. Transport- und Kommunikationskosten, Verluste aus der Veräußerung von Vermögen, psychische Kosten der Trennung von dem sozialen Umfeld, etc. - können als Investition in die Wanderung angesehen werden. ${ }^{30}$ Der Gegenwartswert des erwarteten Einkommenszuwachses muß größer sein als die erwarteten Wanderungskosten, um eine Wanderung auszulösen.

Ursprünglich wurde dabei der Lohnsatz als alleinige Einflußgröße angesehen und so Wanderungen von Niedrig- zu Hochlohnregionen erklärt. Harris und Todaro ${ }^{31}$ haben diesen Ansatz erweitert, indem sie als entscheidende Größe statt des Lohnsatzes das erwartete Einkommen ansahen (Todaro-Modell). So kann die Wahrscheinlichkeit, einen Arbeitsplatz zu finden, oder andersgewendet ausgedrückt, die Wahrscheinlichkeit von Arbeitslosigkeit mit in das Erklärungsmodell aufgenommen werden. ${ }^{32}$ Entscheidend ist der Vergleich des Gegenwartswertes des erwarteten Einkommens im Zielland $H$ mit dem des erwarteten Einkommens im Herkunftsland $N$, wobei die Höhe des erwarteten Einkommens von den länderspezifischen und zeitabhängigen Lohnsätzen $\left(w_{N}(t)\right.$ bzw. $\left.w_{H}(t)\right)$ und den Wahrscheinlichkeiten $\left(p_{N}(t)\right.$ und $\left.p_{H}(t)\right)$, daß keine Arbeitslosigkeit eintritt, abhängt. ${ }^{33}$ Diese Wahrscheinlichkeit wird mit der im Gastland verbrachten Zeit zunehmen, weil der Migrant zunehmend Sprachkenntnisse erwirbt, die Institutionen besser kennenlernt, etc. ${ }^{34}$ Eine Wanderung wird also erfolgen, wenn folgende Ungleichung erfüllt ist:

$$
\int_{t=0}^{T}\left[p_{H}(t) w_{H}(t)\right] e^{-r t} d t-K_{N H}>\int_{t=0}^{T}\left[p_{N}(t) w_{N}(t)\right] e^{-r t} d t
$$

An dem Todaro-Modell ist v.a. von Stark Kritik geübt worden, da die alleinige Konzentration auf erwartete Lohnsatzunterschiede der Komplexität der Wanderungsentscheidung nicht hinreichend gerecht werde. ${ }^{35}$ Er untersucht daher den Einfluß von drei zusätzlichen Wanderungsgründen - Risikodiversifizierung, relative Schlechterstellung und asymmetrische Information - auf die in dieser Reihenfolge im folgenden genauer eingegangen wird.

(i) Ausgangspunkt für die Analyse des Einflusses der Risikodiversifizierung auf internationale Wanderungen ist die Annahme, daß analog zu der Vorgehensweise in der Theorie der

haltungskosten teilweise kompensiert werden. Vgl. Straubhaar (1988) S. 85ff. und Straubhaar/Dhima (1989) S. 67.

30 Wie bei Siebert (1993) sind auch in dieser Arbeit die Wanderungskosten als einmalige Fixkosten modelliert. Denkbar ist aber auch, da $B$ die Wanderungskosten zumindest für die ersten Jahre mit der Zeit ansteigen, z.B. weil eine vorübergehende Trennung von dem alten Umfeld als weniger schlimm empfunden wird als eine endgültige. Dann läßt sich auch eine temporäre Migration erklären. Diese kann dann optimal sein, wenn der Einkommensunterschied die zunächst recht niedrigen Wanderungskosten überwiegt, letztere aber mit zunehmender Zeit in dem Gastland so stark ansteigen, daß schließlich eine Rückwanderung vorteilhaft ist.

31 Vgl. Harris/Todaro (1970).

32 Zu einer knappen Beschreibung der auf das Todaro-Modell aufbauenden Job Search und Job Turnover Modelle vgl. Straubhaar (1995) S. 243.

33 Vgl. Todaro (1971) S. 411.

34 Vgl. Abschnitt 3.2.1.

35 Vgl. Stark (1984) S. 206. 
Portefeuille-Zusammensetzung nicht allein die Einkommenshöhe, sondern auch die Einkommensvariabilität den Nutzen des Einkommens bestimmt. ${ }^{36}$ Stark geht davon aus, daß die Entscheidungseinheit gerade in weniger entwickelten Herkunftsländern oft nicht das Individuum, sondern die Familie ist. ${ }^{37}$ Drei Annahmen werden getroffen, um zu zeigen, wann die internationale Wanderung eines Familienmitgliedes für den Familienverband vorteilhaft ist: ${ }^{38}$ Erstens wird Risikoaversion unterstellt. Zweitens wird davon ausgegangen, daß die lokalen Finanz- und Versicherungsmärkte das gewünschte Absicherungsmaß z.B. gegen Einkommensausfälle aufgrund von Mißernten nicht oder nur zu prohibitiv hohen Kosten bereitstellen. Die dritte Annahme ist, daß die Einkommensschwankungen der Familienmitglieder am Herkunftsort stark positiv miteinander korrelieren, z.B. weil alle in der Landwirtschaft oder in stark von der Landwirtschaft abhängigen Wirtschaftszweigen beschäftigt sind und Ertrags- und Preisschwankungen daher die Einkommen aller Familienmitglieder gleicherweise betreffen. Wandert nun ein Familienmitglied, um in der Zielregion ein Einkommen zu erzielen, dessen Schwankungen unabhängig von den Einkommensschwankungen am Herkunftsort sind, dann wird die Varianz des Familieneinkommens gemindert. Eine Wanderung ist dann - von Wanderungskosten sei abgesehen - auch vorteilhaft, wenn das erwartete Lohnniveau am Zielort nicht höher ist als am Heimatort.

Risikodiversifizierung als Wanderungsmotiv kann größere Bedeutung nur für Wanderungen aus wenig entwickelten Ländern erlangen, da die Voraussetzungen von lokal kaum diversifizierbaren Einkommensrisiken und schlecht funktionierenden Finanz- und Versicherungsmärkten nur dort als gegeben angesehen werden können. Schon für Wanderungen aus Schwellenländern ist das Risikodiversifizierungsmotiv weniger bedeutend, wenn auch lokal die Möglichkeit besteht, durch Arbeitsaufnahme im sekundären oder tertiären Sektor das Einkommensschwankungsrisiko zu mindern. Für die Erklärung von Wanderungen zwischen Industriestaaten ist dieses Motiv ungeeignet. Da nur ein kleiner Teil der Zuwanderer nach Deutschland aus überwiegend agrarisch strukturierten Entwicklungsländern stammt, ist das Risikodiversifizierungsmotiv zumindest in der Vergangenheit ohne größeren Einfluß auf die Wanderungsbewegungen gewesen.

(ii) Als zweites Wanderungsmotiv identifiziert Stark eine relative Schlechterstellung (relative deprivation) im Vergleich zu einer Referenzgruppe. Mit relativer Schlechterstellung wird die Einkommenssituation im Verhältnis zu der Referenzgruppe bezeichnet. ${ }^{39}$ Nach diesem

36 Vgl. Stark (1991) S. 37ff.

37 Vgl. hierzu auch Mincer (1978).

38 Es läßt sich allerdings zeigen, daß in den meisten Fällen die Wanderung sowohl für das wandernde als auch für die zurückbleibenden Familienmitglieder vorteilhaft wird. Der Rückgriff auf den Familienverband ist daher für den hier erläuterten Erklärungsansatz nur nötig, weil durch die sozialen Bindungen sichergestellt wird, daß die unten beschriebenen schwankungsmindernden Ausgleichszahlungen zwischen den Familienmitgliedern auch tatsächlich erfolgen.

39 Der Begriff "relative deprivation" ist nicht einheitlich definiert. Stark (1991) S. 121 stellt unter Rückgriff auf Runciman (1966) S. 10 folgende vier Bedingungen an das Vorliegen einer relativen Schlechterstellung eines Individuums:

- es hat ein bestimmtes Güterbündel $x$ nicht

- andere Personen in der Referenzgruppe haben $x$

- es will $x$ haben

- nach seinem Empfinden liegt es im Bereich des Möglichen, daß es $x$ haben könnte.

Als Beispiel sei eine Operationalisierungsvariante von Stark angeführt, nach der Personen als schlechter gestellt angesehen werden, deren Einkommen um mindestens einen bestimmten Betrag unter dem Durchschnittseinkommen der Referenzgruppe liegt. Vgl. Stark (1984) S. 213. 
Erklärungsansatz sind es also die Mitglieder der ärmeren Bevölkerungsschichten, die besonders stark zu Wanderungen neigen, wobei die Wanderungswahrscheinlichkeit mit zunehmend ungleicher Einkommensverteilung steigt. ${ }^{40}$ Bei Wanderung innerhalb einer Nation, z.B. von einer ländlichen Region in einem Entwicklungsland in die Hauptstadt, ändert sich die Referenzgruppe möglicherweise relativ schnell. Der kulturelle und soziale Unterschied zwischen Land und Stadt ist innerhalb einer Nation nicht sehr groß, so daß ein Wechsel der Referenzgruppe leicht fällt. Als Resultat kann sich die relative Schlechterstellung aufgrund der Änderung der Referenzgruppe sogar noch verstärken. Antizipiert der Migrant diese Entwicklung, so wird er versuchen, durch seine Wanderung nicht nur eine maximale Verbesserung der Einkommenssituation zu erreichen, sondern gleichzeitig auch die Wahrscheinlichkeit für die Übernahme einer neuen Vergleichsgruppe möglichst gering zu halten. Das kann dadurch geschehen, daß der kulturelle Abstand möglichst groß gewählt wird und eine Akkulturation bewußt verzögert wird. ${ }^{41}$ Nach diesem Ansatz erweist sich also eine internationale Wanderung gegenüber einer Wanderung innerhalb eines Landes als vorteilhaft, wenn die zusätzliche Einkommenserhöhung und die geringere Wahrscheinlichkeit der Änderung der Referenzgruppe die zusätzlichen monetären und psychischen Wanderungskosten überwiegen.

Wenn relative Schlechterstellung eine größere Rolle als Wanderungsmotiv spielt, dann mindert dies die Chancen für eine Integrationspolitik im Zielland. Die Zuwanderer sind dann geradezu daran interessiert, sich nicht integrieren zu lassen, um nicht das neue soziale und kulturelle Umfeld mit dem gegenüber dem Herkunftsland wesentlich höheren Einkommensniveau als Referenzgruppe heranziehen zu müssen und durch diesen Wechsel der Referenzgruppe wieder eine relative Schlechterstellung zu erleiden.

(iii) Als dritten Einwand gegenüber dem allein auf die erwartete Lohndifferenz abstellenden Todaro-Modell führt Stark das Vorliegen asymmetrischer Information an. ${ }^{42}$ In einem einfachen statischen Zwei-Länder-Fall wird angenommen, da $B$ das Wohlfahrtsniveau nur von Humankapitalerträgen abhängt und diese wiederum von dem Ausbildungsniveau bestimmt werden. Die Wanderungskosten werden in das Modell eingebaut, indem der Lohnsatz in dem reichen Zielland mit einem Gewichtungsfaktor multipliziert wird, der je nach Höhe dieser Kosten zwischen Null und Eins liegt und auch als abhängig von dem Ausbildungsniveau dargestellt werden kann. Die asymmetrische Information kann sich durch verschiedene Informationsdefizite des Arbeitgebers im reichen Land in bezug auf die Migranten ergeben. Möglicherweise ist es ihm schon unmöglich, überhaupt zwischen Zuwanderern und einheimischen Arbeitskräften zu unterscheiden (z.B. aufgrund gesetzlicher Vorschriften). Wahrscheinlicher ist aber, daß er nicht nach unterschiedlichen Herkunftsländern bzw. unterschiedlichen Ausbildungsniveaus von Zuwanderern aus dem gleichen Herkunftsland unterscheiden kann. In letzterem Fall wird er die Zuwanderer nicht entsprechend ihrem (nicht beobachtbaren) Wertgrenzprodukt entlohnen, sondern entsprechend dem Wert der Durchschnittsproduktivität aller Zuwanderer. Je nach Abhängigkeit des Lohnniveaus vom Humankapitalbestand im Herkunfts- und Zielland kommt es so zu Wanderungsbewegungen bestimmter, keiner oder aller Qualifikationsstufen. Liegt asymmetrische Information vor, lassen sich also nach diesen

40 In einer empirischen Überprifung dieser Hypothese finden Stark/Taylor (1991), daß sowohl die relative Einkommenssituation im Auswanderungsland als auch der durch internationale Wanderung erzielbare absolute Einkommensanstieg einen signifikanten Einfluß auf die Migrationsentscheidung haben (nach Siebert (1993) S. 237). 
Modellen ohne empirische Untersuchungen keine eindeutigen qualitativen oder quantitativen Aussagen über das Wanderungsverhalten treffen.

Interessant ist dieser Ansatz v.a. deshalb, weil sich mit seiner Hilfe erklären läßt, weshalb gewisse Bevölkerungsgruppen wandern, andere hingegen nicht. Auch Rückwanderungen lassen sich erklären, wenn die Arbeitgeber des reichen Landes im Laufe der Zeit aufgrund der beobachteten Arbeitsleistung der Zuwanderer Informationen gewinnen und daher von der allgemeinen Durchschnittsentlohnung zur Entlohnung nach dem Wertgrenzprodukt übergehen. Sinkt der erzielbare Lohnsatz von unterdurchschnittlich Qualifizierten dadurch stark genug, ist für sie eine Rückwanderung in ihr Herkunftsland vorteilhaft und es erfolgt langfristig eine für das Aufnahmeland positive Selektion der Zuwanderer. ${ }^{43}$ Allerdings ist eine empirische Überprüfung der skizzierten Hypothesen kaum möglich, weil der Gewichtungsfaktor für Einkommen im Zielland, der die Wanderungskosten abbildet, kaum zu ermitteln ist.

Die Kritik von Stark an dem Todaro-Modell macht deutlich, daß dieser mikroökonomische Ansatz noch gravierende Schwachpunkte aufweist und auch nicht in der Lage ist, alle Erscheinungsformen internationaler Wanderungen in einem einheitlichen Modellrahmen plausibel zu erklären. So fehlt beispielsweise eine schlüssige und allgemein anerkannte Erklärung für Rückwanderungen, so daß ein gerade für Deutschland in der Vergangenheit besonders bedeutsamer Aspekt der Migrationsbewegungen ausgeklammert bleibt. ${ }^{44}$ Aufgrund der Schwächen in der theoretischen Fundierung kann es nicht verwundern, wenn ökonometrische Schätzungen der Wanderungselastizitäten in bezug auf Einkommens- und Arbeitslosigkeitsunterschiede zu sehr unterschiedlichen und damit wenig befriedigenden Ergebnissen führen. ${ }^{45}$

Die Hauptursache für die Unzulänglichkeiten des Todaro-Modells liegt darin, daß dieses Modell ursprünglich für die Erklärung intranationaler Wanderungen entworfen wurde, dann aber recht unkritisch auf internationale Wanderungsbewegungen übertragen wurde. Im Gegensatz zu Wanderungen innerhalb eines Staates existieren bei grenzüberschreitenden Wanderungen oft Barrieren wie Visumspflicht und Grenzkontrollen, die verhindern, daß alle Wanderungswilligen auch tatsächlich wandern können. Internationale Wanderungsströme werden daher nicht allein durch die Zahl der Wanderungswilligen, also das Angebot an Migranten, bestimmt, sondern zusätzlich durch die Nachfrage des Ziellandes nach Zuwanderern. ${ }^{46}$ Deshalb wird im nachfolgenden Unterkapitel analysiert, wovon die Nachfrage nach Zuwanderern durch das Aufnahmeland abhängt. Ferner wird das Zusammenspiel von Angebot und Nachfrage, das über die tatsächlichen Migrationsströme entscheidet, beleuchtet.

43 Vgl. Stark (1994) S. 67ff.

44 Verschiedene Erklärungsansätze für Rückwanderungen finden sich u.a. bei Djajic/Milbourne (1988), Stark (1991) S. 381ff., Dustmann (1994) und empirische Studien u.a. bei Brecht/Brecht (1991) und Steiner/Velling (1994).

45 Vgl. den Überblicksartikel von Bhagwati/Krugman (1985) S. 372ff. und die Einschätzung von Petersen (1993) S. 10.

46 Straubhaar (1988) S. 114 stellt daher als Ergebnis einer empirischen Untersuchung europäischer Wanderungsbewegungen fest: "An approach which does not take into account the dominance of the demand-determined causes is of restricted value in explaining the causes of international labor migration flows". 


\subsection{Analyse der Nachfrage nach Zuwanderern durch das Einwande- rungsland}

Bisher wurde anhand von mikroökonomischen und makroökonomischen Überlegungen untersucht, wovon das Potential der Zuwanderer abhängt. Inwieweit sich dieses Potential in tatsächlicher Zuwanderung niederschlägt, läßt sich allerdings nur ermitteln, wenn neben der Angebotsseite auch die Nachfrageseite des "immigration market" 47 betrachtet wird. Daher wird nunmehr zu beleuchten sein, wovon die Nachfrage nach Zuwanderem in dem Einwanderungsland abhängt. Letztlich wird die kürzere Marktseite die tatsächlichen Wanderungsströme determinieren, solange kein gleichgewichtsbildender Preismechanismus existiert. Zunächst wird auf ökonomische Bestimmungsfaktoren der Nachfrage nach Zuwanderern eingegangen, in Unterkapitel 2.2.2 dann auf politische bzw. politisch-ökonomische Überlegungen.

\subsection{1 Ökonomische Bestimmungsgründe der Nachfrage}

Folgt man der neoklassischen Theorie der Faktornachfrage, dann hängt die Nachfrage der Unternehmen ${ }^{48}$ nach Arbeitskräften von ihrem Grenzerlösprodukt und dem Lohnsatz ab, denn eine gewinnmaximierende Unternehmung erreicht ihre optimale Beschäftigtenzahl, wenn Lohnsatz und Grenzerlösprodukt ${ }^{49}$ gerade gleich hoch sind. ${ }^{50}$ Ein Anreiz zur Einstellung von Zuwanderem kann aus zwei Gründen entstehen. Erstens kann das Verhältnis von Grenzerlösprodukt zu Lohnsatz für ausländische Arbeitnehmer über dem für Einheimische liegen. Denkbar wäre etwa, daß Zuwanderer bei gleichem Lohnsatz eine höhere Produktivität aufweisen oder daß sie bei vergleichbarer Produktivität einen niedrigeren Arbeitslohn akzeptieren. In diesem Fall lohnt sich die Einstellung von Zuwanderem auch dann, wenn genügend geeignete einheimische Arbeitskräfte zur Verfügung stehen würden. Zweitens können Unternehmen bei ihrer Personalplanung aber auch dann auf ausländische Arbeitskräfte angewiesen sein, wenn ihr Verhältnis von Grenzerlösprodukt zu Lohnsatz demjenigen inländischer Arbeitnehmer entspricht oder sogar geringfügig niedriger liegt. Dieser Fall kann eintreten, wenn - wie v.a. in den sechziger Jahren - nicht genügend einheimische Arbeitskräfte vorhanden sind, um alle offenen Stellen besetzen zu können. Strukturelle Ungleichgewichte zwischen Angebot und Nachfrage auf dem Arbeitsmarkt können dazu führen, daß auch bei hoher Arbeitslosigkeit im

47 Borjas (1989) S. 460.

48 Die Nachfrage öffentlicher und privater Haushalte nach Arbeitskräften soll hier vernachlässigt werden. Die "Nachfrage" nach Selbständigen kann ähnlich modelliert werden wie für Arbeitnehmer. Liegt für selbständige Zuwanderer das Verhältnis von Grenzerlösprodukt zu kalkulatorischem Unternehmerlohn höher als für Einheimische, dann können sie ceteris paribus durch ein günstigeres Pteis-LeistungsVerhältnis einen Teil der Nachfrage auf sich lenken.

49 Die Arbeitsnachfrage hängt damit u.a. von der Marktform ab.

so Eine ausführliche Analyse der Arbeitsnachfrage findet sich z.B. bei Franz (1991) Kap. 4 und bei Hamermesh (1993). 
Einwanderungsland für bestimmte Qualifikationen ein Nachfrageüberhang auf dem Arbeitsmarkt besteht, der Unternehmen bewegt, Zuwanderer einzustellen. ${ }^{51}$

Festzuhalten bleibt, daß mit steigender positiver Differenz zwischen dem Verhältnis von Grenzerlösprodukt zu Lohnsatz von Zuwanderern und Einheimischen und mit einer steigenden Zahl nicht besetzbarer offener Stellen die Nachfrage der Unternehmen nach Zuwanderern steigen wird.

Der Bevölkerungsrückgang in der Bundesrepublik wird voraussichtlich über beide Einflußfaktoren eine verstärkte Nachfrage nach ausländischen Arbeitskräften bewirken. Die Knappheit an Arbeitskräften, die aufgrund der demographischen Entwicklung erwartet wird, ${ }^{52}$ hat zum einen direkt eine Erhöhung der Zahl der offenen Stellen zur Folge. Führt diese Knappheit an Arbeitskräften zu einem Anstieg des Lohnsatzes für inländische Arbeitnehmer, dann wird dadurch zum anderen der infolge der Einstellung von Zuwanderern entstehende Kostenvorteil verstärkt. Für die Zukunft ist daher von einer steigenden ökonomisch bedingten Nachfrage nach Zuwanderern auszugehen. ${ }^{53}$

\subsubsection{Politisch-ökonomische Bestimmungsgründe der Nachfrage}

Die in der Realität beobachtbaren Wanderungsströme werden nicht nur von ökonomischen Faktoren bestimmt, sondern auch die durch politische Entscheidungen entstandene Rechtslage spielt eine bedeutende Rolle. Die Einflüsse politischer Rahmenbedingungen auf das Wanderungspotential wurden schon im Abschnitt 2.1.2.1 behandelt. Im folgenden wird untersucht, welche Wirkung die Einwanderungspolitik der Empfängerländer auf die ökonomische Nachfrage nach Zuwanderern hat. Anschließend ist zu verdeutlichen, wovon die Ausgestaltung der Einwanderungspolitik, die auch als politisch determinierte Nachfrage nach Zuwanderern angesehen werden kann, abhängt. Zuvor wird kurz begründet, warum bei der Behandlung des Wanderungspotentials, also des Angebots, auf eine entsprechende Analyse der Auswanderungspolitik der Herkunftsländer verzichtet wurde.

Kennzeichnend für die Möglichkeit von Auswanderungsländern, über eine Wanderungspolitik die Wanderungsströme zu beeinflussen, ist eine rechtliche Asymmetrie. Während die Einwanderungsländer in ihrer Entscheidung, wen sie als Zuwanderer einlassen, rechtlich ungebunden sind, ist den Auswanderungsländern eine legale Kontrolle über die Zahl und Zusammensetzung der Emigranten durch das als Menschenrecht anerkannte Recht auf Auswanderung genommen. ${ }^{54}$ Faktisch haben verschiedene Staaten dennoch versucht, durch politische Maßnahmen Einfluß auf die Auswanderungsentscheidungen ihrer Bürger zu nehmen. Als augenfälligstes Beispiel mag die Errichtung der Mauer im Jahr 1961 dienen, die zu einem

51 Die Frage inwieweit Zuwanderer und Einheimische auf dem Arbeitsmarkt Substitute oder Komplemente darstellen wird ausführlicher in Unterkapitel 3.1 erörtert.

Die Prognos AG (1995) rechnet beispielsweise in ihrem Gutachten für den Verband Deutscher Rentenversicherungsträger (VDR) mit verbesserten Beschäftigungsaussichten in Deutschland in den nächsten Jahrzehnten. Im Rentenbericht 1995 wird für die mittlere Variante mit einer Erreichung des Vollbeschäftigungsziels im Jahre 2009 gerechnet. Vgl. Bundesregierung (1995) S. 71.

Chruscz/Höpcke (1993) S. 31ff. berechnen den Einwanderungsbedarf für die damals noch zwölf Mitgliedsstaaten der Europäischen Union für verschiedene Szenarien. 
drastischen Rückgang der Übersiedlerzahl von Ost- nach Westdeutschland führte. ${ }^{55}$ Andererseits wird von einigen Staaten die Auswanderung aktiv unterstützt, weil sie sich davon eine Verminderung der Arbeitslosigkeit und Devisenzuflüsse in Form von Transferzahlungen an zurückgebliebene Angehörige erhoffen. Sieht man von drastischen Maßnahmen ab, deren Wiedereinführung nach dem Zusammenbruch der kommunistischen Regime des Warschauer Pakts in allen für die Bundesrepublik wichtigen Herkunftsländern als unwahrscheinlich gelten kann, ist der Einfluß der Auswanderungsländer auf die Wanderungsströme vergleichsweise gering. ${ }^{56}$ Wie die Fluchtbewegungen aus Kuba im Sommer 1994 deutlich vor Augen geführt haben, sind Auswanderungen seitens des Herkunftslandes selbst unter der günstigen Voraussetzung einer Insellage auch durch repressive und kostspielige Maßnahmen nur schwer zu verhindern. Auf den Einfluß einer Auswanderungspolitik braucht deshalb nicht näher eingegangen zu werden.

Ganz anders stellt sich die Situation für die Einwanderungspolitik dar. Rechtliche Beschränkungen der absoluten Zahl der Einwanderer und eine gezielte Selektion nach ökonomischen und anderen Kriterien seitens des Einwanderungslandes werden als legitim angesehen und weltweit praktiziert. Als Beispiel für die Wirksamkeit rechtlicher Maßnahmen des Einwanderungslandes zur Steuerung der Zuwanderung mag der deutliche Rückgang der Asylbewerberzahlen in Deutschland nach der Änderung des Asylrechts im Jahr 1993 oder auch der Rückgang der Zahl der Kuba-Flüchtlinge infolge der geänderten Aufnahmepolitik der USA im Sommer 1994 dienen.

Allerdings ist durch rechtliche Instrumente keine vollständige Kontrolle der Zuwanderung zu erzielen. Besteht aus ökonomischen Gründen eine hohe Nachfrage nach Zuwanderern, der ein entsprechendes Wanderungspotential gegenübersteht, dann wird eine starke Begrenzung der Zuwanderung durch politische Maßnahmen zu einer Verlagerung von legaler zu illegaler Zuwanderung führen. Da die Illegalität - zumindest dann, wenn sie mit einer gewissen Wahrscheinlichkeit auch zu Strafen führt - sowohl für die illegalen Zuwanderer als auch für die sie beschäftigenden Unternehmen mit Kosten verbunden ist, wird aufgrund einer restriktiveren Einwanderungsgesetzgebung sowohl die ökonomische Nachfrage als auch das Angebot an Zuwanderern zurückgehen (vgl. Abb. D.1). Die Summe aus legalen und illegalen Zuwanderern nach einer Verschärfung der Einwanderungsgesetze $\left(Z_{l}\right)$ wird daher in jedem Fall unter der Zahl der legalen Einwanderer liegen, die ohne die Verschärfung des Einwanderungsrechts zugewandert wären $\left(Z_{0}\right)$.

In welchem Ausmaß legale durch illegale Zuwanderung substituiert wird, hängt einerseits von der Höhe der Strafe, der Wahrscheinlichkeit ihrer Durchsetzung und der Risikoneigung der einstellenden Unternehmen und der Zuwanderer ab, also von dem Ausmaß der LinksVerschiebung der Angebots- und Nachfragekurve auf dem Markt für Zuwanderung und andererseits von der Neigung dieser Kurven, die von der Höhe der Gewinnsteigerung, die durch Beschäftigung von illegalen Arbeitnehmern von den Unternehmen realisiert werden

\footnotetext{
55 Auch in anderen Staaten des Warschauer Paktes wurde die Emigration staatlich reguliert. Im Zuge der Reformbewegungen in Osteuropa Ende der achtziger und Anfang der neunziger Jahre sind allerdings in den meisten Ländern dieser Region neue Ausreise- bzw. Auswanderungsbestimmungen erlassen worden, so in Polen 1988, 1989 in Ungarn, der damaligen Tschechoslowakei und Bulgarien, 1990 in Rumänien und 1991 in der früheren Sowjetunion. Vgl. Salt (1993) S. 45.
}

Vgl. Straubhaar (1988) S. 111. 
Abb. D.I: Wirkung einer Verschärfung der Einwanderungspolitik auf die Zahl der Zuwanderer

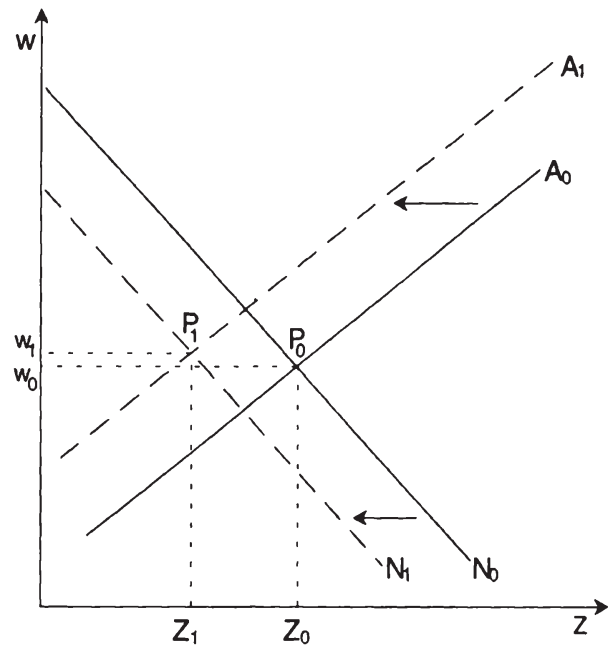

w: Lohnsatz der Zuwanderer im Einwanderungsland ${ }^{57}$

Z: Zahl der Zuwanderer

kann, und der Höhe des durch Zuwanderung zu erreichenden Nutzenzuwachses auf der Seite der ausländischen Erwerbstätigen bestimmt wird (vgl. Abb. D.1). ${ }^{58}$

Nach der Klärung der Wirkungsweise der Einwanderungspolitik bleibt zu untersuchen, von welchen Faktoren ihre Ausrichtung abhängt. Da die Entscheidung über die Bedingungen, unter denen eine Zuwanderung möglich ist, durch politische Organe gefällt wird, eignet sich zu dieser Analyse am besten ein politisch-ökonomischer Ansatz, der die Anreizstruktur verschiedener Wählerschichten und Interessengruppen untersucht.

Vorteile aus einer hohen Zuwanderung von ausländischen Erwerbstätigen haben v.a. die Unternehmen und die Verbraucher. Die Unternehmen werden vornehmlich dann durch ihre

57 Wie in 2.1.2.2 gezeigt wurde, hängt das Wanderungspotential genau genommen von dem seitens der Zuwanderer erwarteten Gegenwartswert des Einkommens im Gastland ab. Hier wurde zur Vereinfachung stattdessen der tatsächliche Lohnsatz verwendet, was ohne Belang ist, so lange zwischen dem tatsächlichem Lohnsatz und erwartetem Gegenwartswert des Einkommens ein hinreichend enger Zusammenhang besteht.

Unter Rückgriff auf einen Vergleich zwischen der Schweiz und den USA sehen Straubhaar/Zimmermann die Möglichkeit einer weitgehenden Reduktion illegaler Einwanderung, sobald die Unternehmen bei Einstellung Illegaler mit empfindlichen Strafen zu rechnen haben. Vgl. Straubhaar/Zimmermann (1992) S. 22. 
Interessenvertretungen auf eine liberale Einwanderungspolitik drängen, wenn sie dadurch große Vorteile in Form von Gewinnsteigerungen durch Kostensenkungen bzw. Produktionserhöhungen erwarten. Das ist nach den Ergebnissen aus 2.2.1 u.a. dann der Fall, wenn die Zahl der nicht besetzbaren offenen Stellen besonders hoch bzw. die Arbeitslosigkeit besonders niedrig ist. Die Verbraucher als zweite Gruppe profitieren zwar von einer liberalen Zuwanderungspolitik, wenn die dadurch entstehenden Kostensenkungen wenigstens teilweise zu Preissenkungen führen. ${ }^{59}$ Diese Vorteile sind aber kaum merklich, da sie sehr indirekt entstehen und quantitativ für den einzelnen Nachfrager wenig Gewicht haben. Ein aktives Eintreten der Verbrauchervertretungen für eine liberale Einwanderungspolitik ist daher kaum zu erwarten.

Die Arbeitnehmer werden sich gegen eine liberale Zuwanderungspolitik wenden, wenn sie befürchten, dadurch Lohneinbußen und/oder Arbeitslosigkeit zu erleiden. ${ }^{60}$ Ein derartiges substitutives Verhältnis zwischen einheimischen und ausländischen Arbeitskräften tritt v.a. bei niedrigen Qualifikationsniveaus und in bestimmten Branchen (Bauwirtschaft, Gaststätten, etc.) auf ${ }^{61}$ und wird besonders in Zeiten hoher Arbeitslosigkeit deutlich wahrgenommen. ${ }^{62}$

Allgemein fallen die Nachteile der Zuwanderung (staatlich finanzierte Eingliederungshilfen, Lohneinbußen, Arbeitsplatzverlust, Konkurrenz auf dem Wohnungsmarkt, etc.) kurzfristig, direkt und auf bestimmte Bevölkerungsgruppen konzentriert an, während die Vorteile wie geringere Produktionskosten und die Schaffung neuer Arbeitsplätze eher langfristig, diffus und breit verteilt eintreten. ${ }^{63}$ Die politischen Entscheidungsträger haben dadurch ähnlich wie bei der Entscheidung für Subventionen einen Anreiz zu einer eher restriktiven Einwanderungspolitik, weil sie so bei den durch diese Politik begünstigten Gruppen Wählerstimmen gewinnen können, ohne bei den Benachteiligten Stimmen zu verlieren. Besonders stark ist dieser Anreiz in

59 In bezug auf die US-amerikanischen Verbraucher schreibt Borjas (1990) S. 222: "For example, it is likely that consumers benefit greatly from the entry of unskilled immigrants. The United States has a comparative advantage at producing relatively skilled labor, and it is costly to attract skilled natives to certain types of occupations, such as the harvesting of seasonal crops. Unskilled immigrants can fill these jobs at a low wage. In effect, the specialization of unskilled immigrants in particular industries reduces the prices of these goods and increases the real income of American consumers." Und weiter: "The practical importance of this increase in real income has not been empirically documented."

60 Ein starkes Interesse der Arbeitnehmer bzw. ihrer Vertreter an restriktiven Einwanderungsregelungen für diesen Fall wird auch von Schmidt (1994a) S. 9 vermutet. In einer der wenigen empirischen Studien auf diesem Gebiet finden Gang/Rivera-Batiz (1994) entgegen dem theoretisch zu erwartenden Ergebnis eine positivere Einstellung gegenüber der Gesamtheit der Ausländer unter Arbeitslosen als unter Erwerbstătigen. Unterscheidet man allerdings bestimmte Herkunftsregionen der Ausländer, dann zeigt sich eine deutlich negativere Einstellung der Arbeitslosen gegenüber allen Gruppen von Ausländern. Gang/Rivera-Batiz erklären die widersprüchlichen Ergebnisse damit, daß Erwerbstätige als Steuerzahler die Auslănder allgemein als Belastung empfinden, während Arbeitslose sich auf dem Arbeitsmarkt v.a. in Konkurrenz zu bestimmten Gruppen sehen. Der Wert der Untersuchung wird dadurch gemindert, da $B$ die Datenbasis recht klein ist und die verwendeten Daten der Eurobarometer Erhebung zudem aus dem Jahr 1988 stammen, also weder die Auswirkungen der jüngsten Wanderungswelle noch die Folgen der Wiedervereinigung der beiden deutschen Staaten abbilden.

61 Eine ausführlichere Diskussion der tatsächlichen Arbeitsmarkteffekte der Zuwanderung, die nicht mit den hier relevanten empfundenen Wirkungen übereinstimmen müssen, findet sich in Kapitel 3.1.

Als Beispiel können die Bemühungen der Gewerkschaften zur Verabschiedung eines Entsendegesetzes gesehen werden, das nach der Allgemeinverbindlichkeitserklärung für die Baubranche sicherstellen soll. $\mathrm{da}$ in Deutschland arbeitenden EU-Ausländern Mindestlöhne gezahlt werden müssen.

63 Vgl. Greenwood/McDowell (1994) S. 187. Nach Petersen sind die "Kosten der Zuwanderung .. folglich gegenwärtig und sicher, die Erträge zukünftig und unsicher." Petersen (1993) S. 14. 
Konjunkturtälern mit hoher Arbeitslosigkeit, ${ }^{64}$ da dann die Unternehmen wenig Interesse an ausländischen Arbeitskräften haben und die Nachteile der Zuwanderung besonders ausgeprägt empfunden werden. ${ }^{65}$

Neben den angeführten ökonomischen Faktoren spielen möglicherweise auch Präferenzen der Wähler für kulturelle, ethnische und weltanschauliche Homogenität der Bevölkerung eine Rolle bei der Ausgestaltung der Einwanderungspolitik im politischen Willensbildungsprozeß.66 In diesem Fall wird der Widerstand gegen eine Liberalisierung der Einwanderungsgesetzgebung wachsen, je mehr Zuwanderer pro Periode aufgenommen werden, je größer die Disparitäten zwischen Einheimischen und Zuwanderern sind und je langsamer eine Angleichung stattfindet. ${ }^{67}$

Als Resultat aus der Untersuchung der Bestimmungsgründe für Wanderungen in diesem Kapitel ist festzuhalten, daß die Höhe der Nettozuwanderung von dem Angebot an Zuwanderungswilligen und der Nachfrage nach Zuwanderern durch das Einwanderungsland abhängt. Auf der Angebotsseite wird das Wanderungspotential durch die Zahl der Personen bestimmt, für die eine Wanderung einen Nutzenzuwachs erwarten läßt. Der Nutzenzuwachs wird dabei nicht ausschließlich, aber doch zu einem großen Teil durch den Unterschied des Barwerts der erwarteten zukünftigen Einkommen im Heimatland und Aufnahmeland bestimmt. Da für die absehbare Zukunft mit einem Fortbestand der wesentlich besseren Einkommenserzielungschancen in Deutschland im Vergleich zu vielen potentiellen Auswanderungsstaaten zu rechnen ist, kann davon ausgegangen werden, daß die Angebotsseite auch in Zukunft nicht die beschränkende Seite auf dem Einwanderungsmarkt sein wird. ${ }^{68}$ Das gilt auch dann, wenn andere

64 In einer ökonometrischen Studie über die europäischen Gastarbeiterwanderungen gelangt Straubhaar (1986) zu dem Ergebnis, daß die Höhe der Arbeitslosigkeit in den Zielländern die Immigrationspolitik dieser Länder beeinflußt und so mittelbar einen großen Einfluß auf den Umfang der Wanderungsbewegungen ausübt. Für die USA kommen Shughart/Tollison/Kimenyi (1986) zu dem Ergebnis, daß sich der Grad der Durchsetzung der Einwanderungsbestimmungen antizyklisch verhält.

65 In dem negativen Zusammenhang zwischen der Höhe der Arbeitslosigkeit und der politischen und ökonomischen Nachfrage nach Zuwanderern kann eine Begründung für den recht hohen empirischen Erklärungswert des Todaro-Modells gesehen werden. In diesem Modell wird nämlich, wie in 2.1.2.2 ausgeführt, gleichfalls ein negativer Zusammenhang zwischen Arbeitslosigkeit und Zuwanderung unterstellt, allerdings entsprechend der Konzentration dieses Modells auf die Erklärung des Wanderungspotentials auf der Angebotsseite des "immigration market". Die nachfrageseitige Wirkung der Arbeitslosigkeit im Einwanderungsland wird dadurch eher zufällig miterfaßt. Vgl. die ökonometrische Arbeit von Straubhaar (1988) S. $111 \mathrm{ff}$.

Hillman (1994) untersucht die Auswirkungen derartiger Präferenzen auf die Einwanderungspolitik im Rahmen einer Erweiterung des Tiebout-Modells.

Vgl. Bernholz (1994).

68 Straubhaar/Zimmermann (1992) S. 3 identifizieren vier Gründe für ein hohes Migrationspotential nach Europa:

- die hohen Bevölkerungswachstumsraten in den nahe zu Europa gelegenen islamischen Ländern,

- den zunehmenden wirtschaftlichen Abstand zwischen der EU und bestimmten Entwicklungsländern,

- Ost-West Wanderungen innerhalb Europas aufgrund politischer und ökonomischer Instabilitäten und

- die Zunahme ökologischer Flüchtlinge (die hier allerdings, wie oben ausgeführt, unter die ökonomisch motivierten Flüchtlinge gefaßt werden).

Allein das Ost-West Wanderungspotential wird von ihnen mit 5-40 Millionen Personen angegeben, von denen ein großer Teil Deutschland als Zielland haben wird. Schewe rechnet daher für Deutschland mit mindestens 500.000 Zuwanderern jährlich allein aus Ost- und Südosteuropa. Vgl. Schewe (1992) S. 254. Frey sieht auch in bezug auf außereuropäische Länder zunehmenden Zuwanderungsdruck nach Deutschland. Vgl. Frey (1992). 
Industriestaaten mit ähnlicher demographischer Entwicklung wie in Deutschland gleichfalls mehr Einwanderer aufnehmen wollen. Um besonders gefragte Einwanderergruppen könnte in diesem Fall allerdings ein verschärfter Wettbewerb zwischen den Aufnahmeländern einsetzen.

Auf der Nachfrageseite des Einwanderungsmarktes ist mit einem Anstieg der Nachfrage der Unternehmen nach Einwanderern zu rechnen, weil die Arbeitslosigkeit durch die demographische Entwicklung längerfristig abgebaut wird, offene Stellen daher längere Zeit nicht zu besetzen sein werden oder aber höhere Löhne gezahlt werden müssen. Die sinkende Arbeitslosigkeit erleichtert gleichzeitig die Lockerung der Einwanderungsgesetzgebung, weil die politischen Entscheidungsträger dann auf weniger Widerstand seitens der Gewerkschaften stoßen und mit größerer Unterstützung durch die Arbeitgeberverbände rechnen können.

Insgesamt ist daher eine verstärkte Einwanderung grundsätzlich erreichbar und die Höhe der jährlichen Nettozuwanderung kann als politisch festlegbare Instrumentvariable angesehen werden. Wird mit Hilfe der Einwanderungspolitik eine Selektion der Einwanderer angestrebt, kann allerdings nicht für alle Zuwanderergruppen davon ausgegangen werden, da $\beta$ die Nachfrage immer durch ein entsprechendes Wanderungspotential gedeckt werden kann.

\section{Kosten-Nutzen-Analyse der Einwanderung}

In diesem Kapitel werden die verschiedenen Kosten- und Nutzenarten, die den Einheimischen durch Einwanderer entstehen, untersucht. Als Referenzmaßstab dient dabei die Wohlfahrt der Einheimischen bei gleicher Bevölkerungsentwickung ohne Einwanderung. Änderungen der Wohlfahrtsposition der Migranten und der in den Herkunftsländern Zurückgebliebenen werden nicht betrachtet. Als relevante Kosten- und Nutzenarten werden bei der Entstehung der Primäreinkommen die Arbeitsmarkteffekte, die von Einwanderern auf die Einheimischen ausgehen (Unterkapitel 3.1), und die Wirkung auf die Kapitaleinkommen (Unterkapitel 3.2) betrachtet. Anschließend werden die Wirkungen analysiert, die sich infolge der Umverteilung dieser Primäreinkommen durch den Staat ergeben (Unterkapitel 3.3). In den Unterkapiteln 3.4 und 3.5 werden schließlich die eher indirekten Einflüsse auf die Produktivitätsentwicklung und makroökonomische Auswirkungen erörtert. Analog zur Vorgehensweise bei der Untersuchung der Kinderkosten in Teil $\mathrm{C}$ dieser Arbeit erfolgt grundsätzlich für jede einzelne Einflußgröße zunächst eine theoretische Analyse, ehe die Größenordnung der Kosten bzw. des Nutzens für die Einheimischen soweit möglich quantifiziert und die Bedeutung der Resultate für ein Einwanderungsgesetz hervorgehoben werden.

$\mathrm{Zu}$ unterscheiden ist bei jeder einzelnen Kostenart zwischen Wirkungen, die aus einer bloßen Bevölkerungserhöhung resultieren und die damit auch bei dem Kinderlastenausgleich als alternativem Instrument zur langfristigen Stabilisierung der Finanzlage der Gesetzlichen Rentenversicherung auftreten, und Wirkungen, die auf unterschiedliche Charakteristika von Einwanderern und Einheimischen zurückgehen. Im Gegensatz zu dem überwiegend in der Literatur vorzufindenden Kostenbegriff interessiert hier nur die zweite, vom Bevölkerungsniveaueffekt unabhängige Wirkung. Lediglich bei einem Vergleich mit weiteren Vorschlägen zur Sicherung des finanziellen Gleichgewichts der Rentenversicherung sind zusätzlich die Kosten und Nutzen der beiden in dieser Arbeit verglichenen Instrumente zu berücksichtigen, die eine relative Bevölkerungszunahme ohne Strukturänderungen hervorruft. 
Die empirischen Ergebnisse bezüglich der Höhe der verschiedenen Kostenarten weichen für einzelne Immigrantengruppen voneinander ab, weil sie in ökonomisch relevanten Charakteristika differieren und weil sie in Deutschland einen unterschiedlichen Rechtsstatus genießen. Für die hier vorliegende Fragestellung erscheint eine Untergliederung der Einwanderer in die drei Gruppen Gastarbeiter, Aussiedler und Asylbewerber zweckmäßig. Das Schwergewicht der empirischen Analyse liegt jeweils bei den Gastarbeitern, da die Merkmale dieser Gruppe wohl am ehesten denjenigen der zukünftig durch ein Einwanderungsgesetz zur Immigration veranlaßten Personen entsprechen. Das Potential an Aussiedlern ist begrenzt, da Anfang der neunziger Jahre nur noch etwa 3 Mio Deutschstämmige in den Aussiedlungsgebieten leben. Dennoch werden auch für Aussiedler soweit möglich empirische Ergebnisse angeführt. Das erlaubt neben der Quantifizierung des Einflusses dieser Immigrantengruppe auf die einheimische Bevölkerung auch eine Abschätzung der Kosten- bzw. Nutzenunterschiede, die durch die gegenüber Gastarbeitern unterschiedlichen Merkmale hervorgerufen werden. Die Zahl der Asylbewerber schließlich ist ebenso wie die durchschnittlichen Kosten bzw. Nutzen pro Kopf weitgehend politisch determiniert. Eine an ökonomischen Kriterien ausgerichtete Einwanderung hat daher kaum Einfluß auf das Kosten/Nutzenverhältnis der Asylbewerber für die Einheimischen, ${ }^{69}$ weshalb empirische Daten für diese Gruppe nur gelegentlich nachrichtlich angeführt werden.

\subsection{Der Einfluß der Einwanderer auf Arbeitseinkommen und Arbeits- losigkeit von Einheimischen}

\subsubsection{Theoretische Analyse der Arbeitsmarktwirkungen von Einwan- derern}

Der Einfluß von Zuwanderern auf Löhne und Arbeitslosigkeit der Einheimischen ist von zentraler Bedeutung für die Kosten der Zuwanderung. Kommt es infolge der Einwanderung zu einem Absinken des Lohnniveaus und/oder einem Anstieg der Arbeitslosigkeit der Einheimischen, müssen die entstehenden Einkommenseinbußen und psychischen Folgen als Kosten der Zuwanderung angesehen werden. Wie in 2.2.2 ausgeführt, haben tatsächliche oder auch nur vermeintliche negative Arbeitsmarkteffekte darüber hinaus über den Prozeß der politischen Willensbildung Rückwirkungen auf die Zahl der Zuwanderer, da befürchtete negative Auswirkungen die Durchsetzbarkeit einer liberalen Einwanderungspolitik erschweren.

Eine erste Annäherung an die Wirkungen von Einwanderung auf die Arbeitsmarktsituation der Einheimischen erlaubt ein partialanalytisches, statisches Zwei-Länder-Modell mit homogener Arbeit als einzigem variablen Produktionsfaktor. ${ }^{70}$ In Abb. D.2 ist der Arbeitsmarkt des Einwanderungslandes dargestellt. Die aggregierte Arbeitsnachfragekurve $N$ der annahmegemäß

69 Allenfalls steht ein leichter Rückgang der Zahl der Asylbewerber für den Fall einer Verstärkung der Einwanderung zu erwarten, weil dann ökonomisch motivierte Einwanderer nicht mehr gezwungen sind, politische Gründe für die Immigration vorzuschieben. 
Abb. D.2: Arbeitsmarkteffekte von Arbeitskräftewanderungen in einem Zwei-Länder-Modell mit homogener Arbeit

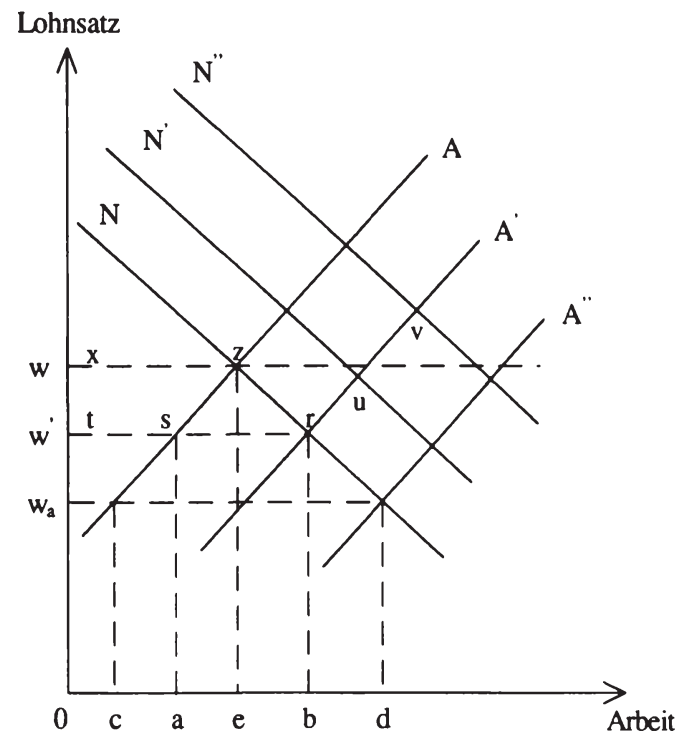

Quelle: Greenwood/McDowell (1994) S. 157

unter vollständiger Konkurrenz handelnden Unternehmen wird bei einer gegebenen Ausstattung mit Human- und Realkapital (es wird zunächst angenommen, daß die Migranten keine Änderung des Kapitalstocks bewirken), konstanten Skalenerträgen und Produktionstechniken durch die Wertgrenzproduktkurve der Arbeit dargestellt. Die Arbeitsangebotskurve $A$ habe für heimische Arbeitskräfte einen ansteigenden Verlauf, während sie für Einwanderer bei dem Lohnsatz $w_{a}$ vollkommen elastisch sei. ${ }^{71}$ Von Wanderungskosten, Staatseinflüssen, Externalitäten und Rigiditäten auf dem Arbeitsmarkt sei abgesehen.

Ohne Arbeitskräftewanderungen wird sich in dem Einwanderungsland ein Arbeitsmarktgleichgewicht bei $z$ mit dem Lohnsatz $w$ und der Arbeitsmenge $e$ einstellen. Werden nun alle Einwanderungsbeschränkungen aufgehoben, verschiebt sich die Arbeitsangebotskurve nach rechts bis zur neuen Kurve $A^{\prime \prime}$. Es kommt zur Einwanderung von Arbeitskräften in Höhe von $c d$ mit der Folge, daß der Lohnsatz auf $w_{a}$ sinkt. Wird statt einer völligen Freigabe der Einwanderung eine begrenzte Zuwanderung z.B. in Höhe von $a b$ erlaubt, verlagert sich die Arbeitsangebotskurve nur nach $A^{\prime}$, der Lohnsatz fällt nur auf $w^{\prime}$ und die Beschäftigung steigt auf $O b$ an. Auf einheimische Arbeitskräfte entfällt von dieser Gesamtbeschäftigung allerdings nur der Teil $O a$, so daß ihre Beschäftigung von $O e$ aus um $a e$ zurückgegangen ist. In diesem

Eine Analyse mit einem gegeben Weltarbeitsangebot findet sich bei Grubel (1994) S. $76 \mathrm{ff}$. 
Umfang haben also Einwanderer Einheimische von dem Arbeitsmarkt verdrängt. Insgesamt sinkt die Lohnsumme der Einheimischen von Oxze auf Otsa. Von diesem Verlust ist nur der Teil txzs zurückzuführen auf den gesunkenen Lohnsatz, während im Umfang von asze den Löhnen, die den Einheimischen entgangenen sind, gestiegene Freizeit im gleichen Wert gegenübersteht.

Den nicht durch den Freizeitzuwachs ausgeglichenen Einkommensverlusten der einheimischen Arbeitskräfte in Höhe von txzs stehen Einkommensgewinne der - annahmegemäß einheimischen - Kapitaleigner im Umfang von $t x z r$ gegenüber, so daß die Einheimischen insgesamt von der Zuwanderung von Arbeitskräften profitieren. ${ }^{72}$ Während die Kapitaleigner Vorteile haben, müssen die Arbeitskräfte allerdings Einkommensseinbußen hinnehmen, deren Höhe von der Zahl der Einwanderer und den Elastizitäten der Arbeitsangebots- und -nachfragekurve abhängt. ${ }^{73}$

Das oben dargestellte neoklassische Modell kann nur als erste Annäherung an die theoretische Analyse der Arbeitsmarktwirkungen von Einwanderern dienen, da recht restriktive Annahmen getroffen wurden. Insbesondere verhindert die Prämisse homogener Arbeit die Analyse von Wirkungen, die sich aus Strukturunterschieden zwischen Einwanderern und Einheimischen ergeben, so daß nur Effekte erfaßt werden, die auf eine reine Bevölkerungserhöhung zurückgehen, sei es durch Einwanderer oder höhere Fertilität der Einheimischen. Im folgenden werden daher drei Erweiterungen des Modells betrachtet. Zunächst wird neben der Angebotsseite auch die Nachfrageseite explizit in die Analyse einbezogen, dann wird der Einfluß von Lohnsatzrigiditäten untersucht und schließlich die Annahme homogener Arbeit aufgegeben und so die Untersuchung der Auswirkungen von Unterschieden zwischen Einwanderern und Einheimischen ermöglicht.

Bisher sind nur die angebotsseitigen Wirkungen der Zuwanderung auf den Arbeitsmarkt in die Betrachtung einbezogen worden. Führt die Einwanderung aber zu einer Erhöhung des Kapitalstocks im Einwanderungsland, verschiebt sich die Nachfragekurve in Abb. D.2 aufgrund der ceteris paribus steigenden Arbeitsproduktivität von $N$ nach $N^{\prime}$ oder $N^{\prime \prime}$. Im letzten Fall ergibt sich statt eines fallenden ein steigender Lohnsatz für die heimischen Erwerbstätigen und ihre Beschäftigung steigt. Die Gesamtwirkung auf die heimischen Arbeitskräfte hängt nun davon ab, ob der nachfrageseitige oder der angebotsseitige Effekt der Einwanderung überwiegt, und ist daher schon in diesem einfachen Modell mit homogener Arbeit allein aufgrund theoretischer Überlegungen nicht zu bestimmen.

72 Diese Schlußfolgerung kann sich ändern, wenn die Einwanderer Vermögen in das Aufnahmeland transferieren und somit an den gestiegenen Kapitaleinkünften teilhaben. Wohlfahrtseffekte werden von Grubel (1994) S. 78ff. untersucht, die Größenordnung dieser Wohlfahrtseffekte versucht Borjas (1995) für die USA abzuschätzen.

73 Ein umfassenderes Ein-Sektoren Wachstumsmodell mit flexiblen Preisen findet sich bei Steinmann (1994). Die Annahme homogener Arbeit schränkt die empirische Relevanz des Ansatzes allerdings stark ein, weshalb hier nur die Ergebnisse referiert werden sollen: In Übereinstimmung mit den oben angeführten Überlegungen erhält Steinmann unter der Prämisse, daß die Einwanderer kein Kapital mitbringen, positive kurzfristige Wirkungen auf die Einkommen der Einheimischen, da die Verluste bei den Arbeitseinkommen durch die Gewinne bei den Kapitaleinkommen überkompensiert werden. Die Einführung von Integrationskosten kann dieses Resultat allerdings schnell ändern (vgl. Steinmann (1994) S. 48). Die langfristigen Effekte, die sich durch eine Änderung der Produktionstechnologie und die Kapitalakkumulation der Einwanderer im steady state ergeben, hängen v.a. von der Sparquote der Einwanderer und der Substitutionselastizität der beiden Produktionsfaktoren Arbeit und Kapital ab, sind in dem Modell aber generell nicht negativ. 
In dem oben erläuterten neoklassischen Grundmodell wird von völliger Lohnsatzflexibilität ausgegangen, so daß die Wirkung eines zusätzlichen Arbeitskräfteangebots sich ausschließlich in sinkenden Löhnen und einem Rückgang der Beschäftigung einheimischer Erwerbstätiger aufgrund "freiwilligem", d.h. auf den geringeren Lohn zurückzuführenden Ausscheiden aus dem Arbeitsmarkt niederschlägt. Existieren dagegen Rigiditäten auf dem Arbeitsmarkt, z.B. weil institutionelle Hemmnisse einer ausreichenden Absenkung des Lohnsatzes entgegenstehen, dann hat das zusätzliche Arbeitsangebot eine Zunahme der Zahl der Arbeitslosen zur Konsequenz. ${ }^{74}$ In der Bundesrepublik Deutschland können aufgrund der kollektiven Lohnverhandlungen solche Rigiditäten angenommen werden, so daß hier stärker als in den USA, für die die meisten empirischen Untersuchungen durchgeführt wurden, auf Änderungen der Arbeitslosenquote der Einheimischen geachtet werden muß.

Als dritte Erweiterung soll die Prämisse der Homogenität der Arbeit aufgegeben werden. Sie ist nur dann gerechtfertigt, wenn die Qualifikationsstruktur und andere arbeitsmarktrelevante Merkmale der Einwanderer denjenigen der Einheimischen genau entsprechen. Realitätsnäher ist die explizite Berücksichtigung der Heterogenität des Produktionsfaktors Arbeit, indem Einheimische und Einwanderer als unterschiedliche Produktionsfaktoren angesehen werden. Nur so können die Wirkungen der Unterschiede zwischen Einheimischen und Einwanderern erfaßt werden. Einwanderer müssen dann nicht mehr, wie noch im Modell mit homogener Arbeit, notwendig Substitute für einheimische Erwerbstätige sein, sondern können auch in einem komplementären ${ }^{75}$ Verhältnis zu ihnen stehen. ${ }^{76}$ In letzterem Fall würde die Grenzproduktivität der einheimischen Arbeitskräfte durch die Einwanderer erhöht und damit die Nachfrage nach ihnen steigen mit der Folge eines höheren Lohnsatzes und höherer Beschäftigung für die Einheimischen. In Abb. D.3 wird die Nachfragekurve auf dem Markt für einheimische Arbeitskräfte im Fall der Komplementarität nach außen von $N$ auf $N^{\prime \prime}$ verschoben, im Fall der Substitutionalität nach innen auf $N^{\prime}$ verlagert. Die Arbeitsmarktwirkungen der Zuwanderung für einheimische Erwerbstätige verkehren sich damit gegenüber dem einfachen Modell mit homogener Arbeit ins Gegenteil, wenn Einheimische und Einwanderer auf dem Arbeitsmarkt Komplemente sind, bleiben aber unverändert, wenn sie Substitute darstellen. Ein großer Teil der empirischen Untersuchungen gilt daher der Klärung der Frage, ob zwischen einheimischen und eingewanderten Arbeitskräften ein komplementäres oder substitutives Verhältnis vorliegt. ${ }^{77}$

Rivera-Batiz/Sechzer ${ }^{78}$ argumentieren allerdings, daß entsprechend des Humankapitalansatzes nicht Geburtsort oder Volkszugehörigkeit die für den Arbeitsmarkt relevanten Unter-

Zimmermann (1995) S. 53 weist darauf hin, daß Einwanderer dazu beitragen können, den unflexiblen institutionellen Rahmen des Arbeitsmarktes aufzuweichen.

Unter Komplementen werden hier Produktionsfaktoren verstanden, bei denen der vermehrte Einsatz des einen Faktors die Grenzproduktivität des anderen erhöht ( $q$-complements). Bei substitutiven Produktionsfaktoren hat dagegen der verstärkte Einsatz eines Faktors ein Reduktion der Grenzproduktivität des anderen zur Folge (q-substitutes). Vgl. Hamermesh (1993) S. $37 f$.

76 Für ein komplementäres Verhältnis spricht die Tatsache, daß die sektorale Beschäftigungsstruktur von Ausländern und Einheimischen in Deutschland sehr unterschiedlich ist. Nach Zimmermann (1994a) S. 231 waren 1992 12,4 Prozent der Beschäftigten im verarbeitenden Gewerbe Ausländer, aber nur 1.7 Prozent im Bereich Banken und Versicherungen.

77 Einen knappen Überblick geben Rürup/Sesselmeier (1994) S. 65ff.

Vgl. Rivera-Batiz/Sechzer (1991) S. 94. 
Abb. D.3: Die Arbeitsmarktwirkung von Einwanderern für Einheimische in einem Modell mit Einheimischen und Einwanderern als unterschiedlichen Produktionsfaktoren

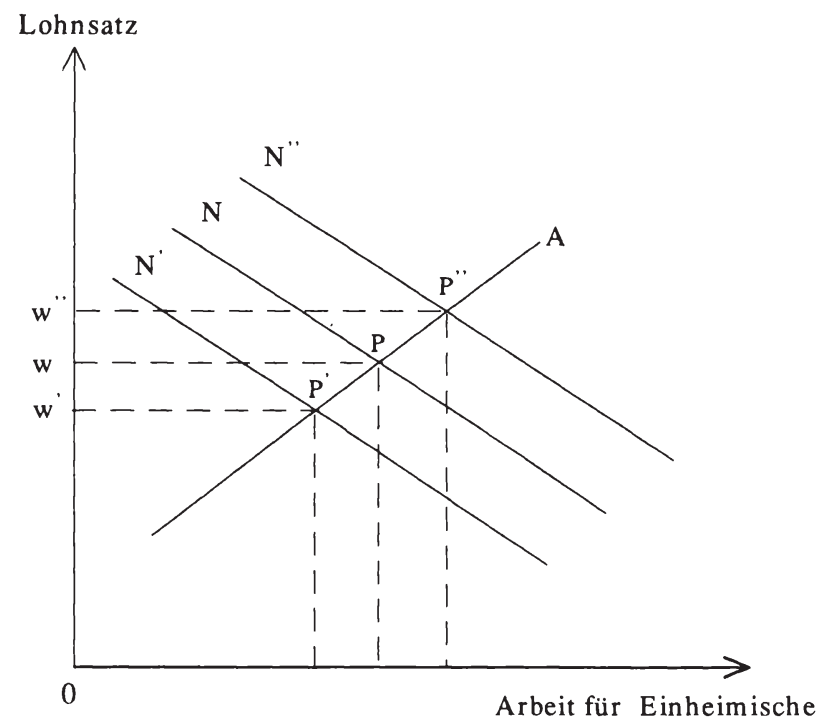

scheidungskriterien sind, sondern die Ausstattung mit Humankapital z.B. in Form von Ausbildung und Berufserfahrung. Dementsprechend unterscheiden manche Autoren nicht zwischen Einheimischen und Einwanderern als unterschiedlichen Produktionsfaktoren, sondern zwischen qualifizierten und unqualifizierten Arbeitskräften. Die Arbeitsmarktwirkung der Einwanderer hängt damit nicht allein davon $a b$, ob zwischen qualifizierten und unqualifizierten Arbeitskräften ein komplementäres oder substitutives Verhältnis besteht, sondern auch von dem Anteil der Qualifizierten unter Einheimischen und Eingewanderten.

Zur Illustration sei angenommen, daß eine komplementäre Beziehung zwischen Qualifizierten und Unqualifizierten bestehe und nur unqualifizierte Arbeitskräfte einwandern. Durch die Einwanderung erhöht sich dann das Angebot auf dem Arbeitsmarkt für Unqualifizierte (Rechtsverschiebung der kompensierten Angebotskurve von $A_{n}$ nach $A_{n}{ }^{\prime}$ in Abb. D.4) und sowohl Lohnsatz als auch Beschäftigung unqualifizierter einheimischer Arbeitskräfte fallen. Auf dem Arbeitsmarkt für Qualifizierte steigt die Nachfrage (Rechtsverschiebung der Nachfragekurve $N_{q}$ auf $N_{q}{ }^{\prime}$ in Abb. D.4), da die Grenzproduktivität der qualifizierten (heimischen) Arbeitskräfte aufgrund des vermehrten Einsatzes an unqualifizierten Kräften (heimischen und eingewanderten) gestiegen ist. Während die qualifizierten heimischen Arbeitskräfte von der Zuwanderung durch höhere Löhne profitieren, sind die Unqualifizierten negativ betroffen. Gang und Rivera-Batiz weisen allerdings darauf hin, daß die Arbeitsmarktwirkungen der Einwanderer von einem Arbeitsmarktsegment in ein anderes verlagert werden können, wenn beispielsweise die Einheimischen in Reaktion auf zunehmende Konkurrenz auf dem Arbeits- 
Abb. D.4: Die Arbeitsmarktwirkung von Einwanderern für Einheimische in einem Modell mit qualifizierten und unqualifizierten Arbeitskräften als unterschiedlichen Produktionsfaktoren
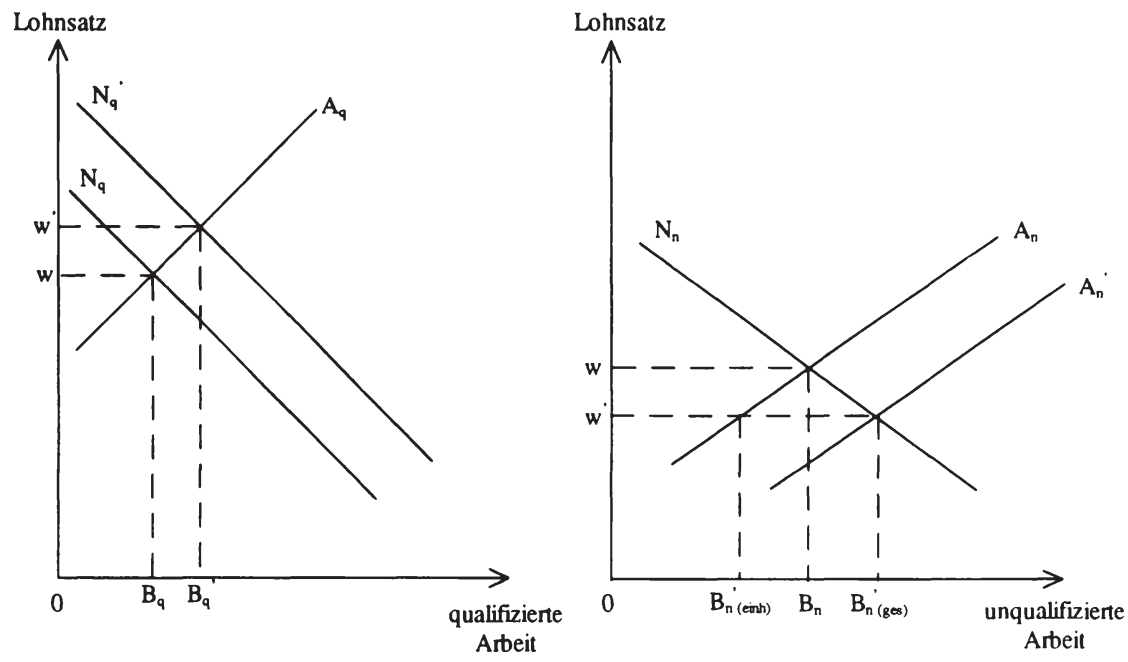

markt für Unqualifizierte eine Qualifizierungsstrategie einschlagen und nach einiger Zeit das Angebot auf dem Arbeitsmarkt für Qualifizierte erhöhen. ${ }^{79}$

\subsubsection{Die empirische Größenordnung der Arbeitsmarktwirkungen von Einwanderern}

Die empirischen Studien zu den Arbeitsmarktwirkungen von Zuwanderern, die die Heterogenität des Produktionsfaktors Arbeit berücksichtigen, gliedern sich in Querschnittsstudien und Längsschnittstudien.

In den meisten Querschnittsstudien wird versucht, die Auswirkungen der Einwanderer auf die Arbeitsmarktsituation der Einheimischen zu erfassen, indem die Arbeitseinkommen und die Arbeitslosigkeit der Einheimischen in bestimmten Regionen (oder auch Branchen) mit unterschiedlichem Anteil eingewanderter Arbeitskräfte verglichen werden. Meistens wird bei dieser Vorgehensweise ein zweistufiges Regressionsverfahren verwandt. Zunächst wird eine Regressionsanalyse für das individuelle Arbeitseinkommen durchgeführt, um die Koeffizienten verschiedener Erklärungsvariablen wie z.B. Ausbildung, Alter, Unterbrechungszeiten etc. zu 
erhalten. ${ }^{80}$ Die geschätzten Koeffizienten dienen dazu, die regionalen Lohn- bzw. Arbeitslosigkeitsdaten von Struktureffekten zu bereinigen. In einem zweiten Schritt werden die bereinigten Daten der verschiedenen Regionen erklärt durch unterschiedliche Merkmale dieser Regionen, zu denen auch ein Maß für den Anteil der Einwanderer zählt. ${ }^{81}$ Aus der Höhe des dieser Variablen zugeordneten Koeffizienten und deren Standardabweichung können dann Schlüsse über den Einfluß der Einwanderer auf die Arbeitsmarktsituation der Einheimischen gezogen werden.

Dieses Vorgehen entspricht dem oben erläuterten Modell mit Einheimischen und Eingewanderten als unterschiedlichen Produktionsfaktoren. Da die Situation auf Arbeitsmärkten mit einem unterschiedlichen Anteil Eingewanderter verglichen wird, werden auch die nachfrageseitigen Effekte berücksichtigt, soweit sie innerhalb der Grenzen einer Arbeitsmarktregion wirksam werden.

Gegen diese Vorgehensweise lassen sich allerdings mehrere Kritikpunkte geltend machen. Eine Grundvoraussetzung für die Aussagefähigkeit der Ergebnisse ist das Vorliegen von regional segmentierten Arbeitsmärkten. ${ }^{82}$ Besteht ein enger Austausch zwischen verschiedenen Regionen, dann kann eine besonders hohe Zuwanderung in einer Region infolge der dadurch hervorgerufenen schlechteren Arbeitsmarktlage für einheimische Arbeitskräfte zu deren Abwanderung führen, mit der Folge, daß sich die Arbeitsmarktwirkungen zumindest teilweise in einer anderen Region niederschlagen als der Region, in die die Zuwanderung aus dem Ausland erfolgt ist. Entscheidend ist also die Frage, ob internationale Zuwanderung in eine Region einen negativen Effekt auf den Binnenwanderungssaldo hervorruft. Die wenigen empirische Studien zu diesem Thema beziehen sich meistens auf die USA und haben außerdem widersprüchliche Resultate. Filer findet ebenso wie White/Hunter ${ }^{83}$ eine starke negative Korrelation zwischen Außen- und Binnenwanderung, die zudem v.a. auf Wanderungen der hauptsächlich mit den Einwanderern konkurrierenden einheimischen Arbeiter mit niedrigeren Ausbildungsstufen beruht. Demgegenüber kommen White/Liang genauso wie Butcher/Card ${ }^{84} \mathrm{zu}$ dem Schluß, daß Außen- und Binnenwanderung in den meisten Fällen positiv miteinander korrelieren, ein Ergebnis, das Pischke/Velling ${ }^{85}$ auch für Deutschland erhalten. In den USA tritt das skizzierte Problem weniger stark auf, da die in den meisten empirischen Studien als regionale Abgrenzung verwandten Standardized Metropolitan Statistical Areas (SMSA) als

80 Vgl. zu diesem Vorgehen Abschnitt 3.2.1.2 in Teil C.

81 Vgl. Schmidt (1994a) S. 9.

82 Vgl. Schmidt (1994a) S. 9.

83 Vgl. Filer (1992) bzw. White/Hunter (1993) (letztere nach Friedberg/Hunt (1995) S. 36). Hunt findet für Frankreich in den sechziger Jahren ebenfalls eine negative Korrelation zwischen Binnen- und Außenwanderungen. Vgl. Hunt (1992) S. 568.

Vgl. Butcher/Card (1991) S. 294 bzw. White/Liang (1994) (letztere nach Friedberg/Hunt (1995) S. 36). Filer (1992) S. 246 weist darauf hin, daß das Ergebnis von Butcher/Card nicht auf Wanderungsdaten beruht, sondern auf Bevölkerungszuwachsraten. Eine mögliche Erklärung für die unterschiedlichen Ergebnisse wäre demnach, daß Einwanderer v.a. in Städte mit einem hohen Anteil früherer Zuwanderer ziehen, die eine überdurchschnittliche Fertilität aufweisen. Die hohen Bevölkerungswachstumsraten in den Städten mit hoher Einwanderung wären dann nicht auf einen positiven Binnenwanderungssaldo, sondern auf eine hohe natürliche Bevölkerungszunahme zurückzuführen.

85 Vgl. Pischke/Velling (1994) S. 17f. Die Autoren weisen allerdings darauf hin, daß ihr Ergebnis auf einer ungenügenden Berücksichtigung der Simultanität der Wanderungen von Ausländern und Einheimischen und der wirtschaftlichen Attraktivität einer Region beruhen könnte. Vgl. Pischke/Velling (1994) S. 19. 
hinreichend abgeschlossen gelten können. ${ }^{86}$ In Deutschland ist dagegen eine einigermaßen zuverlässige Abgrenzung von Arbeitsmarktregionen wesentlich schwieriger.

Aber selbst wenn interne Wanderungen völlig unelastisch in bezug auf Einwanderung sind, die Arbeitsmarktregionen also tatsächlich als abgeschlossen gelten können, werden Güter- und Kapitalbewegungen zwischen den Arbeitsmarktregionen zu einer tendenziellen Angleichung der Faktorpreise führen. ${ }^{87}$ Die Arbeitsmarkteffekte werden sich also nicht auf die Regionen mit hoher Einwanderung beschränken, sondern erstrecken sich infolge von Güter-, Arbeitskräfteund Kapitalbewegungen über das gesamte Staatsgebiet. ${ }^{88}$ Querschnittsanalysen, die sich auf die unterschiedliche Arbeitsmarktsituation in Regionen mit divergierenden Einwandereranteilen stützen, bergen daher die Gefahr einer Unterschätzung der Arbeitsmarkteffekte von Einwanderern.

Eine weitere Schwierigkeit bei der Interpretation der in Querschnittsuntersuchungen gefundenen Ergebnisse ergibt sich, wenn die Einwanderer den Arbeitsort im Gastland gezielt nach den Arbeitsmarktbedingungen aussuchen. Dann nämlich ist das Maß für den Anteil der Einwanderer an der Bevölkerung nicht mehr unabhängig von der zu erklärenden Variablen, so daß ein Endogenitätsproblem entsteht. Tendenziell werden sich mehr Einwanderer in Regionen mit günstigen Arbeitsmarktbedingungen niederlassen, so da $B$ in Querschnittsanalysen negative Effekte der Einwanderung auf die Arbeitsmarktsituation unterzeichnet werden.

Schließlich wird in vielen Studien nicht berücksichtigt, daß die Einwanderer im Durchschnitt weniger verdienen als die Einheimischen. ${ }^{89}$ Ist der Anteil der Einwanderer in einer Region besonders hoch, dann wird das durchschnittliche Einkommen in dieser Region unter dem durchschnittlichen Einkommen in einer Region mit weniger Einwanderern liegen, auch wenn die Einkommen der Einheimischen in beiden Regionen gleich hoch sind..$^{90}$ Durch diesen Zusammensetzungseffekt werden die Wirkungen der Einwanderer auf die Arbeitsmarktsituation der Einheimischen zu hoch ausgewiesen.

Diese Kritikpunkte müssen bei der nachfolgenden Darstellung der Ergebnisse der Querschnittsstudien beachtet werden.

Die Resultate der empirischen Querschnittsuntersuchungen werden zunächst für die USA, dann für Deutschland vorgestellt.91 Wie oben bereits erwähnt, sind die amerikanischen Ergebnisse wegen der unterschiedlichen institutionellen Ausprägung der nationalen Arbeitsıärkte nur schwer auf andere Staaten übertragbar. ${ }^{92}$ Sie werden hier dennoch angeführt, da für Deutschland erst wenige empirische Studien vorliegen.

86

Vgl. Schmidt (1994a) S. 9.

Die in 2.1.1 für unterschiedliche Staaten dargestellte Tendenz von Güter- und Faktorbewegungen zur Faktorpreisangleichung gilt in verstärktem Maße für unterschiedliche Arbeitsmarktregionen, da hier im Vergleich zu internationalen Güter- und Faktorbewegungen geringere Transaktions- und Informationskosten anfallen. (1994), Borjas (1990), Hamermesh (1993) sowie De New/Zimmermann (1994) S. 82ff. und Pischke/Velling (1994) S. 2 f.

In Abschnitt 3.3.1 wird ausführlicher auf die unterschiedlichen Einkommen von Einheimischen und Einwanderem eingegangen.

Vgl. Friedberg/Hunt (1995) S. 31 f.

Vgl. Schmidt (1994a) S. 2. 
Borjas $^{93}$ untersucht Standardized Metropolitan Statistical Areas für das Jahr 1970 mit einer verallgemeinerten Leontief-Produktionsfunktion mit Kapital und sechs nach Volkszugehörigkeit, Geschlecht und Einwanderungsstatus unterschiedlichen Arbeitstypen als Produktionsfaktoren und findet ebenso wie in einer Folgestudie ${ }^{94}$ für das Untersuchungsjahr 1980 mit acht Arbeitstypen sowohl positive als auch negative Komplementaritätselastizitäten ${ }^{95}$ zwischen den einzelnen Arbeitsgruppen, die allerdings alle sehr klein sind. Bean, Lowell und Taylor ${ }^{96}$ finden für das Jahr 1980 mit einem ähnlichen Ansatz auch für illegale Einwanderer nur Komplementaritätselastizitäten mit einem Betrag von weniger als 0,15 . In weiteren Studien finden auch Butcher und Card sowie Simon, Moore und Sullivan ${ }^{97}$ keine negativen Effekte der Zuwanderer auf die Arbeitsmarktsituation der Einheimischen. Eine Ausnahme zu diesen recht einheitlichen empirischen Befunden bildet die Studie von Altonji und Card ${ }^{98}$, nach der ein recht hoher substitutionaler Zusammenhang zwischen vier Gruppen von Einheimischen und Einwanderern besteht. Möglicherweise ist dies darauf zurückzuführen, daß hier versucht wurde, das oben beschriebene Endogenitätsproblem zu lösen, indem der Bestand der Einwanderer als erklärende Variable in die Regression aufgenommen wurde. ${ }^{99}$

Rivera-Batiz und Sechzer differenzieren entsprechend ihrer oben angeführten theoretischen Überlegungen nicht nach der Volkszugehörigkeit, sondern nach dem Humankapitalbestand. ${ }^{100}$ Sie erhalten eine komplementäre Beziehung zwischen den Produktionsfaktoren Berufserfahrung, formale Ausbildung und unqualifizierte Arbeitskraft, wobei die Komplementarität zwischen formaler Ausbildung und den anderen beiden Arbeitstypen besonders ausgeprägt ist. Die berechneten Komplementaritätselastizitäten werden benutzt, um mit Hilfe der in der Vergangenheit festgestellten Humankapitalausstattung verschiedener Gruppen von Einwanderern und Einheimischen die jeweilige Wirkung auf das Lohnniveau festzustellen. Der Einfluß aller Einwanderergruppen auf alle Gruppen von Einheimischen ist gering und weist ein wechselndes Vorzeichen auf. Der größte negative Einfluß wurde mit einer Lohneinbuße von weniger als einem Prozent für einheimische ethnische Mexikaner im Fall der Einwanderung von Mexikanern in Höhe von zehn Prozent der amerikanischen Arbeitskräfte ermittelt. ${ }^{101}$

In Deutschland ist das wissenschaftliche Interesse an den Arbeitsmarktwirkungen von Einwanderern erst mit dem Ende der achtziger Jahre stark anwachsenden Einwanderungsüberschuß gestiegen. Es liegen daher bisher nur wenige empirische Studien vor. Winkelmann

93

Vgl. Borjas (1986).

Vgl. Borjas (1987).

Die Komplementaritätselastizitäten beschreiben die relative Veränderung des Faktorpreisverhältnisses durch eine sie bewirkende relative Veränderung des Faktoreinsatzverhältnisses. Vgl. Hamermesh (1993) S. 27f.

Vgl. Bean/Lowell/Taylor (1988).

Vgl. Butcher/Card (1991) und Simon/Moore/Sullivan (1993). Weitere Studien mit ähnlichen Ergebnissen finden sich in Borjas (1990), Simon (1989) und Winegarden/Khor (1993).

Vgl. Altonji/Card (1991).

Voraussetzung für eine Lösung des Endogenitätsproblems durch diese Instrumentierung ist, daß die Einwanderer sich entsprechend der Hypothese der Kettenwanderungen (chain migration) v.a. in Orten niederlassen, in denen schon früher Eingewanderte wohnen.

Vgl. Rivera-Batiz/Sechzer (1991).

Vgl. Rivera-Batiz/Sechzer (1991) S. 106. 
und Zimmermann ${ }^{102}$ gehen anhand von Daten des Sozio-Ökonomischen Panels der Frage nach, ob Ende der siebziger und Anfang der achtziger Jahre Einwanderung zu Arbeitslosigkeit von Einheimischen geführt hat. In zwei Folgestudien untersuchen De New und Zimmermann den Einfluß auf das Lohnniveau. ${ }^{103}$ Im Gegensatz zu den meisten anderen empirischen Studien benutzen sie allerdings nicht unterschiedliche Anteile ausländischer Arbeitskräfte in verschiedenen Arbeitsmarktregionen, sondern unterschiedliche Anteile in verschiedenen Branchen als erklärende Variable. Sie erhalten einen positiven Einfluß des Anteils der Ausländer in einer Branche auf die Häufigkeit von Arbeitslosigkeit von Einheimischen und negative Einkommenseffekte für Arbeiter, aber positive Einkommenseffekte für Angestellte. Die Einkommensverluste für alle Arbeiter durch einen Anstieg des Ausländeranteils um einen Prozentpunkt oder 9,8 Prozent belaufen sich auf 6,5 Prozent, wobei die größten Einbußen von den bereits länger in Deutschland ansässigen Ausländern mit langjähriger Berufserfahrung und in geringerem $\mathrm{Maße}$ von deutschen Arbeitern mit geringer Berufserfahrung hingenommen werden müssen, während die Löhne deutscher Arbeiter mit hoher Berufserfahrung kaum betroffen sind. ${ }^{104}$ Die Autoren erklären diese Resultate dadurch, daß die Einwanderer sich fast ausschließlich aus Arbeitern zusammensetzen und daher in einer substitutionalen Beziehung zu heimischen Arbeitern stehen, zwischen Arbeitern und Angestellten aber Komplementarität vorliegt. Als Folge werden die Arbeitseinkommen der Arbeiter negativ, die der Angestellten dagegen positiv durch die Einwanderung beeinflußt.

Die oben aufgeführten Kritikpunkte an Querschnittsstudien auf regionaler Basis werden mit dem hier erfolgten Rückgriff auf unterschiedliche Branchen allerdings nicht umgangen. Ebenso wie sich die Arbeitsmarkteffekte über Regionen ausbreiten, findet auch zwischen Branchen eine Übertragung statt. Auch das Endogenitätsproblem ist nicht gelöst. Pischke und Velling vermuten im Gegenteil, daß die in den beiden Studien erhaltenen negativen Effekte darauf zurückgeführt werden können, daß die Gastarbeiter in den sechziger Jahren v.a. in prosperierenden Industriezweigen Beschäftigung fanden, die zwei Jahrzehnte später besonders hart vom Strukturwandel betroffen waren. ${ }^{105}$ Damit ergäbe sich eine positive Korrelation zwischen dem Anteil der Einwanderer und der Arbeitslosenquote in einer bestimmten Branche, ohne da $\beta$ eine Kausalbeziehung besteht. ${ }^{106}$

Pischke und Velling ${ }^{107}$ benutzen Arbeitsmarktdaten der Bundesforschungsanstalt für Landeskunde und Raumordnung aus 319 Kreisen, die sie zu 160 Arbeitsmarktregionen zusammenfassen, um den Einfluß der Einwanderer auf Beschäftigung, Arbeitslosigkeit und Industrielöhne zu ermitteln. Als Maß für die Zuwanderung verwenden sie neben der Änderung

\footnotetext{
102 Vgl. Winkelmann/Zimmermann (1993).

103 Vgl. De New/Zimmermann (1993 und 1994).

104 Vgl. De New/Zimmermann (1994) S. 87ff.
}

105 Empirisch gestützt wird diese Vermutung durch Angaben zu Ausländeranteilen in verschiedenen Branchen in Barabas u.a. (1992) S. 140f. Danach weisen die Wirtschaftszweige Gießereien (24\%), Textilindustrie (17\%), Bergbau (14\%) sowie Eisen- und Stahlindustrie (14\%) weit über dem Durchschnitt (8\%) liegende Ausländeranteile auf. Rivera-Batiz/Sechzer (1991) S. 95 weisen für die USA auf die Tendenz von Einwanderern hin, sich in niedergehenden Industriezweigen eine Beschäftigung zu suchen.

106 Vgl. Pischke/Velling (1994) S. 3. Die in De New/Zimmermann (1993) vorgenommene Instrumentierung mit Hilfe von Dummy- und Trend-Variablen für die einzelnen Branchen halten Pischke/Velling für nicht ausreichend. 
des Ausländeranteils zwischen 1985 und 1989 auch jährliche Brutto- und Nettowanderungsströme, weil das erste Maß durch interne Wanderungen von Ausländern und das Heranwachsen der zweiten Generation von Ausländern verzerrt ist. V.a. die letzte Gruppe ist der übrigen Bevölkerung recht ähnlich und ökonomisch daher wie Einheimische anzusehen, auch wenn sie nach deutschem Recht noch ausländische Staatsbürger sind. Um das Endogenitätsproblem zu entschärfen, benutzen die Autoren in Anlehnung an Altonji und Card den Ausländerbestand der ersten Periode in einer Arbeitsmarktregion als Instrument für die Veränderung des Anteils der Ausländer in dieser Region.

Als Resultat ergibt sich ein implausibel hoher positiver Einfluß der Veränderung des Ausländeranteils auf die Arbeitslosigkeit - die Zunahme der Arbeitslosen wäre stärker als die Zunahme der Ausländer - und auch ein hoher positiver Einfluß auf das Lohnniveau. Die Ursache für diese theoretisch kaum erklärbaren Ergebnisse sehen Pischke/Velling im Fall des Lohnniveaus in der potentiellen Endogenität der Instrumentvariablen. Wenn die Arbeitsmarkttrends sich über längere Zeiträume fortsetzen, kann der Anteil der Ausländer aufgrund günstiger Bedingungen schon 1985 in den Arbeitsmarktregionen besonders hoch gewesen sein, die auch in den folgenden Perioden besonders vorteilhafte Veränderungen des Lohnniveaus aufwiesen. ${ }^{108}$ Die Wirkung der Zuwanderung von Ausländern auf das Lohnniveau ist daher nicht genau feststellbar. Den großen positiven Einfluß auf die Veränderung der Arbeitslosenrate erklären die Autoren mit einer durch ihre Daten bestätigten Tendenz zu einer überproportional starken Abnahme der Arbeitslosigkeit in strukturschwachen ländlichen Gebieten mit hoher Arbeitslosigkeit und niedrigem Lohnniveau während Aufschwungphasen wie dem ihrer Studie zugrundeliegenden Zeitraum von 1985 bis 1989. Dieser Trend zur Angleichung der Arbeitslosenraten in Aufschwungzeiten führt zu einer hohen negativen Korrelation zwischen Ausländeranteil und Veränderung der Arbeitslosenrate, wenn der Ausländeranteil in den Regionen mit besonders günstigen Arbeitsmarktbedingungen höher ist als in strukturschwachen Räumen. Bei einer Einbeziehung dieses Effektes wurde kein Einfluß von Wanderungsströmen auf die Arbeitslosenrate gefunden.

Den Unterschied zu den Ergebnissen von Winkelmann/Zimmermann und De New/Zimmermann erklären Pischke/Velling neben der oben erläuterten Konzentration von Ausländern in schrumpfenden Industrien damit, daß sie im Gegensatz zu den angeführten Autoren eine Aufschwungperiode betrachten, in der die Eingliederung von Einwanderern in den Arbeitsmarkt vergleichsweise einfach möglich ist.

Letztlich ist in allen Querschnittsstudien das Erfassungsproblem, das aus der Übertragung der Wirkungen von Einwanderern aus der Arbeitsmarktregion, in der sie sich niederlassen, auf andere Regionen entsteht, nicht befriedigend gelöst. Deshalb wird im folgenden auf die Ergebnisse aus Längsschnittstudien eingegangen, die die Arbeitsmarktwirkungen eines einmaligen exogenen, d.h. nicht durch die Arbeitsmarktlage im Zielgebiet determinierten Einwanderungsschubs in einer Region über die Zeit betrachten. Da davon ausgegangen werden kann, da $\beta$ die Anpassung an die veränderten Arbeitsmarktbedingungen nicht sofort erfolgt, ${ }^{109}$ müßten Arbeitsmarktwirkungen der Zuwanderung, sofern vorhanden, wenigstens kurzfristig zu Unterschieden zwischen verschieden stark von der Einwanderung betroffenen Regionen führen.

108 Vgl. Pischke/Velling (1994) S. 12.

109 Blanchard/Katz (1992) finden für die USA, daß Nachfrageschocks das Lohnniveau eines Bundesstaates etwa zehn Jahre und die Arbeitslosenrate etwa sechs Jahre lang beeinflussen. Für Europa weisen Decressin/Fatàs (1996) Beschäftigungswirkungen für etwa drei Jahre nach. 
Leider existieren nur wenige Längsschnittstudien. Hier werden im folgenden die Studie von Card ${ }^{110}$ zu den Auswirkungen der Zuwanderung von Kubanern auf den Arbeitsmarkt von Miami im Jahr 1980, die Arbeit von Hunt ${ }^{111}$ über die Konsequenzen der Algerienflüchtlinge für den französischen Arbeitsmarkt und eine Studie von Borjas, Freeman und Katz ${ }^{112}$ für die Vereinigten Staaten dargestellt. ${ }^{113}$

In der sog. Mariel Boatlift landeten von Mai bis September 1980 etwa 125.000 Kubaner mit kleinen Booten in Miami, von denen sich etwa 50 Prozent dauerhaft dort niederließen. Dadurch ergab sich eine Zunahme der Zahl der Arbeitskräfte im Raum Miami um etwa sieben Prozent, die sich v.a. aus wenig qualifizierten Arbeitskräften zusammensetzte. Card findet anhand von Mikro-Daten für den Zeitraum von 1979 bis 1985 im Vergleich zu vier Referenzstädten weder einen negativen Einfluß dieses einmaligen und hohen exogenen Anstiegs des Arbeitskräfteangebots auf die Lohnsätze verschiedener Arbeitskräftegruppen noch auf die Höhe ihrer Arbeitslosenquoten. Selbst für früher eingewanderte Kubaner und wenig qualifizierte Arbeitskräfte, die am ehesten Substitute zu den Einwanderern darstellen, sind keine negativen Arbeitsmarkteffekte feststellbar. Diese erstaunliche Absorptionsfähigkeit des Arbeitsmarktes von Miami führt Card einerseits auf die zur Beschäftigung von wenig qualifizierten Arbeitskräften günstige Industriestruktur zurück, die sich als Folge der langen Einwanderungstradition in der Stadt entwickelt hat, und zum anderen auf möglicherweise in Reaktion auf den Einwanderungsschub unterbliebene Zuwanderung von Einheimischen nach Miami. ${ }^{114}$ $\mathrm{Da}$ die Arbeitsmarktsituation für Einheimische sich durch die Flüchtlingswelle nicht verschlechtert hat, können für eine solche Reduktion der inländischen Zuwanderung allerdings nur außerökonomische Gründe wie beispielsweise die angespannte Sicherheitslage maßgebend sein.

Ein weiteres natürliches Experiment war die Rückkehr von etwa 900.000 Personen nach Frankreich in Folge der Unabhängigkeit Algeriens im Jahr 1962. Wie bei der Mariel Boatlift waren auch hier Zeitpunkt der Wanderungsbewegung und Niederlassungsort im Zielland nicht von der dortigen Arbeitsmarktlage bestimmt. Die sogenannten pieds noirs siedelten sich vielmehr besonders in Regionen an, die klimatisch ähnliche Bedingungen aufweisen wie ihr Herkunftsland. Eine bedeutende Rolle spielte auch die Anwesenheit von bereits früher aus Algerien ausgewanderten Personen. 115 Als Datenbasis dienten Hunt Arbeitsmarktmerkmale von departements mit unterschiedlichen Anteilen von pieds noirs an der Gesamtbevölkerung für die Jahre 1962 und 1968. Im Gegensatz zu der Einwanderungswelle im Zuge der Mariel Boatlift waren die pieds noirs durchschnittlich besser qualifiziert als die übrigen Franzosen. ${ }^{116}$ Dennoch konnte auch in diesem Fall sowohl bezüglich der Arbeitslosigkeit und Erwerbsbeteiligung als auch hinsichtlich des Lohnniveaus anhand der Differenzen zwischen den Daten für 1962 und 1968 nur geringfügige negative Wirkungen auf die Einheimischen festgestellt

\footnotetext{
110 Vgl. Card (1990).

111 Vgl. Hunt (1992).

112 Vgl. Borjas/Freeman/Katz (1992).

113 In einer weiteren Studie untersuchen Carrington/deLima (1994) die Auswirkungen der Rückkehrer aus den Kolonien nach Portugal Mitte der siebziger Jahre, können aber weder positive noch negative Wirkungen belegen (nach Friedberg/Hunt (1995)).

114 Vgl. Card (1990) S. 255f.

115 Vgl. Hunt (1992) S. 562ff.

116 Vgl. Hunt (1992) S. 557f.
} 
werden. ${ }^{117}$ Möglicherweise ist dies allerdings darauf zurückzuführen, daß ein Teil des Einflusses der pieds noirs auf den Arbeitsmarkt nach sechs Jahren durch die wirtschaftlichen Verflechtungen der departements bereits auf das ganze Land verteilt wurden und daher in dem räumlichen Vergleich nicht mehr nachweisbar sind.

Borjas, Freeman und Katz ${ }^{118}$ untersuchen den Einfluß des Außenhandels und der Einwanderung in die Vereinigten Staaten von Amerika in den achtziger Jahren auf die Lohndifferenz zwischen weniger und höher qualifizierten Arbeitskräften mit Hilfe von aus Daten der Zählung von 1980 und des Current Population Surveys gewonnenen Zeitreihen. In einem ersten Schritt schätzen sie für die einzelnen Jahre des Betrachtungszeitraums die Höhe und bildungsmäßige Zusammensetzung des (impliziten) Arbeitsangebots, das in den Außenhandelsströmen enthalten ist bzw. durch Einwanderer entsteht. Aus diesen Daten wurde dann der Prozentsatz berechnet, um den Außenhandel und Einwanderer das Verhältnis von hoch zu weniger qualifizierten Arbeitskräften verändert haben. Die Auswirkungen dieser Änderung auf die Lohndifferenz wurde schließlich unter Rückgriff auf die Substitutionselastitzität zwischen den verschiedenen Qualifikationsniveaus berechnet.

Die Autoren finden einen starken Einfluß des durch das Außenhandelsdefizit hervorgerufenen impliziten Anstiegs des Angebots an wenig qualifizierten Arbeitskräften auf die Lohndifferenz. Für die hier interessierende Fragestellung bedeutsamer ist, daß Borjas, Freeman und Katz im Gegensatz zu den oben dargestellten Ergebnissen der meisten Querschnittsstudien auch einen negativen Einfluß der Einwanderung auf die relative Einkommensposition der wenig Qualifizierten (Schulabbrecher) erhalten. ${ }^{119}$

Die Abweichung der Ergebnisse ist möglicherweise darauf zurückzuführen, daß durch die Vorgehensweise von Borjas, Freeman und Katz auch landesweit wirkende Effekte erfaßt werden. Im Gegensatz zu den oben erläuterten Studien werden damit nicht nur Unterschiede zwischen regionalen Arbeitsmärkten mit abweichenden Einwanderungsquoten erfaßt. Sondern es werden auch dann Wirkungen festgestellt, wenn aufgrund des oben beschriebenen Endogenitätsproblems oder von sehr schnell ablaufenden Anpassungsprozessen - beispielsweise durch ein verändertes Mobilitätsverhalten der Einheimischen oder durch Kapital-, und Güterbewegungen zwischen Regionen - keine regional begrenzten Auswirkungen der Einwanderung feststellbar sind. In diesen Fällen ist die in der Untersuchung von Borjas, Freeman und Katz gewählte Vorgehensweise vorzuziehen. Allerdings werden durch diese Untersuchungsmethode nachfrageseitige Wirkungen der Einwanderung ausgeblendet, so daß nur Aussagen über die Veränderung der Lohndifferenz zwischen verschiedenen Arbeitsmarktgruppen möglich sind, nicht aber Antworten auf die Frage gefunden werden können, ob die Arbeitsmarktsituation einzelner Gruppen durch die Einwanderer absolut verbessert oder verschlechtert wurde. Außerdem wird implizit angenommen, daß unqualifizierte Einwanderer genau gleiche Arbeitsmarktwirkungen haben wie zusätzliche unqualifizierte Einheimische. Sollten zugewanderte Arbeitskräfte keine perfekten Substitute für einheimische Erwerbstätige ohne Qualifikation sein, wird die Wirkung der Zuwanderung überschätzt. ${ }^{120}$ Weiterhin führt das oben erläuterte

117 Ein um einen Prozentpunkt höherer Anteil der pieds noirs an der Bevölkerung hatte einen Anstieg der Arbeitslosenquote unter den Einheimischen von maximal 0,2 Prozentpunkten und einen Rückgang ihres Lohnniveaus um höchstens 0,8 Prozent zur Folge. Vgl. Hunt (1992) S. $564 \mathrm{ff}$.

Vgl. Borjas/Freeman/Katz (1992).

119 Vgl. Borjas/Freeman/Katz (1992) S. 214f.

Vgl. Friedberg/Hunt (1995) S. 39. 
Zusammensetzungsproblem auch bei Längsschnittstudien zu einer Überschätzung der Arbeitsmarktwirkungen von Einwanderern.

Welches Fazit läßt sich aus den Ergebnissen der empirischen Studien zu der Arbeitsmarktwirkung von Einwanderern treffen? Die Ergebnisse der einzelnen Studien unterscheiden sich je nach dem, welche methodische Vorgehensweise gewählt wurde, welches Gebiet und welcher Zeitraum betrachtet wurde. Die meisten Studien finden allerdings nur geringe Arbeitmarkteffekte und die restlichen Arbeiten kommen etwa ebenso häufig zu positiven Wirkungen der Zuwanderung auf die Arbeitseinkommen und die Arbeitslosenquote der Einheimischen wie zu negativen. ${ }^{121}$ Insoferm kann von einem nur geringen Einfluß ausgegangen werden, der in seinem Vorzeichen unbestimmt ist. Allerdings ist zu beachten, da $ß$ die meisten Arbeiten methodischer Kritik ausgesetzt sind, die die Aussagekraft ihrer Ergebnisse schmälem. ${ }^{122}$ Weiterhin ist nochmals darauf hinzuweisen, daß alle Studien die Gesamtwirkung der Einwanderer messen, die sich aus dem Bevölkerungszuwachseffekt und dem Struktureffekt zusammensetzt. In dem Kontext dieser Arbeit interessiert aber nur der isolierte Struktureffekt. Nur insofern beide Effekte das gleiche Vorzeichen aufweisen, kann daher auch für den Struktureffekt allein von nur geringfügigen Wirkungen ausgegangen werden. Anderenfalls bestünde die Möglichkeit, daß sich Bevölkerungszuwachseffekt und Struktureffekt in ihren Wirkungen gegenseitig kompensieren, der Struktureffekt allein aber trotz der geringen Gesamtwirkung eine nicht unbedeutende Größenordnung annimmt. ${ }^{123}$

\subsubsection{Die Bedeutung der Arbeitsmarktwirkungen von Einwanderern für ein Einwanderungsgesetz}

Die Gesamtwirkung von Einwanderern auf Arbeitseinkommen und Arbeitslosigkeit der Einheimischen kann nach den obigen Ergebnissen als gering eingestuft werden. Allerdings bestehen stärkere Unterschiede der Wirkungen auf einzelne Arbeitsmarktgruppen. Verharrt man zunächst auf dem recht hohen Abstraktionsniveau der in 3.1.1 angeführten theoretischen Überlegungen, dann ist festzuhalten, daß die Zuwanderung wenig qualifizierter Arbeitskräfte zu Lohneinbußen und Arbeitsplatzverlusten der unqualifizierten Einheimischen führt, während gut ausgebildete Einheimische von einer derartigen Zuwanderung profitieren. In dem Fall einer v.a. aus schlecht oder gar nicht ausgebildeten Arbeitskräften bestehenden Zuwanderung wird daher mit den wenig Qualifizierten eine Gruppe von Einheimischen negativ betroffen, die

121 Simon (1994) S. 229 stellt mit Blick auf die Gruppen von Einheimischen, die besonders stark in Konkurrenz zu den Zuwanderem stehen, bezüglich negativer Lohnwirkungen fest, daß "the effect impresses all students of the subject as being small relative to 'common sense' expectations and relative to other sources of variation."

122 Borjas (1994) S. 1700 schreibt als Schlußfolgerung aus einem Literaturüberblick: "A fair appraisal of the literature thus suggests that we still do not fully understand how immigrants affect the employment opportunities of natives in local labor markets; nor do we understand the dynamic process through which natives respond to these supply shocks and reestablish labor market equilibrium."

123 Durch einen Vergleich von Studien zu den Arbeitsmarktwirkungen von Einwanderern mit denen überdurchschnittlich großer Kohorten von Einheimischen kommt Friedberg (1995) zu dem Resultat, daß der negative Einfluß der Einwanderer geringer ist als derjenige von zusätzlichen Einheimischen. Dieses Ergebnis spricht dafür, daß der isolierte Struktureffekt für die USA eher positiv als negativ ist. 
ohnehin schon unter besonders hoher Arbeitslosigkeit leidet. Im Rahmen eines Einwanderungsgesetzes sollte daher auf ein ausgeglichenes Verhältnis von wenig und hoch qualifizierten Zuwanderern geachtet werden. So lassen sich Friktionskosten bei den heimischen Arbeitnehmern senken.

Bei einer weiteren Disaggregierung ist festzustellen, daß der deutsche Arbeitsmarkt allgemein durch strukturelle Arbeitslosigkeit gekennzeichnet ist. Die Struktur der Arbeitskräftenachfrage durch die Unternehmen und den Staat entspricht z.B. hinsichtlich der Merkmale Qualifikation und Lokalität nicht immer dem Arbeitskräfteangebot. Ungenügende berufliche und regionale Mobilität ermöglichen das Nebeneinander von Arbeitskräftemangel auf einem und Arbeitskräfteüberschuß auf einem anderen Teilarbeitsmarkt. Im Rahmen eines Einwanderungsgesetzes ist daher dafür Sorge zu tragen, daß die Zuwanderer ein günstiges Arbeitsmarktprofil besitzen und nicht in Konkurrenz zu Einheimischen auf einem bereits durch strukturelle Arbeitslosigkeit gekennzeichneten Arbeitsmarktsegment treten.

Zwei Kriterien für die Selektion der Zuwanderer lassen sich daraus ableiten. Erstens sollte der Einwanderer auf einem Arbeitsmarktsegment tätig werden, das nicht durch strukturelle Arbeitslosigkeit gekennzeichnet ist. Hierfür ist durch ein Einwanderungsgesetzes ein geeignetes Auswahlverfahren bereitzustellen. Zweitens ist eine hohe Mobilität der Einwanderer sicherzustellen. Das kann zum Beispiel durch eine Selektion nach dem Stand der Allgemeinbildung erfolgen, da ein hohes Allgemeinbildungsniveau die berufliche Mobilität für den Fall erleichtert, da $\beta$ in dem gegenwärtigen Beruf eines Einwanderers später ein Arbeitsangebotsüberschuß existiert.

Bei der theoretischen Analyse der Arbeitsmarktwirkungen von Zuwanderern wurde neben der Frage der Substitutionalität oder Komplementarität der Zuwanderer in bezug auf einheimische Arbeitskräfte die Bedeutung der Nachfragewirkungen der Zuwanderer hervorgehoben. Je größer der Kapitalzuwachs aufgrund der Zuwanderung ist, desto höher werden ceteris paribus Arbeitseinkommen und Beschäftigung der Einheimischen liegen. Um diese beschäftigungsinduzierende Wirkung zu erhöhen, ist sicherzustellen, daß die internationale Kapitalmobilität möglichst hoch ist. Dann wird die zuwanderungsbedingt gestiegene Grenzproduktivität des Kapitals zu Kapitalzuflüssen nach Deutschland führen. Da vollständige Kapitalmobilität kaum zu erreichen sein dürfte, ist vom Standpunkt der Arbeitsmarktwirkungen in einem Einwanderungsgesetz zusätzlich ein Selektionsmechanismus vorzusehen, der die Auswahl von Einwanderern fördert, die Vermögen nach Deutschland transferieren und Investitionen vornehmen.

\subsection{Der Einfluß der Einwanderer auf die Kapitaleinkommen der Ein- heimischen}

\subsubsection{Theoretische Analyse der Wirkungen auf die Kapitaleinkommen}

Neben den Auswirkungen, die Einwanderung auf die Arbeitseinkommen und die Arbeitslosigkeit von Einheimischen hat, können den Einheimischen auch durch den Einfluß der Zuwanderung auf die Kapitaleinkommen Kosten oder Nutzen entstehen. 
Abb. D.5: Einfluß der Einwanderer auf das Kapitaleinkommen im Fall ohne Änderung des Kapital-Arbeit-Verhältnisses

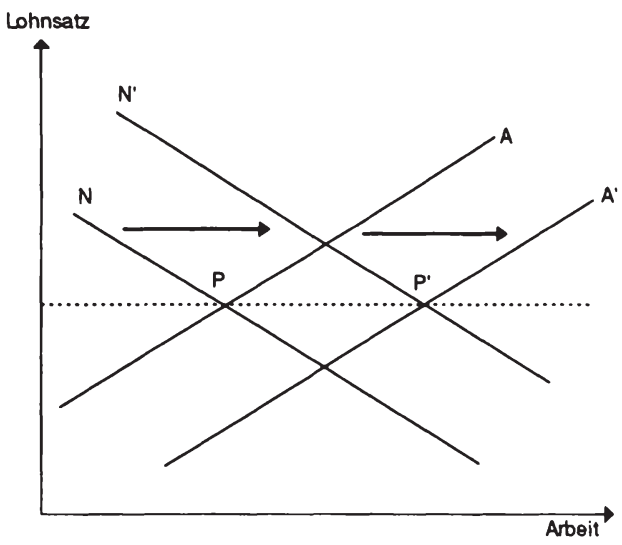

Die theoretischen Überlegungen zu den Wirkungen auf die Kapitaleinkommen der Einheimischen bauen auf dem statischen Zwei-Länder-Modell auf, das schon für die Untersuchung der Arbeitsmarkteffekte im vorangegangenen Abschnitt verwendet wurde. Ausgangspunkt der Überlegung ist die Festlegung des Referenzniveaus in Höhe des durchschnittlich pro Kopf der einheimischen Erwerbstätigen vorhandenen Kapitals. Es kann angenommen werden, daß dieses Pro-Kopf-Kapital auch bei einer Ausweitung des Kinderlastenausgleichs zur Verfügung stehen würde. ${ }^{124}$ Es bildet daher den Nullpunkt für den Vergleich mit der Erhöhung der Bevölkerungszahl durch verstärkte Einwanderung.

Für den Spezialfall, in dem die Einwanderer gerade so viel Kapital mit in ihr neues Heimatland bringen, daß ihre Arbeitsplätze davon durchschnittlich in der gleichen Höhe mit Kapital ausgestattet werden können, wie die Arbeitsplätze der Einheimischen, das Einsatzverhältnis der beiden Produktionsfaktoren Arbeit und Kapital also unverändert bleibt, ergeben sich daher keine relevanten Wirkungen auf die Kapitaleinkommen der Einheimischen (vgl. Abb. D.5). Der gestiegene Kapitalbestand führt zu einer Rechtsverschiebung der durch das Wertgrenzprodukt der Arbeit angegebenen Arbeitsnachfragefunktion, da bei einer gleichhohen Arbeitsmenge jetzt mehr produktives Kapital pro Arbeitskraft eingesetzt werden kann und die Grenzproduktivität der Arbeit dadurch steigt. Gleichzeitig hat sich durch die Einwanderung aber auch die Arbeitsangebotsfunktion nach rechts verschoben. Weist die Produktionsfunktion konstante Skalenerträge auf, hat sich weder der Lohnsatz noch die Rendite des Kapitals durch die Einwanderung verändert. ${ }^{125}$

124 Hier wird angenommen, daß die durch den Kinderlastenausgleich hervorgerufene größere Kinderzahl zu vermehrten Ersparnissen in einem solchen Umfang führt, daß sich das Kapital-Arbeits-Verhältnis in der Volkswirtschaft nicht verändert. Vgl. auch Kap. 1 in Teil C dieser Arbeit.

125 Vgl. Grubel (1994) S. 79. Die Annahme konstanter Skalenerträge ist nur für den Vergleich mit einer Situation ohne Bevölkerungserhöhung notwendig. Im Vergleich zu den Ergebnissen bei Erhöhung des Kinderlastenausgleichs ergeben sich in dem hier betrachteten Spezialfall auch dann keine abweichenden Ergebnisse, wenn abnehmende oder zunehmende Skalenerträge vorliegen, da in beiden Fällen die Arbeitsnachfragekurve und die Arbeitsangebotskurve in gleicher Weise verschoben werden. 
Welche Wirkungen auf die Kapitaleinkommen der Einheimischen sind für den Fall zu erwarten, daß die Einwanderer zu wenig Kapital in ihr Aufnahmeland transferieren, um die durchschnittliche Ausstattung der dortigen Arbeitsplätze mit Kapital konstant zu halten? Zur Veranschaulichung soll der Grenzfall betrachtet werden, in dem die Einwanderer gar kein Kapital in das Einwanderungsland transferieren. In diesem Fall bleibt die Nachfragekurve nach Arbeit unverändert, während sich die Angebotskurve nach rechts verschiebt (vgl. die Bewegung von $A$ nach $A^{\prime}$ bei unveränderter Nachfragekurve $N$ in Abb. D.2). Als Ergebnis ergibt sich ein Anstieg der Kapitaleinkommen in Höhe der Fläche $t x z r$ in Abb. D.2. Da sich der Kapitalbestand nicht verändert hat, ist die Faktorentlohnung pro Kapitaleinheit gestiegen. ${ }^{126}$

Allerdings ist infolge der höheren Faktorentlohnung mit einem Zufluß ausländischen Kapitals zu rechnen, der diesen Faktorpreisanstieg im Grenzfall international völlig mobilen Kapitals vollständig zunichte macht. Kurz gesagt macht es keinen Unterschied, ob die Einwanderer das Kapital zur Ausstattung ihrer Arbeitsplätze selbst mitbringen oder ob dieses Kapital infolge der gestiegenen Rendite durch andere Ausländer in das Einwanderungsland transferiert wird. Vollständige internationale Mobilität des Kapitals ist in der Realität allerdings kaum gegeben. Denn bei überdurchschnittlich vermögenden Immigranten kann angenommen werden, daß ein hoher Prozentsatz von ihnen Selbständige sind, die Direktinvestitionen in Form von Firmengründungen im neuen Heimatland vornehmen werden. Solche Firmengründungen erfordern aber in den überwiegenden Fällen eine Mitarbeit und Kontrolle durch den Eigentümer vor Ort, so daß sie für im Ausland verbleibende Kapitalanleger schon deshalb nicht in Betracht kommen. ${ }^{127}$ Aufgrund eingeschränkter internationaler Kapitalmobilität sind daher zwar für den Fall, da $ß$ die Einwanderer mittellos in das Aufnahmeland einreisen, positive Effekte auf das Kapitaleinkommen der Einheimischen zu erwarten, aber diese werden recht gering ausfallen.

\subsubsection{Empirische Größenordnung der Wirkungen auf die Kapitalein- kommen}

Hinweise auf die Höhe der Kapitalimporte von Einwanderern sind für die Bundesrepublik Deutschland kaum vorhanden. Was das Ausland betrifft, führt Simon einige Studien an, nach denen der durchschnittliche Kapitalimport nach Kanada und Australien 1984 mit $\$ 30.000$ bis $\$ 50.000$ in etwa dem Kapital entspricht, mit dem ein durchschnittlicher Arbeitsplatz in den USA ausgestattet war. ${ }^{128}$ Allerdings schwankt die Höhe der Kapitalimporte stark in Abhängigkeit von der Einwanderungsgruppe. Während über die Familienzusammenführung Eingewan-

126 Diese Ergebnis ändert sich nicht, wenn man Komplementarität zwischen zugewanderten und einheimischen Arbeitskräften zuläßt, indem man die Annahme homogener Arbeit aufgibt. Auf dem Arbeitsmarktsegment mit einer substitutionalen Beziehung steigen die Kapitaleinkommen wegen gesunkener Löhne, auf dem Arbeitsmarktsegment mit einer komplementären Beziehung zwischen einheimischen und zugewanderten Arbeitskräften aufgrund der gestiegenen Produktivität der einheimischen Erwerbstätigen (vgl. Abb. D.4).

127 Auch bei reinen Portfolioinvestitionen wird vollständige internationale Kapitalmobilität durch Informationsdefizite, das Wechselkursrisiko (bzw. die Absicherungskosten dagegen) und das politische Risiko (Enteignungen, Kapitalverkehrskontrollen, etc.) verhindert. Vgl. die Diskussion zur Mackenroth-These in Teil B, Abschnitt 3.2.2.

Vgl. Simon (1989) S. 146. 
derte in Kanada 1983/84 durchschnittlich etwa $\$ 18.000$ importierten, lag der Mittelwert für 'business immigrants' (Unternehmer und Selbständige) bei fast $\$ 400.000$, eine Größenordnung, die auch in Australien erreicht wurde. In einer statistischen Auswertung von Daten zwischen 1900 und 1975 kommt Baker für Australien zu dem Ergebnis, daß Veränderungen der Nettowanderungen gleichgerichtete Veränderungen der Pro-Kopf-Investitionen verursachen. ${ }^{129}$ Die Änderung der Investitionen reicht nach seinen Berechnungen aus, um den Pro-Kopf-Bestand an privatem Kapital außerhalb des Wohnungssektors auch bei verstärkter Einwanderung mindestens konstant zu halten. Für die Einwanderungsländer Australien und Kanada, die beide nach ökonomischen Kriterien eine gezielte Selektion der Immigranten vornehmen, kann aufgrund dieser Daten davon ausgegangen werden, daß die Zuwanderer das für ihre Arbeitsplätze benötigte Kapital selbst mitbringen und deshalb keinen Einfluß auf die Kapitaleinkommen der Einheimischen ausüben.

Für Deutschland sah die Situation in der Vergangenheit grundlegend anders aus. Der größte Teil der Einwanderer waren Gastarbeiter, die ursprünglich oft gerade mit dem Ziel nach Deutschland kamen, sich mit dem hier verdienten Geld in ihrem Heimatland selbständig zu machen. Einen nennenswerten Kapitalimport nach Deutschland durch die Gastarbeiter wird es wohl nicht gegeben haben. Im Gegenteil transferieren Gastarbeiter als zumindest der ursprünglichen Absicht nach temporäre Migranten im Gegensatz zu den permanenten Einwanderern oft einen Teil ihres Einkommens zur Unterstützung ihrer Familien in ihr Heimatland. Mit der Anfang der siebziger Jahre einsetzenden Familienzusammenführung entfiel der Grund für diese Zahlungen zwar mehr und mehr, aber zu höheren Kapitalimporten aus den Heimatländern wird es dennoch kaum gekommen sein, wie die Zahlen für die unter die Rubrik Familienzusammenführung fallenden kanadischen Immigranten nahelegen. Für Deutschland kann daher für die Vergangenheit von einem positiven Einfluß auf die Kapitaleinkommen ausgegangen werden, der in der Größenordnung allerdings durch die infolge der liberalen Kapitalverkehrsbestimmungen Deutschlands hohe internationale Kapitalmobilität reduziert wird.

\subsubsection{Bedeutung der Wirkungen auf die Kapitaleinkommen für ein Einwanderungsgesetz}

Aufgrund der internationalen Kapitalmobilität ist die Bedeutung des Einflusses der Einwanderer auf die Kapitaleinkommen der Einheimischen als gering anzusehen. Allenfalls ergeben sich geringe positive Effekte für die einheimischen Kapitaleigner, wenn durch ein Einwanderungsgesetz die Zuwanderung auf Gastarbeiter beschränkt wird, die nach dem Rotationsprinzip eine kurze durchschnittliche Verweildauer in Deutschland haben. In diesem Fall werden die Zuwanderer kaum Kapital nach Deutschland importieren, da sich Investitionen in Deutschland bei der kurzen Verweildauer nicht lohnen. Im Gegenteil ist durch Überweisungen in die Heimatländer mit Kapitalabflüssen zu rechnen.

Wird dagegen durch ein Einwanderungsgesetz eine Selektion zugunsten von Selbständigen vorgenommen, die in Deutschland Investitionen tätigen wollen, dann ist mit - wiederum geringfügigen - negativen Auswirkungen auf die einheimischen Kapitaleinkommen zu rechnen, da die Faktorentlohnung für das Kapital sinkt. 
Ist das Einwanderungsgesetz so konstruiert, daß das durchschnittlich von einem einwandernden Erwerbstätigen mitgebrachte Kapital der durchschnittlichen Kapitalausstattung eines Arbeitsplatzes in Deutschland entspricht, entstehen im Vergleich mit der Situation bei Ausweitung des Kinderlastenausgleichs keine Auswirkungen auf die Kapitaleinkommen der Einheimischen.

\subsection{Der Einfluß von Einwanderern auf die öffentlichen Haushalte}

Für die Wirkung der Einwanderer auf die öffentlichen Haushalte des Gastlandes spielt die von Ökonomen als Assimilationszeitraum bezeichnete Phase bis zur Angleichung der Einkommens- und Erwerbssituation der Einwanderer an diejenige der Einheimischen eine bedeutende Rolle. Erstens ist die Dauer bis zur ökonomischen Integration der Einwanderer von entscheidender Bedeutung für die Höhe der durch den Staat zu tragenden Aufwendungen für die Zuwanderer wie Integrationshilfen, Sozialhilfe, Arbeitslosengeld, etc. Zweitens bestimmt die Position der Zuwanderer auf dem Arbeitsmarkt aber auch die Höhe ihrer Beiträge zur Finanzierung dieser Kosten und der allgemeinen Staatsausgaben. Je höher das Durchschnittseinkommen und die Erwerbsbeteiligung der Einwanderer und je niedriger ihre Arbeitslosigkeitsrate, desto höher sind ihre Steuern und Sozialabgaben. Die direkte Nettowirkung der Einwanderer auf die öffentlichen Haushalte hängt also entscheidend von ihrer Integration in den Arbeitsmarkt ab. Daher wird im folgenden zunächst auf die Assimilation der Einwanderer in den Arbeitsmarkt eingegangen (3.2.1), ehe ihr Einfluß auf die Haushalte der Gebietskörperschaften (3.2.2) und der Sozialversicherungen (3.2.3) untersucht wird.

\subsubsection{Die Assimilation der Einwanderer in den Arbeitsmarkt des Gast- landes}

Wie schon bei der Behandlung der Auswirkungen der Einwanderer auf die Arbeitsmarktsituation der Einheimischen soll hier auch die auf die Einwanderung folgende Entwicklung der Arbeitsmarktsituation der Zuwanderer selbst zunächst theoretisch beleuchtet werden, ehe auf die Ergebnisse empirischer Studien und die Bedeutung für ein Einwanderungsgesetz eingegangen wird.

\subsubsection{Theoretische Analyse der Assimilation}

Die theoretische Basis nahezu aller Studien zur Assimilation der Einwanderer bildet ein zuerst von Chiswick entwickelter Humankapitalansatz. ${ }^{130}$ Danach verfügen die Einwanderer auch bei gleicher formaler Ausbildung im Zeitpunkt der Wanderung über einen geringeren

130 Vgl. Chiswick (1978). Eine auf kontrakttheoretische Überlegungen aufbauende Erklärung des Assimilationsvorgangs liefert Schmidt (1993). 
Humankapitalbestand als die Einheimischen des Gastlandes, da ein Teil des Humankapitals an die besonderen Bedingungen des jeweiligen Landes geknüpft ist. Das länderspezifische Humankapital für das Gastland muß von den eingewanderten Arbeitskräften erst im Laufe der Zeit erworben werden, während die einheimischen Arbeitskräfte dieses Humankapital seit ihrer Geburt ansammeln konnten. Das länderspezifische Humankapital, zu dem die Sprachfertigkeit ebenso gehört wie die Kenntnis von Institutionen und Umgangsformen des Landes, erhöht die Grenzproduktivität der Arbeitskräfte, die es besitzen. Auf einem polypolistischen Arbeitsmarkt, auf dem die Entlohnung der Arbeitskräfte entsprechend ihrem Wertgrenzprodukt erfolgt, werden die einheimischen Arbeitskräfte daher einen höheren Lohnsatz erzielen als kürzlich Eingewanderte. ${ }^{131}$ In dem Maße, in dem die Einwanderer im Zeitablauf (länderspezifisches) Humankapital bilden, wird diesem Ansatz zur Folge ihr Lohnsatz steigen und sich demjenigen der Einheimischen annähern. Diese Angleichungstendenz wird noch verstärkt, wenn Einwanderer nach dem Immigrationszeitpunkt im Vergleich zu Einheimischen höhere Investitionen in nicht-länderspezifisches Humankapital wie den Erwerb formaler Qualifikationen und on-thejob training, in geographische Mobilität innerhalb des Landes und in die Arbeitsplatzsuche tätigen. ${ }^{132}$ Für die Einwanderer besteht ein höherer Anreiz zur Akkumulation solchen Humankapitals als für die Einheimischen, weil sie niedrigere Opportunitätskosten in Form entgangener Einkommen haben. Für temporäre Migranten ist dieser Anreiz allerdings geringer als für dauerhafte Einwanderer, weil für erstere die Erträge dieser Investitionen über einen kürzeren Zeitraum anfallen. Temporäre Migranten werden daher zwar anfänglich einen geringeren Einkommensrückstand gegenüber Einheimischen haben als permanente Migranten, weil sie weniger Zeit auf Humankapitalinvestitionen verwenden und damit mehr arbeiten. Dafür verzeichnet man bei ihnen allerdings auch geringere Einkommenswachstumsraten, so daß der Assimilationsproze $ß$ langsamer verläuft. ${ }^{133}$

Oft wird dieser Humankapitalansatz mit einer Selbstselektions-Hypothese bezüglich der Einwanderer verbunden. Ursprünglich wurde dabei unterstellt, daß nicht ein zufällig zusammengesetzter Teil der Bevölkerung des Herkunftslandes auswandert, sondern v.a. überdurchschnittlich ambitionierte, fähige und hart arbeitende Personen. Als Begründung wurde angeführt, daß sie eher als der Rest der Bevölkerung in der Lage seien, die Schwierigkeiten, die sich einer internationalen Migration entgegenstellen, zu überwinden. Ist das Verhältnis der Einkommen von sehr produktiven zu denjenigen von weniger produktiven Arbeitskräften in beiden Ländern gleich, dann haben produktivere Personen außerdem einen größeren absoluten Einkommensvorteil von einer Wanderung in ein Hochlohnland. Für sie wird sich daher die Wanderung auch dann noch lohnen, wenn die Fixkosten der Migration die Wanderung weniger produktiver Personen schon verhindern. ${ }^{134}$

131 Diskriminierung - verstanden als geringere Entlohnung von Einwanderern gegenüber Einheimischen mit gleicher Grenzproduktivität - spielt in Deutschland nach den Ergebnissen von Velling (1995) kaum eine Rolle. Es kann daher davon ausgegangen werden, daß Lohnsatzunterschiede tatsächlich Produktivitätsunterschiede widerspiegeln.

132 Vgl. Borjas (1994) S. 1684. Der Einfluß unterschiedlicher Investitionen in das nicht-länderspezifische Humankapital auf die Assimilationsrate ist bisher kaum untersucht worden. $\mathrm{Zu}$ den Auswirkungen der Fertigkeiten in der Landessprache auf die Einkommenshöhe und -entwicklung vgl. z.B. Chiswick (1991) und Chiswick/Miller (1992) sowie zu einer Kritik an der Konzeption dieser Studien Borjas (1994) S. $1684 f$.

133 Vgl. Dustmann (1993) S. 154ff.

134 Vgl. Chiswick (1994) S. 97ff. Zusätzlich sind die Fixkosten der Migration für besonders produktive Personen möglicherweise geringer als für den Durchschnitt der Bevölkerung, weil sie die Wanderung effizienter organisieren können. 
Der Selbstselektionsproze $ß$ kann allerdings auch ein gegenteiliges Ergebnis haben. Ausgangspunkt dieser Überlegung ist der in 2.1.2 erläuterte mikroökonomische Kalkül, nach dem die Individuen wandern, die aus einer internationalen Migration einen Vorteil ziehen. Personengruppen, die einen besonders hohen Nutzen aus der Wanderung ziehen, werden daher unter den Migranten überrepräsentiert sein. Um genauere Aussagen über den Selektionsprozeß machen zu können, nimmt Borjas an, daß (i) das Nutzenniveau ausschließlich durch die Einkommenshöhe festgelegt wird, (ii) das durch formale Ausbildung erworbene Humankapital weitgehend international übertragbar ist und (iii) diese Qualifikationen sowohl im Herkunftsals auch im Aufnahmeland die Höhe des Einkommens bestimmen. ${ }^{135}$ Unter diesen Voraussetzungen werden qualifizierte Arbeitskräfte in der Gruppe der Migranten überproportional vertreten sein, wenn die Einkommensunterschiede zwischen ihnen und weniger qualifizierten Arbeitskräften im Herkunftsland geringer sind als im Gastland. Ist allerdings umgekehrt die Entlohnung für formale Ausbildung im Herkunftsland größer als im Gastland, ist mit einem überdurchschnittlichen Anteil von weniger produktiven Arbeitskräften unter den wandernden Personen zu rechnen. ${ }^{136}$

Das gleiche Ergebnis kann sich einstellen, wenn man neben der Einkommenshöhe auch die Wahrscheinlichkeit von Arbeitslosigkeit mit in den Nutzenmaximierungskalkül einfließen läßt. ${ }^{137}$ Besteht im Aufnahmeland ein Nachfrageüberschuß nach weniger qualifizierten Arbeitskräften, während im Emigrationsland in diesem Arbeitsmarktsegment eine hohe Arbeitslosigkeit herrscht und sind gleichzeitig die Einstellungschancen für qualifizierte Arbeitskräfte in beiden Ländern etwa gleich gut, dann haben weniger qualifizierte Arbeitskräfte ceteris paribus einen höheren Migrationsanreiz als Qualifizierte.

$\mathrm{Da}$ die beschriebenen Selbstselektionsmechanismen von der EinkommensmaximierungsHypothese ausgehen, betreffen sie v.a. ökonomisch motivierte Migranten. Für Flüchtlinge steht daher eine weniger ausgeprägte positive oder auch negative Selbstselektion zu erwarten. ${ }^{138}$

\subsubsection{Empirische Analyse der Assimilation}

Die meisten empirischen Arbeiten zur Assimilation der Einwanderer in den Arbeitsmarkt des Gastlandes sind Querschnittsstudien. In ihnen werden mit Hilfe von Regressionsanalysen die Einkommen von einheimischen und eingewanderten Arbeitskräften durch ihre formale Ausbildung, ihre Berufserfahrung und eine Reihe anderer unabhängiger Variablen erklärt, zu denen für die Einwanderer auch die Zahl der Jahre im Gastland gehört. ${ }^{139}$ Durch diese Vorgehens-

135 Vgl. Borjas (1989) S. 461ff. Borjas weist darauf hin, daß ähnliche Selektionseffekte auch bezüglich unbeobachtbarer arbeitsmarktrelevanter Fähigkeiten (beispielsweise besondere Einsatzbereitschaft und andere Persönlichkeitsmerkmale sowie außergewöhnliche Fertigkeiten) auftreten, die neben beobachtbaren Größen wie der Berufserfahrung und der formalen Ausbildung die Höhe des Einkommens entscheidend mitbestimmen.

136 Eine formalisierte Darstellung des hier beschriebenen Selbstselektionsprozesses findet sich bei Borjas (1994) S. 1687ff.

137 Vgl. Dustmann (1993) S. 156ff.

138 Vgl. Chiswick (1994) S. 101.

139 Eine genauere Darstellung einer derartigen Regression findet sich in Abschnitt 3.2.1.2 des Teils C dieser Arbeit. 
Abb. D.6: Die Einkommensentwicklung von Einheimischen und Einwanderern gleicher Ausbildung und Berufserfahrung im Zeitablauf

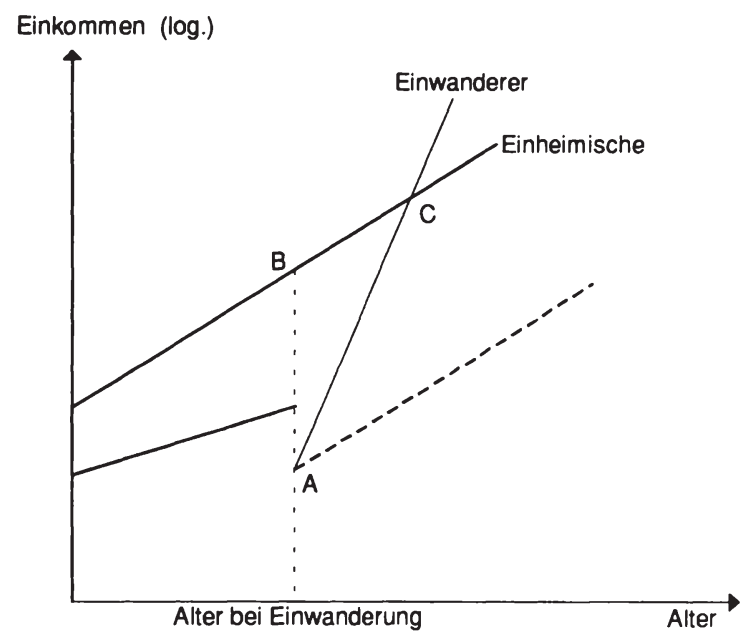

Quelle: Pischke (1992) S. 34 Abb. 1

weise läßt sich für beide Gruppen getrennt die Einkommensentwicklung im Laufe des Erwerbslebens nachzeichnen. In Abbildung D.6 wird ein derartiger Einkommensverlauf für Immigranten und Einheimische gleichen Qualifikationsniveaus exemplarisch dargestellt. ${ }^{140}$ Aufgrund der theoretischen Überlegungen steht zu vermuten, daß die Einkommen der Einwanderer zum Zeitpunkt der Einreise unter denjenigen der Einheimischen liegen, das absolute Glied der Regressionsgleichung also für Einwanderer kleiner ist als für Einheimische. Der nachfolgende Einkommenszuwachs liegt dann aber bei erfolgreicher Assimilation über dem der Einheimischen, was in einem größeren Anstieg der Einkommenskurve der Einwanderer zum Ausdruck kommt. Die Differenz zwischen den Steigungen der beiden Kurven, also die Differenz der jährlichen Einkommenswachstumsraten von Einheimischen und Eingewanderten, wird als Assimilationsrate bezeichnet.

Bei Gültigkeit der These positiver Selbstselektion der Einwanderer liegen die Einkommen der Einwanderer schließlich sogar über denen der Einheimischen mit gleicher Ausbildung und Berufserfahrung. Die empirischen Arbeiten konzentrieren sich daher zum einen auf die Frage, ob die absoluten Glieder der Regressionsgleichungen für Einheimische und Einwanderer voneinander abweichen und zum anderen auf den Koeffizienten der Variablen "Zahl der Jahre seit der Einwanderung", der die Differenz der Steigungen der beiden Einkommensverläufe angibt. ${ }^{141}$

140 Der Einfachheit halber wurden in der Abbildung lineare Zusammenhänge unterstellt. Viele Studien berücksichtigen aber die im höheren Alter abnehmenden Einkommenszuwächse.

141 Damit wird das Wachstum der Einkommen der Einheimischen als Referenzgröße für die Messung der Assimilation gesetzt. Vgl. Pischke (1992) S. 4. 
Abb. D.7: Der Kohorteneffekt bei sinkender Qualität der Einwanderer

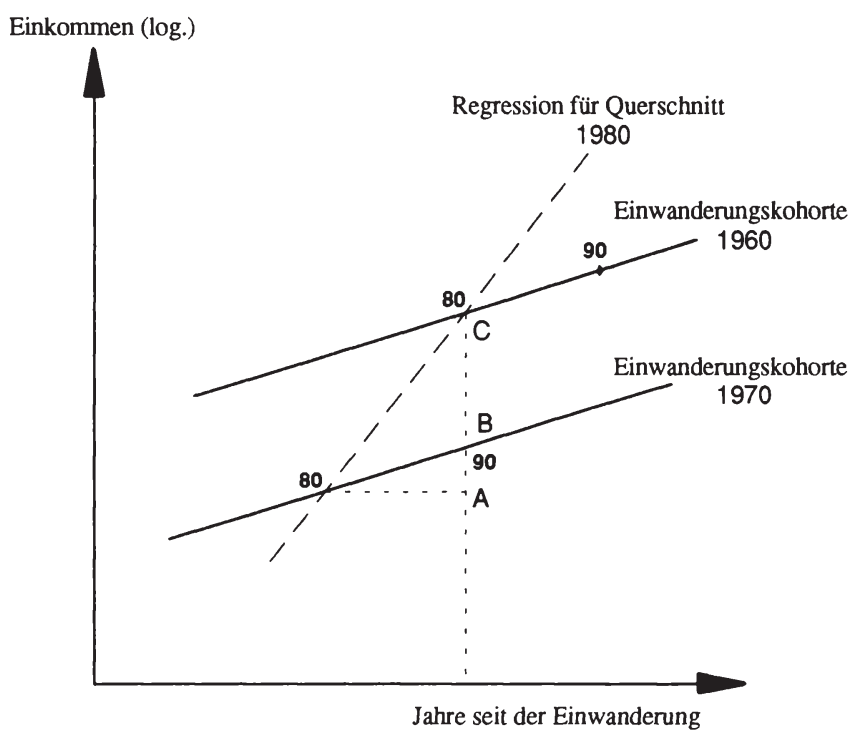

Dieses Vorgehen stößt allerdings auf mehrere Kritikpunkte. So wird eine sinkende "Qualität" der Einwanderer, d.h. ein Rückgang der Produktivität aufeinanderfolgender Einwanderungskohorten, zu einer Überzeichnung der Assimilationsrate führen. In diesem Fall ist die den Querschnittsanalysen implizite Unterstellung, daß die heute Einwandernden in zehn Jahren das gleiche Einkommen haben werden, wie heute die vor zehn Jahren Eingewanderten, nicht zutreffend. ${ }^{142}$ In Abb. D.7 ist dieser als Kohorteneffekt bezeichnete Zusammenhang graphisch dargestellt. Die tatsächlichen Einkommensverläufe von 1960 und 1970 eingewanderten Personen sind durch die durchgezogenen Linien dargestellt. Durch Querschnittsanalysen wird aufgrund des Kohorteneffekts allerdings die weit größere Steigung der gestrichelten Linie ausgewiesen. Wenn man mindestens zwei zu unterschiedlichen Zeiten erhobene Querschnittsdatenreihen zur Verfügung hat, lassen sich diese Kohorteneffekte ausschließen, indem man Dummy-Variablen für die Kohorten einfügt. ${ }^{143}$

Eine weitere Schwierigkeit entsteht, wenn der Einkommensunterschied zwischen Einheimischen und Immigranten von dem Alter der Migranten zum Zeitpunkt der Einwanderung abhängt. Da jeder Migrant irgendwann aus dem Arbeitsleben ausscheidet, werden die noch berufstätigen Migranten, die bereits seit langem in ihrem Gastland sind, sehr jung eingewandert sein. Sinkt das Einkommen der Einwanderer mit steigendem Alter bei der Einwanderung, dann wird der Assimilationseffekt durch die zu hoch ausgewiesenen Einkommen der v.a. in jungen

142 Diese Kritik an den Querschnittsstudien wurde v.a. von Borjas vorgetragen. Vgl. Borjas (1985).

143 Vgl. Pischke (1992) S. 5f. Auch unterschiedliche Assimilationsraten für verschiedene Kohorten können prinzipiell berücksichtigt werden. 
Jahren eingewanderten älteren Einwanderungskohorten überzeichnet. ${ }^{144}$ Ein gemessen an den Einheimischen niedrigeres Einkommen älterer Einwanderer kann mit dem niedrigeren Bestand an aufnahmelandspezifischem Humankapital und mit größeren Anpassungsschwierigkeiten im Alter begründet werden. Besonders bei Immigranten, die bereits im Kindesalter eingewandert sind, kann dagegen von einem den Einheimischen recht ähnlichen Bestand an Humankapital und daher auch einem ähnlichen Einkommensverlauf ausgegangen werden. In neueren Studien wird deshalb das Alter bei der Einwanderung als weitere unabhängige Variable mit in die Regressionsgleichung aufgenommen.

Schließlich bereitet noch der potentielle Selektionseffekt durch Rückwanderungen Probleme. Kehren hauptsächlich solche Migranten in ihre Heimatländer zurück, die aufgrund unterdurchschnittlicher Einkommen im Gastland ihre Erwartungen enttäuscht sehen, dann weisen die älteren Einwanderungskohorten eine höhere qualitative Zusammensetzung auf als die erst kürzlich eingewanderten. Der Assimilationseffekt wird dann überzeichnet, weil ein Teil des gemittelten Einkommenszuwachses auf das Ausscheiden der unterdurchschnittlich verdienenden Rückkehrer aus dem Datensatz zurückzuführen ist. Allerdings ist auch genau der gegenteilige Effekt denkbar. Unterstellt man nämlich, daß das Motiv der Wanderung die Bildung einer bestimmten Sparsumme ist - eine Annahme, die gerade für die Gastarbeiter in Deutschland häufig zutrifft -, dann kehren die Einwanderer mit überdurchschnittlichen Einkommen früher in ihr Heimatland zurück als weniger erfolgreiche Landsleute. ${ }^{145}$ In diesem Fall wird der Assimilationseffekt aufgrund der selektiven Rückwanderung nicht über- sondern unterzeichnet. ${ }^{146}$ Dieses Problem kann durch die nachfolgend beschriebenen Panelstudien umgangen werden.

Zuvor sollen aber die Ergebnisse der Querschnittsanalysen kurz referiert werden. Die meisten Untersuchungen wurden für die USA durchgeführt und finden eine anfängliche Einkommensdifferenz zu Lasten der Einwanderer, die nach ein bis zwei Jahrzehnten durch höhere Einkommenszuwachsraten der Einwanderer umgekehrt wird. So findet etwa Chiswick in seiner für viele weitere Untersuchungen grundlegenden Studie positive Assimilationsraten von anfänglich 1,5 Prozent pro Jahr, die bei einem anfänglichen Einkommensunterschied von etwa 16 Prozent nach 13 Jahren erstmals zu einem höheren Einkommen der Immigranten führen. ${ }^{147}$ Inwieweit diese Ergebnisse auf tatsächliche Assimilation oder auf MeBfehler infolge der oben beschriebenen ökonometrischen Probleme zurückzuführen sind, ist in der Literatur strittig. ${ }^{148}$ Borjas $^{149}$ findet eine sinkende Qualität jüngerer Einwanderungskohorten, Fried-

144 Vgl. Borjas (1994) S. 1678ff.

145 Vgl. Pischke (1992) S. 9.

146 Die Ergebnisse einer Untersuchung von Velling sprechen für eine positive Selektionswirkung der Rückwanderungen, da er in Deutschland eine mit steigendem Haushaltseinkommen sinkende Rückkehrneigung findet (vgl. Velling (1993) S. 18). Borjas sieht aufgrund empirischer Ergebnisse für dic USA seine Hypothese bestätigt, daß die ursprüngliche Selektionswirkung durch Rückwanderungen verstärkt wird (vgl. Borjas (1994) S. 1691f.).

147 Vgl. Chiswick (1978) S. 906.

148 Vgl. Schmidt (1993) S. 5.

149 Vgl. Borjas (1985, 1987 und 1994). Die sinkende Qualität der späteren Einwanderungskohorten in den USA und die daraus resultierenden niedrigeren Assimilationsraten sind nach Borjas (1994) S. $1685 \mathrm{zu}$ über 90\% auf eine Änderung der Anteile verschiedener Herkunftsnationen zurückzuführen. Die 1965 vorgenommene Änderung des Immigration and Nationality Acts hob das bis dahin gültige System der Nationalitäten-Quoten auf, das die Einwanderung von Europäern stark begünstigte. Seit dieser Änderung hat der Anteil der Nationen mit geringerem durchschnittlichen Ausbildungsstand und 
berg ${ }^{150}$ erhält ebenso wie Borjas ${ }^{151}$ eine negative Korrelation zwischen dem Alter bei der Einwanderung und dem Einkommen, beides Faktoren, die für eine Überzeichnung der in den Querschnittsanalysen gefundenen Assimilationsraten sprechen. Auch selektive Rückwanderung hat nach den Ergebnissen von Borjas einen zu hohen Ausweis der Angleichungsrate zur Folge. ${ }^{152}$ Im allgemeinen wird jedoch nicht bestritten, daß für die USA positive Assimilationsraten vorliegen, also mit zunehmendem Zeitraum seit dem Einwanderungszeitpunkt zumindest eine Verringerung des anfänglich bestehenden Einkommensunterschiedes zwischen Einheimischen und Einwanderern stattfindet. ${ }^{153}$

Analysen, die für nach Deutschland eingewanderte Arbeitskräfte durchgeführt wurden, sind überwiegend Panelstudien, da für die Bundesrepublik mit dem Sozio-Ökonomischen Panel eine entsprechende Datenbasis zur Verfügung steht. Die Vorgehensweise entspricht zunächst derjenigen für Querschnittsdaten: Durch eine Regressionsanalyse werden der Achsenabschnitt und die Koeffizienten für die unabhängigen Variablen der Einkommensfunktion für Einheimische und Eingewanderte getrennt ermittelt und dann die Einkommensdifferenz zwischen den beiden Gruppen unter Ausschaltung des Einflusses anderer einkommensrelevanter Charakteristika geschätzt. Dabei gehen in die Regressionsanalyse jetzt aber nur die Daten einer bestimmten Kohorte ein. Für die gleiche Kohorte wird auf dieselbe Weise auch in den Folgejahren die geschätzte Einkommensdifferenz bestimmt. Die Differenz der Differenzen, also der Unterschied der Einkommenswachstumsraten zwischen Einheimischen und Eingewanderten, ergibt bezogen auf ein Jahr die gesuchte Assimilationsrate. ${ }^{154}$

Durch die Verwendung von Panel-Daten können bei entsprechender Spezifizierung der Schätzung die verzerrenden Effekte unterschiedlicher Qualitäten von Kohorten, von eintrittsalterabhängigen Einkommen und von selektiver Rückwanderung vermieden werden. Allerdings treten bei Panel-Analysen andere ökonometrische Schwierigkeiten auf. So können Periodeneffekte zu Mißinterpretationen des Schätzergebnisses führen. ${ }^{155}$ Zur Illustration sei von einer im Zeitraum des Panels zunehmend ungleicher werdenden Einkommensverteilung ausgegangen, so daß die Wachstumsraten der unteren Einkommensgruppen unter denen der oberen liegen. Verdienen die Immigranten zunächst unterdurchschnittlich, dann kann auch eine gegenüber den Einheimischen der gleichen Einkommensgruppe höhere Wachstumsrate noch geringer sein als die mittlere Wachstumsrate der Einheimischen. In diesem Fall wird keine positive Assimilationsrate ausgewiesen, obwohl die Einwanderer höhere Einkommenswachstumsraten aufweisen als vergleichbare Einheimische. LaLonde und Topel errechnen aufgrund der in den

geringerem Pro-Kopf-Einkommen stark zugenommen, während derjenige der hochentwickelten Industrieländer gesunken ist.

Vgl. Friedberg (1991) (nach Pischke (1992) S. 8).

151 Vgl. Borjas (1994) S. 1678ff. Borjas erhält einen Rückgang der auf 20 Jahre bezogenen Assimilationsrate von 1970 in die USA eingewanderten 25- bis 34jährigen von 33 Prozentpunkten in einer reinen Querschnittsuntersuchung auf 18 Prozentpunkte bei ausschließlicher Berücksichtigung des Kohorteneffektes und auf 10 Prozentpunkte bei zusätzlicher Berücksichtigung des Alters der Immigranten bei ihrer Einwanderung.

152 Vgl. Borjas (1989) S. 476.

153 Vgl. Chiswick (1994) S. 107f.

154 Vgl. Pischke (1992) S. 7.

155 Vgl. zu einer Darstellung dieses Problems beispielsweise Borjas (1994) S. 1675f. 
USA in den siebziger Jahren zunehmend ungleicher werdenden Einkommensverteilung eine Unterschätzung der Assimilationsrate von Mexikanern um etwa acht Prozentpunkte bei NichtBerücksichtigung der Periodeneffekte. ${ }^{156} \mathrm{Da}$ in Deutschland die Einkommensverteilung im Zeitablauf aber relativ konstant geblieben ist, dürften durch Einkommensdifferenzierung hervorgerufene Zeiteffekte für auf Deutschland bezogene Studien kaum eine Rolle spielen. ${ }^{157}$

Die Resultate der für Deutschland durchgeführten Panelanalysen sind unterschiedlich, je nachdem, ob die Assimilation deutscher Migranten oder von Gastarbeitern untersucht wurde. Auf letztere wird zuerst eingegangen. Schmidt findet positive, aber sehr geringe Assimilationsraten (0,7 Prozentpunkte pro Jahr), so daß der durchschnittliche Gastarbeiter erst nach 17 Jahren Einkommensgleichheit mit vergleichbaren Einheimischen, d.h. mit unterdurchschnittlich verdienenden Arbeitern, erreicht. ${ }^{158}$ Licht und Steiner erhalten dagegen keine positiven Assimilationsraten, aber entgegen den Aussagen des Humankapitalansatzes höhere Einkommen für Einwanderer mit kurzem Aufenthalt als für längerfristig in Deutschland bleibende Personen. ${ }^{159}$ Dustmann findet dagegen eine empirische Bestätigung für die theoretisch abgeleitete Vermutung, daß temporäre Migranten geringere Assimilationsraten haben als dauerhafte. ${ }^{160}$ Gleichzeitig unterstellt er eine negative Selektion der Migranten, weil die Arbeitsmarktbedingungen in den Anwerbejahren der Gastarbeiter für wenig Qualifizierte in ihren Heimatländern ungünstig, in Deutschland dagegen recht vorteilhaft waren. Beide Faktoren können eine Erklärung dafür liefern, daß die Assimilation der Gastarbeiter in Deutschland offenbar wesentlich ungünstiger verlief als die Einkommensentwicklung der überwiegend permanenten Einwanderer der USA, Kanadas und Australiens und auch der dauerhaft nach Deutschland zuwandernden Aussiedler und Übersiedler.

Auch Pischke findet in den meisten Spezifikationen keine positiven Assimilationsraten. ${ }^{161} \mathrm{Er}$ führt dieses Ergebnis erstens darauf zurück, da $B$ für Gastarbeiter in Deutschland schon zum Zeitpunkt ihrer Einwanderung kaum eine Einkommensdifferenz zu Deutschen mit gleichen arbeitsmarktrelevanten Charakteristika besteht und insofern auch keine Angleichung stattfinden kann. Das geringere Durchschnittseinkommen der Zuwanderer nach Deutschland ist allein durch ihren geringeren (Berufs-)Ausbildungsstand erklärbar (vgl. Tab. D.1) und offenbar nicht auf fehlendes länderspezifisches Humankapital zurückzuführen. Zweitens sind die höchsten Assimilationsraten in den ersten Jahren nach der Zuwanderung zu erwarten. Die Gruppe kürzlich Zugewanderter ist in der auch von Pischke verwendeten Datenbasis des Sozio-Ökonomischen Panels aber unterrepräsentiert, so daß eventuell vorhandene Assimilationseffekte nicht vollständig erfaßt werden. ${ }^{162}$

\footnotetext{
156 Vgl. LaLonde/Topel (1992) S. 86f.
}

157 Vgl. Pischke (1992) S. 8. Andere Zeiteffekte, wie beispielsweise je nach Konjunkturlage unterschiedliche Einkommenszuwachsraten für Immigranten und Einheimische, sind aber eine potentielle Fehlerquelle auch für deutsche Panelstudien.

158 Vgl. Schmidt (1993) S. 20.

159 Vgl. Licht/Steiner (1993) S. $16 f$.

160 Vgl. Dustmann (1993).

161 Vgl. Pischke (1992) S. $17 \mathrm{ff}$.

162 Für Zuwanderer nach 1977 findet Pischke sowohl eine hohe anfängliche Einkommensdifferenz von 50 Prozent, als auch eine anfangs mit 5.5 Prozentpunkten recht hohe Assimilationsrate. Vgl. Pischke (1992) S. 18. 
Tab. D.I: Arbeitsplatztypen deutscher und ausländischer Arbeitnehmer

\begin{tabular}{l|c|c} 
Arbeitsplatztyp & $\begin{array}{c}\text { Deutsche } \\
\text { (in Prozent) }\end{array}$ & $\begin{array}{c}\text { Ausländer } \\
\text { (in Prozent) }\end{array}$ \\
\hline ungelernte Arbeitskraft & 4,3 & 21,5 \\
angelernter Arbeiter & 12,5 & 46,7 \\
Geselle & 20,8 & 21,0 \\
Meister & 7,9 & 4,3 \\
Selbständiger & 7,0 & 3,4 \\
Angestellter & 32,0 & 2,8 \\
einfacher öffentlicher & 1,2 & 0,0 \\
$\quad$ Dienst & & \\
mittlerer und höherer & 14,3 & 0,3 \\
öffentlicher Dienst & &
\end{tabular}

Quelle: Pischke (1992) S. 13

Als Ergebnis der angeführten Studien ist festzuhalten, daß die Gastarbeiter zwar relativ schnell gleiche Einkommen erzielen wie Deutsche mit entsprechenden Qualifikationen, ihr durchschnittliches Einkommen aber dennoch dauerhaft hinter dem mittleren Einkommen der Deutschen zurückbleibt, weil die Gastarbeiter ganz überwiegend in unterdurchschnittlich bezahlten Arbeiterpositionen beschäftigt werden.

Für deutsche Migranten, d.h. für die Vertriebenen, Flüchtlinge, Übersiedler und Aussiedler, die nach dem Zweiten Weltkrieg nach Westdeutschland kamen, konstatiert Schmidt dagegen eine wesentlich bessere Assimilation als für die Gastarbeiter. ${ }^{163}$ Der ursprüngliche Einkommensrückstand von $16 \%$ für Aussiedler und $9 \%$ für Übersiedler aus der DDR verringert sich im Zeitablauf, so daß nach 36 bzw. 21 Jahren Einkommensgleichheit mit den Einheimischen erreicht wird. ${ }^{164}$ Die im Vergleich zu den Gastarbeitern positivere Entwicklung bei den Übersiedlern kann entsprechend des Humankapitalansatzes mit deren besserer Ausbildung und mit Sprachvorteilen erklärt werden. ${ }^{165}$ Weiterhin beabsichtigte der überwiegende Teil der deutschen Migranten von vornherein dauerhaft in (West-)Deutschland zu bleiben, während die Gastarbeiter zumindest der Intention nach überwiegend als temporäre Migranten einzustufen sind und deshalb weniger Anreize zur Humankapitalbildung hatten. Hier mag auch eine Rolle spielen, daß die deutschstämmigen Immigranten im Unterschied zu den Gastarbeitern sofort bei der Einreise die deutsche Staatsbürgerschaft zuerkannt bekamen. Schließlich spricht die relativ geringe Entlohnung produktivitätssteigernder Charakteristika in den Herkunftsländern der deutschen Immigranten verbunden mit den Widerständen, die sich einer Ausreise entgegen-

163 Vgl. Schmidt (1994b). Hinweise auf eine gleichfalls recht erfolgreiche Integration der Ende der achtziger Jahre einsetzenden zweiten großen Welle der Aus- und Übersiedler in den deutschen Arbeitsmarkt finden sich bei Klös (1992), Schulz/Seiring (1994) und o.V. (1994).

164 Vgl. Schmidt (1994b) S. 19f.

165 Da ein großer Teil der Aussiedler, die seit Ende der achıiger Jahre einwanderten, nicht über die guten Deutschkenntnisse der früheren Gruppen verfügten, sind die hier wiedergegebenen Ergebnisse nicht ohne Einschränkungen auf sie übertragbar. Vgl. Schmidt (1994b) S. 22. 
stellten, für eine positive Selbstselektion dieser Gruppe, während für die Gastarbeiter, wie oben ausgeführt, eher eine negative Selektion zu vermuten ist.

\subsubsection{Bedeutung der Assimilation für ein Einwanderungsgesetz}

Welche Schlußfolgerungen für ein Einwanderungsgesetz lassen sich aus den Ergebnissen der Untersuchungen über die Assimilation von Einwanderern ziehen? Offenbar spielt länderspezifisches Humankapital für die untersuchte Gruppe von Gastarbeitern in Deutschland nur eine sehr geringe Rolle, so daß gegenüber Einheimischen mit gleichen arbeitsmarktrelevanten Charakteristika schon bald nach dem Zeitpunkt der Zuwanderung keine Einkommensunterschiede mehr bestehen. Aufgrund der gleichen Einkommen könnten Einwanderer daher als einfache Erhöhung des einheimischen Arbeitsangebots angesehen werden, wenn ihre Qualifikationsstruktur nicht ungünstiger wäre als diejenige der Einheimischen. Gelingt es mit Hilfe eines Einwanderungsgesetzes die Qualifikationen der Einwanderer so zu beeinflussen, daß sie im Durchschnitt ein den Einheimischen vergleichbar hohes Einkommen erzielen, steht zunächst zu vermuten, daß sie zumindest von der Finanzierungsseite des Staatshaushaltes gesehen sich nicht wesentlich von Einheimischen unterscheiden. Der Einfluß der Einwanderer auf die staatlichen Haushalte wäre dann v.a. in einer möglicherweise unterschiedlichen Inanspruchnahme staatlicher Leistungen zu sehen. Die positiveren Erfahrungen mit den Aus- und Übersiedlern sprechen dafür, daß die ungünstige Einkommensentwicklung der Gastarbeiter nicht auf institutionelle Besonderheiten des deutschen Arbeitsmarktes zurückzuführen ist, sondern auf die beobachtbaren und nicht-beobachtbaren produktivitätsrelevanten Merkmale der Gastarbeiter. Durch entsprechende Selektionsmechanismen im Rahmen eines Einwanderungsgesetzes erscheint eine bessere Assimilation zukünftiger Einwanderer daher grundsätzlich erreichbar.

Dem stehen allerdings zwei Einwände entgegen. Sobald im Rahmen eines Einwanderungsgesetzes das durchschnittliche Qualifikationsniveau der Einwanderer angehoben wird, steht zu erwarten, daß länderspezifisches Humankapital eine größere Bedeutung gewinnt als bei den wenig qualifizierten Gastarbeitern vergangener Einwanderungskohorten. Damit sind dann ähnlich zu den Ergebnissen für die Aus- und Übersiedler und für die USA auch höhere anfängliche Einkommensunterschiede und Assimilationsraten zu erwarten und daher auch im Vergleich zu Einheimischen zumindest anfänglich geringere Beiträge der Einwanderer zur Finanzierung der Staatshaushalte. Der zweite, fundamentalere Einwand bezieht sich auf die alleinige Konzentration fast aller empirischer Untersuchungen auf die Einkommensentwicklung der Einwanderungskohorten. Die Bedeutung der Einwanderer für die Staatshaushalte hängt neben der Einkommenshöhe aber entscheidend auch von der Erwerbsquote, der Arbeitslosenquote und der Einkommensverteilung ab. Über die Entwicklung dieser Einflußfaktoren in Abhängigkeit von dem Zeitraum seit der Einwanderung ist empirisch kaum etwas bekannt. ${ }^{166}$

166 Eine Zusammenfassung der spärlichen Ergebnisse für die USA gibt Chiswick (1994) S. 108ff. 


\subsubsection{Der Einfluß der Einwanderung auf die Haushalte der Gebiets- körperschaften}

Die Analyse der Konsequenzen von Immigration für die Finanzsituation der Gebietskörperschaften erfolgt getrennt für die Einnahmen- und die Ausgabenseite. So wird zunächst auf den Einfluß der Einwanderer auf die Einnahmen, d.h. im wesentlichen auf die Steuerzahlungen der Einwanderer, eingegangen und dann die Wirkung verstärkter Immigration auf die verschiedenen Ausgabenposten der Gebietskörperschaften untersucht.

\subsubsection{Die Einnahmenseite}

Die Einnahmen der Gebietskörperschaften setzen sich im wesentlichen aus Steuern, Beiträgen und Gebühren zusammen. Für Beiträge und Gebühren kann weitgehend die Realisierung des Äquivalenzprinzips, also der Entsprechung von Abgabenhöhe und (erwarteter) Leistungserbringung durch die öffentliche Hand, unterstellt werden. Damit geht von Beiträgen und Gebühren der Immigranten keine Nettowirkung auf die Einheimischen aus und sie können daher hier ebenso wie die ihnen gegenüberstehenden staatlichen Leistungen aus der Betrachtung ausgeklammert werden. Die Höhe der zusätzlichen Steuern, die den Gebietskörperschaften aufgrund von Einwanderungen zufließen, wird entscheidend bestimmt durch die rechtliche Position der Einwanderer und ihre wirtschaftliche Situation. In bezug auf die rechtliche Position kann mit Blick auf die grundsätzlich für Einwanderer und Einheimische einheitlich geltenden Steuerbemessungsgrundlagen ${ }^{167}$ von gleichen Voraussetzungen ausgegangen werden.

Unterschiedliche durchschnittliche Steuerzahlungen von Einheimischen und Eingewanderten beruhen daher v.a. auf Unterschieden in ihrer wirtschaftlichen Situation. Insbesondere das geringe durchschnittliche Einkommen der Eingewanderten und der gegenläufige Effekt der höheren Erwerbsbeteiligung spielen eine große Rolle, da die aufkommensstärksten Steuern das Einkommen oder eine einkommensabhängige Größe (beispielsweise den Konsum) als Bemessungsgrundlagen haben. Ulrich benutzt neben allgemein zugänglichen Informationen Mikrodaten des Sozio-Ökonomischen Panels aus dem Jahr 1984 zur Ermittlung der durchschnittlichen Steuerzahlungen. ${ }^{168} \mathrm{Da}$ im Rahmen des Sozio-Ökonomischen Panels nicht zwischen Einheimischen und Eingewanderten unterschieden wird, beschränkt sich seine Untersuchung auf Unterschiede zwischen Deutschen und Ausländern ${ }^{169}$. Die Analyse bezieht nur die beiden aufkommensstärksten Steuern, die Einkommensteuer und die Umsatzsteuer, ein.

167 Geringfügige Abweichungen ergeben sich z.B. durch Pauschbeträge für Unterhalt an im Ausland lebende Familienangehörige und dadurch, daß das Ehegattensplitting an den Aufenthalt beider Ehepartner in Deutschland gebunden ist. Weiterhin sind Ausländer, die ihren Wohnsitz für weniger als sechs Monate in Deutschland haben, nur beschränkt steuerpflichtig. Vgl. Ulrich (1992) S. 201.

Vgl. zu der folgenden Darstellung Ulrich (1992).

Unter die Gruppe der Ausländer fast Ulrich Einwanderer aus den typischen Herkunftsländern der Gastarbeiter (Türkei, Griechenland, Italien, Jugoslawien, Spanien), während andere Nationalitäten der Gruppe der Deutschen zugeordnet werden. Im folgenden wird daher von Gastarbeitern gesprochen. Vgl. Ulrich (1994a) S. 66. 
Da die im Sozio-Ökonomischen Panel enthaltenen Angaben bezüglich der Einkommensteuerhöhe als zu unzuverlässig gelten, mußten die Werte mit Hilfe eines Steuersimulationsmodells von Berntsen synthetisch generiert werden. ${ }^{170}$ Auf diese Art und Weise ermittelt Ulrich durchschnittliche Einkommensteuerzahlungen von 6.032 DM (1984) für Haushalte mit einem deutschen Haushaltsvorstand und Einkommensteuerzahlungen von jährlich 5.266 DM für Haushalte mit einem Gastarbeiter als Vorstand. Nicht berücksichtigt ist dabei, daß Ausländer, die weniger als ein halbes Jahr ihren Wohnsitz oder gewöhnlichen Aufenthalt in Deutschland haben, nur beschränkt steuerpflichtig sind und daher nach den Doppelbesteuerungsabkommen mit den Heimatländern der Gastarbeiter ihre Lohnsteuer an das Herkunftsland abführen. Die progressive Ausgestaltung der Einkommensteuer führt v.a. bei hoher Fluktuation der ausländischen Bevölkerung über die Möglichkeit des Einkommensteuer-Jahresausgleichs zu einer weiteren Verringerung der Einkommensteuerschuld von Ausländern. ${ }^{171}$

Die Mehrwertsteuerlast wurde unter der Annahme vollständiger Überwälzung unter Zugrundelegung einer Studie von Kaiser ermittelt, in der der Anteil der Mehrwertsteuer am Einkommen für unterschiedliche Einkommenshöhen festgestellt wird. ${ }^{172}$ So sinkt der Mehrwertsteueranteil am Einkommen von 6,6 Prozent für Haushalte mit monatlichen Nettoeinkommen zwischen 1.000 DM und 1.500 DM auf 4,9 Prozent für Haushalte mit Einkommen zwischen 7.500 DM und 10.000 DM. Aus den Einkommensdaten für Haushalte mit deutschem bzw. Gastarbeiter-Vorstand berechnet Ulrich eine durchschnittliche Mehrwertsteuerbelastung von 2.018 DM bzw. 1.863 DM (1984). Der Wert für Gastarbeiter-Haushalte ist allerdings dann zu hoch angesetzt, wenn man davon ausgeht, daß Gastarbeiter einen größeren Teil ihres Einkommens als Deutsche ins Ausland transferieren, und dieses Einkommen somit nicht zu Mehrwertsteuerzahlungen in Deutschland führt. ${ }^{173} \mathrm{Da}$ die Werte auf Haushaltsebene ermittelt wurden, überwiegt offenbar der Einfluß des geringeren Durchschnittseinkommens der Gastarbeiter den Effekt der (damals noch) höheren Erwerbsbeteiligung der Gastarbeiter, so daß sich insgesamt die Einkommen- und Mehrwertsteuerzahlungen der Gastarbeiter auf 89 Prozent des Niveaus der Deutschen belaufen.

Da die Einkommen- und Mehrwertsteuer zusammen über 70 Prozent des gesamten Steueraufkommens ausmachen, kann davon ausgegangen werden, daß auch in bezug auf das gesamte Steueraufkommen die Steuerzahlungen von Gastarbeitern knapp neun Zehntel des Niveaus derjenigen der Deutschen betragen.

Dieses Ergebnis wird durch eine Studie von Simon und Akbari nur für Einwanderungskohorten nach 1980 in etwa bestätigt, während vorher zugewanderte Ausländer sogar höhere Steuerzahlungen pro Haushalt aufweisen als Einheimische. ${ }^{174}$ Allerdings sind die Ergebnisse von Simon und Akbari hinsichtlich der Steuerzahlungen als wenig verläßlich anzusehen, weil der als Datengrundlage dienende Mikrozensus 1989 aufgrund der größeren Fallzahl zwar die Unterscheidung verschiedener Einwanderungskohorten ermöglicht, andererseits aber keine Daten über die Steuerzahlungen der Haushalte enthält. Deshalb waren die Autoren gezwungen, die Steuern indirekt zu bestimmen. Dazu ermittelten sie in einem ersten Schritt das durch-

\footnotetext{
170 Vgl. Berntsen (1989) (nach Ulrich (1992) S. 194).

171 Vgl. Wehrmann (1989) S. 258.

172 Vgl. Kaiser (1989).

173 Vgl. Wehrmann (1989) S. 260.

174 Vgl. Simon/Akbari (1995).
} 
schnittliche Bruttoeinkommen der Haushalte. Sie erhielten einen weit von den Ergebnissen der volkswirtschaftlichen Gesamtrechnung abweichenden Wert, was Zweifel an der Zuverlässigkeit des Ergebnisses angebracht scheinen läßt. In einem zweiten Schritt wurden die Bruttoeinkommen dann mit einem einheitlichen Durchschnittssteuersatz multipliziert. Die Progressivität des Steuertarifs fand also keine Berücksichtigung.

Eine Schätzung der durch Aussiedler zusätzlich entstehenden Steuereinnahmen mit Hilfe von Mikrodaten wurde meines Wissens bisher noch nicht vorgenommen. Gieseck, Heilemann und Loeffelholz berechnen als Primärwirkung der 1,2 Mio zwischen 1988 und 1991 nach Deutschland zugewanderten Aussiedler zusätzliche Steuereinnahmen von etwa 4 Mrd DM 1992. ${ }^{175}$ Sie unterstellen dabei, daß 400.000 dieser Aussiedler bis Ende 1991 einen Arbeitsplatz gefunden hatten und das durchschnittliche Einkommen eines westdeutschen Arbeitnehmers erhielten. Aufgrund der letztgenannten Annahme, der verschiedenen Erhebungszeiträume und der Verwendung von Personen statt Haushalten als Basis ist ein direkter Vergleich mit den von Ulrich gewonnenen Daten für Gastarbeiter nicht möglich.

\subsubsection{Die Ausgabenseite}

Bei den Ausgaben der Gebietskörperschaften lassen sich zwei große Bereiche unterscheiden, zum einen die Kosten der Bereitstellung und Unterhaltung von Infrastruktur im weiteren Sinne und zum anderen die steuerfinanzierten Sozialleistungen.

$\mathrm{Zu}$ den Ausgaben für Erstellung und Instandhaltung von Infrastruktur zählen nach dem weiten Infrastrukturbegriff von Jochimsen nicht nur Kosten für materielle Infrastruktur (Verkehrseinrichtungen, Versorgungseinrichtungen für Elektrizität, Gas und Wasser, Entsorgungsanlagen, etc.), sondern auch Ausgaben für personelle und immaterielle Infrastruktur, also für Humankapital und Institutionen zur Förderung einer effizienten Allokation der Ressourcen. ${ }^{176}$ Unter den so definierten Infrastrukturbegriff fallen nahezu alle nach dem Abzug von Transfers verbleibenden Staatsausgaben.

In der Literatur wird bei der Analyse der Kosten von Einwanderern für die einheimische Bevölkerung oft eine Unterteilung in sogenannte Fixkosten (z.B. Verteidigung, Auswärtiges und staatlicher Schuldendienst) und bevölkerungszahlabhängige staatliche Leistungen vorgenommen, um die durch die Zuwanderer zusätzlich verursachten Ausgaben isolieren zu können. Diese Unterscheidung ist hier nicht notwendig, weil auch bei dem Kinderlastenausgleich als dem anderen in dieser Arbeit betrachteten Instrument zur Stabilisierung der Gesetzlichen Rentenversicherung eine Bevölkerungszunahme bzw. ein geringerer Bevölkerungsrückgang auftreten werden. Betrachtet werden muß für diesen Vergleich daher nur ein eventuell unterschiedlicher Infrastrukturbedarf von Einheimischen und Eingewanderten. ${ }^{177}$

175 Eigene Berechnung nach Gieseck/Heilemann/Loeffelholz (1992) S. 271f. Vgl. auch die ausführlichere Darstellung in Barabas u.a. (1992).

176 Vgl. Jochimsen (1966) S. 99ff.

177 Um eine Vorstellung von der Größenordnung der Infrastrukturkosten zu vermitteln und den Vergleich mit anderen Maßnahmen zur Stabilisierung der Finanzlage der Gesetzlichen Rentenversicherung zu erleichtern, sollen hier trotz der Irrelevanz für die vorliegende Fragestellung kurz einige Indizien bezüglich der durch eine relative Bevölkerungszunahme hervorgerufenen Kosten - sei es durch höhere 
$\mathrm{Zu}$ vermuten ist, daß bei dem Instrument des Kinderlastenausgleichs der Bedarf an Kindergärten, Schulen, etc. höher sein wird, als bei verstärkter Einwanderung, da die Einwanderer ihre Kindheit überwiegend noch im Heimatland verbracht haben. ${ }^{178}$ Wehrmann geht aber ebenso wie Miegel davon aus, daß sich die Nutzungsintensität von Infrastruktureinrichtungen durch Ausländer und Deutsche in den achtziger Jahren nicht mehr voneinander unterscheidet. ${ }^{179}$ Als Determinanten der Infrastrukturnutzung durch Immigranten stellt Wehrmann die Aufenthaltsdauer, die Altersstruktur, den Familienstand, die Kinderzahl und das Anspruchsniveau der Zuwanderer heraus. ${ }^{180}$ Mit zunehmender Integration der Ausländer, d.h. langen Aufenthaltsdauern, Familiennachzug, etc. steigt demnach der Bedarf an staatlich bereitgestellten Infrastruktureinrichtungen. Für dauerhafte Einwanderer unterscheiden sich die Infrastrukturausgaben daher nach der Assimilationsphase nur unwesentlich von denen der Einheimischen. Als Empfehlung für die Ausgestaltung eines Einwanderungsgesetzes läßt sich daraus entsprechend des ursprünglich von Regierungsseite angestrebten Rotationsprinzips ${ }^{181}$ ein Einwirken auf eine möglichst kurze Verweildauer im Gastland ableiten.

Auch über die steuerfinanzierten Transferzahlungen an Einheimische und Gastarbeiter liegen nur wenige Informationen bezüglich der tatsächlichen Inanspruchnahme vor. Nur für die Bereiche Kindergeld und Sozialhilfe sind entsprechende Daten verfügbar, weshalb sich der folgende Vergleich zwischen Einheimischen und Gastarbeitern auf diese beiden Transferarten beschränkt.

Hinsichtlich der Rechtsposition gilt in fast allen Sozialleistungsbereichen das Territorialitätsprinzip, so daß die Leistungsgewährung nicht von der Nationalität, sondern von dem Wohnsitz oder ständigen Aufenthaltsort des Berechtigten abhängt. ${ }^{182}$ Beim Kindergeld bestehen aber insofem Ausnahmen von diesem Prinzip, als für Kinder von in Deutschland als Erwerbspersonen registrierten EU-Staatsangehörigen auch dann Kindergeld in voller Höhe

Geburten oder durch Zuwanderung - zusammengetragen werden. Gieseck/Heilemann/Loeffelholz (1992) S. 272f. setzen den bevölkerungszahlunabhängigen Teil der Staatsausgaben mit etwa einem Drittel an. Sie ermitteln für die über eine halbe Million zwischen 1988 und 1991 zugewanderten Kinder und Jugendlichen im Alter von 6 bis 18 Jahren durch Multiplikation mit einem Pro-Kopf-Ausgabensatz für einheimische Kinder von 7.500 DM zusätzliche laufende und investive Ausgaben an allgemeinbildenden Schulen von jährlich knapp 4 Mrd DM. Im Verkehrsbereich ermitteln die Autoren für die 2.7 Mio zwischen 1988 und 1991 nach Deutschland zugewanderten Personen zusätzliche Ausgaben von etwa 2 Mrd DM pro Jahr. Wehrmann (1989) S. 246 sieht schon vor der letzten großen Wanderungswelle in den besonders betroffenen Ballungsgebieten die "Kapazitätsgrenzen in wichtigen Infrastrukturbereichen bereits erreicht, wenn nicht sogar längst überschritten" und schließt daher auf eine nachhaltige Belastung der offentlichen Haushalte. Eine genauere Abschätzung der durch eine relative Bevölkerungszunahme verursachten Infrastrukturkosten erweist sich wegen des nur äußerst spärlich vorhandenen statistischen Materials als unmöglich. Außerdem werden diese Infrastrukturkosten für den Fall einer bloßen Verlangsamung des Bevölkerungsrückgangs wegen der dann weiterhin nutzbaren Kapazitäten geringer ausfallen als bei einer absoluten Zunahme der Bevölkerung, die eine Ausweitung bestehender Infrastruktur erfordert.

Dieser Transfer von Humankapital aus den Herkunftsländern in die Aufnahmeländer wurde in den siebziger und achtziger Jahren in der Entwicklungsökonomik unter dem Begriff des "brain drain" stark diskutiert. Vgl. beispielsweise Bhagwati (1985) S. 303-361.

Vgl. Wehrmann (1989) S. 247f. sowie Miegel (1984) S. 167.

Vgl. Wehrmann (1989) S. 243.

181 Als Rotationsprinzip bezeichnet man den ständigen Austausch der jeweils im Inland befindlichen Ausländer durch Rückwanderungen und Zuzug anderer Ausländer. 
gezahlt wird, wenn sie weder einen Wohnsitz noch ihren ständigen Aufenthalt in Deutschland haben. Ein (geringeres) Kindergeld wird aufgrund bilateraler Abkommen auch Arbeitnehmern und vorübergehend Arbeitslosen aus der Türkei, Jugoslawien, Marokko und Tunesien gewährt, deren Kinder sich im Heimatland aufhalten.

Demographische und ökonomische Unterschiede führen dazu, daß Gastarbeiter trotz dieser rechtlichen Regelungen mehr Kindergeldzahlungen erhalten, als es ihrem Anteil an der Bevölkerung entspricht. Als erster Grund ist die überdurchschnittliche Fertilität der in der Bundesrepublik Deutschland lebenden Gastarbeiter anzuführen. ${ }^{183}$ Verstärkt wird die Wirkung der höheren Kinderzahl pro Anspruchsberechtigtem dadurch, daß die Kindergeldzahlungen pro Kind mit der Parität steigen. ${ }^{184}$ Weiterhin führt die höhere Fruchtbarkeit der Gastarbeiter und der hohe Anteil von Gastarbeitern im mittleren Alter auch zu einem höheren Anteil von Anspruchsberechtigten unter Gastarbeitern als unter Deutschen. Schließlich hat der mit steigendem Einkommen sinkende Kindergeldanspruch in Verbindung mit dem geringeren Durchschnittseinkommen der Gastarbeiter zur Folge, daß auch bei gleicher Kinderzahl Gastarbeiter im Mittel mehr Kindergeld erhalten als Deutsche. Alle Faktoren zusammengenommen führten nach den Berechnungen von Ulrich dazu, daß auf Haushaltsebene Gastarbeiter 1984 mit 1.143 DM fast drei mal höhere Kindergeldzahlungen erhielten als Deutsche (390 DM). ${ }^{185}$ Die überschlägige Berechnung von Simon und Akbari bestätigt diese Größenordnung in etwa. ${ }^{186}$

Einen Rechtsanspruch auf Gewährung von Sozialhilfe haben in Deutschland lebende Ausländer in der Regel unter den gleichen Bedingungen wie Deutsche, sofern der Bezug von Sozialhilfe nicht Zweck ihrer Einreise gewesen ist (§ 120 Abs 1 BSHG). Die nach dem Ausländerrecht mögliche Ausweisung von Ausländern, die ihren Lebensunterhalt nicht bestreiten können, ist faktisch kaum relevant.

Die Anzahl der Sozialhilfeempfänger pro 1000 Einwohner lag für Ausländer in den sechziger Jahren deutlich unter derjenigen für Deutsche. Die Ursache dafür dürfte in der bis 1973 hohen Rückwanderungsquote bei Arbeitslosigkeit zu suchen sein. Die nach dem Anwerbestopp sinkende Rückkehrneigung und die für Ausländer überproportional steigende Arbeitslosenquote infolge der verschlechterten Wirtschaftslage haben ab der zweiten Hälfte der siebziger Jahre zu einem drastischen Anstieg des Anteils der Sozialhilfeempfänger unter den Ausländern geführt. 1989 bezogen über 130 von 1000 ausländischen Einwohnern Sozialhilfe, während es bei den Deutschen nur etwa 50 von 1000 waren. Gerade für Ausländer, die sich erst wenige Jahre in Deutschland aufhalten, errechnen Simon und Akbari sehr hohe Sozialhilfeleistungen (bis zu 3.750 DM im Jahr 1989 für einen Haushalt der Einwanderungskohorte 1987$88 \mathrm{im}$ Vergleich zu 125 DM für Einheimische). ${ }^{187}$ Allerdings dürften diese Werte gerade für die jüngeren Kohorten durch den steigenden Prozentsatz von Asylbewerbern unter den zuwandernden Ausländern stark erhöht worden seien, denn die Asylbewerber waren aufgrund des damals noch bestehenden Arbeitsverbotes fast ausschließlich auf die Sozialhilfe zur

\footnotetext{
183 Die Kindergeldzahlungen pro Anspruchsberechtigtem betrugen 1994 im Durchschnitt aller Kindergeldberechtigten etwa 2.120 DM, für ausländische Berechtigte aber 2.770 DM. Eigene Berechnungen nach Angaben der Bundesanstalt für Arbeit.

Vgl. dazu im einzelnen Anhang 2.

Vgl. Ulrich (1992) S. 203.

Vgl. Simon/Akbari (1995) S. 13.

Vgl. Simon/Akbari (1995) S. 13.
} 
Bestreitung ihres Lebensunterhaltes angewiesen. Für die hier interessierende Gruppe der Gastarbeiter dürften die durchschnittlichen Sozialhilfeleistungen pro Haushalt dagegen auch für jüngere Einwanderungskohorten wesentlich geringer gewesen sein. Anhand der von Wehrmann angeführten Zahlen ergeben sich damit für 1986 durch die ungünstigere Struktur der Ausländer zusätzliche Sozialhilfezahlungen von knapp 1,3 Mrd DM. ${ }^{188}$ Zukünftig ist aufgrund der geringeren Rentenanwartschaften der Ausländer mit dem Eintritt der ersten Gastarbeitergeneration in das Rentenalter mit einem Anstieg der sozialhilfeberechtigten Ausländer zu rechnen, wenn nicht andere Faktoren diese Entwicklung kompensieren.

\subsubsection{Bedeutung für ein Einwanderungsgesetz}

Da die Bemessungsgrundlagen der aufkommensstärksten Steuern in der Bundesrepublik Deutschland am Einkommen bzw, am Konsum (welcher wiederum entscheidend durch die Einkommenshöhe determiniert wird) ansetzen, sollte die Auswahl der ökonomisch motivierten Immigranten im Rahmen eines Einwanderungsgesetzes unter dem Gesichtspunkt des Einflusses auf die Haushalte der Gebietskörperschaften so erfolgen, daß ein möglichst hohes durchschnittliches Einkommen der Einwanderer zu erwarten ist. Die durchschnittliche Einkommenshöhe der Zuwanderer hängt wiederum ab von der Höhe ihrer Erwerbsbeteiligung, von der Arbeitslosenquote und von dem durchschnittlichen Einkommen eines Erwerbstätigen.

Die Erwerbsbeteiligung der Zuwanderer wird stark von ihrem Altersaufbau und von ihrer Familienstruktur beeinflußt. Mit einer besonders hohen Erwerbsquote ist zu rechnen, wenn die Zuwanderer sich im arbeitsfähigen Alter zwischen etwa 20 und 60 Jahren befinden und keine Familienangehörigen in Deutschland haben. Temporäre Migration mit Verwirklichung des Rotationsprinzips hat gegenüber permanenter Migration den Vorteil, daß die Erwerbsquote dauerhaft hoch bleiben wird, da fortlaufend eine günstige Alters- und Familienstruktur gewahrt werden kann. Bei dauerhafter Einwanderung wird dagegen der Familiennachzug und das Ausscheiden aus dem Erwerbsleben nach und nach zu einer Verringerung der Erwerbsquote führen.

Die Arbeitslosenquote der Zuwanderer kann durch die schon unter 3.1.3 beschriebenen Auswahlkriterien gering gehalten werden: Das Arbeitsmarktprofil der Zuwanderer sollte so beschaffen sein, daß diese zum einen nicht in Konkurrenz zu Einheimischen auf durch strukturelle Arbeitslosigkeit gekennzeichneten Teilarbeitsmärkten treten und zum anderen aufgrund einer hohen Allgemeinbildung eine hohe berufliche Mobilität sowie aufgrund geringer familiärer Bindungen auch eine hohe regionale Mobilität aufweisen.

Die Höhe des durchschnittlichen Einkommens der erwerbstätigen Zuwanderer schließlich hängt vor allem von ihrem durchschnittlichen formalen Qualifikationsniveau ab, aber auch von ihrer Einsatzbereitschaft und ihren sonstigen Fähigkeiten. Durch ein Einwanderungsgesetz sollten daher unter diesem Gesichtspunkt bevorzugt gut ausgebildete, fähige und ambitionierte Personen zugelassen werden. Ob temporäre oder permanente Einwanderer bei sonst gleichen Charakteristika höhere durchschnittliche Einkommen erzielen, ist kaum feststellbar. Einerseits haben temporäre Einwanderer möglicherweise einen höheren Anreiz in der begrenzten Zeit im Gastland besonders viel Vermögen anzusammeln (Sparmotiv). Andererseits hat die Diskussion in Unterkapitel 3.1 gezeigt, daß v.a. qualifizierte Erwerbstätige in den ersten Jahren im Gastland verglichen mit ähnlich ausgebildeten Einheimischen unterdurchschnittlich verdienen und 
daher eine längere durchschnittliche Aufenthaltsdauer ein höheres Durchschnittseinkommen der Zuwanderer zur Folge hat.

Auch die Inanspruchnahme von staatlich bereitgestellter Infrastruktur und von steuerfinanzierten Sozialleistungen wird bei vorübergehendem Aufenthalt in Deutschland geringer sein als bei dauerhafter Einwanderung, da bei vorübergehendem Aufenthalt überwiegend einzelne Erwerbspersonen ohne ihre Familienangehörigen zuwandern. Damit wird ein Teil der staatlich bereitgestellten Infrastruktur wie z.B. Schulen und Kindergärten nicht genutzt und im Fall längerer Arbeitslosigkeit, die zur Inanspruchnahme von Sozialhilfeleistungen führen kann, besteht ein starker Anreiz zur Rückwanderung, die wiederum einen Verlust der Anspruchsberechtigung nach sich zieht.

Auf Integrationsmaßnahmen wie Sprachkurse und Qualifizierungen sollte verzichtet werden, wenn die daraus entstehenden Belastung der Haushalte der Gebietskörperschaften (und auch der Sozialversicherungen) ihren Nutzen übersteigen. Dieser Nutzen besteht in einem höheren Barwert der Abgaben der (permanenten) Einwanderer, weil durch die Integrationsmaßnahmen ihr durchschnittliches Einkommen ansteigt. $\mathrm{Zu}$ berücksichtigen ist dabei, daß durch Integrationsmaßnahmen der Wanderungsanreiz für Migranten mit nachteiligen Charakteristika ansteigt und sie daher einer vorteilhaften Selektion entgegenstehen. Weiterhin werden die Vorbehalte der Einheimischen gegen die Zuwanderung zunehmen, wenn sie eine mißbräuchliche Inanspruchnahme der Integrationsmaßnahmen befürchten. Es scheint daher angeraten, auf Integrationsmaßnahmen im Zweifelsfall lieber ganz zu verzichten.

Insgesamt ist also aus Sicht der Finanzlage der öffentlichen Haushalte die temporäre Zuwanderung von gut ausgebildeten Personen im Erwerbsalter vorteilhaft, die in einem Arbeitsmarktsegment tätig werden, das nicht durch ein strukturelles Überangebot an Arbeitskräften gekennzeichnet ist. Inwieweit dieses Ergebnis durch die Wirkungen der Eingewanderten auf die Haushalte der Sozialversicherungen zu revidieren ist, wird im folgenden untersucht.

\subsubsection{Der Einfluß der Einwanderung auf die Finanzlage der Sozialver- sicherungen}

\subsubsection{Der Einfluß der Gastarbeiter auf die Sozialversicherungen}

Zunächst wird auf die rechtliche Stellung von Gastarbeitern in dem System der Sozialversicherungen eingegangen, ehe demographische und wirtschaftliche Unterschiede zwischen Deutschen und Gastarbeitern untersucht werden, die zu abweichenden Beitragszahlungen und Leistungsansprüchen führen.

Im Sozialversicherungsrecht gilt grundsätzlich das Territorialitätsprinzip, d.h. alle im Inland beschäftigten Personen werden sowohl bezüglich der Beitragspflicht als auch hinsichtlich der Höhe und Dauer der Leistungsansprüche gleichbehandelt. ${ }^{189}$ Allerdings impliziert das Territorialitätsprinzip auch eine Beschränkung der Leistungspflicht auf das Inland. Um zu verhindern, daß ausländische Arbeitnehmer bei der Rückkehr in ihr Heimatland alle Ansprüche an die deutschen Sozialversicherungen verlieren, wurden eine Reihe von internationalen 
Verträgen geschlossen, in denen der Ersatz des Territorialitätsprinzips durch das Personalitätsprinzip verankert wurde. Damit haben Ausländer auch dann Leistungsansprüche, wenn sie ihren Wohnsitz in einem der Vertragsstaaten haben. Insbesondere regeln die zwischenstaatlichen Abkommen, daß unter bestimmten Voraussetzungen Versicherungszeiten aus den anderen Vertragsstaaten auf die Erfüllung der Anwartschaftszeit und die Festsetzung der Anspruchsdauer angerechnet werden. Soweit für einen einzelnen Versicherungszweig spezielle Regelungen greifen, werden diese bei der Behandlung von Arbeitslosen-, Kranken-, und Rentenversicherung weiter unten erläutert.

Die wohl umfangreichste Schätzung des Einflusses wirtschaftlicher und demographischer Charakteristika der Gastarbeiter auf die Finanzlage des Sozialversicherungssystems wurde von Ulrich durchgeführt. ${ }^{190}$ Anhand von Daten des Sozio-Ökonomischen Panels über die Einkommen von Haushalten mit deutschen bzw. Gastarbeiter-Vorständen berechnet Ulrich zunächst die entsprechenden Sozialversicherungsbeiträge und Transferzahlungen mit Hilfe des bereits oben bei der Behandlung der Einnahmen der Gebietskörperschaften erwähnten Simulationsmodells von Berntsen. ${ }^{191}$ Als Ergebnis erhält Ulrich für deutsche Haushalte einen mit 4.249 DM wesentlich geringeren Beitrag zu den Sozialversicherungen als für GastarbeiterHaushalte. Dem stehen Arbeitslosengeld- und Rentenzahlungen von 1.505 DM an Gastarbeiter-Haushalte und 6.380 DM an deutsche Haushalte gegenüber (vgl. Tab. D.2). ${ }^{192}$ Über Leistungen der Gesetzlichen Krankenversicherungen konnten keine Angaben gemacht werden, da es sich größtenteils um Sachleistungen handelt, deren Wert den Versicherten im Normalfall nicht bekannt ist.

Das im Vergleich zu deutschen Haushalten wesentlich positivere Bild ist vor allem auf die günstigere Alters- und Erwerbsstruktur der Gastarbeiter-Haushalte zurückzuführen. Schaltet man diese Einflüsse aus, indem man nur Vollzeitbeschäftigte gleicher Altersgruppen miteinander vergleicht, dann ergeben sich nur noch geringfügige Unterschiede zwischen den Sozialversicherungsbeiträgen von Deutschen und Gastarbeitern (vgl. Abb. D.8).

Tab. D.2: Transfers und Beiträge zu den Sozialversicherungen pro Haushalt in DM (1984)

\begin{tabular}{c|r|r} 
& Deutsche & Gastarbeiter \\
\hline Sozialversicherungsbeiträge & 4.249 & 6.550 \\
Arbeitslosenversicherung & 560 & 874 \\
Ges. Krankenversicherung & 1.435 & 2.159 \\
Ges. Rentenversicherung & 2.254 & 3.517 \\
ausgewählte Transfers & 6.380 & 1.505 \\
Arbeitslosengeld & 463 & 1.140 \\
Renten & 5.917 & 365
\end{tabular}

Quelle: Ulrich (1994) S. 67

\footnotetext{
190 Vgl. Ulrich (1992) und Ulrich (1994a).

191 Vgl. Berntsen (1989) (nach Ulrich (1992) S. 194).

192 Simon/Akbari (1995) S. 13f. errechnen gleichfalls sehr geringe Rentenzahlungen an Ausländer (nur für die Einwanderungskohorten vor 1961 liegen die Rentenzahlungen geringfügig über denjenigen an Einheimische). Dagegen erhalten alle Einwanderungskohorten mehr Arbeitslosengeld als der Durchschnitt der einheimischen Haushalte.
} 
Abb.D.8: Sozialversicherungsbeiträge von Vollzeit-Erwerbstätigen 1984 in DM

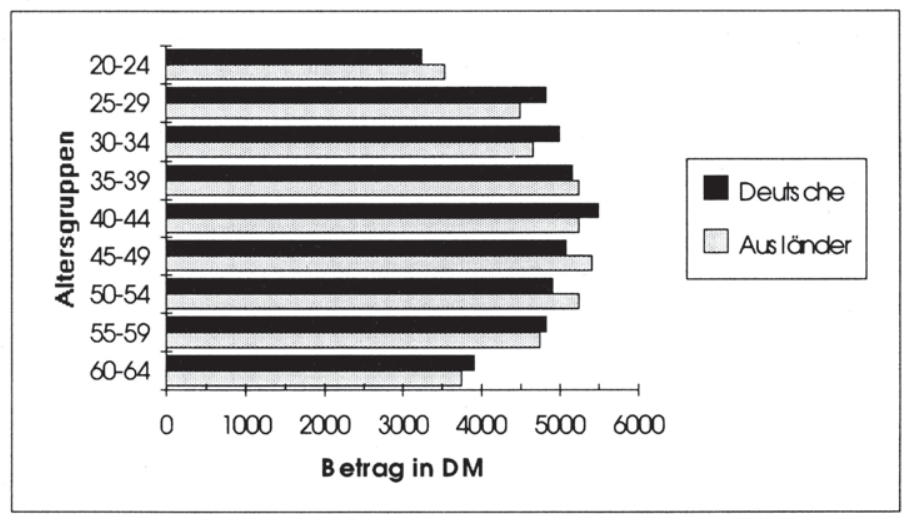

Quelle: Ulrich (1994) S. 68

Der isolierte Einfluß der Alters- bzw. der Erwerbsstruktur wird in Tabelle D.3 deutlich, in der die Beitragszahlungen und Transfers unter der Annahme ausgewiesen werden, daß die Gastarbeiter die gleiche Altersstruktur (Spalte 4) bzw. die gleiche Erwerbsstruktur besitzen wie die Deutschen (Spalte 5). Dazu werden beispielsweise die Produkte aus den Beiträgen zur Arbeitslosenversicherung, die von Gastarbeitern einer bestimmten Altersgruppe durchschnittlich geleistet werden, und dem Anteil dieser Altersgruppe in der deutschen Bevölkerung gebildet. Die Summe dieser Produkte über alle Altersgruppen ergibt dann die durchschnittlichen Beiträge der Gastarbeiter zur Arbeitslosenversicherung, wenn sie die gleiche Altersstruktur wie die deutsche Bevölkerung aufweisen würden.

Die Beiträge der Gastarbeiter zu den einzelnen Sozialversicherungszweigen wären wesentlich geringer, wenn sie die Altersstruktur der Deutschen hätten. Eine noch stärkere Angleichung der durchschnittlichen Beitragszahlungen von Gastarbeitern an die der Deutschen ist für die Standardisierung der Erwerbsstruktur festzustellen. ${ }^{193}$ Gleichzeitig steigen die Rentenzahlungen an Gastarbeitern durch die Standardisierung der Altersstruktur stark an, liegen aber immer noch bei nur wenig mehr als einem Drittel der durchschnittlichen Rentenzahlungen an Deutsche.

Als Ergebnis der auf der Mikroebene durchgeführten Untersuchung von Ulrich läßt sich daher festhalten, daß vor allem die vorteilhafte Alters- und Erwerbsstruktur der Gastarbeiter dazu führt, daß der negative Einfluß ihres geringeren Durchschnittseinkommens überkompensiert wird und Haushalte mit einem Gastarbeiter als Vorstand wesentlich höhere Sozialversicherungsbeiträge zahlen, aber weniger Leistungen in Form von Renten und Arbeitslosengeld erhalten.

193 Hier liegt die wesentliche Schwäche der Ergebnisse von Simon/Akbari (1995) S. 8ff. Sie unterstellen implizit eine gleiche Erwerbsstruktur für ausländische und einheimische Haushalte und können so den Effekt unterschiedlicher Erwerbsbeteiligungen auf die Beitragszahlungen nicht erfassen. 
Tab. D.3: Transfers und Beiträge zu den Sozialversicherungen pro Person in DM (1984)

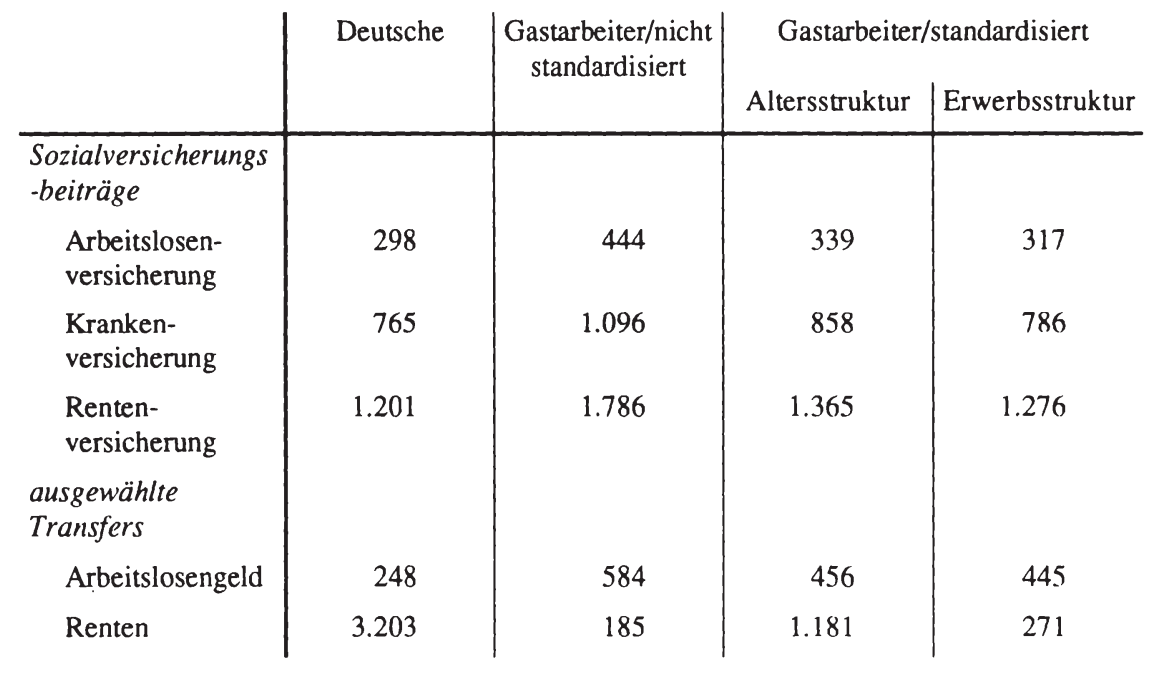

Quelle: Ulrich (1994) S. 69

Leider beziehen sich die oben dargestellten Resultate auf das Jahr 1984. Das Ausscheiden von einzelnen Haushalten aus den Datensätzen späterer Wellen des Sozio-Ökonomischen Panels macht wegen der dann zu geringen Fallzahl auch eine Wiederholung der Untersuchung für spätere Jahre unmöglich. ${ }^{194}$ Ulrich versucht daher die weitere Entwicklung der Sozialversicherungsbeiträge von Gastarbeitern und der Zahlungen von Sozialversicherungsleistungen an sie durch Zuhilfenahme von Makrodatenreihen abzuschätzen. Ausgangspunkt ist die Überlegung, daß Zahlungen und Leistungen von zwei Faktoren abhängen: zum einen von dem Anteil der deutschen bzw. der Gastarbeiter-Haushalte, die Beiträge zahlen bzw. Leistungen empfangen an allen Haushalten dieser Gruppe und zum anderen von der durchschnittlichen Höhe der Beiträge bzw. Leistungen pro deutschem bzw. Gastarbeiter-Haushalt. Da die Einflußfaktoren auf diese beiden Komponenten zwischen den einzelnen Sozialversicherungszweigen unterschiedlich sind, werden Arbeitslosenversicherung, Kranken-, Pflege- und Rentenversicherung im folgenden gesondert untersucht.

Die Höhe der Leistungen der Arbeitslosenversicherung ist für jeden Versicherten proportional zu den gezahlten Beiträgen. Daher können sich Unterschiede zwischen Gastarbeitern und Deutschen nicht durch abweichende durchschnittliche Zahlungen und Leistungen ergeben, sondern nur durch unterschiedliche Häufigkeiten des Empfangs von Leistungen.

Die Wahrscheinlichkeit des Empfangs von Leistungen hängt wiederum von der Arbeitslosenquote ab. Betrachtet man die Arbeitslosenquote von Gastarbeitern und Deutschen seit 1960, dann fällt, wie bereits bei der Behandlung der Sozialhilfeausgaben erwähnt, auf, daß die Quote für Ausländer bis zum Anwerbestopp 1973 stets unter derjenigen der Deutschen lag, sie

194 Vgl. Ulrich (1994a) S. 66. 
danach aber mit zunehmendem Abstand überstieg. Ein Grund dafür ist darin zu sehen, daß viele Gastarbeiter im Fall der Arbeitslosigkeit nicht mehr in ihr Heimatland zurückgekehrt sind, weil eine Wiedereinreise nach Deutschland dann eventuell nicht mehr möglich gewesen wäre. Die höhere Arbeitslosenrate unter Ausländern läßt Wehrmann auf eine Belastung dieses Sozialversicherungszweigs durch die Gastarbeiter schließen. ${ }^{195}$ Dagegen ist einzuwenden, daß nicht alle Arbeitslosen auch Leistungen aus der Arbeitslosenversicherung erhalten, da der Anspruch auf Arbeitslosengeld an die Erfüllung einer zwölfmonatigen Anwartschaft gebunden ist und auch darüber hinaus die Anspruchsdauer an die Beschäftigungszeit geknüpft ist. ${ }^{196}$ Die Fluktuation der Ausländer führt dazu, daß der Anteil der Arbeitslosen, die keinen Anspruch auf Arbeitslosengeld haben, bei ihnen wesentlich höher ist als bei Deutschen. ${ }^{197}$ Der höhere Anteil von Arbeitslosen unter den Ausländern zieht also nicht zwangsläufig auch einen höheren Anteil von Empfängern von Arbeitslosengeld nach sich. Die oben angeführte Auswertung von MikroDaten für 1984 durch Ulrich ergab dennoch höhere durchschnittliche Leistungen an Gastarbeiter als an Deutsche. Da sich das Verhältnis zwischen den Arbeitslosenquoten von Ausländern und Deutschen seither nur wenig verändert hat, ist unter der Annahme gleichbleibender Raten von anspruchsberechtigten Arbeitslosen davon auszugehen, daß die Gastarbeiter nach wie vor eine Belastung der Arbeitslosenversicherung darstellen.

Die Nettoposition der Gastarbeiter in der Gesetzlichen Krankenversicherung herauszufinden ist weitaus schwieriger als in der Arbeitslosenversicherung. Die Leistungen sind nicht proportional zu den Beiträgen und außerdem können mit den Familienangehörigen auch Personen Leistungen beziehen, die gar keine Beiträge entrichten. Folgende Einflußfaktoren sind zu berücksichtigen: das Verhältnis der Zahl der Beitragszahler zur Zahl der Anspruchsberechtigten, die durchschnittliche Höhe der Beiträge, der Anteil der kranken Personen an allen Anspruchsberechtigten und die durchschnittlichen Kosten pro Krankheitsfall. ${ }^{198}$

Das Verhältnis von Beitragszahlern zu Anspruchsberechtigten war in den sechziger Jahren zunächst bei den Ausländern sehr günstig. Der Anteil der ausländischen Beitragszahler an allen Beitragszahlern lag etwa doppelt so hoch wie der Anteil der Ausländer an der Bevölkerung. Mit dem in den siebziger Jahren verstärkt einsetzenden Familiennachzug näherte sich die Relation von Beitragszahlern zu Anspruchsberechtigten bei den Ausländern aber immer mehr derjenigen der Deutschen an und unterschied sich Mitte der achtziger Jahre nicht mehr wesentlich von ihr. ${ }^{199}$

Die durchschnittliche Beitragshöhe als zweite Einflußgröße ist abhängig vom durchschnittlichen Einkommen, das nach den oben angeführten Resultaten der Analyse der Daten des

195 Vgl. Wehrmann (1989) S. 335ff.

196 Vgl. § 106 AFG. Ausländische Arbeitnehmer haben zwar - wie Wehrmann (1989) S. 336 betont - über bi- und multilaterale Verträge oft die Möglichkeit sich Anwartschaftzeiten aus den Herkunftsländern anrechnen zu lassen, dennoch liegt der Anteil von Arbeitslosengeldempfängern unter den Arbeitslosen bei Ausländern weit niedriger als bei Deutschen. Vgl. Bach (1987) S. 171.

197 Bach (1987) S. 171 gibt den Anteil der Arbeitslosengeldempfänger an allen arbeitslosen Ausländern mit 25,9 Prozent, für Deutsche aber mit 35,1 Prozent im Jahr 1984 an.

198 Vgl. Ulrich (1994a) S. 73.

199 Vgl. Ulrich (1994a) S. 74. Wehrmann (1989) S. 321ff. weist darauf hin, daß die im Heimatland verbliebenen Familienangehörigen aufgrund internationaler Sozialversicherungsabkommen Ansprüche gegen die Gesetzlichen Krankenkassen haben. Allerdings bemessen sich diese Ansprüchen hinsichtlich Art und Umfang nach dem jeweiligen Krankenversicherungsrecht des Auslands und sind daher regelmäßig niedriger als nach deutschem Recht. 
Sozio-Ökonomischen Panels durch Ulrich und auch nach den in Abschnitt 3.3.1 dargestellten Ergebnissen für Gastarbeiter deutlich unter dem für Deutsche liegt. Demnach wären bei annähernd gleichem Verhältnis von Beitragszahlern zur Bevölkerung niedrigere durchschnittliche Beitragszahlungen der Gastarbeiter zu erwarten. Wie aus den Tabellen D.2 und D. 3 zu entnehmen ist, lagen die durchschnittlichen Beiträge der Gastarbeiter zur Gesetzlichen Krankenversicherung aber sowohl auf Haushalts- als auch auf Personenebene deutlich über denen der Deutschen. Ulrich erklärt diesen scheinbaren Widerspruch mit dem höheren Anteil von Rentnern unter den Deutschen, da diese keine oder nur geringe Beiträge zahlen. ${ }^{200}$ Diese Deutung ist allerdings nicht ausreichend, weil auch die altersstrukturbereinigten Beiträge der Gastarbeiter über denen der Deutschen liegen. Eine weitere Ursache für die höheren Beiträge der Gastarbeiter liegt möglicherweise in einer geringeren Privatversicherungsquote. Zum einen sind Gastarbeiter in der nicht versicherungspflichtigen, überdurchschnittlich verdienenden Beamtenschaft kraß unterrepräsentiert. Zum anderen lohnt sich der Wechsel zu einer privaten Krankenversicherung für Gastarbeiter mit Einkommen über der Beitragsbemessungsgrenze weniger häufig als für Deutsche, weil sie aufgrund der höheren Kinderzahl stärker von der Familienmitversicherung der Gesetzlichen Krankenkassen profitieren.

Auf der Beitragsseite ist also ein positiver Einfluß der Gastarbeiter festzustellen. Inwiefern er durch eine höhere Inanspruchnahme von Leistungen durch Gastarbeiter kompensiert wird, hängt von den beiden letzten der oben aufgeführten Einflußfaktoren ab, dem Anteil der kranken Personen an allen Anspruchsberechtigten und den durchschnittlichen Kosten pro Krankheitsfall.

Da Daten über den Prozentsatz der Kranken an allen Anspruchsberechtigten nicht verfügbar sind, zieht Ulrich ebenso wie Wehrmann als Ersatzgröße den Krankenstand der erwerbstätigen Personen heran. Auch hier ist wieder ab Mitte der siebziger Jahre eine deutliche Verschlechterung bei den Gastarbeitern zu erkennen. Lag bis dahin der Anteil der arbeitsunfähigen Gastarbeiter unter dem der Deutschen, kehrte sich diese Relation in der folgenden Zeit um. Die Ursache des hohen Krankenstandes der Gastarbeiter ist teilweise in der Beschäftigungsstruktur der Gastarbeiter zu sehen, die eher als Deutsche gesundheitsbelastende Tätigkeiten ausüben.201 Allerdings ist die starke Zunahme seit Mitte der siebziger Jahre dadurch ebenso wenig zu erklären wie durch eine angeblich höhere Mißbrauchsneigung der Gastarbeiter.

Einen gegenläufigen Einfluß auf die Inanspruchnahme von Leistungen der Gesetzlichen Krankenversicherung hat die günstigere Altersstruktur der Gastarbeiter. Zum einen liegt nämlich die Häufigkeit von Erkrankungen bei älteren Personen wesentlich höher als bei jüngeren, so daß der höhere Anteil der Älteren unter den Nicht-Erwerbstätigen bei den Deutschen die Krankenhäufigkeit gegenüber dem nur auf Erwerbstätige bezogenen Krankenstand erhöht. Zum anderen liegen die Behandlungskosten pro Erkranktem für ältere Personen höher als für Jüngere. ${ }^{202}$ Damit kann davon ausgegangen werden, daß die durchschnittlichen Kosten pro Krankheitsfall bei Gastarbeitem unter denen für Deutsche liegen.

Geht man davon aus, daß sich die Kostenbelastung durch eine höhere Krankheitshäufigkeit von Gastarbeitern und die geringeren Kosten pro Krankheitsfall in etwa ausgleichen, so daß auf der Leistungsseite kein großer Unterschied zu den Deutschen besteht, sind die höheren Beitragszahlungen der Gastarbeiter ausschlaggebend für einen insgesamt positiven Einfluß der

200 Vgl. Ulrich (1994a) S. 73.

201 Vgl. Wehrmann (1989) S. 331f. und Rürup/Sesselmeier (1994) S. 74.

202 Vgl. Fickel (1994) S. 47ff. und Wehrmann (1989) S. 329f. 
Gastarbeiter auf die Finanzsituation der Gesetzlichen Krankenversicherung. Damit kann aufgrund der ausländischen Versicherten der Beitragssatz zur Gesetzlichen Krankenversicherung geringer ausfallen, als wenn nur Deutsche Mitglieder in diesem Sozialversicherungszweig wären. Alternativ wäre die Ansparung eines Kapitalstocks möglich.

Allerdings muß darauf hingewiesen werden, daß dieses Ergebnis anhand von Mikrodaten für ein bestimmtes Jahr gewonnen und die weitere Entwicklung in einzelnen Jahren durch Makrozeitreihen abgeschätzt wurde. Diese Vorgehensweise erlaubt nur Aussagen über die Auswirkungen der Gastarbeiter in diesem Jahr und sagt wenig über ihre periodenübergreifende Gesamtwirkung unter Einschluß aller vergangenen und zukünftigen Jahre. Wehrmann unterscheidet in diesem Zusammenhang zwischen der Liquiditätsebene, also der Beeinflussung der Finanzsituation in einem bestimmten Kalenderjahr, und der Rentabilitätsebene, d.h. der Gegenüberstellung der Barwerte der in der Vergangenheit von den Gastarbeitern entrichteten und zukünftig noch zu zahlenden Krankenkassenbeiträge mit den von ihnen empfangenen und noch zu empfangenden Leistungen. ${ }^{203}$ Für die Arbeitslosenversicherung spielte die Rentabilitätsebene keine Rolle, da dort kaum ein Risikoausgleich zwischen verschiedenen Altersgruppen stattfindet. ${ }^{204}$ Für die Gesetzlichen Krankenkasse ist aber festzuhalten, daß Rentner geringere Beiträge bezahlen als Jüngere, obwohl die durchschnittliche Höhe der Leistungen an ältere Personen um ein Vielfaches über den Leistungen an Jüngere liegt. ${ }^{205}$

Der Unterschied zwischen Liquiditätsebene und Rentabilitätsebene wird deutlich, wenn man die Wirkung einer einmaligen Einwanderungswelle von kinderlosen Personen am Beginn ihrer Erwerbskarriere betracht, die sich nur in ihrem Alter von den Einheimischen unterscheiden. Zunächst werden diese Personen in unterdurchschnittlichem Umfang Leistungen beanspruchen, da sie noch recht jung und gesund sind. Im Alter werden dann ihre durchschnittlichen Beiträge mit dem im Rentenalter geringeren Einkommen sinken und gleichzeitig die durch sie verursachten Kosten steigen. Damit ergibt sich für die ersten Jahrzehnte ein positiver Einfluß auf die Finanzlage der Gesetzlichen Krankenversicherung, der dann einem zunehmend negativen Einfluß weicht. Entscheidend für die Rentabilitätsebene ist, ob der Barwert der Einzahlungs- und Leistungsdifferenzen gegenüber Deutschen positiv oder negativ ist. Eine in allen Perioden positive Wirkung ist bei der hier einfachheitshalber unterstellten Homogenität von Immigranten und Einheimischen nur dann zu erreichen, wenn der zugewanderte Bevölkerungsteil dauerhaft eine jüngere Altersstruktur aufweist als die Einheimischen. ${ }^{206}$ Vorteilhaft aus Sicht der Finanzlage der Gesetzlichen Krankenkasse wäre daher eine Rückwanderung im höheren Alter, da die implizit gebildeten Altersrückstellungen im Gegensatz zu Anwartschaften aus der Gesetzlichen Rentenversicherung ersatzlos verfallen.

203 Vgl. Wehrmann (1989) S. 281. Wehrmann sieht die Notwendigkeit der Betrachtung der Rentabilitätsebene allerdings nur für die Gesetzliche Rentenversicherung und übersieht, daß das Risiko des Alterns auch in der Gesetzlichen Krankenversicherung versichert wird. Vgl. Wehrmann (1989) S. 324.

Insofern ältere Personen eine höhere Arbeitslosenrate aufweisen und aufgrund der oben beschriebenen beitragszeitabhängigen Dauer des Anspruchs auf Zahlung von Arbeitslosengeld auch einen höheren Anteil von Arbeitslosengeldempfängern an allen Arbeitslosen haben, findet auch in diesem Versicherungszweig ein Risikoausgleich zwischen verschiedenen Altersgruppen statt.

205 Das in der Gesetzlichen Krankenversicherung praktizierte Umlageverfahren führt deshalb in Verbindung mit dem zunehmenden Durchschnittsalter wie in der Gesetzlichen Rentenversicherung auch hier $\mathrm{zu}$ in der Zukunft steigenden Finanzierungslasten.

Als Vergleichsmaßstab ist die Altersstruktur der Einheimischen heranzuziehen, die sich bei einer Erhöhung des Kinderlastenausgleichs ergeben würde. Anderenfalls wird neben dem Bevölkerungsstruktureffekt auch der Bevölkerungszuwachseffekt erfaßt. 
Die Auswirkungen von Eingewanderten im Rahmen der Gesetzlichen Pflegeversicherung läßt sich nicht mit Hilfe von Vergangenheitsdaten abschätzen, weil dieser Zweig des Sozialversicherungssystems erst 1995 eingeführt wurde. Grundsätzlich sind aber ähnliche Wirkungen wie bei der Gesetzlichen Krankenversicherung zu vermuten, weil Beitragszahlungen und Leistungsansprüche einem vergleichbaren Muster folgen. Allenfalls wird eine jüngere Altersstruktur der Ausländer bei diesem Versicherungszweig noch positivere Konsequenzen für die Einheimischen haben, weil Pflegefälle noch stärker als Krankheitsfälle auf ältere Personen konzentriert sind.

Ähnlich wie für die Arbeitslosenversicherung besteht auch für den vierten Zweig des Sozialversicherungssystems, die Gesetzliche Rentenversicherung, eine - wenngleich stärker eingeschränkte - Proportionalität zwischen Beiträgen und Leistungen. Allerdings tritt hier der Unterschied zwischen der auf ein bestimmtes Jahr bezogenen Liquiditätsbetrachtung und der Rentabilitätsebene besonders deutlich hervor, weil Beiträge und Rentenzahlungen um Jahrzehnte auseinanderfallen können.

Rehfeld untersucht die Liquiditätsebene für das Jahr 1989 und findet einen Überschuß der Beiträge der Ausländer (12,8 Mrd DM) über ihre Renten (3,7 Mrd DM) von 9,1 Mrd DM.207 Für die vor 1989 liegenden Jahre kann aufgrund der Altersstruktur der Ausländer von einem noch günstigeren Verhältnis von Beiträgen zu Renten ausgegangen werden. Aus dem gleichen Grund ist aber für die Zukunft mit einem geringeren Überschuß zu rechnen. Wenn die Einwanderungszahlen nicht exponential zunehmen, wird der Anteil der über 60jährigen Ausländer an allen Ausländern steigen. Die finanzielle Entlastung der Gesetzlichen Rentenversicherung durch die Gastarbeiter in den vergangenen drei Jahrzehnten muß als einmaliger Liquiditätsschub gewertet werden, dessen Wirkung in dem Maße nachläßt, wie sich die Altersstrukturen von versicherten Gastarbeitern und Deutschen annähern. ${ }^{208}$ Diese Wirkung eines positiven Wanderungssaldos ist wiederum vergleichbar mit der Wirkung eines zeitlich versetzten Geburtenanstiegs, so daß die altersstrukturbedingten Effekte der Einwanderung hier nicht betrachtet zu werden brauchen. ${ }^{209}$

Für den Vergleich mit dem Instrument des Kinderlastenausgleichs auf der Rentabilitätsebene ist vielmehr entscheidend, ob Unterschiede zwischen den Beitragszahlungen und Rentenhöhen von Gastarbeitern und Deutschen gleicher Altersklassen bestehen. Als Referenzgröße werden hier also Beitrags- bzw. Rentenzahlungen von Deutschen und Gastarbeitern gleichen Alters herangezogen und nicht wie bei Wehrmann (1989) der Kapitalwert der Ein- und Auszahlungen von Gastarbeitern gewählt.

Der Kapitalwert weist als Vergleichsmaßstab mehrere Schwachpunkte auf. So ist er aufgrund der großen Zeiträume stark abhängig von dem gewählten Diskontierungszinssatz, der mehr oder weniger willkürlich festgelegt werden muß. Weiterhin wird nicht berücksichtigt, daß

\footnotetext{
207 Vgl. Rehfeld (1991) S. 491.
}

208 Dieser Liquiditätsschub hat möglicherweise mit zu den Leistungsausweitungen beigetragen, die bis in die siebziger Jahre hinein vorgenommen wurden und in Zukunft die demographisch bedingten Finanzierungsprobleme noch verschärfen. Vgl. Wehrmann (1989) S. 263 und Ulrich (1994a) S. 77.

In Abschnitt 3.3.2 des Teils B dieser Arbeit wurde festgestellt, daß die Wirkung einer permanenten Einwanderung von 15jährigen in etwa der Wirkung eines gleich hohen Geburtenanstiegs vor 15 Jahren entspricht, wenn die altersspezifischen Fertilitäts- und Mortalitätsraten von Zuwanderem und Einheimischen identisch sind. 
der Kapitalwert entscheidend durch die gegenwärtige Rechtslage beeinflußt wird und Änderungen der Leistungsansprüche wie durch das Rentenreformgesetz 1992 den Barwert der Rentenanwartschaften deutlich reduzieren können. Weitere Änderungen des Rentenrechts sind aufgrund der in Teil B beschriebenen Entwicklung der Finanzlage der Gesetzlichen Rentenversicherung aber schon heute absehbar. Schließlich dürfte nicht nur der Kapitalwert für Gastarbeiter negativ sein - Wehrmann berechnet einen Fehlbetrag von 62,2 Mrd DM zum 1. Januar 1986 - sondern auch für Deutsche. Der gemeinsame Fehlbetrag wird zum Teil über den Bundeszuschuß finanziert, also aus Steuermitteln, an deren Aufbringung auch die Gastarbeiter beteiligt sind, und zum anderen Teil müssen Deckungsmöglichkeiten noch gesucht werden. Sicher ist aber, daß die ausländischen Mitglieder der Gesetzlichen Rentenversicherung an der Schließung dieser Deckungslücke ebenso beteiligt werden wie deutsche Mitglieder. Das Vorgehen von Wehrmann impliziert dagegen, daß allein die Deutschen zur Finanzierung ihrer eigenen Deckungslücke und zusätzlich derjenigen der Gastarbeiter herangezogen werden. ${ }^{210}$

Nachdem die Beitrags- bzw. Rentenzahlungen von Deutschen gleichen Alters als geeignetes Referenzmodell für die Messung des Einflusses der Gastarbeiter auf die Rentabilitätsebene der Gesetzlichen Rentenversicherung erkannt wurden, ist nun festzustellen, ob Beiträge und Renten für Deutsche und Gastarbeiter gleichen Alters voneinander abweichen und welche Gründe hierfür anzuführen sind. Bezüglich der Beitragsseite ist festzustellen, daß nach den in Tab. D.3 aufgeführten Ergebnissen von Ulrich Gastarbeiter mit 1.365 DM altersstrukturbereinigt höhere Beiträge zur Gesetzlichen Rentenversicherung leisten als Deutsche (1.201 DM). Ausschlaggebend hierfür ist die 1984 noch höhere Erwerbsbeteiligung der Gastarbeiter, die sich inzwischen derjenigen der Deutschen allerdings weitgehend angeglichen hat, ${ }^{211}$ so daß nicht mehr von nennenswerten Unterschieden auf der Beitragsseite ausgegangen werden kann.

Die Ausgabenseite wird beeinflußt durch die durchschnittlich etwas geringere Lebenserwartung der Ausländer, die zu einer geringeren durchschnittlichen Rentenbezugsdauer führt. Ein weiterer Faktor, der die durchschnittlichen Ausgaben der Gesetzlichen Rentenversicherung für Renten an Gastarbeiter gegenüber den Renten für Deutsche senkt, ist der geringere Prozentsatz der Versicherten, der die Anspruchsvoraussetzungen erfüllt. Außerdem werden die Rentenansprüche von Gastarbeitern, die ihren Wohnsitz in einem Land haben, mit dem kein entsprechendes zwischenstaatliches Abkommen besteht, auf 70 Prozent gekürzt (vgl. §113 SGB VI). Insgesamt ist daher eher mit einer leichten Entlastung der Gesetzlichen Rentenversicherung zu rechnen, wenn anstelle von Deutschen Gastarbeiter gleichen Alters Mitglieder sind.

210 Wehrmann (1989) S. 319 schreibt selbst, daß die deutschen Versicherten das Defizit der Ausländer nur dann zu tragen haben, wenn sich die Zahl der ausländischen Beitragszahler reduziert. "Bleibt dagegen der Ausländeranteil an der Versichertengesamtzahl konstant, finanzieren die in der Folgezeit beschäftigten Ausländer allein, d.h. ohne Belastung der deutschen Versicherten, die Rentenzahlungen an ihre 'Vorgänger' und damit auch das auf diese entfallende Defizit - allerdings nur unter der Voraussetzung, $\mathrm{da} B$ das durchschnittliche Defizit bzw. die Eigenfinanzierungsquote bei den Deutschen und Ausländern gleich hoch ist." Wehrmann (1989) S. 319.

Vgl. Franz (1993) S. 7. 


\subsubsection{Der Einfluß der Aussiedler auf die Sozialversicherungen}

Bisher sind nur die Wirkungen der Einwanderung von Ausländern bzw. von Gastarbeitern auf das Sozialversicherungssystem der Bundesrepublik Deutschland dargestellt worden. Im folgenden soll analog hierzu nun auch die Gruppe der Aussiedler in die Untersuchung einbezogen werden.

Die rechtliche Stellung von Aussiedlem unterscheidet sich von derjenigen der Ausländer insofern, als erstere mit ihrer Anerkennung als Aussiedler die deutsche Staatsbürgerschaft besitzen und somit auch alle daraus resultierenden Rechte. Die wichtigsten Unterschiede in ökonomischer Hinsicht ergeben sich daraus, daß Aussiedler im Gegensatz zu Ausländern Anspruch auf eine Reihe von Eingliederungshilfen haben und in der Gesetzlichen Rentenversicherung so gestellt werden, als ob sie Beiträge geleistet hätten, die von dem ihrem Qualifikationsniveau entsprechenden Einkommen in Deutschland zu zahlen gewesen wären. ${ }^{212}$

Welche Wirkung aufgrund dieser Rechtslage sowie der demographischen und ökonomischen Charakteristika von der Zuwanderung von Aussiedlem auf das System der Sozialversicherungen in Deutschland ausgeht, wurde von Buttler, Winterstein und Jäger untersucht. ${ }^{213}$ Naturgemäß ist das Ergebnis der Untersuchung stark von den Annahmen abhängig, von denen hier aber nur die wichtigsten kurz angeführt werden können. Die Autoren gehen von einem Zuzug von 200.000 Aussiedlern jährlich in der Zeit von 1991 bis 2001 aus. In den Jahren 1989 und 1990 liegt die Zahl höher, nach 2001 sinkt sie auf 50.000 ab und im Jahr 2010 ist das Wanderungspotential von etwa 3,5 Mio Personen erschöpft. Weiterhin wird unterstellt, daß die junge Altersstruktur der Aussiedler im Jahr 1988 ebenso wie die um 30 Prozent über dem Wert der einheimischen Bevölkerung liegende Fruchtbarkeit in Zukunft konstant bleiben. ${ }^{214}$ Hinsichtlich der Arbeitslosenquote wird ausgehend von einem sehr hohen Niveau von 45 Prozent im Jahr 1989 eine Angleichung an das Niveau der Einheimischen bis zum Ende dieses Jahrzehnts unterstellt. Erwerbstätige Aussiedler haben durchschnittlich das gleiche Einkommen wie Einheimische.

Aus dieser Konstellation ergeben sich für die einzelnen Versicherungszweige unterschiedliche Konsequenzen.

Für die Arbeitslosenversicherung entsteht zunächst durch die weit überdurchschnittliche Arbeitslosenquote der Aussiedler eine hohe Belastung von kumuliert etwa 11 Mrd DM von 1990 bis zum Jahr 2000. Erst mit zunehmender Assimilation kommen Buttler, Winterstein und Jäger für die Zeit nach der Jahrtausendwende zu einem positiven Saldo von Beitragseinnahmen und Unterstützungszahlungen. ${ }^{215}$

In der Gesetzlichen Krankenversicherung sieht die Situation günstiger aus, weil wie anfangs bei den Gastarbeitern der günstigere Altersaufbau und die günstigere Erwerbsstruktur zu

212 Diese Regelung führte in Verbindung mit der damals über dem Bundesdurchschnitt liegenden fachlichen Qualifikation der Aussiedler dazu, daß bei den Rentenzugängen des Jahres 1988 Aussiedler gegenüber dem Durchschnitt um 14 Prozent höhere Renten erhielten. Vgl. Winterstein (1991) S. 135.

213 Vgl. Buttler/Winterstein/Jäger (1989) und den Beitrag von Winterstein (1991), der sich auf diese Studie bezieht.

214 Vgl. Buttler/Winterstein/Jäger (1989) S. 233.

215 Vgl. Buttler/Winterstein/Jäger (1989) S. 235. 
steigenden jährlichen Einzahlungsüberschüssen führen, die sich für die letzte Dekade dieses Jahrhunderts auf über 13 Mrd DM kumulieren.

Die gleichen Gründe haben Einzahlungsüberschüsse auch in der Gesetzlichen Rentenversicherung zur Folge. Ohne Berücksichtigung der Rentenreform 1992 und unter der Annahme durchschnittlicher Häufigkeit des Rentenbezugs und durchschnittlicher Rentenhöhe ergibt sich für das Jahrzehnt von 1991 bis 2000 ein Überschuß von etwa 40 Mrd DM, der erst nach dem Jahr 2030 in ein jährliches Defizit umschlägt. ${ }^{216}$

Die insgesamt gegenüber den Gastarbeitern günstiger erscheinende Situation ist einerseits auf das höhere Qualifikationsniveau der Aussiedler zurückzuführen, ${ }^{217}$ andererseits muß aber auch berücksichtigt werden, daß hier der besonders vorteilhafte Zeitraum zu Beginn der Auswirkungen einer Einwanderungswelle betrachtet wird, während die Hauptwanderungswelle für die Gastarbeiterbevölkerung schon Jahrzehnte zurückliegt.

Insgesamt kommen Buttler, Winterstein und Jäger zu dem Ergebnis eines eindeutig positiven Einflusses der Aussiedler auf das System der Sozialversicherungen. ${ }^{218}$

\subsubsection{Bedeutung für ein Einwanderungsgesetz}

In bezug auf die Arbeitslosenversicherung wirkte sich in der jüngeren Vergangenheit v.a. die häufigere Inanspruchnahme der Leistungen durch Einwanderer negativ aus. Die Einkommenshöhe spielt dagegen kaum eine Rolle, da die Leistungshöhe weitgehend proportional zu den gezahlten einkommensabhängigen Beiträgen ist. Wie schon zur Minderung von negativen Arbeitsmarkteffekten auf Einheimische und bezüglich der Finanzlage der Gebietskörperschaften ist daher eine Selektion der Ausländer vorteilhaft, die eine schnelle Eingliederung in den Arbeitsmarkt erwarten läßt und eine geringe Arbeitslosenquote sicherstellt. Voraussetzung der Zuwanderung sollte daher die Betätigung des Einwanderers auf einem Arbeitsmarktsegment sein, daß nicht durch strukturelle Arbeitslosigkeit gekennzeichnet ist. Dadurch kann auch weitgehend verhindert werden, daß im Zuge einer Substitution von Einheimischen durch Einwanderer zwar die Arbeitslosenquote der Zuwanderer niedrig ist, durch die Zuwanderung aber die Arbeitslosenquote der Einheimischen ansteigt. Besonders bei dauerhaft Einwandernden ist darüber hinaus eine hohe regionale und berufliche Mobilität positiv zu bewerten, da dadurch die Anpassung an die sich im Strukturwandel ständig ändernden Anforderungen an die Arbeitskräfte reibungsloser erfolgen kann. Bei temporären Einwanderern ist bei konsequenter Verwirklichung des Rotationsprinzips eine schnelle Anpassung an die im Konjunkturverlauf schwankende Beschäftigungssituation möglich, indem beispielsweise bei Vorgabe einer bestimmten Höchstaufenthaltsdauer (z.B. ein Jahr) die Rückwanderer nicht vollständig durch neue temporäre Zuwanderer ersetzt werden (Konjunkturpufferfunktion). Gleichzeitig kann die Aufenthaltserlaubnis bei ihnen an das Vorliegen eines Beschäftigungsverhältnisses geknüpft werden. Weber/Straubhaar weisen darauf hin, daß allgemein die Informiertheit über die Möglichkeiten der Inanspruchnahme von Sozialleistungen mit der Aufenthaltsdauer steigt und

\footnotetext{
216 Vgl. Buttler/Winterstein/Jäger (1989) S. 235.

217 Zum Qualifikationsniveau der Aussiedler vgl. Ulrich (1994b).

218 Diese Tendenz wird von Winterstein (1991) S. 142 nochmals bestätigt.
} 
gleichzeitig auch die Wahrscheinlichkeit der Anspruchsberechtigung durch die Erfüllung von Anwartschaftszeiten zunimmt. ${ }^{219}$

Hinsichtlich der Einnahmenseite der Gesetzlichen Krankenversicherung und der Gesetzlichen Pflegeversicherung ist eine Auswahl der Zuwanderer nach ihrem erwarteten durchschnittlichen Einkommen vorteilhaft, weil die Höhe der Beiträge einkommensabhängig ist. $\mathrm{Da}$ das gleiche Auswahlkriterium sich auch günstig auf die Haushalte der Gebietskörperschaften auswirkt, kann auf die Ausführungen unter 3.3.2.3 verwiesen werden. Als besonders vorteilhaft wurde dort die Zuwanderung von Gruppen mit hoher Erwerbsbeteiligung, niedriger Arbeitslosenquote und hohem Qualifikationsniveau herausgestellt. Ein weiterer Vorteil einer hohen Erwerbsbeteiligung ergibt sich dadurch, daß so das Verhältnis von Beitragszahlern zu Anspruchsberechtigten günstig bleibt. Bei einem hohen Anteil nicht erwerbstätiger Zuwanderer hat die Familienmitversicherung dagegen eine ungünstige Versichertenstruktur zur Folge. Aus dieser Sicht ist daher der Familiennachzug aber auch permanente Einwanderung weniger günstig als temporäre Zuwanderung, die sich gerade durch einen hohen Anteil von Erwerbstätigen und wenige mitversicherte Familienangehörige auszeichnet. ${ }^{220}$ Weiterhin wirkt sich ein niedriges Alter der Zuwanderer positiv aus, weil sowohl die Krankheitshäufigkeit als auch die Behandlungskosten pro Erkranktem für junge Personen wesentlich niedriger liegen als für Ältere. Die dauerhaft junge Altersstruktur der temporären Einwanderer ist hier vorteilhafter als permanente Einwanderung, weil bei letzterer im Laufe der Jahre auch dann eine Alterung eintritt, wenn die jeweils neuen Immigranten eines Jahres sehr jung sind. Eine Selektion der Einwanderer nach ihrem Gesundheitszustand könnte zwar niedrigere Ausgaben der Krankenkassen zur Folge haben, wäre aber gleichzeitig mit hohen Transaktionskosten verbunden. ${ }^{221}$

In der Gesetzlichen Rentenversicherung wirkt sich eine hohe Erwerbsbeteiligung der Zuwanderer positiv aus, weil eigene Rentenansprüche auf die Witwenrente angerechnet werden. Bei temporären Migranten werden häufiger die Anspruchsvoraussetzungen für den Bezug einer Rente nicht erfüllt sein als bei dauerhaft Einwandernden. Dennoch ist aus Sicht der Rentenkasse permanente Einwanderung weitaus vorteilhafter als temporäre Migration. Bei permanenter Einwanderung werden nämlich die Kinder der Einwanderer die Renten ihrer Eltern bezahlen und die Beitragszahlungen der Eltern können insofern als gegenleistungsloser ${ }^{222}$ Liquiditätsschub für die Gesetzliche Rentenversicherung gewertet werden. Wenn ein dauerhafter Einwanderer die Altergrenze erreicht, werden nicht die Einheimischen zur Finan-

219 Vgl. Weber/Straubhaar (1994) S. 7.

220 Dies gilt nur insoweit die im Ausland verbliebenen Familienangehörigen temporärer Einwanderer nicht durch zwischenstaatliche Verträge auch Ansprüche gegenüber der Gesetzlichen Krankenversicherung in gleicher Höhe haben wie inländische Familienangehörige. Vgl. hierzu den vorangegangenen Unterabschnitt.

221 Auch aus ethischer Sicht kann eine an den Gesundheitszustand der Antragsteller geknüpfte Erlaubnis der Einwanderung problematisch erscheinen.

222 Der Begriff "gegenleistungslos" bezieht sich hier auf die Tatsache, daß die Einheimischen von den Beiträgen der ersten Generation der Einwanderer in Form von Beitragssatzsenkungen profitieren, ohne daB sie dafür unter bestimmten Voraussetzungen zu irgendeinem Zeitpunkt einen Transfer an die Einwanderer oder deren Nachkommen leisten müssen. Die Einwanderer selbst erwerben natürlich durch ihre Beitragszahlungen einen Anspruch auf Gegenleistung in Form von späteren Rentenzahlungen. 
zierung seiner Rente herangezogen, sondern seine in Deutschland geborenen Kinder. ${ }^{223}$ Der gleiche Effekt ergibt sich zeitverschoben auch bei einer steigenden Fertilität der Einheimischen und stellt die Ursache der entlastenden Wirkung demographischer Instrumente auf die Finanzlage der Gesetzlichen Rentenversicherung dar. Bei temporärer Migration verbleiben die Kinder der Einwanderer dagegen überwiegend im Herkunftsland oder wandern mit ihren Eltern dorthin zurück. Sie werden also als Erwerbstätige keine Beiträge zur deutschen Rentenversicherung leisten, so daß die Renten der temporären Migranten, die ja auch nach ihrer Rückkehr größtenteils einen Anspruch auf Leistungen der Gesetzlichen Rentenversicherung besitzen, durch dann in Deutschland erwerbstätige Migranten erbracht werden müssen. Reichen diese Beitragszahlungen der vorübergehend Eingewanderten zur Deckung der Rentenansprüche ihrer Vorgänger nicht aus, muß auf die Beitragszahlungen der Einheimischen zurückgegriffen werden. Während bei permanenter Immigration also ein ständiger gegenleistungsloser Einzahlungsstrom in Höhe der Beitragszahlungen der ersten Generation der Einwanderer den einheimischen Mitgliedern der Gesetzlichen Rentenversicherung zugute kommt, ist bei temporärer Migration nur für den Fall einer Ausweitung der Zahl der in Deutschland anwesenden Einwanderer mit entlastenden, im Fall eines Rückgangs aber mit belastenden Effekten für die Einheimischen zu rechnen. In bezug auf die Gesetzliche Rentenversicherung ist dauerhafte Einwanderung daher eindeutig einer temporären Wanderung vorzuziehen.

\subsection{Der Einfluß der Einwanderer auf die Produktivitätsentwicklung}

Bisher sind mit den Auswirkungen der Einwanderung auf die Arbeits- und Kapitaleinkommen der Einheimischen sowie die öffentlichen Haushalte Beeinflussungen des Niveaus des ProKopf-Einkommens der Einheimischen durch die Einwanderer behandelt worden. Langfristig wird das Pro-Kopf-Einkommen in einer Volkswirtschaft aber auch durch eine Veränderung der Wachstumsraten des Einkommens, d.h. v.a. durch die Produktivitätsentwicklung, bestimmt. Verändern die spezifischen Charakteristika der Einwanderer den Verlauf der Produktivitätsentwicklung und verändert sich dementsprechend das durchschnittliche Einkommen der Einheimischen, dann entstehen letzteren Kosten in Höhe des Barwerts einer Abnahme des ProKopf-Einkommens bzw. Nutzen in Höhe des Barwerts einer Zunahme. ${ }^{224}$ Die Produktivitätsentwicklung hängt von einer Vielzahl von Faktoren ab, zu denen neben dem Kapitalbestand pro Erwerbstätigem, der in Abschnitt 4.2 untersucht wurde, dem Humankapitalbestand und der Effizienz, mit der diese Kapitalarten eingesetzt werden, auch die eingesetzte Produktionstechnik und Organisationsform gehören. In diesem Abschnitt soll untersucht werden, welchen Einfluß Einwanderer langfristig auf die Produktivitätsentwicklung ausüben, indem sie die Produktionsfunktion durch technischen und organisatorischen Wandel verändern.

223 Insofern die Fertilität der permanenten Einwanderer höher liegt als die der Deutschen, stellen sogar die Beitragszahlungen der zweiten Generation noch teilweise einen Transfer zugunsten der Einheimischen dar, wenn die durchschnittliche Beitragshöhe beider Gruppen gleich ist.

224 Hier wird angenommen, daß keine vollständige Aneignung der Produktivitätsänderung durch die Einwanderer erfolgt, sondem Einheimische und Zuwanderer gleichermaßen von ihr betroffen sind. 


\subsubsection{Theoretische Analyse der Produktivitätseffekte}

Grundsätzlich sind zwei Wege denkbar, auf denen Immigranten die Produktivitätsentwicklung in dem Einwanderungsland beeinflussen können. Zum einen können sie in ihrem Herkunftsland bereits vorhandenes technisches und organisatorisches Wissen in das neue Heimatland übertragen und zum anderen können sie die Rate, mit der neues Wissen im Einwanderungsland geschaffen wird, senken oder erhöhen.

Die Bedeutung von Migranten als Transferträgern von Wissen hängt von verschiedenen Faktoren ab. Je höher der Wissensstand im Herkunftsland im Vergleich zum Zielland ist, desto höher ist ceteris paribus die Wahrscheinlichkeit eines Wissens- oder Technologietransfers. Diese Wahrscheinlichkeit steigt weiterhin mit der Unterschiedlichkeit des Wissensbestandes und der Übertragbarkeit auf die lokalen Verhältnisse im Zielland. Auch die Selektion der Einwanderer spielt eine Rolle. Hier kann zunächst eine vorteilhafte Selbstselektion der Einwanderer unterstellt werden, weil die Personen mit einem hohen Stand an übertragbarem Wissen überdurchschnittliche Einkommenserzielungschancen im Aufnahmeland und damit einen hohen Anreiz zur Migration haben. Aber auch der durchschnittliche Bestand der Einwanderer an allgemeinem Humankapital spielt eine Rolle, denn grundsätzlich kann von gut ausgebildeten Personen ein höherer Wissenstransfer erwartet werden als von unqualifizierten Arbeitskräften.

Der Einfluß, den Einwanderer auf die Generierung neuen Wissens im Einwanderungsland haben, ist schon auf theoretischer Ebene schwer faßbar, da die Einflußfaktoren auf die Inventions- und Innovationsrate nicht hinreichend bekannt sind. In der neoklassischen Wachstumstheorie wird die Generierung technischen Fortschritts als exogen angesehen, weshalb sie hier wenig hilfreich ist. In der neuen Wachstumstheorie wird zwar eine Endogenisierung des Entstehungsprozesses von neuem Wissen angestrebt, aber wie in diesen Modellen die Wanderung von Arbeitskräften die Rate der Wissensakkumulation beeinflußt, ist noch weitgehend ungeklärt. ${ }^{225}$ Am ehesten bietet hier die evolutorische Ökonomik einen gedanklichen Ausgangspunkt. ${ }^{226}$ Nach ihr vollzieht sich der technische und organisatorische Fortschritt in einem fortwährenden Prozeß der Variation und Selektion. ${ }^{227}$ Im Wettbewerb stehende Unternehmen verfolgen verschiedene Strategien zur Erreichung des Unternehmensziels. Das unterschiedliche Ergebnis der Strategien führt zu einer Übernahme der besonders erfolgreichen Strategien durch andere Unternehmen, wodurch die durchschnittliche Produktivität aller Unternehmen steigt. Durch die dem Menschen eigene Kreativität werden ständig neue Organisationsformen und Techniken erfunden. Die so fortlaufend entstehende Variation der Wettbewerbsstrategien führt über die Selektion in einem kontinuierlichen Versuch-Irrtum-Verfahren zu ständigen Produk-

225 Vgl. zu dieser Einschătzung auch Straubhaar (1995) S. 248. Erste Gedanken zu den Wirkungen von Immigranten auf Produktionsniveau und Wachstum finden sich bei Ichino (1993) S. 154ff.. Galor/Stark (1994), Grubel (1994) S. 82ff., Felderer (1994b) S. 74ff. und bei Friedberg/Hunt (1995) S. 39ff. Dolado/ Goria/Ichino (1994) kommen anhand eines um Humankapital und Wanderungen erweiterten SolowWachstumsmodells zu dem Ergebnis, daß die Wachstumsrate und das Pro-Kopf-Einkommen durch Einwanderer weniger gemindert werden als durch eine gleich hohe natürliche Bevölkerungszunahme.

226 Ein Hinweis in diese Richtung findet sich bei Simon (1989) S. 166, der ohne auf die evolutorische Ökonomik Bezug zu nehmen feststellt, daß "[v]ariety is a key ingredient of invention."

227 Für eine vertiefende Auseinandersetzung mit diesem evolutorischen Ansatz vgl. etwa Hesse (1990), Vanberg/Kerber (1994) oder Koch (1996). 
tivitätsfortschritten. Wird nun die Variation der verschiedenen Wettbewerbsstrategien durch den andersartigen Erfahrungshintergrund der Einwanderer und ihre Humankapitalausstattung erhöht, kommt es zu einer Beschleunigung des Produktivitätswachstums. Unterstellt wird dabei, daß die durch Einwanderer induzierten neuen Strategien eine gleich hohe Erfolgswahrscheinlichkeit haben wie diejenigen der Einheimischen.

Simon weist darauf hin, daß möglicherweise die Produktivität der Einheimischen auch auf direktem Weg durch die Einwanderer verändert wird. ${ }^{228}$ Einerseits können sie durch die Begegnung mit der abweichenden Lebens- und Arbeitsweise der Immigranten zu produktivitätssteigernden Ideen angeregt werden. Andererseits kann aber die Zusammenarbeit mit vielen an zeitintensive Vorgehensweisen gewöhnte Einwanderern die Produktivität der Einheimischen auch solange reduzieren, bis erstere sich durch Erwerb informellen Humankapitals an die Arbeitsweise der Einheimischen angepaßt haben.

Gemäß diesen Gedankengängen werden also sowohl der Wissenstransfer durch die Immigranten als auch die durch sie erhöhte Wissensakkumulationsrate zu einem Anstieg des Produktivitätswachstums im Einwanderungsland führen. ${ }^{229}$ Allenfalls wenn das Humankapital der Einwanderer sehr gering ist und sie daher weder in nennenswertem Umfang Wissen aus ihren Heimatländern übertragen können noch in durchschnittlichem Ausmaß neue Wettbewerbsstrategien kreieren und eventuell sogar die Produktivität der einheimischen Arbeitskräfte senken, ist mit negativen Auswirkungen auf die Produktivitätsentwicklung zu rechnen.

Zur Vermeidung einer Doppelzählung ist hier nochmals darauf hinzuweisen, daß für die vorliegende Fragestellung nur diejenigen Produktivitätsunterschiede relevant sind, die zu einem von der Situation ohne Einwanderung abweichenden Einkommen der Einheimischen führen. Produktivitätsunterschiede, die sich allein in einem höheren Einkommen der Eingewanderten niederschlagen, sind für die Wohlfahrt der Einheimischen ohne Belang. ${ }^{230}$

\subsubsection{Empirische Größenordnung der Produktivitätseffekte}

Statistisch abgesicherte empirische Belege für die aufgrund der theoretischen Betrachtung vermuteten Produktivitätswirkungen der Einwanderer lassen sich nicht finden, da nicht feststellbar ist, wie die Produktivitätsentwicklung ohne Einwanderung verlaufen wäre. Deshalb muß hier die Anführung einiger Fallbeispiele genügen, die unterstreichen, daß Migranten sowohl als Träger von produktivitätssteigerndem Wissen als auch als Produzenten neuen Wissens in der Vergangenheit für viele Einwanderungsländer eine große Rolle gespielt haben.

Als Beispiel für den Wissenstransfer mag der Weinanbau in der Umgebung von Adelaide im Süden Australiens dienen, der durch die Emigration deutscher Winzer entstanden ist. Grubel führt als Beispiel jüngeren Datums die Errichtung von Lachsfarmen in den Fjorden British Columbias durch norwegische Emigranten in den achtziger Jahren dieses Jahrhunderts an. ${ }^{231}$

228

Vgl. Simon (1989) S. 182ff.

Zu dem gleichen Ergebnis gelangt auch Simon (1989) S. 186.

Die Auswirkungen der Einkommensentwicklung der Eingewanderten auf die Wohlfahrtsposition der Einheimischen, die sich über ihren Einfluß auf die öffentlichen Haushalte ergeben, wurden bereits in Unterkapitel 3.3 behandelt und werden hier daher nicht nochmal aufgegriffen.

Vgl. Grubel (1994) S. 82. 
Auch die Entsendung von Spezialisten in ausländische Produktionsstandorte durch multinationale Unternehmen kann genannt werden. Sie macht einerseits deutlich, daß die modernen Kommunikationsmittel den Wissenstransfer mittels Arbeitskräftewanderung bisher nicht ersetzen konnten und zeigt andererseits auch, daß diese Art des Wissenstransfers offenbar produktivitätssteigernde Wirkungen hervorruft, die ihn trotz der Kosten für das Unternehmen lohnend erscheinen lassen.

Als Hinweis auf eine überdurchschnittliche Leistung von in die USA Eingewanderten in bezug auf die Kreation neuer Wettbewerbsstrategien kann der über dem Prozentsatz der Einheimischen liegende Anteil der Immigranten gesehen werden, die sich in ihrem Gastland selbständig machen. ${ }^{232}$ Die in Tab. D.1 angeführten negativeren Zahlen für Deutschland belegen gleichzeitig die Wirkung unterschiedlicher Selektionsmechanismen.

Aufgrund dieser Indizien kann ein negativer Einfluß der Immigranten auf die Produktivitätsentwicklung mit einiger Wahrscheinlichkeit ausgeschlossen werden. Ob ein positiver Einfluß besteht und wie hoch dieser gegebenenfalls ausfällt, ist bei dem gegenwärtigen Stand der Forschung nicht feststellbar.

\subsubsection{Bedeutung der Produktivitätseffekte für ein Einwanderungs- gesetz}

Die theoretische Analyse der Wirkungen, die Einwanderer über Wissenstransfer sowie über die Erzeugung neuen technischen und organisatorischen Wissens auf das Pro-Kopf-Einkommen der Einheimischen ausüben, steckt noch in den Anfängen. Die empirischen Belege müssen als anekdotenhaft bezeichnet werden. Deshalb steht jede Aussage bezüglich der Konsequenzen, die sich für ein Einwanderungsgesetz ableiten lassen, auf recht unsicherem Fundament.

Dennoch kann die Wahrscheinlichkeit negativer Auswirkungen auf den technischen Fortschritt minimiert werden, wenn eine Selektion der Einwanderer nach dem Humankapitalbestand erfolgt. Möglicherweise können Einwanderer aus wirtschaftlich und kulturell besonders andersartigen Ländern in größerem Umfang verwertbares Wissen aus ihrer Heimat übertragen und durch eine größere Variation der Unternehmensstrategien zu einer höheren Wissensakkumulationsrate führen als Einwanderer aus sehr ähnlichen Kulturkreisen. ${ }^{233}$ Allerdings erscheint eine Konzentration der Einwanderungsströme auf Herkunftsländer mit hohem Wissensstand vorteilhaft, weil dadurch die Rate des Wissenstransfers angehoben werden kann und negative Auswirkungen auf die Produktivität der einheimischen Arbeitskräfte verringert werden.

232 Vgl. Straubhaar/Zimmermann (1992) S. 17.

233 Hier besteht ein Konflikt mit dem Ziel einer raschen Assimilation der Eingewanderten, weil eine Einkommenangleichung umso eher zu erwarten ist, je weniger Humankapital der Immigranten durch bedeutende kulturelle Unterschiede entwertet wird. Auch die immateriellen gesellschaftlichen Kosten der Zuwanderung steigen mit der Fremdartigkeit der Zuwanderer. 


\subsection{Makroökonomische Auswirkungen der Zuwanderung}

Bisher ist in diesem Kapitel eine Untersuchung der verschiedenen Kostenarten erfolgt, die den Einheimischen durch die Änderung der Bevölkerungsstruktur entstehen, wenn bei gleicher Entwicklung der absoluten Bevölkerungszahl Geburten Einheimischer durch Zuwanderung von außen ersetzt werden. Dabei wurde allerdings nur die Primärwirkung betrachtet, d.h. es wurde der partielle Einfluß der Einwanderer beispielsweise auf die Steuereinnahmen untersucht, ohne zu analysieren, welche Auswirkungen diese Veränderung des Steueraufkommens auf wichtige gesamtwirtschaftliche Größen hat.

In Abschnitt 3.1 ist bei der Behandlung der Arbeitsmarkteffekte von Einwanderern mit einer möglichen Erhöhung des Kapitalbestandes infolge einer Zuwanderung eine makroökonomische Rückwirkung angedeutet worden. Aber auch in den dort vorgestellten empirischen Studien ist überwiegend vernachlässigt worden, daß Rückwirkungen infolge der über die Region hinausgreifenden Effekte auf Faktor- und Gütermärkte entstehen.

Rückwirkungen der Veränderung der Kapitaleinkommen wurden in Kapitel 3.2 nur angedeutet und die Folgewirkungen der Veränderung der Finanzlage der öffentlichen Haushalte in Abschnitt 3.3 vollständig ausgeklammert. Die langfristig zu erwartende Veränderung der gesamtwirtschaftlichen Produktionsfunktion durch den in Abschnitt 3.4 untersuchten Einfluß der Einwanderer auf den technischen und organisatorischen Fortschritt konnte schon in der Primärwirkung kaum erfaßt werden. Der Versuch einer Abschätzung der dadurch hervorgerufenen Sekundärwirkungen wäre daher hoch spekulativ. Die folgenden Ausführungen beschränken sich deshalb auf die Analyse der Folgewirkungen der Kostenarten, die in den Kapiteln 3.1 bis 3.3 behandelt wurden.

\subsubsection{Theoretische Analyse der makroökonomischen Auswirkungen}

Um die Sekundäreffekte zu erfassen, die sich aus makroökonomischen Rückkoppelungen ergeben, muß auf eine Modellierung der Volkswirtschaft der Bundesrepublik Deutschland zurückgegriffen werden, die hinreichend detailliert ist, um die durch Einwanderer verursachten Änderungen abzubilden. Im Rahmen des gewählten Modells werden zwei Berechnungen durchgeführt, einmal mit den exogenen Werten, die sich ohne Zuwanderung für den Fall einer erhöhten Fertilität der Einheimischen ergeben (Variante 1) und zum zweiten mit den Werten bei einer bestimmten Nettoeinwanderung und gleicher absoluter Bevölkerungsentwicklung (Variante 2). Als Ergebnis erhält man Zeitreihen, die den Verlauf ausgewählter makroökonomischer Größen unter den Annahmen der Variante 1 bzw. der Variante 2 darstellen. Aus der Differenz der einzelnen Werte für die beiden Varianten lassen sich dann die gesamten Kosten oder Nutzen der Zuwanderung inklusive der Sekundäreffekte errechnen.

Die Entwicklung eines aussagefähigen Modells stößt allerdings auf eine Reihe von Schwierigkeiten, die den Wert der Ergebnisse stark relativieren. Im folgenden werden zunächst einige der gravierendsten Probleme angeführt, um die Beurteilung der Aussagekraft der nachfolgend dargestellten Simulationen zu erleichtern.

Ein erstes Problem ist die Auswahl der einbezogenen exogenen und endogenen Parameter und der Modellstruktur. Der funktionale Zusammenhang der einzelnen Variablen variiert je nachdem, welche makroökonomische Schule als Grundlage herangezogen wird. Geringere 
Probleme bereitet die Beschaffung der Vergangenheitsdaten, ${ }^{234}$ die zur Schätzung von Verhaltensparametern unerläßlich sind. Das Verhalten der Wirtschaftssubjekte kann sich allerdings eventuell gerade in Reaktion auf die unterschiedliche Nettoeinwanderung - in der Zukunft ändern, so daß die prognostizierten Verhaltensparameter eine potentielle Fehlerquelle darstellen. Auch die Prognose der exogenen Variablen bereitet Probleme: Welche Annahme soll beispielsweise bezüglich der zukünftigen Fiskal- und Geldpolitik getroffen werden? Aber auch die Primärwirkungen des Einwanderungsszenarios sind schwer abschätzbar, wie die Ausführungen in den Abschnitten 3.1 bis 3.3 gezeigt haben. Da die Gefahr von Verhaltensänderungen und Prognosefehlern bezüglich exogener Variablen mit der Länge des Prognosezeitraums zunimmt, ist nur für recht kurzfristige Zeiträume eine Prognose mit hinlänglicher Genauigkeit möglich.

\subsubsection{Empirische Größenordnung der makroökonomischen Auswir- kungen}

Die großen Hindernisse auf dem Weg zu einer brauchbaren Simulation der makroökonomischen Auswirkungen von Einwanderern haben dazu geführt, daß nur wenige empirische Studien zu dieser Fragestellung existieren. Im folgenden werden zwei auf Deutschland bezogene Studien vorgestellt, um exemplarisch die Vorgehensweise und die aus ihr resultierenden Ergebnisse einer makroökonomischen Simulation zu erläutern.

Barabas u.a. benutzen das Konjunkturmodell des RWI als Grundlage für die Untersuchung der gesamtwirtschaftlichen Auswirkungen der Zuwanderung zwischen 1988 und 1991 auf West-Deutschland. ${ }^{235}$ Ansatzpunkt ist der Vergleich der Ergebnisse des Modells für die Situation ohne Zuwanderung und die Situation mit den insgesamt 3,6 Mio Zuwanderern in dem betrachteten Zeitraum. Es wird also keine Prognose über die zukünftige Entwicklung gegeben, sondern der tatsächlichen Entwicklung in der Vergangenheit ein Szenario gegenübergestellt, das beschreibt, wie die Entwicklung ohne Zuwanderer verlaufen wäre. ${ }^{236}$ Gegenüber der oben beschriebenen Vorgehensweise hat dieser Ansatz den Vorteil, daß weniger Schätzungen erforderlich sind, aber dieser Vorteil wird mit dem Nachteil erkauft, daß die für die Vergangenheit errechneten Kosten auch bei gleichhoher Einwanderung nicht ohne weiteres in die Zukunft fortgeschrieben werden können.

Zunächst seien die angenommenen Primärwirkungen auf Arbeitsmarkt und öffentliche Haushalte dargestellt. Für die einzelnen Zuwanderergruppen (Übersiedler, Aussiedler, Ausländer) werden verschiedene Erwerbsquoten unterstellt und so das Erwerbspersonenpotential insgesamt errechnet. Von den insgesamt etwa 1,7 Mio potentiell erwerbstätigen Zuwanderern wird angenommen, daß bis Ende 1991 etwa 1,3 Mio einen Arbeitsplatz gefunden haben. Da die Arbeitslosigkeit in dem betrachteten Zeitraum trotzdem um etwa 540.000 Personen zurück-

234 Das gilt allerdings nicht für die Erwartungen der Wirtschaftssubjekte, denen eine entscheidende Rolle in bezug auf ihr beobachtbares Verhalten zukommt.

235 Vgl. Barabas u.a. (1992). Eine Analyse anhand eines Ungleichgewichtsmodells findet man bei Franz/Oser/Winker (1994), eine Simulationsstudie für die USA bei Simon (1989) S. 199ff.

236 Hier interessiert genau genommen der Vergleich mit einer gleichhohen Bevölkerungszunahme aufgrund eines Geburtenüberschusses. Eine Isolierung der Effekte, die Einwanderer infolge ihrer spezifischen Charakteristika bzw. als zusätzliche Personen haben, ist nachträglich nicht durchführbar. 
gegangen ist, sind wohl keine nennenswerten Substitutionseffekte zu Lasten einheimischer Arbeitskräfte zu verzeichnen. ${ }^{237}$

Die Gebietskörperschaften und Sozialversicherungen wurden nach der Schätzung von Barabas u.a. zwischen 1988 und 1991 durch die Einwanderungswelle in der Primärwirkung um etwa 14 Mrd DM entlastet. ${ }^{238}$

Die Effekte höherer Ordnung ergeben sich durch die Einflüsse der Primärwirkungen auf Nachfrage- und Angebotsseite der Volkswirtschaft. Auf die Nachfrageseite wirken die Transferzahlungen an die Zuwanderer (Kindergeld, Renten, Eingliederungshilfen) und ihre Entlohnung. Die Transferzahlungen, die nach Angabe der Autoren der Studie 1991 etwa 17 Mrd DM ausmachten, ${ }^{239}$ schlugen sich direkt in einem Anstieg des privaten Verbrauchs nieder. Auch die Arbeitseinkommen der Zuwanderer hatten eine Anhebung des privaten Konsums zur Folge. Dabei gehen Barabas u.a. davon aus, daß sich die Wirkungen einer unterdurchschnittlichen Entlohnung der Zuwanderer und einer gegenüber den Einheimischen höheren Konsumquote gerade kompensierten, so daß der Konsum pro Zuwanderer gerade dem der einheimischen Bevölkerung entsprach.

Die Beschäftigung der Einwanderer hatte natürlich auch Wirkungen auf der Angebotsseite der Volkswirtschaft. Die Autoren gehen davon aus, daß das infolge der gestiegenen Beschäftigung höhere Güterangebot aufgrund der im Betrachtungszeitraum sehr hohen Kapazitätsauslastung nachfrageseitig vollständig absorbiert wurde, indem die Importe zurückgingen und die Exporte stiegen. Es wird also unterstellt, daß vor der Zuwanderungswelle ein Teil der Nachfrage wegen des Mangels an geeigneten Arbeitskräften nicht befriedigt werden konnte (Barabas u.a. ${ }^{240}$ sprechen in diesem Zusammenhang von "restringierter Nachfrage"). Erleichtert wurde die Substitution von Importen und die Expansion der Exporte durch einen geringeren Anstieg der Lohnkosten, der die internationale Wettbewerbsfähigkeit der in Deutschland erzeugten Güter gestärkt hat. Bezüglich der Arbeitsproduktivität wurde von einem negativen Einfluß der Zuwanderer ausgegangen, weil sie zum einen über ein geringeres Qualifikationsniveau verfügten als der Durchschnitt aller Beschäftigten und zum anderen, weil das zusätzliche Arbeitsangebot in Verbindung mit der hohen Kapazitätsauslastung die Wiederinbetriebnahme weniger produktiver Anlagen lohnend erscheinen ließ.

Als Ergebnis der makroökonomischen Simulation der Zuwanderungswelle von 1988 bis 1991 ergibt sich eine gegenüber der Situation ohne Zuwanderung um 0,2 Prozentpunkte geringere Arbeitslosenquote, ein Anstieg des Bruttosozialprodukts von knapp unter und des Volkseinkommens etwas über 5 Prozent, wozu v.a. die stark gestiegenen Einkommen aus Unternehmertätigkeit und Vermögen beitragen. Die zusätzlichen Einnahmen des Staates betragen 1991 50,8 Mrd DM, die sich zusammensetzen aus etwa 30 Mrd DM durch Primäreffekte und rund $20 \mathrm{Mrd}$ DM durch Sekundärwirkungen. Diesen Einnahmen stehen Ausgaben von 1991 37,4 Mrd DM gegenüber, so daß der Finanzierungsaldo des Staates nach anfänglich geringen Defiziten für das Jahr 1991 einen Überschuß von 13,4 Mrd DM ausweist (vgl. zu den Ergebnissen Tab. D.4).

\footnotetext{
237 Vgl. Barabas u.a. (1992) S. 142ff.

238 Vgl. Barabas u.a. (1992) S. 145.

239 Vgl. Barabas u.a. (1992) S. 148.

240 Vgl. Barabas u.a. (1992) S. 147.
} 
Tab. D.4: Gesamtwirtschaftliche Wirkungen der Zuwanderung (Abweichungen gegenüber Szenario ohne Zuwanderung)

\begin{tabular}{|c|c|c|c|c|}
\hline Annahmen & 1988 & 1989 & 1990 & 1991 \\
\hline $\begin{array}{l}\text { Erwerbspersonenpotential } \\
\text { der Zuwanderer ( } 1.000 \\
\text { Personen) }\end{array}$ & 129 & 528 & 1.078 & 1.547 \\
\hline $\begin{array}{l}\text { erwerbstätige Zuwanderer } \\
\text { (1.000 Personen) }\end{array}$ & 21 & 183 & 618 & 1.133 \\
\hline $\begin{array}{l}\text { Transfereinkommen an } \\
\text { Zuwanderer (Mrd DM) }\end{array}$ & 3,0 & 9,7 & 14,9 & 17,1 \\
\hline Wirkungen & & & & \\
\hline $\begin{array}{l}\text { Erwerbstätige ( } 1.000 \\
\text { Personen) }\end{array}$ & 22 & 186 & 645 & 1.218 \\
\hline Arbeitslosenquote (Prozent) & 0,1 & 0,3 & 0,3 & 0,2 \\
\hline $\begin{array}{l}\text { Privater Verbrauch } \\
\text { (Prozent) }\end{array}$ & 0,4 & 1,6 & 3,6 & 5,6 \\
\hline $\begin{array}{l}\text { Bruttosozialprodukt } \\
\text { (Prozent) }\end{array}$ & 0,2 & 1,1 & 2,9 & 4,9 \\
\hline Volkseinkommen (Prozent) & 0,3 & 1,2 & 3,2 & 5,4 \\
\hline $\begin{array}{l}\text { Einnahmen des Staates } \\
\text { (Mrd DM) }\end{array}$ & 1,4 & 8,8 & 26,1 & 50,8 \\
\hline $\begin{array}{l}\text { Ausgaben des Staates (Mrd } \\
\text { DM) }\end{array}$ & 3,2 & 11,4 & 22,1 & 37,4 \\
\hline $\begin{array}{l}\text { Finanzierungssaldo des } \\
\text { Staates (Mrd DM) }\end{array}$ & $-1,8$ & $-2,6$ & 4,0 & 13,4 \\
\hline
\end{tabular}

Quelle: Barabas u.a. (1992) S. 151

Wie sensitiv diese Ergebnisse auf eine Änderung der Prämissen reagieren, wird deutlich, wenn man die Konsumquote der Zuwanderer nicht mit $100 \%$, sondern wie für einheimische Haushalte mit 85\% ansetzt. Für 1991 ergibt sich dann gegenüber den in Tabelle D.2 ausgewiesenen Ergebnissen ein um 0,4 Prozentpunkte niedrigeres Bruttosozialprodukt, ein um einen Prozentpunkt niedrigerer Privater Verbrauch und eine um 80.000 niedrigere Zahl der Beschäftigten. Noch gravierenderen Einfluß hat eine Modifizierung der Prämissen bezüglich der Produktivitätsentwicklungen. Wird unterstellt, da $\beta$ die Produktivität durch die Zuwanderer nicht verändert wird, erhalten Barabas u.a. ein gleichfalls um 0,4 Prozentpunkte niedrigeres Bruttosozialprodukt, aber eine um 430.000 Personen geringere Beschäftigung und einen um 16,5 Mrd DM geringeren Finanzierungssaldo. 
Es muß nochmals darauf hingewiesen werden, daß die Ergebnisse der Arbeit von Barabas entscheidend von den Prämissen abhängen. Wäre beispielsweise nicht das Arbeitsangebot als begrenzender Faktor für das wirtschaftliche Wachstum angesehen worden, sondern die Nachfrage, ${ }^{241}$ dann steht zu vermuten, daß die Integration der Zuwanderer in den Arbeitsmarkt bedeutend schwieriger gewesen wäre. Weiterhin hängen die Resultate stark von den Charakteristika der Zuwanderer v.a. hinsichtlich Altersstruktur, Erwerbsbeteiligung und Qualifikation ab. Börsch-Supan weist darauf hin, daß die Ergebnisse von Barabas u.a. allein schon deshalb mit Vorsicht zu betrachten sind, weil der Effekt der Einwanderer durch die tiefgreifenden Veränderungen infolge der Wiedervereinigung der beiden deutschen Staaten schwer zu identifizieren sei. ${ }^{242}$ Schließlich stellt die Studie einen Vergleich zur Situation ohne Zuwanderung auf, während in dieser Arbeit die Differenzwirkung zur Situation mit einer gleich großen Anhebung des natürlichen Bevölkerungswachstums interessiert.

Die wesentlichen Ergebnisse der Studie werden aber durch die mit einem völlig anderen Ansatz gewonnenen Resultate von Börsch-Supan gestützt und können daher trotz der angeführten Mängel als Anhaltspunkt für die Sekundäreffekte betrachtet werden. ${ }^{243}$ Börsch-Supan benutzt ein neoklassisches langfristiges Wachstumsmodell, in dem der Konsum als Wohlfahrtsindikator dient. Im Basisszenario mit sehr geringer Nettoeinwanderung steigt der Pro-KopfKonsum zunächst an, weil der Rückgang der Zahl der Arbeitskräfte eine Rückführung der Bruttoinvestitionen ermöglicht. Ab dem Jahr 2010 dominiert dann allerdings ein entgegengerichteter Effekt, der dadurch entsteht, daß der steigende Rentnerquotient das Verhältnis von Arbeitkräften zu Konsumenten verschlechtert. ${ }^{244}$ Ein positiver Wanderungssaldo von 300.000 Personen jährlich senkt den Pro-Kopf-Konsum zunächst leicht ab, weil die zusätzlichen Arbeitskräfte mit Kapital ausgestattet werden müssen. Langfristig führt die Verbesserung des Verhältnisses von Arbeitskräften zu Konsumenten aber dazu, daß der Pro-Kopf-Konsum deutlich über dem Basisszenario liegt. Interessant ist, daß unter Annahme einer geschlossenen Volkswirtschaft zwar die Bruttolöhne infolge des gestiegenen Arbeitsangebots niedriger sind, die Nettolöhne aufgrund der entlastenden Wirkung der Migranten in den Sozialversicherungssystemen aber dennoch höher liegen als im Referenzfall. Ersetzt man die Arbeitskräftewanderungen durch internationale Kapitalbewegungen und Handel mit einem Land, dessen Bevölkerungsstruktur weniger stark altert, erhält man zwar ähnlich positive Effekte in bezug auf den Pro-Kopf-Konsum, aber die Nettolöhne sind dann ebenso wie die Bruttolöhne niedriger als im Referenzfall. Dieses Ergebnis ist darauf zurückzuführen, da $\beta$ die Sozialversicherungssysteme wegen der Lohnabhängigkeit der Beiträge nur durch Arbeitseinkommen, nicht aber durch Kapitaleinkommen entlastet werden.

Diese positiven Ergebnisse hinsichtlich der Auswirkungen verstärkter Zuwanderung sind allerdings zu relativieren, da sie auf der Annahme von Homogenität zwischen Zuwanderern und Einheimischen beruhen. Ist der Assimilationszeitraum für die Immigranten sehr lang oder haben sie z.B. aufgrund geringeren Humankapitals dauerhaft eine niedrigere Produktivität, sind die Auswirkungen weniger günstig. Vor allem aber treten durch die Homogenitätsannahme die Auswirkungen einer reinen Bevölkerungserhöhung in den Vordergrund, während hier der Vergleich zur Situation bei verstärktem Kinderlastenausgleich, also bei anderer Bevölkerungs-

241 Aufgrund der sinkenden Kapazitätsauslastung unterstellen Barabas u.a. ab dem dritten Quartal 1991 die Nachfrage als restriktiven Faktor. Vgl Barabas u.a. (1992) S. $149 f$.

Vgl. Börsch-Supan (1994) S. 131.

243 Vgl. zu diesem Absatz Börsch-Supan (1994).

Vgl. Börsch-Supan (1994) S. 135f. 
struktur interessiert. In dem Modell von Börsch-Supan unterscheiden sich die Auswirkungen von höherer Einwanderung und höherer Fertilität der Einheimischen aber nur noch durch die daraus resultierende unterschiedliche Altersstruktur der Bevölkerung.

Als Fazit der beiden vorgestellten Studien muß festgehalten werden, daß die für die Einheimischen vorteilhaften Ergebnisse bezüglich der makroökonomischen Sekundäreffekte von Einwanderern noch als recht unsicher angesehen werden müssen. Dies gilt umso mehr, als hier der Vergleich mit der Situation bei höherer Fertilität der Einheimischen interessiert und nicht wie in den oben angeführten Arbeiten der Vergleich mit einer Situation, in der ein geringeres Bevölkerungswachstum vorliegt. Da die makroökonomischen Rückwirkungen in den allermeisten Fällen aber nur zu einer Verstärkung oder Abschwächung der Primäreffekte führen, kann zumindest die Einflußrichtung der Primärwirkungen auch unter Einbezug der Sekundärwirkungen als einigermaßen gesichert betrachtet werden.

\subsubsection{Die Bedeutung der makroökonomischen Auswirkungen für ein Einwanderungsgesetz}

Die recht große Unsicherheit über die makroökonomischen Wirkungen einer verstärkten Nettozuwanderung lassen kaum Rückschlüsse auf eine besonders vorteilhafte Gestaltung eines Einwanderungsgesetzes zu. Die Bedeutung der Primärwirkungen der Zuwanderer wird insofern unterstrichen, als makroökonomische Rückwirkungen diese Primärwirkungen in den meisten Fällen nicht beeinträchtigen werden. Die Ergebnisse von Barabas u.a. sprechen im Gegenteil dafür, daß die Anstoßwirkung durch makroökonomische Wirkungsketten zumindest in positivem konjunkturellen Umfeld verstärkt werden. Damit kommt einer an den Primärwirkungen orientierten Ausrichtung des Einwanderungsgesetzes verstärktes Gewicht zu.

Gleichzeitig erscheint eine eingehendere Erforschung der Makrowirkungen notwendig. Eine Politik der kleinen Schritte ermöglicht es, Erfahrungen zu sammeln, ohne dabei ein zu hohes Risiko einzugehen. Die Ergebnisse der Forschungen sind dann durch Korrekturen im Einwanderungsgesetz zu berücksichtigen.

\subsection{Zusammenfassung}

Ziel dieses Kapitels war es, die Kosten (und Nutzen) zu untersuchen, die den Einheimischen durch Einwanderer entstehen. Als Vergleichsmaßstab wurde dabei die Situation bei einer gleich hohen relativen Bevölkerungszunahme durch eine erhöhte Fertilität der Einheimischen herangezogen, um so die Auswirkungen einer verstärkten Einwanderung gegenüber einem Anstieg des natürlichen Bevölkerungswachstums durch pronatalistische Instrumente herauszuarbeiten. Betrachtet wurde also nicht der Bevölkerungsniveaueffekt, der aus einer relativen Bevölkerungszunahme infolge der Zuwanderung entsteht, sondern der Bevölkerungsstruktureffekt, der auf die unterschiedlichen Merkmale von Einwanderern und Einheimischen zurückgeht.

Für die Prognose der Auswirkungen zukünftiger Einwanderung sind die in der Vergangenheit durch Gastarbeiter hervorgerufenen Effekte besonders aufschlußreich, weil ihre Merkmale am ehesten denen der potentiellen Migranten ähneln, die durch ein Einwanderungsgesetz zur 
Immigration nach Deutschland veranlaßt werden könnten. Um den Einfluß unterschiedlicher Merkmale zu verdeutlichen, wurde darüber hinaus, sofern möglich, auch auf die Wirkungen der Aussiedler eingegangen. Asylbewerber spielten demgegenüber für die hier vorliegende Fragestellung eine untergeordnete Rolle, weil ihre Zahl und die durch sie pro Kopf verursachten Kosten weitgehend politisch determiniert sind.

Als relevante Auswirkungen der Einwanderung auf die Einheimischen wurden die Arbeitsmarkteffekte und Wirkungen auf die Kapitaleinkommen der Einheimischen, die Wirkungen infolge staatlicher Umverteilung, Produktivitätseffekte und makroökonomische Rückwirkungen analysiert. ${ }^{245}$

Hinsichtlich der Arbeitsmarktwirkungen ergaben sich weder in bezug auf die Höhe der Arbeitseinkommen noch auf die Arbeitslosigkeit von Einheimischen größere Änderungen durch Einwanderer. Allenfalls ergeben sich durch die Zuwanderung wenig qualifizierter Arbeitskräfte negative Auswirkungen für einheimische Arbeitskräfte mit ähnlichen ökonomischen Charakteristika, während qualifizierte Arbeitskräfte infolge einer Komplementarität mit Unqualifizierten von einer derart strukturierten Zuwanderung profitieren. Allerdings sind die Studien zu den Arbeitsmarktwirkungen Kritikpunkten ausgesetzt, die ihre Aussagefähigkeit einschränken. Eine Quantifizierung der den Einheimischen über die Wirkungen der Zuwanderer auf dem Arbeitsmarkt entstehenden Kosten konnte daher nicht sinnvoll durchgeführt werden.

Der Einfluß von Einwanderern auf die Kapitaleinkommen der Einheimischen darf aufgrund der hohen internationalen Kapitalmobilität als gering angesehen werden. Allenfalls bei der Einwanderung von Arbeitskräften, die wie die meisten temporären Migranten kaum Kapital in das Aufnahmeland transferieren, ist ein geringfügiger Anstieg der Kapitaleinkommen der Einheimischen möglich.

Die Wirkungen von Einwanderern auf Einheimische, die durch umverteilende Staatsaktivitäten entstehen, wurden in drei Schritten untersucht. Da sowohl die Höhe der Sozialabgaben und der Steuerzahlungen als auch die Inanspruchnahme staatlicher Leistungen stark einkommensabhängig sind, wurde zunächst untersucht, ob eingewanderte Arbeitskräfte, wie nach dem Humankapitalansatz zu erwarten, anfänglich durchschnittlich ein niedrigeres Einkommen erzielen und ob sich dieser Einkommensrückstand mit zunehmender Verweildauer im Gastland abbaut. So läßt sich die zeitliche Entwicklung der den Einheimischen durch die öffentlichen Haushalte entstehenden Kosten der Einwanderung abschätzen. Für die Gastarbeiter stellte sich im Durchschnitt ein Einkommensrückstand gegenüber den Einheimischen heraus, der auch mit zunehmender Länge des Aufenthalts in Deutschland nicht vermindert wird. Allerdings sind diese Ergebnisse v.a. auf die geringere durchschnittliche Qualifikation der Gastarbeiter zurückzuführen, was sich darin zeigt, daß gegenüber gleich qualifizierten einheimischen Arbeitskräften kein Einkommensnachteil feststellbar ist. Gestützt wird dieser Befund weiterhin auch dadurch, daß für die im Mittel besser qualifizierten (und mit Eingliederungshilfen unterstützten) Aus- und Übersiedler zwar ein anfänglicher Einkommensrückstand gegenüber den Einheimischen besteht, dieser aber im Laufe der Zeit vollständig abgebaut werden kann. Aus diesen Resultaten ist zu schließen, daß durch eine Selektion der Zuwanderer nach ihrem Humankapitalbestand die Kosten für Einheimische gesenkt werden können. Allerdings wird das Verhältmis von Steuern und Beiträgen der Eingewanderten zu den von ihnen empfangenen Leistungen nicht nur von der Einkommensentwicklung beeinflußt, sondern auch von der

245 Inwieweit neben diesen wirtschaftlichen auch gesellschaftliche Kosten bzw. Nutzen - etwa in Form von Fremdenfeindlichkeit und zunehmender Gewalt - durch eine verstärkte Einwanderung verursacht werden, wurde nicht untersucht, weil die subjektiven Bewertungen der Zuwanderung durch die Einheimischen kaum feststellbar und schon gar nicht intersubjektiv aggregierbar sind. 
Entwicklung ihrer Erwerbs- und Arbeitslosenquote und ihrer Einkommensverteilung. Über die Entwicklung dieser Einflußfaktoren im Zeitablauf liegen aber bisher kaum empirische Untersuchungen vor.

Als Grundlage für die Ermittlung der Höhe der Kosten, die den Einheimischen durch die Staatsaktivität in einem bestimmten Zeitpunkt aus der Einwanderung erwachsen, wurde eine Studie von Ulrich über Gastarbeiter herangezogen. Danach überwog auf der Einnahmenseite der Einfluß des geringeren durchschnittlichen Einkommens der Gastarbeiter die Wirkungen der höheren Erwerbsbeteiligung, so daß sie nur etwa neun Zehntel der Steuerzahlungen der Einheimischen leisten. Auf der Ausgabenseite hingegen entstehen den Gebietskörperschaften für Infrastruktur niedrigere Ausgaben als für Einheimische, während sich die Infrastrukturausgaben für dauerhafte Einwanderer kaum von denen für Einheimische unterscheiden. Anders stellt sich die Situation bei den steuerfinanzierten Transferleistungen dar. Zumindest bei den Kindergeldzahlungen und der Sozialhilfe lagen die Leistungen an Ausländer deutlich über den Leistungen an deutsche Haushalte. Die Belastung der Einheimischen durch die niedrigeren Steuerzahlungen und die höhere Inanspruchnahme von Leistungen durch Eingewanderte läßt sich reduzieren, indem v.a. die Zuwanderung von jungen temporären Migranten gefördert wird, die über hohes Humankapital verfügen und in einem Arbeitsmarktsegment tätig werden, das sich nicht durch ein strukturelles Überangebot an Arbeitskräften auszeichnet.

Im Rahmen der Haushalte der Sozialversicherungen wurde demgegenüber ein positiver Einfluß der Einwanderer für die Einheimischen festgestellt. Die günstige Alters- und Erwerbsstruktur der Zuwanderer hat in der Vergangenheit dazu geführt, daß sie pro Haushalt höhere Beiträge leisten, aber weniger Leistungen beanspruchen. Hinsichtlich der Gesetzlichen Krankenversicherung und der Gesetzlichen Pflegeversicherung ist eine Rückwanderung im Alter vorteilhaft, weil dadurch die Altersstruktur der Versicherten verbessert wird. Anders stellt sich die Situation bezüglich der Gesetzlichen Rentenversicherung dar. Hier ist permanente Zuwanderung von Migranten mit einer hohen Kinderzahl für die Einheimischen günstiger, da bei temporärer Migration die Renten der in ihre Heimatländer zurückgewanderten Migranten von der nächsten Generation der Zuwanderer aufgebracht werden müssen, während bei permanenter Migration die Kinder der Migranten über das Umlageverfahren die Renten ihrer Eltern bezahlen. Die Beiträge der nachfolgenden Einwanderungskohorten sind bei dauerhafter Einwanderung also wieder eine einmalige Liquiditätsspritze für die Gesetzliche Rentenversicherung.

Auf die Produktivitätsentwicklung der Einheimischen wirken die Eingewanderten auf zweifache Weise. Zum einen treten sie als Transferträger von organisatorischem und technischem Wissen auf, zum anderen generieren sie neues Wissen. Obschon sowohl die theoretische als auch die empirische Basis auf diesem Gebiet noch sehr dünn ist, kann davon ausgegangen werden, daß sich durch Zuwanderung eher positive als negative Produktivitätseffekte bei den Einheimischen einstellen werden. Vorteilhaft erscheint eine Selektion der Migranten nach dem Humankapitalbestand und nach Herkunftsländern mit besonders andersartiger Kultur und Wirtschaft.

Bei der Abschätzung der makroökonomischen Sekundärwirkungen von Zugewanderten bereiteten die große Unsicherheit bezüglich der Höhe der Primärwirkungen und die Modellierung Probleme. Im allgemeinen verstärken die makroökonomischen Rückwirkungen aber die Primärwirkungen, so daß einer an den Anstoßwirkungen orientierten Selektion der Zuwanderer ein verstärktes Gewicht zukommt. 


\section{Die Kosteneffizienz einer verstärkten Einwanderung}

Die Ausführungen in Kapitel 3 hatten neben dem Ziel, die Kosten zu bestimmen, die den Einheimischen durch die Einwanderer entstehen, den Zweck, die Wirkungsketten aufzuzeigen, die von der Zuwanderung zu den Kosten für die Einheimischen führen. Dadurch wurde die Möglichkeit eröffnet, von bestimmten Merkmalen der Einwanderer auf die Höhe der durch sie verursachten Kosten zu schließen. Am Ende jedes Unterkapitels konnten so erste Schlußfolgerungen für ein Einwanderungsgesetz gezogen werden, das auf eine Minimierung dieser Kosten abstellt. Die Überlegungen des vorangegangenen Kapitels dienen daher hier als Grundlage zur Untersuchung der Kosteneffizienz eines an dem Ziel der Maximierung der Wohlfahrt der Bürger des Aufnahmelandes ${ }^{246}$ orientierten Verstärkung der Einwanderung.

Dazu müssen zunächst in Unterkapitel 4.1 Kriterien entwickelt werden, bei deren Anlegung eine möglichst kostengünstige Zuwanderung erreicht wird. In Kapitel 4.2 wird dann untersucht, welche Instrumente eine derartige Selektion von Zuwanderern mit ökonomisch besonders vorteilhaften Merkmalen am ehesten gewährleisten. In Kapitel 4.3 finden sich schließlich einige Überlegungen zu den Kosten der Zuwanderung bei einer nach ökonomischen Kriterien vorteilhaften Struktur der Einwanderer.

\subsection{Kriterien für eine verstärkte Einwanderung}

Bei der Konstruktion eines Einwanderungsgesetzes können nicht ausschließlich ökonomische Erwägungen einfließen, sondern es sind auch politische, humanitäre und rechtliche Überlegungen zu berücksichtigen. Sinnvoll erscheint eine Unterteilung der Zuwanderungswilligen in die drei Gruppen Flüchtlinge, Familienangehörige und ökonomisch motivierte Einwanderer. Auch wenn sich ein Einwanderungswilliger nicht immer eindeutig einer Gruppe zuordnen läßt, liegen der Einwanderungserlaubnis seitens des Gastlandes für jede Gruppe doch unterschiedliche Motive zugrunde, die eine für alle Gruppen einheitliche Regelung ungeeignet erscheinen lassen. So wird die Einwanderung eines Bürgerkriegsflüchtlings oder eines Verfolgten aus humanitären Gründen eventuell auch dann gestattet, wenn den Einheimischen dadurch hohe Kosten entstehen. Bei ökonomisch motivierter Einwanderung können hohe Kosten für das Einwanderungsland dagegen ein legitimer Ablehnungsgrund sein.

Über die Aufnahmeregelungen für Flüchtlinge kann nur auf politischer Ebene entschieden werden, da hier v.a. humanitäre Gesichtspunkte eine Rolle spielen. ${ }^{247}$ Allerdings ist der Handlungsspielraum in diesem Bereich durch internationale Abkommen und das Grundgesetz eingeschränkt. Art. 3 der Europäischen Menschenrechtskonvention (EMRK) verbietet die Abschiebung eines Ausländers in ein Land, in dem er der ernsthaften Gefahr von Folter oder unmenschlicher oder erniedrigender Behandlung oder Bestrafung ausgesetzt ist. Art. 1, 33 der Genfer Flüchtlingskonvention untersagt die Zurückweisung von Schutzsuchenden an der Grenze, wenn sie durch diese Maßnahme in Gefahr gebracht werden, unmittelbar oder mittel-

246 Prinzipiell kann auch die Erhöhung der Wohlfahrt der Wandernden und der Bürger des Herkunftlandes Ziel einer Verstärkung der Einwanderung sein. 
bar in das Land zurückkehren zu müssen, in dem ihnen Verfolgung droht (non-refoulement). Schließlich ist das Grundrecht auf Asyl im Grundgesetz (Art. 16 GG) festgeschrieben. Eine Kontingentierung oder Quotierung der einwanderungsberechtigten Flüchtlinge oder Verfolgten ist mit der humanitären Zielsetzung der Einreiseerlaubnis für diesen Personenkreis kaum vereinbar. Dennoch bleibt gerade im Bereich der (Bürger-) Kriegsflüchtlinge ein erheblicher Spielraum für diskretionäre Entscheidungen. Bei der Nutzung dieses Spielraumes sollte beachtet werden, daß Flüchtlinge weniger vorteilhafte Charakteristika aufweisen als ökonomische Einwanderer. Der Selbstselektionsmechanismus bezüglich der Fähigkeiten, Einsatzbereitschaft und Ambition ist bei ihnen weniger stark ausgeprägt, die Alters- und Familienstruktur ist im allgemeinen weniger günstig. ${ }^{248}$ Weiterhin ist eine Anrechnung der Zahl der zugewanderten Flüchtlinge auf die Kontingente anderer Zuwanderergruppen möglich, um die Gesamtzahl der Zuwanderer konstant zu halten. Da die Zahl der in einem bestimmten Jahr aufzunehmenden Flüchtlinge nicht im vorhinein bestimmbar ist, bietet sich eine Anrechung auf das Gesamtkontingent des jeweils nächsten Jahres an. ${ }^{249}$

Auch die Familienzusammenführung beruht hauptsächlich auf humanitären Beweggründen. Die rechtlichen Vorgaben sind hier allerdings wesentlich unschärfer - allenfalls aus Art. 8 EMRK und Art. 6 GG kann sich eine gewisse Einschränkung ergeben ${ }^{250}$-, so daß der Legislative hier bezüglich der Gestaltung der Einwanderungserlaubnis weitgehend freie Hand gelassen ist. Eine Kontingentierung und Quotierung ist daher ebenso rechtlich möglich, wie eine Verzahnung mit den Regelungen für ökonomische Einwanderer. Das könnte z.B. geschehen, indem abgestuft nach dem Verwandtschaftsgrad Angehörigen von Inländern im Rahmen eines Punktesystems Sonderpunkte zuerkannt werden. Die Aufnahme von Familienangehörigen von Ausländern, denen bereits ein Aufenthaltsrecht zuerkannt wurde, kann ebenso geregelt werden. Der gegenüber Inländern geringeren Dringlichkeit des Aufenthalts von aufenthaltsberechtigen Ausländern im Inland kann beispielsweise durch eine geringere Punktezahl Rechnung getragen werden. Die Integration der Familienzusammenführung in die Regelungen für ökonomische Zuwanderer hat den Vorzug, daß die anderenfalls recht nachteiligen Charakteristika der nachziehenden Familienangehörigen (niedrige Erwerbsbeteiligung, geringe Durchschnittseinkommen, etc.) zum Vorteil der Einheimischen beeinflußt werden können.

Bezüglich der ökonomisch motivierten Einwanderer bestehen keine rechtlichen Bindungen, so daß hier an den Kosten der Einwanderer für das Aufnahmeland orientierte Auswahlkriterien zur Geltung kommen können.

Zunächst ist zu klären, wieviel Einwanderer insgesamt pro Jahr zugelassen werden sollen. Ein völlig liberales Regime ohne Einwanderungsbeschränkungen, bei dem die Höhe der Einwanderung hauptsächlich durch den Arbeitsmarkt bestimmt wird, ist wegen der (pekuniären) externen Effekte auf die Einheimischen für diese nicht wohlfahrtsmaximierend und zudem aufgrund der hohen sozialen und gesellschaftlichen Kosten und der daraus resultierenden politischen Widerstände auch unrealistisch. Da die Höhe dieser Kosten kaum bestimmbar ist, ${ }^{251}$ muß die Entscheidung über den Gesamtumfang der Einwanderung auf politischer Ebene

\footnotetext{
248 Vgl. Simon (1989) S. 313f.

249 Vgl. Gusy (1994b) S. 147.
}

250 Der Art. 8 der Europäischen Menschenrechtskonvention (EMRK) regelt den Anspruch auf Achtung des Privat- und Familienlebens, Art. 6 des Grundgesetzes (GG) sichert Ehe und Familie den besonderen Schutz des Staates zu. 
fallen. ${ }^{252}$ Die im folgenden angeführten Selektionskriterien dienen dann einer ökonomisch effizienten Allokation der Zuwanderungsrechte auf die einwanderungswilligen Personen.

Die bisherigen Ausführungen zu den Bedeutungen der einzelnen Kostenarten für ein Einwanderungsgesetz legen die Selektion der ökonomisch motivierten Einwanderer nach folgenden Kriterien nahe:

- Erwerbstätigkeit

- bisherige Bindung an den Aufnahmestaat einschließlich der Sprachkenntnisse

- berufliche Qualifikation

- Humankapitalbestand

- Fähigkeiten, Einsatzbereitschaft und Ambition

- Dauerhaftigkeit der Zuwanderung

- Alter

- Vermögenslage, Investitionsbereitschaft

Grundsätzlich ist ein möglichst hoher Anteil von Erwerbstätigen unter den Einwanderern anzustreben. Die negativen Auswirkungen zusätzlicher Arbeitskräfte sind gering und betreffen nur einen Teil der einheimischen Arbeitskräfte, während Arbeitskräfte, deren Eigenschaften komplementär zu denen der Zuwanderer sind, möglicherweise sogar durch steigende Arbeitseinkommen und geringere Arbeitslosigkeit von der Einwanderung profitieren. Besonders positiv wirkt sich eine hohe Erwerbsbeteiligung der Zuwanderer auf die öffentlichen Haushalte aus. Die Gebietskörperschaften profitieren dadurch, daß die Steuerzahlungen von Erwerbstätigen höher sind als diejenigen von Nicht-Erwerbstätigen und gleichzeitig auf der Ausgabenseite weniger Infrastrukturausgaben und steuerfinanzierte Sozialleistungen für Erwerbstätige anfallen. Bei den Sozialversicherungen profitieren v.a. die Krankenkassen und die Rentenversicherung von einer hohen Erwerbsbeteiligung der Zuwanderer, da beitragslose Mitversicherung von Familienmitgliedern bzw. Witwen-/Witwerrente reduziert werden.

Entscheidend ist dabei nicht allein die Erwerbstätigkeit im Zeitpunkt der Aufnahme, sondern das für die Aufenthaltszeit in Deutschland zu erwartende Verhältnis von Erwerbsphasen zu Nicht-Erwerbsphasen. So wäre die dauerhafte Einwanderung eines kurz vor seinem Abschluß stehenden Studenten unter dem Gesichtspunkt der Erwerbsbeteiligung positiv zu beurteilen, die Aufnahme eines 60jährigen Werktätigen ohne ausreichende Alterssicherung dagegen negativ.

Eine hohe bisherige Bindung an Deutschland, z.B. wiederholte Urlaubs- und Sprachaufenthalte, aber auch Verwandte in der Bundesrepublik und eine hohe Sprachfertigkeit in Deutsch erleichtern dem Einwanderer die Assimilation. ${ }^{253}$ Ein Teil des länderspezifischen Humankapitals ist bereits vorhanden bzw. kann durch Verwandte oder Bekannte schnell vermittelt werden, so daß eine vergleichsweise schnelle Angleichung des Einkommens an das Niveau von Einheimischen mit gleichen Charakteristika erwartet werden kann.

Eine berufliche Qualifikation, die zu einer Tätigkeit auf einem Arbeitsmarktsegment befähigt, das nicht durch strukturelle Unterbeschäftigung gekennzeichnet ist, trägt dazu bei, die negativen Auswirkungen auf Arbeitseinkommen und Arbeitslosigkeit der Einheimischen gering zu halten. Die Substitution Einheimischer durch Einwanderer wird durch eine Qualifikation auf

252 Vgl. Straubhaar (1991) S. 213f.

253 Vgl. Simon (1989) S. 327. 
einem Gebiet, das in Deutschland durch einen Nachfrageüberschuß nach Arbeitskräften gekennzeichnet ist, vermindert. Gleichzeitig wird bei einer derartigen Qualifikation die Assimilation durch einen einfacheren Einstieg in das Arbeitsleben erleichtert, so daß auch schneller höhere Einkommen und damit Steuer- und Beitragszahlungen an den Staat zu erwarten sind.

Da die Einkommenshöhe stark von dem Bestand an Humankapital determiniert wird, wirkt sich ein hohes Bildungsniveau der Einwanderer positiv für die Einheimischen aus. Je höher die Einkommen der Zuwanderer, desto höher sind ceteris paribus auch ihre Steuerzahlungen, während mit zunehmendem Einkommen eher mit einer abnehmenden Inanspruchnahme öffentlich bereitgestellter Infrastruktur und steuerfinanzierter Sozialleistungen zu rechnen ist. Auch im Bereich der Sozialversicherungen wirken sich hohe Einkommen der Zuwanderer positiv aus. V.a. bei den Gesetzlichen Krankenkassen stehen höheren Beitragseinnahmen keine höheren Ausgaben gegenüber. ${ }^{254}$ Aber auch über die Arbeitslosenversicherung profitieren die Einheimischen von einem hohen Humankapitalbestand der Zuwanderer, solange die Arbeitslosenquote aufgrund der höheren beruflichen und regionalen Mobilität mit zunehmender Bildung abnimmt. Darüber hinaus werden Einwanderer mit hohem Humankapitalbestand eher einen positiven Einfluß auf die Fertigkeiten der Einheimischen ausüben und deren Produktivität erhöhen als Zuwanderer mit geringer Ausbildung. ${ }^{255}$

Auch die nicht durch formale Ausbildung erfaßten arbeitsmarktrelevanten Fähigkeiten, die Einsatzbereitschaft und die Ambition der Einwanderer beeinflussen längerfristig ihre Einkommensentwicklung im Aufnahmeland. Im Normalfall wird aufgrund von asymmetrischer Information eine gewisse Zeit vergehen, bis der Arbeitgeber diese die Produktivität beeinflussenden Eigenschaften eines Arbeitnehmers erkennt. Bei Entlohnung nach dem erwarteten Wertgrenzprodukt wird schließlich aber das Einkommen besonders fähiger und einsatzfreudiger Arbeitskräfte über das durchschnittliche Niveau ansteigen. Bei Selbständigen wird dieser Unterschied im Einkommensniveau sofort zu beobachten sein, da bei ihnen naturgemäß keine asymmetrische Information vorliegt. Die Auswirkungen höherer Einkommen der Einwanderer auf die Kosten der Zuwanderung für die Einheimischen wurden bereits im vorangehenden Absatz dargestellt.

Die Dauerhaftigkeit des Aufenthalts von Zuwanderern im Aufnahmeland hat Konsequenzen für die Arbeitsmarktwirkung, die Kapitaleinkommen und die öffentlichen Haushalte. Wenn die Aufenthaltserlaubnis temporärer Zuwanderer an den Besitz eines Arbeitsplatzes gebunden ist oder wenn die Aufenthaltserlaubnisse nur mit sehr kurzen Laufzeiten vergeben werden, können die Migranten als Konjunkturpuffer dienen, indem bei einer Rezession das Arbeitsangebot durch einen Rückgang der Zahl der Zuwanderer reduziert wird und so die Beschäftigung der Einheimischen stabilisiert werden kann.256

Für die Haushalte der Gebietskörperschaften ist temporäre Migration gleichfalls vorteilhafter als dauerhafte Einwanderung von Personen gleichen Qualifikationsniveaus. Temporäre Migranten weisen oft eine höhere Erwerbsbeteiligung, eine geringere Arbeitslosenquote und eine günstigere Alters- und Familienstruktur auf, so daß sie pro Kopf höhere Steuern zahlen, gleichzeitig aber weniger staatliche Leistungen in Form von Infrastruktur und steuerfinanzier-

254 Die mit dem Einkommen steigenden Ausgaben für die Lohnfortzahlung ab dem 42. Krankheitstag fallen kaum ins Gewicht.

$255 \mathrm{Vgl}$. zu dem letzten Punkt Simon (1989) S. 321.

256 Vgl. Straubhaar/Zimmermann (1992) S. 20. 
ten Sozialleistungen beanspruchen. Die mit längerer Aufenthaltsdauer steigenden Einkommen können diese positiven Effekte temporärer Migration nicht kompensieren, legen aber eine Differenzierung in temporäre und dauerhafte Einwanderung schon bei der Einreise nahe, damit für die permanenten Einwanderer sofort ein Anreiz zur Humankapitalbildung besteht.

Bei den Haushalten der Sozialversicherungen sind die Einflüsse widersprüchlich. Für die Arbeitslosenversicherung und die Krankenversicherung sind temporäre Migranten aufgrund der niedrigeren Arbeitslosenquote bzw. der besseren Familien-, Erwerbs-, und Altersstruktur vorteilhaft. Entscheidend ist aber, daß dauerhafte Einwanderung für die Gesetzliche Rentenversicherung weitaus vorteilhafter ist als temporäre Einwanderung. Während bei dauerhafter Einwanderung die Kinder der Einwanderer die Renten ihrer Eltern durch Beiträge finanzieren und die Beiträge der Einwanderer-Generation allein den Einheimischen in Form einer Beitragssenkung zugute kommen, zahlen die Kinder der temporären Migranten keine Beiträge an die Gesetzliche Rentenversicherung in Deutschland, da sie gar nicht erst in die Bundesrepublik mitgenommen wurden oder diese zusammen mit ihren Eltern wieder verlassen haben. Nur durch eine ständige Ausweitung der temporären Migration läßt sich ein dauerhafter Vorteil für die Einheimischen erzielen, durch eine Rückführung entstehen ihnen allerdings auch Nachteile, so daß temporäre Migration allenfalls zu einer zeitlichen Verschiebung der Finanzierunglasten geeignet ist. Der Vorteil der dauerhaften gegenüber der temporären Migration in der Gesetzlichen Rentenversicherung ist so hoch, ${ }^{257}$ daß er die gegenläufigen Wirkungen auf die Arbeitslosen- und Krankenversicherung sowie auf die Haushalte der Gebietskörperschaften und die Arbeitsmarktsituation der Einheimischen weit überkompensieren dürfte. Insgesamt ist daher eine dauerhafte Einwanderung temporären Wanderungen vorzuziehen.

Je niedriger das durchschnittliche Alter der Einwanderer ist, desto vorteilhafter ist dies für die Einheimischen, sofern die Einwanderer bereits erwerbstätig sind und somit keine Kosten für ihre Ausbildung anfallen. Junge Erwerbstätige sind auf dem Arbeitsmarkt flexibler, sie erwerben schneller länderspezifisches Humankapital und zahlen so mittelfristig höhere Steuern und Sozialbeiträge. Gleichzeitig ist die Beanspruchung von steuerfinanzierten Sozialleistungen bei jungen Personen ebenso niedriger als bei Älteren ${ }^{258}$ wie auch die Ansprüche an die Krankenund Arbeitslosenversicherung. Besonders die Rentenversicherung profitiert von jungen Zuwanderern. Im Gegensatz zu älteren Zuwanderern bringen sie häufig ihre Kinder mit in die neue Heimat oder bekommen sie dort. Dadurch können die Rentenzahlungen an die Einwanderer-Generation im Umlageverfahren durch die Beiträge der eigenen Nachfahren gesichert werden, so daß die Summe der Beiträge der ersten Generation (die aufgrund der langen Erwerbsphase bei jungen Einwanderern besonders hoch ist) ganz den Einheimischen zufließt. Bei älteren Einwanderern, die ihre Fertilitätsphase bereits abgeschlossen haben und deren Kinder im Heimatland verblieben sind, haben die Einheimischen wie bei den temporären Einwanderern nur dann Vorteile im Rahmen der Gesetzlichen Rentenversicherung, wenn die Zahl der Einwanderer dauerhaft erhöht wird. Besonders vorteilhaft ist daher die Zuwanderung junger Personen am Beginn ihres Erwerbs- und Familienlebens. 259

257 Vgl. Tab. D.2 und die Diskussion in 3.3.3.1.

258 Eine Ausnahme bildet das Kindergeld, da Einwanderer, die sich am Beginn ihrer Erwerbskarriere befinden, auch am Anfang ihrer Familienbildung stehen.

259 Diese Einschätzung wird u.a. geteilt von Simon (1989) S. 326. Arthur/Espenshade (1988) S. 324 stellen als Ergebnis einer Simulationsanalyse fest, da $B$ "changes in immigrants' ages exert a substantial effect, but one that takes a considerable time to manifest itself fully, especially when a realistic range of migrants' ages is assumed." 
Die Vermögenslage und Investitionsbereitschaft der Einwanderer hat widersprüchliche Konsequenzen für die Kosten, die den Einheimischen durch die Zuwanderung entstehen. ${ }^{260}$ Einerseits werden die Kapitaleinkommen der Einheimischen durch das steigende Angebot an Kapital ceteris paribus gemindert, wobei dieser negative Effekt aufgrund der hohen internationalen Kapitalmobilität recht gering sein dürfte. Andererseits erhöht der durch Einwanderung induzierte Kapitalzufluß, soweit er nicht durch den Abfluß inländischer Vermögen ins Ausland kompensiert wird, die Produktivität der einheimischen Arbeitskräfte und verbessert so deren Beschäftigungsaussichten und Einkommensentwicklung. Dieser Arbeitsmarkteffekt ist bei Investitionen in Produktivkapital stärker als bei reinen Finanzanlagen ${ }^{261}$, weil bei ersteren die internationale Mobilität geringer ist. Insgesamt ist daher ein hohes Vermögen der Zuwanderer, v.a. wenn es in inländisches Produktivkapital angelegt wird, für die Einheimischen vorteilhaft.

Auch in Abhängigkeit von der Nationalität der Einwanderer entstehen den Einheimischen unterschiedliche Kosten. Nach Borjas erklärt die Änderung der Nationalitätenstruktur der Einwanderer in die USA den Rückgang des Bildungsstands zwischen den Einwanderungskohorten 1955-60 und 1975-80 zu etwa 85\% und die Erhöhung des Einkommensabstands zu den Einheimischen zu über 100\%.262 Dieser Rückgang der Qualität der Einwanderer infolge der Aufhebung des Systems der Nationalitäten-Quoten in den sechziger Jahren ist allerdings überwiegend durch die dadurch bewirkte Veränderung der Einwandererstruktur bezüglich der oben angeführten Kriterien (v.a. des Bildungsstandes) erklärbar. Für Immigranten in die USA aus Ländern mit niedrigem Pro-Kopf-Einkommen sind die Assimilationskosten höher als für Einwanderer aus hoch entwickelten Industriestaaten, weil zwischen Industrienationen eine größere Ähnlichkeit des länderspezifischen Humankapitals besteht. ${ }^{263}$ Hinsichtlich der nicht quantifizierbaren gesellschaftlichen Kosten, die durch die kulturelle Andersartigkeit der Einwanderer entstehen, und der im Gegensatz dazu positiven Produktivitätswirkung durch größere Variation der Kulturkreise kann der Nationalität eine gewisse Bedeutung zugemessen werden.

Eine Auswahl nach der Nationalität erscheint aus drei Gründen dennoch nicht gerechtfertigt. ${ }^{264}$ Erstens wird eine Auswahl nach der Nationalität aus ethischer Sicht als rassistisch angesehen werden. Zweitens sind die Unterschiede in den Kosten für die Einheimischen wohl eher gering und drittens kann eine Diskriminierung im Aufnahmeland die Ursache für das schlechtere Abschneiden einiger Nationalitäten sein.

\subsection{Instrumente für eine verstärkte Einwanderung}

Im vorangegangenen Unterkapitel ist geklärt worden, welche Zuwanderergruppen zu unterscheiden sind und welche Auswahlkriterien zur Erreichung einer für die Einheimischen beson-

260 Eine leicht abweichende Einschätzung findet sich bei Simon (1989) S. $327 \mathrm{f}$.

261 Auch Portfolioinvestitionen führen zur Bildung von Produktivkapital, solange die Schuldner die Finanzmittel nicht zu Konsumzwecken verwenden.

Vgl. Borjas (1992) S. 41.

263 Vgl. Borjas (1994) S. 1687.

264 Vgl. Simon (1989) S. 320. 
ders günstigen Struktur der ökonomischen Zuwanderer zu beachten sind. Jetzt wird untersucht, welches von verschiedenen Instrumenten einer Einwanderungspolitik zur Erfüllung der oben angeführten Kriterien am geeignetsten erscheint. Flüchtlinge und Verfolgte werden - wie oben ausgeführt - nach überwiegend politisch orientierten Kriterien zugelassen, so daß sie für die Diskussion in diesem Kapitel ausgeklammert werden können. In welchem Ausmaß ökonomische Kriterien für die Einwanderung von Familienangehörigen maßgeblich sein sollen, muß gleichfalls auf politischer Ebene entschieden werden. Prinzipiell lassen sich Elemente der Familienzusammenführung mit jedem der unten angeführten Instrumente kombinieren.

Grundsätzlich sollen diese Instrumente dazu dienen, den Nutzen für die Einheimischen zu maximieren. Analog zum Gewinnmaximierungskalküls eines Unternehmens ist die Einwanderung so zu steuern, daß die Grenzerlöse der Einwanderung den Grenzkosten entsprechen. ${ }^{265}$ Die Erlöse ergeben sich dabei z.B. aus der Erhebung einer Einwanderungsabgabe oder der Versteigerung von Einwanderungsrechten, ${ }^{266}$ die Kosten als - annahmegemäß negativer Saldo aus den in Kapitel 3 dargestellten Nutzen und Kosten der Einwanderung einschließlich kultureller und gesellschaftlicher Kosten. Da die Höhe der Grenzkosten nicht beobachtbar sind, muß die Entscheidung über die Zahl der Zuwanderer allerdings auf politischer Ebene fallen. Ein weiteres Problem entsteht daraus, daß die Einwanderer nicht homogen sind, sondern aufgrund unterschiedlicher Charakteristika verschieden hohe Nettokosten bzw. -nutzen haben. Deshalb ist zusätzlich dafür Sorge zu tragen, daß zunächst die Einwanderer mit den vorteilhaftesten Merkmalen zugelassen werden. Als Instrumente einer verstärkten Einwanderung werden Einwanderungskontingente, Quoten, Abgaben und Einwanderungszertifikate vorgeschlagen. Welches dieser Instrumente zur Erreichung des oben angeführten Ziels am geeignetsten ist, wird im folgenden untersucht.

Ein Einwanderungskontingent setzt nur die Gesamtzahl der pro Periode zugelassenen Einwanderer fest. Nach welchem Allokationsmechanismus die Einwanderungserlaubnisse den Einwanderungswilligen zugeteilt werden, ist damit noch nicht bestimmt. Normalerweise wird dies mit Hilfe einer Warteschlange geschehen, so daß nicht die potentiellen Einwanderer zugelassen werden, die das beste Kosten-Nutzen-Verhältnis für die Einheimischen aufweisen, sondern diejenigen, die am längsten gewartet haben. ${ }^{267}$ Durch diesen Zuteilungsmechanismus wird nicht einmal eine zufällige, d.h. durchschnittliche Auswahl unter den Einwanderungswilligen getroffen. Denn sofern andere Staaten eine erfolgreiche Selektionspolitik betreiben, werden Migranten mit besonders vorteilhaften Charakteristika dort ohne Wartezeit Aufnahme finden und daher als potentielle Einwanderer nach Deutschland ausscheiden. Außerdem hat die Wartezeit ein hohes Alter der Einwanderer zur Folge, was für die Einheimischen (und auch für die Zuwanderer) von Nachteil ist. Einwanderungskontingente ohne Quotierungen führen zu vermeidbaren Effizienzverlusten und sind daher ein wenig taugliches Mittel für eine die Wohlfahrt der Einheimischen steigernde Einwanderungspolitik.

265 Straubhaar (1991) sieht die Internalisierung externer Effekte von Zuwanderem als vorrangige Aufgabe eines Einwanderungsgesetzes an. Ein solcher Ansatz ist nur dann sinnvoll, wenn die Maximierung der Wohlfahrt sowohl der Einheimischen als auch der Einwanderer angestrebt wird.

266 Inwieweit sich die Grenzerlöse und die Höhe der Abgabe bzw. der Preis der Einwanderungsrechte unterscheiden, hängt von der Intensität des Wettbewerbs zwischen den Einwanderungsländern um die Einwanderer $\mathbf{a b}$.

267 Alternativ kann die Auswahl auch durch eine Lotterie erfolgen, ein Verfahren, das in begrenztem Umfang in den USA praktiziert wird. Vgl. Zimmermann (1994a) S. 240. 
Unter Quotierung wird eine Aufteilung der durch ein Kontingent festgelegten Gesamtzahl der Einwanderer auf verschiedene Einwanderergruppen verstanden, ${ }^{268}$ wobei zunächst offen ist, nach welchen Kriterien die Gruppen gebildet werden. Die Verwirklichung eines Punktesystems erlaubt die Kombination verschiedener Selektionskriterien. Theoretisch ist daher die Aufnahme aller oben angeführten Kriterien möglich. Insbesondere kann die Altersstruktur der Einwanderer durch entsprechende Quotenfestsetzungen günstig beeinflußt werden. Probleme bereitet aber die Gewichtung der verschiedenen Kriterien im Rahmen des Punktesystems, und auch die Skalierung für ein Kriterium ist letztendlich nur willkürlich möglich. So erfordert beispielsweise das Kriterium "Qualifikation" die Abgrenzung einzelner Arbeitsmarktsegmente und die Feststellung, ob in ihnen strukturelle Arbeitslosigkeit vorliegt. Dies verursacht zumindest einen hohen administrativen Aufwand und ist möglicherweise durch eine Behörde gar nicht leistbar. ${ }^{269}$ Hinsichtlich einiger Kriterien muß mit strategischem Verhalten der Einwanderer bei Beantwortung der Fragen gerechnet werden. Falsche und subjektiv gefärbte Antworten zu den Kriterien "Fähigkeiten, Einsatzbereitschaft und Ambition" und "Aufenthaltsdauer" können durch die Einwanderungsbehörden nicht erkannt werden. Da nur etwa ein Drittel der Einkommensvariation beobachtbaren Merkmalen zugeordnet werden kann, ${ }^{270}$ ist hierin ein entscheidender Nachteil eines Punktesystems zu sehen.

Im Gegensatz zu Kontingenten und Quoten setzen Einwanderungsabgaben nicht bei der Menge an, sondern beim Preis. Verschiedene Varianten sind denkbar: Wird die Abgabe als einmalige Zahlung bei der Einreise von dem Migranten eingefordert, stellt sich das Problem, $\mathrm{da}$ ß viele Immigranten mit vorteilhaften Merkmalen zu dem günstigsten Wanderungszeitpunkt nicht über die nötigen finanziellen Mittel verfügen. Eine Beleihung ihres Humankapitals scheitert an dessen mangelnder Aneigenbarkeit durch den Gläubiger im Fall des Zahlungsverzugs, so daß als einziger Ausweg ein staatlich gesicherter oder bereitgestellter Kredit in Verbindung mit der Ausweisung bei Zahlungsverzug bleibt. Diese Erkenntnis hat zu dem Vorschlag geführt, die Abgabe von vornherein nicht als einmalige Summe bei der Einreise, sondern als Sondersteuer auf das später im Inland erzielte Einkommen der Zuwanderer zu erheben, die bei einem bestimmten kumulierten Steuerbetrag ausläuft. ${ }^{271}$ Dann ist allerdings mit verzerrenden Substitutionseffekten zu rechnen, weil die Arbeitsangebotsentscheidung der Migranten möglicherweise durch den zusätzlichen Steuerkeil verändert wird. Erfolgt die Besteuerung nicht bei dem Einwanderer selbst, sondern bei seinem inländischen Arbeitgeber, birgt das bei einer einmaligen Abgabe ein hohes Risiko für den Arbeitgeber, da der Steuerbetrag bei einer Kündigung des Einwanderers verloren wäre. Bei periodischer Steuerzahlung ergibt sich in bezug auf die Arbeitsangebotsentscheidung kein Unterschied zu dem Fall, in dem die Zahllast bei den Einwanderern liegt, da die Traglast der Steuer unabhängig davon ist, welche Partei als Steuerschuldner bestimmt wird.

268 Vgl. zu dieser Definition Gusy (1994b) S. 143. Ist die Gesamtzahl der Einwanderer nicht festgelegt, dann kann unter bestimmten Annahmen eine Erhöhung der Minimumanforderungen an die Qualifikation der Einwanderer zu einer Senkung des tatsächlichen durchschnittlichen Qualifikationsstandes der Zuwanderer führen, wie Djajic (1989) in einem Zwei-Länder-Modell zeigt.

269 Auch hinsichtlich der Bewertung des Humankapitalbestandes der Zuwanderer entstehen durch mangelnde Vergleichbarkeit der Abschlüsse Probleme bei der Implementierung. Vgl. Simon (1989) S. 322.

270 Vgl. die in Franz (1991) S. 107f. aufgeführten Studien für Deutschland und die Studie von Borjas (1990) S. 212 für die USA.

271 Alternativ ist auch eine periodisch zu erhebende Kopf-Steuer für Immigranten denkbar. 
Gegenüber den oben angeführten Quoten hat die Einwanderungsabgabe den Vorteil, daß das individuelle Kosten-Nutzen-Kalkül der Einwanderer stärker in die Migrationsentscheidung eingeht. Bei einer Kontingentierung oder Quotierung wollen alle Personen wandern, die sich unter Berücksichtigung der Wanderungskosten von der Migration einen Nutzenzuwachs versprechen, und die tatsächliche Auswahl ist unabhängig von der Höhe dieses Nutzenzuwachses. Bei Erhebung einer Einwanderungsabgabe rechnen die Migranten deren Höhe zu den Kosten der Migration hinzu. Damit sinkt die Zahl der potentiellen Migranten und zwar aufgrund des Wegfalls derjenigen Wanderungswilligen, deren erwarteter positiver Nutzen aus der Wanderung nicht ausreicht, um den negativen Nutzen aus der Abgabe auszugleichen. Da der erwartete Nutzen einer Wanderung stark mit dem erwarteten Einkommen im Zielland zusammenhängt, ${ }^{272}$ kann angenommen werden, daß eine Einwanderungsabgabe zu einer Selektion führt, die das durchschnittliche erwartete Einkommen der Immigranten im Aufnahmestaat anhebt. So kann eine hinsichtlich aller acht im vorigen Unterkapitel angeführten Kriterien günstige Auswahl unter den Einwanderungswilligen erreicht werden, und zwar einschließlich derjenigen, bei denen aufgrund von strategischem Antwortverhalten bei Implementierung eines Punktesystems keine Selektionswirkung erreichbar ist. ${ }^{273}$ Als positiver Nebeneffekt fallen Steuereinnahmen an, die z.B. in Form einer (Einkommen-)Steuersenkung an die Einheimischen weitergegeben werden können oder auch zur Kompensation der Herkunftsländer für den Abfluß an Humankapital dienen können. Um zu verhindern, daß ökonomisch motivierte Einwanderer versuchen, die Steuer zu vermeiden, indem sie zunächst den Weg über eine Anerkennung als Flüchtling wählen und erst im Falle einer Ablehnung die Einwanderungssteuer zahlen, ist der Antrag auf Einwanderung als Flüchtling mit einem - eventuell befristeten - Ausschluß der Einwanderung aus ökonomischen Motiven zu verbinden.

Nachteilig ist bei einer Einwanderungsabgabe, daß die Festsetzung des Steuerbetrags willkürlich erfolgen muß, weil die tatsächliche Höhe der Kosten der Einwanderer für die Einheimischen nicht feststellbar ist. Aus der Höhe der Abgabe und den anderen für die Wanderungsentscheidung relevanten Einflußfaktoren ergibt sich dann die Zahl der tatsächlich einwandernden Personen. Die Gesamtzahl der Einwanderer ist aufgrund der Unkenntnis über die individuellen Nutzenfunktionen ex ante unbekannt und bietet daher anders als die mengenfixierende Kontingents- oder Quotierungslösung keine verläßliche Planungsgrundlage. Es besteht zwar die Möglichkeit einer Korrektur der Steuersatzhöhe, um so durch ein Versuch-IrrtumVerfahren die Abgabenhöhe zu finden, die einer gewünschten Einwandererzahl entspricht. Zum einen unterliegen aber die wanderungsbestimmenden Faktoren recht kurzfristigen Schwankungen und zum anderen ist das Verfahren einer Steuersatzänderung in der Regel recht langwierig. Durch eine Einwanderungsabgabe ist daher keine auch nur annähernd präzise Steuerung der Mengengröße (Zahl der Einwanderer pro Periode) möglich.

Diese Schwäche einer Einwanderungsabgabe wird durch das gleichfalls marktkonforme Instrument der Einwanderungszertifikate vermieden. ${ }^{274}$ Bei ihnen wird nicht der Preis der Einwanderung auf politischem Wege fixiert, sondern die Menge, d.h. die Zahl der gewünschten

272 Vgl. die Diskussion in Kapitel 2.

273 Vgl. Borjas (1990) S. 224. Eine gezielte Beeinflussung der Altersstruktur wie im Rahmen eines Punktesystems ist hier nicht möglich. Da aber junge Einwanderer einen längeren Zeitraum haben, um die "Investitionskosten" in Form der Steuerzahlung wiederzugewinnen, ist mit einer positiven Selbstselektion der Zuwanderer zu rechnen.

274 Eine ausführliche Diskussion dieses Instruments findet sich in Simon (1989) S. 329. Er führt den Vorschlag, Einwanderungszertifikate zu versteigem, auf Chiswick (1982) und Becker (1987) zurück. 
Einwanderer pro Periode. Angebot und Nachfrage entscheiden dann über den Preis für ein Einwanderungszertifikat. Die Zusammensetzung der Einwanderer wird also wie bei der Steuerlösung durch den Markt bestimmt und es stellt sich die gleiche vorteilhafte Selbstselektion der Zuwanderer ein. ${ }^{275}$ Bei Gleichheit von Steuersatzhöhe und Zertifikatspreis sind die Ergebnisse der beiden Instrumente identisch.

Der Vorteil der Zertifikatslösung gegenüber der Steuerlösung ist v.a. in der flexiblen Anpassung des Zertifikatspreises an Nachfrageschwankungen zu sehen. Dabei besteht nach wie vor die Möglichkeit, Änderungen der Kosten der Zuwanderer für die Einheimischen (etwa durch den Eintritt in eine andere Konjunkturphase) durch eine Veränderung der Zahl der pro Periode ausgegebenen Zertifikate zu berücksichtigen. So kann in Hochkonjunkturphasen mit besonders starkem Bedarf an ausländischen Arbeitskräften die Gesamtzahl der Zertifikate erhöht, in einer Rezession dann aber wieder zurückgefahren werden. Allerdings stellt sich wie schon bei der Abgabenlösung das Problem, daß einige Einwanderungswillige mit besonders vorteilhaften Charakteristika zum Zeitpunkt der Einreise nicht in der Lage sein werden, den Preis für ein Einwanderungszertifikat aufzubringen. Wie oben erläutert, ist diese aus der eingeschränkten Funktionstüchtigkeit der Kapitalmärkte resultierende Ineffizienz durch staatlich garantierte Kredite in Verbindung mit einer Ausweisung bei Nicht-Erfüllung der Annuitätszahlungen vermeidbar. Dadurch entstehen dem Staat jedoch recht hohe Verwaltungs- und Durchsetzungskosten. Ist das Einwanderungszertifikat im Gegensatz zu der nicht erstattungsfähigen Steuer bei Rückkehr in das Heimatland wieder verkaufbar, ${ }^{276}$ ist ein Verzicht auf staatlich garantierte Kredite möglich, weil dann die Arbeitgeber der Immigranten oder andere Gläubiger eine Sicherheit besitzen. Für eine Wiederveräußerbarkeit der Lizenzen spricht zudem, daß so die Zahl der im Ausland geborenen Personen in Deutschland langfristig geplant werden kann. Eine Rückwanderung führt dann über eine Preissenkung für die Einwanderungslizenzen zu einer genau gleichhohen Zunahme der Zuwanderung, ${ }^{277}$ so daß die Höhe der Nettowanderung steuerbar ist.

Gerade unter der Zielsetzung der Stabilisierung der Gesetzlichen Rentenversicherung über eine Beeinflussung der Bevölkerungsentwicklung sind Einwanderungslizenzen daher als das Instrument einer Einwanderungspolitik anzusehen, das am ehesten geeignet ist, die Kosten der Zuwanderung für die Einheimischen zu minimieren. ${ }^{278}$

Ethische Bedenken gegen dieses Instrument unter dem Vorwurf des Menschenhandels sind nicht gerechtfertigt, solange man von der Legitimität der Wohlfahrtsmaximierung der Einheimischen als gesellschaftlich anerkanntem Ziel ausgeht. ${ }^{279}$ Letztlich ist irgendeine Form der

275 Wie bei der Steuerlösung ist die Selbstselektion nur dann vorteilhaft für die Einheimischen, wenn die Kosten für die Einheimischen mit steigender Zahlungsbereitschaft der Einwanderungswilligen sinken. Ist die Einkommensverteilung im Herkunftsland wesentlich ungleicher als im Aufnahmeland, haben Individuen mit geringem erwarteten Einkommen im Aufnahmeland möglicherweise stärkere Wanderungsanreize als Personen mit hohem erwarteten Einkommen.

276 In diesem Fall sind die Einwanderungszertifikate mit einer Laufzeit auszustatten. Denkbar wäre z.B. eine Gültigkeitsdauer von 10 Jahren, an deren Ende die Möglichkeit zur Einbürgerung eröffnet wird.

277 Vorausgesetzt ist hier, daB immer mehr Einwanderungswillige als Lizenzen vorhanden sind.

278 Simon (1994) S. 247 schreibt: "The best way to ration immigration and to select the most economically productive people is to allow them to select themselves according to their willingness to invest in their own talent and capital. This implies an auction. A poor second choice is a point system." Zimmermann (1994b) S. 270 befürwortet demgegenüber ein Quotierungssystem. 
Selektion erforderlich, will man nicht völlige Freizügigkeit zulassen. Die Selektion über die (zukünftige) Zahlungsfähigkeit und -bereitschaft der Einwanderer ist aber im Vergleich zu anderen Kriterien sehr transparent und damit nicht so willkürlich wie beispielsweise ein Punktesystem. ${ }^{280}$ Niemand wird gegen seinen Willen gezwungen zu wandern, ${ }^{281}$ sondern jedes Individuum kann in freier Entscheidung abwägen, ob es bereit ist, den Preis für eine Lizenz zu zahlen oder nicht.

Jedes Einwanderungsgesetz ist aber nur so gut wie seine Durchsetzung. Dabei scheitert die Sicherstellung einer ausschließlich legalen Zuwanderung an den prohibitiv hohen Kosten einer solchen Politik. ${ }^{282}$ Die grüne Grenze weitgehend gegen illegale Grenzübertritte abzusichern, wäre mit sehr hohen Aufwendungen verbunden. Aber selbst wenn es gelänge, illegale Grenzübertritte zu verhindern, hat jeder Einwanderungswillige immer noch die Möglichkeit, als Tourist oder Transitreisender die Grenze auf legale Weise zu passieren und dann einfach die zulässige Höchstaufenthaltsdauer zu überschreiten. Die Zahl der illegal beschäftigten Arbeitnehmer kann man zwar über die Androhung von Strafen für die Arbeitgeber ${ }^{283}$ reduzieren, aber nur mit polizeistaatlichen Mitteln weitgehend unterbinden. Darüber hinaus weisen illegale Einwanderer Charakteristika auf, die die Last für die Einheimischen sehr gering halten und die ihnen Vorteile verschaffen. ${ }^{284}$ Nach den Erfahrungen in den USA sind sie fast ausnahmslos erwerbstätig, nehmen kaum Sozialleistungen in Anspruch, halten sich meistens nur temporär im Gastland auf und erledigen oft Aufgaben, für die einheimische Arbeitskräfte nicht zur Verfügung stehen. ${ }^{285}$ Andererseits stellt eine zu große Zahl an illegalen Einwanderern eine Ungerechtigkeit gegenüber den legalen Einwanderern dar, die den Preis für eine Einwanderungslizenz entrichtet haben.

Eine Möglichkeit, die Zahl der illegalen Einwanderer zu reduzieren, ohne hohe Überwachungskosten in Kauf nehmen zu müssen, bietet die Einführung eines Wanderarbeiterprogramms. ${ }^{286}$ Arbeitnehmer und Arbeitgeber profitieren gleichermaßen von der höheren Sicherheit des Arbeitsverhältnisses, so daß die Nachfrage nach illegalen Arbeitskräften zurückgeht. ${ }^{287}$ Um die Käufer von Zertifikaten nicht zu benachteiligen, müßte allerdings auf strikte Einhaltung des Rotationsprinzips geachtet werden. Nur so ist auch sichergestellt, daß den Einheimischen keine hohen Kosten aus der Anwesenheit von Wanderarbeitern entstehen und der Umfang von Kettenwanderungen gering bleibt. Gleichzeitig fördert die institutionelle Tren-

280 Ähnlich äußern sich Simon (1989) S. 332 und Zimmermann (1994a) S. 241.

281 Wie oben ausgeführt, sind Flüchtlinge und Verfolgte von der Einwanderungsregelung für ökonomisch motivierte Migranten ausgenommen.

Vgl. Abschnitt 2.2.2.

Eine Bestrafung der illegalen Arbeitnehmer hat nur eine geringe Wirkung, weil diese selten über konfiszierbare Aktiva verfügen und im Falle einer Deportation sofort wieder illegal einwandern können. Eine Bestrafung mit Freiheitsentzug wäre aber für die Einheimischen sehr kostspielig. Eine hohe Strafe für Arbeitgeber, die illegale Einwanderer beschäftigen, ist in Verbindung mit einer entsprechenden Entdeckungswahrscheinlichkeit dagegen erfolgversprechender wie das Beispiel der Schweiz zeigt.

Vgl. Simon (1989) S. 285ff.

Vgl. Simon (1989) S. 288ff.

Gegenwärtig bestehen bereits derartige Abkommen mit einigen osteuropäischen Staaten, die 1992 116.000 Arbeitnehmern aus diesen Ländern Beschäftigung in Deutschland verschafften. Vgl. Zimmermann (1994a) S. 249f.

Vgl. Simon (1994) S. 247 und allgemein zu einem Wanderarbeiterprogramm Simon (1989) S. $310 f$. 
nung von temporären Einwanderern (Wanderarbeitern) und dauerhaften Einwanderern die frühzeitige Humankapitalbildung bei letzteren.

Allerdings ist die Bundesrepublik Deutschland hinsichtlich der Gestaltung ihrer Einwanderungspolitik nicht ungebunden. Faktisch hat sie die Interessen der anderen Mitgliedstaaten der Europäischen Union zu beachten, da diese infolge der Abschaffung der Personenkontrollen an den meisten EU-Binnengrenzen mittelbar durch die Einwanderungsgesetzgebung in Deutschland betroffen werden. ${ }^{288}$ Einem nationalen Alleingang Deutschlands in der Einwanderungsgesetzgebung ist daher eine Regelung im Rahmen der Europäischen Union vorzuziehen. ${ }^{289}$

Besondere Bedeutung könnte in diesem Zusammenhang die Aufnahme neuer Mitglieder in die EU erlangen. Wenn die Bürger der Visegrad Staaten, der Baltischen Republiken und v.a. der Türkei mit der Mitgliedschaft dieser Staaten in der EU das Recht auf freie Wahl des Wohnund Arbeitsplatzes erhalten, entsteht ein großes Wanderungspotential, das durch ein Einwanderungsgesetz nicht beeinflußt werden kann. ${ }^{290}$ Bereits jetzt zeigt die Diskussion um das Entsendegesetz, welche Widerstände und Probleme die innerhalb der Europäischen Union praktizierte laissez-faire Lösung hervorruft. 291

\subsection{Die Kosten der Zuwanderung bei Realisierung eines Einwande- rungsgesetzes}

Nachdem in den vorangegangenen beiden Unterkapiteln geklärt worden ist, welche Kriterien einer für die Einheimischen vorteilhaften Auswahl der Zuwanderer zugrundegelegt werden sollten und eine Versteigerung von handelbaren Einwanderungszertifikaten als geeignetstes Instrument zur Erfüllung dieser Kriterien erkannt wurde, werden in diesem Unterkapitel die Kosten untersucht, die den Einheimischen bei Realisierung eines Einwanderungsgesetzes durch die Zuwanderung entstehen. Da sich bisher kein Land des Instruments der Einwanderungszertifikate bedient hat, liegen allerdings keinerlei Erfahrungswerte vor, die die Überprüfung der Richtigkeit und eine Abschätzung der Größenordnung der theoretisch vermuteten Wirkungen dieses Instruments erlauben würden.

Zwei Auswege bleiben: Erstens der Rückgriff auf einen Vergleich von Staaten mit unterschiedlichen Einwanderungspolitiken (Querschnittsvergleich). Sind beispielsweise die Kosten pro Einwanderer in Deutschland wesentlich höher als in Ländern, die wie Kanada oder Australien ein nach ökonomischen Kriterien gestaltetes Punktesystem zur Selektion der ökonomischen Zuwanderer verwenden, dann können die Kosten dieser Länder als Höchstgrenze für

288 Vgl. Zimmermann (1994a) S. 242. Formell haben Bürger aus Drittstaaten zwar nicht wie EU-Bürger das Recht auf freie Wahl des Wohnsitzes und Arbeitsplatzes innerhalb der EU, aber erstens sind Wanderungen innerhalb der EU schwer zu kontrollieren, zweitens sind sie spätestens mit Erwerb der Staatsbürgerschaft eines EU-Mitgliedstaates auch legal möglich und drittens kann die Zuwanderung von Drittstaatlem in ein Mitgliedsland entgegengerichtete Wanderungen von EU-Bürgern auslösen.

289 Vorschläge zu der konkreten Ausgestaltung eines an einem Quotierungssystem orientierten europăischen Einwanderungsgesetzes wurden von Gusy (1994a) und Wollenschläger (1994) unterbreitet.

290 Vgl. Zimmermann (1994b) S. 258.

291 Mit dem Entsendegesetz sollen ausländische Bauunternehmen verpflichtet werden, ihren in Deutschland arbeitenden Bauarbeitem für allgemeinverbindlich erklărte Mindestlöhne zu zahlen. Vgl. zu einer Diskussion Straubhaar (1996). 
die in Deutschland zu erwartenden Kosten bei Realisierung der Zertifikatslösung angesehen werden, wenn nicht andere Faktoren für die Kostendifferenz verantwortlich sind. Als zweite Möglichkeit kommt ein zeitlicher Vergleich in Betracht, indem untersucht wird, wie sich die Kosten der Zuwanderung infolge einer Änderung der Einwanderungspolitik entwickelt haben (Längsschnittsvergleich). Auch hier ist wieder zu beachten, daß neben der anderen Einwanderungspolitik auch weitere Faktoren zu der veränderten Kostenhöhe beigetragen haben können. Beiden Methoden gemeinsam ist die Schwierigkeit, die Kosten der Einwanderung überhaupt zu bestimmen. Wie in Kapitel 3 deutlich geworden ist, sind nur wenige Kostenarten mit hinlänglicher Verläßlichkeit quantifizierbar, während bei anderen noch nicht einmal die qualitativen Auswirkungen als gesichert angesehen werden können. Der Kostenvergleich muß daher auf wenige Kostenarten beziehungsweise auf vorgelagerte Einflußgrößen der Kostenhöhe beschränkt bleiben. Ein Längsschnittvergleich ist meines Wissens bisher für kein Land vorgenommen worden, so daß nur Querschnittsvergleiche zur Analyse der Kosteneffizienz von Einwanderungsinstrumenten herangezogen werden können.

Die Querschnittsstudien lassen sich unterteilen in Vergleiche verschiedener Einwanderungsinstrumente auf nationaler Ebene und internationale Vergleiche. Einen Vergleich auf nationaler Ebene zur Abschätzung des Einflusses der Einwanderungsgesetzgebung auf die Assimilation der Zuwanderer im Aufnahmeland führen Duleep und Regets für die USA durch. Sie nehmen dazu eine Variable in die Regressionsgleichung zur Erklärung des Einkommens der Einwanderer auf, die erfaßt, ob ein Einwanderer unter der Familienzusammenführung oder als ökonomischer Immigrant in die USA aufgenommen wurde. ${ }^{292} \mathrm{Als}$ Ergebnis der Regression erhalten die Autoren das auf theoretischer Basis antizipierte Ergebnis. Mit steigendem Anteil der Familienzusammenführung sinken die Einkommen der Immigranten ceteris paribus. Gleichzeitig weisen diese Immigranten aber in den Jahren nach der Einwanderung höhere Einkommenswachstumsraten auf als ökonomische Migranten, so daß sie nach etwa 16 bis 17 Jahren deren Einkommensniveau erreichen. ${ }^{293}$

Im folgenden wird auf internationale Vergleiche im Rahmen von Querschnittsbetrachtungen eingegangen. Die Arbeitsmarkteffekte bieten sich für einen internationalen Vergleich der durch Einwanderung entstehenden Kosten nicht an, weil die institutionellen Rahmenbedingungen von Land zu Land so unterschiedlich sind, daß eventuell auf unterschiedliche Charakteristika der Immigranten zurückzuführende Differenzen in den Arbeitsmarktwirkungen der Einwanderer nicht isoliert werden können. Bei den Kapitaleinkommen ist der Grad der Einbindung in den internationalen Kapitalmarkt von Land zu Land unterschiedlich. Zudem sind kaum Daten über die von Einwanderern in ihr Aufnahmeland transferierten Vermögen erhältlich, so daß auch hier ein internationaler Vergleich nicht vorgenommen werden kann. Auch ein internationaler Vergleich des Einflußes der Einwanderer auf die Sozialversicherungen und die Ausgaben der Gebietskörperschaften ist wenig sinnvoll, weil die nationalen Regelungen zu weit voneinander abweichen.

292 Da Daten über die Zulassungskategorie und das Einkommen auf individueller Ebene nicht kombiniert vorliegen, haben Duleep/Regets (1992) S. 419ff. die Wahrscheinlichkeit dafür, daß ein Immigrant einer bestimmten Herkunftsnation unter die Kategorie "ökonomischer Zuwanderer" fiel, in die Regressionsgleichung aufgenommen.

Werden alle Immigranten einer Herkunftsnation aufgrund der Familienzusammenführung zugelassen, erreichen sie nach diesen Ergebnissen durchschnittliche Einkommenswachstumsraten von etwa 5,5\% pro Jahr, während bei einem Anteil der Familienzusammenführung von sechzig Prozent nur noch Einkommenswachstumsraten von $0,6 \%$ erzielt werden. Vgl. Duleep/Regets (1992) S. 423. 
Vielversprechender sind internationale Vergleiche der Assimilation der Einwanderer und ihrer Steuerzahlungen an die öffentlichen Haushalte. Zwar sind auch die Steuersysteme und Abgabenniveaus international unterschiedlich, aber direkt oder indirekt ist der Großteil der jeweiligen Steuern einkommensabhängig. Außerdem genügt ein Vergleich des jeweiligen Unterschiedes zu den Einheimischen, um Schlußfolgerungen hinsichtlich der Selektionswirkungen verschiedener Einwanderungskonzepte ziehen zu können. Ulrich erhält für Gastarbeiter in Deutschland Einkommens- und Umsatzsteuerzahlungen von zusammen 7.129 DM pro Haushalt (1984), was 89\% der entsprechenden Steuerzahlungen der einheimischen Haushalte entspricht. ${ }^{294}$ Demgegenüber erhält Akbari für Kanada 1980 um Can $\$ 602$ oder 7\% höhere Steuerzahlungen der Einwanderer. ${ }^{295}$ Es kann daher angenommen werden, daß das an ökonomischen Kriterien ausgerichtete Einwanderungskonzept Kanadas einen Selektionseffekt ausübt, der zu einer Entlastung der öffentlichen Haushalte führt, bzw. daß die Regelungen in Deutschland zu einer für die Einheimischen ungünstigen Auswahl geführt haben.

Weitere Hinweise auf die Wirkungen unterschiedlicher Einwanderungspolitiken lassen sich aus einem von Duleep und Regets durchgeführten Vergleich zwischen den USA und Kanada hinsichtlich der Assimilation der Einwanderer gewinnen. ${ }^{296}$ Beide Staaten berücksichtigten in dem untersuchten Zeitraum zwischen 1960 und 1980 in ihren Einwanderungspolitiken sowohl Elemente der Familienzusammenführung als auch ökonomische Kriterien. Der Unterschied zwischen beiden Staaten bestand in der Gewichtung. Während in Kanada die meisten Einwanderer nach den Änderungen der Einwanderungsgesetzgebung in den Jahren 1962 und 1967 sowie vor einer Aufwertung der Familienzusammenführung im Jahr 1976 aufgrund eines an der Länge der Ausbildung, dem Niveau der beruflichen Qualifikation, dem Alter, der Sprachfertigkeit, der Motivation, der spezifischen Arbeitsnachfrage in Kanada, dem Vorhandensein eines Arbeitsvertrages und den Beschäftigungsaussichten in der Zielregion ausgerichteten Punktesystems selektiert wurden, dominierte in den USA die Familienzusammenführung. ${ }^{297}$ Tatsächlich zeigt sich, daß die Einwanderer nach Kanada im betrachteten Zeitraum für die meisten Herkunftsregionen (China, übriges Asien, Europa und Großbritannien) und Einwanderungszeitpunkte ${ }^{298}$ jünger sind als für die USA und zudem über bessere Sprachkenntnisse verfügen (vgl. Tab D.5). In bezug auf den Humankapitalbestand ist dagegen kein großer Unterschied festzustellen. ${ }^{299}$

Allerdings führen die vorteilhafteren Charakteristika der kanadischen Immigranten weder zu einem konsistent höheren Einkommen relativ zu einheimischen Weißen noch zu höheren

294 Vgl. die im Unterabschnitt 3.3.2.1 dargestellte Studie von Ulrich (1992).

295 Vgl. Akbari (1989) S. 431.

296 Vgl. Duleep/Regets (1992).

297 Zu einer Beschreibung der unterschiedlichen Einwanderungspolitiken vgl. Borjas (1990) S. 202ff. und Duleep/Regets (1992) S. 410ff. sowie zu neueren Entwicklungen Chapman (1994), Ruddick (1994) und Jasso/Rosenzweig (1994).

298 Das relative hohe Alter der kanadischen Einwanderungskohorten zwischen 1975 und 1980 hat seine Ursache möglicherweise in einer Verlagerung der kanadischen Einwanderungspolitik in Richtung Familienzusammenführung im Jahre 1976.

299 Vgl. Duleep/Regets (1992) S. 425f. Die Autoren weisen auf die eingeschrănkte Vergleichbarkeit der USamerikanischen und kanadischen Daten zu Sprachfertigkeit und Humankapitalbestand hin. 
Tab. D.5: Durchschnittliches Alter, Ausbildung und Sprachfertigkeit amerikanischer und kanadischer Einwanderer nach Herkunftsregion und Einwanderungsperiode

\begin{tabular}{|c|c|c|c|c|}
\hline \multirow{3}{*}{$\begin{array}{l}\text { China } \\
\text { Alter }\end{array}$} & $1975-80$ & $1970-74^{\mathrm{a}}$ & $1965-69^{b}$ & $1960-64^{c}$ \\
\hline & & & & \\
\hline & \multicolumn{4}{|c|}{ Alter } \\
\hline & 36,83 & 39,31 & 43,20 & 46,42 \\
\hline Kanada & 38,19 & 36,62 & 39,00 & 38,07 \\
\hline \multicolumn{5}{|l|}{ Ausbildung } \\
\hline USA & 13,01 & 13,76 & 14,16 & 14,34 \\
\hline Kanada & 12,16 & 14,23 & 14,60 & 13,93 \\
\hline \multicolumn{5}{|c|}{ Sprachfertigkeit } \\
\hline USA & 60,08 & 71,94 & 73,99 & 74,28 \\
\hline Kanada & 73,38 & 89,70 & 94,04 & 90,74 \\
\hline \multicolumn{5}{|c|}{ Übriges Asien } \\
\hline \multicolumn{5}{|c|}{ Alter } \\
\hline USA & 36,32 & 37,97 & 41,23 & 43,77 \\
\hline Kanada & 35,75 & 36,00 & 40,41 & 44,25 \\
\hline \multicolumn{5}{|l|}{ Ausbildung } \\
\hline USA & 13,95 & 15,73 & 16,06 & 16,44 \\
\hline Kanada & 13,39 & 14,03 & 14,72 & 14,74 \\
\hline \multicolumn{5}{|c|}{ Sprachfertigkeit } \\
\hline USA & 75,16 & 92,44 & 96,31 & 97,18 \\
\hline Kanada & 94,36 & 99,29 & 99,29 & 100,00 \\
\hline \multicolumn{5}{|l|}{ Europa } \\
\hline \multicolumn{5}{|l|}{ Alter } \\
\hline USA & 34,48 & 42,83 & 45,43 & 48,03 \\
\hline Kanada & 35,35 & 37,75 & 39,89 & 41,43 \\
\hline \multicolumn{5}{|l|}{ Ausbildung } \\
\hline USA & 12,63 & 10,56 & 10,32 & 11,49 \\
\hline Kanada & 12,46 & 10,75 & 11,26 & 10,39 \\
\hline \multicolumn{5}{|c|}{ Sprachfertigkeit } \\
\hline USA & 65,71 & 63,16 & 75,29 & 85,52 \\
\hline Kanada & 87,07 & 89,63 & 91,34 & 92,80 \\
\hline \multicolumn{5}{|c|}{ Großbritannien } \\
\hline \multicolumn{5}{|l|}{ Alter } \\
\hline USA & 33,42 & 38,50 & 45,39 & 48,50 \\
\hline \multirow{2}{*}{\multicolumn{5}{|c|}{ Ausbildung }} \\
\hline & & & & \\
\hline USA & 15,37 & 14,83 & 14,22 & 13,50 \\
\hline Kanada & 14,17 & 13,92 & 14,05 & 13,92 \\
\hline \multicolumn{5}{|c|}{ Sprachfertigkeit } \\
\hline USA & 100,00 & 100,00 & 100,00 & 100,00 \\
\hline & 100,00 & 100,00 & 100,00 & 100,00 \\
\hline \multicolumn{5}{|c|}{ a Für Kanada 1971-74. } \\
\hline b Für $\mathrm{l}$ & $6-70$ & & & \\
\hline c Für 1 & $1-65$ & & & \\
\hline
\end{tabular}

Quelle: Duleep/Regets (1992) S. 425f. 
Assimilationsraten für die einzelnen Herkunftsregionen. ${ }^{300}$ Die Autoren der Studie geben als mögliche Erklärung für die Ähnlichkeit der Einkommensprofile v.a. der asiatischen Immigranten in den USA und in Kanada an, daß die Einwanderer in die USA durch die Unterstützung der ethnischen Kommunen und Familien die Auswirkungen des höheren Durchschnittsalters und der geringeren Sprachfertigkeit auf das Einkommen kompensieren können. ${ }^{301}$

Eine andere Begründung für den geringen Unterschied der Einkommensprofile amerikanischer und kanadischer Immigranten liegt in der Vorgehensweise von Duleep und Regets. Sie vergleichen nämlich jeweils isoliert die relative Einkommensposition von Immigranten einer Herkunftsregion in den USA mit der relativen Einkommensposition von aus der gleichen Region nach Kanada Eingewanderten. Führt das kanadische Punktesystem zu einem höheren Anteil von Einwanderern aus Herkunftsregionen, die besonders vorteilhafte Charakteristika aufweisen, wird der daraus resultierende Effekt durch die Ergebnisse von Duleep und Regets nicht erfaßt. Letztendlich sind für die Kosten der Einwanderung aber nicht die Kosten der Einwanderer eines bestimmten Herkunftlandes entscheidend, sondern die den Einheimischen im Durchschnitt durch Immigranten aus allen Herkunftsgebieten zusammengenommen entstehenden Kosten. Eine Untersuchung von Borjas ${ }^{302}$ zeigt, daß das durchschnittliche Ausbildungsniveau der kanadischen Immigranten durch das Punktesystem positiv beeinflußt wurde, wenn man nicht isoliert einzelne Herkunftsländer betrachtet. Während in der ersten Hälfte der sechziger Jahre die amerikanischen Immigranten um etwa ein halbes Jahr längere Ausbildungszeiten aufwiesen, kehrte sich die Situation ab Mitte der sechziger Jahre ins Gegenteil. ${ }^{303}$ Dieser Bildungsvorsprung der kanadischen Immigranten geht einher mit im Vergleich zu US-amerikanischen Einwanderern höheren relativen Einkommen bei der Einwanderung. ${ }^{304}$

Erklärbar ist die im Vergleich zu den USA positivere Entwicklung des durchschnittlichen Ausbildungsniveaus und der durchschnittlichen Einkommen der kanadischen Immigranten größtenteils durch die Änderung der Anteile einzelner Herkunftsländer. Das Punktesystem Kanadas führte in dem betrachteten Zeitraum offenbar nicht zu einer Anhebung der durchschnittlichen Bildung der Einwanderer eines bestimmten Herkunftslandes, sondern zu einer im Vergleich zu den USA vorteilhafteren Nationalitätenstruktur der Immigranten. ${ }^{305}$

Dieses Ergebnis wird durch die Einbeziehung von Australien in den internationalen Vergleich bestätigt. Mit Aufgabe der "White Australia Policy" wurde dort 1972 ein ähnliches Punktesystem wie in Kanada eingeführt. Die Nationalitätenstruktur der Einwanderer blieb aber noch weit vorteilhafter als in Kanada, so daß australische Einwanderer Ende der siebziger

300 Die europäischen Migranten, die auch in den USA zu einem großen Teil in die Kategorie der ökonomischen Zuwanderer fallen, bilden eine Ausnahme. Vgl. Duleep/Regets (1992) S. 427ff.

301 Vgl. Duleep/Regets (1992) S. 431.

302 Vgl. Borjas (1991).

303 Vgl. Borjas (1991) S. 9ff.

304 Während kanadische Immigranten der Jahre 1975 bis 1980 bei der Einwanderung etwa 15 Prozent weniger verdienten als Kanadier, wiesen die amerikanischen Immigranten eine Verdienstdifferenz gegenüber den Einheimischen von ungefahr 29 Prozent auf. Diese Differenz wird weitgehend durch die unterschiedlichen Ausbildungsniveaus erklärt, so daß unbeobachtbare Faktoren wie Fähigkeiten, Einsatzbereitschaft, Ambition und Transferierbarkeit des Humankapitals der Einwanderer offenbar keine Rolle spielten. Vgl. Borjas (1991) S. 11. 
Jahre schon zum Zeitpunkt der Einwanderung keine Einkommensdifferenz zu den Einheimischen aufwiesen. ${ }^{306}$

Der Einfluß der kanadischen Einwanderungspolitik auf die Selbstselektion der Zuwanderer kann anhand der bilateralen Wanderungsströme zwischen den USA und Kanada erfaßt werden. Die US-amerikanischen Einwanderer nach Kanada (zwischen 1975 und 1980) hatten zwar eine um 4,5 Jahre längere Ausbildungszeit als Kanadier, verdienten 1981 aber geringfügig weniger. Kanadische Einwanderer in die USA hatten demgegenüber eine nur um zwei Jahre längere Ausbildungszeit als ein durchschnittlicher US-Amerikaner, verdienten aber etwa 20 Prozent mehr. ${ }^{307}$ Während das kanadische Punktesystem also durchaus erfolgreich in der Selektion besonders gut ausgebildeter US-Amerikaner ist, muß die Einkommenssituation dieser Einwanderergruppe als enttäuschend angesehen werden. Als Erklärung der abweichenden Einkommensprofile können unterschiedliche nicht-beobachtbare Eigenschaften der jeweiligen Immigrantengruppen dienen, die durch das kanadische Punktesystem nicht erfaßt werden können. $\mathrm{Zu}$ vermuten ist, daß in den USA aufgrund der ungleicheren Einkommensverteilung eine höhere Entlohnung für besondere Fähigkeiten, Einsatzfreude, etc. vorzufinden ist als in Kanada. Die Wanderungsanreize sind daher für Kanadier mit vorteilhaften unbeobachtbaren Charakteristika besonders hoch, während für US-Amerikaner das Gegenteil zutrifft. ${ }^{308}$ Die in Unterkapitel 4.2 geäußerte Kritik an der mangelnden Fähigkeit eines Punktesystems, auf unbeobachtbare Kriterien Einfluß nehmen zu können, wird durch diese Ergebnisse gestützt. Inwieweit handelbare Einwanderungszertifikate eine bessere Selbstselektion der Zuwanderer gewährleisten, kann aufgrund fehlender Erfahrungen mit diesem Instrument nicht beurteilt werden.

Als Ergebnis des nationalen und internationalen Vergleichs der Kosten der Einwanderer in Abhängigkeit von der Ausgestaltung der Einwanderungspolitik kann die Vorteilhaftigkeit einer ökonomischen Selektion der Einwanderer als bestätigt angesehen werden. Allerdings ist zu beachten, daß im wesentlichen nur die Assimilation der Zuwanderer betrachtet werden konnte und der Einfluß der Einwanderungsgesetzgebung auf die anderen in Kapitel 3 vorgestellten Kostenarten ausgeklammert blieb. Außerdem mußte auf die Erfahrungen der traditionellen Einwanderungsländer (USA, Kanada, Australien) zurückgegriffen werden, weil in Deutschland keine Erfahrungswerte bezüglich der Wirkungen ökonomischer Selektion permanenter Einwanderer vorliegen und auch die Wirkungen des Anwerbestopps für Gastarbeiter im Jahre 1973 auf die Kosten für die Deutschen bisher nicht untersucht worden sind. Unter Umständen ist ein höherer Anteil von Einwanderern mit hohem Humankapital für Deutschland schon deshalb schwerer zu erreichen, weil diese Einwanderergruppe oft schon vor der Wanderung über Englischkenntnisse verfügt und daher die Migrationskosten bei einer Wanderung in englischsprachige Aufnahmeländer geringer ausfallen als bei einer Einwanderung nach Deutschland. Da auch für die angelsächsischen Einwanderungsländer keine Erfahrungen mit

\footnotetext{
306 Vgl. Borjas (1990) S. 205.

307 Vgl. Borjas (1991) S. 19f.

308 Vgl. Borjas (1990) S. 215. Für Deutschland stellt sich dieses Problem noch schärfer, weil die Umverteilung durch den Staat bedeutend ausgeprägter ist als in Kanada. Deshalb muß damit gerechnet werden, daß nach Deutschland bei Einführung eines Punktesystems v.a. Migranten mit besonders negativen nicht-beobachtbaren Merkmalen kommen würden, wăhrend diejenigen mit vorteilhaften Ausprägungen dieser Charakteristika die höheren Einkommenschancen in Ländern mit höherer Entlohnung dieser Merkmale vorziehen werden.
} 
handelbaren Einwanderungszertifikaten vorliegen, kann die konkrete Höhe der Kosten der Immigration bei Anwendung dieses Instruments in Deutschland nicht abgeschätzt werden. Es muß bei der qualitativen Prognose bleiben, daß die Kosten der Einwanderung durch eine konsequent an ökonomischen Kriterien ausgerichtete Einwanderungskonzeption gegenüber dem status quo wahrscheinlich deutlich gesenkt werden können.

\section{Zusammenfassung und Schlußfolgerung}

Ziel dieses Teils der Arbeit war es, die Höhe der Kosten festzustellen, die eine verstärkte Einwanderung für die einheimische Bevölkerung mit sich bringen würde, um so einen Vergleich mit den Kosten zu ermöglichen, die zur Erhöhung der Fertilität der Einheimischen mittels eines Ausbaus des Kinderlastenausgleichs aufgebracht werden müßten.

Dazu wurden zunächst verschiedene Ansätze zur Erklärung internationaler Wanderungsbewegungen erläutert. Bei der Analyse des Wanderungspotentials erwiesen sich die auf das Heckscher-Ohlin-Samuelson Modell aufbauenden makroökonomischen Theorien als wenig hilfreich. Die am individuellen Nutzenkalkül ansetzenden mikroökonomischen Erklärungsansätze wurden in Kombination mit ökonomischen und politisch-ökonomischen Bestimmungsgründen der Nachfrage nach Zuwanderem für die folgende Kosten-Nutzen-Analyse als fruchtbarer erkannt. Gleichwohl muß die theoretische Basis zur Bestimmung von internationaler Migration als noch recht fragmentarisch bezeichnet werden. Dieser Mangel fällt hier allerdings weniger ins Gewicht, weil die Schaffung von Anreizen zur Einwanderung auf absehbare Zeit zweitrangig ist, da davon ausgegangen werden kann, daß die Zahl der Wanderungswilligen die Zahl der erwünschten Zuwanderer übersteigt.

Kosten der Zuwanderung entstehen im Gegensatz zum Kinderlastenausgleich nicht durch die zur Schaffung von Anreizen notwendigen staatlichen Ausgaben, sondern durch die kurzund langfristigen Auswirkungen der Zuwanderer auf die Wohlfahrt der Einheimischen. Betrachtet wurden nur die Kosten, die aufgrund anderer Charakteristika der Einwanderer also aufgrund einer Änderung der Bevölkerungsstruktur - entstehen, nicht dagegen Kosten, die durch die Änderung der absoluten Bevölkerungsgröße infolge der Zuwanderung entstehen. So ist ein direkter Vergleich mit der Situation bei einer gleichen Entwicklung der absoluten Bevölkerungsgröße infolge einer Erhöhung des Kinderlastenausgleichs möglich. Entsprechend des ökonomischen Ansatzes dieser Arbeit wurde auf eine Einbeziehung der gesellschaftlichen und kulturellen Folgen der Zuwanderung verzichtet, ohne deren Bedeutung leugnen zu wollen. Stattdessen erfolgte eine Eingrenzung der Analyse auf die Änderung der Wohlfahrtsposition der Einheimischen, die sich durch eine Veränderung ihrer (Netto-)Einkommen ergibt. Trotz dieser Eingrenzung konnte wegen der großen Schwierigkeiten, die sich einer Quantifizierung der verschiedenen Kostenarten entgegenstellen, keine Abschätzung der Höhe der Kosten der Zuwanderung vorgenommen werden.

Die Arbeitseinkommen werden durch die Zuwanderer möglicherweise ebenso wie die Höhe der Arbeitslosigkeit geringfügig in einer für die Einheimischen ungünstigen Weise beeinflußt. Wandern wie in der Vergangenheit hauptsächlich unterdurchschnittlich qualifizierte Arbeitskräfte zu, ist allerdings mit sinkenden Löhnen für gleichfalls wenig qualifizierte Einheimische zu rechnen, während einheimische Arbeitskräfte mit hohem Humankapitalbestand von der 
Einwanderung profitieren. Die Kapitaleinkommen der Einheimischen werden durch die Einwanderung gleichfalls kaum betroffen.

Während die Primäreinkommen der Einheimischen durch die Zuwanderer zumindest als Aggregatgröße wenig beeinflußt werden, ergibt sich bei den verfügbaren Einkommen aufgrund der umverteilenden Staatsaktivität ein anderes Bild. Das unterdurchschnittliche Qualifikationsniveau der Einwanderer hat in der Vergangenheit dazu geführt, daß sie von ihrem Einwanderungszeitpunkt an im Durchschnitt einen Einkommensrückstand gegenüber den Einheimischen hatten und diesen mit zunehmender Aufenthaltsdauer in Deutschland auch nicht verringern konnten. Da die höhere Erwerbsbeteiligung der Einwanderer nicht ausreichte, um die Auswirkungen des geringeren Durchschnittseinkommens zu kompensieren, leisteten EinwandererHaushalte geringere Steuerzahlungen als Haushalte Einheimischer. Gleichzeitig nahmen die Einwanderer aber überproportional Transferleistungen der Gebietskörperschaften in Anspruch, so daß sie die Nettoeinkommen der Einheimischen durch ceteris paribus höhere Steuern bzw. niedrigere Leistungen minderten.

Im Unterschied zu diesem Ergebnis für die Umverteilung durch die Gebietskörperschaften ergab sich eine positive Wirkung der Einwanderer auf die Nettoeinkommen der Einheimischen durch die Umverteilung im Rahmen der Sozialversicherungen. V.a. die günstigere Erwerbsund Altersstruktur der Zuwanderer führte dazu, daß sie pro Haushalte höhere Beiträge leisteten, aber im Durchschnitt weniger Leistungen erhielten als ein von einem Einheimischen geführter Haushalt.

Langfristig wird die Einkommensposition der Einheimischen durch die Produktivitätsentwicklung bestimmt. Die Erforschung des Einflusses der Einwanderer auf den technischen und organisatorischen Fortschritt und damit die Produktivität der Einheimischen steckt allerdings noch in den Anfängen. Zu vermuten ist aber ein eher positiver Einfluß auf die langfristige Einkommensentwicklung der Einheimischen.

Makroökonomische Rückwirkungen können die Größenordnung der beschriebenen Primäreffekte beeinflussen, qualitative Änderungen der Auswirkungen der Zuwanderer auf die Einkommensposition der Einheimischen sind dagegen unwahrscheinlich.

Die bei der Analyse der Kosten (und Nutzen) von Einwanderern gewonnenen Erkenntnisse wurden im folgenden benutzt, um Kriterien aufzustellen, deren Anlegung an ökonomische Zuwanderer zu einer für die Einheimischen möglichst vorteilhaften Gestaltung der Einwanderung führen. Als besonders bedeutend erwiesen sich eine junge Altersstruktur der Zuwanderer, nachgefragte berufliche Qualifikationen und hoher Humankapitalbestand, eine hohe Erwerbsbeteiligung und nicht-beobachtbare Merkmale wie besondere Fähigkeiten, Einsatzbereitschaft und Ambition. Als das Instrument, das am besten geeignet ist, eine entsprechende Selektion der Zuwanderer vorzunehmen, empfiehlt sich die Versteigerung von Einwanderungszertifikaten. Aufgrund der Erfahrungen der angelsächsischen Einwanderungsnationen mit an ökonomischen Kriterien ausgerichteten Einwanderungspolitiken kann davon ausgegangen werden, daß die Kosten der Zuwanderung für Deutschland bei einer Einführung von Einwanderungszertifikaten deutlich geringer ausfallen würden als in der Vergangenheit. 
Teil E:

\section{ABSCHLIEßENDER VERGLEICH VON KINDERLASTEN- AUSGLEICH UND VERSTÄRKTER EINWANDERUNG}

Ziel dieser Arbeit war es, Instrumente zu identifizieren, die geeignet sind, die zukünftigen Belastungen der Mitglieder der Gesetzlichen Rentenversicherung, die aus der prognostizierten Bevölkerungsentwicklung entstehen, zu reduzieren und diese Instrumente anschließend hinsichtlich ihrer Kosteneffizienz miteinander zu vergleichen. Den Ausgangspunkt bildete eine Untersuchung des Zusammenhangs zwischen Bevölkerungsentwicklung und Finanzlage der umlagefinanzierten Gesetzlichen Rentenversicherung, die zu dem Ergebnis führte, da $\beta$ der prognostizierte Anstieg des Beitragssatzes auf 26 bis 29 Prozent im Jahr 2030 die tatsächlichen Belastungen aus der vorhergesagten demographischen Entwicklung noch unterzeichnet. Die anschließende Analyse verschiedener Vorschläge zur Entschärfung dieser demographischen Krise ergab, daß sowohl Reformansätze, die an den grundsätzlichen Merkmalen des bisherigen Alterssicherungssystems festhalten, als auch die betrachteten Alternativen zu diesem System letztlich nur eine Umverteilung der zukünftigen Lasten zwischen Beitragszahlern, Steuerpflichtigen und Rentnern bewirken. Eine wirkliche Reduzierung der Lasten ist nur durch eine Korrektur der demographischen Ursachen zu erreichen, also durch eine Verminderung des prognostizierten Anstiegs der Altenquotienten.

Als gangbare Wege zur Erreichung dieses Ziels bieten sich zwei Alternativen an: eine Erhöhung der Fertilität durch eine Ausweitung des Kinderlastenausgleichs und eine gegenüber der angenommenen Entwicklung erhöhte Einwanderung. Dabei wirkt sich - wie in Teil B herausgefunden wurde - eine Erhöhung der zusammengefaßten Geburtenrate um 0,3 Kinder pro Frau im Jahr 2030 mit einer Beitragssatzsenkung um etwa 1,5 Prozentpunkte ähnlich aus wie eine Erhöhung des Einwanderungssaldos um 200.000 Personen pro Jahr (bei gleichbleibender Altersstruktur der Migranten). Welches der beiden Instrumente eine kostengünstigere Belastungsreduzierung verspricht, wurde in den Teilen $\mathrm{C}$ und $\mathrm{D}$ der Arbeit untersucht.

Zur Analyse der Wirkung des Kinderlastenausgleichs auf die Fertilitätsentwicklung wurde auf die neoklassische Theorie der Fertilität zurückgegriffen. Danach bestimmt sich die Nachfrage nach Kindern ähnlich wie die Nachfrage nach Konsumgütern. Die nachgefragte Menge wird ceteris paribus solange ausgedehnt bis die Grenzkosten dem Grenznutzen entsprechen. Sieht man von Präferenzänderungen ab, wird die Nachfrage nach Kindern neben der Einkommenshöhe vor allem durch ihre Grenzkosten bestimmt. Diese setzen sich nach den Erkenntnissen der New Home Economics aus den Kosten der zur Produktion des Basisgutes "Kind" erforderlichen Marktgüter, d.h. den direkten Kinderkosten, und dem bewerteten Zeiteinsatz, also den indirekten Kinderkosten, zusammen. Als durchschnittliche Höhe der indirekten Kosten wurde anhand eines Humankapitalansatzes ein Wert von etwa 300.000 DM pro Kind errechnet, wobei diese Zahl als Barwert der indirekten Kosten im Jahr 1992 zu verstehen ist. Der drastische Anstieg dieser Opportunitätskosten von Kindern aufgrund des steigenden Frauenlohnsatzes wurde als Hauptursache des beobachteten Geburtenrückgangs identifiziert. Demgegenüber ist die Wirkungsrichtung einer Einkommenserhöhung auf die Kinderzahl infolge einer Änderung des Schattenpreises der Kinderzahl, die durch den Zusammenhang zwischen Kinderqualität und -quantität hervorgerufen wird, weder theoretisch noch empirisch eindeutig feststellbar, bewirkt aber möglicherweise eine weitere Reduzierung der Kinderzahl. Für die direkten Kinderkosten wurde ein Wert von durchschnittlich 180.000 DM pro Kind im 
Jahr 1992 berechnet, die Veränderung der direkten Kinderkosten im Zeitablauf war allerdings eher gering.

Der starke Anstieg der indirekten Kosten in der Vergangenheit und der für die Zukunft anzunehmende weitere Anstieg machen eine auch nur teilweise Kompensation der Kinderkosten durch eine Aufstockung der Mittel des Kinderlastenausgleichs sehr kostspielig. Als Resultat der mit Hilfe unterschiedlicher Methoden durchgeführten Wirkungsanalyse wurde ein Betrag von rund $72.000 \mathrm{DM}$ pro Kind oder etwa $110 \mathrm{Mrd}$ DM pro Jahr für erforderlich befunden, um die zusammengefaßte Geburtenrate um 0,3 Kinder pro Frau ansteigen zu lassen. Durch eine stärkere Ausrichtung des Kinderlastenausgleichs auf Maßnahmen zur besseren Vereinbarkeit von Familie und Beruf ließe sich die Kosteneffizienz dieses Instruments allerdings steigern.

Die durch verstärkte Einwanderung hervorgerufenen Kosten bestehen im Gegensatz zu den Kosten, die durch eine Ausweitung des Kinderlastenausgleichs entstehen, nicht in staatlichen Ausgaben zur Schaffung von Anreizen. Es kann vielmehr davon ausgegangen werden, daß die Zahl der Zuwanderungswilligen auf absehbare Zeit die gewünschte Zuwandererzahl übersteigt, so daß die Schaffung von Einwanderungsanreizen überflüssig ist. Es spielte daher im Rahmen dieser Arbeit eine untergeordnete Rolle, daß die theoretische Basis zur Erklärung der Größe und Struktur von Migrationsströmen als noch recht fragmentarisch bezeichnet werden muß. Die Kosten (und auch Nutzen) der Einwanderung für die Einheimischen ergeben sich aus den gegenüber den Einheimischen andersartigen Charakteristika der Migranten und nicht aus den Kosten zur Schaffung von Einwanderungsanreizen. Eine eingehendere Analyse der Motivation zur Wanderung ist nur erforderlich, um Kostenänderungen zu erfassen, die sich infolge von Änderungen der Charakteristika der Einwanderer ergeben, wenn beispielsweise eine Reform der Einwanderungsgesetzgebung andere Anreizstrukturen schafft.

Die Kosten der Einwanderung wurden anhand der Einflüsse auf die Nettoeinkommen der Einheimischen untersucht. Bezüglich der Arbeitsmarktsituation der Einheimischen ergaben sich nur geringe Effekte. Vor allem für weniger qualifizierte einheimische Arbeitskräfte ist ein Rückgang des Lohnniveaus und ein Anstieg der Arbeitslosigkeit nicht auszuschließen. Eine Quantifizierung erwies sich aber als unmöglich, weil die einschlägigen empirischen Studien gravierenden Kritikpunkten ausgesetzt sind. Der Einfluß verstärkter Zuwanderung auf die Kapitaleinkommen der Einheimischen ist gleichfalls als gering einzuschätzen. Durch die Umverteilungstätigkeit der Gebietskörperschaften ergeben sich durch die Steuerzahlungen und die steuerfinanzierten Transferleistungen nachteilige Wirkungen für die Einheimischen. Zurückzuführen ist dieses Ergebnis auf das geringere Durchschnittseinkommen von Einwanderer-Haushalten, welches wiederum in ihrem unterdurchschnittlichen Humankapitalbestand begründet liegt. Im Bereich der Sozialversicherungen ergeben sich demgegenüber im Vergleich zur Situation mit einem verstärkten Kinderlastenausgleich zumindest durch permanente Zuwanderung keine Nachteile für Einheimische, weil sich hier die günstigere Altersstruktur der Einwanderer auswirkt und eine stärkere Äquivalenz zwischen Beiträgen und Transferzahlungen vorliegt. Langfristige Einkommensänderungen der Einheimischen über eine Beeinflussung des technischen und organisatorischen Fortschritts durch die Einwanderung sind nur schwer erfaßbar. Die angeführten theoretischen Argumente und einige empirische Hinweise sprechen aber für einen eher positiven Einfluß bei Zuwanderung gut ausgebildeter Arbeitskräfte.

Welche Schlußfolgerungen lassen sich hinsichtlich des Vergleichs der Kosteneffizienz von Kinderlastenausgleich und verstärkter Einwanderung ziehen? Obwohl eine genauere Quantifizierung der Kosten auch aufgrund der ungesicherten makroökonomischen Rückwirkungen 
nicht möglich ist, sprechen die gewonnenen Ergebnisse dafür, daß die Kosten der Einwanderung deutlich unter den Kosten für eine Aufstockung des Kinderlastenausgleichs liegen. Eine Anhebung der zusammengefaßten Geburtenziffer um 0,3 Kinder pro Frau hat nach dem in Teil B abgeleiteten Ergebnis etwa die gleiche Wirkung auf den Beitragssatz zur Gesetzlichen Rentenversicherung im Jahr 2030 wie eine jährliche Netto-Zuwanderung von 200.000 Personen. Um eine derartige Anhebung des Fertilitätsniveaus zu erreichen, müßten zusätzliche Mittel von ungefähr 110 Mrd DM pro Jahr für den Kinderlastenausgleich bereit gestellt werden. Würde man dieses Geld verwenden, um die Kosten der Zuwanderung auszugleichen, stünde pro Zuwanderer ein Betrag von rund 550.000 DM zur Verfügung. Es ist aber aufgrund der Ergebnisse aus Teil D dieser Arbeit kaum vorstellbar, daß den Einheimischen durch einen repräsentativen Zuwanderer Kosten entstehen, die auch nur entfernt an diese Größenordnung heranreichen.

Nun läßt sich einwenden, daß die Kosteneffizienz des Kinderlastenausgleichs noch steigerungsfähig ist, indem hauptsächlich Maßnahmen mit dem Ziel einer besseren Vereinbarkeit von Familie und Beruf getroffen werden. Gleiches gilt allerdings auch für die Zuwanderungsvariante, denn die Kosten der Einwanderung wurden überwiegend anhand der in der Vergangenheit beobachteten Auswirkungen der Zuwanderung von Gastarbeitern abgeleitet. Wie im vorangegangenen Kapitel gezeigt wurde, lassen sich diese Kosten aber bei Einführung eines Einwanderungsgesetzes, das beispielsweise durch die Versteigerung von Einwanderungszertifikaten eine Selektion nach ökonomischen Kriterien vornimmt, noch deutlich senken. Selbst wenn es gelingen sollte, die Kosteneffizienz des Kinderlastenausgleichs zu steigern, bleibt die Zuwanderungsvariante damit vorteilhafter.

Weiterhin kann zugunsten des Kinderlastenausgleichs angeführt werden, daß dieser im Jahr 2030 noch nicht seine volle beitragssenkende Wirkung entfaltet hat. Die Kosteneffizienz dieses Instruments wird daher in den nachfolgenden Jahrzehnten noch zunehmen. Hierin liegt allerdings gleichzeitig ein gewichtiger Nachteil des Kinderlastenausgleichs gegenüber einer verstärkten Einwanderung. Das Jahr 2030 wurde schließlich gerade deshalb als Vergleichsjahr herangezogen, weil die Finanzlage der Gesetzlichen Rentenversicherung in diesem Zeitraum besonders stark durch die demographische Entwicklung angespannt wird. Die lange Wirkungsverzögerung, die dadurch entsteht, daß die Neugeborenen erst nach etwa zwei Jahrzehnten in das Erwerbsleben eintreten, verhindert eine schnelle Entlastung der Gesetzlichen Rentenversicherung. Die indirekte Wirkungsweise des Kinderlastenausgleichs macht zudem eine Feinsteuerung unmöglich. Mit Hilfe einer verstärkten Einwanderung kann dagegen gezielt die Belastung in den besonders kritischen Perioden reduziert werden und es ist eine flexible Reaktion auf neue Entwicklungen möglich. Wegen der Möglichkeit von Verhaltensänderungen und der hohen Unsicherheit langfristiger Bevölkerungsprognosen liegt in der besseren Steuerbarkeit ein nicht zu unterschätzender Vorteil einer verstärkten Einwanderung.

Welche Politikempfehlungen lassen sich aus diesen Ergebnissen ableiten? Nach dem bisher Gesagten ist eine Ausweitung des Kinderlastenausgleichs eindeutig nachteilig gegenüber einer Verstärkung der Einwanderung. Der Kinderlastenausgleich hat sich als wenig effektives Instrument zur Erreichung demographischer Ziele erwiesen. Eine Ausweitung des Kinderlastenausgleichs aus demographischen Gründen ist daher nicht sinnvoll. Allenfalls sollte eine Umschichtung der Mittel, die aufgrund anderer Zielsetzungen für den Kinderlastenausgleich bereitgestellt werden, vorgenommen werden. Durch eine verstärkte Konzentration auf Maßnahmen zur besseren Vereinbarkeit von Familie und Beruf kann verhindert werden, daß die 
Kosteneffizienz des Kinderlastenausgleichs im Hinblick auf demographische Ziele mit steigendem Frauenlohnsatz in Zukunft weiter sinkt.

Die Verabschiedung eines Gesetzes zur Regelung der ökonomisch bedingten Zuwanderung ist nach den bisherigen Ausführungen weitaus vorteilhafter als eine Ausweitung des Kinderlastenausgleichs. Allerdings muß beachtet werden, daß gesellschaftliche und kulturelle Kosten nicht mit in die Kosten-Nutzen-Analyse der Einwanderung einbezogen wurden und die Höhe der wirtschaftlichen Kosten nicht endgültig geklärt werden konnte. Außerdem wurde kein direkter Vergleich mit der Situation ohne Eingriffe vorgenommen, so da $\beta$ nicht ausgeschlossen werden kann, daß eine Ausweitung der Zuwanderung zwar vorteilhafter ist als eine Erhöhung des Kinderlastenausgleichs, aber trotzdem nachteilig gegenüber einer Politik des laissez-faire. Eine solche Situation ist jedoch nicht sehr wahrscheinlich. Wenn die Auswirkungen einer absoluten Bevölkerungsänderung gering sind - wofür nach der knappen Diskussion in der Einleitung zu Teil $\mathrm{C}$ einiges spricht - haben die Ergebnisse aus Teil D auch im Vergleich mit einer laissez-faire Lösung Bestand. Trotzdem ist es ratsam, zunächst recht vorsichtig mit dem Instrument der ökonomisch motivierten Einwanderung umzugehen und die Zahl der Einwanderer nicht sofort drastisch zu erhöhen. Eine schrittweise Ausweitung hat den Vorteil, daß die Anpassungsvorgänge erleichtert werden und bei der Realisierung der ersten Schritte gewonnene Erkenntnisse verarbeitet werden können. Da sich die Finanzlage der Gesetzlichen Rentenversicherung nur allmählich verschlechtert, ist genügend Zeit für eine Experimentier- und Erprobungsphase bei niedrigeren Einwanderungszahlen vorhanden. Denkbar wäre etwa die Versteigerung von 100.000 Einwanderungszertifikaten im ersten Jahr, die dann jährlich um 100.000 aufgestockt wird. Die Aufstockung sollte beendet werden, wenn das Kontingent die Zahl der Wanderungswilligen mit vorteilhaften Charakteristika überschreitet oder wenn die gesellschaftlichen und kulturellen Kosten als zu hoch empfunden werden.

Allein durch eine verstärkte Einwanderung läßt sich der prognostizierte Anstieg des Beitragssatzes zur Gesetzlichen Rentenversicherung allerdings nicht verhindern, wenn man nicht Wanderungssalden von bis zu mehreren Millionen Personen pro Jahr akzeptieren will. Um effizienzmindernde Ausweichreaktionen der Beitragszahler zu verringern, muß deshalb zusätzlich auf umverteilende Maßnahmen zurückgegriffen werden, die über das Rentenreformgesetz 1992 hinausgehen. Die Einführung eines Teilkapitaldeckungsverfahrens kann nur noch einen geringen Beitrag leisten, weil nur noch wenige Jahre mit relativ niedrigen Beitragssätzen als Ansparzeitraum verbleiben. Stattdessen bietet sich v.a. eine Heraufsetzung des Renteneintrittsalters an, weil ein Teil des prognostizierten Anstiegs des Altenquotienten nicht auf den Geburtenrückgang, sondern auf die zunehmende Lebenserwartung zurückgeht. Die gestiegene Lebenserwartung und Vitalität macht eine Erwerbstätigkeit auch im höheren Alter zumutbar. Selbst wenn man die durchschnittliche Rentenbezugsdauer konstant läßt, ist deshalb eine sukzessive Anhebung des Renteneintrittsalters möglich. Außerdem hat sich eine Anhebung des Renteneintrittsalters als recht wirksames Instrument zur Beitragssatzsenkung erwiesen. Die genaue Kombination von höherer Einwanderung, Heraufsetzung des Renteneintrittsalters und Anstieg des Beitragssatzes muß allerdings im politischen Willensbildungsproze $\beta$ entschieden werden, weil neben Effizienz auch Distributionsaspekte berührt werden. 


\section{Anhang 1: Die biographische Theorie der Fertilität}

Die biographische Theorie der Fertilität sieht ebenso wie die neoklassische Theorie die Geburtenrate als Ergebnis individueller Wahlakte an. Während aber die neoklassische Theorie zu ergründen sucht, welche Kinderzahl bei vorgegebenen Restriktionen und unveränderter Nutzenfunktion für potentielle Eltern optimal ist, setzt die v.a. von Birg entwickelte biographische Theorie schon vorher an. Sie untersucht nämlich, wie die Menge aller alternativen Entscheidungsmöglichkeiten, unter denen dann die beste herausgesucht wird, zustandekommt und geht insofern über den neoklassischen Ansatz hinaus. Es steht also nicht mehr sie Suche des optimalen Elements einer vorgegebenen Wahlmenge im Zentrum des Interesses, sondern vielmehr das Zustandekommen der Wahlmenge selbst. Erklärt werden soll, weshalb einige Elemente in der Wahlmenge eines Individuums enthalten sind, nicht aber in der eines anderen.

Ob ein Element zur Wahlmenge gehört oder nicht, ist nicht auf eine gegenwärtige Entscheidung des betreffenden Individuums zurückzuführen, sondern nach der biographischen Theorie Ergebnis von biographieinternen und biographieexternen Faktoren.

Unter ersteren sind v.a. Entscheidungen in früheren Lebensphasen zu verstehen, wie beispielsweise die Wahl der Ausbildung, des Berufs und der Arbeitsstelle, des Wohnortes, des Partners, etc. Durch die Festlegung solcher Elemente oder Grundbausteine des Lebenslaufs in der Vergangenheit, zu denen neben ökonomischen auch entwicklungspsychologische, soziale und demographische Phasen und Zustände zählen, wird die Zahl aller potentiell realisierbaren Kombinationen dieser Elemente eingeschränkt. Eine geordnete Menge solcher biographischen Elemente bezeichnet Birg als biographische Sequenz, die Gesamtheit aller mathematisch konstruierbaren Sequenzen als biographisches Universum. Da die einzelnen Elemente intertemporal miteinander verknüpft sind, wird die handlungsrelevante Teilmenge des biographischen Universums, die sogenannte virtuelle Biographie, durch frühere Elemente bestimmt. Beispielsweise ist für einen 40jährigen Mann mit Hauptschulabschluß das biographische Grundelement "Internist" wohl kaum Teil seines Möglichkeitsraumes für biographische Entscheidungen, seiner virtuellen Biographie also. Ebenso ist für einen katholischen Priester (noch?) das Element "Kinder" nicht in der Wahlmenge enthalten.

Zu den biographieexterenen Faktoren zählt z.B. die Veränderung des biographischen Universums selbst aufgrund der ökonomischen und gesellschaftlichen Entwicklung.

Eine Grundannahme der biographischen Fertilitätstheorie besteht nun in der Behauptung, daß sich das biographische Universum und auch die virtuelle Biographie seit der industriellen Revolution ständig vergrößert haben. Zurückgeführt wird diese Entwicklung auf den Wegfall sozialer, normativer und ökonomischer Beschränkungen einerseits ${ }^{t}$ und das Entstehen neuer biographischer Grundelemente u.a. durch die zunehmende Arbeitsteilung und Spezialisierung andererseits. Durch die größere virtuelle Biographie nimmt die Zahl der Alternativen zu, die durch eine biographische Festlegung - beispielsweise durch die Geburt eines Kindes - ausgeschlossen werden. Die Opportunitätskosten (Birg spricht auch von "Risiko") nehmen also zu, und zwar besonders bei der Entscheidung für ein Kind, denn hier handelt es sich um eine besonders langfristige und irreversible Festlegung. Während es noch relativ leicht sein mag, die

1 Vgl. Birg (1992) S. 203. Birg sieht als Konsequenz des Wegfalls dieser Beschränkungen eine Erweiterung des biographischen Universums. Genaugenommen handelt es sich aber um eine Erweiterung der virtuellen Biographie, da sich die Zahl der mathematisch möglichen Kombinationen von biographischen Grundelementen nicht geändert hat. 
Entscheidung für einen Ausbildungsgang zu revidieren, indem man später einen Abschluß in einer anderen Ausbildungsrichtung nachholt, ${ }^{2}$ ist die Entscheidung für ein Kind aus ethischen Gründen nicht mehr veränderbar. Hinzu kommt, daß sich die Fertilitätsentscheidung relativ leicht aufschieben läßt, während sich berufliche Festlegungen nicht in gleichem Maße hinauszögern lassen. Infolgedessen führt die Ausdehnung der virtuellen Biographie zu im Vergleich mit beruflichen Festlegungen überproportional steigenden Opportunitätskosten eines Kindes. Als Folge ergibt sich, daß "...die aufschiebbaren bzw. vermeidbaren Festlegungen in der Familienbiographie (Partnerbindung, Eheschließung, Kindgeburten) mehr und mehr zugunsten der nicht aufschiebbaren Festlegungen in der Erwerbsbiographie (Entscheidung für eine bestimmte Ausbildung, Berufswahl, Arbeitsplatzwahl) zurücktreten". ${ }^{3}$

Für ein erstes Kind ist die Einschränkung der virtuellen Biographie besonders groß. Ist dagegen bereits ein Kind vorhanden, so sind die zusätzlichen Opportunitätskosten - also die zusätzlich wegfallenden Alternativen - geringer, so daß die bedingte Geburtenwahrscheinlichkeit für zweite Kinder höher ist als die für erste Kinder. Es besteht also nicht etwa die Tendenz zur Ein-Kind-Familie, sondern zu völliger Kinderlosigkeit oder mehreren Kindern. Die Bevölkerung läßt sich dann in einen reproduktiven und einen nichtreproduktiven Teil gliedern.

In ersten empirischen Untersuchungen ${ }^{4}$ wurden die Grundhypothesen der biographischen Theorie der Fertilität bestätigt. Insgesamt ist die Theorie aber noch zu wenig gefestigt und nicht operational genug, um hier als Grundlage für die Untersuchung des Einflusses des Kinderlastenausgleichs auf die Kinderzahl zu dienen.

2 Aufgrund der fortschreitenden Spezialisierung ist allerdings diese nachträgliche Laufbahnänderung auch zunehmend schwieriger geworden.

3 Birg (1992) S. 206.

4 Vgl. Birg/Filip/Flöthmann (1990). 


\section{Anhang 2: Überblick über wichtige Änderungen im System des Kinder- lastenausgleichs der Bundesrepublik Deutschland}

Zeittafel zu wichtigen Änderungen des Kinderlastenausgleichs in der Bundesrepublik Deutschland ${ }^{1}$

1955 Einführung eines einkommensabhängigen Kindergeldes von 25 DM für dritte und weitere Kinder

1959 Erhöhung des Kindergeldes auf 40 DM/Monat

1961 Einbeziehung zweiter Kinder mit 25 DM/Monat

1964 Änderung der Beträge auf 25 DM/Monat für ein zweites Kind, 50 DM/Monat für ein drittes Kind, 60 DM/Monat für ein viertes Kind und $70 \mathrm{DM}$ für fünfte und weitere Kinder

1973 Erhöhung der Einkommensgrenze auf 18.360 DM/Jahr für das Kindergeld

1975 Neugestaltung der Monatsbeträge und Einführung eines Kindergeldes von 50 DM/Monat schon für das erste Kind; für zweite Kinder jetzt 70 DM/Monat, für dritte und weitere Kinder 120 DM/Monat; Aufhebung der Einkommensgrenze; Wegfall der Kinderfreibeträge im Rahmen der Einkommensteuer

1981 Erhöhung der Monatsbeträge auf 120 DM/Monat für ein zweites Kind und 240 DM/Monat für weitere Kinder

1982 Minderung der Monatsbeträge auf 100 DM/Monat für zweite Kinder und 220 DM/Monat für dritte Kinder (weitere Kinder nach wie vor 240 DM/Monat)

1983 Wiedereinführung von Einkommensgrenzen beim Kindergeld ab dem zweiten Kind (Sockelbeträge von $70 \mathrm{DM}$ für das zweite und $140 \mathrm{DM}$ für weitere Kinder für hohe Einkommen); Wiedereinführung eines Kinderfreibetrages (432 DM je Kind), dafür aber Streichung der Absetzmöglichkeit von Kinderbetreuungskosten in Höhe von bis zu $1.200 \mathrm{DM}$

1984 Steuerliche Berücksichtigung von Betreuungskosten Alleinerziehender bis 4.000 DM für das erste und bis $2.000 \mathrm{DM}$ für jedes weitere Kind

1986 Starke Erhöhung der Kinderfreibeträge auf 2.484 DM je Kind; Aufhebung der Kindererhöhungsbeträge bei den Vorsorgeaufwendungen; Einführung des Zusatzkindergeldes für Familien, die durch die Kinderfreibeträge nicht entlastet werden von maximal 46 DM je Kind; Überführung des Mutterschaftsurlaubsgeldes für sozialversicherungspflichtige Arbeitnehmerinnen vom 3. bis 6. Monat nach der Geburt eines Kindes in einen zehnmonatigen Erziehungsurlaub und Erziehungsgeld von 600 DM/Monat für den betreuenden Elternteil. Ab dem siebten Monat wird das

1 Änderungen der Realtransfers sind nicht aufgeführt. 
Erziehungsgeld oberhalb einer kinderzahlabhängigen Einkommensgrenze mit steigendem Einkommen vollständig abgebaut; Anrechnung von Erziehungszeiten von einem Jahr pro Kind in der GRV falls im ersten Jahr nach der Geburt keiner Arbeit nachgegangen wurde (zunächst nur für ältere Rentnerinnen, bis 1990 stufenweise für alle Rentenempfänger); Erhöhung des Haushaltsfreibetrages für Alleinerziehende auf 4.536 DM

1987 Baukindergeld von 600 DM für jedes Kind

1988 Ausdehnung des Erziehungsurlaubs und des Bezugszeitraums für Erziehungsgeld auf 12 Monate; Erhöhung des Haushaltsfreibetrages für Alleinerziehende auf 4.752 DM Verdoppelung der steuerlichen Ausbildungsfreibeträge

1989 Weitere Ausdehnung des Erziehungsurlaubs und des Bezugszeitraums für Erziehungsgeld auf 15 Monate

1990 Weitere Erhöhung des Kinderfreibetrages auf 3.024 DM je Kind; Erhöhung des Zusatzkindergeldes auf maximal $48 \mathrm{DM}$ je Kind; Erhöhung des Kindergeldes für das 2. Kind auf $130 \mathrm{DM}$ bei unverändertem Sockel von $70 \mathrm{DM}$; Erhöhung des Baukindergeldes auf 750 DM je Kind; Sonderausgabenabzug bis zu $12.000 \mathrm{DM}$ für Aufwendungen für Beschäftigungen zur Kinderbetreuung; Verbesserung der Ausbildungsförderung im Rahmen des BAFöG u.a. durch Umstellung auf Halbdarlehen; nochmalige Ausdehnung des Erziehungsurlaubs und des Bezugszeitraums für Erziehungsgeld auf 18 Monate; Erhöhung des Haushaltsfreibetrages für Alleinerziehende auf 5.616 DM

1991 Erhöhung des Baukindergeldes auf 1.000 DM je Kind und Ermöglichung eines Rückbzw. Vortrags

1992 Erhöhung des Kinderfreibetrags auf 4.104 DM; Erhöhung des Kindergeldzuschlags auf bis zu 65 DM je Kind; Erhöhung des Kindergeldes für das erste Kind von 50 DM auf 70 DM pro Monat; Ausdehnung des Erziehungsurlaubs auf 36 Monate; Berücksichtigung von drei Erziehungsjahren je Kind in der GRV für Geburten ab 1992

1993 Ausdehnung des Bezugszeitraums für Erziehungsgeld auf 24 Monate

1994 Reduzierung des Sockelbetrags des Kindergelds für dritte und weitere Kinder auf 70 DM pro Monat; Einführung einer sogenannten Gutverdienergrenze beim Erziehungsgeld von 100.000 DM Jahresnettoeinkommen bei Ehepaaren mit dem ersten Kind und von 75.000 DM bei Alleinerziehenden mit dem ersten Kind, oberhalb dieser Grenze entfällt der Anspruch auf Erziehungsgeld vollständig

1996 Wahlmöglichkeit zwischen einem auf $200 \mathrm{DM} / \mathrm{Monat}$ für das erste und zweite, 300 DM/Monat für das dritte und $350 \mathrm{DM} /$ Monat für das vierte Kind erhöhten, einkommensunabhängigem Kindergeld und einem steuerlichen Kinderfreibetrag von 6.264 DM/Jahr; Erhöhung des Baukindergeldes auf 1.500 pro Jahr 


\section{Anhang 3: Bevölkerung und Gesundheitskosten nach Altersklassen}

Abb. A3.1: Bevölkerung und Gesundheitskosten nach Altersklassen in der Bundesrepublik Deutschland für die Jahre 1990, 2010 und 2050 (alle DM-Werte beziehen sich auf Preise von 1990)

\section{0}

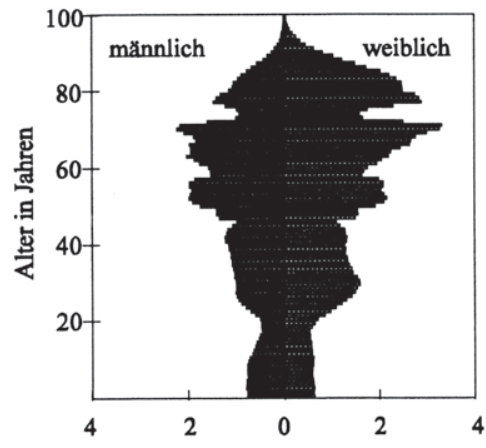

Gesundheitskosten in Mrd. Mark

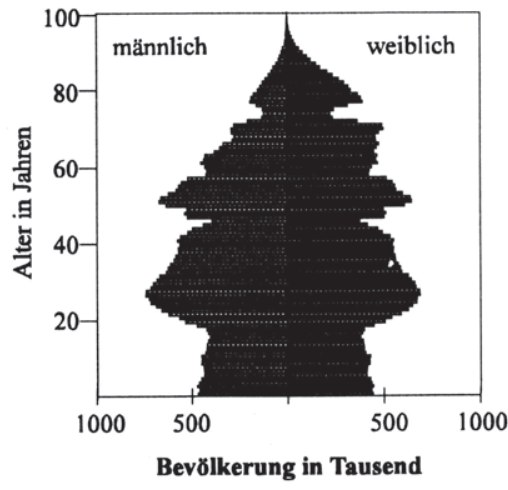




\section{0}

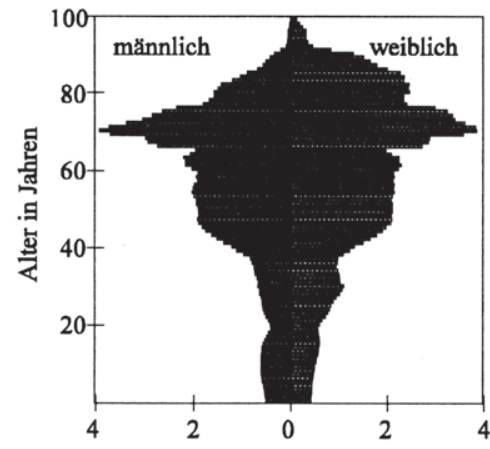

Gesundheitskosten in Mrd. Mark

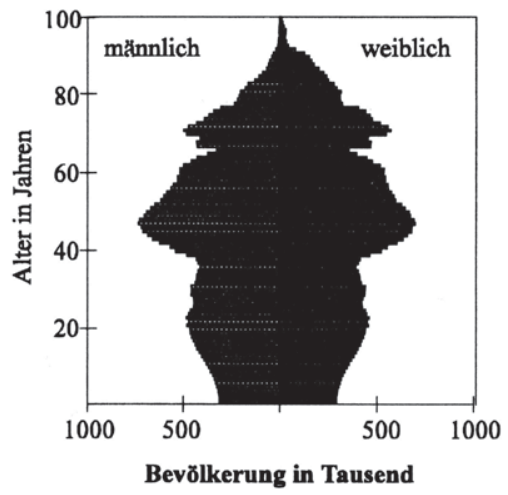

\section{0}

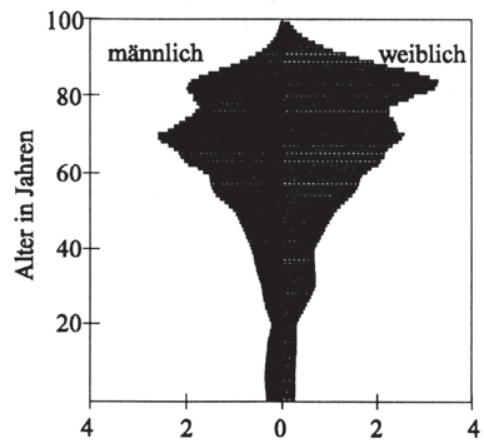

Gesundheitskosten in Mrd. Mark

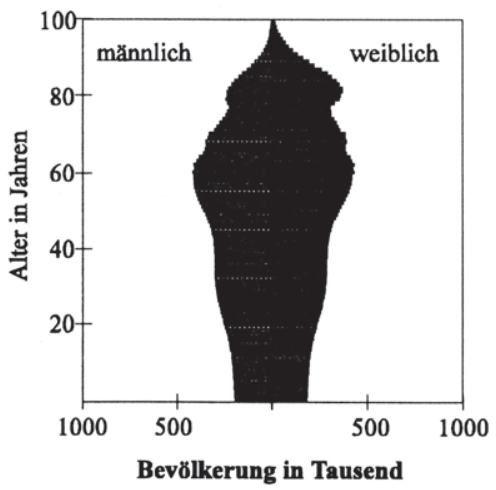

Quelle: Die zugrundeliegenden Daten wurden freundlicher Weise von N. Fickel zur Verfügung gestellt und mit Hilfe von Angaben zu den Gesundheitskosten aus dem Sozialbericht 1993 (vgl. Bundesregierung (1994)) umgerechnet. 


\section{Anhang 4: Klassifizierung der Zuwanderer}

In der tagespolitischen Diskussion werden viele in Zusammenhang mit der Zuwanderung stehende Begriffe unpräzise oder gar widersprüchlich verwendet. Daher werden in diesem Anhang die verschiedenen Gruppen von Zuwanderern gegeneinander abgegrenzt und so gleichzeitig ein Überblick über die gegenwärtigen rechtlichen Möglichkeiten des Aufenthaltes von Zuwanderern in Deutschland gegeben. Grundsätzlich lassen sich entprechend der Rechtslage sechs Gruppen unterscheiden, wobei Überschneidungen zwischen den einzelnen Gruppen möglich sind: ${ }^{1}$ politisch motivierte Zuwanderung von Ausländern, ökonomisch motivierte Zuwanderung von Ausländern, Familienzusammenführung, Aussiedler, EU-Ausländer und illegale Zuwanderung.

Zunächst sei die Gruppe der aus politischen Motiven zuwandernden Ausländer ${ }^{2}$, d.h. der Flüchtlinge, betrachtet. Ihnen steht das Asylverfahren als Weg offen, um eine Aufenthaltsgenehmigung in Deutschland zu erlangen.

Als Asylbewerber werden Ausländer bezeichnet, die einen Asylantrag gestellt haben und deren Verfahren noch nicht rechtskräftig entschieden ist. Sie dürfen den Bezirk, in dem sie den Asylantrag gestellt haben, nicht verlassen und ihre Unterbringung erfolgt in der Regel in Sammelunterkünften. Wenn die Arbeitsmarktsituation es zuläßt, können sie eine Arbeitserlaubnis erhalten, anderenfalls haben sie Anspruch auf (eingeschränkte) Sozialhilfe, die in verschiedenen Bundesländern als Sachleistung gewährt wird.

Wird der Asylbewerber rechtskräftig als politisch Verfolgter anerkannt, dann handelt es sich ab diesem Zeitpunkt um einen Asylberechtigten mit unbefristeter Aufenthaltserlaubnis und dem Recht, eine Arbeit oder Ausbildung aufzunehmen, mit dem Recht auf Freizügigkeit und mit Anspruch auf eine Reihe staatlicher Leistungen wie Sprachkursen, Sozialhilfe, Wohngeld und Zahlungen auf Grundlage des Bundesausbildungsförderungsgesetzes und des Arbeitsförderungsgesetzes.

Als De-facto-Flüchtlinge werden Personen bezeichnet, deren Asylantrag abgelehnt worden ist, oder die einen Asylantrag gar nicht erst gestellt haben, die aber aus rechtlichen, politischen oder humanitären Gründen dennoch nicht abgeschoben werden. Sie erhalten keine Aufenthaltserlaubnis, sondern nur eine jederzeit widerrufbare Aufenthaltsduldung, haben keinen Anspruch auf Integrationshilfen (Sprachkurse, BAFöG, Arbeitsförderung), dürfen aber eine Arbeit aufnehmen sobald sie eine Aufenthaltsbefugnis erhalten haben.

Die Bona-fide-Flüchtlinge teilen sich in zwei Gruppen: Zum einen Personen, die der United Nations High Commissioner for Refugees nach der Genfer Konvention als Flüchtlinge betrach-

Die Erläuterungen zu den verschiedenen Zuwanderungsgruppen entstammen im wesentlichen Bade (1994) S. 9ff. und S. 285ff.

Als Auslănder gilt nach dem Ausländergesetz ( 11 Abs. 2) jeder, der nicht Deutscher im Sinne des Grundgesetzes ist. Deutscher in diesem Sinne ist, "wer die deutsche Staatsangehörigkeit besitzt oder als Flüchtling oder Vertriebener deutscher Volkszugehörigkeit oder als dessen Ehegatte oder Abkömmling in dem Gebiet des Deutschen Reiches nach dem Stande vom 31. Dezember 1937 Aufnahme gefunden hat" (Art. 116 Abs. $1 \mathrm{GG}$ ). Als Ausländer gelten aufgrund dieses Abstammungsprinzips (jus sanguinis) in Deutschland auch Zuwanderer in der zweiten und dritten Generation und damit Personen, die sich in Lebensform und Selbstverständnis von der restlichen Bevölkerung kaum noch unterscheiden (vgl. Bade (1994) S. 10). In Staaten, die das Territorialprinzip (jus soli) verwirklicht haben, kann dagegen die Staatsangehörigkeit auch durch Geburt im Staatsgebiet erworben werden. 
tet ${ }^{3}$ und zum anderen Asylberechtigte, gegen deren Anerkennung Rechtsmittel eingelegt wurden.

Flüchtlinge, die im Rahmen humanitärer Hilfsaktionen bis zu einer bestimmten Gesamtzahl aufgenommen wurden (z.B. die vietnamesischen Boatpeople), werden als Kontingentflüchtlinge bezeichnet. Aufgrund des Gesetzes über Maßnahmen für im Rahmen humanitärer Hilfsaktionen aufgenommene Flüchtlinge haben sie einen besonderen Rechtsstatus und erhalten sofort eine Aufenthaltsgenehmigung und Arbeitserlaubnis.

Die zweite Gruppe der Zuwanderer umfaßt mit den sogenannten Gastarbeitern die aus ökonomischen Motiven Zuwandernden. Nach dem Anwerbestopp im Jahre 1973 ist die Einreise von Nicht-EU-Ausländern zur Arbeitsaufnahme in Deutschland nicht mehr möglich. Aufenthaltserlaubnis und Beschäftigungserlaubnis sind für Gastarbeiter eng miteinander verbunden. Erst nach fünf Jahren wird die Aufenthaltserlaubnis unbefristet erteilt.

Neben dem Asylverfahren ${ }^{4}$ bleibt für ökonomisch motivierte Zuwanderung damit nur noch die dritte Kategorie der Zuwanderung, nämlich die Familienzusammenführung, als Eintrittstor. Der Nachzug von Ehegatten und Kindern von in Deutschland arbeitenden Ausländern ist auch nach dem Anwerbestopp möglich. Der Anteil der Erwerbstätigen an allen Zuwanderern hat sich daher seit 1973 vermindert.

Die vierte Gruppe der Zuwanderer besteht aus den Aussiedlern bzw. ab 1993 den Spätaussiedlern, d.h. aus "deutsche[n] Staatsangehörige[n] oder Volkszugehörige[n], die vor dem 8. Mai 1945 ihren Wohnsitz in den zur Zeit unter fremder Verwaltung stehenden deutschen Ostgebieten bzw. in Polen, der Sowjetunion, der Tschechoslowakei, Ungarn, Rumänien, Jugoslawien, Danzig, Estland, Lettland, Litauen, Bulgarien, Albanien oder China gehabt und diese Länder nach Abschluß der allgemeinen Vertreibungsmaßnahmen verlassen haben oder verlassen." ${ }^{5}$ Bis zur Wiedervereinigung der beiden deutschen Staaten bildeten die Übersiedler und Sperrbrecher eine weitere Zuwanderungsgruppe. Während die Übersiedler regulär aus der DDR in die Bundesrepublik umgezogen sind, waren die Sperrbrecher auf der Flucht Gefahr für Leib und Leben ausgesetzt. Da diesen Zuwanderern sofort die Staatsbürgerschaft zuerkannt wird, haben sie die gleichen Grundrechte wie die Einheimischen und Anspruch auf Sozialleistungen.

Für Ausländer aus Mitgliedstaaten der Europäischen Union gibt es keinerlei Zuzugsbeschränkungen, jeder Bürger eines EU-Staates hat das Recht, in Deutschland eine Arbeit aufzunehmen. Die auf Deutsche beschränkten Grundrechte (z.B. Wahlrecht) gelten für sie allerdings nicht. ${ }^{6}$

Als letzte Gruppe sind schließlich die illegalen Zuwanderer zu erwähnen, d.h. die steigende Zahl von Personen, die sich ohne Aufenthaltserlaubnis in der Bundesrepublik Deutschland aufhalten.

3 Nach der Genfer Flüchtlingskonvention ist der Flüchtlingsbegriff weiter gefaßt als im deutschen Asylrecht, so daß auch Personen, die nicht als Asylberechtigte anerkannt worden sind, durch die Genfer Konvention vor Abschiebung geschützt werden.

4 Straubhaar/Zimmermann (1992), S. iv führen aus, daß "[i]t is the impossibility of applying for permission to work in the host country that drives the abuse of asylum rights in some EC member countries."

Vgl. Tichy (1993) S. 42. 


\section{Literaturverzeichnis}

ABOWD, John M./FrEEMAN, Richard B. (Hrsg.) (1991): Immigration, Trade, and the Labor Market, Chicago, London, 1991.

AKBARI, Ather (1989): The Benefits of Immigrants to Canada: Evidence on Tax and Public Services, in: Canadian Public Policy, Jg. 15 (1989), Heft 4, S. 424-435.

ALBERS, Willi (1988): Die Beeinflussung der Verhaltensweisen der Familie mit ökonomischen Mitteln, in: FELDERER, Bernhard (Hrsg.), Familienlastenausgleich und demographische Entwicklung, Berlin, 1988, S. 53-66.

ALBRECHT, Gerhard (Hrsg.) (1952): Verhandlungen auf der Sondertagung in Berlin (Schriften des Vereins für Sozialpolitik), Berlin, 1952.

ALTONJ, Joseph/CARD, David (1991): The Effects of Immigration on the Labor Market Outcomes of Less-skilled Natives, in: ABOwD, John M./FrEEMAN, Richard B. (Hrsg.), Immigration, Trade, and the Labor Market, Chicago, London, 1991, S. 201-234.

ARISTOTELES (1978): Politik, 3. Aufl., Band 2, München, 1978.

ARNOLD, Fred/FAWCETT, James T. (1974): The Value of Children, Band 3, Hawaii, 1974.

ARTHUR, W. Brian/ESPENSHADE, Thomas J. (1988): Immigration policy and immigrants' ages, in: Population and Development Review, Jg. 14 (1988), S. 315-326.

BACH, Hans-Uwe (1987): Entwicklung und Struktur der Ausländerarbeitslosigkeit in der Bundesrepublik Deutschland, in: HöNEKOPP, Elmar (Hrsg.), Aspekte der Ausländerbeschäftigung in der Bundesrepublik Deutschland (Beiträge zur Arbeitsmarkt und Berufsforschung 114), Nürnberg, 1987, S. 144-178.

BADE, Klaus J. (1994): Ausländer - Aussiedler - Asyl, München, 1994

BAKER, Lyle (1987): Immigration and Per Capita Investment, unveröffentlichtes Manuskript, 1987 (zitiert nach SimON, Julian L. (1989): The Economic Consequences of Immigration, Cambridge, 1989).

BARABAS, György u.a. (1992): Gesamtwirtschaftliche Effekte der Zuwanderung 1988 bis 1991, in: RWI-Mitteilungen, Jg. 43 (1992), Heft 2, S. 133-154.

BARTH, Siegrun/HAIN, Winfried/MÜLLER, Horst-Wolf (1994): Langfristige demographische Bestimmungsfaktoren der Rentenfinanzen - Eine Vorausberechnung mit dem neuen Rentenmodell 1993, in: Deutsche Rentenversicherung, (1994), S. 228-258.

BEAN, Frank D./LOWELL, B. Lindsay/TAYLOR, Lowell J. (1988): Undocumented Mexican Immigrants and Earnings of Other Workers in the United States, in: Demography, Jg. 25 (1988), S. 35-49.

BECKER, Gary S. (1960): An Economic Analysis of Fertility, in: NATIONAL BUREAU OF ECONOMIC RESEARCH (Hrsg.), Demographic and Economic Change in Developed Countries, Princeton, 1960, S. 209-231.

BeCKeR, Gary S. (1987): A Radical Proposal to Improve Immigration Policy, mimeo., 1987 (zitiert nach SimON, Julian L. (1989): The Economic Consequences of Immigration, Cambridge, 1989). 
BECKER, Gary S. (1991): A Treatise on the Family, 2. erw. Aufl., Cambridge, London, 1991.

BECKER, Gary S. (1993): Der ökonomische Ansatz zur Erklärung menschlichen Verhaltens, in: BECKER, Gary S. (Hrsg.), Der ökonomische Ansatz zur Erklärung menschlichen Verhaltens (The Economic Approach to Human Behavior, 1976), 2. Aufl., übersetzt von Monika und Viktor Vanberg, Tübingen, 1993, S. 1-15.

BECKER, Gary S. (Hrsg.) (1993): Der ökonomische Ansatz zur Erklärung menschlichen Verhaltens. (The Economic Approach to Human Behavior, 1976), 2. Aufl., übersetzt von Monika und Viktor Vanberg, Tübingen, 1993.

BECKER, Gary S./BARRO, Robert J. (1988): A Reformulation of the Economic Theory of Fertility, in: Quarterly Journal of Economics, Jg. 103 (1988), S. 1-25.

BECKER, Gary S./LANDES, Elisabeth M./MichaEL, Robert T. (1977): An Economic Analysis of Marital Instability, in: Journal of Political Economy, Jg. 85 (1977), S. 1141-87.

BeHRMANN, S.J./CORSA, Leslie/FreEdMAN, Ronald (Hrsg.) (1969): Fertility and Family Planning: A World View, Ann Arbor, 1969.

BEN-PORATH, Yoram (1975): First-Generation Effects on Second-Generation Fertility, in: Demography, Jg. 12 (1975), Heft 3, S. 397-405.

BERNHOLZ, Peter (1994): Comment on Arye L. Hillman, "The Political Economy of Migration Policy", in: SEEBERT, Horst (Hrsg.), Migration: A Challenge for Europe, Tübingen, 1994, S. 283-88.

BERNTSEN, Roland (1989): Einkommensanalysen mit den Daten des Sozio-ökonomischen Panels unter Verwendung von generierten Einkommensdaten (Sonderforschungsbereich 3, Arbeitspapier Nr. 291), Frankfurt, Mannheim, 1989 (zitiert nach ULRICH, Ralf (1992): Der Einfluß der Zuwanderung auf die staatlichen Einnahmen und Ausgaben in Deutschland, in: Acta Demographica, (1992), S. 189208).

BERTRAM, Hans (Hrsg.) (1991): Die Familie in Westdeutschland. Stabilität und Wandel familiarer Lebensformen, Opladen, 1991.

BHAGWATI, Jagdish N. (Hrsg.) (1985): Dependence and Interdependence, Cambridge, 1985.

BHAGWATI, Jagdish N. (1985): Taxation and International Migration: Recent Policy Issues, in: Bhagwati, Jagdish N. (Hrsg.), Dependence and Interdependence, Cambridge, 1985 , S. 347-361.

Bhagwati, Jagdish N./Krugman, Paul R. (1985): The Decision to Migrate: A Survey, in: BhaGWaTI, Jagdish N. (Hrsg.), Dependence and Interdependence, Cambridge, 1985, S. 362-382.

BIEDENKOPF, Kurt (1993): Verstaatlichung der Nächstenliebe, in: DIE ZEIT, (1993), Nr. 23 vom 4.6.93, S. 22.

BIRG, Herwig (1992): Differentielle Reproduktion aus der Sicht der biographischen Theorie der Fertilität, in: VolAND, Eckart v. (Hrsg.), Fortpflanzung: Natur und Kultur im Wechselspiel: Versuch eines Dialogs zwischen Biologen und Sozialwissenschaftlern, Frankfurt/Main, 1992, S. 189-215. 
BIRG, Herwig/FIlIP, D./FlÖthmanN, E.-J. (1990): Paritätsspezifische Kohortenanalyse des generativen Verhaltens in der Bundesrepublik Deutschland nach dem 2. Weltkrieg (IBS-Materialien Nr. 30), Bielefeld, 1990.

BIRG, Herwig/FlötHMANN, E.-J. (1993): Bevölkerungsprojektionen für das vereinigte Deutschland bis zum Jahr 2100, Bielefeld, 1993.

BIRG, Herwig/MACKENSEN, Rainer (Hrsg.) (1990): Demographische Wirkungen politischen Handelns, Frankfurt/Main, New York, 1990.

BLAKE, Judith (1966): Ideal Family Size among White Americans: A Quarter of a Century's Evidence, in: Demography, Jg. 3 (1966), S. 154-73.

BLAKE, Judith (1967): Income and Reproductive Motivation, in: Population Studies, Jg. 21 (1967), Heft 3, S. 185-206.

BlanchaRD, Olivier/KatZ, Lawrence (1992): Regional Evolutions, in: Brookings Papers on Economic Activity, (1992), Heft 1, S. 1-61.

BLOSSER-REISEN, Lore/SEIFERT, Margit (1984): Arbeitszeit- und Geldaufwand für die Lebenshaltung von Kindern verschiedener Altersgruppen in Familienhaushalten, in: Hauswirtschaft und Wissenschaft, (1984), Heft 3, S. 135.

BOMSDORF, Eckart (1994): Die ältere Bevölkerung Deutschlands - ihre Entwicklung bis zum Jahr 2050, in: Zeitschrift für Wirtschaftspolitik, Jg. 43 (1994), Heft 1, S. 89-103.

BORCHERT, Jürgen (1981): Die Berücksichtigung familiärer Kindererziehung im Recht der gesetzlichen Rentenversicherung, Berlin, 1981.

BORCHERT, Jürgen (1989): Innenweltzerstörung, Frankfurt/Main, 1989.

BORCHERT, Jürgen (1993): Renten vor dem Absturz, Frankfurt/Main, 1993.

BORJAS, George J. (1985): Assimilation, Changes in Cohort Quality, and the Earnings of Immigrants, in: Journal of Labor Economics, Jg. 3 (1985), Heft Oct., S. 463-489.

BORJAS, George J. (1986): The Demographic Determinants of the Demand for Black Labor, in: FREEMAN, Richard B./HOLZER, Harry J. (Hrsg.), The Black Youth Employment Crisis, Chicago, 1986, S. 191-232.

BORJAS, George J. (1987): Self-Selection and the Earnings of Immigrants, in: American Economic Review, Jg. 77 (1987), S. 521-553.

BORJAS, George J. (1989): Economic Theory and International Migration, in: International Migration Review, Jg. 23 (1989), Heft 3, S. 457-485.

BORJAS, George J. (1990): Friends or Strangers: the Impact of Immigrants on the U.S. Economy, New York, 1990.

BORJAS, George J. (1991): Immigration Policy, National Origin, and Immigrant Skills: a Comparison of Canada and the US (National Bureau of Economic Research, Working Paper No. 3671), Cambridge, 1991.

BORJAS, George J. (1992): National Origin and the Skills of Immigrants in the Postwar Period, in: BORJAS, George J./FrEEMAN, Richard B. (Hrsg.), Immigration and the Work Force, Chicago, London, 1992, S. 17-47.

BORJAS, George J. (1994): The Economics of Immigration, in: Journal of Economic Literature, Jg. 32 (1994), S. 1667-1717. 
BORJAS, George J. (1995): The Economic Benefits from Immigration, in: The Journal of Economic Perspectives, Jg. 9 (1995), Heft 2, S. 3-22.

BORJAS, George J./FrEEMAN, Richard B. (Hrsg.) (1992): Immigration and the Work Force, Chicago, London, 1992.

BORJAS, George J./FREEMAN, Richard B./KATZ, Lawrence F. (1992): On the Labor Market Effects of Immigration and Trade, in: BORJAS, George J./FrEEMAN, Richard B. (Hrsg.), Immigration and the Work Force, Chicago, London,1992, S. 213-244.

BÖRSCH-SUPAN, Axel (1994): Migration, Social Security Systems, and Public Finance, in: SIEBERT, Horst (Hrsg.), Migration: A Challenge for Europe, Tübingen, 1994, S. 119-147.

BRECHT, Beatrix/BRECHT, Leo (1991): Analysis of Remigrant Behavior with the Grouped Cox Model (Diskussionsbeiträge des Sonderforschungsbereichs 178 "Internationalisierung der Wirtschaft"), Konstanz, 1991.

BRENTANO, Lujo (1909): Die Malthus'sche Lehre und die Bevölkerungsbewegung der letzten Dezennien, in: Abhandlungen der historischen Klasse der königlich bayerischen Akademie der Wissenschaften, Band 24, (1909), S. 565-625.

BREYER, Friedrich (1990): Das Äquivalenzprinzip in der Rentenversicherung aus wohlfahrtsökonomischer Sicht, in: Finanzarchiv, N.F., (1990), S. 127-142.

BREYER, Friedrich (1992): Die Zukunft umlagefinanzierter Alterssicherungssysteme bei schrumpfender Bevölkerung, in: Wirtschaftswissenschaftliches Studium, (1992), Heft 3, S. 106-112.

BREYER, Friedrich/SCHUlenbURG, J.-Matthias Graf v.d. (1987): Voting on Social Security: The Family as Decision Making Unit, in: Kyklos, Jg. 40 (1987), Heft 4, S. 529547.

BREYER, Friedrich/STRAUB, Martin (1993): Welfare Effects of unfunded Pension Systems when Labor Supply is endogenous, in: Journal of Public Economics, Jg. 50 (1993), Heft 1, S. 77-91.

BROWNING, Edgar K. (1975): Why the Social Insurance Budget is too Large in a Democracy, in: Economic Inquiry, Jg. 13 (1975), S. 373-388.

Buhr, Petra/Strack, Peter/StrohmeIER, K. Peter (1987): Lebenslage und Alltagsorganisation junger Familien in Nordrhein-Westfalen, Bielefeld, 1987.

BUNDESREGIERUNG (1993): Rentenversicherungsbericht 1993, (Deutscher Bundestag (Hrsg.), Bundestag Drucksache 12/5470), Bonn, 1993.

BUNDESREGIERUNG (1994): Sozialbericht 1993, (Deutscher Bundestag (Hrsg.), Bundestag Drucksache 12/7130), Bonn, 1994.

BUNDESREGIERUNG (1995): Rentenversicherungsbericht 1995, (Deutscher Bundestag (Hrsg.), Drucksache 13/2017), Bonn, 1995.

BUNDESMINISTERIUM FÜR ARBEIT UND SOZIALORDNUNG (1993): Frauen und Beruf, Bonn, 1993.

BURKHARDT, Wilfried (1985): Drei-Generationen-Solidarität in der gesetzlichen Rentenversicherung als zwingende Notwendigkeit, Berlin, 1985. 
BUSLEI, Hermann/SCHMIDT, Peter (1994): Staatliche Alterssicherung im demographischen Wandel, in: ZEW Newsletter, (1994), Heft 2, S. 15-20.

BUTCHER, Kristin F./CARD, David (1991): Immigration and Wages: Evidence from the 1980's, in: American Economic Review, Jg. 81 (1991), S. 292-296.

BUTTLER, Günter/JÄGER, Norbert (1988): Reform der gesetzlichen Rentenversicherung durch ein Teilkapitaldeckungsverfahren, in: Zeitschrift für die gesamte Versicherungswissenschaft, (1988), Heft 3, S. 385-406.

BUTTLER, Günter/WINTERSTEIN, Helmut/JÄGER, Norbert (1989): Aussiedler - ein Gewinn oder Verlust für das System der Sozialen Sicherung?, in: Arbeit und Sozialpolitik, Jg. 43 (1989), Heft 8/9, S. 232-237.

CALOT, Gérard (1978): Der französische Weg zum Geburtenüberschuß, in: FRANKE, Lutz/JÜRGENS, Hans W. (Hrsg.), Keine Jugend - Keine Zukunft?, Boppard, 1978, S. 67-75.

CARD, David (1990): The Impact of the Mariel Boatlift on the Miami Labor Market, in: Industrial and Labor Relations Review, Jg. 45 (1990), Heft Jan., S. 245-257.

CARrington, William/DeLima, Pedro (1994): Large-Scale Immigration and Labor Markets: An Analysis of the Retornados and Their Impact on Portugal (Working Paper, John Hopkins University), 1994 (zitiert nach FRIEDBERG, Rachel M./HUNT, Jennifer (1995): The Impact of Immigrants on Host Country Wages, Employment and Growth, in: Journal of Economic Perspectives, Jg. 9 (1995), Heft 2, S. 23-44).

CHAmIE, Joseph (1981): Religion and Fertility. Arab Christian - Muslim Differentials, Cambridge u.a., 1981.

CHAPMAN, Laura (1994): Main Features of the Immigration System in Canada, in: OrGANISATION FOR ECONOMIC CO-OPERATION AND DEVEIOPMENT (Hrsg.), Migration and Development, Paris, 1994, S. 37-40.

CHISwICK, Barry R. (1978): The Effects of Americanization on the Earnings of Foreign-Born Men, in: Journal of Political Economy, Jg. 86 (1978), S. 897-921.

CHIswick, Barry R. (Hrsg.) (1982): The Gateway: U.S. Immigration Issues and Policies, Washington, D.C., 1982.

CHISwICK, Barry R. (1991): Speaking, Reading, and Earnings Among Low-Skilled Immigrants, in: Journal of Labor Economics, Jg. 9 (1991), Heft 2, S. 149-170.

Chiswick, Barry R. (Hrsg.) (1992a): Immigration, Language, and Ethnicity, Washington, D.C., 1992.

CHISwICK, Barry R. (1992b): Review of "Immigration, Trade, and the Labor Market" by Abowd and Freeman, in: Journal of Economic Literature, Jg. 30 (1992), S. 212213.

CHIswick, Barry R. (1993): Review of "Immigration and the Work Force: Economic Consequences for the United States and Source Areas" by Borjas and Freeman, in: Journal of Economic Literature, Jg. 31 (1993), S. 910-911.

CHISwICK, Barry R. (1994): The Performance of Immigrants in the United States Labor Market, in: GIERSCH, Herbert (Hrsg.), Economic Aspects of International Migration, Berlin u.a., 1994, S. 95-114. 
CHISwick, Barry R./MLLER, Paul (1993): The Endogeneity between Language and Earnings: An International Analysis, (Centre for Economic Policy Research (CEPR), Discussion Paper 93.03), o.O., 1993.

CHRUSCZ, Detlev/HÖPCKE, Hauke (1993): Alterung und Schrumpfung des Erwerbspotentials in der Europäischen Gemeinschaft. Möglichkeiten und Grenzen ihrer Kompensation (Schmid, Josef (Hrsg.), Bamberger Materialien zur Bevölkerungswissenschaft), Bamberg, 1993.

CignO, Alessandro/ERMISCH, John (1989): A Microeconomic Analysis of the Timing of Births, in: European Economic Review, Jg. 33 (1989), S. 737-760.

CoAle, Ansley J. (1975): The Demographic Transition, in: UnITED NaTiONS (Hrsg.), The Population Debate: Dimensions and Perspectives, New York, 1975, S. 347-355.

CORNELIUS, Ivar/LINDER, Peter/RÜCKERT, Gerd-Rüdiger (1986): Ökonomische Rahmenbedingungen der Familien (Statistisches Landesamt Baden-Württemberg (Hrsg.), Materialien und Berichte der Familienwissenschaftlichen Forschungsstelle), Stuttgart, 1986.

Darvish-Lecker, Tikva/Kahana, Nava (1992): The Destination Decision of Political Migrants: An Economic Approach, in: Journal of Population Economics, Jg.5 (1992), S. 145-153.

DAvIS, Kingsley/BLAKE, Judith (1956): Social Structure and Fertility: An Analytic Framework, in: Economic Development and Cultural Change, Jg. 4 (1956), S. 211-235.

DE NEW, John P./ZiMmERMANN, Klaus F. (1993): Native Wage Impacts of Foreign Labor: A Random Effects Panel Analysis (Münchener Wirtschaftswissenschaftliche Beiträge 93-19), München, 1993.

DE NEW, John P./ZimmermanN, Klaus F. (1994): Blue Collar Labor Vulnerability: Wage Impacts of Migration, in: StEINMANN, Gunter/UlRICH, Ralf E. (Hrsg.), The Economic Consequences of Immigration to Germany, Heidelberg, 1994, S. 81-100.

Deaton, Angus S./Muellbauer, John (1986): On Measuring Child Costs, in: Journal of Political Economy, Jg. 94 (1986), Heft 4, S. 720-44.

DECRESSIN, Jörg/FATÀs, Antonio (1996): Regional Labour Market Dynamics in Europe, in: European Economic Review, Jg. 39 (1996), S. 1627-1655.

DELBRÜCK, Christopher/RAFFELHÜSCHEN, Bernd (1993): Die Theorie der Migration, in: Jahrbücher für Nationalökonomie und Statistik, Band 212/3-4 (1993), S. 341-356.

DE TRAY, Dennis (1978): Child Schooling and Family Size: An Economic Analysis, Santa Monica, 1978.

DEUTSCHE GESELLSCHAFT FÜR BEVÖLKERUNGSWISSENSCHAFTEN (Hrsg.) (1984): Zusammenhänge zwischen Bevölkerungs- und Wirtschaftsentwicklung in der Bundesrepublik Deutschland, Wiesbaden, 1984.

DIE GRÜNEN (1985): Grundrecht statt Altersarmut (BT-Drucksache 10/3496), Bonn, 1985.

DINKEl, Reiner H. (1984a): Die Auswirkungen eines Geburten- und Bevölkerungsrückgangs auf Entwicklung und Ausgestaltung von gesetzlicher Alterssicherung und Familienlastenausgleich, Berlin, 1984. 
DINKEl, Reiner H. (1984b): Ökonomische versus soziologische Ansätze zur Erklärung des generativen Verhaltens, in: DEUTSCHE GESELLSCHAFT FÜR BEVÖLKERUNGSWISSENSCHAFTEN (Hrsg.), Zusammenhänge zwischen Bevölkerungs- und Wirtschaftsentwicklung in der Bundesrepublik Deutschland, Wiesbaden, 1984, S. 159190.

DINKEl, Reiner H. (1985): Kann es in einer Demokratie eine "rationale" Familienpolitik geben?, in: OPPENLÄNDER, Karl Heinrich/WAGNER, Adolf (Hrsg.), Ökonomische Verhaltensweisen und Wirtschaftspolitik bei schrumpfender Bevölkerung, München, 1985, S. 315-348.

DINKEl, Reiner H. (1986): Die Zukunftsprobleme der Rentenversicherung: Eine Folge des Versicherungsverfahrens?, in: Wirtschaftsdienst, Jg. 66 (1986), Heft 2, S. 79-86.

DINKEl, Reiner H. (1989): Demographie, Band 1, München, 1989.

DINKEl, Reiner H. (1990): Der Einfluß von Wanderungen auf die langfristige Bevölkerungsdynamik, in: Acta Demographica, (1990), Heft 1, S. 47-62.

DINKEl, Reiner H./LEBOK, Uwe (1993): Könnten durch Zuwanderung die Alterung der Bevölkerung und die daraus resultierenden Zusatzlasten der Sozialen Sicherung aufgehalten oder gemildert werden?, in: Deutsche Rentenversicherung, (1993), Heft 6 , S. 388-400.

DINKEL, Reiner H./LEBoK, Uwe (1994): Demographische Aspekte der vergangenen und zukünftigen Zuwanderung nach Deutschland, in: Aus Politik und Zeitgeschichte. Beilage zur Wochenzeitung "Das Parlament", (1994), Heft 48, S. 27-36.

DIXIT, Avinash/NORMAN, Victor (1980): Theory of International Trade, Cambridge, 1980.

DJAJIC, Slobodan (1989): Skills and the Pattern of Migration: The Role of Qualitative and Quantitative Restrictions on International Labor Mobility, in: International Economic Review, Jg. 30 (1989), Heft 4, S. 795-809.

DJAJIC, Slobodan/MIbOURNE, Ross (1988): A General Equilibrium Model of Guest Workers Migration. The Source Country Perspective, in: Journal of International Economics, Jg. 25 (1988), S. 335-351.

DOLADO, Juan/GoRIA, Alessandra/ICHINO, Andrea (1994): Immigration, Human Capital and Growth in the Host Country. Evidence from pooled Country Data, in: Journal of Population Economics, Jg. 7 (1994), S. 193-215.

DULEeP, Harriet Orcutt/Regets, Mark C. (1992): Some Evidence of the Effects of Admissions Criteria on Immigrant Assimilation, in: CHIswick, Barry R. (Hrsg.), Immigration, Language, and Ethnicity, Washington, D.C., 1992, S. 410-437.

DUSTMANN, Christian (1993): Earnings Adjustment of Temporary Migrants, in: Journal of Population Economics, Jg. 6 (1993), S. 153-168.

DUSTMANN, Christian (1994): Return Intentions of Migrants: Theory and Evidence (Centre for Economic Policy Research (CEPR) Discussion Paper No. 906), London, 1994.

EASTERLIN, Richard A. (1969): Towards a Socioeconomic Theory of Fertility: A Survey of Recent Research on Economic Factors in American Fertiliy, in: BEHRMANN, S.J./CORSA, Leslie/FrEedMaN, Ronald (Hrsg.), Fertility and Family Planning: A World View, Ann Arbor, 1969, S. 127-156. 
EASTERLIN, Richard A. (1973): Relative Economic Status and the American Fertility Swing, in: SHELDON, Eleanor Bernert (Hrsg.), Family Economic Behaviour: Problems and Prospects, Toronto, Philadelphia, 1973, S. 170-223.

EBERT, Thomas (1993): Frauen, Kinder, Renten. Zur Notwendigkeit einer Reform der Alterssicherung, in: Soziale Sicherheit, Jg. 42 (1993), Heft 4, S. 97-104.

ENGEL, Ernst (1883): Der Kostenwert des Menschen, Berlin, 1883 (zitiert nach TURCH, Boone A. (1984): The Monetary Cost of a Child, in: SteInManN, Gunter (Hrsg.), Economic Consequences of Population Change in Industrialized Countries, Berlin u.a., 1984, S. 258-277).

ENGEL, Ernst (1895): Die Lebenskosten Belgischer Arbeiter-Familien Früher und Jetzt, in: Internationales Statistisches Institut Bulletin, (1895), Heft 1, S. 1-74 (zitiert nach Deaton, Angus S./Muellbauer, John (1986): On Measuring Child Costs, in: Journal of Political Economy, Jg. 94 (1986), Heft 4, S. 720-44).

ENGELBERT, Angelika (1992): Wie "teuer" sind Kinder? Wert und Kosten von Kindern für Familien und Gesellschaft, in: Diskurs, (1992), Heft 1, S. 12-21.

ENQUETE-KOMMISSION "DEMOGRAPHISCHER WANDEL" (1994): Zwischenbericht - Herausforderungen unserer älter werdenden Gesellschaft an den einzelnen und die Politik (Bundestags-Drucksache 12/7876), Bonn, 1994.

ERMISCH, John (1989): Purchased Child Care, Optimal Family Size, and Mother's Employment. Theory and Econometric Evidence, in: Journal of Population Economics, Jg. 2 (1989), S. 79-102.

EsPENSHADE, Thomas J. (1984): Investing in Children: New Estimates of Parental Expenditure, Washington, 1984.

EsPenshade, Thomas J./CALHOUN, Charles A. (1986): The Dollars and Cents of Parenthood, in: Journal of Policy Analysis and Management, Jg. 5 (1986), Heft 4, S. 813-817.

EULER, Manfred (1974): Zur Problematik der Ermittlung des Unterhaltsbedarfs und der Unterhaltskosten eines Kindes, in: Wirtschaft und Statistik, (1974), S. 320-324.

EulER, Manfred (1993): Aufwendungen für Kinder, in: Wirtschaft und Statistik, (1993), Heft 10, S. 759-769.

EUROSTAT (1992): Messung und Entwicklung der Fruchtbarkeit in der Europäischen Gemeinschaft, Luxemburg, 1992.

Feichtinger, Gustav (1990): Demographische Prognosen und populationsdynamische Modelle, in: FELDERER, Bernhard (Hrsg.), Bevölkerung und Wirtschaft, Berlin, 1990, S. 71-92.

FELDERER, Bernhard (Hrsg.) (1986): Beiträge zur Bevölkerungsökonomie, Berlin, 1986.

FELDERER, Bernhard (Hrsg.) (1988): Familienlastenausgleich und demographische Entwicklung, Berlin, 1988.

FELDERER, Bernhard (Hrsg.) (1990): Bevölkerung und Wirtschaft, Berlin, 1990.

FELDERER, Bernhard (1994a): Can Immigration Policy Help to Stabilize Social Security Systems?, in: GIERSCH, Herbert (Hrsg.), Economic Aspects of International Migration, Berlin, u.a., 1994, S. 197-226. 
FELDERER, Bernhard (1994b): Immigration, the Labor Market, and Structural Adjustment: The Case of Germany, in: SIEBERT, Horst (Hrsg.), Migration: A Challenge for Europe, Tübingen, 1994, S. 71-84.

FELDERER, Bernhard/SAUGA, Michael (1988): Bevölkerung und Wirtschaftsentwicklung, Frankfurt/Main, New York, 1988.

FICKEL, Norman (1995): Auswirkungen der Bevölkerungsentwicklung in der Bundesrepublik Deutschland auf die Ausgaben für Gesundheit: eine Simulationsanalyse, Frankfurt/Main u.a., 1995.

FICKL, Stephan (Hrsg.) (1991): Bevölkerungsentwicklung und öffentliche Haushalte, Frankfurt/Main, New York, 1991.

FLER, Randall (1992): The Effect of Immigrant Arrivals on Migratory Patterns of Native Workers, in: BORJAS, George J./FrEEMAN, Richard B. (Hrsg.), Immigration and the Work Force: Economic Consequences for the United States and Source Areas, Chicago, London, 1992, S. 245-270.

FRANKE, Lutz/JÜRGENS Hans W. (Hrsg.) (1978): Keine Jugend - keine Zukunft?, Boppard, 1978.

FRANZ, Wolfgang (1991): Arbeitsmarktökonomik, Berlin u.a., 1991.

FRANZ, Wolfgang (1991): International Migratory Movements: The German Experience (Diskussionspapiere Universität Konstanz, Serie II, Nr. 160), Konstanz, 1991.

FRANZ, Wolfgang (1993): Zur ökonomischen Bedeutung von Wanderungen und den Möglichkeiten und Grenzen einer Einwanderungspolitik (CILE-Diskussionspapier 3-1993, Universität Konstanz), Konstanz, 1993.

FRANZ, Wolfgang/OSER, Ursula/WINKER, Peter (1993): Migratory Movements in a Disequilibrium Macroeconometric Model for West Germany (Diskussionsbeiträge des Sonderforschungsbereichs 178 "Internationalisierung der Wirtschaft"), Konstanz, 1993.

FREEDMAN, Deborah S. (1963): The Relation of Economic Status to Fertility, in: American Economic Review, Jg. 53 (1963), S. 414-426.

FreEMAN, Richard B./HolzeR, Harry J. (Hrsg.) (1986): The Black Youth Employment Crisis, Chicago, 1986.

FREJKA, Tomas (1983): Induced Abortion and Fertility: A Quarter Century of Experience in Eastern Europe, in: Population and Development Review, Jg. 9 (1983), Heft 3, S. 494-520.

FREY, Bruno S. (1981): Theorie demokratischer Wirtschaftspolitik, München, 1981.

FREY, Martin (1992): Die künftigen Wanderungsbewegungen zwischen dem geeinten Deutschland und aussereuropäischen Ländern, in: Informationen zur Raumentwicklung, (1992), Heft 9/10, S. 783-788.

FRIED, Ellen Shapiro/UDRY, J. Richard (1979): Wives' and Husbands' Expected Costs and Benefits of Childbearing as Predictors of Pregnancy, in: Social Biology, Jg. 26 (1979), Heft 4, S. 265-274. 
FRIEDBERG, Rachel M. (1991): The Labor Market Assimilation of Immigrants in the United States: the Role of Age at Arrival, mimeo., Cambridge, 1991 (zitiert nach PISCHKE, Jörn-Steffen (1992): Assimilation and the Earnings of Guestworkers in Germany (ZEW Discussion Paper No. 92-17), Mannheim, 1992).

FRIEDBERG, Rachel M./HUNT, Jennifer (1995): The Impact of Immigrants on Host Country Wages, Employment and Growth, in: Journal of Economic Perspectives, Jg. 9 (1995), Heft 2, S. 23-44.

FRITSCHE, Ute (1984): Sozialpolitische Maßnahmen und Geburtenhäufigkeit. Analyse der Fruchtbarkeitsentwicklung in der DDR 1970-1982, in: Soziologie und Sozialpolitik, (1984), S. 111-118.

GAAG, Jacques v.d. (1982): On Measuring the Cost of Children, in: Children and Youth Services Review, Jg. 4 (1982), Heft 1/2, S. 77-109.

GaLLER, Heinz Peter (1988): Familiale Lebenslagen und Familienlastenausgleich. Zu den Opportunitätskosten familialer Entscheidungen, in: FELDERER, Bernhard (Hrsg.), Familienlastenausgleich und demographische Entwicklung, Berlin, 1988, S. 83112.

GALLER, Heinz Peter (1991): Opportunitätskosten der Entscheidung für Familie und Haushalt, in: GRÄBE, Sylvia (Hrsg.), Der Private Haushalt als Wirtschaftsfaktor, Frankfurt, 1991, S. 118-152.

GaLlER, Heinz Peter/OTT, Notburga (1990): Zur Bedeutung familienpolitischer Maßnahmen für die Familienbildung - eine verhandlungstheoretische Analyse familiarer Entscheidungsprozesse, in: FELDERER, Bernhard (Hrsg.), Bevölkerung und Wirtschaft, Berlin, 1990, S. 111-134.

GaLlON, Thomas-Peter (1993): Logische Inkonsistenzen des "Elternrenten"-Modells, in: Sozialer Fortschritt, Jg. 42 (1993), Heft 1, S. 10-16.

GALOR, Oded/STARK, Oded (1994): Migration, Human Capital Formation, and Long-Run Output, in: SIEBERT, Horst (Hrsg.), Migration: A Challenge for Europe, Tübingen, 1994, S. 59-68.

GaNG, Ira N./Rivera-BATZ, Francisco L. (1994): Unemployment and Attitudes Towards Foreigners in Germany, in: STEINMANN, Gunter/UlRICH, Ralf E. (Hrsg.), The Economic Consequences of Immigration to Germany, Heidelberg, 1994, S. 121154.

GAUGLER, E. u.a. (1984): Wiedereingliederung von Frauen in qualifizierte Berufstätigkeit nach längerer Berufsunterbrechung (Forschungsbericht im Auftrag des Ministeriums für Soziales, Gesundheit und Umwelt Rheinland-Pfalz), Mannheim, 1984 (zitiert nach GALLER, Heinz Peter (1988): Familiale Lebenslagen und Familienlastenausgleich. $\mathrm{Zu}$ den Opportunitätskosten familialer Entscheidungen, in: FELDERER, Bernhard (Hrsg.), Familienlastenausgleich und demographische Entwicklung, Berlin, 1988, S. 83-112).

GENOSKO, Joachim/WEBER, Reinhard (1992): The Impact of Family Allowances on Demographic Change: A Case Study for Germany (Diskussionsbeiträge der Wirtschaftswissenschaftlichen Fakultät Ingolstadt, Nr. 18), Ingolstadt, 1992. 
GIERSCH, Herbert (Hrsg.) (1994): Economic Aspects of International Migration, Berlin u.a., 1994.

GIESECK, Arne/HEILEMANN, Ullrich/LOEFFELHOLZ, Hans Dietrich v. (1992): Implikationen der Zuwanderung aus Ost- und Südosteuropa für die öffentlichen Finanzen und das Wirtschaftswachstum in der Bundesrepublik, in: Sozialer Fortschritt, Jg. 41 (1992), Heft 11, S. 271-274.

GLATZER, Wolfgang (1983): Haushaltsproduktion in der modernen Gesellschaft (Sonderforschungsbereich 3, Arbeitspapier Nr. 86), Frankfurt, Mannheim, 1983 (zitiert nach VOTTLERER, Martin (1987): Die Aufwendungen der Familien für ihre minderjährigen Kinder - Eine theoretische und empirische Studie auf der Grundlage einer Zusatzerhebung zur Einkommens- und Verbrauchsstichprobe 1983 in BadenWürttemberg (Materialien und Berichte der Familienwissenschaftlichen Forschungsstelle im Statistischen Landesamt Baden-Württemberg), Stuttgart, 1987).

GRÄBE, Sylvia (Hrsg.) (1991): Der Private Haushalt als Wirtschaftsfaktor, Frankfurt, 1991.

GreENwoOd, Michael J./MCDowell, John M. (1994): The National Labor Market Consequences of U.S. Immigration, in: GIERSCH, Herbert (Hrsg.), Economic Aspects of International Migration, Berlin u.a., 1994, S. 155-194.

GroHmanN, Heinz (1987): Probleme einer Abschätzung des für ein Kapitaldeckungsverfahren in der gesetzlichen Rentenversicherung notwendigen Deckungskapitals - theoretische Überlegungen, in: FELDERER, Bernhard (Hrsg.), Kapitaldeckungsverfahren versus Umlageverfahren, Berlin, 1987, S. 67-90.

GRUBEL, Herbert G. (1994): The Economics of International Labor and Capital Flows, in: GIERSCH, Herbert (Hrsg.), Economic Aspects of International Migration, Berlin u.a., 1994, S. 75-92.

GuSY, Christoph (1994a): Entwurf einer Einwanderungsverordnung für die Europäische Union, in: WEIDENFELD, Wemer (Hrsg.), Das Europäische Einwanderungskonzept, Gütersloh, 1994, S. 155-160.

GUSY, Christoph (1994b): Möglichkeiten und Grenzen eines effektiven und flexiblen europäischen Einwanderungsrechts, in: WEIDENFELD, Werner (Hrsg.), Das Europäische Einwanderungskonzept, Gütersloh, 1994, S. 127-154.

HAMERMESH, Daniel S. (1993): Labor Demand, Princeton, 1993.

HARRIS, John R./TODARO, Michael P. (1970): Migration, Unemployment and Development: A Two-Sector-Analysis, in: American Economic Review, Jg. 60 (1970), S. 126-142.

HARRISON, M. Elizabeth/RAFFELHUESCHEN, Bernd (1989): Die Reform der staatlichen Alterssicherung in den Vereinigten Staaten, in: Versicherungswirtschaft, Jg. 44 (1989), Heft 7, S. 419-426.

HASE, Friedhelm (1992): Benachteiligung der Familie durch die Rentenversicherung?, in: Wirtschaftsdienst, Jg. 72 (1992), Heft 10, S. 526-530.

HAVEMAN, Robert/Wolfe, Barbara (1995): The Determinants of Children's Attainments: A Review of Methods and Findings, in: Journal of Economic Literature, Jg. 33 (1995), Heft 4, S. 1829-1878. 
HECK, Bruno (Hrsg.) (1988): Sterben wir aus? Die Bevölkerungsentwicklung in der Bundesrepublik Deutschland, Freiburg, 1988.

HELBERGER, Christof (1984): Humankapital, Berufbiographie und die Einkommen von Männern und Frauen (Sonderforschungsbereich 3, Arbeitspapier Nr. 129), Frankfurt, Mannheim, 1984.

HEMMER, Hans-Rimbert (1988): Wirtschaftsprobleme der Entwicklungsländer, 2. Aufl., München, 1988.

HESSE, Günter (1990): Evolutorische Ökonomik oder Kreativität in der Theorie, in: WITT, Ulrich (Hrsg.), Studien zur Evolutorischen Ökonomik I, Berlin, 1990, S. 49-74.

Hillman, Arye L. (1994): The Political Economy of Migration Policy, in: SIEBERT, Horst (Hrsg.), Migration: A Challenge for Europe, Tübingen, 1994, S. 263-282.

HILZENBECHER, Manfred (1985): Die Richtigstellung des Drei-Generationenvertrages in der gesetzlichen Rentenversicherung durch eine Beitragsstaffelung nach der Kinderzahl, in: Sozialer Fortschritt, Jg. 34 (1985), Heft 12, S. 281-288.

HIRSCH, Jürgen (1992): Einkommen und Kinderzahl, Wirtschaftswachstum und Bevölkerungsentwicklung, Tübingen, 1992.

HÖNEKOPP, Elmar (Hrsg.) (1987): Aspekte der Ausländerbeschäftigung in der Bundesrepublik Deutschland (Beiträge zur Arbeitsmarkt und Berufsforschung 114), Nürnberg, 1987.

HoFFMANN-NowOTNY, Hans-Joachim/FuX, Beat (1992): Die Zukunft der Familienpolitik: Zusammenfassung und Ausblick, in: HoFFMANN-NowOTNY, Hans-Joachim/HöHN, Charlotte/Fux, Beat (Hrsg.), Kinderzahl und Familienpolitik im Drei-Länder-Vergleich, Boppard am Rhein, 1992, S. 174-179.

HoFFMANN-NowotNY, Hans-Joachim/HöHN, Charlotte/FuX, Beat (Hrsg.) (1992): Kinderzahl und Familienpolitik im Drei-Länder-Vergleich, Boppard am Rhein, 1992.

HÖHN, Charlotte/SCHUBNELL, Hermann (1986a): Bevölkerungspolitische Maßnahmen und ihre Wirksamkeit in ausgewählten europäischen Industrieländern (I), in: Zeitschrift für Bevölkerungswissenschaft, Jg. 12 (1986), Heft 1, S. 3-51.

HÖHN, Charlotte/SCHUBNELL, Hermann (1986b): Bevölkerungspolitische Maßnahmen und ihre Wirksamkeit in ausgewählten europäischen Industrieländern (II), in: Zeitschrift für Bevölkerungswissenschaft, Jg. 12 (1986), Heft 2, S. 185-219.

HÖHN, Charlotte (1992): Kenntnis und Bewertung familienpolitischer Maßnahmen, in: HoFFMANN-NowOTNY, Hans-Joachim/HÖHN, Charlotte/FuX, Beat (Hrsg.), Kinderzahl und Familienpolitik im Drei-Länder-Vergleich, Boppard am Rhein, 1992, S. $150-173$.

HOMBURG, Stefan (1988): Theorie der Alterssicherung, Berlin, 1988.

HOMBURG, Stefan (1990): The Efficiency of Unfunded Pension Schemes, in: Journal of Institutional and Theoretical Economics, Jg. 146 (1990), Heft 4, S. 640-647.

HOMBURG, Stefan/GRÄFF, Carsten (1988): Zur ökonomischen Begründbarkeit eines Familienlastenausgleichs, in: FELDERER, Bernhard (Hrsg.), Familienlastenausgleich und demographische Entwicklung, Berlin, 1988, S. 13-28. 
HUNT, Jennifer (1992): The Impact of the 1962 Repatriates from Algeria on the French Labor Market, in: Industrial and Labor Relations Review, Jg. 45 (1992), S. 556-572.

Hutchinson, E.P. (1967): The Population Debate, Boston u.a., 1967.

ICHINO, Andrea (1991): The Economic Impact of Immigration on the Host Country, (Centre for Economic Policy Research (CEPR) Discussion Paper), o.O., 1991.

INSTTTUT NATIONAL D'ETUDES DÉMOGRAPHIQUES (Hrsg.) (1982): Natalité et politiques de population en France et en Europe de l'Est; Traveaux et Documents, Band 98, Paris, 1982.

JASSO, Guillermina/RosENZWEIG, Mark R. (1994): Labour Immigration to the United States and Family Preference, in: ORGANISATION FOR ECONOMIC CO-OPERATION AND DEVELOPMENT (Hrsg.), Migration and Development, Paris, 1994, S. 90-99.

JOCHIMSEN, Reimut (1966): Theorie der Infrastruktur, Tübingen, 1966.

JOHNSON, Paul/ZIMMERMANN, Klaus F. (Hrsg.) (1993): Labour Markets in an Ageing Europe, Cambridge, 1993.

JÜRGENS, Hans W./POHL, Katharina (1975): Kinderzahl - Wunsch und Wirklichkeit, Stuttgart, 1975.

KÄLIN, Walter/MOSER, Rupert (Hrsg.) (1989): Migrationen aus der Dritten Welt, Bern, 1989.

KAISER, Helmut (1989): Die Mehrwertsteuerbelastung privater Haushalte in der Bundesrepublik Deutschland, in: DIW-Vierteljahreshefte, Jg. 58 (1989), Heft 1, S. 24-61.

KAUFMANN, Franz-Xaver (1990): Ursachen des Geburtenrückgangs in der Bundesrepublik Deutschland und Möglichkeiten staatlicher Gegenmaßnahmen, in: Zeitschrift für Bevölkerungswissenschaft, Jg. 16 (1990), Heft 3/4, S. 383-396.

KELMAN, Nico Willem (1990): Uncertainty in National Population Forecasting. Issues, Backgrounds, Analyses, Recommendations, Amsterdam, 1990.

KLös, Hans-Peter (1992): Integration der Einwanderer aus Ost-/Südosteuropa in den deutschen Arbeitsmarkt, in: Sozialer Fortschritt, Jg. 41 (1992), Heft 11, S. 261-270.

КосH, Lambert T. (1996): Evolutorische Wirtschaftspolitik: Eine elementare Analyse mit entwicklungspolitischen Beispielen, Tübingen, 1996.

KÖPP, Günter (1994): Finanzierungsprobleme der Rentenversicherung: Die langfristige Entwicklung der Finanzlage in der gesetzlichen Rentenversicherung der Arbeiter und Angestellten, Bern u.a., 1994.

KöSSLER, Richard (1984): Arbeitszeitbudgets ausgewählter privater Haushalte in BadenWürttemberg (Statistisches Landesamt Baden-Württemberg (Hrsg.), Materialien und Berichte der Familienwissenschaftlichen Forschungsstelle, Heft 12), Stuttgart, 1984.

KROMBHOLZ, H. (1991): Arbeit und Familie: Geschlechtsspezifische Unterschiede in der Erwerbstätigkeit und die Aufteilung der Erwerbstätigkeit in der Partnerschaft, in: BERTRAM, Hans (Hrsg.), Die Familie in Westdeutschland. Stabilität und Wandel familiarer Lebensformen, Opladen, 1991, S. 193-231. 
KüLP, Bernhard (1981): Zur Diskussion um den Generationenvertrag, in: SCHWEITZER, Rosemarie v. (Hrsg.), Leitbilder für Familie und Familienpolitik, Berlin, 1981, S. 131143.

KÜLP, Bernhard (1993): Das Mackenroth-Theorem, in: Wirtschaftswissenschaftliches Studium, Jg. 22 (1993), Heft 2, S. 82-84.

LALONDE, Robert J./TOPEL, Robert H. (1992): The Assimilation of Immigrants in the U.S. Labor Market, in: BORJAS, George J./FREEMAN, Richard B. (Hrsg.), Immigration and the Work Force, Chicago, London, 1992, S. 67-92.

LAMPERT, Heinz (1986): Ordnungspolitische und verteilungspolitische Aspekte der Familienpolitik in der Bundesrepublik Deutschland, in: LAMPERT, Heinz/WINGEN, Max (Hrsg.), Familien und Familienpolitik, Köln, 1986, S. 9-49.

LAMPERT, Heinz (1992): Der Beitrag von Familien mit Kindern zur Humanvermögensbildung (Sozialpolitik und Wissenschaft: Positionen zur Theorie und Praxis der sozialen Hilfen. Schriftenreihe des Deutschen Vereins für öffentliche und private Fürsorge, Band 269), Frankfurt/Main, 1992, S. 130-141.

LAMPERT, Heinz/WINGEN, Max (Hrsg.) (1986): Familien und Familienpolitik, Köln, 1986.

LEIBENSTEIN, Harvey (1957): Economic Backwardness and Economic Growth, New York, London, 1957.

LEIBENSTEIN, Harvey (1974): An Interpretation of the Economic Theory of Fertility: Promising Path or Blind Alley?, in: Journal of Economic Literature, Jg. 12 (1974), S. 457479.

LEIBENSTEIN, Harvey (1975): The Economic Theory of Fertility Decline, in: Quarterly Journal of Economics, Jg. 89 (1975), Heft 1, S. 1-31.

LENGSFELD, Wolfgang (1987): Meinungen und Einstellungen zur Bevölkerungsentwicklung in der Bundesrepublik Deutschland: Ergebnisse einer Meinungsumfrage (Bundesinstitut für Bevölkerungsforschung (Hrsg.), Materialien zur Bevölkerungswissenschaft, Sonderheft 14), Wiesbaden, 1987.

LesthaEge, Ron/PAGE, Hilary/SuRKYN, Johan (1991): Sind Einwanderer ein Ersatz für Geburten, in: Zeitschrift für Bevölkerungswissenschaft, Jg. 17 (1991), Heft 3, S. 281314.

LICHT, Georg/STEINER, Viktor (1993): Assimilation, Labour Market Experience, and Earnings Profiles of Temporary and Permanent Immigrant Workers in Germany (ZEW Discussion Paper No. 93-06), Mannheim, 1993.

LuCAS, Robert E. Jr. (1990): Why Doesn't Capital Flow from Rich to Poor Countries?, in: American Economic Review, Papers and Proceedings, Jg. 80 (1990), Heft 2, S. 92-96.

LÜSCHER, K. (Hrsg.) (1979): Sozialpolitik für das Kind, Stuttgart, 1979.

LUTZ, Wolfgang (Hrsg.) (1991): Future Demographic Trends in Europe and North America, London, San Diego, 1991.

MACKENROTH, Gerhard (1952): Die Reform der Sozialpolitik durch einen deutschen Sozialplan, in: ALBRECHT, Gerhard (Hrsg.), Verhandlungen auf der Sondertagung in Berlin (Schriften des Vereins für Sozialpolitik), Berlin, 1952, S. 39-76. 
MACKENSEN, Rainer/WEWER, Heinz (Hrsg.) (1973): Dynamik der Bevölkerungsentwicklung. Strukturen - Bedingungen - Folgen, München, 1973.

MADGE, Charles (Hrsg.) (1943): War-Time Pattern of Saving and Spending, Cambridge, 1943.

MalthUS, Thomas R. (1798): An Essay on the Principle of Population, in: Wrigley, E.A., Souden, D. (Hrsg.), The Works of Thomas Robert Malthus 1986, Band 1, London, 1798.

Malthus, Thomas R. (1826): An Essay on the Principle of Population, in: Wrigley, E.A., Souden, D. (Hrsg.), The Works of Thomas Robert Malthus 1986, the sixth edition with variant readings from the second edition (1803), Band 2, Part I , London, 1826.

MARKUSEN, James R. (1983): Factor Movements and Commodity Trade as Complements, in: Journal of International Economics, Jg. 14 (1983), S. 341-356.

MIEGEl, Meinhard (1984): Arbeitsmarktpolitik auf Irrwegen, Bonn, 1984.

MIEGEl, Meinhard (1989): Die eigentliche Reformaufgabe liegt noch vor uns: Grundsicherung im Alter, in: Arbeit und Sozialpolitik, Jg. 43 (1989), Heft 4, S. 74-78.

MIEGEl, Meinhard/WaHL, Stefanie (1985): Gesetzliche Grundsicherung, Private Vorsorge. Der Weg aus der Rentenkrise, 1. Aufl., Bonn, 1985.

MILL, John Stuart (1881): Grundsätze der Politischen Ökonomie (Neudruck der Ausgabe Leipzig 1881, übersetzt von Adolf Soetbeer, 4. Aufl.), Aalen, 1986.

MINCER, Jacob (1978): Family Migration Decisions, in: Journal of Political Economy, Jg. 86 (1978), S. 749-773.

MITTERAUER, Michael/SIEDER, Reinhard (1991): Vom Patriachat zur Partnerschaft: Zum Strukturwandel der Familie, 4. Aufl., München, 1991.

MoON, Marylin (Hrsg.) (1984): Economic Transfers in the United States, Chicago, 1984.

MOORE, Kristin A./HOFFERTH, Sandra L. (1979): Women and their Children, in: SMITH, Ralph E. (Hrsg.), The Subtle Revolution: Women at Work, Washington, D.C., 1979, S. 125-158.

MüLLER, Horst-Wolf (1992): Zu den Kosten möglicher Verbesserungen bei der Anrechnung oder Bewertung von Kindererziehungszeiten im Rentenrecht, in: Deutsche Rentenversicherung, (1992), Heft 10/11, S. 620-640.

MÜLLER, Horst-Wolf/ROPPEL, Ulrich (1990): Eine Abschätzung des Kapitalbedarfs bei einer vollständigen Kapitaldeckung der gesetzlichen Rentenversicherung, in: FELDERER, Bernhard (Hrsg.), Bevölkerung und Wirtschaft, Berlin, 1990, S. 425-440.

MUNDELL, Robert A. (1957): International Trade and Factor Mobility, in: American Economic Review, Jg. 47 (1957), S. 321-335.

MYRDAL, Gunnar (1959): Ökonomische Theorie und unterentwickelte Regionen, Stuttgart, 1959.

National Bureau of Economic Research (Hrsg.) (1960): Demographic and Economic Change in Developed Countries, Princeton, 1960.

NELL-BREUNING, Oswald v. (1978): Vertrag zwischen drei Generationen, in: Wirtschaftswoche, Jg. 32 (1978), Heft 23, S. 77-80. 
NELL-BREUNING, Oswald v. (1980): Soziale Rentenversicherung aus familien- und bevölkerungspolitischer Sicht, in: SCHENKE, Klaus/SCHMÄHL, Winfried (Hrsg.), Alterssicherung als Aufgabe für Wissenschaft und Politik, Stuttgart u.a., 1980, S. 369378.

NELL-BREUNING, Oswald v. (1986): Die optische Täuschung in der Rentendiskussion, in: Stimmen der Zeit, (1986), Heft 11, S. 737-743.

NOTESTEIN, Frank W. (1945): Population - the Long View, in: SCHULTZ, Theodore W. (Hrsg.), Food for the World, Chicago, 1945, S. 36-57.

ORGANISATION FOR ECONOMIC CO-OPERATION AND DEVELOPMENT (Hrsg.) (1994): Migration and Development, Paris, 1994.

O.V. (1993a): Bevölkerungsentwicklung in Deutschland bis zum Jahr 2010 mit Ausblick auf 2040, in: DIW Wochenberichte, Jg. 60 (1993), Heft 29, S. 393-404.

o.V. (1993b): Zur ökonomischen Rationalität des Geburtenrückgangs in Ostdeutschland, in: DIW-Wochenberichte, Jg. 60 (1993), Heft 45, S. 662-663.

O.V. (1994): Integration deutscher Zuwanderer in den westdeutschen Arbeitsmarkt, in: DIWWochenberichte, Jg. 61 (1994), Heft 35, S. 609-617.

OBERHAUSER, Alois (1989): Familie und Haushalt als Transferempfänger, Frankfurt, New York, 1989.

OETER, Ferdinand (1992): Familienlastenausgleich und Einkommensteuer, in: Sozialer Fortschritt, Jg. 41 (1992), Heft 2, S. 49-50.

OPPENLÄNDER, Karl Heinrich/WAGNER, Adolf (Hrsg.) (1985): Ökonomische Verhaltensweisen und Wirtschaftspolitik bei schrumpfender Bevölkerung, München, 1985.

PELZER, Helmut (1994): Bürgergeld, Stuttgart, 1994.

Petersen, Hans-Georg (1993): Politische Ökonomie von Nationalismus und Migration, Gießen, 1993.

PISCHKE, Jörn-Steffen (1992): Assimilation and the Earnings of Guestworkers in Germany (ZEW Discussion Paper No. 92-17), Mannheim, 1992.

PISCHKE, Jörn-Steffen/VELLING, Johannes (1994): Wage and Employment Effects of Immigration to Germany: An Analysis Based on Local Labour Markets (ZEW Discussion Paper No. 94-03), Mannheim, 1994.

PROGNOS AG (1987): Zur langfristigen Entwicklung der gesetzlichen Rentenversicherung (Verband Deutscher Rentenversicherungsträger (Hrsg.), DRV-Schriften), Frankfurt/Main, 1987.

PROGNOS AG (1995): Prognos-Gutachten 1995 (Verband Deutscher Rentenversicherungsträger (Hrsg.), DRV-Schriften, Band 4), Frankfurt/Main, 1995.

RASCH, Hans Georg (1980): Zur Neuberechnung des Preisindexes für die einfache Lebenshaltung eines Kindes auf Basis von 1976, in: Wirtschaft und Statistik, (1980), Heft 4, S. 236-238.

REHFELD, Uwe (1991): Ausländische Arbeitnehmer und Rentner in der gesetzlichen Rentenversicherung, in: Deutsche Rentenversicherung, (1991), Heft 7, S. 468-492. 
RENESSE, Margot v. (1992): Eigenständige Rentenansprüche der Frauen, in: Soziale Sicherheit, Jg. 41 (1992), Heft 10, S. 306-308.

RICHTER, Wolfram F./WEIMANN, Joachim (1987): Kinderjahre: Ein Vorschlag zur Rentenreform, in: Wirtschaftsdienst, Jg. 67 (1987), Heft 5, S. 260-262.

RIVERA-BATZ, Francisco L./SECHZER, Selig L. (1991): Substitution and Complementarity between Immigrant and Native Labor in the United States, in: RIVERA-BATZ, Francisco L./SECHZER, Selig L./GANG, Ira N. (Hrsg.), U.S. Immigration Policy Reform in the 1980s: A Preliminary Assessment, New York, 1991, S. 89-116.

RIVERA-BATZ, Francisco L./SECHZER, Selig L./GANG, Ira N. (Hrsg.) (1991): U.S. Immigration Policy Reform in the 1980s: A Preliminary Assessment, New York, 1991.

RobBINS, Lionel (1972): An Essay on the Nature and Significance of Economic Science, London, Basingstoke, 1972.

Robinson, Warren C. (1987): The Time Cost of Children and Other Household Production, in: Population Studies, Jg. 41 (1987), S. 313-323.

ROSE, Klaus/SAUERNHEIMER, Karlhans (1992): Theorie der Außenwirtschaft, 11. völlig überarb. und erw. Aufl., München, 1992.

RoSEN, Harvey S./WINDISCH, Rupert (1992): Finanzwissenschaft I, München, Wien, 1992.

ROSENZWEIG, Mark R./WOLPIN, Kenneth I. (1980): Testing the Quantity-Quality Fertility Model: The Use of Twins as a Natural Experiment, in: Econometrica, Jg. 48 (1980), Heft 1, S. 227-240.

Ross, Hartmut (1990): Familienlastenausgleich - quo vadis?, in: Deutsche Steuerzeitung, (1990), Heft 24, S. 611-618.

ROTHBARTH, E. (1943): Note on a Method of Determining Equivalent Income for Families of Different Composition, in: MADGE, Charles (Hrsg.), War-Time Pattern of Saving and Spending, Cambridge, 1943, App. 4.

RoTHSCHILD, Michael/STIGLITZ, Joseph (1976): Equilibrium in Competitive Insurance Markets: An Essay on the Economics of Imperfect Information, in: Quarterly Journal of Economics, Jg. 90 (1976), S. 629-650.

RUDDICK, Elizabeth (1994): The Selection and Management of Immigration to Canada, in: ORganization fOR ECONOMIC CO-OPERATION AND DEVELOPMENT (Hrsg.), Migration and Development, Paris, 1994, S. 85-89.

RÜRUP, Bert/SESSELMEIER, Werner (1994): Zu den wichtigsten Auswirkungen von Einwanderung auf Arbeitsmarkt und Sozialversicherung, in: forum demographie und politik, (1994), Heft 5, S. 64-89.

RUNCIMAN, W.G. (1966): Relative Deprivation and Social Justice: A Study of Attitudes to Social Inequality in Twentieth-Century England, Berkeley, 1966.

SALT, John (1993): Migration and Population Change in Europe, New York, 1993.

SCHEMMEL, Lothar (1991): Die verfassungsgerichtlichen Maßstäbe für das Existenzminimum eines Kindes, in: Deutsche Steuerzeitung, (1991), Heft 16, S. 481-491.

SCHENKE, Klaus/SCHMÄHL, Winfried (Hrsg.) (1980): Alterssicherung als Aufgabe für Wissenschaft und Politik, Stuttgart u.a., 1980. 
SCHEWE, Dieter (1992): Die Zuwanderungen aus Ost-/Südosteuropa nach Deutschland - Stand und künftige Möglichkeiten, in: Sozialer Fortschritt, Jg. 41 (1992), Heft 11, S. 251-256.

SCHMÄHL, Winfried (1988): Beiträge zur Reform der Rentenversicherung, Tübingen, 1988.

SCHMÄHL, Winfried (1990): Beitragsfinanzierte Mindestsicherung im Alter, in: Hamburger Jahrbuch für Wirtschafts- und Gesellschaftspolitik, Band 35 (1990), S. 187-205.

SCHMÄHL, Winfried (1994a): Perspektiven der Alterssicherung in Deutschland, in: Wirtschaftsdienst, Jg. 74 (1994), Heft 8, S. 390-395.

SCHMÄHL, Winfried (1994b): Strategien und Maßnahmen künftiger Alterssicherungspolitik in Deutschland, in: Wirtschaftsdienst, Jg. 74 (1994), Heft 10, S. 507-514.

SCHMIDT, Christoph (1993): The Earnings Dynamics of Immigrant Labour (Centre for Economic Policy Research (CEPR) Discussion Paper No. 763), o.O., 1993.

SCHMIDT, Christoph (1994a): Immigration Countries and Migration Research: The Case of Germany, in: STEInManN, Gunter/UlRICH, Ralf E. (Hrsg.), The Economic Consequences of Immigration to Germany, Heidelberg, 1994, S. 1-20.

SCHMIDT, Christoph (1994b): The Economic Performance of Germany's East European Immigrants, (Centre for Economic Policy Research (CEPR) Discussion Paper No. 963), o.O., 1994.

SCHMIDT, Henning/FrANK, Udo/MüLLER-ROHR, Iris (1985): Kritische Bemerkungen zum System des Kinderlastenausgleichs - zugleich ein Vorschlag zur Neugestaltung der gesetzlichen Rentenversicherung, in: Finanzarchiv, (1985), S. 28-66.

SCHNEIDER, Dieter (1991): Investition, Finanzierung und Besteuerung, 6. Aufl., Wiesbaden, 1991.

SCHUBNELL, Hermann (1973): Der Bevölkerungsrückgang in der Bundesrepublik Deutschland, in: MACKENSEN, Rainer/WEWER, Heinz (Hrsg.): Dynamik der Bevölkerungsentwicklung. Strukturen - Bedingungen - Folgen, München, 1973, S. 40-79.

SCHUBNELL, Hermann (1990): Bevölkerungs- und Familienpolitik - Definitionen, Bezug zur Gesamtpolitik und Probleme der Wirkungsanalyse, in: BIRG, Herwig/MACKENSEN, Rainer (Hrsg.), Demographische Wirkungen politischen Handelns, Frankfurt/Main, New York, 1990, S. 33-54.

SCHULTHEIS, Franz (1988): Familien- und Bevölkerungspolitik in europäischen Ländern, in: HECK, Bruno (Hrsg.), Sterben wir aus? Die Bevölkerungsentwicklung in der Bundesrepublik Deutschland, Freiburg, 1988, S. 113-127.

SCHULTHEIS, Franz (1990): Die pronatalistische Bevölkerungspolitik in Frankreich, in: BIRG, Herwig/MACKENSEN, Rainer (Hrsg.), Demographische Wirkungen politischen Handelns, Frankfurt/Main, New York, 1990, S. 303-356.

SCHULTZ, Theodore W. (Hrsg.) (1945): Food for the World, Chicago, 1945.

SchulTZ, Theodore W. (Hrsg.) (1974): Economics of the Family, Chicago, 1974.

SCHULZ, Erika/SEIRING, Kerstin (1994): Analyse der beruflichen Eingliederung deutscher Zuwanderer - Ein Beispiel für die logistische Regressionsanalyse mit SPSS (DIW, Diskussionspapier Nr. 102), Berlin, 1994. 
SCHUMACHER, Astrid (1982): Zum Kinderwunsch von Jugendlichen, in: Zeitschrift für Bevölkerungswissenschaft, Jg. 8 (1982), Heft 4, S. 559-574.

SCHWARZ, Karl (1982): Kinderzahl der Ehen nach Bevölkerungsgruppen im Jahr 1981, in: Zeitschrift für Bevölkerungswissenschaft, Jg. 8 (1982), Heft 4, S. 575-587.

SCHWARZ, Karl (1988): Familienpolitik und demographische Entwicklung in den Bundesländern nach dem Zweiten Weltkrieg (Bundesinstitut für Bevölkerungsforschung (Hrsg.), Materialien zur Bevölkerungswissenschaft, Band 1), Wiesbaden, 1988.

SCHWARZ, Karl (1990): Demographische Wirkungen der Familienpolitik in der Bundesrepublik Deutschland und in den Bundesländern nach dem Zweiten Weltkrieg, in: FELDERER, Bernhard (Hrsg.), Bevölkerung und Wirtschaft, Berlin, 1990, S. 495-518.

SCHWEITZER, Rosemarie v. (1979): Kinder und ihre Kosten, in: LÜSCHER, K. (Hrsg.), Sozialpolitik für das Kind, Stuttgart, 1979, S. 113-142.

SCHWEITZER, Rosemarie v. (Hrsg.) (1981): Leitbilder für Familie und Familienpolitik, Berlin, 1981.

SHELDON, Eleanor Bernert (Hrsg.) (1973): Family Economic Behaviour: Problems and Prospects, Toronto, Philadelphia, 1973.

Shughart, William F./Tollison, Robert D./KIMENYI, Mwangi S. (1986): The Political Economy of Immigration Restrictions, in: Yale Journal on Regulation, Jg. 4 (1986), Heft 1, S. 79-97.

SIEBERT, Horst (1993): Internationale Wanderungsbewegungen: Erklärungsansätze und Gestaltungsfragen, in: Schweizerische Zeitschrift für Volkswirtschaft und Statistik, (1993), S. 229-255.

SIEBERT, Horst (Hrsg.) (1994): Migration: A Challenge for Europe, Tübingen, 1994.

SIKORA, Joachim (Hrsg.) (1991): Aussiedler als Herausforderung und Auftrag für die deutsche Gesellschaft, Bad Honnef, 1991.

SIMON, Julian L. (1989): The Economic Consequences of Immigration, Cambridge, 1989.

SIMON, Julian L. (1994): On the Economic Consequences of Immigration: Lessons for Immigration Policies, in: GIERSCH, Herbert (Hrsg.), Economic Aspects of International Migration, Berlin u.a., 1994, S. 227-248.

SimON, Julian L./AKBAR, Ather (1995): The Effects of In-Migrants on the German Public Purse (unveröffentlichtes Manuskript, under review by International Migration Review), 0.0, 1995.

SimON, Julian L./MoORE, Stephen/Sullivan, Richard (1993): The Effect of Immigration on Aggregate Native Unemployment: An Across-City Estimation, in: Journal of Labor Research, Jg. 14 (1993), S. 299-316.

SJAASTAD, Larry A. (1962): The Costs and Returns of Human Migration, in: Journal of Political Economy, Jg. 70 (1962), S. 80-93.

SMTrH, Adam (1776): Der Wohlstand der Nationen. Eine Untersuchung seiner Natur und seiner Ursachen (Deutsche Übersetzung nach der 5. Aufl. (1783) der Londoner Originalausgabe (1776)), München, 1994. 
SMITH, D.P. (1981): A Reconsideration of Easterlin Cycles, in: Population Studies, Jg. 35 (1981), S. 247-264.

SMITH, Ralph E. (Hrsg.) (1979): The Subtle Revolution: Women at Work, Washington, D.C., 1979.

SOMMER, Bettina (1992): Entwicklung der Bevölkerung bis 2030. Ergebnis der siebten koordinierten Bevölkerungsvorausberechnung, in: Wirtschaft und Statistik, (1992), Heft 4, S. 217-222.

SOMMER, Bettina (1994): Entwicklung der Bevölkerung bis 2040, in: Wirtschaft und Statistik, (1994), Heft 7, S. 497-503.

SOWELL, Thomas (1979): Classical Economics Reconsidered, Princeton, 1979.

STARK, Oded (1984): Discontinuity and the Theory of International Migration, in: Kyklos, Jg. 37 (1984), Heft 2, S. 206-222.

STARK, Oded (1991): The Migration of Labor, Oxford, Cambridge, 1991.

STARK, Oded (1994): Market Environment, Trade Technology, and Migrants' Performance, in: SEBERT, Horst (Hrsg.), Migration: A Challenge for Europe, Tübingen, 1994, S. 45-52.

STARK, Oded/TAYloR, J.E. (1991): Relative Deprivation and Migration: Theory, Evidence and Policy Implications (World Bank Working Papers No. 656 (Policy, Research and External Affairs)), Washington, D.C., 1991 (zitiert nach SIEBERT, Horst (1993): Internationale Wanderungsbewegungen: Erklärungsansätze und Gestaltungsfragen, in: Schweizerische Zeitschrift für Volkswirtschaft und Statistik, (1993), S. 229-255).

STATISTISCHES BUNDESAMT (1993): Statistisches Jahrbuch 1993, Stuttgart, 1993.

STEINER, Viktor/VELLING, Johannes (1994): Re-Migration Behavior and Expected Duration of Stay of Guest Workers in Germany, in: STEINMANN, Gunter/UlRICH, Ralf E. (Hrsg.), The Economic Consequences of Immigration to Germany, Heidelberg, 1994, S. 101-120.

SteinmanN, Gunter (Hrsg.) (1984): Economic Consequences of Population Change in Industrialized Countries, Berlin u.a., 1984.

STEINMANN, Gunter (1991): Immigration as a Remedy for the Birth Dearths, in: LUTZ, Wolfgang (Hrsg.), Future Demographic Trends in Europe and North America, London, San Diego, 1991, S. 337-357.

STEINMANN, Gunter (1993): Zusammenhang zwischen Alterungsprozeß und Einwanderung. Expertise für die Enquete-Kommission "Demographischer Wandel", Halle, 1993.

STEINMANN, Gunter (1994): The Effects of Immigrants on the Income of Natives, in: SteInManN, Gunter/Ulrich, Ralf E. (Hrsg.), The Economic Consequences of Immigration to Germany, Heidelberg, 1994, S. 45-60.

STEINMANN, Gunter/UlRICH, Ralf E. (Hrsg.) (1994): The Economic Consequences of Immigration to Germany, Heidelberg, 1994.

STIGLER, George J./BECKER Gary S. (1977): De Gustibus non est Disputandum, in: American Economic Review, Jg. 67 (1977), Heft 2, S. 76-90. 
STRAUbHAAR, Thomas (1986): The Causes of Labor Migration: A Demand-Determined Approach, in: International Migration Review, Jg. 20 (1986), S. 835-855.

STRAUBHAAR, Thomas (1988): On the Economics of International Labor Migration, Bern, 1988.

STRAUBHAAR, Thomas (1991): Einwanderungspolitische Instrumente im Dienst der Bevölkerungspolitik: Ethische Probleme, Wirksamkeit, Erfahrungen, in: FicKL, Stephan (Hrsg.), Bevölkerungsentwicklung und öffentliche Haushalte, Frankfurt/Main, New York, 1991, S. 205-226.

STRAUBHAAR, Thomas (1992): Internationale Faktorwanderungen: Substitutionalität versus Komplementarität, in: Wirtschaftswissenschaftliches Studium, Jg. 21 (1992), Heft 11, S. 563-570.

STRAUBHAAR, Thomas (1995): Neuere Entwicklungen in der Migrationstheorie, in: Wirtschaftswissenschaftliches Studium, Jg. 24 (1995), Heft 5, S. 243-248.

STRAUBHAAR, Thomas (1996): Wie sich das Baugewerbe unverblümt eine Schutzwand mauert, in: Wirtschaftswissenschaftliches Studium, Jg. 25 (1996), Heft 2, S. 53 , 71.

STRAUBHAAR, Thomas/DHIMA, Giorgio (1989): Migration im Spannungsfeld zwischen Mikroökonomie und politischer Ökonomie, in: KÄLIN, Walter/MOSER, Rupert (Hrsg.), Migrationen aus der Dritten Welt, Bern, 1989, S. 65-91.

StraubhaAR, Thomas/ZimmermanN, Klaus F. (1992): Towards a European Migration Policy (Centre for Economic Policy Research (CEPR) Discussion Paper No. 641), London, 1992.

SUNTUM, Ulrich v. (1989): Möglichkeiten und Grenzen privatwirtschaftlicher Altersvorsorge, in: Wirtschaftsdienst, Jg. 69 (1989), Heft 4, S. 207-216.

Thompson, Warren S. (1929): Population, in: American Journal of Sociology, Jg. 34 (1929), S. 959-975.

TICHY, Roland (1993): Ausländer rein!, 3. völlig überarb. Aufl., München, 1993.

TIEBOUT, Charles M. (1956): A Pure Theory of Local Expenditures, in: Journal of Political Economy, Jg. 64 (1956), S. 416-424.

TIETZEL, Manfred/WEBER, Marion (1993): Autokratische Mobilitätspolitik, in: ORDO, (1993), Heft 44, S. 291-305.

ToBIN, James (1974): Comment, in: SchulTZ, Theodore W. (Hrsg.), Economics of the Family, Chicago, 1974, S. 293-294.

TODARO, Michael P. (1971): Income Expectations, Rural-Urban Migration and Employment in Africa, in: International Labour Review, Jg. 104 (1971), Heft 5, S. 391-395 und 411-413.

TRANSFER-ENQUETE-KOMMISSION (1979): Zur Einkommenslage der Rentner, Bonn, 1979.

TREBICI, Vladimir (1982): Situation démographique de la Roumanie, in: INSTITUT NATIONAL D'ETUDES DÉMOGRAPHIQUES (Hrsg.), Natalité et politiques de population en France et en Europe de l'Est; Traveaux et Documents, Band 98, Paris, 1982, S. 109-160. 
TURCHI, Boone A. (1984): The Monetary Cost of a Child, in: STEINMANN, Gunter (Hrsg.), Economic Consequences of Population Change in Industrialized Countries, Berlin u.a., 1984, S. 258-277.

UlRICH, Ralf E. (1992): Der Einfluß der Zuwanderung auf die staatlichen Einnahmen und Ausgaben in Deutschland, in: Acta Demographica, (1992), S. 189-208.

ULRICH, Ralf E. (1994a): Foreigners and the Social Insurance System in Germany, in: SteInManN, Gunter/UlRICH, Ralf E. (Hrsg.), The Economic Consequences of Immigration to Germany, Heidelberg, 1994, S. 61-80.

ULRICH, Ralf E. (1994b): Vertriebene and Aussiedler - The Immigration of Ethnic Germans, in: STEINMANN, Gunter/UlRICH, Ralf E. (Hrsg.), The Economic Consequences of Immigration to Germany, Heidelberg, 1994, S. 155-177.

UNTED NATIONS (Hrsg.) (1975): The Population Debate: Dimensions and Perspectives, New York, 1975.

VANBERG, Viktor/KeRBER, Wolfgang (1994): Competition among Institutions: Evolution within Constraints, (Paper to be presented at the 1994 Friedrich August von Hayek-Symposium on "Competition among Institutions"), Fairfax, Freiburg, Bochum, 1994.

VELLING, Johannes (1993): Immigration to Germany in the Seventies and Eighties - The Role of Family Reunification (ZEW Discussion Paper No. 93-18), Mannheim, 1993.

VELLING, Johannes (1995): Wage Discrimination and Occupational Segregation of Foreign Male Workers in Germany (ZEW Discussion Paper No. 95-04), Mannheim, 1995.

VOLAND, Eckart v. (Hrsg.) (1992): Fortpflanzung: Natur und Kultur im Wechselspiel. Versuch eines Dialogs zwischen Biologen und Sozialwissenschaftlern, Frankfurt/Main, 1992.

VOTTLERER, Martin (1987): Die Aufwendungen der Familien für ihre minderjährigen Kinder Eine theoretische und empirische Studie auf der Grundlage einer Zusatzerhebung zur Einkommens- und Verbrauchsstichprobe 1983 in Baden-Württemberg (Materialien und Berichte der Familienwissenschaftlichen Forschungsstelle im Statistischen Landesamt Baden-Württemberg), Stuttgart, 1987.

WANDER, Hilde (1980): Ursachen des Geburtenrückgangs in ökonomischer Sicht. Theoretische Ansätze und empirische Ergebnisse unter Berücksichtigung der Verhältnisse in der Bundesrepublik Deutschland (Kieler Diskussionsbeiträge Nr. 71), Kiel, 1980.

WARLICK, Jennifer L./BURKHAUSER, Richard V. (1984): Raising the Normal Retirement Age under Social Security: A Life-Cycle Analysis, in: MooN, Marylin (Hrsg.), Economic Transfers in the United States, Chicago, 1984, S. 359-79.

WEBER, Réne/STRAUBHAAR, Thomas (1994): Budget Incidence of Immigration into Switzerland: A Cross-Section Analysis of the Public Transfer System (Centre for Economic Policy Research (CEPR) Discussion Paper No. 934), o.O., 1994.

WEHRMANN, Martin (1989): Auswirkungen der Ausländerbeschäftigung auf die Volkswirtschaft der Bundesrepublik Deutschland in Vergangenheit und Zukunft, BadenBaden, 1989. 
WEIDENFELD, Werner (Hrsg.) (1994): Das Europäische Einwanderungskonzept, Gütersloh, 1994.

WEIZSÄCKER, Robert K. v. (1990): Population Aging and Social Security: A Politico-Economic Model of State Pension Financing, in: Public Finance, Jg. 45 (1990), Heft 3, S. 491-509.

WHITE, Michael J./HUNTER, Lori (1993): The Migratory Response of Native-Born Workers to the Presence of Immigrants in the Labor Market (Brown University Working Paper No. 93-08), 0.O., 1993 (zitiert nach FRIEDBERG, Rachel M./HUNT, Jennifer (1995): The Impact of Immigrants on Host Country Wages, Employment and Growth, in: Journal of Economic Perspectives, Jg. 9 (1995), Heft 2, S. 23-44).

WHITE, Michael J./LIANG, Zai (1994): The Effect of Immigration on the Internal Migration of the Native Born Population 1981-90 (mimeo, Brown University), o.O., 1994 (zitiert nach FRIEDBERG, Rachel M./HUNT, Jennifer (1995): The Impact of Immigrants on Host Country Wages, Employment and Growth, in: Journal of Economic Perspectives, Jg. 9 (1995), Heft 2, S. 23-44).

WILLEKE, Franz-Ulrich/ONKEN, Ralph (1991): Familienlastenausgleich mit variablem Kindergeld, in: Statistik und Wirtschaft, (1991), Heft 1, S. 3-16.

WILLIS, Robert J. (1987): What have we learned from the Economics of the Family?, in: American Economic Review, Papers and Proceedings, Jg. 77 (1987), S. 68-81.

WINEGARDEN, C.R./KHOR, Lay Boon (1993): Undocumented Immigration and Income Inequality in the Native-Bom Population of the US: Econometric Evidence, in: Applied Economics, Jg. 25 (1993), Heft 2, S. 157-163.

WinkelmanN, Rainer/ZimmermanN, Klaus F. (1993): Ageing, Migration and Labour Mobility, in: JoHNSON, Paul/ZimMERMANN, Klaus F. (Hrsg.), Labour Markets in an Ageing Europe, Cambridge, 1993, S. 255-283.

WINTERSTEIN, Helmut (1991): Aussiedler - Auswirkungen auf das Soziale Sicherungssystem, in: SIKORA, Joachim (Hrsg.), Aussiedler als Herausforderung und Auftrag für die deutsche Gesellschaft, Bad Honnef, 1991, S. 113-147.

WISSENSCHAFTLICHER BEIRAT FÜR FAMILIENFRAGEN (1992): Zur Berechnung des steuerfreien Existenzminimums für den Lebensunterhalt eines Kindes (Schriftenreihe des Bundesministeriums für Familie und Senioren, Band 15), Stuttgart, Berlin, Köln, 1992.

WrTT, Ulrich (Hrsg.) (1990): Studien zur evolutorischen Ökonomik I, Berlin, 1990.

WOLLENSCHLÄGER, Michael (1994): Entwurf einer Einwanderungsverordnung für die Europäische Union, in: WEIDENFELD, Werner (Hrsg.), Das Europäische Einwanderungskonzept, Gütersloh, 1994, S. 184-192.

ZAMECK-GLYSCINSKI, Walburga v. (1985): Neoklassische Bevölkerungsökonomik, München, 1985.

ZIMMERMANN, Klaus F. (1985): Familienökonomie. Theoretische und empirische Untersuchungen zur Frauenerwerbstätigkeit und Geburtenentwicklung, Berlin u.a., 1985.

ZIMMERMANN, Klaus F. (1986): Die ökonomische Theorie der Familie, in: Felderer, Bernhard (Hrsg.), Beiträge zur Bevölkerungsökonomie, Berlin, 1986, S. 11-63. 
ZimmermanN, Klaus F. (1989): Die Konkurrenz der Genüsse: ein Brentano-Modell des Geburtenrückgangs, in: Zeitschrift für Wirtschafts- und Sozialwissenschaften, Jg. 109 (1989), Heft 3, S. 467-483.

ZimmeRMANN, Klaus F. (1991): Migration Pressure in Germany: Past and Future (Münchener wirtschaftswissenschaftliche Beiträge Nr. 91/24), München, 1991.

ZIMMERMANN, Klaus F. (1994a): Immigration Policies in Europe: An Overview, in: SieberT, Horst (Hrsg.), Migration: A Challenge for Europe, Tübingen, 1994, S. 227-258.

ZimmermanN, Klaus F. (1994b): Some General Lessons for Europe's Migration Problem, in: GIERSCH, Herbert (Hrsg.), Economic Aspects of International Migration, Berlin u.a., 1994, S. 249-271.

ZimmermanN, Klaus F. (1995): Tackling the European Migration Problem, in: Journal of Economic Perspectives, Jg. 9 (1995), Heft 2, S. 45-62. 


\section{FINANZWISSENSCHAFTLICHE SCHRIFTEN}

Band 1 Werner Steden: Finanzpolitik und Einkommensverteilung. Ein Wachstums- und Konjunkturmodell der Bundesrepublik Deutschland. 1979.

Band 2 Rainer Hagemann: Kommunale Finanzplanung im föderativen Staat. 1976.

Band 3 Klaus Scherer: Maßstäbe zur Beurteilung von konjunkturellen Wirkungen des öffentlichen Haushalts. 1977.

Band 4 Brita Steinbach: "Formula Flexibility" - Kritische Analyse und Vergleich mit diskretionärer Konjunkturpolitik. 1977.

Band 5 Hans-Georg Petersen: Personelle Einkommensbesteuerung und Inflation. Eine theoretisch-empirische Analyse der Lohn- und veranlagten Einkommensteuer in der Bundesrepublik Deutschland. 1977.

Band 6 Friedemann Tetsch: Raumwirkungen des Finanzsystems der Bundesrepublik Deutschland. Eine Untersuchung der Auswirkungen der Finanzreform von 1969 auf die Einnahmenposition der untergeordneten Gebietskörperschaften und ihrer regionalpolitischen Zieladäquanz. 1978.

Band 7 Wilhelm Pfähler: Normative Theorie der fiskalischen Besteuerung. Ein methodologischer und theoretischer Beitrag zur Integration der normativen Besteuerungstheorie in der Wohlfahrtstheorie. 1978.

Band 8 Wolfgang Wiegard: Optimale Schattenpreise und Produktionsprogramme für öffentliche Unternehmen. Second-Best Modelle im finanzwirtschaftlichen Staatsbereich. 1978.

Band 9 Hans P. Fischer: Die Finanzierung des Umweltschutzes im Rahmen einer rationalen Umweltpolitik. 1978.

Band 10 Rainer Paulenz: Der Einsatz finanzpolitischer Instrumente in der Forschungs- und Entwicklungspolitik. 1978.

Band 11 Hans-Joachim Hauser: Verteilungswirkungen der Staatsverschuldung. Eine kreislauftheoretische Inzidenzbetrachtung. 1979.

Band 12 Gunnar Schwarting: Kommunale Investitionen. Theoretische und empirische Untersuchungen der Bestimmungsgründe kommunaler Investitionstätigkeit in NordrheinWestfalen 1965-1972. 1979.

Band 13 Hans-Joachim Conrad: Stadt-Umland-Wanderung und Finanzwirtschaft der Kemstädte. Amerikanische Erfahrungen, grundsätzliche Zusammenhänge und eine Fallstudie für das Ballungsgebiet Frankfurt am Main. 1980.

Band 14 Cay Folkers: Vermögensverteilung und staatliche Aktivität. Zur Theorie distributiver Prozesse im Interventionsstaat. 1981.

Band 15 Helmut Fischer: US-amerikanische Exportförderung durch die DISC-Gesetzgebung. 1981.

Band 16 Günter Ott: Einkommensumverteilungen in der gesetzlichen Krankenversicherung. Eine quantitative Analyse. 1981.

Band 17 Johann Hermann von Oehsen: Optimale Besteuerung. (Optimal Taxation). 1982.

Band 18 Richard Kössler: Sozialversicherungsprinzip und Staatszuschüsse in der gesetzlichen Rentenversicherung. 1982.

Band 19 Hinrich Steffen: Zum Handlungs- und Entscheidungsspielraum der kommunalen Investitionspolitik in der Bundesrepublik Deutschland. 1983.

Band 20 Manfred Scheuer: Wirkungen einer Auslandsverschuldung des Staates bei flexiblen Wechselkursen. 1983. 
Band 21 Christian Schiller: Staatsausgaben und crowding-out-Effekte. Zur Effizienz einer Finanzpolitik keynesianischer Provenienz. 1983.

Band 22 Hannelore Weck: Schattenwirtschaft: Eine Möglichkeit zur Einschränkung der öffentlichen Verwaltung? Eine ökonomische Analyse. 1983.

Band 23 Wolfgang Schmitt: Steuern als Mittel der Einkommenspolitik. Eine Ergänzung der Stabilitätspolitik? 1984.

Band 24 Wolfgang Laux: Erhöhung staatswirtschaftlicher Effizienz durch budgetäre Selbstbeschränkung? Zur Idee einer verfassungsmäßig verankerten Ausgabengrenze. 1984.

Band 25 Brita Steinbach-van der Veen: Steuerinzidenz. Methodologische Grundlagen und empirisch-statistische Probleme von Länderstudien. 1985.

Band 26 Albert Peters: Ökonomische Kriterien für eine Aufgabenverteilung in der Marktwirtschaft. Eine deskriptive und normative Betrachtung für den Allokationsbereich. 1985.

Band 27 Achim Zeidler: Möglichkeiten zur Fortsetzung der Gemeindefinanzreform. Eine theoretische und empirische Analyse. 1985.

Band 28 Peter Bartsch: Zur Theorie der längerfristigen Wirkungen 'expansiver' Fiskalpolitik. Eine dynamische Analyse unter besonderer Berücksichtigung der staatlichen Budgetbeschränkung und ausgewählter Möglichkeiten der öffentlichen Defizitfinanzierung. 1986.

Band 29 Konrad Beiwinkel: Wehrgerechtigkeit als finanzpolitisches Verteilungsproblem. Möglichkeiten einer Kompensation von Wehrungerechtigkeit durch monetäre Transfers. 1986.

Band 30 Wolfgang Kitterer: Effizienz- und Verteilungswirkungen des Steuersystems. 1986.

Band 31 Heinz Dieter Hessler: Theorie und Politik der Personalsteuern. Eine Kritik ihrer Einkommens- und Vermögensbegriffe mit Blick auf die Leistungsfähigkeitstheorie. 1994.

Band 32 Wolfgang Scherf: Die beschäftigungspolitische und fiskalische Problematik der Arbeitgeberbeiträge zur Rentenversicherung. Eine Auseinandersetzung mit der Kritik an der lohnbezogenen Beitragsbemessung. 1987.

Band 33 Andreas Mästle: Die Steuerunion. Probleme der Harmonisierung spezifischer Gütersteuern. 1987.

Band 34 Günter Ott: Internationale Verteilungswirkungen im Finanzausgleich der Europäischen Gemeinschaften. 1987.

Band 35 Heinz Haller: Zur Frage der zweckmäßigen Gestalt gemeindlicher Steuern. Ein Diskussionsbeitrag zur Gemeindesteuerreform. 1987.

Band 36 Thomas Kuhn: Schlüsselzuweisungen und fiskalische Ungleichheit. Eine theoretische Analyse der Verteilung von Schlüsselzuweisungen an Kommunen. 1988.

Band 37 Walter Hahn: Steuerpolitische Willensbildungsprozesse in der Europäischen Gemeinschaft. Das Beispiel der Umsatzssteuer-Harmonisierung. 1988.

Band 38 Ulrike Hardt: Kommunale Finanzkraft. Die Problematik einer objektiven Bestimmung kommunaler Einnahmemöglichkeiten in der gemeindlichen Haushaltsplanung und im kommunalen Finanzausgleich. 1988.

Band 39 Jochen Michaelis: Optimale Finanzpolitik im Modell überlappender Generationen. 1989.

Band 40 Bernd Raffelhüschen: Anreizwirkungen der sozialen Alterssicherung. Eine dynamische Simulationsanalyse. 1989.

Band 41 Berend Diekmann: Die Anleihe- und Darlehenstransaktionen der Europäischen Gemeinschaften. 1990.

Band 42 Helmut Kaiser: Konsumnachfrage, Arbeitsangebot und optimale Haushaltsbesteuerung. Theoretische Ergebnisse und mikroökonometrische Simulation für die Bundesrepublik Deutschland. 1990. 
Band 43 Rüdiger von Kleist: Das Gramm-Rudman-Hollings-Gesetz. Ein gescheiterter Versuch der Haushaltskonsolidierung. 1991.

Band 44 Rolf Hagedorn: Steuerhinterziehung und Finanzpolitik. Ein theoretischer Beitrag unter besonderer Berücksichtigung der Hinterziehung von Zinserträgen. 1991.

Band 45 Cornelia S. Behrens: Intertemporale Verteilungswirkungen in der gesetzlichen Krankenversicherung der Bundesrepublik Deutschland. 1991.

Band 46 Peter Saile: Ein ökonomischer Ansatz der Theorie der intermediären Finanzgewalten Die Kirchen als Parafisci. 1992.

Band 47 Peter Gottfried: Die verdeckten Effizienzwirkungen der Umsatzsteuer. Eine empirische allgemeine Gleichgewichtsanalyse. 1992.

Band 48 Andreas Burger: Umweltorientierte Beschättigungsprogramme. Eine Effizienzanalyse am Beispiel des "Sondervermögens Arbeit und Umwelt". 1992.

Band 49 Jeanette Malchow: Die Zuordnung verteilungspolitischer Kompetenzen in der Europäischen Gemeinschaft. Eine Untersuchung aufgrund einer Fortentwicklung der ökonomischen Theorie des Föderalismus. 1992.

Band 50 Barbara Seidel: Die Einbindung der Bundesrepublik Deutschland in die Europäischen Gemeinschaften als Problem des Finanzausgleichs. 1992.

Band 51 Ralph Wiechers: Markt und Macht im Rundfunk. Zur Stellung der öffentlich-rechtlichen Rundfunkanstalten im dualen Rundfunksystem der Bundesrepublik Deutschland. 1992.

Band 52 Klaus Eckhardt: Probleme einer Umweltpolitik mit Abgaben. 1993.

Band 53 Oliver Schwarzkopf: Die Problematik unterschiedlicher Körperschaftsteuersysteme innerhalb der EG. 1993.

Band 54 Thorsten Giersch: Bergson-Wohlfahrtsfunktion und normative Ökonomie. 1993.

Band 55 Li-Fang Chou: Selbstbeteiligung bei Arzneimitteln aus ordnungspolitischer Sicht. Das Beispiel der Bundesrepublik Deutschland. 1993.

Band 56 Harald Schlee: Einkommensteuerliche Behandlung von Transferzahlungen. Zur Neuordnung der Familienbesteuerung sowie der Besteuerung von Versicherungsleistungen und Sozialtransfers. 1994.

Band 57 Alexander Spermann: Kommunales Krisenmanagement. Reaktionen baden-württembergischer Stadtkreise auf steigende Sozialhilfekosten und Einnahmenausfälle (198092). 1993.

Band 58 Otto Roloff / Sibylle Brander / Ingo Barens / Claudia Wesselbaum: Direktinvestitionen und internationale Steuerkonkurrenz. 1994.

Band 59 Claudia Wesselbaum-Neugebauer: Internationale Steuerbelastungsvergleiche. 1994.

Band 60 Stephanie Miera: Kommunales Finanzsystem und Bevölkerungsentwicklung. Eine Analyse des kommunalen Finanzsystems vor dem Hintergrund der sich abzeichnenden Bevölkerungsentwicklung am Beispiel Niedersachsens unter besonderer Berūcksichtigung des Landkreises Wolfenbüttel und seiner Gemeinden. 1994.

Band 61 Wolfgang Scherf: Die Bedeutung des kaldorianischen Verteilungsmechanismus für die gesamtwirtschaftlichen Wirkungen der staatlichen Neuverschuldung. 1994.

Band 62 Rainer Volk: Vergleich der Vergünstigungseffekte der verschiedenen investitionsfördernden Maßnahmen. 1994.

Band 63 Hans-Georg Napp: Kommunale Finanzautonomie und inre Bedeutung für eine effiziente lokale Finanzwirtschaft. 1994. 2., unveränderte Auflage 1994.

Band 64 Bernd Rahmann / Uwe Steinborn / Günter Vornholz: Empirische Analyse der Autonomie lokaler Finanzwirtschaften in der Europäischen Gemeinschaft. 1994. 
Band 65 Carsten Kühl: Strategien zur Finanzierung der Altlastensanierung. 1994.

Band 66 Stephan Boll: Intergenerationale Umverteilungswirkungen der Fiskalpolitik in der Bundesrepublik Deutschland. Ein Ansatz mit Hilfe des Generational Accounting. 1994.

Band 67 Karl Justus Bernhard Neumärker: Finanzverfassung und Staatsgewalt in der Demokratie. Ein Beitrag zur konstitutionellen Finanztheorie. 1995.

Band 68 Christian Haslbeck: Zentrale versus dezentrale Internalisierung externer Effekte bei unvollständiger Information. 1995.

Band 69 Regina Müller: Horizontale oder vertikale Transfers zur Durchsetzung eines horizontalen Finanzausgleichs. 1995.

Band 70 Christian Hockenjos: Öffentliche Sportförderung in der Bundesrepublik Deutschland. Darstellung und finanztheoretische Analyse. 1995.

Band 71 Manfred Rosenstock: Die Kontrolle und Harmonisierung nationaler Beihilfen durch die Kommission der Europäischen Gemeinschaften. 1995.

Band 72 Christian Rüsch: Wohnungsbau- und Wohneigentumspolitik im Rahmen der Einkommensteuer. Eine Analyse unter steuersystematischen, verteilungspolitischen und fiskalischen Aspekten. 1996.

Band 73 Stephan Winters: Die kollektive Vorsorge für den Pflegefall im Alter. Eine Untersuchung am Beispiel der gesetzlichen Pflegeversicherung in den Niederlanden. 1996.

Band 74 Knut Blind: Allokationsineffizienzen auf Sicherheitsmärkten: Ursachen und Lösungsmöglichkeiten. Fallstudie: Informationssicherheit in Kommunikationssystemen. 1996.

Band 75 Barbara Petrick-Rump: Ökonomische Wirkungen von Steueramnestien. Untersuchung konkreter Erfahrungen ausgewählter Länder mit dem Einsatz von Steueramnestien anhand eines effizienten Steueramnestieprogramms. 1996.

Band 76 Georg Hirte: Effizienzwirkungen von Finanzausgleichsregelungen. Eine Empirische Allgemeine Gleichgewichtsanalyse für die Bundesrepublik Deutschland. 1996.

Band 77 Ulrike Kirchhoff: Die rheinland-pfälzischen Gemeinden im System des Finanzausgleichs. 1996.

Band 78 Kerstin Keil: Der soziale Mietwohnungsbau: Mängel und Alternativen. 1996.

Band 79 Bernhard Manzke: Kinderlastenausgleich versus verstärkte Einwanderung. Alternative Ansätze zur langfristigen Sicherung der Gesetzlichen Rentenversicherung. 1997. 